\title{
DISCLAIMER
}

This report was prepared as an account of work sponsored by an agency of the United States Government. Neither the United States Government nor any agency thereof, nor any of their employees, makes any warranty, express or implied, or assumes any legal liability or responsibility for the accuracy, completeness, or usefulness of any information, apparatus, product, or process disclosed, or represents that its use would not infringe privately owned rights. Reference herein to any specific commercial product, process, or service by trade name, trademark, manufacturer, or otherwise does not necessarily constitute or imply its endorsement, recommendation, or favoring by the United States Government or any agency thereof. The views and opinions of authors expressed herein do not necessarily state or reflect those of the Utitited States Government or any agency thereof.

WSRC-TR- $-90-222$

DE92

015322 \section{F-AREA SEEPAGE BASINS, GROUNDWATER QUALITY
ASSESSMENT REPORT, FIRST QUARTER 1990 (U)}

This is an EPA/SCDHEC \& General Public

Publication Date: June, 1990 acknowledges the U.S. Government's right to retain of this paper, the publisher and/or recipient reproduce all covering this paper, along with the right to reproduc, royalty-free license in and to produce all or part of the copyrighted paper.

\section{MASIIER}

OISTRIBUTION OF TIII

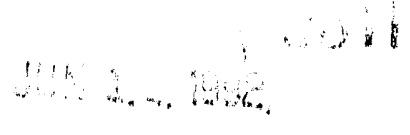




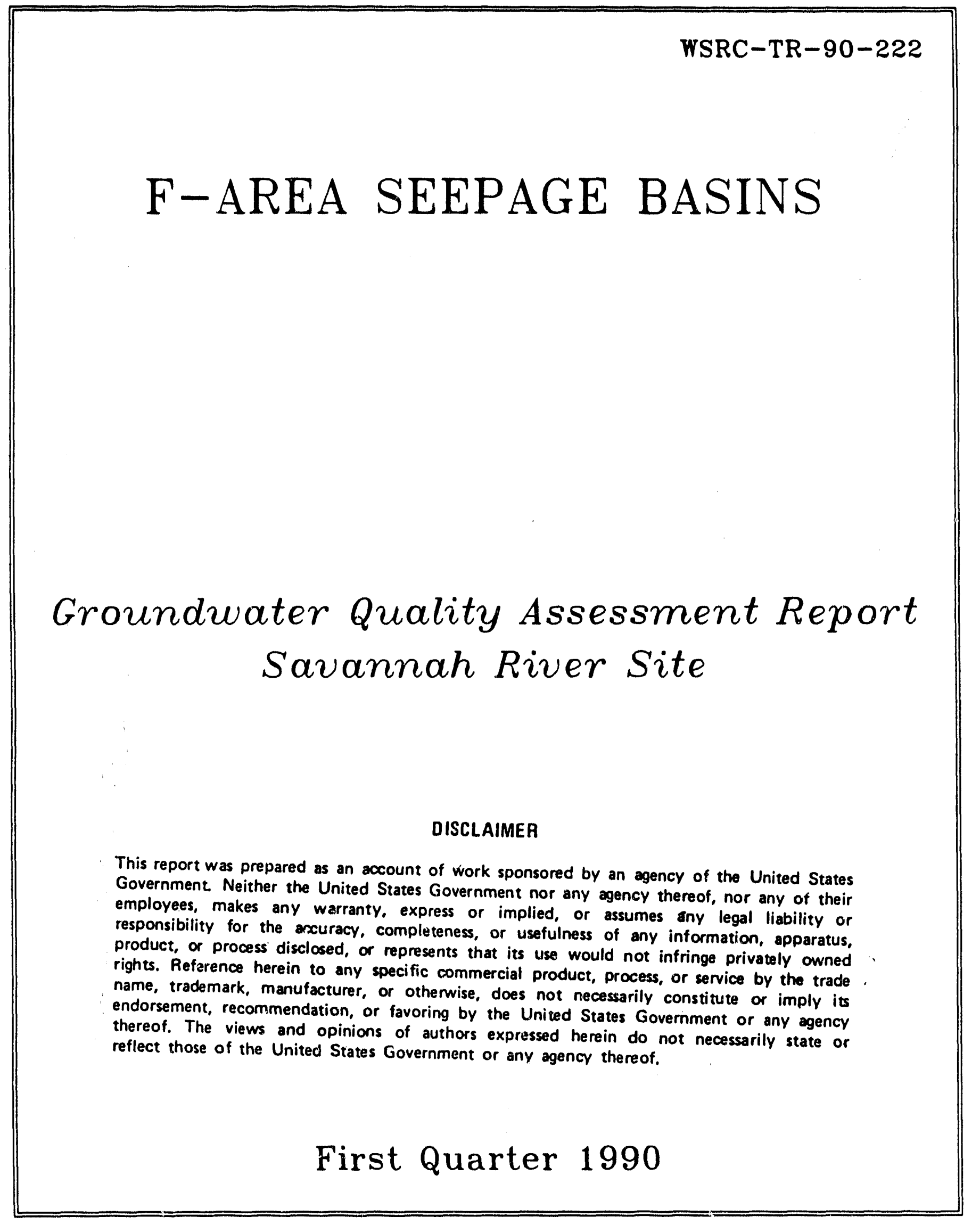




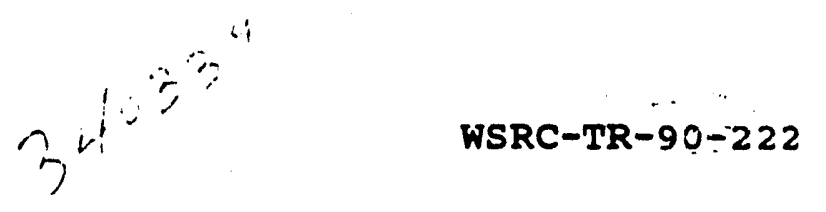

F-Area Seepage Basins (F-HWhF)

First Quarter 1990

Groundwater Quality Assessment Report (U)

Authorized Derivative Classifier:

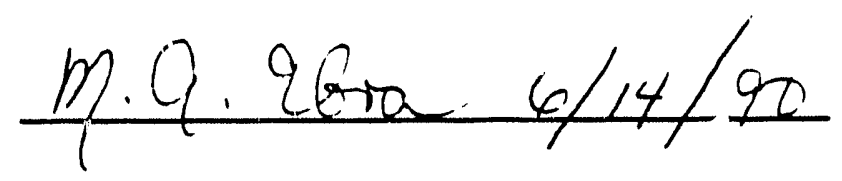

Publication Date: June 1990

Westinghouse Savannah River cert oo $\mathrm{m}_{\mathrm{g}}$

Savannah River ito Aiken, SC $2980 \%$ 
TABT: OF CONFENTY

$\begin{array}{lrl}\text { ABSTRACT } & \text { PAGE } 1 \\ \text { EXECUTIVE SUMMARY } & \text { PAGE } 2 \\ \text { HYDROLOGIC UNITS AND } & \\ \text { STRATIGRAPHIC UNITS } & \text { PAGE } 3 \\ \text { FACILITY DESCRIPTION } & \text { PAGE } 4 \\ \text { MONITORING WEIL NETWORK } & \text { PAGE } 4 \\ \text { MONITORING DATA } & \text { PAGE } 6 \\ \text { WATER LEVELS } & \text { PAGE } 9 \\ \text { FLOW RATES AND DIRECTIONS } & \text { PAGE } 9 \\ \text { NITRATE TIME TREND DATA } & \text { PAGE } 10 \\ \text { TRITIUM TIME TREND DATA } & \text { PAGE } 11 \\ \text { CONCLUSIONS } & \text { PAGE } 11 \\ \text { TABIEB } & & \\ \text { TABLE } 1 & \text { FIOW RATE CALCULATIONS } & \text { PAGE } 29 \\ \text { TABLE 2 } & \text { SCHEDULE FOR REHABILITATION } & \\ & \text { PAGE } 30\end{array}$

APPENDICES

APPENDIX A

TABLES OF MONITORING DATA

APPENDIX B

MAPS OF CONSTITUENTS

APPENDIX C

HYDROGRAPHS

APPENDIX D

NITRATE TIME TREND PLOTS

APPENDIX E

TRITIUM TIME TREND PLOTS

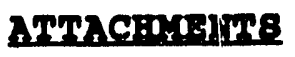

ATTACHMENT 1

WELL REHABILITATION PLAN OF THE MONITORING WELL NETWORK

ATTACHEMENT 2 STATUS OF THE REHABILITATION and ABANDONMENT/REPLACEMENT PLANS 


\begin{tabular}{|c|c|c|c|}
\hline FIGURE & 1 & HYDROLOGIC UNITS & $\frac{\text { PAGE }}{12}$ \\
\hline FIGURE & 2 & WELL LOCATIONS & 13 \\
\hline FIGURE & 3 & WATER TABIE WELIS & 14 \\
\hline FIGURE & 4 & MCBEAN WELIS & 15 \\
\hline FIGURE & 5 & UPPER CONGAREE WELLS & 16 \\
\hline FIGURE & 6 & IOWER CONGAREE WELIS & 17 \\
\hline FIGURE & 7 & WATER TABLE ELEVATION MAP & 18 \\
\hline FIGURE & $\mathbf{8}$ & MCBEAN POTENTIOMETRIC SURFACE & 19 \\
\hline FIGURE & 9 & UPPER CONGAREE POTENTIOMETRIC SURFACE & 20 \\
\hline FIGURE & 10 & LOWER CONGAREE POTENTIOMETRIC SURFACE & 21 \\
\hline FIGURE & 11 & FLOW RATES IN THE WATER TABLE & 22 \\
\hline FIGURE & 12 & FLOW RATES IN THE MCBEAN & 23 \\
\hline FIGURE & 13 & FLOW RATES IN THE UPPER CONGAREE & 24 \\
\hline FIGURE & 14 & FLOW RATES IN THE IOWER CONGAREE & 25 \\
\hline FIGURE & 15 & WATER ELEVATION CROSS-SECTION & 26 \\
\hline FIGURE & 16 & NITRATE CROSS-SECTION & 27 \\
\hline FIGURE & 17 & CROSS SECTION LOCATION & 28 \\
\hline
\end{tabular}




\section{AB8TRACY}

During the first quarter of 1990, wells which make up the F-Area Seepage Basins ( $F$-HWMF) ${ }^{1}$ monitoring network were sampled. Laboratory analyses were performed to measure levels of hazardous constituents, indicator parameters, tritium, gross alpha, and nonvolatile beta. The primary contaminants observed at wells monitoring the F-Area Seepage Basins are tritium, nitrate, cadmium, lead, total radium, gross alpha, and nonvolatile beta.

Concentrations of at least one of the following constituents: tritium, nitrate, total radium, gross alpha, cadmium, lead, tetrachloroethylene, nonvolatile beta, endrin, lindane, barium, fluoride, mercury, and trichloroethylene in excess of the primary drinking water standard (PDWS) were observes in at least one well monitoring the F-Area seepage Basins. Tritium concentrations above the PDWS occur in fortyfour of the fifty-nine (758) groundwater monitoring wells. Nitrate concentrations above the PDWS occur in thirty-four of the fifty-nine (598) groundwater wells. The radionuclides, total radium, gross alpha, and nonvolatile beta, exceed the PDWS in over twenty-five percent of the groundwater wells. Heavy metals, cadmium and lead in particular, exceed the PDWS in over twelve percent of the wells. Since 1987, tritium and nitrate concentrations have been steadily declining in a majority of the wells. However, tritium concentrations, from fourth quarter 1989 to first quarter 1990, have increased.

1 F-HWMF is the acronym for "F-Area Hazardous Waste Management Facility". 
F-Area Seepage Basins

First Quarter 1990

Groundwater Quality Assessment Report

Page 2

June 14,1990

\section{Excourtos 80uangy}

During the first quarter of 1990 wells which make up the F-Area Seepage Basins (F-HWMF) monitoring network were sampled. Laboratory analyses were performed to measure levels of hazardous constituents, indicator parameters, tritium, gross alpha and nonvolatile beta. A Gas Chromatograph Mass spectrometer (GCMS) scan was performed on all well samples to determine any hazardous organic constituents present in the groundwater. Notable events are as follows:

The primary contaminants that exceed the PDWS observed at wells monitoring the F-Area seepage basins are tritium, nitrate, cadmium, lead, total radium, gross alpha, and nonvolatile beta. A plume of tritium and nitrate extends south from the seepage basins to the seepline at Four Mile creek in the Water Table and McBean hydrologic units although the plume of contaminated water is mainly contained in the Water Table and McBean hydrologic units. Tritium concentrations ranging from one to twenty-five times the PDWS occur in $s i x$ out of ten of the Upper Congaree groundwater wells. Tritium concentrations in the Iower congaree groundwater wells are below the primary drinking water standard except for HSB 87A which exhibits a concentration of $20.7 \mathrm{pCi} / \mathrm{ml}$.

The spatial occurrence of hazardous metals above the primary drinking water standard is restricted as compared to that of nitrate and tritium. The primary metal constituent observed is cadmium. In the first quarter of 1990 seven wells exhibited levels of cadmium in excess of the primary drinking water standard. of those wells, three are water table wells and four are screened in the McBean. Only one well (FSB 105D) has pumped dry prior to collecting a representative sample. This well, along with FSB 98A, 95C, and 94D, are slated for abandonment/replacement later this year. Table two gives a brief synopsis of the rehabilitation and replacement plans.

Meásurements of elevated levels of organic constituents appear to be spurious. Levels of trichloroethylene (TCE) above the primary drinking water standard were reported in one well during the first quarter of 1990. Elevated levels of endrin and lindane concentrations were also exhibited in two wells (FSB 76 and 79B). During 1989, there were sporadic occurrences of TCE concentrations in the groundwater monitoring well network. No other organic constituents were detected until the first quarter of 1990 with endrin and lindane. Based on these observations, measurements of organic constituents seem to be sporadic and it is expected that 
F-Area Seepage Basins

First Quarter 1990

Groundwater Quality Assessment Report

Page. 3

June 14,1990

continued sampling and analysis will demonstrate that there are no hazardous organic contaminants in groundwater downgradient of the F-Area seepage basins.

A proposal to drill fifty-one new plume assessment wells at the $F$ and $H$ Area Seepage Basins has been approved. Installation of the new groundwater monitoring wells has begun.

\section{IXYRODUCTIOU}

\section{HYDROIOGIC UNITS AND STRATIGRAPHIC UNITS}

Four distinct hydrogeologic zones are monitored by the FArea Seepage Basins (F-HWMF) well notwork. Historically Ground Water Quality Assessment Reports for facilities in the General Separations Areas (which include the Mixed Waste Management Facility [MWKF] and the $F$ and $H$ area seepage basins) have used the names, "Water Table", "McBean", and "Upper and Lower Congaree" as hydrologic unit names. In order to preserve continuity in the groundwater quality assessment report for the F-HWWF these same names are used in confunction with the most recently adopted nomenclature for hydrologic units at SRS. Figure 1 is a simplified correlation chart showing the commonly used names for water bearing units in the General Separations Areas as well as the SRS official hydrologic unit designations and their correlation to stratigraphic units of the South Carolina coastal plain. The intent of using a hydrologic zone nomenclature is to reduce confusion between the concepts of lithostratigraphic and hydrostratigraphic units, and to make SRs nomenclature consistent with that of the regional geology.

The RCRA Part B Post Closure permit application Volume V includes a detailed description of the geology and hydrogeologic regime at the F-Area seepage basins. In summary, four hydrogeologic zones are monitored in the uppermost aquifer. The uppermost zone 18 comprised of the water Table which occurs in zone $7 \mathrm{C}$ and is supported by the Tan clay. Below the Tan clay is the McBean unit which contains some carbonate (zone 6). Underlying the McBean is the Green Clay which separates the McBean from the gemiconfined Upper and Iower Congaree (zone 5) units. Below the Congaree is the Ellenton formation. The base of the uppermost aquifer is defined as the uppermost confining unit of the Ellenton formation which lies approximately 300 feet below the facility. 
F-Area Seepage Basins

First Quarter 1990

Groundwater Quality Assessment Report

Page 4

June 14, 1990

\section{FACILITY DESCRIPTION}

Use of the $F$ Area Seepage Basins for the disposal of waste water was discontinued on November 7,1988 . The basins are currently being prepared for closure under RCRA. Closure plans include the emplacement of a low permeability cap with a vegetative cover; the Post-closure care package is being prepared. The Part B application which was submitted in April 1988 proposed alternate concentration limits (ACL) for hazardous constituents in lieu of groundwater remediation. The ACL demonstration has since been denied and SRS is conducting an extensive hydrogeologic investigation and research of remediation techniques in order to prepare a groundwater remediation program at the F-Area seepage Basins.

\section{DI8C088I0y}

\section{INTEGRITY OF THE MONITORING WELC NETWORK}

The F-Area Seepage Basins (F-HWMF) groundwater monitoring well network is intended to provide representative samples of the groundwater in the four hydrologic units which are monitored in the uppermost aquifer below the facility. Anomalous values of water elevation and $\mathrm{pH}$ were recorded at some wells during the round of sampling which directly followed installation. Initially it was speculated that these anomalous measurements were the result of the newness of the wells. It was supposed that the screens were clogged with residual drilling mud frow well construction and would be cleared after several sampling episodes. After several months, representative samples were expected to be obtained from the wells. However, problems with some wells still exist after more than a year of sampling.

A program is in place to rehabilitate and replace wells which do not produce representative sanples from the units being monitored. A brief overview of the problems and intended corrective actions follows:

-Three wells have exhibited unusually high pH values through more than four quarters of monitoring. They are FSB 95C, 96A, and 98A. Unusually high $\mathrm{pH}$ and specific conductance values are associated with grout contaminating the sample water. These wells have been slated for abandonment/replacement since the attempt to rehabilitate these wells was unsuccessful. 
-There are twenty three Water Table wells, two of which (FSB 87D and 94D) have been reported as dry for the past several quarters: One well (FSB 76), yields a sample, but no water level measurement. All of these wells appear to be screened such that part of the screen is below the phreatic surface of the water table. Water level measuring pipes are typically set so that the bottom of the pipe rests on the top of the pump. It is possible for the pump intake to be submerged while the top of the pump is dry, thus explaining how a well with no water level will sometimes yield a watur sample. There is in program ongoing to attempt to lower the pumps in these two wells. In FSB 87D, the pump was successfully lowered, ard the well will now yield a groundwater sample. This was done after sampling for first quarter 1990 was completed; therefore, there are no data for FSB 87D for first quarter 1990. The pump was lowered in FSB 94D but it $5^{+-11}$ yields no groundwater sample and has therefore been slaced for replacement.

-Six of the water Table wells (FSB 90D, 93D, 97D, 105D, 108D, and 109D) pumped dry prior to collecting a representative sample. These same wells have also pumped dry over previous quarters. of the six Water Table wells that pumped dry, one well (FSB 105D) displayed values of cadmium above the primary drinking water standard. Similarly, that same well (FSB 105D) displayed lead above the primary drinking water standard. Mercury concentrations above the primary drinking water standard were exhibited in two wells (FSB 78 and 89D). Barium values exceeded the PDWS consistently for two quarters and during the first quarter of 1990 in one well (FSB 98D). The last time that elevated levels of barium were detected was during the firet quarter of 1989 in two different wells (FSB 90D, 98A) and in the same well (FSB 98D) that exhibits elevated barium levels in the first quarter of 1990 .

-There are twenty-three MCBean wells of which five (FSB 90C, 91C, 94C, 95C, and 97C) pumped dry before a representative sample could be collected. of the five McBean wells that pumped dry, one (FSB 97C) had values for cadmium and barium above the PDWS.

-There are fourteen, ten Upper and four Lower, Congaree wells, three of which (FSB 96A, 98A, and 100A) pumped dry prior to collecting a representative sample. None of the Congaree wells exhibited heavy metals above the prima $y$ drinking water standard. FSB $98 \mathrm{~A}$ is slated for abandonment/replacement since attempts to rehabilitate it were unsuccessful. FSB 96A and $100 A$ were successfully rehabilitated prior to sampling for first quarter 1990 groundwater assessment. 


\section{MONITORING DATA}

The F-Area Seepage Basins (F-HWWF) groundwater quality data include field and $1 \mathrm{ab}$ analysis data from 59 monitoring wells sampled during the first quarter of 1990. These wells monitor the four hydrogeologic zones which comprise the uppermost aquifer. A map lindicating the locations of the 28 well clusters monitoring the F-Area seepage basins is shown in Figure 2. The location of wells in each hydrologic zone are indicated in Figures $3-6$. All field and analytical data collected at these wells during the first quarter of 1990 are provided in the Tables in Appendix $A$. Maps indicating the lateral distribution of measured values of $\mathrm{pH}$, conductivity, nitrate, lead, cadmium and tritium are provicied in Appendix $B$.

Flow in the Water Table aquifer is to the southwest towards Four Mile Creek. As indicated on the water table surface elevation map, wells FSB 76, FSB 99D, 108D, and 109D monitor upgradient water quality in the Water Table aquifer (Figures 3 and 7). All remaining Water Table wells are downgradient and monitor downgradient water quality. Flow in the MCBean is also southwest towards Four Mile Creek as shown by the potentiometric surface map (Figure 8 ). There is one well which monitors upgradient water quality in the McBean, FSB 76C. All of the other MCBean wells monitor downgradient water quality.

Flow in the Upper and Lower Congaree is to the northwest towards Upper Three Runs. Unlike the shallower units, the Congaree is not incised by Four Mile Creek, but is incised by Upper Three Runs, so Congaree flow directions are affected by Upper Three Runs but not Four Mile creek. Upgradient wells for the Upper Congaree are FSB 96A, 78B, and 79B. For the Lower Congaree the upgradient wells are FSB 78A, and FSB 79A. Wells between the basins and the seepline are upgradient of the facility, but way not represent "background" water quality because of the downward flow potential from the contaminated shallower units, into the conjaree. Figures F-9 and F-10 depict the potentiometric surfaces of the Upper and Lower Congaree, respectively.

Monitoring wells at the F-Area Seepage Basins (lp-HWMF) displayed at least one of the contaminants tritium, nitrate, gross alpha, lead, cadmium, fluoride, mercury, barium, total radium, trichloroethylene, lindane, and endrin in excess of the primary drinking water standards during first quarter 1990. 
For wells screened in the Water Table and McBean units, elevated levels of tritium, nitrate, gross alpha, cadmium, and total radium are consistently present in downgradient wells as opposed to upgradient wells.' For first quarter of 1990 at least one measurement from samples taken from the following wells exceeded the primary drinking water standard for the indicated constituents:

\section{FIRST QUARTER 1990}

A) TRITIUN: $F S B 76,77,78,78 \mathrm{~B}, 78 \mathrm{C}, 79,79 \mathrm{C}, 87 \mathrm{~A}, 87 \mathrm{~B}$ $87 \mathrm{C}, 88 \mathrm{C}, 88 \mathrm{D}, 89 \mathrm{C}, 89 \mathrm{D}, 90 \mathrm{C}, 90 \mathrm{D}, 91 \mathrm{C}, 91 \mathrm{D}, 92 \mathrm{D}$, 93C, 93D, 94C, 95C, 97A, 97C, 97D, 98A, 98C, 98D, 99A, 99C, 99D, 100A, 102C, 103C, 104C, 104D, 105C, 105D, 106C, 107C, 107D, 109D, 110C. 110D.

B) NITRATE: FSB 76, 76C, 77, 78, 78C, 79, 79C, 87C, 88D, 89D, 90C, 90D, 91C, 91D, 92D, 93C, 93D, 94C, 95C, 97A, 97C, 97D,98C, 98D, 99C, 102C, 103C, 104C, $104 \mathrm{D}, 105 \mathrm{C}, 105 \mathrm{D}, 106 \mathrm{C}, 107 \mathrm{C}, 107 \mathrm{D}, 110 \mathrm{C}$.

C) GROSS ALPHA: FSB 77, 78, 79,79C, 88D, 89D, 92D, 93D, 94C, 97C, 97D, 98A, 98C, 98D, 99D, 105C, 105D, 110D.

D) LEAD: $\quad$ FSB 76, 88D, 90D, 93D, 97C, 97D, 98D, $105 \mathrm{D}$.

E) TOTAL RADIUM: FSB 77, 79, 79C, 89D, 91D, 92D, 93D, 97C, 97D, 98A, 98C, 98D, 105D, 110D.

F) CADMIUK: FSB 78C, 79C, 91D, 92D, 105C, 105D, 106C.

G) BARIUY: FSB 97C, 98D.

H) FIUORIDE: FSB 98A, 101A.

I) MERCURY: FSB 78, 89D.

J) NONVOLATILE BETA: FSB 77, 78,78C, 79, 79C, 88D, 89D, 90D, 91C, 91D, 92D, 93D, 94C, 95C, 97D, 98C, 98D, 104D, 105C, 105D, 107C, 110D.

K) TRICHLOROETHYLENE: FSB 76B.

L) ENDRIN: FSB 76, 79B.

M) LINDANE: FSB 76, 79B.

N) TETRACHLOROETHYLENE: FSB 88C, 89C, 90D. 
In general, wells which exhibit constituents above the PDWS are downgradient of the seepags basins. Most of the contamination is found in the Water Table and McBean zones indicating that the horizontal component of groundwater flow is the dominant component for this particular site.

Heavy metal concentrations were exhibited in over twelve percent of the lls monitoring the 8 -Area Seepage Basins. In general, cadmium, barium, and lead, tend to occur togethener in wells which provide very little water and sporadicaliy in wells which are more productive. For instance, in FSB 97C, only thirty-two gallons of water were evacuated from the well before sampling, therefore, a representative sample colld not have been taken, and it exceeded the PDWS for lead and barium. Wells which aze screened in clayey zones (the water table aquifer resides in clayey zones in some locations) seerps to be associated with some of the elevated levels of these constituents; this may be the effect of low $\mathrm{pH}$ water from the basins leaching metals from the native clays and drill:-ng mud. Mercury was exhibited in two wells (FSB 78 and 89D). Fhe concentrations for each were 1,5 times and 3 times the PDWS, respectively.

Tritium concentrations above the PDWS were axhibit:ed in forty-five of the fifty-nine (75\&) groundwater wells. of those forty-five groundwater wells, eighteen were water Table wells, twenty were McBean wells, six wire Upper Congarase wells, and one was a Lower Congaree well. Since 1987, tritium concentrations have been decreasing, however, comparing first guarter 1990 data to fourth quarter 1989 data, the majority of the wells have increased in concentrations. More than half of the Upper Congaree wells show tritium concentrations gj:eater than the primary drinking water standard. One well (F\$B 98A) has consistently exhibited elevated levels of total radium.

Nitrate concentrations above the PDWS were exhibit:ed in thirty-four of the fifty-nine (59\%) groundwater wells. of the thirty-four groundwater wells, fifteen were Water Tabla, eighteen were McBean, and one was Upper Congaree. Ovejall, nitrate concentrations have decreased but there are fe's clear trends. There are a few wells in which nitrate conccnt:rations have increased (e.g., FSB 98C).

Radionuclides (gross alpha, nonvolatile beta, and total radium) concentrations above the primary drinking water: standard were exhibited in over twenty-five percent of the wells monitoring the groundwater in the F-Area Seepage Basins.

Three organic contaminants (trichloroethylene (TCls), endrin, and lindane) were exhibited in first quarter o: 1990. 
Levels of TCE above the PDWs were detacted in one well (FSB 76B) white lindane and endrin levele exceeded the PDWS in two wells (FSB 76 and 79B). Elevated TCE concentrations occurred sporadically throughout 1989 while concentrations of lindane and endrin were not detected above the PDWS in 1989. These measurements of organic contaminants seem to be unreliable and continual sampling and analysis is expected to demonstrate that there are no hazardous organic contaminants in the groundwater at the F-Area Seepage Basins.

\section{WATER IEVELS}

Hydrographs showing the water elevations through time for each well cluster are provided in Appendix C. In general, water levels in the Water Table unit, have allen an average of $0.52 \mathrm{ft}$. since the fourth quarter 0.21989 . Water levels in the McBean have fallen an average of $0.24 \mathrm{ft}$. and water levels in the congaree have risen an average of 0.14 feet since the fourth quarter of 1989.

There is a consistent vertical head relationship between the hydrologic zones mnnit rored at the F-area Seepage Basins. Flow potential is downward from the Water Table unit (zone 7C) to the McBean (zone 6 ), and downward from the McBean to the Congaree (zone 5). A head reversal exists between the Lower and Upper Congaree at the $\mathbf{F}$ seepage basins which indicates the potential for upward flow between those two units. This vertical head relationship between the different monitored zones remains essentially unchanged through the past several quarters.

\section{GROUNDWATER FIOW RATES AND DIRECTIONS}

Groundwater flow directions in the vicinity of the F-Area seepage basins in the Water Table, (zone 7C), McBean (zone 6) and Upper and Lower Congaree (zone 5) have remained relatively unchanged by closure activities. Potentiometric surface maps (Figures 7 - 10) for all four monitored zones illustrate these flow patterns. Flow in the water Table and McBean is generally southwest toward Four Mile creek. Flow in the Congaree is northwest directed towards Upper Three Runs.

In order to estimate the maximum transport rate of any potential contamination originating in the $F-H W M F$, the maximum horizontal flow rate of groundwater has been estimated for each zone monitored. Flow rate calculations are 
F-Area Seepage Basins

Firet Quarter 1990

Groundwater Quality Assessment Report

Page 10

June 14, 1990

approximations based on inferred or estimated parameters; the resulting rates shuuls be considered order of magnitude estimations. These estimations are based on the simple onedimensional flow equation: Flow rate $=($ hydraulic conductivity) $\times($ gradient $) /$ effective
porosity

Gradients input to the equation have been inferred from the water table and potentiometric surface maps. The arrows in Figures 11 - 14 indicate the locations that correspond to the flow estimations listed in Tab).e F-1. The gradient in each calculation represents the change in head divided by the horizontal distance along each arrow.

The value of effective porosity (20\%) used in each case is that cited in the Environmental Information Document: $F-$ Area Se page Basing (DPST-85-704). This value is an assumed conservative estimate.

The values of hydraulic conductivity (Kh) of each zone have been taken from the most recent groundwater modeling work in the area of the F-HWMF, (Evaluation of Integrated Waste Facility closure capping on Groundwater Flow and solute Transport in General Separations Area savannar River site-Elow Model and Particle Tracking Analysis: Draft Report, prepared by Geraghty-Miller Modeling Group, May 31, 1989.) Hydraulic conductivity values used in the calculations are as follows:

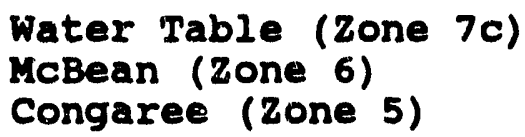

Maximum groundwater flow rates in the water table and McBean are estimated to be approximately 50 feet per year with a range of $43-93 \mathrm{ft} / \mathrm{yr}$.. Flow rates in the upper and lower Congaree are estimated in the range of $270 \mathrm{ft} / \mathrm{Yr}$ and 166 $\mathrm{ft} / \mathrm{yr}$, respectively.

NITRATE TIME TREND DATA

Graphs of the levels of nitrate concentrations measured at each well since the first quarter of 1987 to the present are presented in Appendix D. Most of the plots show no clear trend with time. However some wells do show a trend in nitrate concentrations. For example, FSB $98 C(D-15)$ shows a 
systematic increase in nitrate in the McBean unit with time. The highest values of nitrate are observed in the Water Table.

\section{TRITIUM TIKE TREND DATA}

Graphs of the levels of tritium concentrations measured at each well since the first quarter of 1987 to the present are presented in Appendix $\mathrm{E}$. Most of the plots show no clear trend with time. However, some wells do show a trend (e.g., FSB 76). A majority of the wells exhibiting tritium concentrations have increased since the fourth quarter 1989. The highest value of tritium is in the Water Table at FSB 78 with $44,800 \mathrm{pCi} / \mathrm{ml}$.

\section{CONCLU8I0:8}

Concentrations of tritium and nitrate in excess of the primary drinking water standard were observed in over thirty four wells. Also concentrations of total radium, gross alpha, and nonvolatile beta in excess of the PDWS were observed in over fifteen wells. Trichloroethylene concentrations above the PDWS were not observed in the fourth quarter 1989 but were observed in one well (FSB 76B) in the first quarter 1990. The extent of hazardous constituents in the groundwater is restricted, as compared to the plumes of tritium and nitrate present (Appendix B). Some wells are exhibiting values of barium, cadmium, and lead above the PDWS when the wells pump dry during purging, and therefore a representative sample could be collected. This has been a continuous trend over the year of 1989 and into 1990. There is a program plan in progress to abandon/replace these wells if rehabilitation is unsuccessful. Attached at the end of the report is a listing the status of the rehabilitation and abandonment/replacement programs. Water elevations for the first quarter have decreased in the Water Table and McBean units, while in the Congaree it has risen. A RCRA post-closure permit application is being prepared by SRs which will propose a plan to remediate groundwater contamination at the F-Area seepage basins. 


\begin{tabular}{|c|c|c|c|}
\hline FORYER NAME & ZONE 8 & & UPLAND TOBACCO ROAD \\
\hline TAN CLAY & \multirow{2}{*}{ ZONE 7} & $\frac{7 C}{78}$ & \multirow[t]{2}{*}{ DRY BRANCH } \\
\hline BARNWELL & & $7 A$ & \\
\hline McBEAN & \multicolumn{2}{|l|}{ ZONE 6} & \multirow{2}{*}{ SANTEE } \\
\hline GREEN CLAY & \multirow{2}{*}{ ZONE 5} & $5 B$ & \\
\hline CONGAREE & & $5 A$ & CONGAREE \\
\hline \multirow[t]{2}{*}{ ELLENTON } & \multirow[t]{2}{*}{ ZONE 4} & & \multirow[t]{2}{*}{ WILLIAKSBURG } \\
\hline & & & \\
\hline \multirow[b]{2}{*}{$\begin{array}{c}\text { UPPER } \\
\text { TUSCALOSSA } \\
\text { AQUIFER }\end{array}$} & \multirow[b]{2}{*}{ ZONE 3} & 38 & \multirow[b]{2}{*}{$P E E \quad D E E$} \\
\hline & & 3A & \\
\hline MIDDLE T. CONF. UNIT & \multirow{4}{*}{ ZONE 2} & $2 C$ & \multirow[t]{2}{*}{ BLACK CREEK } \\
\hline \multirow{2}{*}{$\begin{array}{c}\text { LOWER } \\
\text { TUSCALOOSA } \\
\text { AQUIFER }\end{array}$} & & 2B & \\
\hline & & \multirow[b]{2}{*}{ 2A } & \multirow[b]{2}{*}{ MIDDENDORF } \\
\hline & & & \\
\hline $\begin{array}{c}\text { BASAL } \\
\text { CONFINING } \\
\text { UNIT }\end{array}$ & \multirow[t]{2}{*}{ ZONE ! } & & $C A P E F E A R$ \\
\hline & & & $B A S E M E N T$ \\
\hline
\end{tabular}

FIGURE 1: Hydrologic Units and Stratigraphic Units 


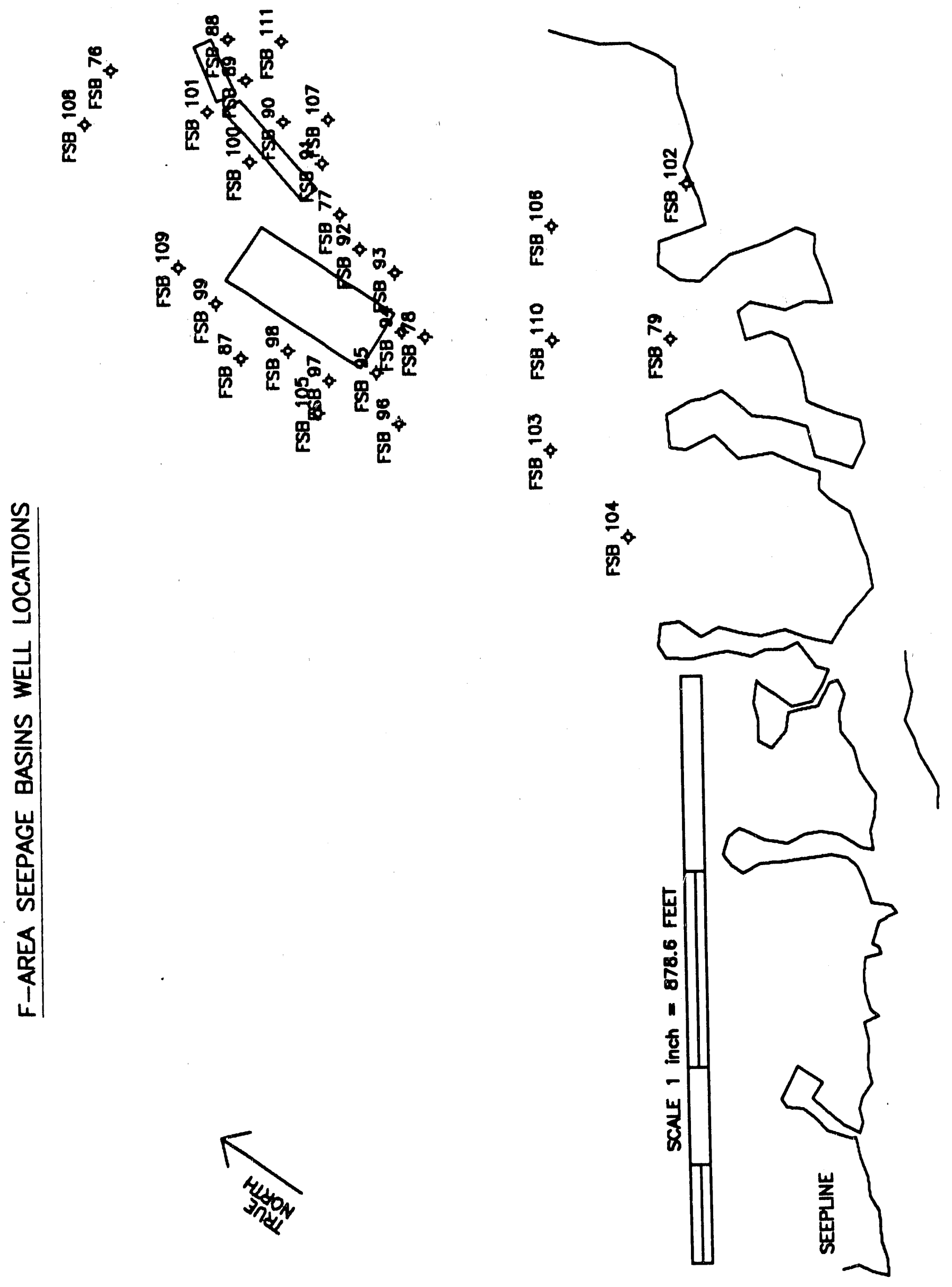



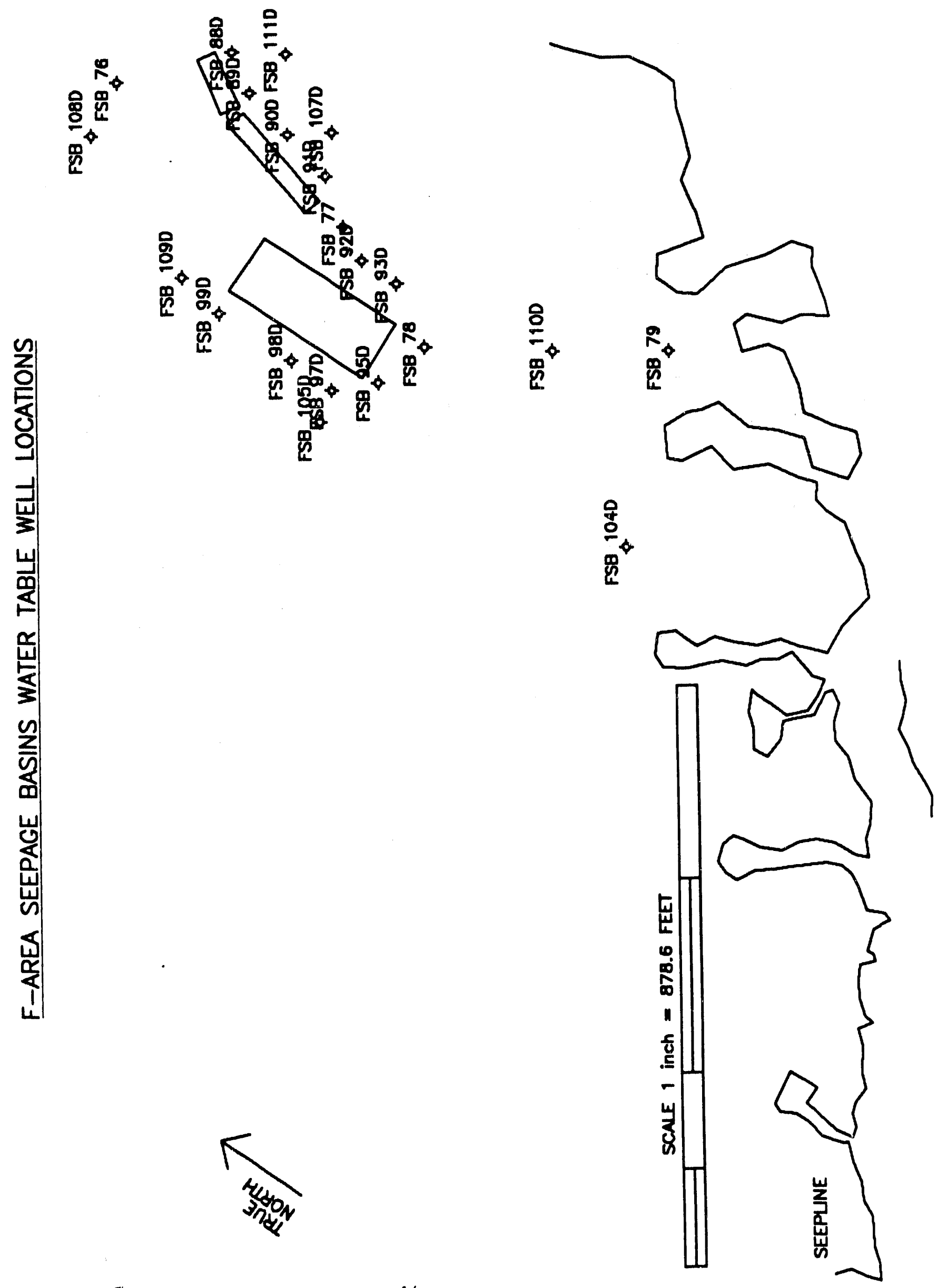


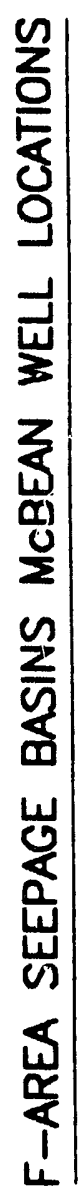
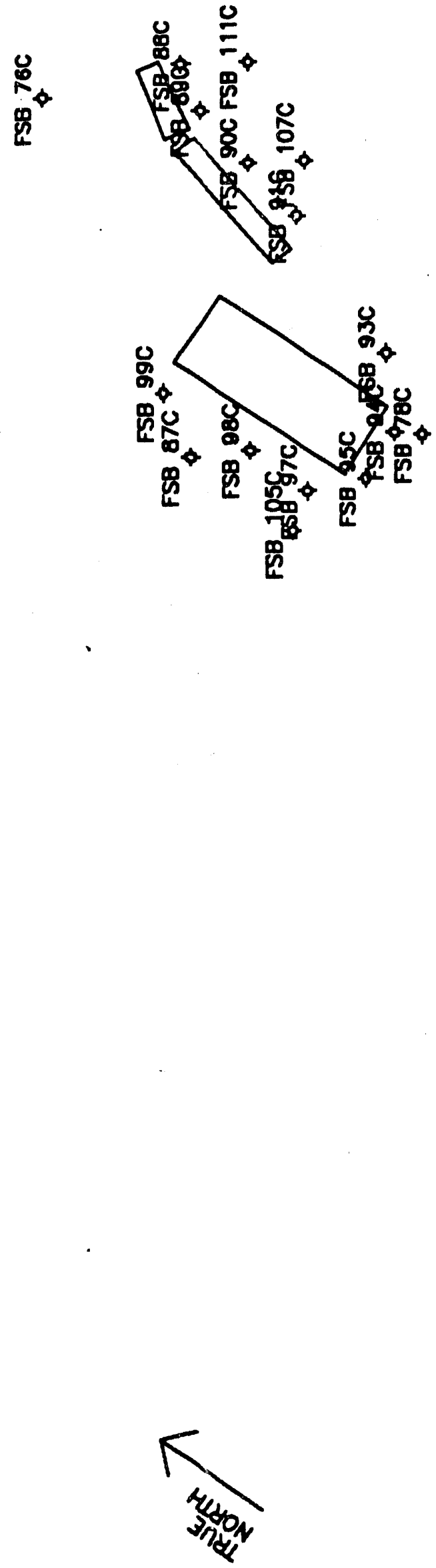

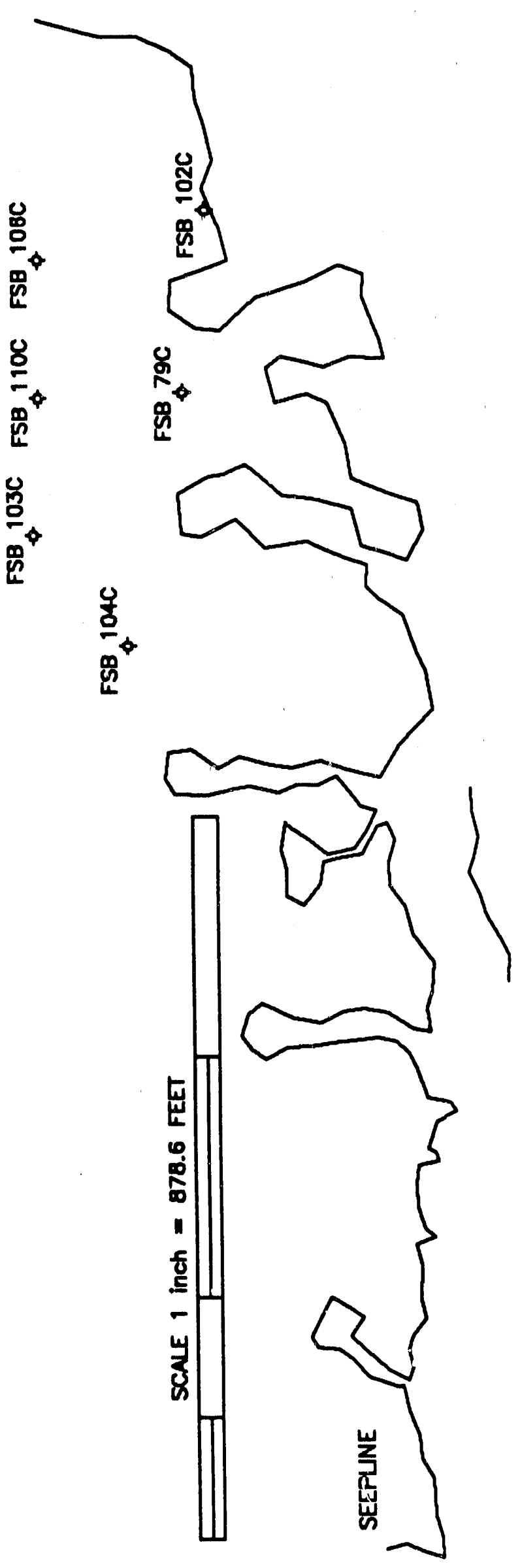




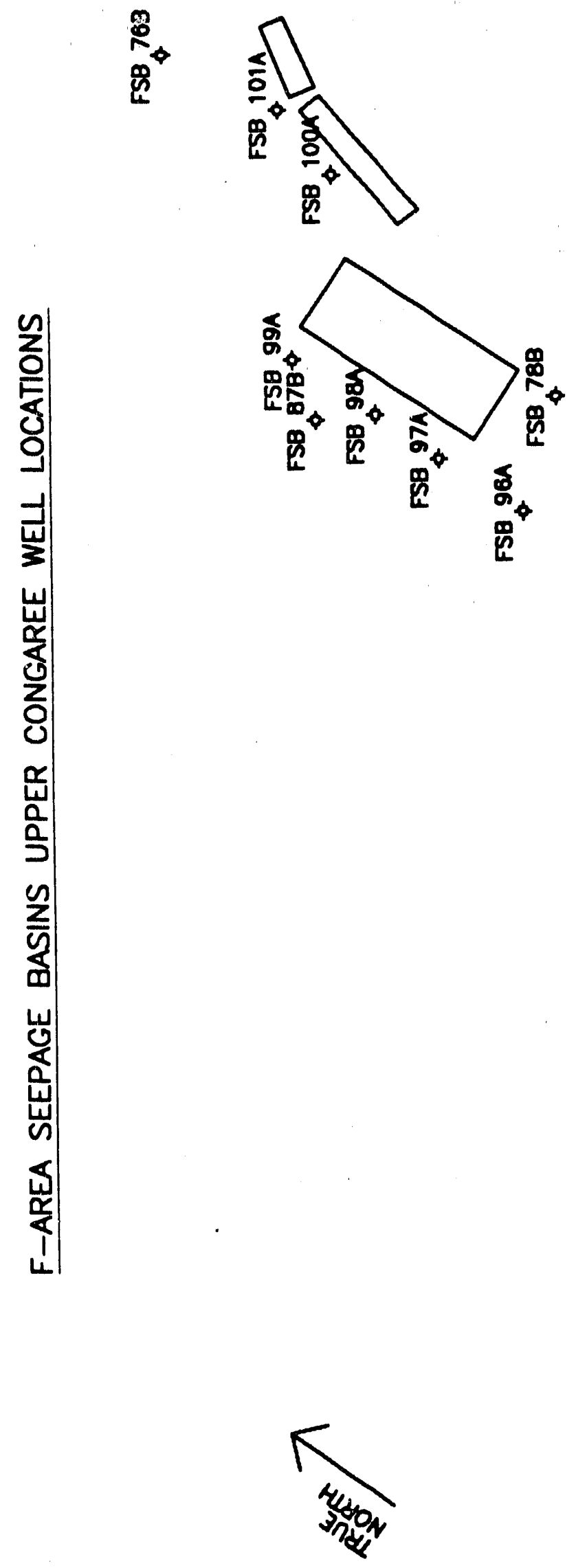



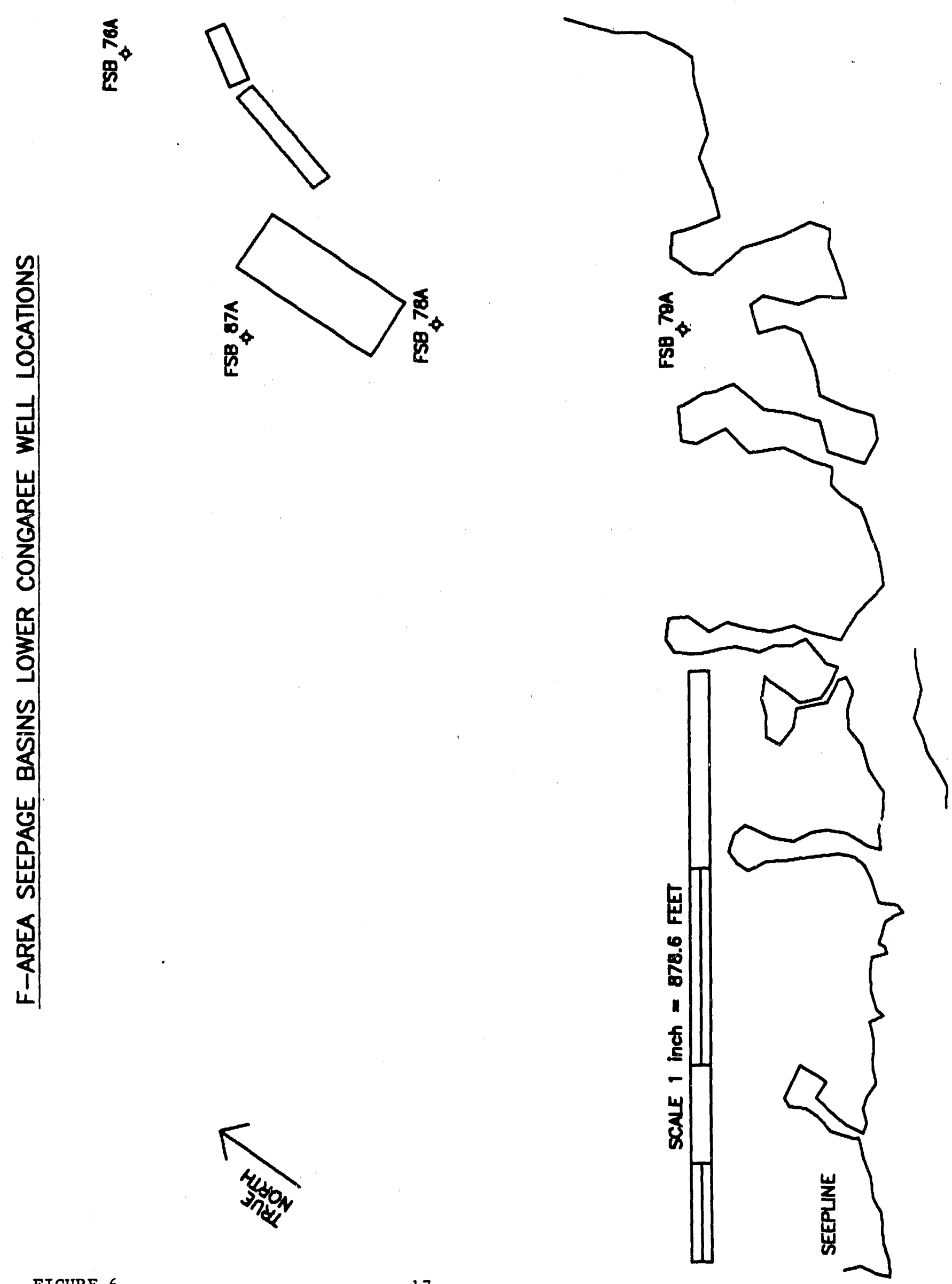


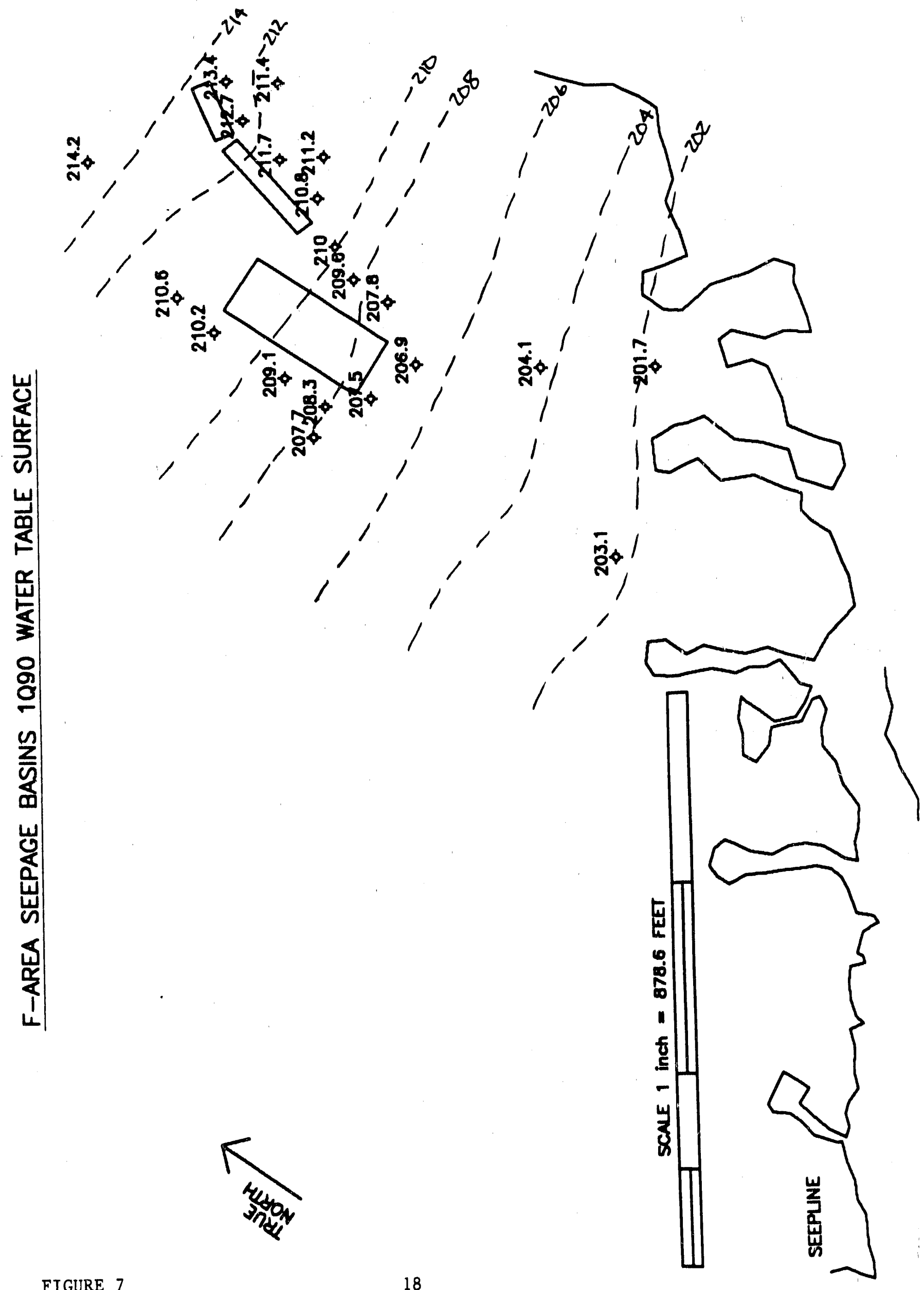




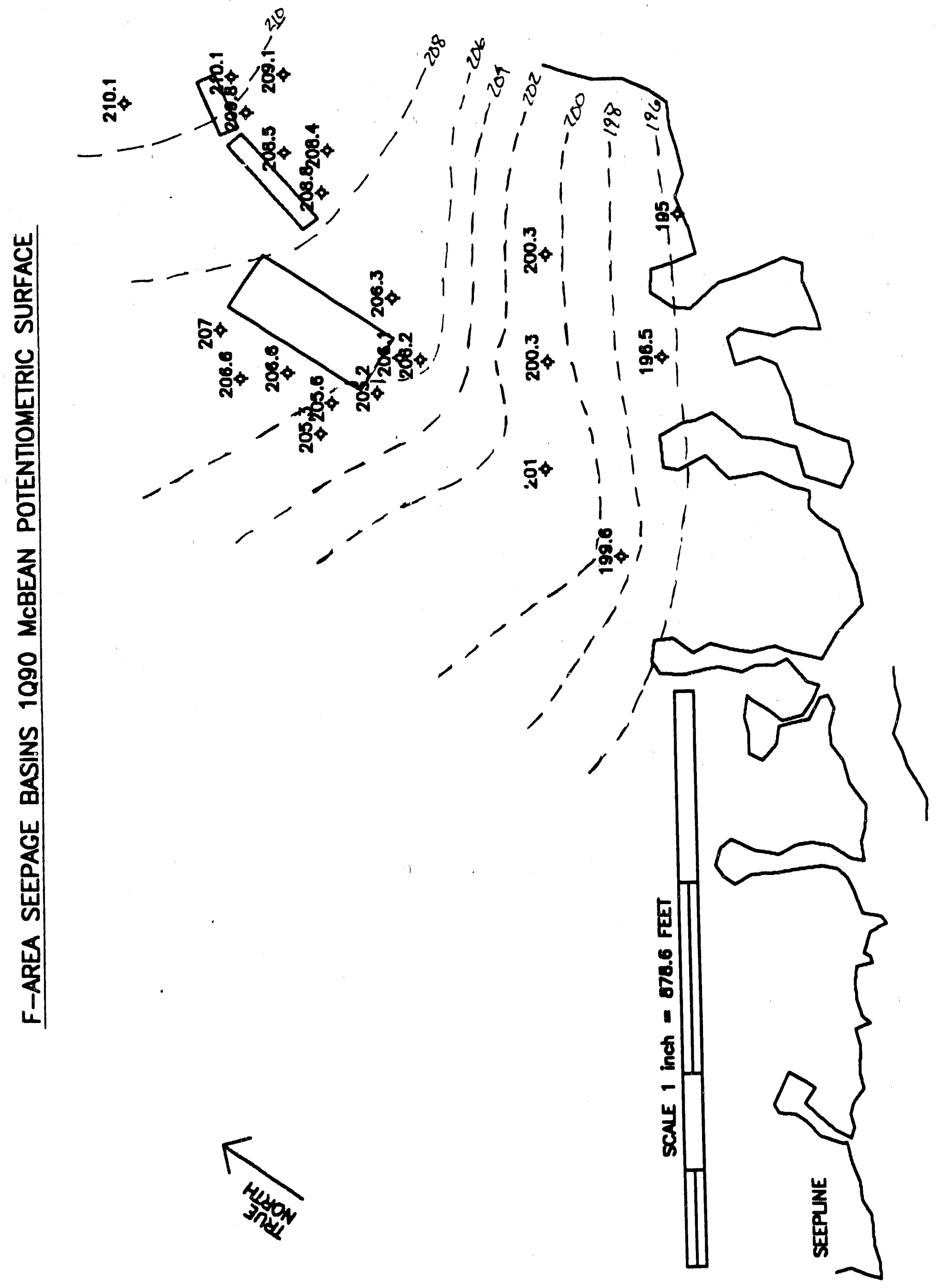

FIGURE 8 


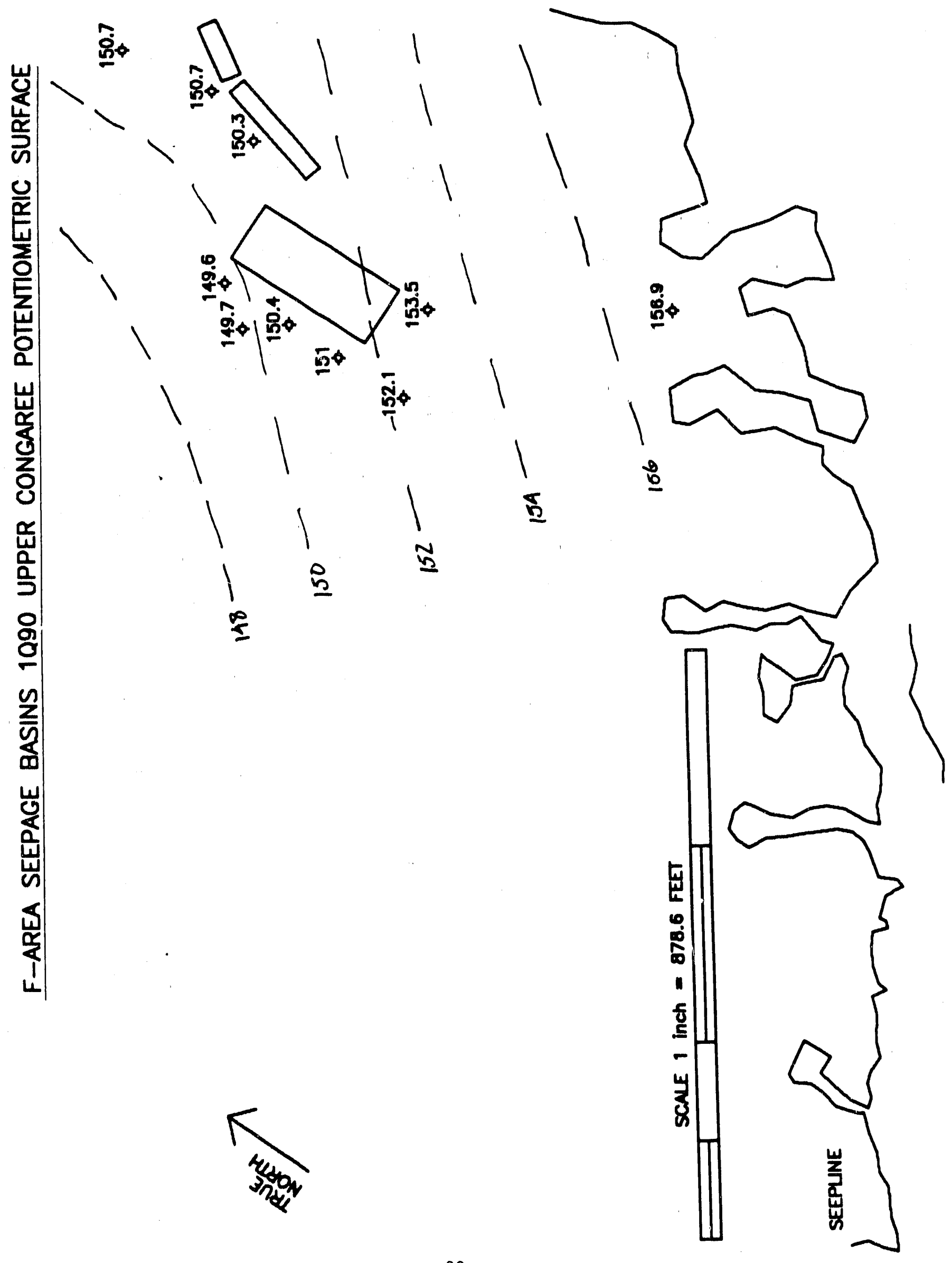




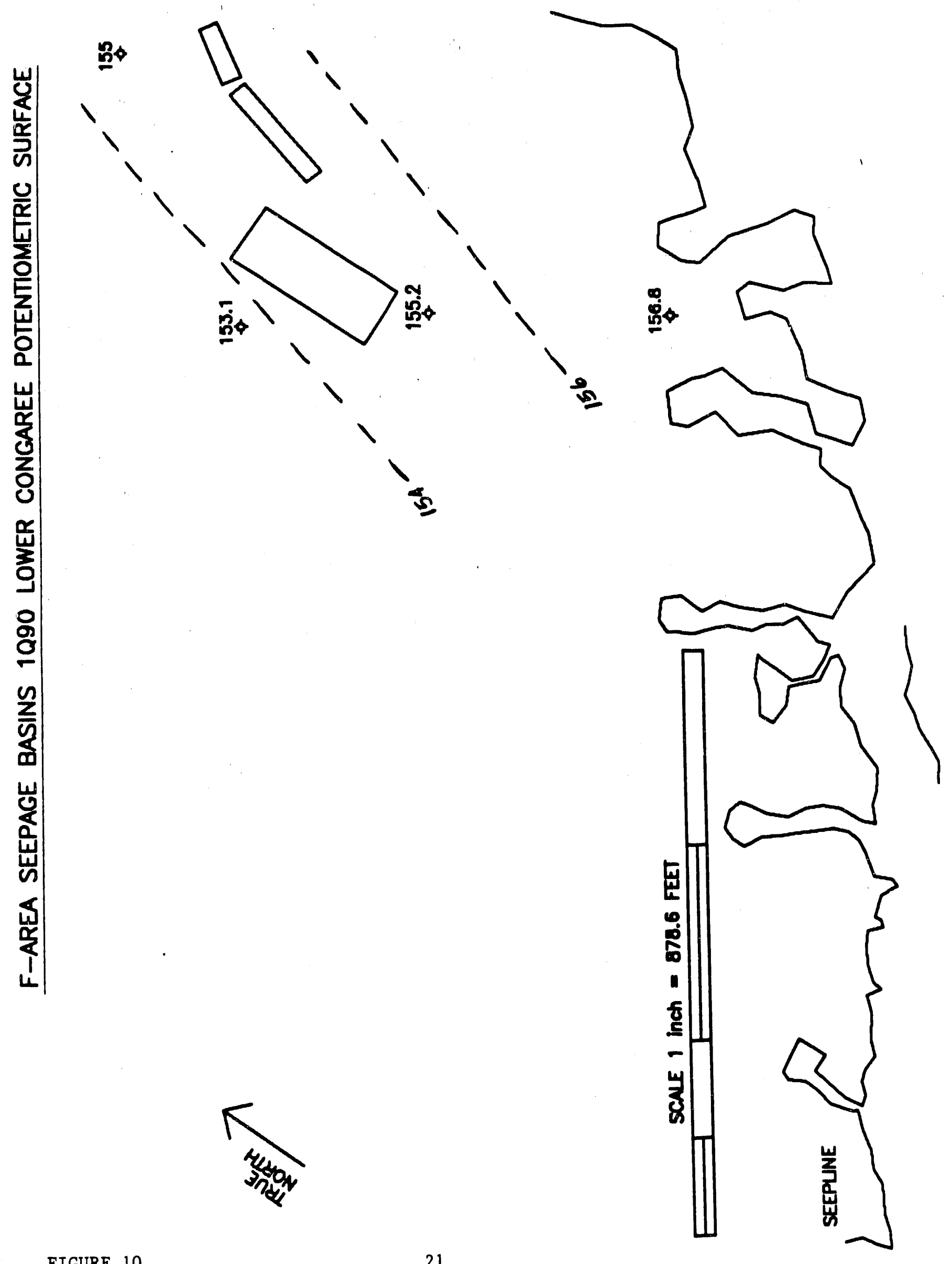




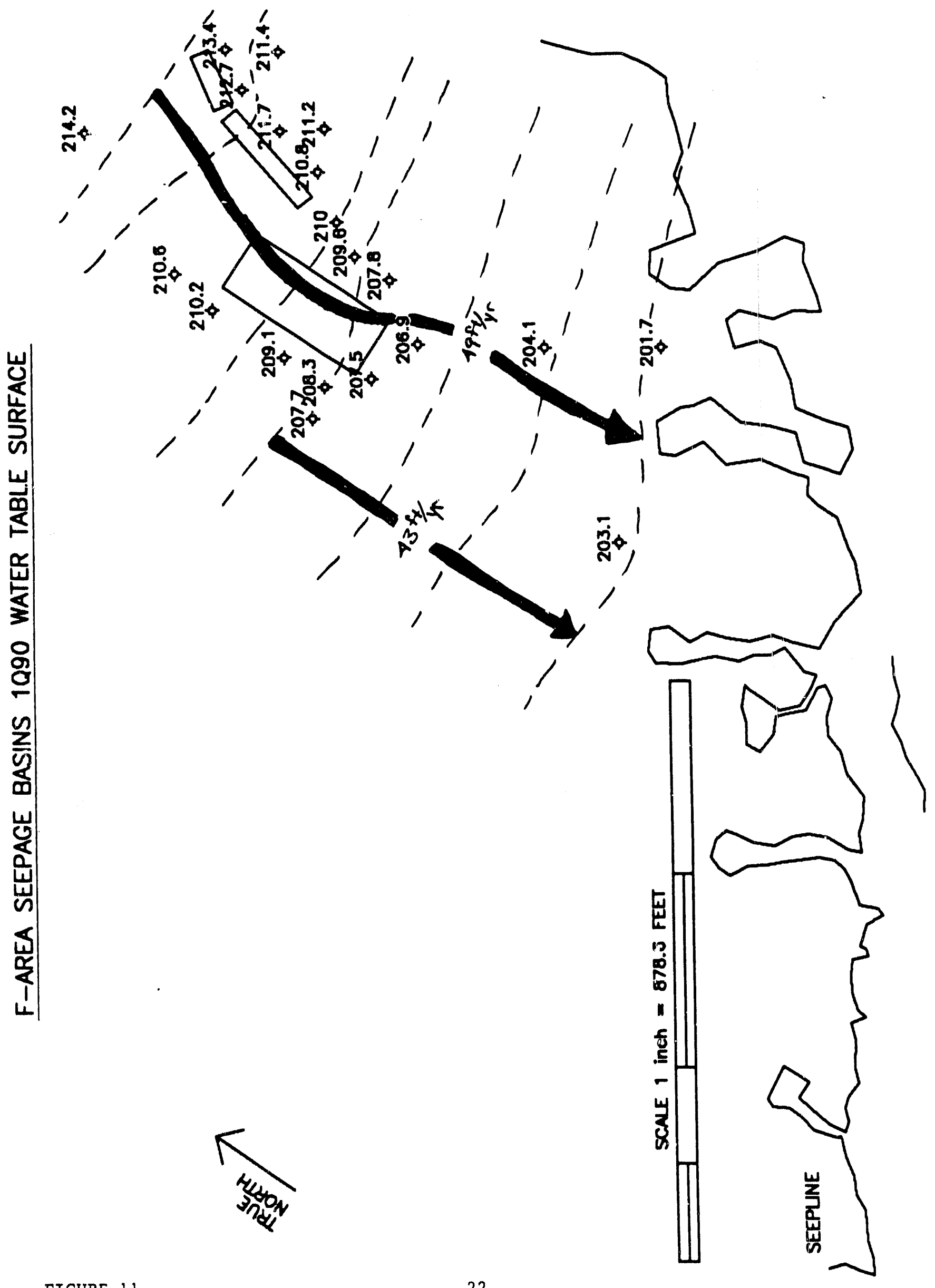




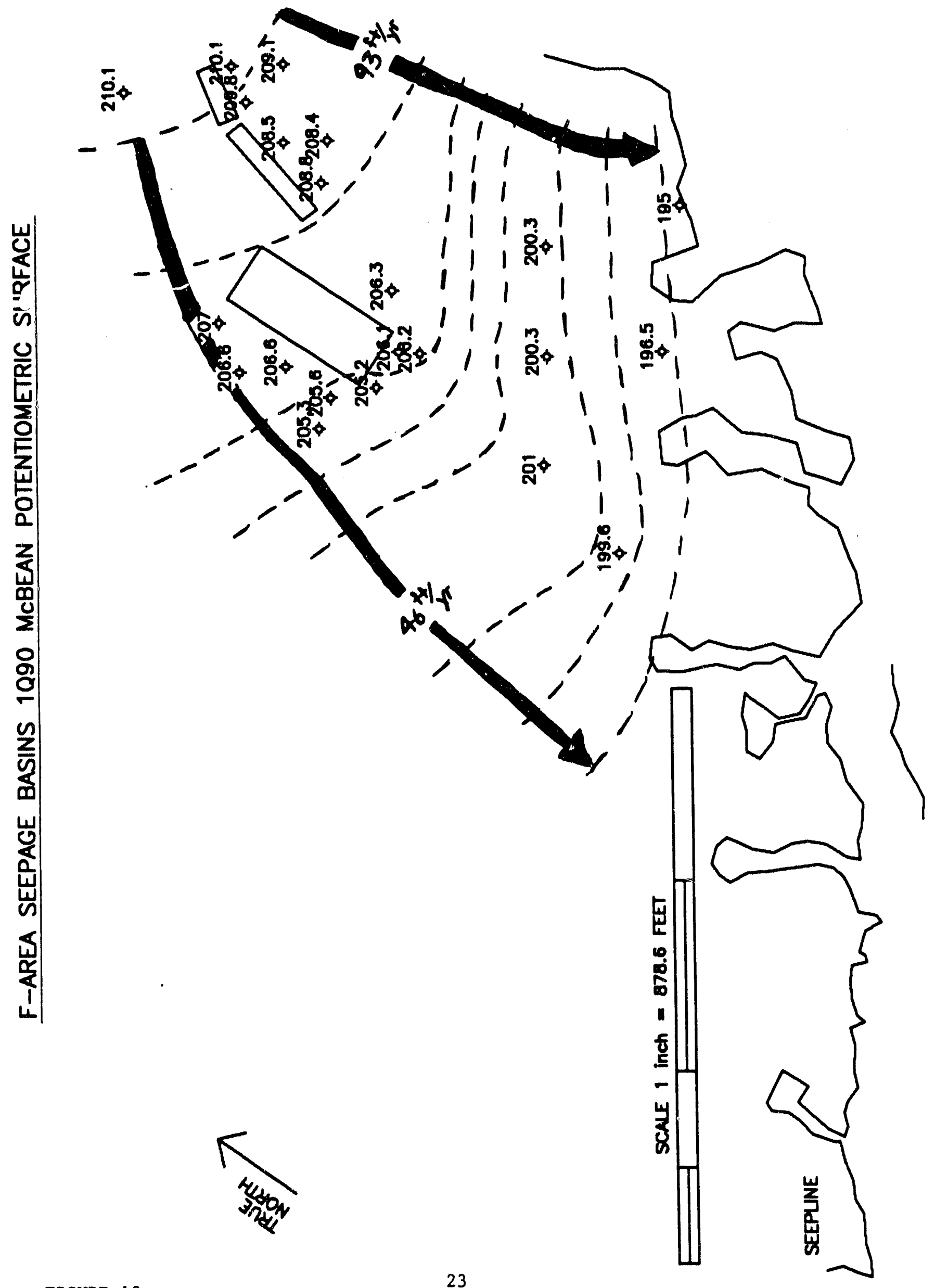




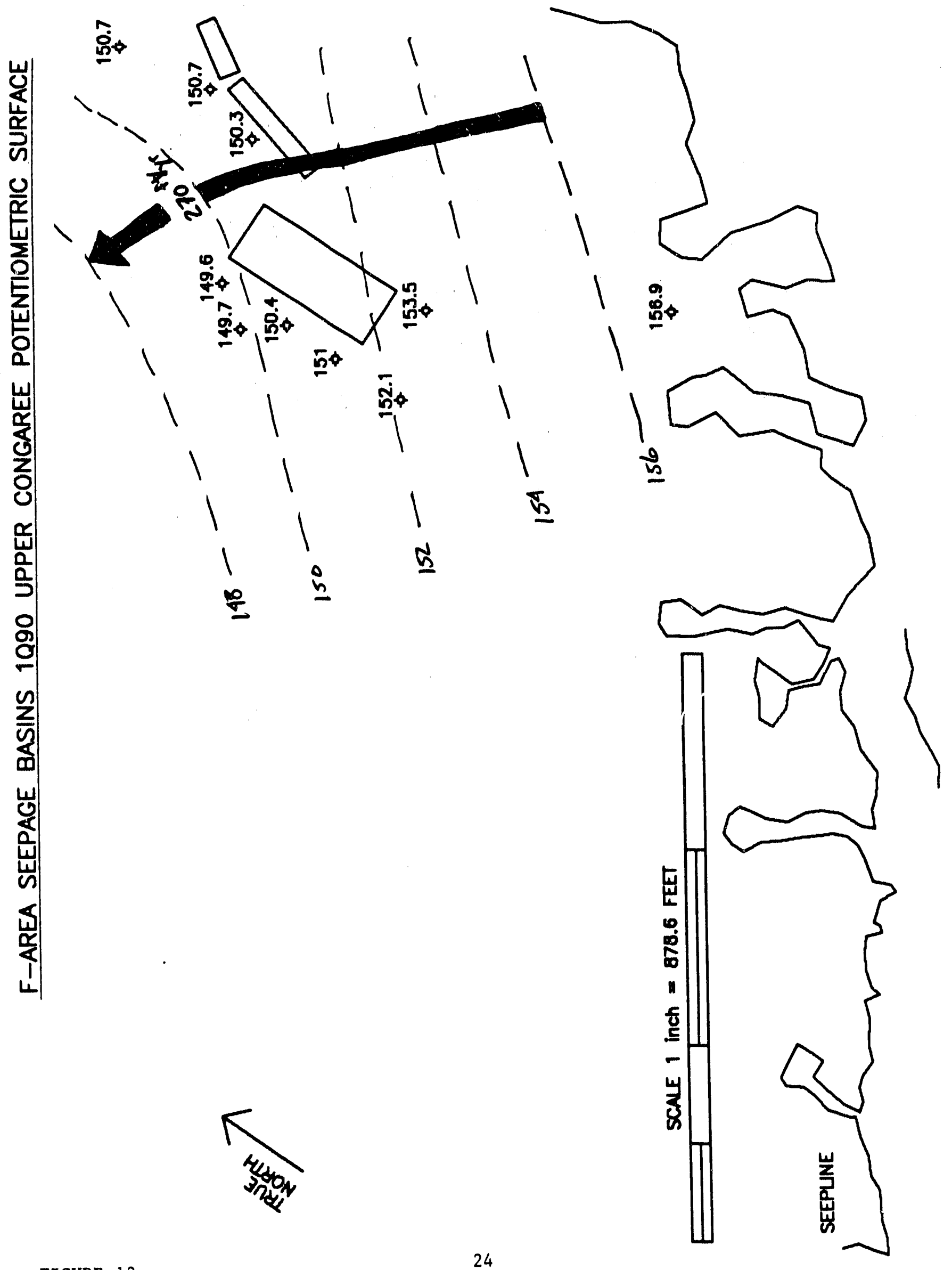




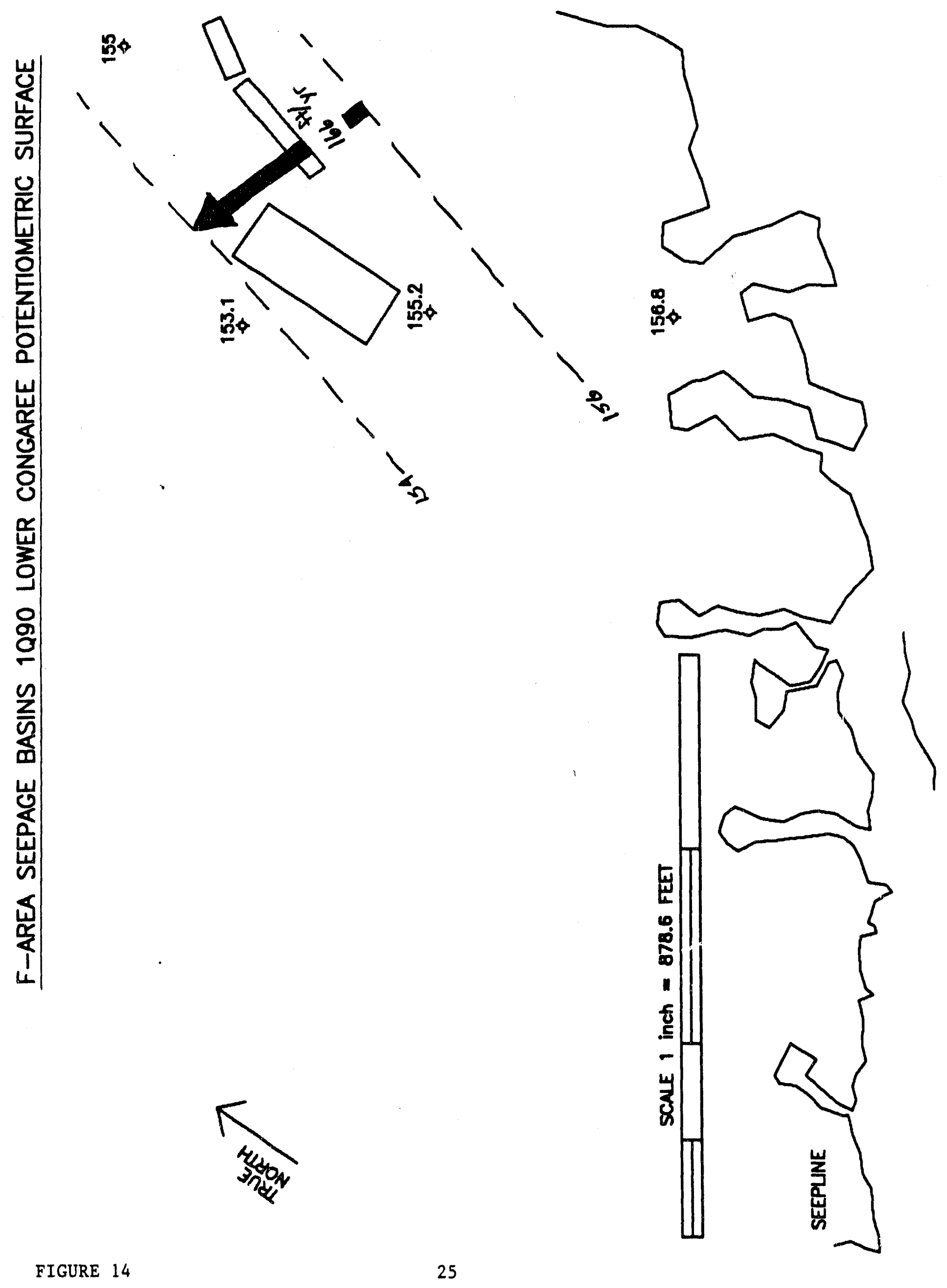




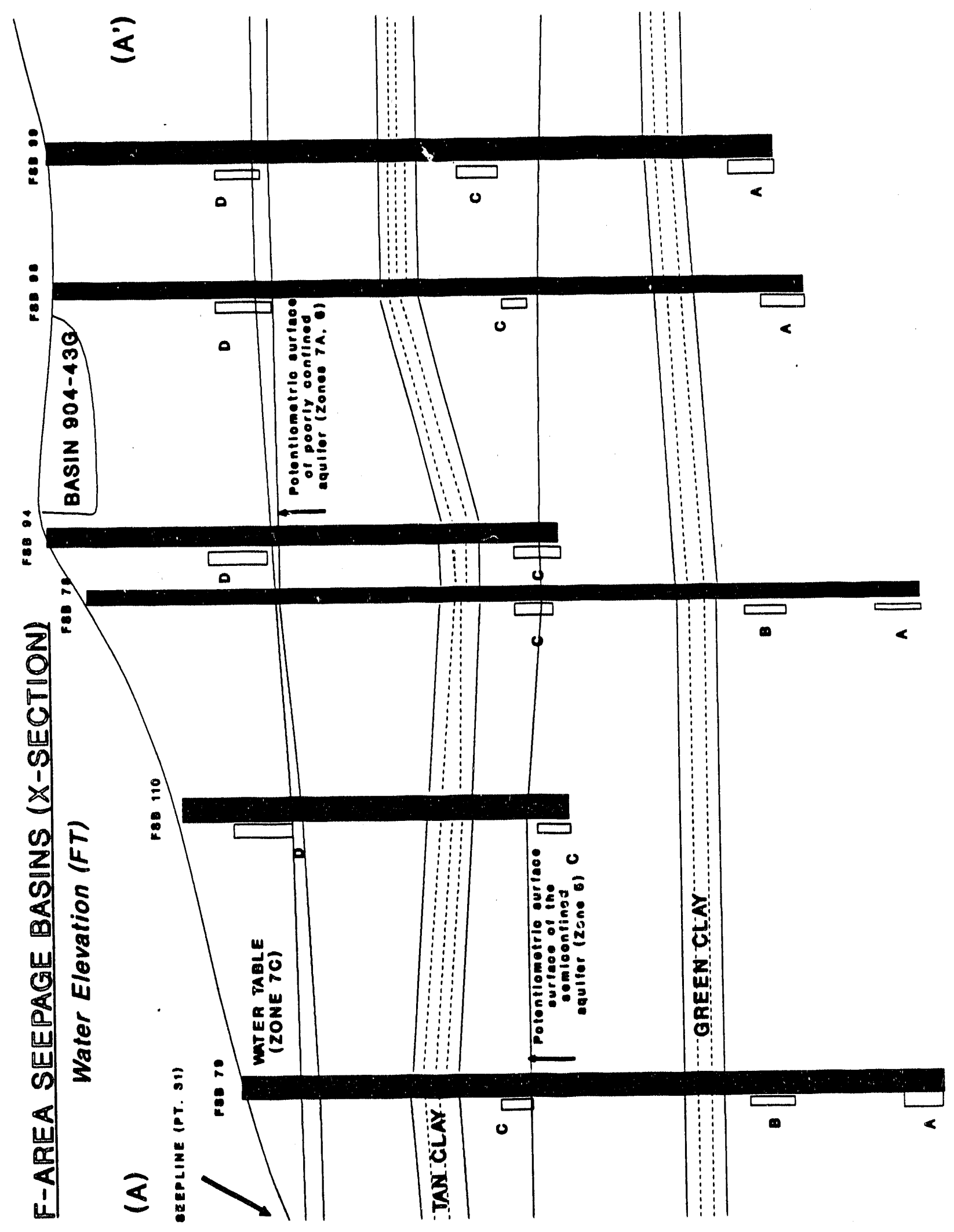




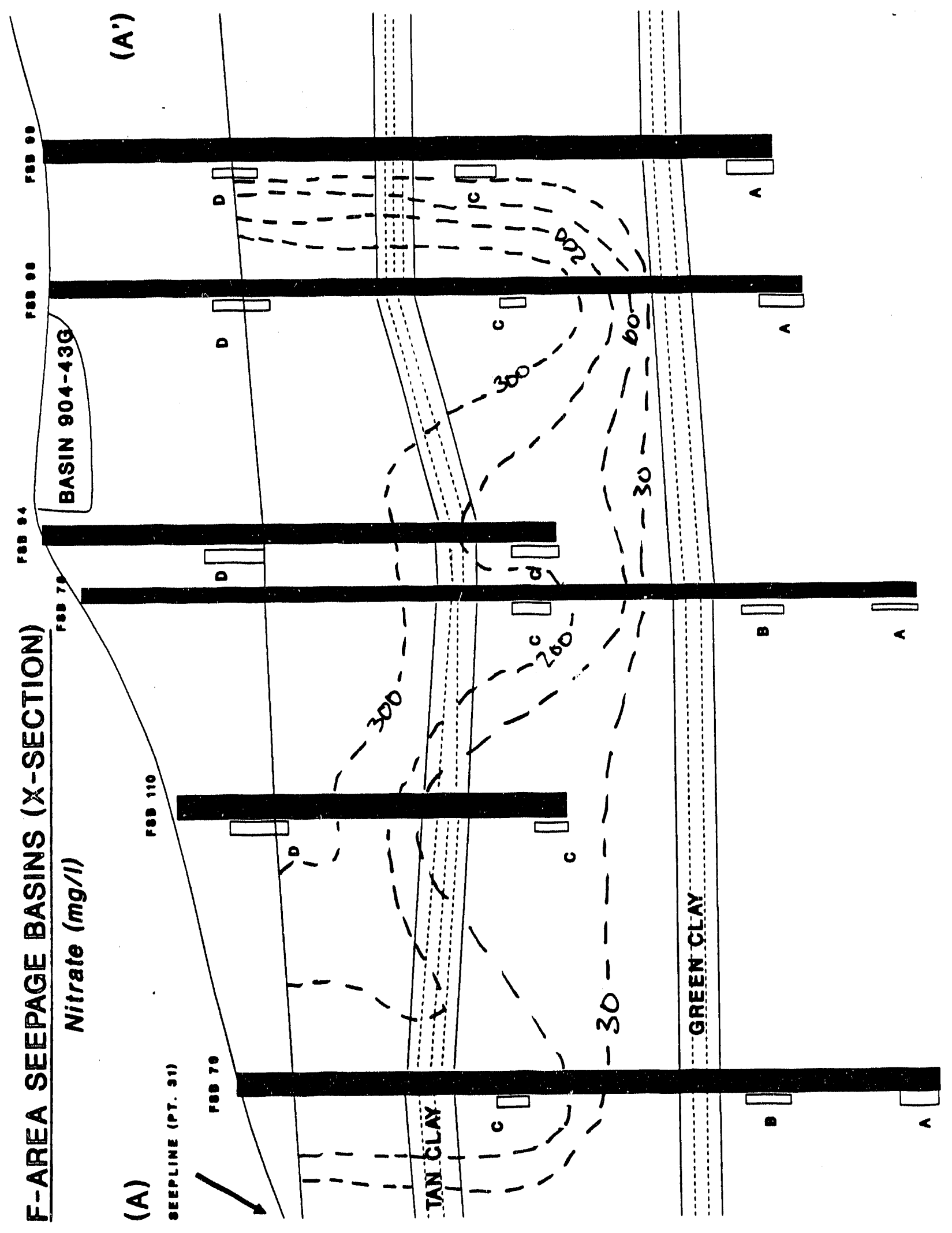




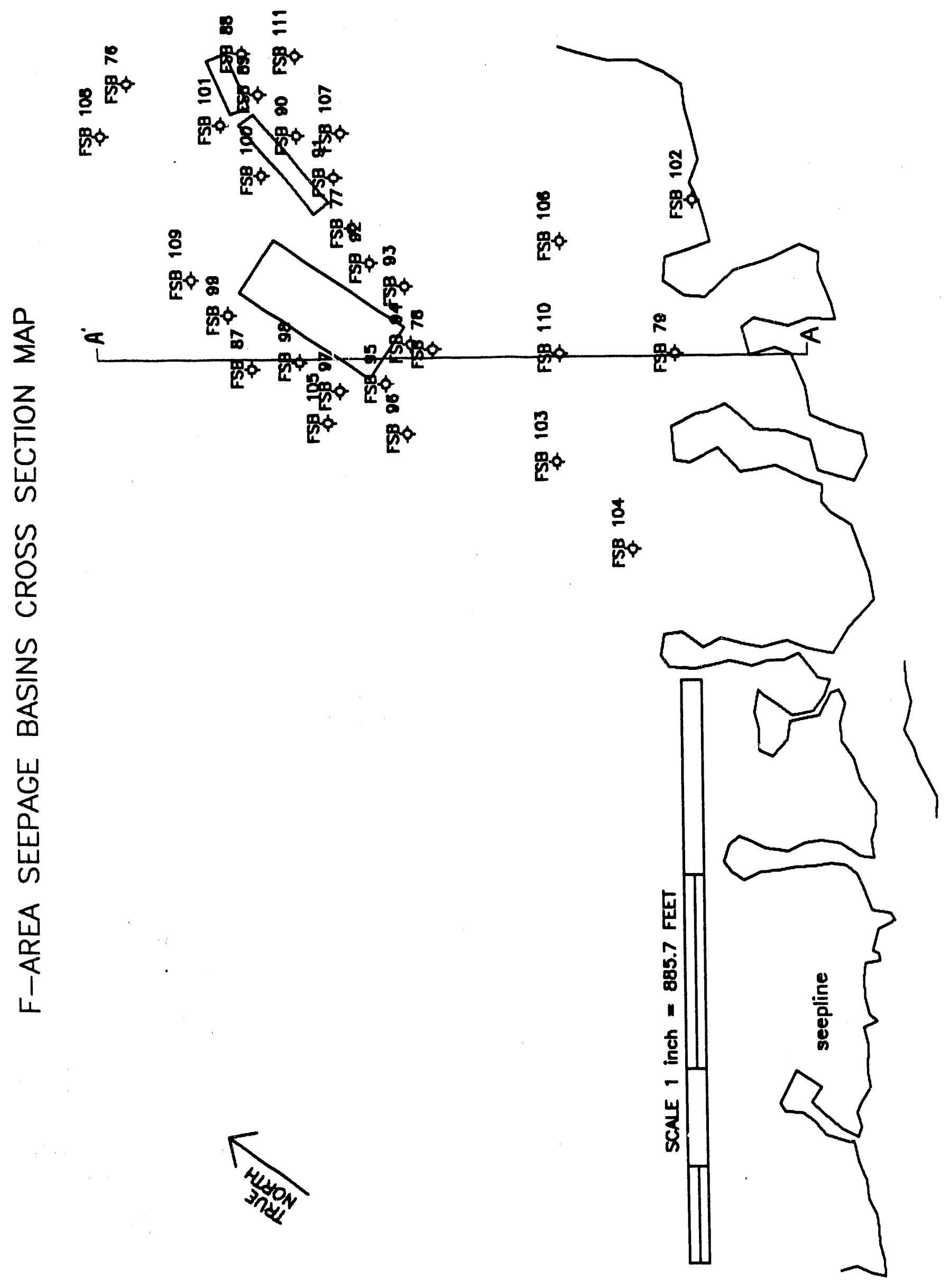


TABLE 1

\section{F-MREA}

FLOW RATE ESTIMATIONS

1090

FION $=\frac{\text { HYDRAULIC CONDUCTIVITY }}{\text { POROSITY }} \quad x \quad \frac{\mathrm{dh}}{\mathrm{dl}}$
$\mathrm{ft} / \mathrm{day}=(\mathrm{ft} / \mathrm{day} /$ unitless $) \mathrm{x}(\mathrm{ft} / \mathrm{ft})$

mATER TABLE (zONE 7C)

$$
\begin{aligned}
& \frac{10}{0.20} \times \frac{6}{2526}= \\
& \frac{10}{0.20} \times \frac{12}{4393}=
\end{aligned}
$$

YCBEAN (ZONE 6)

\section{ET/DAY ET/YR}

$\begin{array}{ll}0.12 & 43 \\ 0.14 & 50\end{array}$

DPPER CONGARFE (2ONE 5B)

$\frac{65}{0.20} \times \frac{8}{3514}=0.74 \quad 270$

\section{IONGR CONGNRFE (RONE $5 \mathrm{~N}$}

$\frac{65}{0.20} \times \frac{2}{1428}=0.45 \quad 166$




\section{CHEDULE FOR REHABIIITATION AND ABNWDONLENT/RERIACENENT FOR F/B BEEPAGE BABINS AND THE KIXED WAETE MNAGEKENT FACIIITY (KWNF) GROONDWATER KONITORIYG WELI NETWORX}

A) Wells which were successfully rehabilitated.

\begin{tabular}{ll} 
FSB & HSB \\
\hline $87 D$ & 68 \\
76 & $115 C$ \\
$107 D$ & $102 D$ \\
$94 C$ & $104 C$ \\
$98 D$ & $119 A$ \\
$99 A$ & $120 A$ \\
$100 A$ & $121 A$ \\
$110 C$ & $123 A$ \\
& $127 C$ \\
& $133 C$
\end{tabular}

B) Wells which need to be replaced.

\begin{tabular}{llc} 
FSB & HSB & MWNF \\
\hline $94 D$ & $124 A$ & $8 A$ \\
$95 D$ & & $10 A$ \\
$95 \mathrm{C}$ & & $10 D$ \\
$96 \mathrm{~A}$ & & $12 \mathrm{~A}$ \\
$98 \mathrm{~A}$ & & $13 \mathrm{D}$ \\
& & $14 \mathrm{D}$ \\
& & $16 \mathrm{~A}$ \\
& & $22 \mathrm{D}$
\end{tabular}

C) *Wells which need to be rehabilitated.

$$
\begin{aligned}
& \text { MWTYF } \\
& \hline \text { 4D } \\
& 12 C \\
& 14 A \\
& 14 C \\
& 16 D \\
& 24 D
\end{aligned}
$$

*Post closure activity in the Mixed Waste Management Facility has hindered the accessibility of these wells for rehabilitation. Every effort is being made to attempt to rehabilitate these wells when the area is free of obstacles.

Replacement of the groundwater wells in the $F / H$ Seepage Basins and the Mixed Waste Management Facility will begin 
approximately in January of 1991. Approximate time till. completion of this task is about two months. This will be conducted after the installation of the fifty-one new groundwater monitoring wells in the $F / H$ Seepage Basins. 
EIRST OUARTER 2990 YONTTORING DATA - F-HWME

All field data and results of laboratory analyses from samples taken from each well in the F-HWMF network are presented. Wells are arranged in alphabetic/numeric order. Results of analyses which exceed the primary drinking water standard are flagged with an asterisk. 
WELL FSB 76

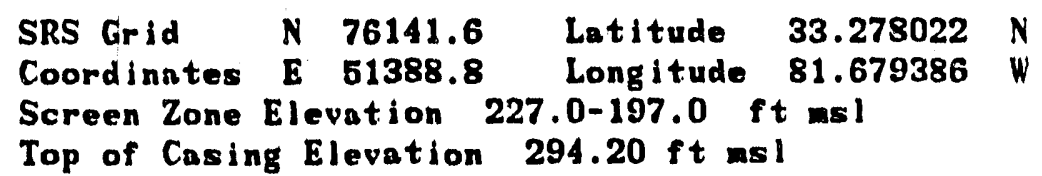

MEASUREMENTS CONDUCTED IN THE FIELD

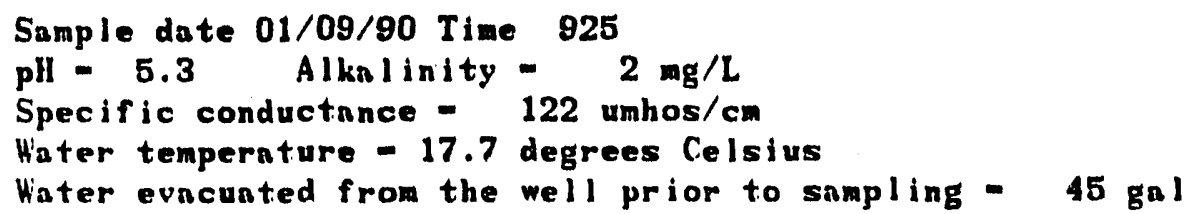

SPECIFIC CONDUCTANCE

SPECIFIC CONDUCTANCE

$\mathrm{PH}$

PII

SILVER

SILVER

ARSENIC

ARSENIC

BARILM

BARIUM

BRONOD I CIILOROMETHANE

BROMOD I CILLOROMETIIANE

CALCIVM

CALCIUM

TR I CIL OROFLUOROMETHANE

TR ICHLOROFLUOROMETHANE

CARBON TETRACHLORIDE

CARBOY TETRACHLORIDE

CADMIUM

CADMIUM

BROMOFORY

BROMOFORM

CIILOROFORM

CILOROFORY

METIIYLENE CILORIDE

METIIYLENE CHLORIDE

BROMOMETIIANE

BROMOMETIIANE

CHLOROMETIIANE

CILLOROMETHANE

CHILORIDE

CIILORIDE

CILLOROBENZENE

CILORORENZENE

COBALT

COBALT

CHROMILM

CONTINUED
$110.0 \mathrm{umh} / \mathrm{cm}$ Gen. Eng.

$110.0 \mathrm{umh} / \mathrm{cm}$ Gen. Eng.

5.52 pll

$5.51 \mathrm{pH}$

$2 \mathrm{ug} / \mathrm{L}$

$2 \mathrm{ug} / \mathrm{L}$

$2 \mathrm{ug} / \mathrm{L}$

$2 \mathrm{ug} / \mathrm{L}$

$12 \mathrm{ug} / \mathrm{L}$

$13 \mathrm{ug} / \mathrm{L}$

LT

LT

$1 \mathrm{ug} / \mathrm{L}$

$1 \mathrm{ug} / \mathrm{L}$

$1370 \mathrm{ug} / \mathrm{L}$

$1510 \mathrm{ug} / \mathrm{L}$

$1 \mathrm{ug} / \mathrm{L}$

$1 \mathrm{ug} / \mathrm{L}$

$1.00 \mathrm{ug} / \mathrm{L}$

$1.00 \mathrm{ug} / \mathrm{L}$

$2 \mathrm{ug} / \mathrm{L}$

$2 \mathrm{ug} / \mathrm{L}$

$1 \mathrm{ug} / \mathrm{L}$

$1 \mathrm{ug} / \mathrm{L}$

$1 \mathrm{ug} / \mathrm{L}$

$1 \mathrm{ug} / \mathrm{L}$

$3 \mathrm{ug} / \mathrm{L}$

$2 \mathrm{ug} / \mathrm{L}$

$1 \mathrm{ug} / \mathrm{L}$

$1 \mathrm{ug} / \mathrm{L}$

$1 \mathrm{ug} / \mathrm{L}$

$1 \mathrm{ug} / \mathrm{L}$

$2600 \mathrm{ug} / \mathrm{L}$

$2600 \mathrm{ug} / \mathrm{L}$

$1 \mathrm{ug} / \mathrm{L}$

$1 \mathrm{ug} / \mathrm{L}$

$4 \mathrm{ug} / \mathrm{L}$

$4 \mathrm{ug} / \mathrm{L}$

$4 \mathrm{ug} / \mathrm{L}$
Gen. Eng.

Gen. Eng.

Gen. Eng.

Gen. Eng.

Gen. Eng.

Gen. Eng.

Gen. Eng.

Gen. Eng.

Gen. Eng.

Gen. Eng.

Gen. Eng.

Gen. Eng.

Gen. Eng.

Gen. Eng.

Gen. Eng.

Gen. Eng.

Gen. Eng.

Gen. Eng.

Gen. Eng.

Gen. Eng.

Gen. Eng.

Gen. Eng.

Gen. Eng.

Gen. Eng.

Gen. Eng.

Gen. Eng.

Gen. Eng.

Gen. Eng.

Gen. Eng.

Gen. Eng.

Gen. Eng.

Gen. Eng.

Gen. Eng.

Gen. Eng.

Gen. Eng. 
WELL FSB 76 COLLECTED ON 01/09/90 LABORATORY ANALYSES CONIINUED

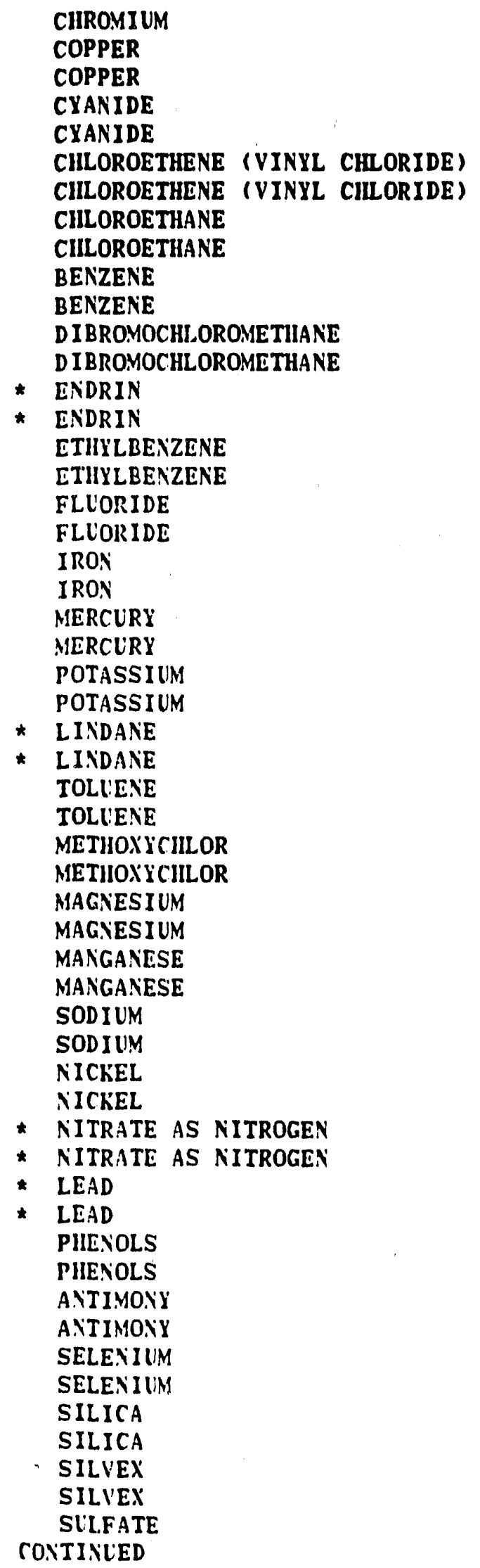

LT

LI

LT

LT

LT

LT

LT

LT

LT

LT

LT

LT

LT

LT

LT

LT

LT

LT

LT

LT

LT

LI

LT

LT

LT

LT

LT

LT

LT

LT

LT

LT

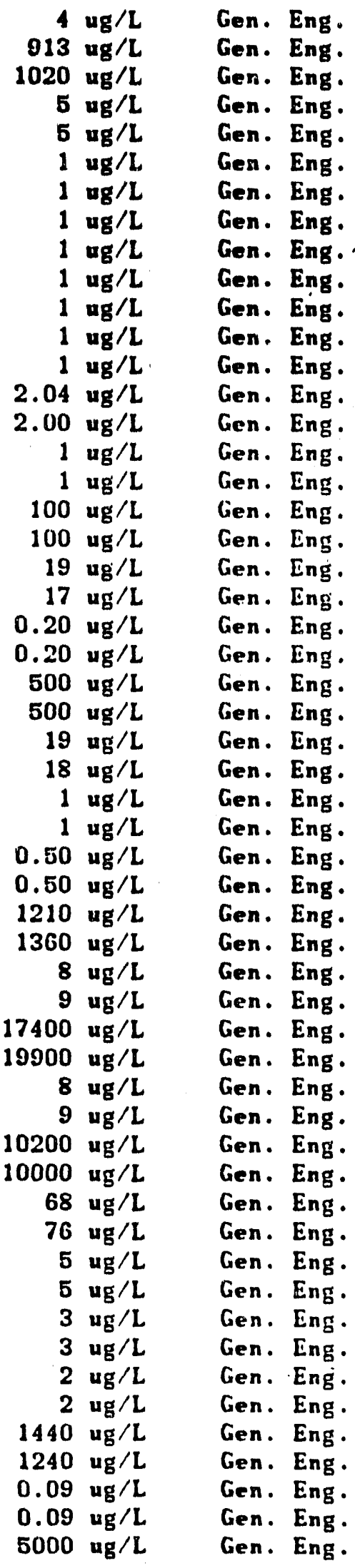

Gen. Eng.

Gen. Eng.

Gen. Eng.

Ger. Eng.

Gen. Eng.

Gen. Eng.

Gen. Eng.

Gen. Eng.

Gen. Eng .

Gen Eng.

Gen. Eng.

Gen. Eng.

Gen. Eng.

Gen. Eng.

Gen. Eng.

Gen. Eng.

Gen. Eng.

Gen. Eng.

Gen. Eng.

Gen. Eng.

Gen. Eng.

Gen. Eng.

Gen. Eng.

Gen. Eng.

Gen. Eng.

Gen. Eng.

Gen. Eng.

Gen. Eng.

Gen. Eng.

Gen. Eng.

Gen. Eng.

Gen. Eng.

Gen. Eng.

. Eng.

Gen. Eng.

Gen. Eng.

Gen. Eng.

Gen. Eng.

Gen. Eng.

Gen. Eng.

Gen. Eng.

Gen. Eng.

Gen. Eng.

Gen. Eng.

Gen. Eng. 


\section{SULFATE}

$1,1,2,2$-TETRACHLOROETHANE

$1,1,2,2$-TETRACIL OROETHANE

TETRACHLOROETHYLENE

TETRACHLOROETHYLENE

TOTAL DISSOLVED SOLIDS

TOTAL DISSOLVED SOLIDS

THALL IUM

THALLIUM

TOTAL ORGANIC CARBON

TOTAL ORGANIC CARBON

TOTAL ORGANIC HALOGENS

TOTAL ORGANIC HALOGENS

TOTAL PHOSPHATES

TOTAL PIIOSPHATES

TRICIILOROETHYLENE

TRICUI.OROETHYLENE

TOXAPIIENE

TOXAPHENE

TRANS - 1,2-DICHLOROETHENE

TRANS-1,2-DICHLOROETHENE

LRANIUM

IRATILIM

VANADIUM

VANADIUM

1,1-D ICIILOROETINLENE

1,1-D ICIILOROETIIYLENE

1,1-DICILLOROETIIANE

1,1-DICHLOROETHANE

$1,1,1$-TRICHLOROETIIANE

$1,1,1$-TRICHLOROETHANE

$1,1,2$-TRICILLOROETHANE

$1,1,2$-TRICHLOROETHANE

1,2-DICIILOROETHANE

1,2-DICIILOROETHANE

1,2-DICILLOROPROPANE

1,2-DICIILOROPROPANE

CIS-1, 3-DICHLOROPROPENE

CIS-1, 3-DICHLOROPROPENE

TRA.YS-1,3-DICILLOROPROPENE

TRANS-1,3-DICILLOROPROPENE

2-CILOROETIIYLYINYL ETHER

2-CILOROETHYLVINYL ETHER

2,4-D ICILOROPIIENONYACETIC ACID

2,4-DICILOROPHENOXYACETIC ACID

ZINC

ZINC

GROSS ALPIIA

GROSS ALPIIA

NONVOLATILE BETA

NONYOLATILE BETA

TOTAL RADIUM

TOTAL RADIUM

* tRITIUM

* TRITILIM
LT

LI

LT

LT

LT

LT

LT

LT

LT

LT

LI

LT

LT

LT

LT

LT

LT

LI

LT

LT

LT

LT

LT

LI

LT

LT

LT

LT

LT

LT

LT

LT

LT

LT

LT

LT

LT

LT

LT

LT

LT

LT

LT

\section{LT}

LT

$16.50+-4.90 \mathrm{pCi} / \mathrm{L}$

$14.50+-4.70 \mathrm{pCi} / \mathrm{L}$

$1.60+-3.30 \mathrm{pCi} / \mathrm{L}$

$2.10+-3.30 \mathrm{pCi} / \mathrm{L}$

$921+-3.10 \mathrm{pCi} / \mathrm{mL}$

$907+-3.00 \mathrm{pCi} / \mathrm{mL}$
Gen. Eng.

Gen. Eng.

Gen. Eng.

Gen. Eng.

Gen. Eng.

Gen. Eng.

Gen. Eng.

Gen. Eng.

Gen. Eng.

Gen. Eng.

Gen. Eng .

Gen. Eng.

Gen. Eng.

Gen. Eng.

Gen. Eng.

Gen. Eng.

Gen. Eng.

Gen. Eng.

Gen. Eng.

Gen. Eng.

Gen. Eng.

Gen. Eng.

Gen. Eng.

Gen. Eng.

Gen. Eng.

Gen. Eng.

Gen. Eng.

Gen. Eng.

Gen. Eng.

Gen. Eng.

Gen. Eng.

Gen. Eng.

Gen. Eng.

Gen. Eng.

Gen. Eng.

Gen. Eng.

Gen. Eng.

Gen. Eng.

Gen. Eng.

Gen. Eng.

Gen. Eng.

Gen. Eng.

Gen. Eng.

Gen. Eng.

Gen. Eng.

Gen. Eng.

Gen. Eng.

Gen. Eng.

Gen. Eng.

Gen. Eng.

Gen. Eng.

Gen. Eng.

Gen. Eng.

Gen. Eng .

Gen. Eng. 
WELL FSB 76A

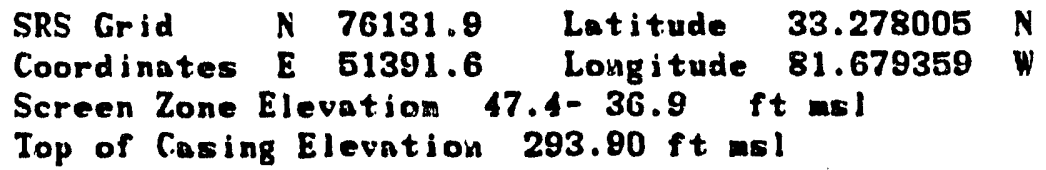

MEASUREMENTS CONDUCTED IN THE FIELD

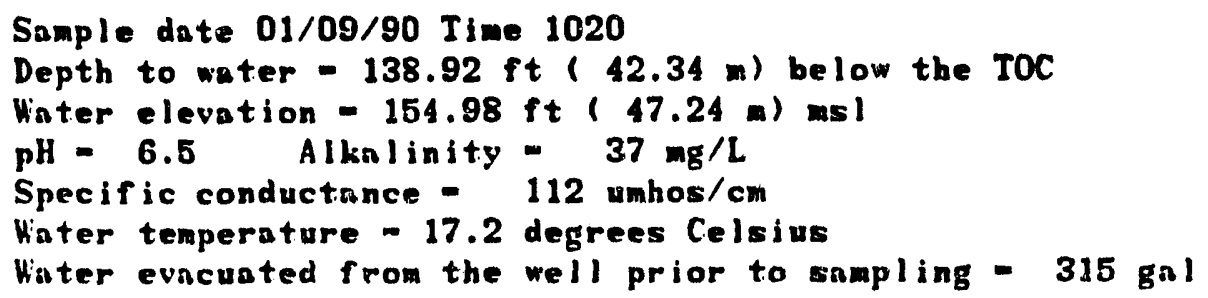

LABORATORY ANALYSES

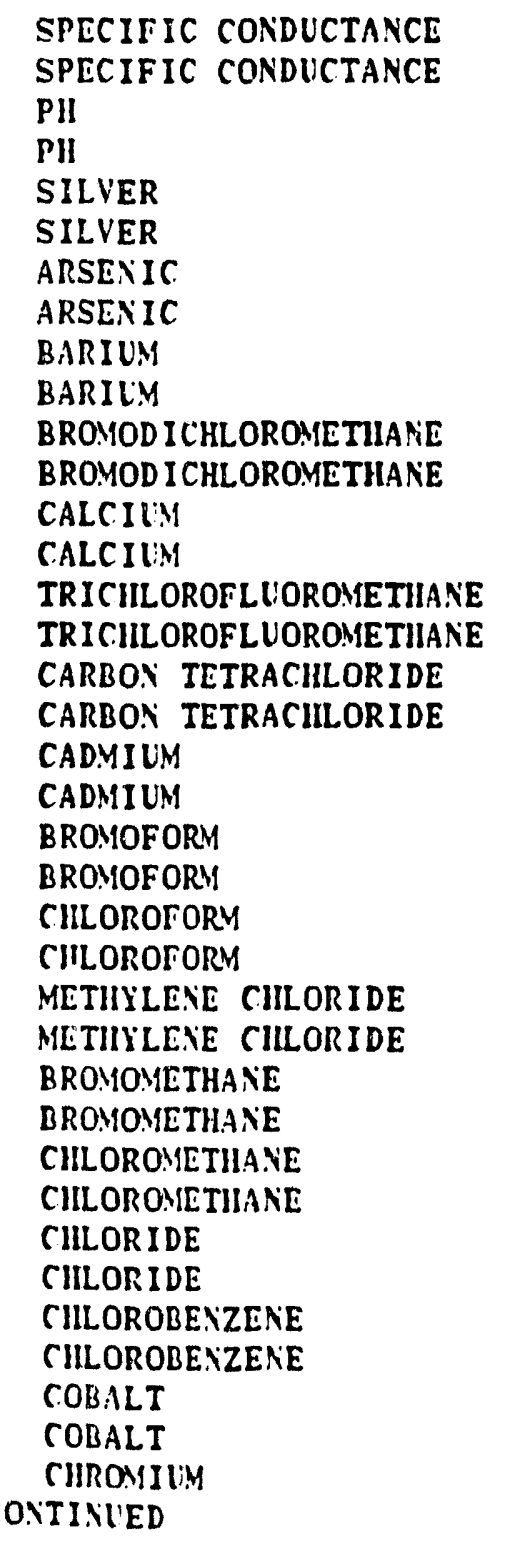

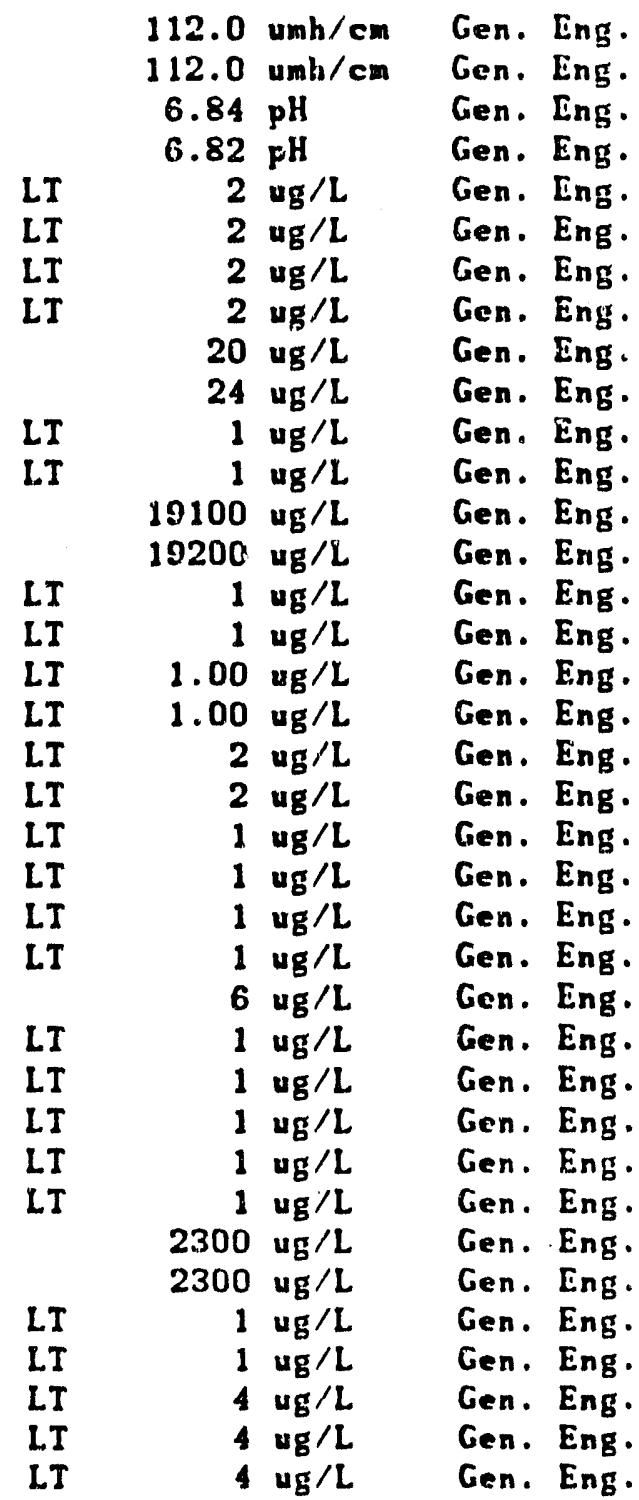


VELL FSB 76A COLLECTED ON 01/09/90 LABORATORY ANALYSES CONTINUED

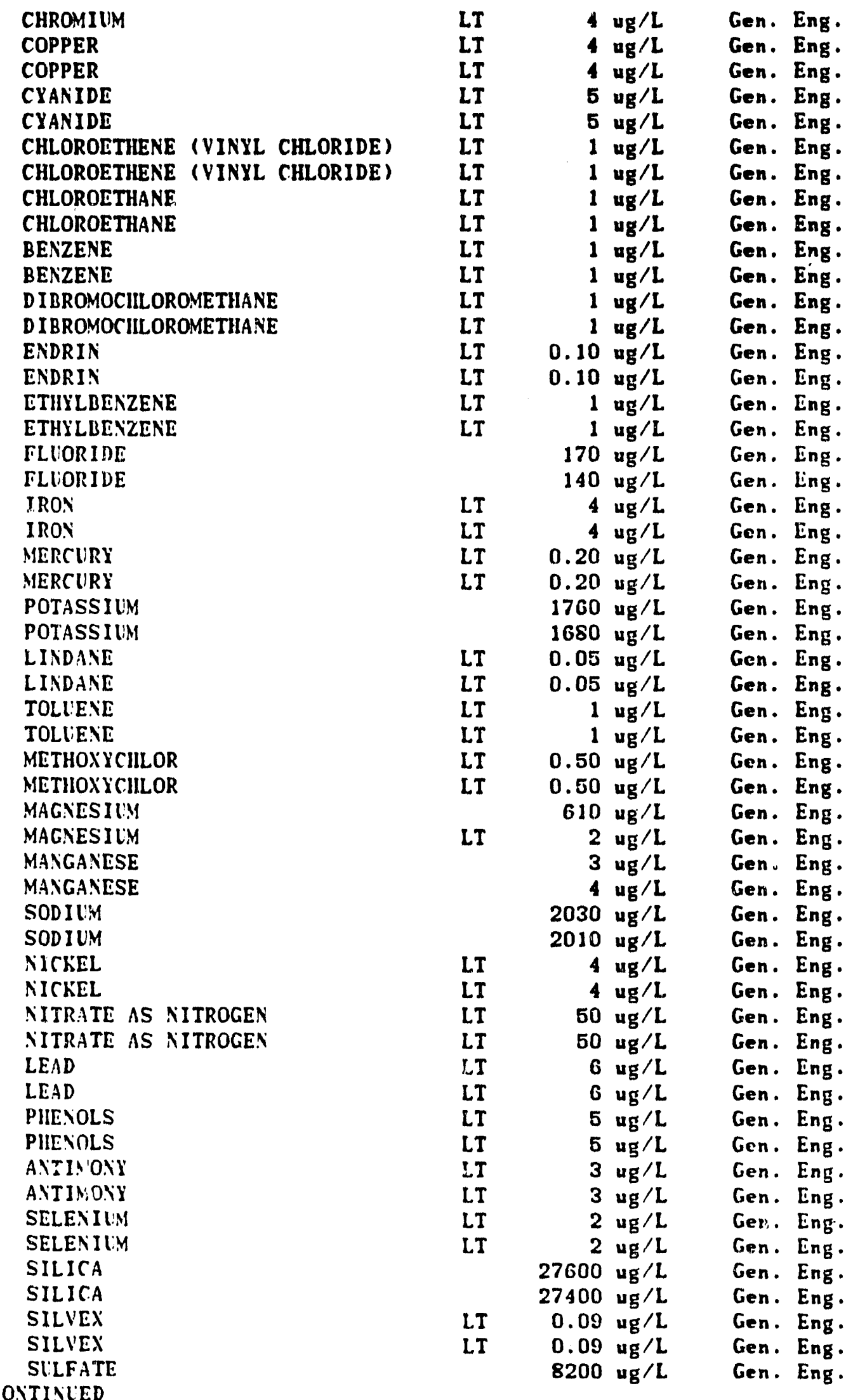




\begin{tabular}{|c|c|c|c|c|c|}
\hline $\begin{array}{l}\text { SLLFATE } \\
1,1,2,2-\text { TETRACHLOROETHANE } \\
1,1,2,2-\text { TETRACHLOROETHANE } \\
\text { TETRACIILOROETHYLENE } \\
\text { TETRACHLOROETHYLNE } \\
\text { TOTAL DISSOLVED SOLIDS } \\
\text { TOTAL DISSOLVED SOLIDS }\end{array}$ & $\begin{array}{l}\text { LT } \\
\text { LT } \\
\text { LT } \\
\text { LT }\end{array}$ & $\begin{array}{r}8500 \\
1 \\
1 \\
1.00 \\
1.00 \\
100000 \\
104000\end{array}$ & $\begin{array}{l}\operatorname{ug} / L \\
\operatorname{ug} / L \\
\operatorname{ug} / L \\
\operatorname{ug} / L \\
\operatorname{ug} / L \\
\operatorname{ug} / L \\
\operatorname{ug} / L\end{array}$ & $\begin{array}{l}\text { Gen. } \\
\text { Gen. } \\
\text { Gen. } \\
\text { Gen. } \\
\text { Gen. } \\
\text { Gen. } \\
\text { Gen. }\end{array}$ & $\begin{array}{l}\text { Eng. } \\
\text { Eng. } \\
\text { Eng. } \\
\text { Eng. } \\
\text { Eng. } \\
\text { Eng. } \\
\text { Eng. }\end{array}$ \\
\hline THAL.LIUM & LT & 2 & ug/L & Gen. & Eng \\
\hline THALLIUM & LT & 2 & $\mathrm{rg} / \mathrm{L}$ & Gen. & ing \\
\hline $\begin{array}{l}\text { TOTAL ORGANIC CARBON } \\
\text { TOTAL ORGANIC CARBON }\end{array}$ & & $\begin{array}{l}3000 \\
2000\end{array}$ & ug/L & & $n$ \\
\hline TOTAL ORGANIC HALOGENS & LI & $\begin{array}{r}2000 \\
5\end{array}$ & $\begin{array}{l}\text { ug } / 2 \\
\text { ug } / 2\end{array}$ & $\begin{array}{l}\text { Gen. } \\
\text { Gen. }\end{array}$ & Eng. \\
\hline TOTAL ORGANIC HALOGENS & LT & 5 & $\mathrm{ag} / \mathrm{L}$ & Gen. & cong. \\
\hline TOTAL PHOSPIIATES & & 350 & ug $/ \mathrm{L}$ & Gen. & Eng. \\
\hline TOTAL PHOSPHATES & & 350 & ug $/ \mathrm{L}$ & Gen. & Eng \\
\hline TRICIILOROETINLENE & LT & 1.00 & ug $/ L$ & en. & $n_{\mathrm{g}}$ \\
\hline TRICHI.OROETHYLENE & LT & 1.00 & ug/L & Gen. & ing. \\
\hline TOXAPIIENE & LT & 1 & ug $/ \mathrm{L}$ & Gen. & ng \\
\hline TOXAPIIENE & LT & 1 & ug $/ L$ & . & Eng. \\
\hline TRA.SS-1,2-DICILLOROETHENE & LT & 1 & ug $/ L$ & n. & $n g$. \\
\hline TRANS-1,2-DICHLOROETHENE & LT & 1 & ug/L & en. & Eng. \\
\hline I:RANIUM & LT & 1000 & ug $/ L$ & Gen. & ig. \\
\hline URANIUM & LT & 1000 & ug $/ \mathrm{L}$ & Gen. & En \\
\hline VANADIUM & LT & 20 & ug/L & Gen. & Ex \\
\hline VANADILM & LT & 20 & $\operatorname{ug} / \mathrm{L}$ & Gen. & 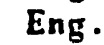 \\
\hline 1,1-DICIILOROETIIYLENE & LT & 1 & $\mathrm{ug} / \mathrm{L}$ & Gen. & \\
\hline 1,1-DICHLOROETHYLENE & $\mathbf{L}$ & 1 & $\operatorname{ug} / \mathrm{L}$ & n. & Eng \\
\hline 1,1-DICILLOROETIIANE & LT & 1 & $\mathrm{ug} / \mathrm{L}$ & Gen. & b \\
\hline 1,1-DICHLOROETHANE & LT & 1 & ug/L & Gen. & Eng. \\
\hline $1,1,1$-TRICHLOROETHANE & LT & 1 & $\operatorname{vg} / \mathrm{L}$ & Gen. & \\
\hline $1,1,1$-TRICHLOROETHANE & $L^{\prime}$ & 1 & ug/L & Gen. & Eng \\
\hline $1,1,2-$ TRICHLOROETHANE & LT & 1 & ug $/ L$ & Gen. & 27 \\
\hline 1,1,2-TRICILLOROETHANE & LT & 1 & ug $/ \mathrm{L}$ & Gen. & Eng. \\
\hline 1,2-DICHLOROETHANE & LT & 1 & ug $/ L$ & Gen. & Eng \\
\hline 1,2-D ICHLOROETIIANE & $L$ & 1 & $u g / L$ & Gen. & Eni \\
\hline 1,2-DICHLOROPROPANE & $\mathbf{L}^{\prime}$ & 1 & $\operatorname{ug} / \mathrm{L}$ & Gen. & $\mathbf{E}$ \\
\hline 1,2-DICHLOROPROPANE & LI & 1 & $\operatorname{ug} / \mathrm{L}$ & Gen. & $\mathbf{E}$ \\
\hline CIS-1,3-DICHLOROPROPENE & LT & 1 & ug/L & Gen. & 19 \\
\hline CIS-1,3-DICILLOROPROPENE & LI & 1 & $u g / L$ & Gen. & $\mathbf{E}$ \\
\hline TRANS-1,3-DICILLOROPROPENE & LT & 1 & $\operatorname{ug} / \mathrm{L}$ & Gen. & \\
\hline TRANS-1,3-DICHLOROPROPENE & LT & 1 & $\operatorname{ug} / \mathrm{L}$ & Gen. & Eng \\
\hline 2-CIILOROETIIYLVINYL ETHER & LT & 1 & $\operatorname{ug} / \mathrm{L}$ & Gen. & ng. \\
\hline 2-CHLOROETHYLYINYL ETIIER & LI & 1 & ug $/ \mathrm{L}$ & Gen. & \\
\hline 2,4-DICHLOROPIENONYACETIC AC & LT & 0.30 & ug/L & Gen & \\
\hline 2,4-DICHLOROPHENONYACETIC ACID & LT & 0.30 & ug/L & Gen. & \\
\hline ZINC & LI & 2 & ug $/ \mathrm{L}$ & & \\
\hline $21 \times C$ & L & 2 & $\mathrm{ug} / \mathrm{L}$ & Gen. & \\
\hline GROSS ALPHA & LI & 3 & $p C i / L$ & Gen. & \\
\hline GROSS ALPHIA & LI & 3 & $\mathrm{pCi} / \mathrm{L}$ & Gen. & \\
\hline NONVOLATILE BETA & LT & 2 & $\mathrm{PC} / \mathrm{L}$ & Gen. & \\
\hline NONVOLATILE BETA & LT & 2 & $\mathrm{pC} i / \mathrm{L}$ & Ger & \\
\hline TOTAL RADIUM & LT & 1 & $\mathrm{PC} i / \mathrm{L}$ & Gen. & \\
\hline TOTAL RADIUM & LT & 1 & $\mathrm{p} C_{i} / L$ & Ger & \\
\hline TRITILM & LT & 0.70 & $\mathrm{pCi} / \mathrm{mL}$ & Ger & \\
\hline TRIIIUM & LI & 0.70 & $\mathrm{pCi} / \mathrm{mL}$ & Gen. & \\
\hline & A- & & & & \\
\hline
\end{tabular}


WELL FSB 76B

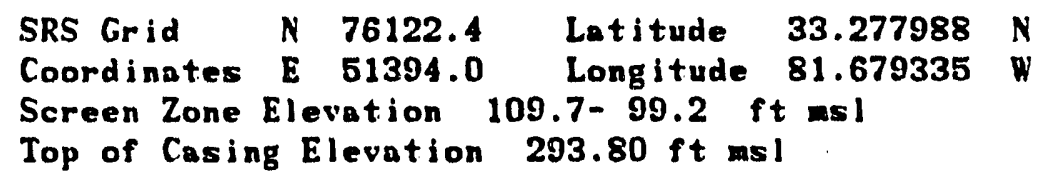

MEASUREMENTS CONDUCTED IN THE FIELD

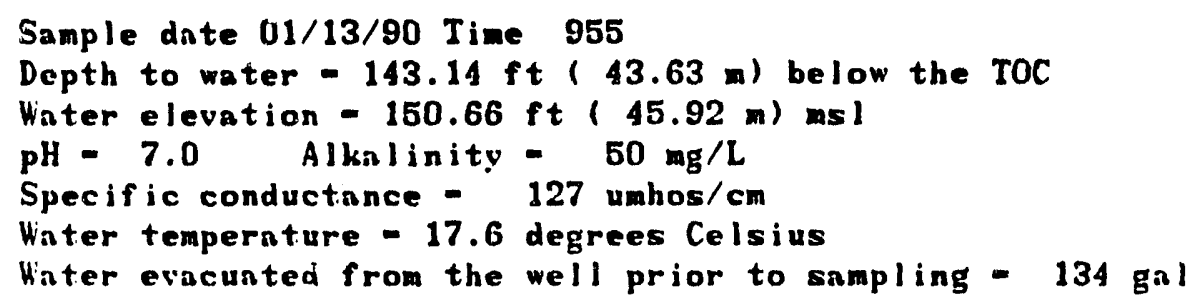

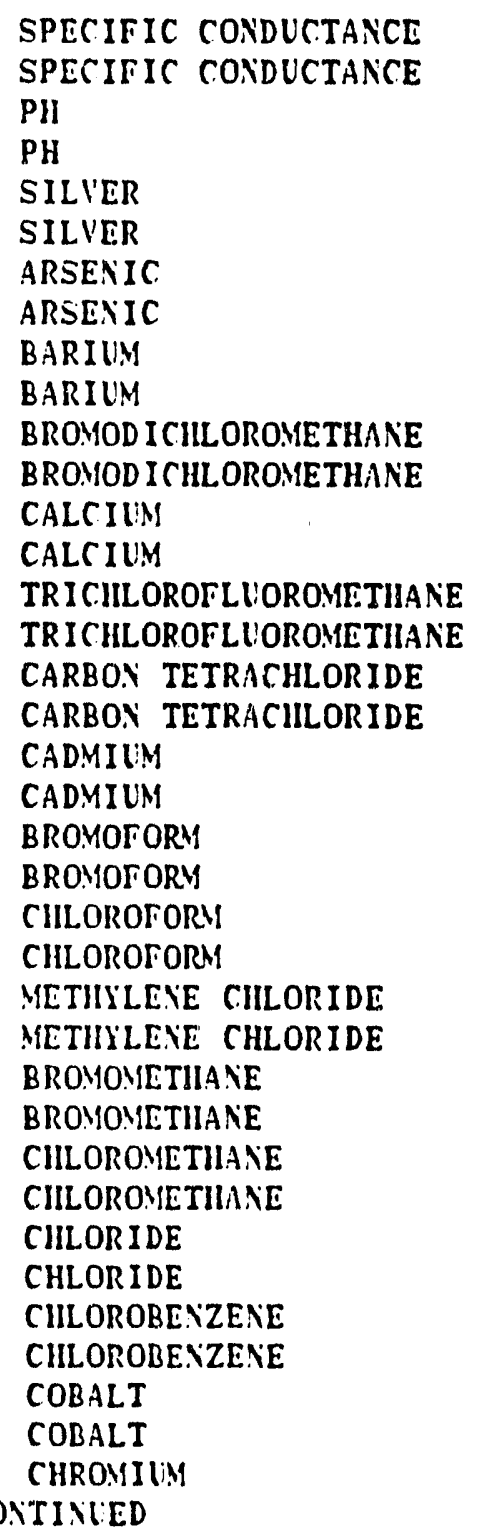

\begin{tabular}{|c|c|c|c|}
\hline & $\begin{array}{r}130.0 \\
110.0 \\
6.91 \\
6.87\end{array}$ & $\begin{array}{l}u \operatorname{unh} / \mathrm{cm} \\
u m h / c m \\
\mathrm{pH} \\
\mathrm{pH}\end{array}$ & $\begin{array}{l}\text { MetaTrace } \\
\text { Gen. Eng. } \\
\text { MetaTrace } \\
\text { Gen. Eng. }\end{array}$ \\
\hline LT & 2 & ug $/ \mathrm{L}$ & MetaTrace \\
\hline$L T$ & 2 & ug $/ L$ & Gen, Eng. \\
\hline LI & 3 & ug $/ \mathrm{L}$ & MetaTrace \\
\hline LT & 2 & $\mathrm{ug} / \mathrm{L}$ & Gen. Eng. \\
\hline & 18 & ug $/ L$ & MetaTrace \\
\hline & 17 & $\operatorname{ug} / \mathrm{L}$ & Gen. Eng. \\
\hline LT & 5 & ug $/ L$ & MetaTrace \\
\hline & 1 & ug $/ L$ & Gen. Eng. \\
\hline & 21900 & ug $/ \mathrm{L}$ & MetaTrace \\
\hline & 28200 & ug $/ \mathrm{L}$ & Gen. Eng. \\
\hline LT & 5 & ug $/ \mathrm{L}$ & MetaTrace \\
\hline LI & 1 & ug $/ L$ & Gen. Eng. \\
\hline L.T & 5.00 & ug $/ L$ & MetaTrace \\
\hline LT & 1.00 & ug $/ \mathrm{L}$ & Gen. Eng. \\
\hline LT & 3 & $u g / L$ & MetaTrace \\
\hline LT & 2 & ug $/ L$ & Gen. Eng. \\
\hline LI & 5 & $u g / L$ & MetaTrace \\
\hline LT & 1 & ug $/ L$ & Gen. Eng. \\
\hline LT & 5 & $u_{g} / L$ & MetaTrace \\
\hline LT & $\begin{array}{l}1 \\
3\end{array}$ & $\begin{array}{l}\operatorname{ug} / \mathrm{L} \\
\mathrm{ug} / \mathrm{L}\end{array}$ & $\begin{array}{l}\text { Gen. Eng. } \\
\text { MetaTrace }\end{array}$ \\
\hline LT & 1 & ug $/ \mathrm{L}$ & Gen. Eng. \\
\hline LT & 10 & ug $/ \mathrm{L}$ & MetaTrace \\
\hline LT & 1 & ug $/ \mathrm{L}$ & Gen. Eng. \\
\hline LT & 10 & $\mathrm{ug} / \mathrm{L}$ & Metatrace \\
\hline LT & 1 & $u g / L$ & Gen. Eng. \\
\hline & 2300 & ug $/ L$ & MetaTrace \\
\hline & 2200 & $u g / L$ & Gen. Eng. \\
\hline & 5 & ug/l & MetaTrace \\
\hline LI & 1 & ug/L & Gen. Eng. \\
\hline LT & 15 & $\operatorname{vg} / \mathrm{L}$ & MetaTrace \\
\hline$L T$ & 4 & ug/L & Gen. Eng. \\
\hline LT & 3 & ug $/ \mathrm{L}$ & MetaTrace \\
\hline
\end{tabular}


WELL FSB 7GB COLLECTED ON 01/13/90 LABORATORY ANALYSES CONTINUED

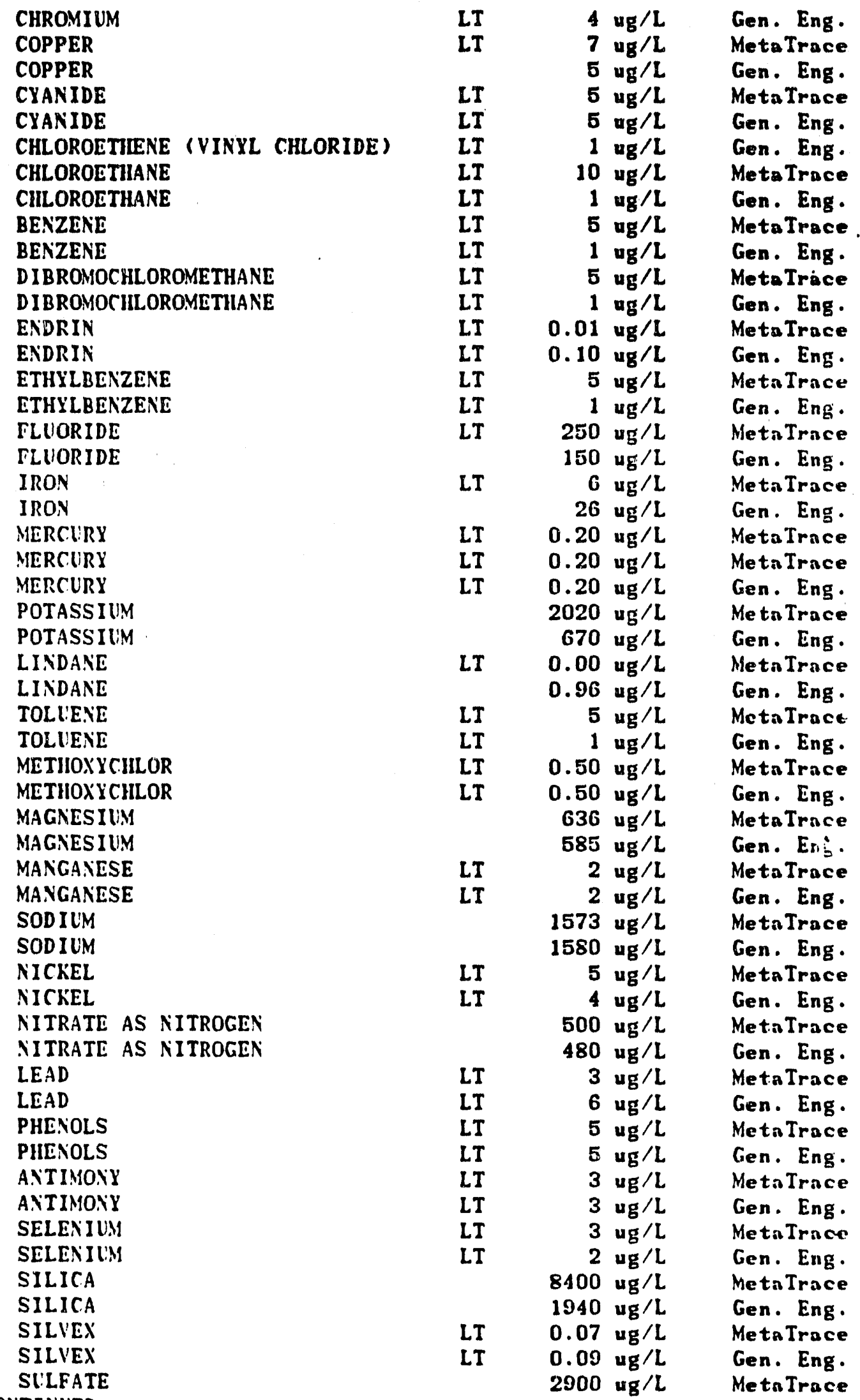




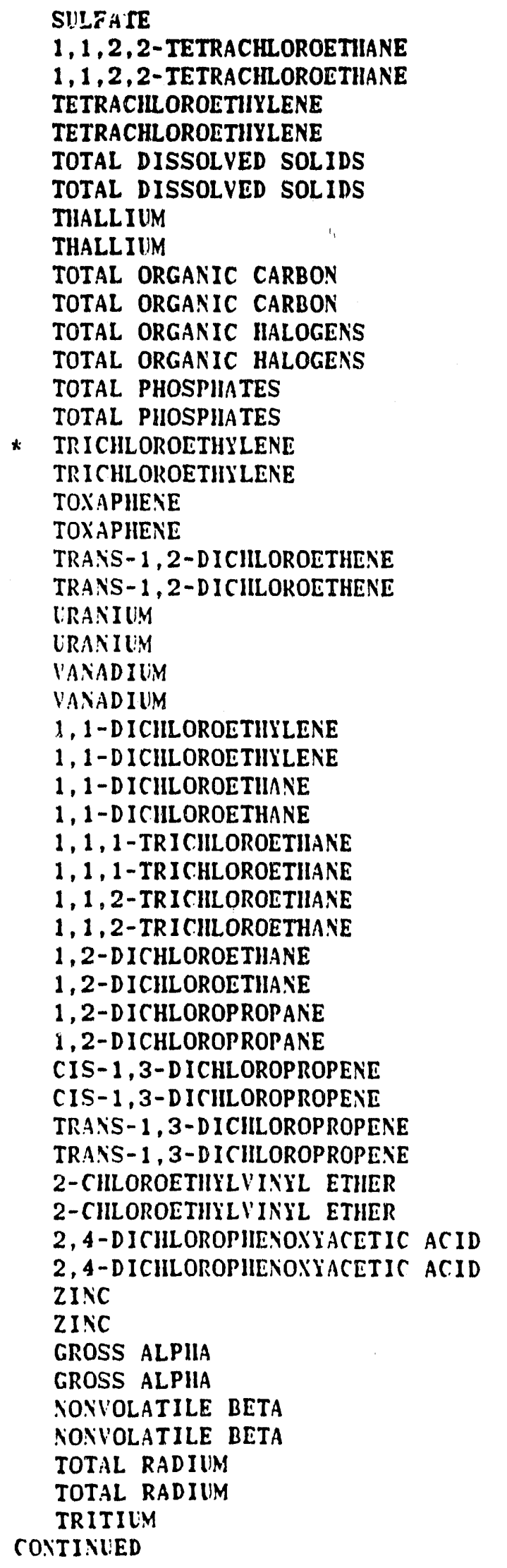

\begin{tabular}{|c|c|c|c|}
\hline LT & $\begin{array}{r}5000 \\
5\end{array}$ & $\operatorname{ug} / \mathrm{L}$ & $\begin{array}{l}\text { Gen. Eng. } \\
\text { MetaTrace }\end{array}$ \\
\hline LT & 1 & ug/L & Gen. Eng \\
\hline LT & 5.00 & ug/L & MetaTrnce \\
\hline LI & $\begin{array}{r}1.00 \\
81000\end{array}$ & $\begin{array}{l}u g / L \\
u g / L\end{array}$ & $\begin{array}{l}\text { Gen. Eng } \\
\text { MetnTrac }\end{array}$ \\
\hline LI & $\begin{array}{r}96000 \\
3\end{array}$ & $\begin{array}{l}\mathrm{ag} / L \\
\mathrm{ag} / \mathrm{L}\end{array}$ & $\begin{array}{l}\text { Gen. Eng } \\
\text { MetaTrace }\end{array}$ \\
\hline LT & 2 & ug $/ L$ & Gen. Eng. \\
\hline LT & 1000 & ug $/ L$ & MetaTrac \\
\hline & $\begin{array}{r}1000 \\
24\end{array}$ & $\begin{array}{l}\mathrm{ug} / \mathrm{L} \\
\mathrm{ug} / \mathrm{L}\end{array}$ & $\begin{array}{l}\text { Gen. Eng. } \\
\text { MetaTrace }\end{array}$ \\
\hline LT & & ug $/ L$ & Gen. Eng \\
\hline & $\begin{array}{l}423 \\
410\end{array}$ & ug/h & $\begin{array}{l}\text { Metin Irac } \\
\text { Gen. Eng }\end{array}$ \\
\hline & 14.0 & $u g / L$ & MetaTrac \\
\hline LI & 1.00 & $\mathrm{ug} / \mathrm{L}$ & Gen. Ing \\
\hline LI & 0.24 & $u g / L$ & MetaTrac \\
\hline LT & 1 & ugु $/ L$ & Gen. Eng \\
\hline LT & 5 & $\mathrm{ug} / \mathrm{L}$ & MetnTrace \\
\hline LT & 1 & $u g / L$ & Gen. Eng \\
\hline LI & 84 & $\mathbf{u g} / \mathrm{L}$ & MetaTrace \\
\hline LI & 1000 & ug $/ L$ & Gen. Eng \\
\hline LI & 3 & $u g / L$ & MetaTrace \\
\hline LI & 20 & ug $/ L$ & Gen. Eng \\
\hline LT & 5 & ug/L & MetaTrace \\
\hline LT & 1 & ug $/ L$ & Gen. Eng \\
\hline LI & 5 & $\operatorname{ug} / \mathrm{L}$ & MetaTrace \\
\hline LT & 1 & ug $/ L$ & Gen. Eng \\
\hline LT & 5 & $u g / L$ & MetaTrace \\
\hline LI & 1 & $u g / L$ & Gen. Eng \\
\hline LT & 5 & $u g / L$ & MetnTr.e \\
\hline$L I$ & 1 & ug $/ L$ & Gen, Eng \\
\hline LI & 5 & $u g / L$ & MetaTrace \\
\hline LT & 1 & $u_{g} / L$ & Gen. Eng \\
\hline LT & 5 & $u g / L$ & MetaTrace \\
\hline LI & 1 & $u g / L$ & Gen. Eng \\
\hline LT & 5 & $u g / L$ & MetaTrace \\
\hline LT & 1 & $u g / L$ & Gen. Eng \\
\hline LI & 5 & $u g / L$ & MetaTrace \\
\hline LI & 1 & $u g / L$ & Gen. Eng \\
\hline LI & 5 & $u g / L$ & MetaTrace \\
\hline LI & 1 & $\operatorname{ug} / \mathrm{L}$ & Gen. Eng \\
\hline LI & 0.46 & ug /L & MetaTrace \\
\hline L' & 0.30 & $\operatorname{ug} / \mathrm{L}$ & Gen. Eng \\
\hline LT & 9 & $u_{g} / L$ & MetaTrace \\
\hline & 5 & $u g / l$ & Gen. En \\
\hline LI & 3 & $p C i / L$ & MetaTrace \\
\hline LI & 3 & $\mathrm{pC} \mathrm{j} / \mathrm{L}$ & Gen. En \\
\hline LI & 5 & $p C i / L$ & MetaTrac \\
\hline & -2.70 & $p C i / L$ & Gen. Eng \\
\hline & +-0.40 & $\mathrm{PCi} / \mathrm{L}$ & MetaTrace \\
\hline LI & 1 & $\mathrm{p} \mathrm{C}_{\mathrm{i}} / \mathrm{L}$ & Ge \\
\hline LI & 1 & $\mathrm{pC} i / m L$ & $T$ \\
\hline
\end{tabular}


WELL FSB 76B COLLECTED ON 01/13/90 LABORATORY ANALYSES CONTINUED

\begin{tabular}{|c|}
\hline $\begin{array}{l}\text { TRITIUM } \\
\text { TRITIUM }\end{array}$ \\
\hline
\end{tabular}

NELL FSB 76B

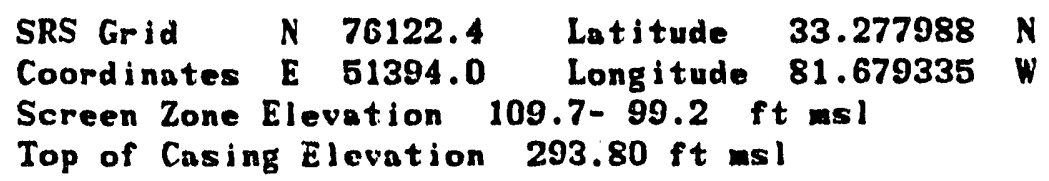

MEASUREMENTS CONDUCTED IN THE FIELD

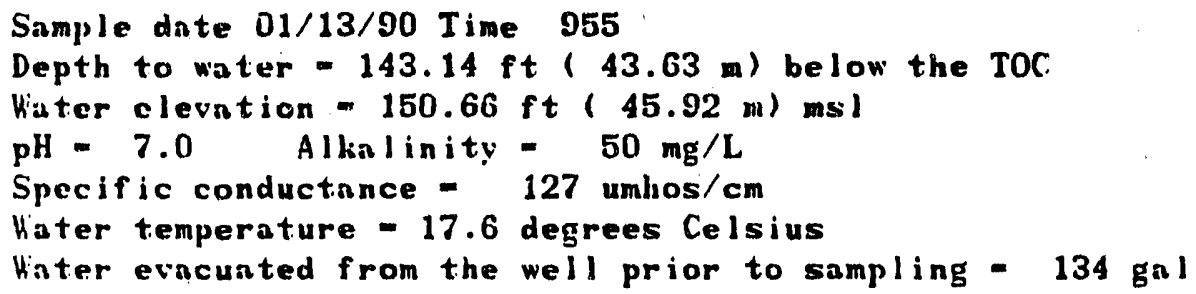

LABORATORY ANALYSES

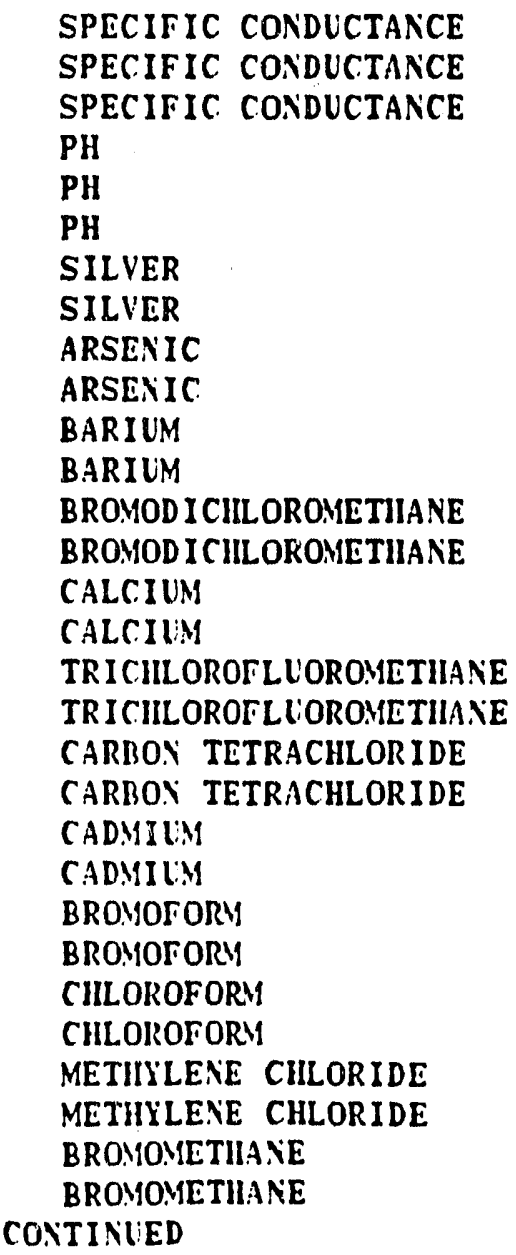

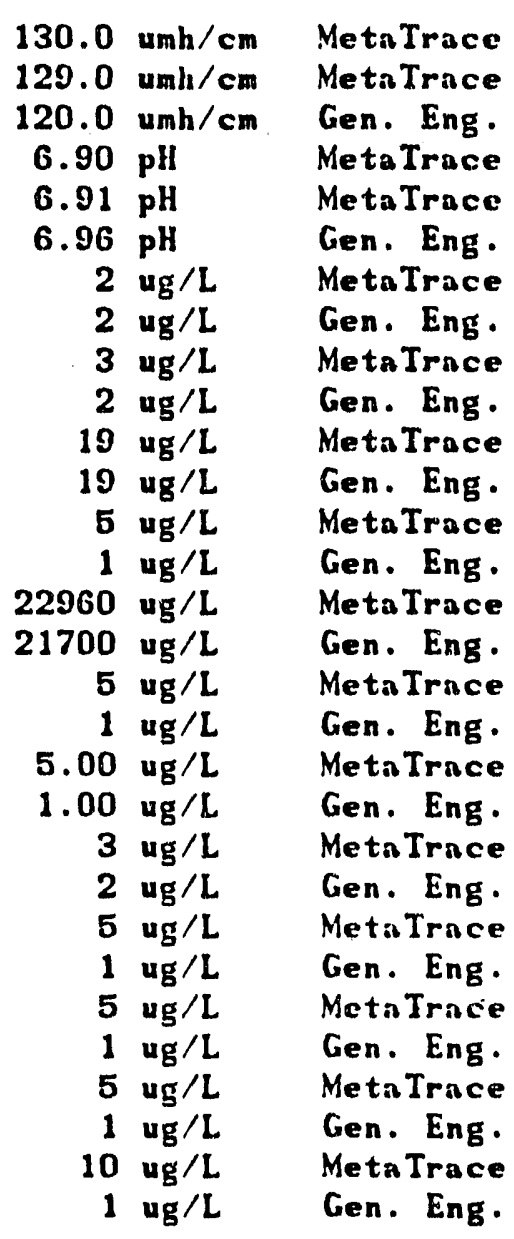


WELL FSB 76B COLLECTED ON 01/13/90 LABORATORY ANALYSES CONTINUED

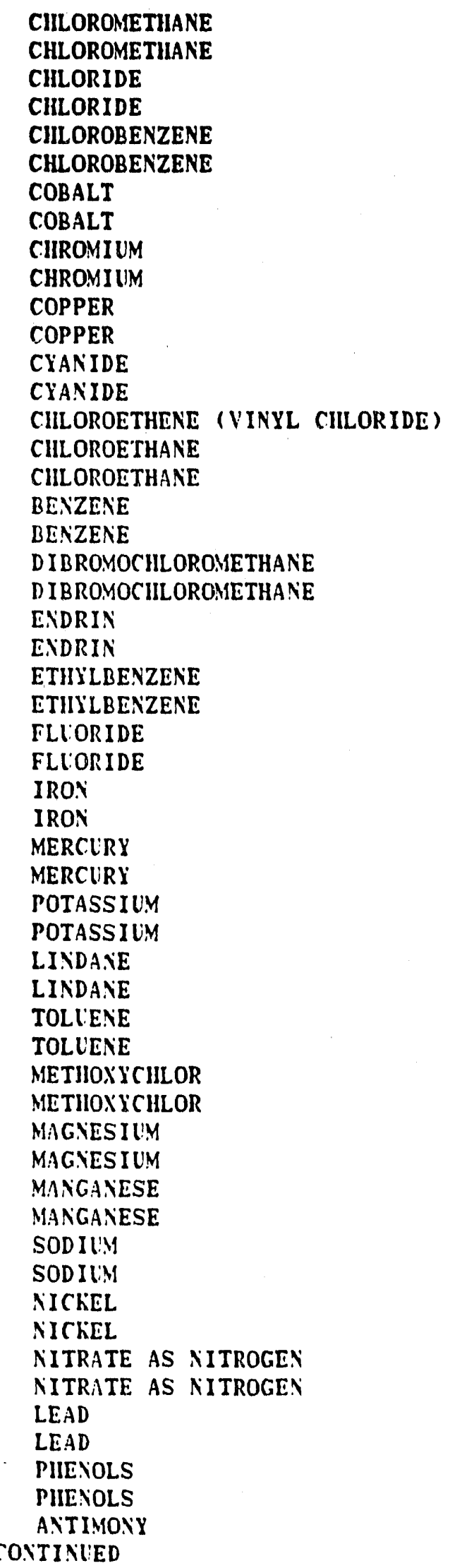

LI

LT

LT

LT

LT

LT

LT

LT

LT

LT

LT

LI

LT

LT

LT

LI

LT

LT

LT

LT

LT

LT

LT

LT

LT

LI

LI

LT

LI

LI

LT

LT

LT

LT

LT

LT

LT

LT

LT

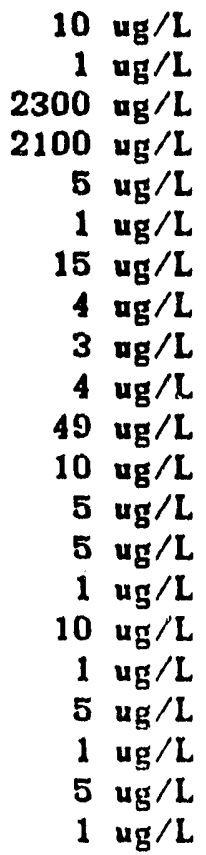

$0.01 \mathrm{ug} / \mathrm{L}$

$0.10 \mathrm{ug} / \mathrm{L}$

$5 \mathrm{ug} / \mathrm{L}$

$1 \mathrm{ug} / \mathrm{L}$

$250 \mathrm{ug} / \mathrm{L}$

$150 \mathrm{ug} / \mathrm{L}$

$6 \mathrm{ug} / \mathrm{L}$

$13 \mathrm{ug} / \mathrm{L}$

$0.20 \mathrm{ug} / \mathrm{L}$

$0.20 \mathrm{ug} / \mathrm{L}$

$1620 \mathrm{ug} / \mathrm{L}$

$743 \mathrm{ug} / \mathrm{L}$

$0.00 \mathrm{ug} / \mathrm{L}$

$0.50 \mathrm{ug} / \mathrm{L}$

$5 \mathrm{ug} / \mathrm{L}$

$1 \mathrm{ug} / \mathrm{L}$

$0.50 \mathrm{ug} / \mathrm{L}$

$0.50 \mathrm{ug} / \mathrm{L}$

$604 \mathrm{ug} / \mathrm{L}$

$652 \mathrm{ug} / \mathrm{L}$

$2 \mathrm{ug} / \mathrm{L}$

$2 \mathrm{ug} / \mathrm{L}$

$1597 \mathrm{ug} / \mathrm{L}$

1720 ug/L

$5 \mathrm{ug} / \mathrm{L}$

$9 \mathrm{ug} / \mathrm{L}$

520 ug $/ L$

$510 \mathrm{ug} / \mathrm{L}$

$3 \mathrm{ug} / \mathrm{L}$

$6 \mathrm{ug} / \mathrm{L}$

$5 \mathrm{ug} / \mathrm{L}$

5 ug/L

$3 \mathrm{ug} / \mathrm{L}$
MetaTrace

Gen. Eng .

MetaTrace

Gen. Eng.

MetaTrace

Gen. Eng.

MetaTrace

Gen. Eng.

MetaTrace.

Gen. Eng .

Metalrace

Gen. Eng.

MetaTrace

Gen. Eng.

Gen. Fng' .

MetaTrace

Gen. Fing.

MetaTrace

Gen. Ing.

MetaTrace

Gen. Eng.

MetuTrace

Gen. Eng.

MetaTrace

Gen. Eng.

MetaTrace

Gen. Eng.

MetaTrace

Gen. Eng .

MetaTrace

Gen. Eng .

MetaIrace

Gen. Eng.

MetaTrace

Gen. Eng.

MetaTrace

Gen. Eng.

MetaTrace

Gen. Eng -

MetaTrace

Gen. Eng.

MetnTrace

Gen. Eng .

MetaTrace

Gen. Eng.

MetaTrace

Gen. Eng.

Meta? ince

Gen. Eng.

MetaTrace

Cen. Eng.

MetaTrace

Gen. Eng.

MetaTrace 
VELL FSB 76B COLLECTED ON 01/13/90 LABORATORY ANALYSES CONTINUED

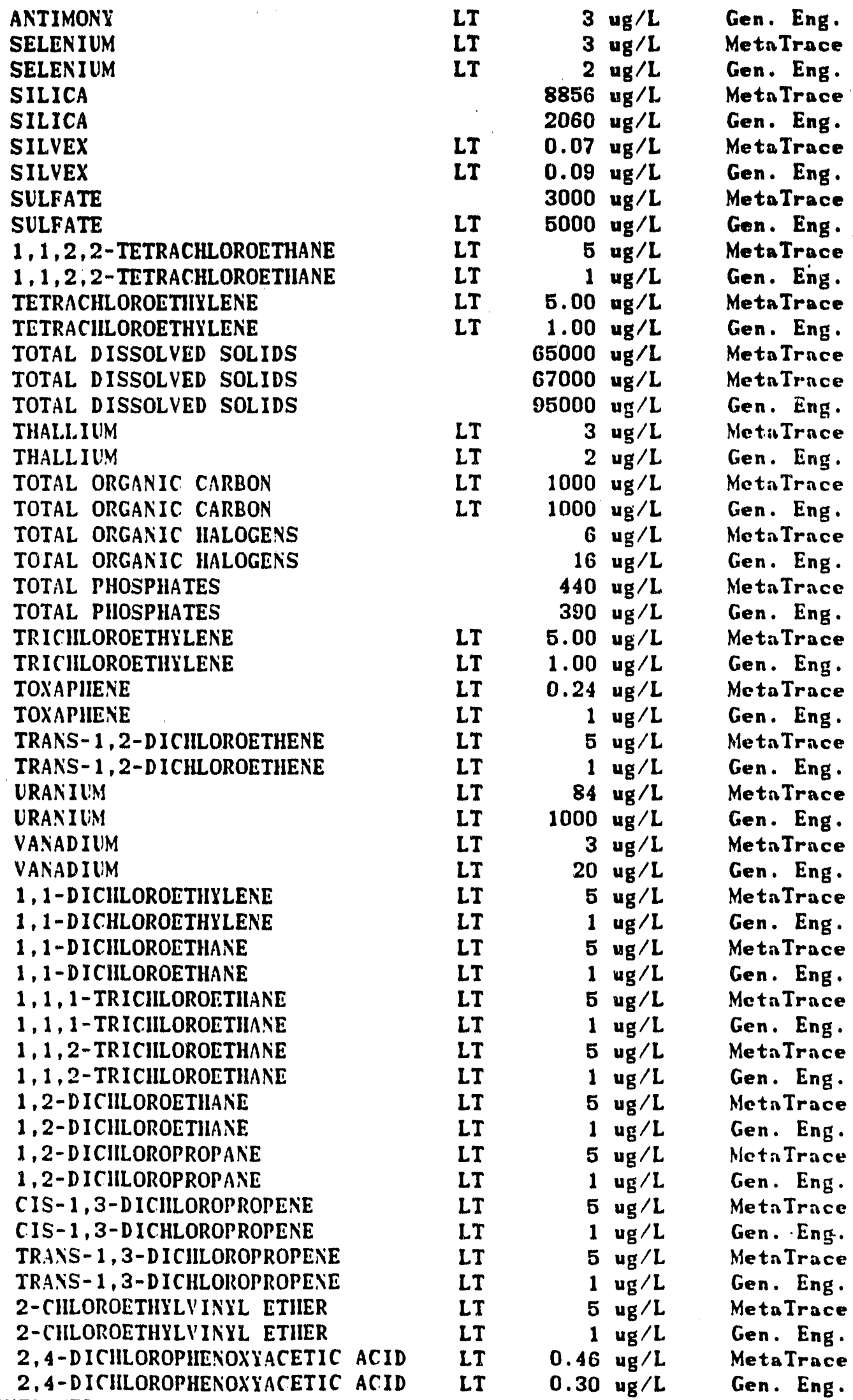


NELL FSB 7GB COLLECTED ON 01/13/90 LABORATORY ANALYSES CONTINUED

\author{
ZINC \\ ZINC \\ GROSS ALPHA \\ GROSS ALPHA \\ NONVOLATILE BETA \\ NONVOLATILE BETA \\ TOTAL RADIUM \\ TOTAL RADIUM \\ TRITIUM \\ TRITIUM
}

$\begin{array}{lrll} & 18 \mathrm{ug} / \mathrm{L} & \text { MetaTrnce } \\ & 8 \mathrm{ug} / \mathrm{L} & \text { Gen. Eng. } \\ \mathrm{LT} & 3 \mathrm{pCi} / \mathrm{L} & \text { MetaTrace } \\ \mathrm{LT} & 3 \mathrm{pCi} / \mathrm{L} & \text { Gen. Eng. } \\ \mathrm{LT} & 5 \mathrm{pCi} / \mathrm{L} & \text { MetaTrace } \\ \mathrm{LT} & 2 \mathrm{pCi} / \mathrm{L} & \text { Gen. Eng. } \\ 1.10+-0.40 \mathrm{pCi} / \mathrm{L} & \text { MetaTrace } \\ \mathrm{LT} & 1 \mathrm{pCi} / \mathrm{L} & \text { Gen. Eng. } \\ \mathrm{LT} & 1 \mathrm{pCi} / \mathrm{mL} & \text { MetaTrace. } \\ \mathrm{LT} & 0.70 \mathrm{pCi} / \mathrm{mL} & \text { Gen. Eng. }\end{array}$

KELL FSB 76C

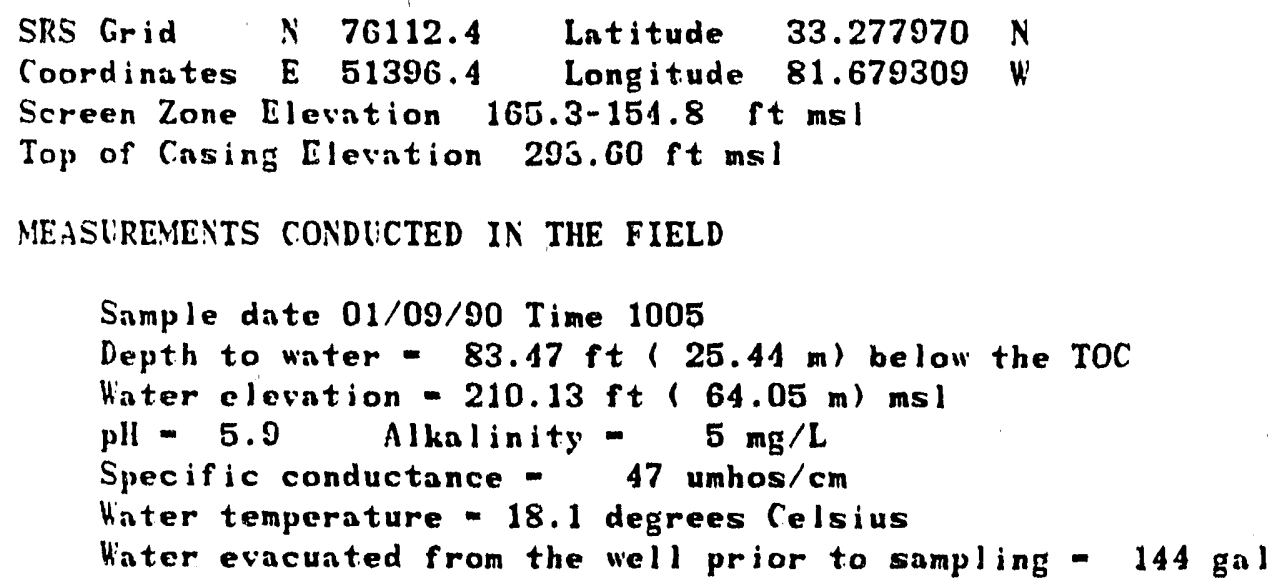

LABORATORY ANALYSES

\begin{tabular}{|c|c|c|c|c|}
\hline $\begin{array}{l}\text { SPECIFIC CONDLCTANCE } \\
\text { PH }\end{array}$ & & $\begin{array}{r}47.00 \\
6.01\end{array}$ & $\operatorname{umh}_{\mathrm{pH}} / \mathrm{cm}$ & $\begin{array}{l}\text { Gen. } \\
\text { Gen. }\end{array}$ \\
\hline SILVER & LT & 2 & ug $/ \mathrm{L}$ & Gen. \\
\hline ARSENIC & LT & 2 & $4 \mathrm{~g} / \mathrm{L}$ & Gen. \\
\hline BARILIM & & 5 & ug $/ L$ & Gen. \\
\hline BROMOD I CIILOROMETHANE & LT & 1 & ug $/ L$ & Gen. \\
\hline CALCIUM & & 5850 & ug $/ L$ & Gen. \\
\hline TRICIILOROFLUOROMETHANE & LT & 1 & ug $/ L$ & Gen. \\
\hline CARBON TETRACIILORIDE & LT & 1.00 & $\operatorname{ug} / \mathrm{L}$ & Gen. \\
\hline CADIIILY & LT & 2 & ug $/ \mathrm{L}$ & Gen. \\
\hline BROMOFORM & LT & 1 & ug $/ L$ & Gen. \\
\hline CIILOROFORY & LT & 1 & ug $/ \mathrm{L}$ & Gen. \\
\hline METHYLENE CIILORIDE & & 7 & $\operatorname{ug} / \mathrm{L}$ & Gen. \\
\hline BROMOMETIIANE & LT & 1 & $\mathrm{ug} / \mathrm{L}$ & Gen. \\
\hline CIILOROMETIIANE & LT & 1 & $u g / L$ & Gen. \\
\hline CHLORIDE & & 2200 & $\operatorname{ug} / \mathrm{L}$ & Gen. \\
\hline CULOROBENZENE & LT & 1 & $\mathrm{ug} / \mathrm{L}$ & Gen. \\
\hline COBALT & LT & 4 & ug $/ \mathrm{L}$ & Gen. \\
\hline CHRONI IM & LT & 4 & ug/L & Gen. \\
\hline COPPER & $L T$ & 4 & $\mathrm{ug} / \mathrm{L}$ & Gen. \\
\hline CYAIIDE & LT & 5 & ug/L & Gen. \\
\hline CIILOROETUENE (VINYL CHLORIDE) & LT & 1 & $\mathrm{ug} / \mathrm{L}$ & Gen. \\
\hline
\end{tabular}


WELL FSB 7GC COLLECTED ON 01/09/90 LABORATORY ANALYSES CONTINUED

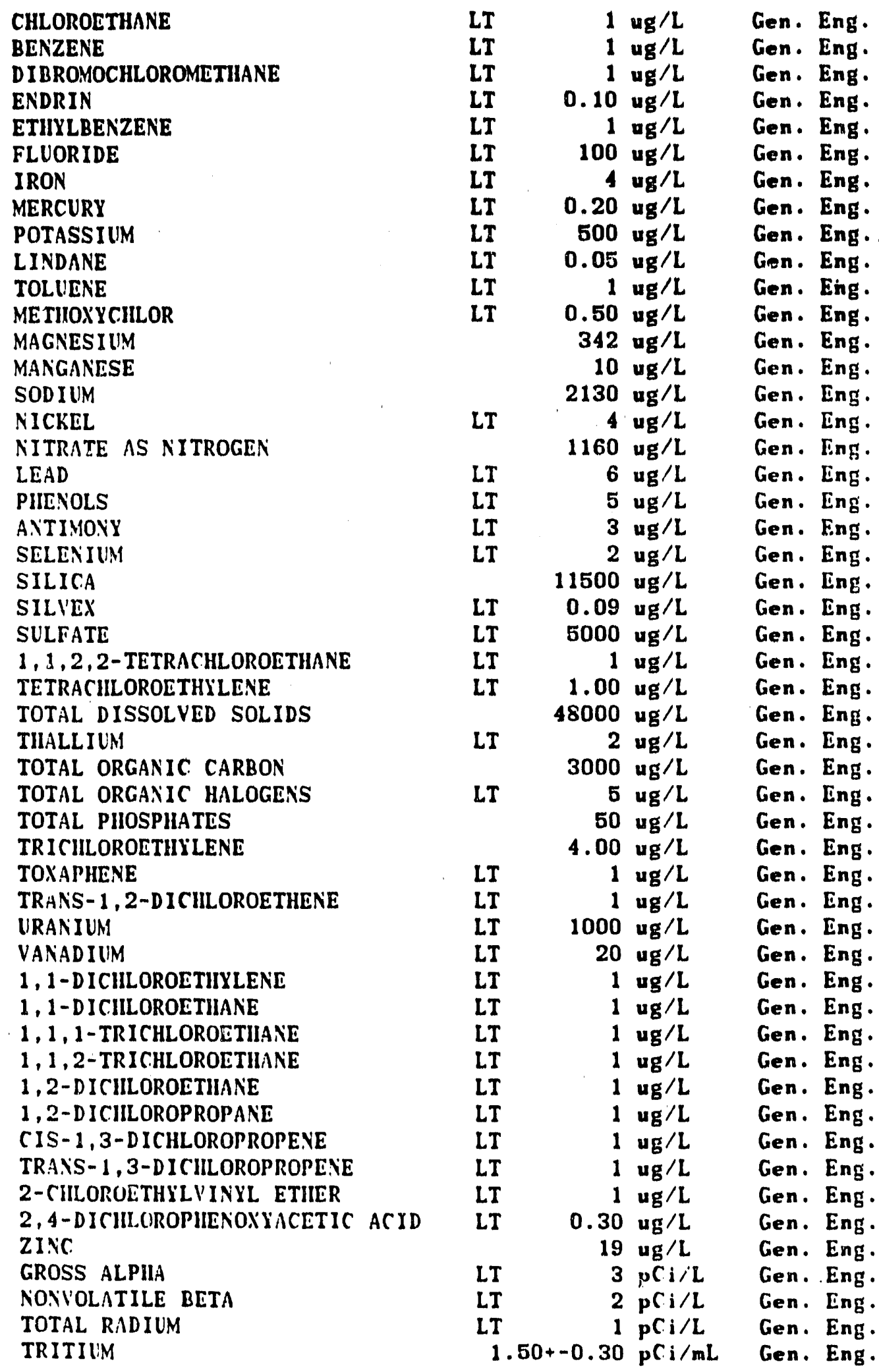


WELL FSB 77

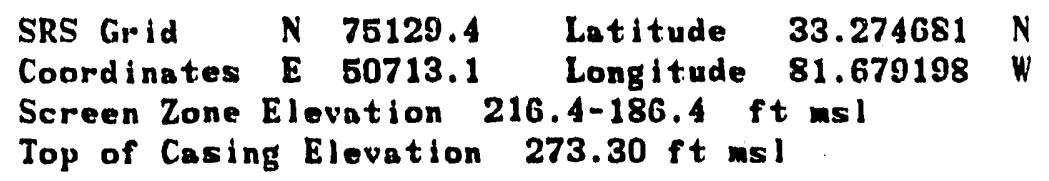

MEASUREMENTS CONDUCTED IN THE FIELD

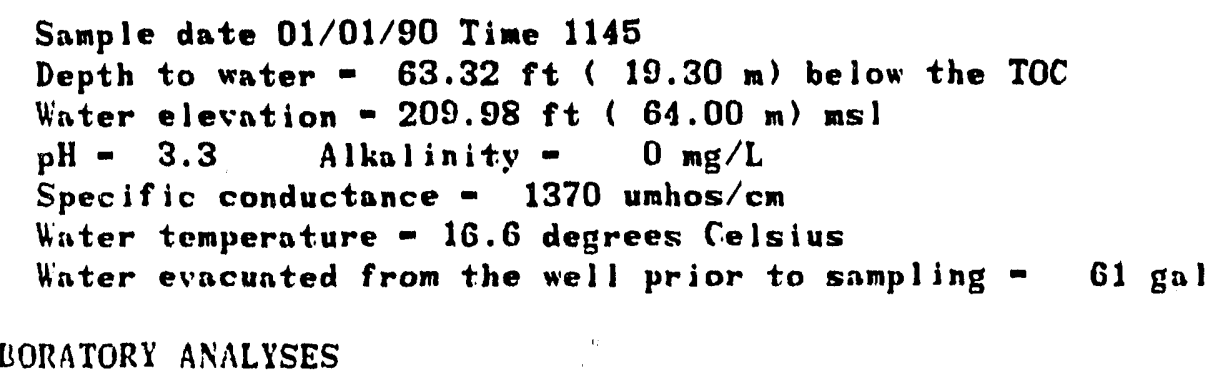

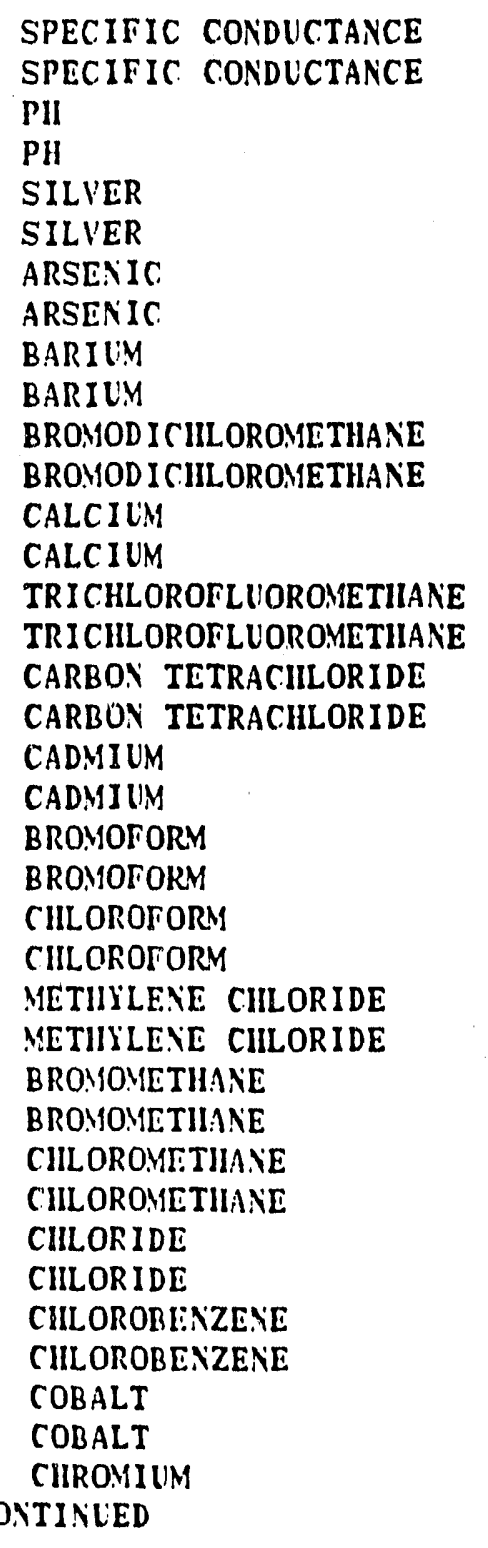

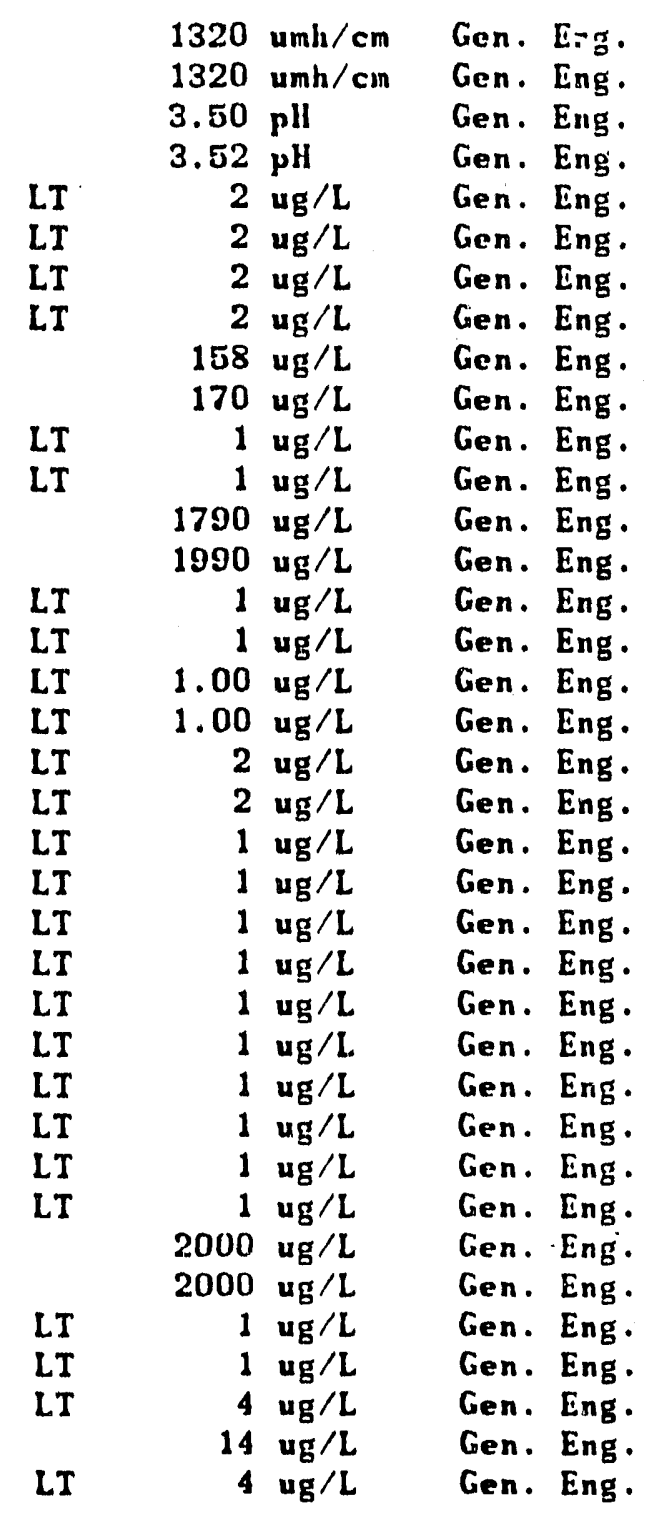


NELL ISB 77 COLLECIED ON 01/01/90 LABORATORY ANALYSES CONTINUED

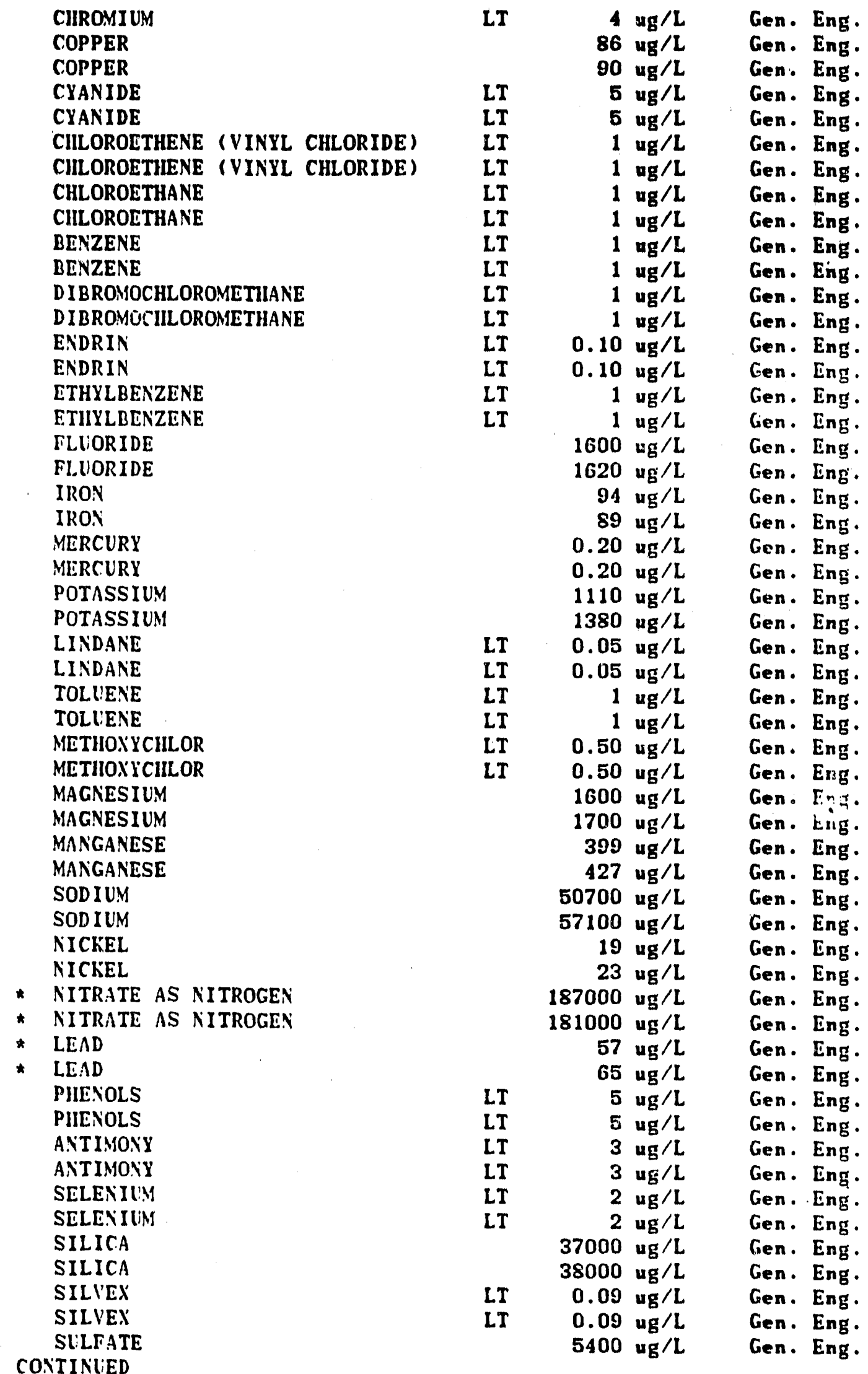




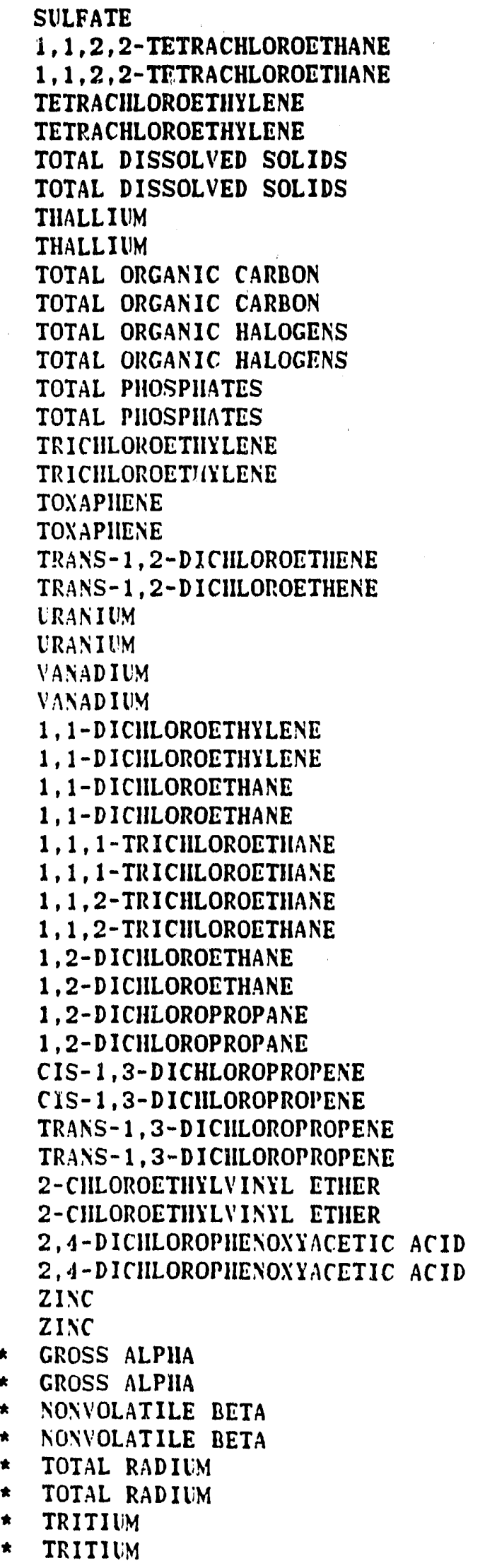

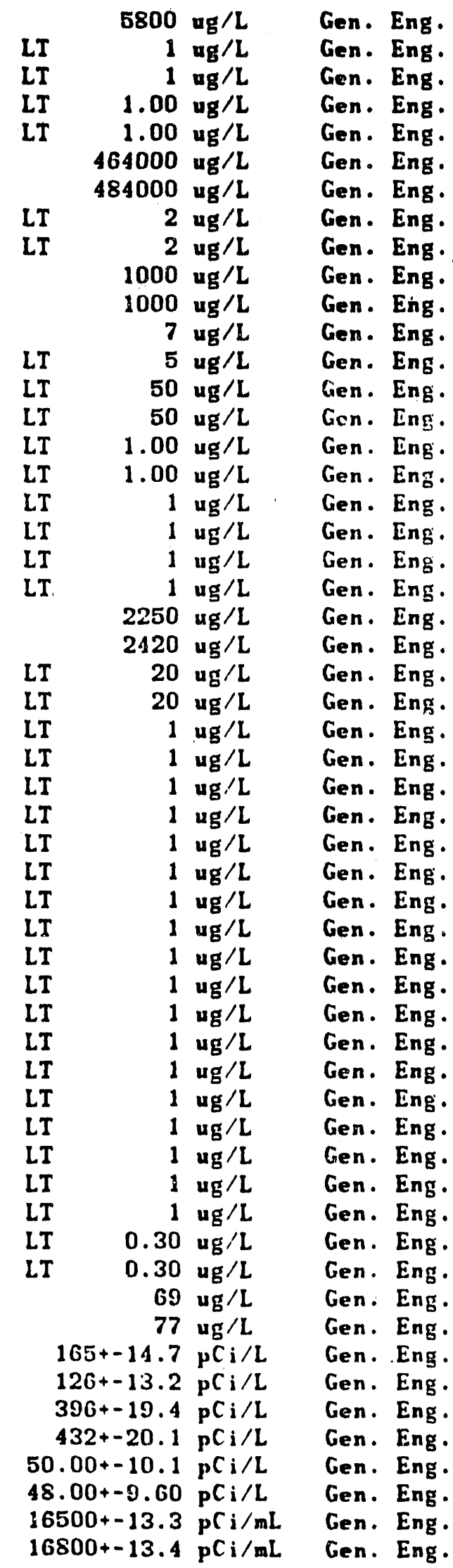


WELL FSB 78

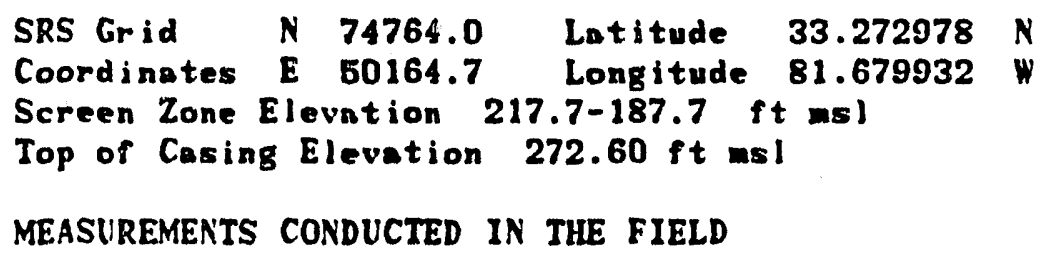

LABORATORY ANALYSES

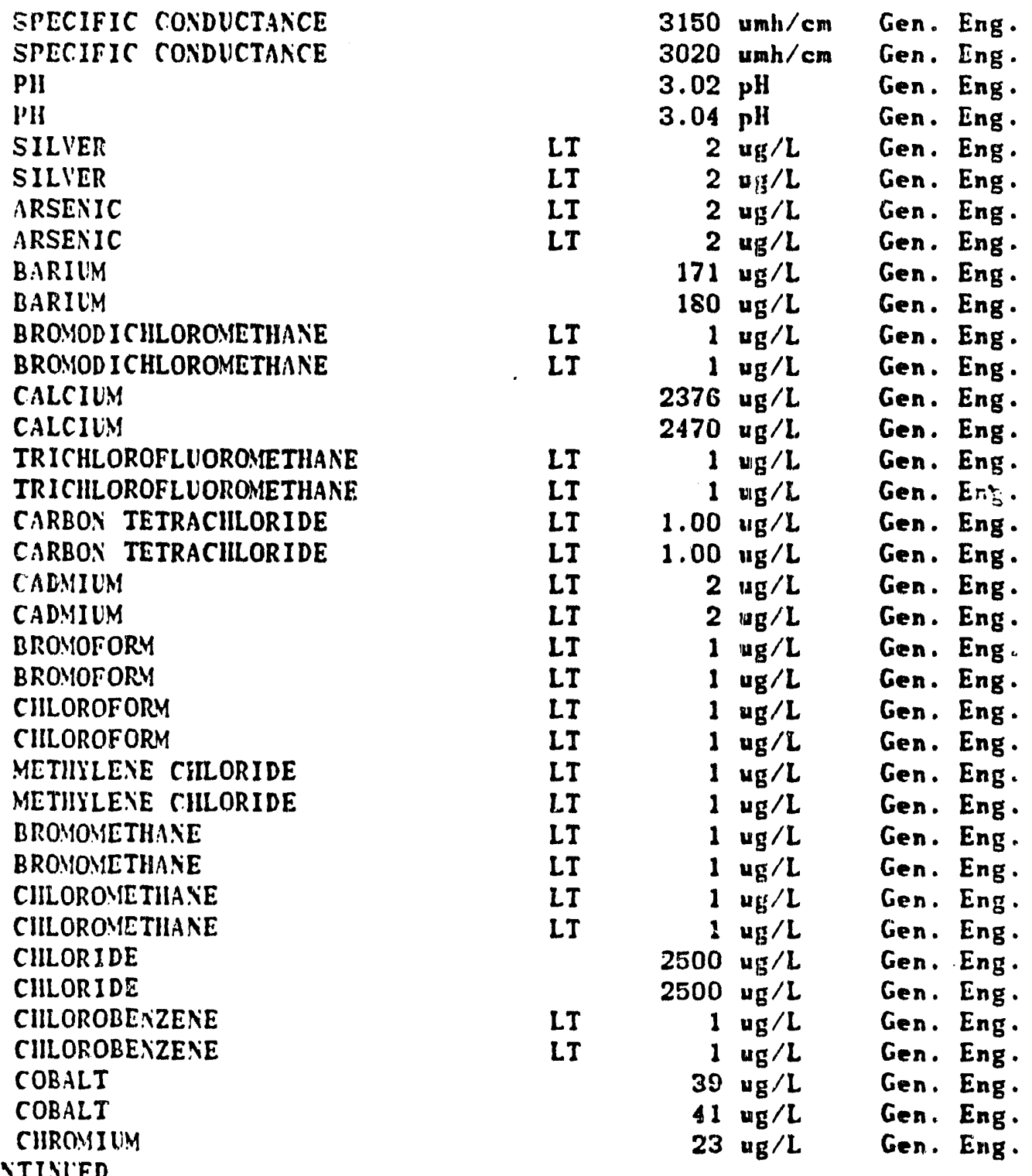


NELL FSB 78 COLLECTED ON 01/06/90 LABORATORY ANALYSES CONTINUED

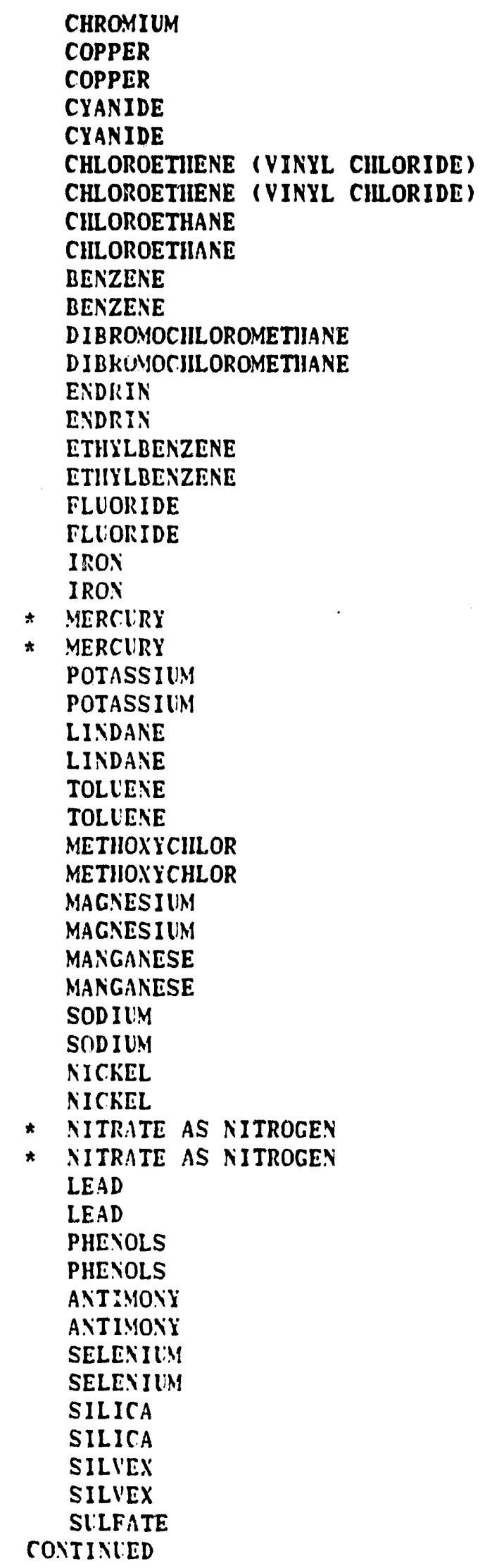

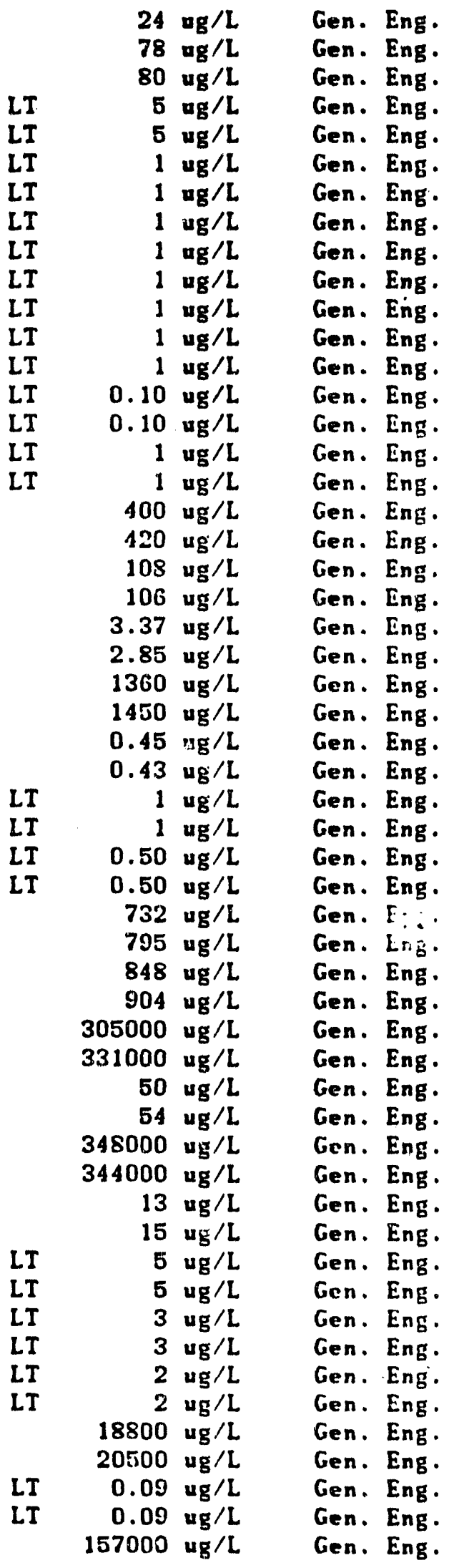


WELL FSB 78 COLLECTED ON 01/06/90 LABORATORY ANALYSES CONTINUED

\section{SULFATE}

$1,1,2,2$-TETRACHLOROETIIANE

1,1,2,2-TETRACIILOROETILANE TETRACHLOROETIIYLENE

TETRACHLOROETHYLENE TOTAL DISSOLVED SOLIDS TOTAL DISSOLVED SOLIDS

THALLIUM

THALLIUM

TOTAL ORGANIC CARBON

TOTAL ORGANIC CARBON

TOTAL ORGANIC HALOGENS

TOTAL ORGANIC HALOGENS

TOTAL PHOSPIIATES

TOTAL PHOSPHATES

TRICHLOROETHYLE! :

TRICIILOROETHYLENE

TOXAPIIENE

TOXAPIIENE

TRANS-1,2-DICHLOROETHENE

TRANS-1, 2-DICHLOROETHENE

IRANIUM

URANIUM

VANADIUM

VANADILM

1,1-DICHLOROETIIYLENE

1,1-DICHLOROETIIILENE

1,1-DICHLOROETHANE

1,1-DICHLOROETHANE

1,1,1-TRICHLOROETHANE

1,1,1-TRICIILOROETHANE

1,1,2-TRICHLOROETHANE

1,1,2-TRICIILOROETHANE

1,2-DICHLOROETIIANE

1,2-DICILLOROETHANE

1,2-DICHLOROPROPANE

1,2-DICHLOROPROPANE

CIS-1,3-DICHLOROPROPENE

CIS-1, 3-DICHLOROPROPENE

TRAIS-1,3-DICHLOROPROPENE

TRAIS-1,3-DICILOROPROPENE

2-CILOROETIIYLIINIL ETHER

2-CIILOROETIIILVINYL ETIER

2,4-DICILOROPIIENOXYACETIC ACID

2,4-DICHLOROPIENOKYACETIC ACID

ZIIC

ZINC

* Gross alpila

* Gross alphla

* Nofivolatile beta

* Noivolatile beta

TOTAL RADIUM

TOTAL RADIUM

- TRITIUM

- tritium

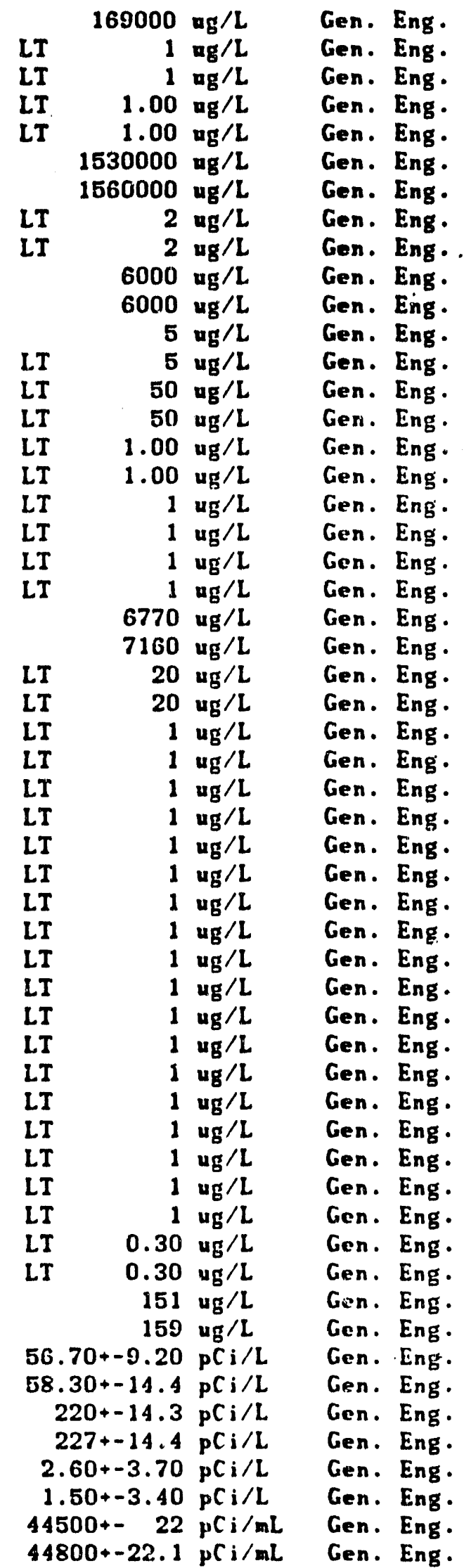


WELL FSB 78

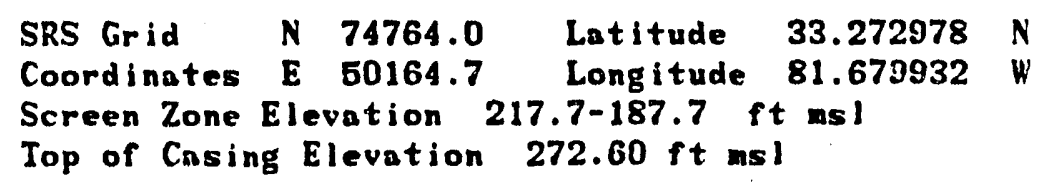

MEASUREMENTS CONDUCTED IN THE FIELD

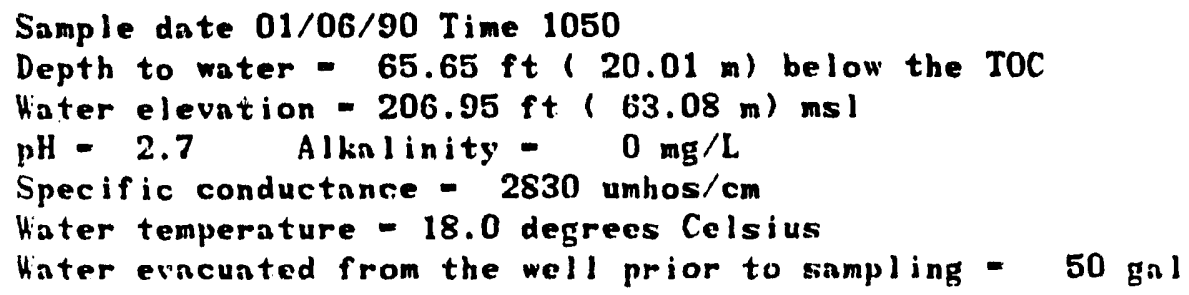

WELL FSB 78A

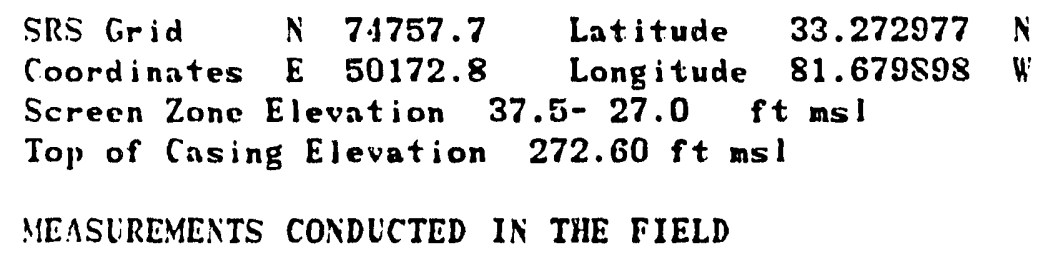

LABORATORY ANALYSES

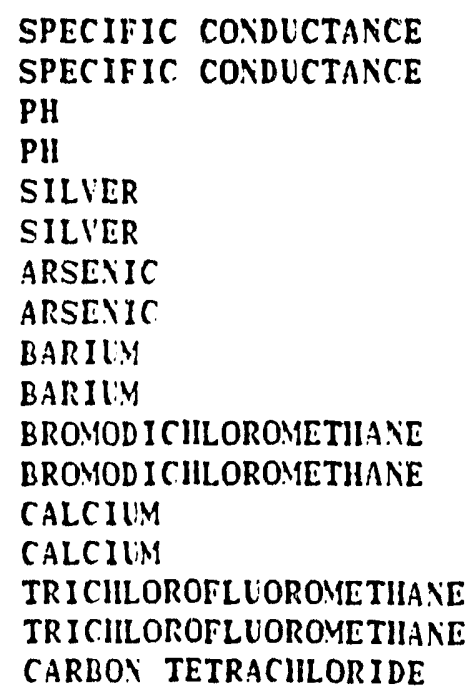

LT

LT

LT

LT

LT

LT

LT

LT

LI
$104.0 \mathrm{umh} / \mathrm{cm}$ $104.0 \mathrm{umh} / \mathrm{cm}$ $6.55 \mathrm{pH}$ $6.54 \mathrm{pH}$ $2 \mathrm{ug} / \mathrm{L}$

$2 \mathrm{ug} / \mathrm{L}$

$2 \mathrm{ug} / \mathrm{L}$ $2 \mathrm{ug} / \mathrm{L}$ $20 \mathrm{mg} / \mathrm{L}$ $19 \mathrm{\mu g} / \mathrm{L}$ $1 \mathrm{ug} / \mathrm{L}$ $1 \mathrm{ug} / \mathrm{L}$ $16600 \mathrm{ug} / \mathrm{L}$ $16100 \mathrm{ug} / \mathrm{L}$ $1 \mathrm{ug} / \mathrm{L}$ $1 \mathrm{ug} / \mathrm{L}$ $1.00 \mathrm{ug} / \mathrm{L}$
Gen. Eng. Gen. Eng. Gen. Eng. Gen. Eng. Gen. Eng. Gen. Eng. Gen. Eng. Gen. Eng. Gen. Eng. Gen. Eng. Gen. Eng. Gen. Eng. Gen. Eng. Gen. Eng. Gen. Eng. Gen. Eng. Gen. Eng. 
NELL FSB 78A COLLECTED ON 01/06/90 LABORATORY ANALYSES CONTINUED

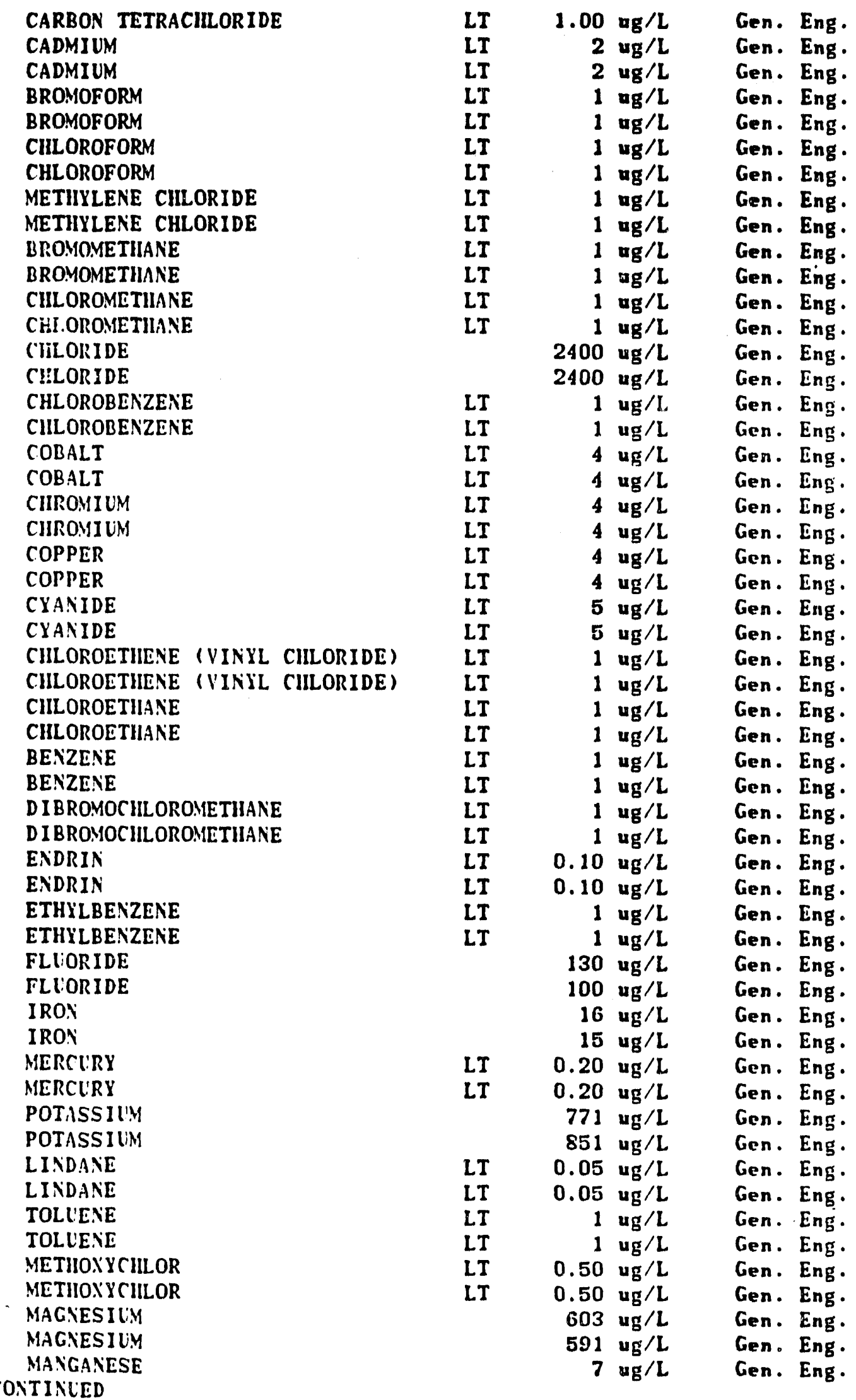


HELL FSB 78A COLLECTED ON 01/06/90 LABORATORY ANALYSES CONTINUED

MANGANESE
SODIUM
SODIUM
NICKEL
NICKEL
NITRATE AS NITROGEN
NITRATE AS NITROGEN
LEAD
LEAD
PIIENOLS
PHENOLS
ANTIMONY
ANTIMONY
SELENIUM
SELENIUM
SILICA
SILICA
SILVEX
SILVEX
SLIFATE
SLLFATE

$1,1,2,2$-TETRACILLOROETIIANE $1,1,2,2$-TETRACILLOROETIIANE TETRACIILOROETIYLENE TETRACIILOROETIIYLENE TOTAL DISSOLVED SOLIDS TOTAL DISSOLVED SOLIDS TIIALLIIM

TIIALLIUM

TOTAL ORGANIC CARBON TOTAL ORGANIC CARBON TOTAL ORGANIC HALOGENS TOTAL ORGANIC IIALOGENS TOTAL PIIOSPHATES TOTAL PHOSPHATES TRICIILOROETIIYLENE TRICIILOROETIYYLENE TOXAPHENE TOXAPHENE

TRANS-1,2-DICHLOROETHENE TRANS-1,2-DICILOROETHENE LRANIIMY LRANIIM VANIDILM VANADILY

1,1-D ICHLOROETHILENE 1,1-D ICILLOROETIIYLENE 1,1-DICILLOROETIIANE 1,1-DICILLOROETIIANE $1,1,1$-TRICHLOROETHANE $1,1,1$-TRICHLOROETIIANE 1,1,2-TRICHLOROETIIANE 1,1,2-TRICHLOROETHANE 1,2-DICILLOROETIIANE CONTINIED

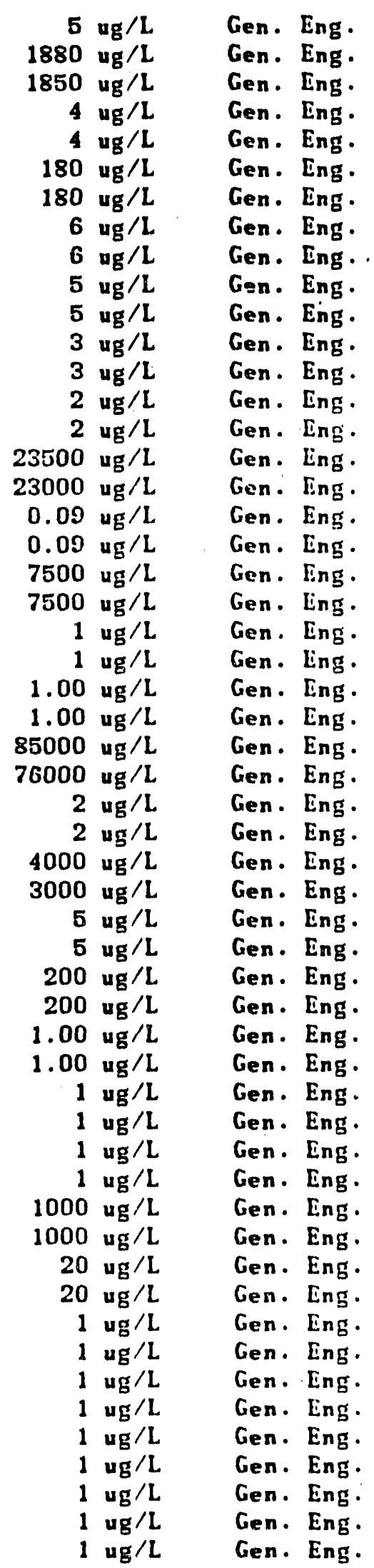

LT

LT

LT

LT

LT

LT

LT

LT

LT

LT

LT

LT

LT

LT

LT

LT

LT

LI

LT

LT

LT

LT

LT

LI

LT

LT

LI

L.T

LI

LT

LT

LT

LT

LT

LT

LT

LT

LT

LT 
WELL FSB 78A COLLECTED ON 01/06/90 LABORATORY ANALYSES CONTINUED

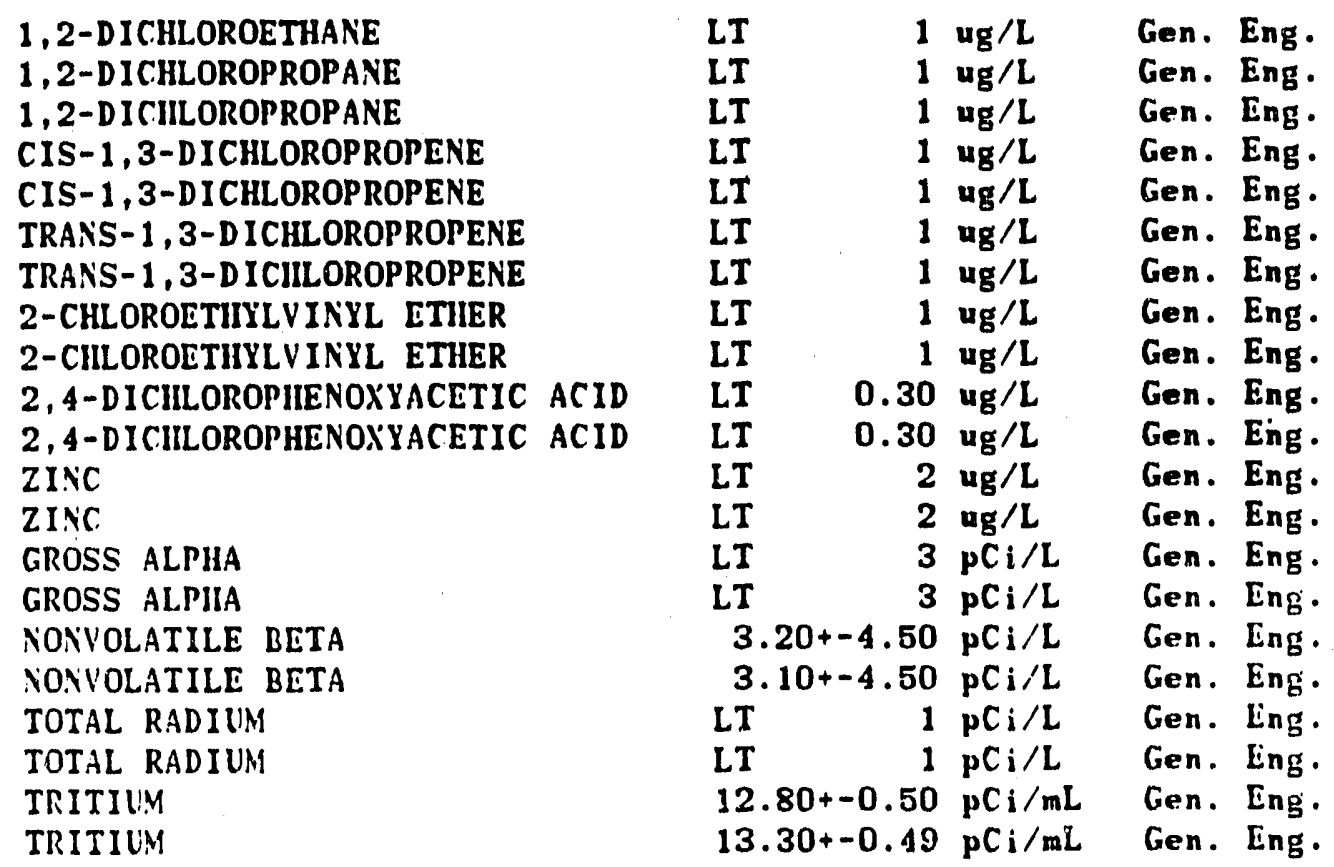

HELL FSB 78B

SRS Grid N 74765.9 Latitude $33.273005 N$

Coordinates E 50178.8 Longitude 81.679898. W'

Screen Zone Elevation 92.8- 82.4 ft msl

Top of Casing Elevation $272.80 \mathrm{ft} \mathrm{ms}$ !

MEASUREMENTS CONDUCTED IN THE FIELD

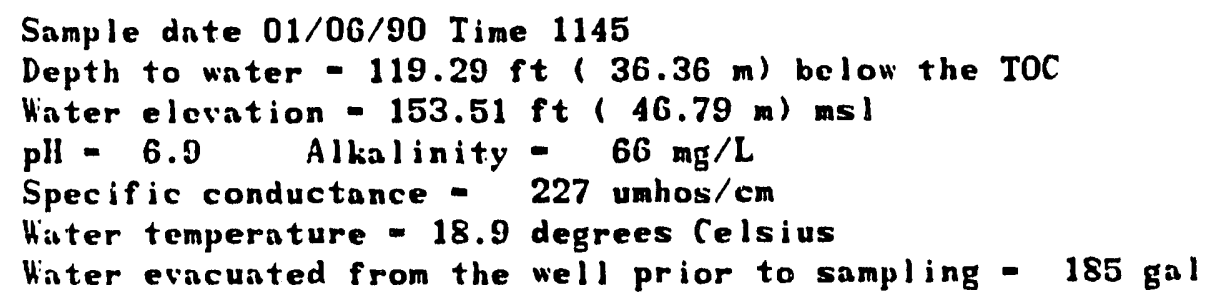

\section{LABORITORY ANALYSES}

SPECIFIC CONDUCTANCE

PII

SILVER

ARSENIC

BARIUM

BROMODICILORONETHANE

CALCIUM

IRICILLOROFLLORONETIIANE

CARBON TETRACILLORIDE

CADMIIM

BROSOFORM

CONTINLED

$\begin{array}{lrll} & 200.0 \mathrm{umh} / \mathrm{cm} & \text { Gen. Eng. } \\ & 7.70 \mathrm{pH} & \text { Gen. Eng. } \\ \text { LT } & 2 \mathrm{ug} / \mathrm{L} & \text { Gen. Eng. } \\ \text { LT } & 2 \mathrm{ug} / \mathrm{L} & \text { Gen. Eng. } \\ & 44 \mathrm{ug} / \mathrm{L} & \text { Gen. Eng. } \\ \text { LT } & 1 \mathrm{ug} / \mathrm{L} & \text { Gen. Eng. } \\ & 38300 \mathrm{ug} / \mathrm{L} & \text { Gen. Eng. } \\ \text { LT } & 1 \mathrm{ug} / \mathrm{L} & \text { Gen. Eng. } \\ \text { LT } & 1.00 \mathrm{ug} / \mathrm{L} & \text { Gen. Eng. } \\ \text { LT } & 2 \mathrm{ug} / \mathrm{L} & \text { Gen. Eng. } \\ \text { LT } & 1 \mathrm{ug} / \mathrm{L} & \text { Gen. Eng. }\end{array}$


NELL FSB 78B COLLECTED ON 01/06/90 LABORATORY ANALYSES CONTINLED

\begin{tabular}{|c|c|c|c|c|c|}
\hline CHLOROFORM & LI & 1 & $u g / L$ & Gen. & Eng. \\
\hline METHYLENE CHLORIDE & LT & 1 & ug $/ \mathrm{L}$ & Gen. & Eng. \\
\hline BROMOMETHANE & LT & 1 & ug $/ \mathrm{L}$ & Gen. & Eng. \\
\hline CILLOROMETHANE & LT & 1 & ug $/ L$ & Gen. & Eng. \\
\hline CIILORIDE & & 2000 & ug $/ L$ & Gen. & Eng. \\
\hline CHLOROBENZENE & LT & 1 & $\mu \mathrm{gg} / \mathrm{L}$ & Gen. & Eng. \\
\hline COBALI & LT & 4 & $\operatorname{ug} / \mathrm{L}$ & Gen. & Eng. \\
\hline CHROMIUM & LT & 4 & $u g / L$ & Gen. & Eng. \\
\hline COPPER & LT & 4 & ug $/ \mathfrak{L}$ & Gen. & Eng. \\
\hline CYANIDE & LI & 5 & ug $/ L$ & Gen. & Eng. \\
\hline CIILOROF:TIIENE (VINYL CHLORIDE) & LT & 1 & $u g / L$ & Gen. & Eng. \\
\hline CIILOROETHANE & LT & 1 & ug $/ L$ & Gen. & Eng. \\
\hline BENZENE & LT & 1 & ug $/ L$ & Gen. & Eng. \\
\hline DIBROMOCHLOROMETIIANE & LI & 1 & $\mathrm{ug} / \mathrm{L}$ & Gen. & Eng. \\
\hline ENDRIN & LT & 0.10 & $\mathrm{ng} / \mathrm{L}$ & lien. & Eng. \\
\hline ETIIYLBENZENE & LT & 1 & ug $/ L$ & Gen. & Eng. \\
\hline FLUORIDE & LT & 100 & ug $/ \mathrm{L}$ & Gen. & Eng. \\
\hline IROX & & 12 & $u_{g} / L$ & Gen. & Eng. \\
\hline MERCURY & I.T & 0.20 & $\operatorname{ug} / \mathrm{L}$ & Gen. & Eng. \\
\hline POTASSILY & & 560 & ug $/ \mathrm{L}$ & Gen. & Eng. \\
\hline LINDANE & LT & 0.05 & ug $/ \mathrm{L}$ & Gen. & Eng. \\
\hline TOLUENE & LT & 1 & ug $/ L$ & Gen. & Eng. \\
\hline METHOXYCHLOR & LT & 0.50 & $\mathrm{ug} / \mathrm{L}$ & Gen. & Eng. \\
\hline MAGNESIUM & & 783 & $4 \mathrm{gg} / \mathrm{L}$ & Gen. & Eng. \\
\hline MANGANESE & & 4 & ug $/ \mathrm{L}$ & Gen. & Eng. \\
\hline SODIUM & & 4110 & ug $/ L$ & Gen. & Eng. \\
\hline NICKEL & LT & 4 & ug $/ L$ & Gen. & Eng. \\
\hline NITRATE AS NITROGEN & & 7400 & ug $/ \mathrm{L}$ & Gen. & Eng. \\
\hline LEAD & LT & $\mathbf{G}$ & ug $/ \mathrm{L}$ & Gen. & Eng. \\
\hline PIIENOLS & LT & $\mathbf{5}$ & ug $/ \mathrm{L}$ & Gen. & Eng. \\
\hline ANTIMONY & LT & 3 & ug $/ \mathrm{L}$ & Gen. & Eng. \\
\hline SELENIUIM & $\operatorname{LT}$ & 2 & ug $/ \mathrm{L}$ & Gen. & Eng. \\
\hline SILICA & & 17800 & $\mathrm{ug} / \mathrm{L}$ & Gen. & Eng. \\
\hline SILVEX & LT & 0.09 & $4 \mathrm{~g} / \mathrm{L}$ & Gen. & Eng. \\
\hline SULFATE & LT & 5000 & ug/L & Gen. & Eng. \\
\hline $1,1,2,2$-TETRACHLOROETHANE & LT & 1 & ug $/ \mathrm{L}$ & Gen. & Eng. \\
\hline TETRACILOROETHYLENE & LT & 1.00 & ug $/ \mathrm{L}$ & Gen. & Eng \\
\hline TOTAL DISSOLVED SOLIDS & & 159000 & $\operatorname{ug} / \mathrm{L}$ & Gen. & Eng \\
\hline TIIALLIVIM & LI & 2 & ug/L & Gen. & Eng \\
\hline TOTAL ORGANIC CARBON & & 14000 & $4 \mathrm{~g} / \mathrm{L}$ & Gen. & Eng \\
\hline TOTAL ORGANIC HALOGENS & LT & $\mathbf{5}$ & ug $/ \mathrm{L}$ & Gen. & Eng. \\
\hline TOTAL PHOSPIIITES & & 70 & 4g/L & Gen. & $E$ \\
\hline TRICHLOROETIILENE & LT & 1.00 & ug/L & Gen. & Eng \\
\hline TOXAPIIENE & LT & 1 & $u g / L$ & Gen. & Eng. \\
\hline TRANS-1, 2-DICIILOROETHENE & LT & 1 & $4 \mathrm{~g} / \mathrm{L}$ & Gen. & Eng \\
\hline IRANILY & LT & 1000 & $4 \mathfrak{g} / L$ & Gen. & $F_{n}$ \\
\hline VANADIUM & LT & 20 & $\operatorname{ug} / \mathrm{L}$ & Gen. & $\mathrm{E}$ \\
\hline 1,1-DICHLOROETHILENE & LT & 1 & $4 \mathrm{~g} / \mathrm{L}$ & Gen. & Eng \\
\hline 1,1-DICILLOROETHANE & LT & 1 & ug $/ \mathrm{L}$ & Gen. & $\Gamma$ \\
\hline 1,1,1-TRICILLOROETIIANE & LT & 1 & $\mathrm{ug} / \mathrm{L}$ & Gen. & \\
\hline $1,1,2$-TRICHLOROETHANE & LT & 1 & $\operatorname{ug} / \mathrm{L}$ & Gen. & En \\
\hline 1,2-D ICILLOROETHANE & LT & 1 & ug/L & Gen. & $\Gamma$ \\
\hline 1,2-DICIILOROPROPANE & LT & 1 & ug $/ \mathrm{L}$ & Cen. & En \\
\hline CIS-1,3-DICILOROPROPENE & L.T & 1 & ug/L & Gen. & En \\
\hline
\end{tabular}


WELL FSB 78B COLLECTED ON 01/06/90 LABORATORY ANALYSES CONTINUED

$\begin{array}{llrl}\text { TRANS-1,3-DICHLOROPROPENE } & \text { LT } & 1 \mathrm{ug} / \mathrm{L} & \text { Gen. Eng. } \\ \text { 2-CHLOROETHYLVINYL ETHER } & \text { LT } & 1 \mathrm{ug} / \mathrm{L} & \text { Gen. Eng. } \\ \text { 2,4-DICKLOROPHENOXYACETIC ACID } & \text { LT } & 0.30 \mathrm{ug} / \mathrm{L} & \text { Gen. Eng. } \\ \text { ZINC } & \text { LT } & 2 \mathrm{ug} / \mathrm{L} & \text { Gen. Eng. } \\ \text { GROSS ALPHA } & \text { LT } & 3 \mathrm{pCi} / \mathrm{L} & \text { Gen. Eng. } \\ \text { NONVOLATILE BETA } & \text { LT } & 2 \mathrm{pCi} / \mathrm{L} & \text { Gen. Eng. } \\ \text { TOTAL RADIUM } & \mathrm{LT} & 1 \mathrm{pCi} / \mathrm{L} & \text { Gen. Eng. } \\ \text { * TRITIUM } & & 264+-1.66 \mathrm{pCi} / \mathrm{mL} & \text { Gen. Eng. }\end{array}$

WELL FSB $78 C$

SRS Grid N 74772.5 Latitude 33.273006 N

Coordinates E 50170.2 Longitude 81.679934 W

Screen Zone Elevation 151.4-141.6 ft msl

Top of Casing Elevation $273.50 \mathrm{ft} \mathrm{ms}$ l

MFASUREMENTS CONDUCTED IN THE FIELD

Sample date 01/07/90 Time 1030

Depth to water - $67.32 \mathrm{ft}(20.52 \mathrm{~m})$ below the TOC

Hater elevation $-206.18 \mathrm{ft}(62.84 \mathrm{~m}) \mathrm{ms}$ )

pll $=4.2$ Alkalinity $=0 \mathrm{mg} / \mathrm{L}$

Specific conductance - 1805 umhos/cm

Hater tempernture - 18.0 degrees Celsius

Hater exncuated from the well prior to sampling - $25 \mathrm{gal}$

The well went dry during purging.

LABORATORI ANALYSES

SPECIFIC. CONDUCTANCE

PH

SILVER

ARSENIC

BARIUM

BROMOD ICHLOROMETHANE

CALCIUM

TRICIILOROFLUOROMETIIANE

CARBON TETRACIILORIDE

* CADMIUM

BROMOFORM

CILLOROFORM

METIILENE CIILORIDE

BROMOMETIIANE

CHLOROMETHANE

CHLORIDE

CHLOROBENZENE

COBALT

CHROMIUM

COPPER

CYANIDE

CHLOROETHENE (VINIL CHLORIDE)

CILLOROETIIANE

BENZENE

CONTINLED

$\begin{aligned} 1890 \mathrm{umh} / \mathrm{cm} & \text { Gen. Eng. } \\ 4.32 \mathrm{pH} & \text { Gen. En. } \\ 2 \mathrm{ug} / \mathrm{L} & \text { Gen. Eng. } \\ 3 \mathrm{ug} / \mathrm{L} & \text { Gen. Eng. } \\ 463 \mathrm{ug} / \mathrm{L} & \text { Gen. Eng. } \\ 1 \mathrm{ug} / \mathrm{L} & \text { Gen. Eng. } \\ 157000 \mathrm{ug} / \mathrm{L} & \text { Gen. Eng. } \\ 1 \mathrm{ug} / \mathrm{L} & \text { Gen. Eng. } \\ 1.00 \mathrm{ug} / \mathrm{L} & \text { Gen. Eng. } \\ 14 \mathrm{ug} / \mathrm{L} & \text { Gen. Eng. } \\ 1 \mathrm{ug} / \mathrm{L} & \text { Gen. Eng. } \\ 1 \mathrm{ug} / \mathrm{L} & \text { Gen. Eng. } \\ 1 \mathrm{ug} / \mathrm{L} & \text { Gen. Eng. } \\ 1 \mathrm{ug} / \mathrm{L} & \text { Gen. Eng. } \\ 1 \mathrm{ug} / \mathrm{L} & \text { Gen. Eng. } \\ 2600 \mathrm{ug} / \mathrm{L} & \text { Gen. Eng. } \\ 1 \mathrm{ug} / \mathrm{L} & \text { Gen. Eng. } \\ 201 \mathrm{ug} / \mathrm{L} & \text { Gen. Eng. } \\ 1 \mathrm{ug} / \mathrm{L} & \text { Gen. Eng: } \\ 16 \mathrm{ug} / \mathrm{L} & \text { Gen. Eng. } \\ 5 \mathrm{ug} / \mathrm{L} & \text { Gen. Eng. } \\ 1 \mathrm{ug} / \mathrm{L} & \text { Gen. Eng. } \\ 1 \mathrm{ug} / \mathrm{L} & \text { Gen. Eng. } \\ 1 \mathrm{ug} / \mathrm{L} & \text { Gen. Eng. }\end{aligned}$


NELL FSB 78C COLLECTED ON 01/07/90 LABORATORY ANALYSES CONTINUED

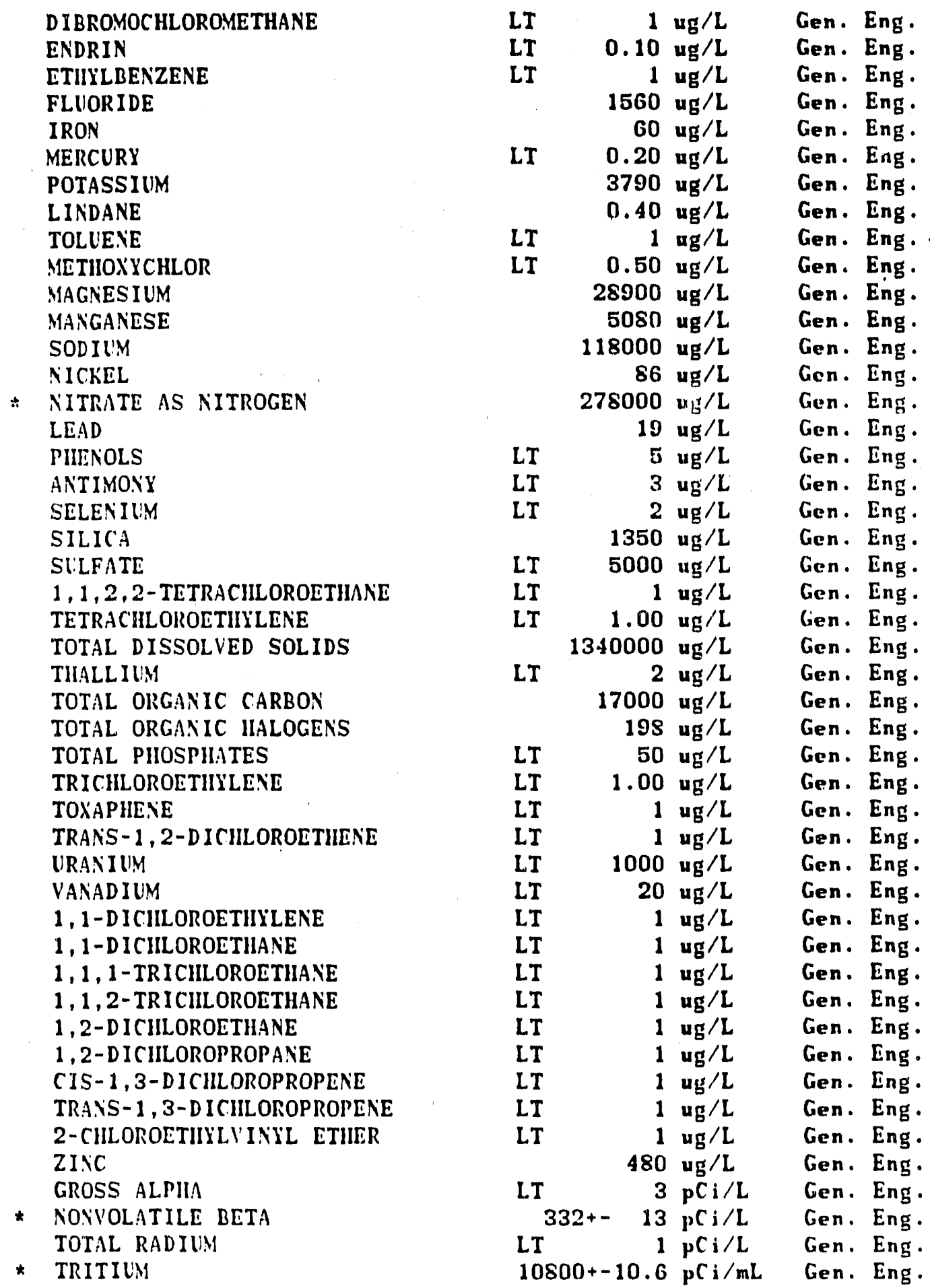




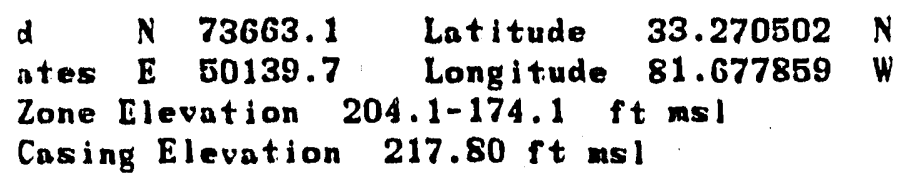

B 79

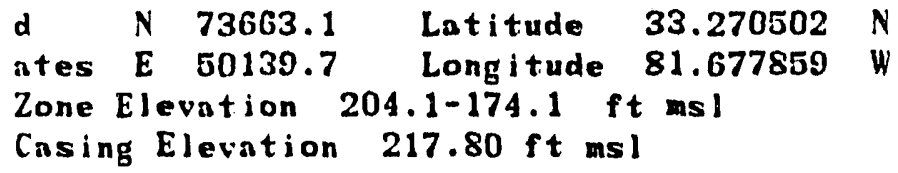

CIFIC CONDUCTANCE

CIFIC CONDICTANCE

IER
VER
IENIC
IENIC
IILM
IILM
JMODICHLOROMETHANE
JMODICHLOROMETHANE
GIIM
VIED

$\begin{aligned} 1730 \mathrm{umh} / \mathrm{cm} & \text { Gen. Eng. } \\ 1700 \mathrm{umh} / \mathrm{cm} & \text { Gen. Eng. } \\ 3.21 \mathrm{pll} & \text { Gen. Eng. } \\ 3.20 \mathrm{pH} & \text { Gen. Eng. } \\ 2 \mathrm{ug} / \mathrm{L} & \text { Gen. Eng. } \\ 2 \mathrm{ug} / \mathrm{L} & \text { Gen. Eng. } \\ 2 \mathrm{ug} / \mathrm{L} & \text { Gen. Eng. } \\ 2 \mathrm{ug} / \mathrm{L} & \text { Gen. Eng. } \\ 385 \mathrm{ug} / \mathrm{L} & \text { Gen. Eng. } \\ 393 \mathrm{ug} / \mathrm{L} & \text { Gen. Eng. } \\ 1 \mathrm{ug} / \mathrm{L} & \text { Gen. Eng. } \\ 1 \mathrm{ug} / \mathrm{L} & \text { Gen. Eng. } \\ 3030 \mathrm{ug} / \mathrm{L} & \text { Gen. Eng. }\end{aligned}$


NELL FSB 79 COLLECTED ON 01/28/90 LABORATORY ANALYSES CONTINUED

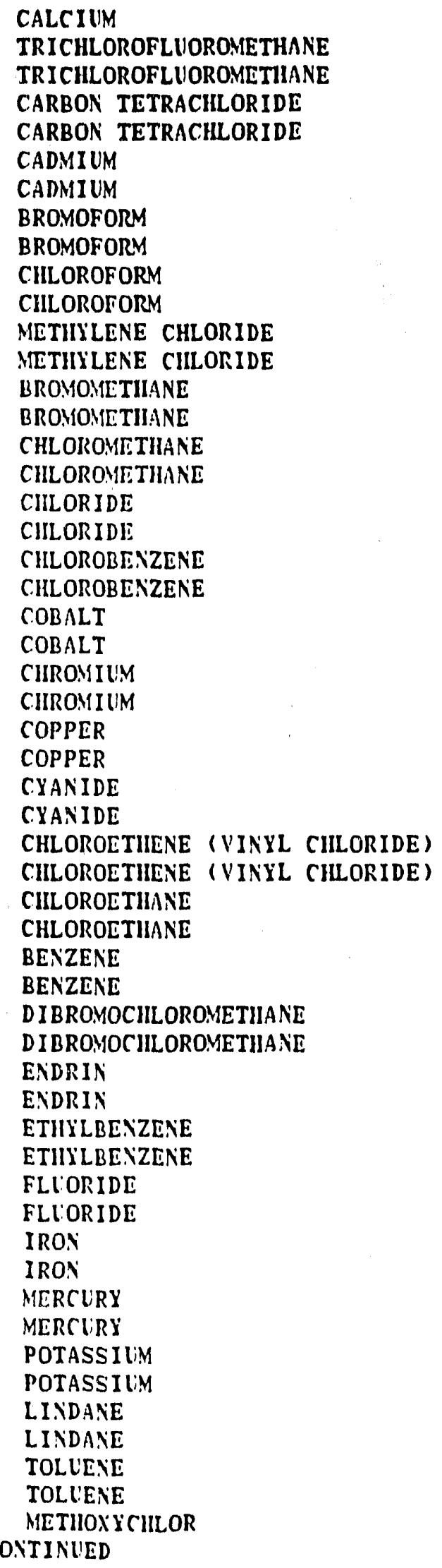

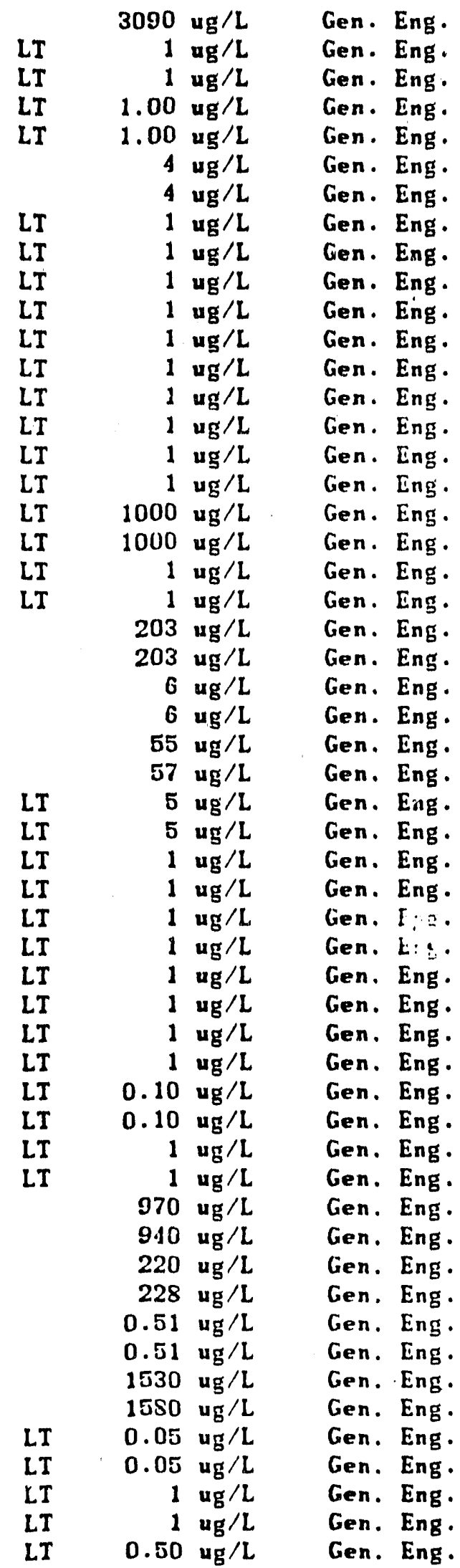


HELL FSB 79 COLLECTED ON 01/28/90 LABORATORY ANALYSES CONTINUED

METIIOXYCIILOR
MAGNESIUM
MAGNESIUM
MANGANESE
MANGANESE
SODIUM
SODIUM
NICKEL
NICKEL

* Nitrate as NITROGEN

* NITRATE AS NITROGEN LEAD

LEAD

PHIEOLS

PIIINOLS

ANTIMONY

ANTIMONY

SELEXIUM

SELEXIUM

SILICA

SILICA

SILVEX

SILVEX

SLLFATE

SLLFATE

$1,1,2,2$-TETRACILLOROETIIANE

$1,1,2,2$-TETRACIILOROETHANE

TETRACILOROETHYLENE

TETRACIILOROETHYLENE

TOTAL DISSOLVED SOLIDS

TOTAL DISSOLVED SOLIDS

THALLILM

THALLILM

TOTAL ORGANIC CARBON

TOTAL ORGANIC CARBON

TOTAL ORGANIC IIALOGENS

TOTAL ORGANIC HALOGENS

TOTAL PHOSPIITES

TOTAL PIIOSPHATES

TR I CIILOROETIILLENE

TRI CIILOROF.THILENE

TOXAPIIENE

TOXAPIIENE

TRANS-1,2-D ICHILOROETIIENE

TRANS-1,2-DICIILOROETHENE

URANILM

URANILY

VANADILIM

VANADILM

1, 1-D I CIILOROETIILENE

1,1-DICIILOROETHYLENE

1,1 -D ICIILOROLTIIANE

1,1-D ICIILOROETIIANE

$1,1,1$-TRICHLOROETHANE

CONTINUED
LT

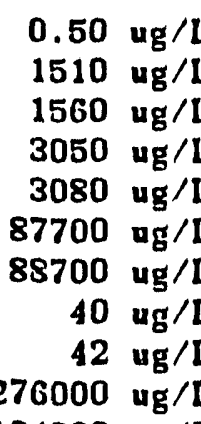

LT

LT

LT

LT

LT

LT

LT

LT

LT

LT

LT

LT

LT

$194000 \mathrm{ug} / \mathrm{L}$

$6 \mathrm{ug} / \mathrm{L}$

$7 \mathrm{ug} / \mathrm{L}$

$5 \mathrm{ug} / \mathrm{L}$

$5 \mathrm{ug} / \mathrm{L}$

$3 \mathrm{ug} / \mathrm{L}$

$3 \mathrm{ug} / \mathrm{L}$

$2 \mathrm{ug} / \mathrm{L}$

$2 \mathrm{ug} / \mathrm{L}$

$89100 \mathrm{ug} / \mathrm{L}$

$91400 \mathrm{ug} / \mathrm{L}$

$0.09 \mathrm{vg} / \mathrm{L}$

$0.09 \mathrm{ug} / \mathrm{L}$

$62700 \mathrm{mg} / \mathrm{L}$

$63700 \mathrm{ug} / \mathrm{L}$

$1 \mathrm{ug} / \mathrm{L}$

$1 \mathrm{ug} / \mathrm{L}$

$1.00 \mathrm{ug} / \mathrm{L}$

$1.00 \mathrm{ug} / \mathrm{L}$

$772000 \mathrm{ug} / \mathrm{L}$

$791000 \mathrm{ug} / \mathrm{L}$

$2 \mathrm{ug} / \mathrm{L}$

LT

LT

LT

LT

LT

LT

LT

LT

LT

LT

LT

LT

LT

LT

LI

LT

LT

LT

LT

LT
$2 \mathrm{ug} / \mathrm{L}$

$1000 \mathrm{ug} / \mathrm{L}$

$1000 \mathrm{ug} / \mathrm{L}$

$5 \mathrm{ug} / \mathrm{L}$

$5 \mathrm{ug} / \mathrm{L}$

$50 \mathrm{ug} / \mathrm{L}$

$50 \mathrm{ug} / \mathrm{L}$

$1.00 \mathrm{ug} / \mathrm{L}$

$1.00 \mathrm{ug} / \mathrm{L}$

$1 \mathrm{ug} / \mathrm{L}$

$1 \mathrm{ug} / \mathrm{L}$

$1 \mathrm{ug} / \mathrm{L}$

$1 \mathrm{ug} / \mathrm{L}$

$1240 \mathrm{ug} / \mathrm{L}$

$1300 \mathrm{ug} / \mathrm{L}$

$20 \mathrm{ug} / \mathrm{L}$

$20 \mathrm{ug} / \mathrm{L}$

$1 \mathrm{ug} / \mathrm{L}$

$1 \mathrm{ug} / \mathrm{L}$

$1 \mathrm{ug} / \mathrm{L}$

$1 \mathrm{ug} / \mathrm{L}$

$1 \mathrm{ug} / \mathrm{L}$
Gen. Eng .

Gen. Eng .

Gen. Eng.

Gen. Eng.

Gen. Eng.

Gen. Eng.

Gen. Eng.

Gen. Eng.

Gen. Eng.

Gen. Eng.

Gen. Eng.

Gen. Eng.

Gen. Eng .

Gen. Eng .

Gen. Eng.

Gen. Ing.

Gen. Eng.

Gen. Eng.

Gen. Eng.

Gen. Eng.

Gen. Eng.

Gen. Engू.

Gen. Eng.

Gen. Eng.

Gen. Eng.

Gen. Eng.

Gen. Eng.

Gen. Eng.

Gen. Eng.

Gen. Eng.

Gen. Eng.

Gen. Eng.

Gen. Eng.

Gen. Eng.

Gen. Eng.

Gen. Eng.

Gen. Eng .

Gen. Eng .

Gen. Eng .

Gen. Eng.

Gen. Eng .

Gen. Eng.

Gen. Eng.

Gen. Eng.

Gen. Eng.

Gen. Eng.

Gen. Eng.

Gen. Eng.

Gen. Eng.

Gen. Eng.

Gen. Eng .

Gen. Eng .

Gen. Eng.

Gen. Eng. 
WELL FSB 79 COLLECTED ON 01/28/90 LABORATORY ANALYSES CONTINUED

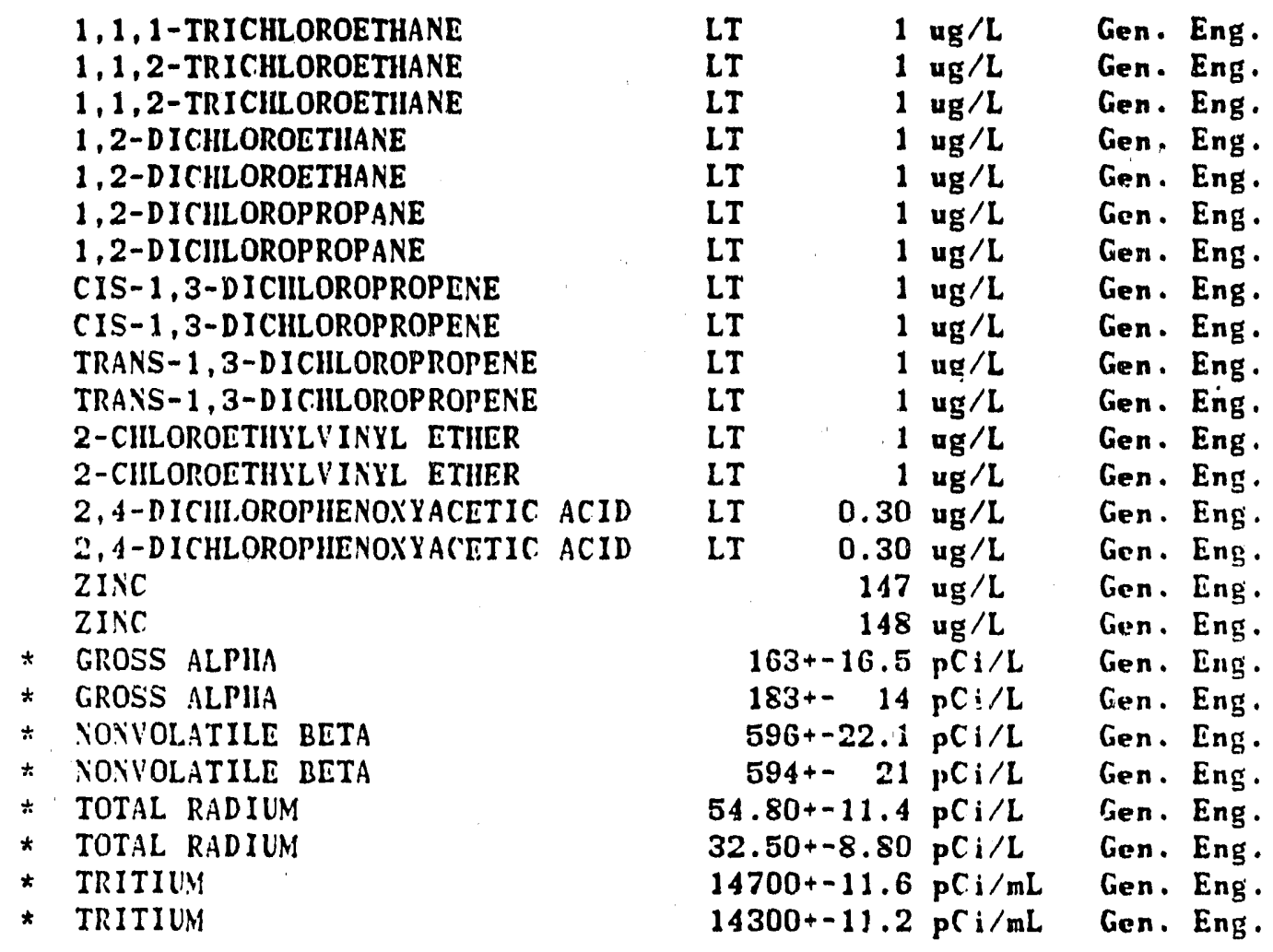

HELL TSB 79A

SRS Grid N 73664.5 Latitude $33.270522 \mathrm{~N}$

Coordinates E 50149.6 Longitude 81.677836 W

Screen Zone Elevation 34.4-24.0 ft msl

Top of Casing Elevation $218.10 \mathrm{ft} \mathrm{msl}$

MEASUREMENTS CONDUCTED IN THE FIELD

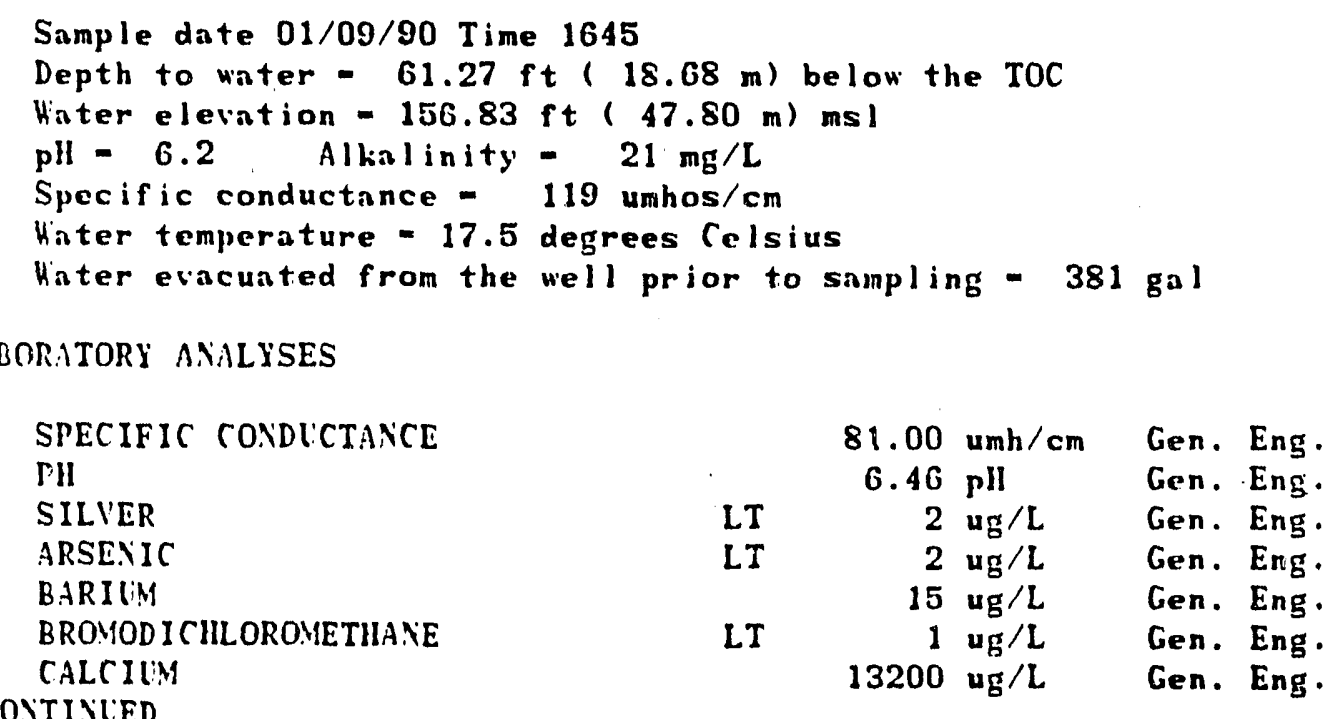


NELL FSB 79A COLLECTED ON 01/09/90 LABORATORY ANALYSES CONTINUED

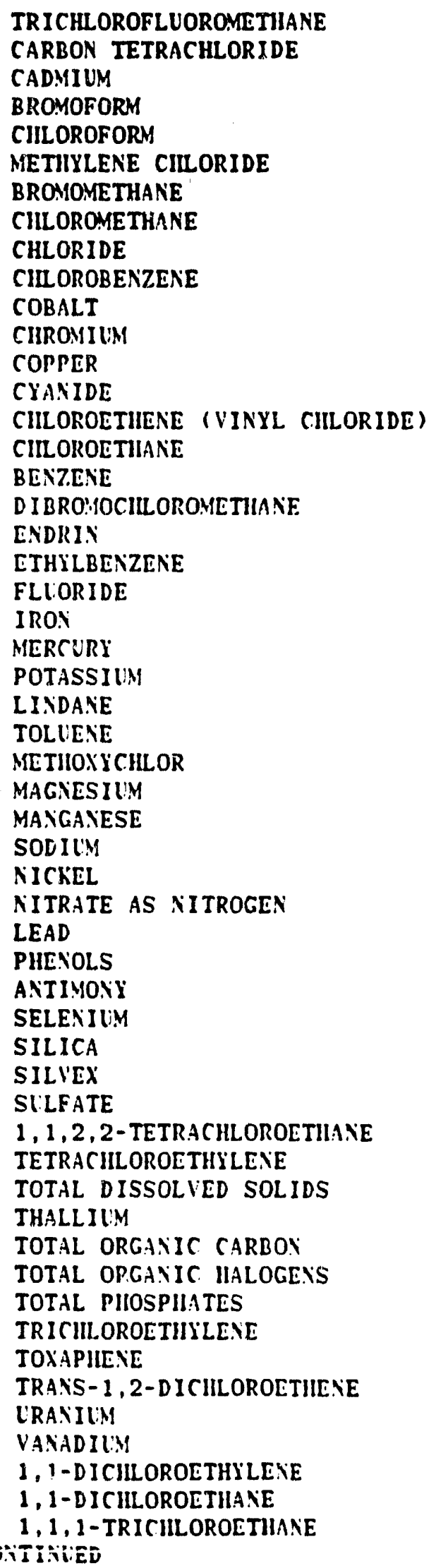

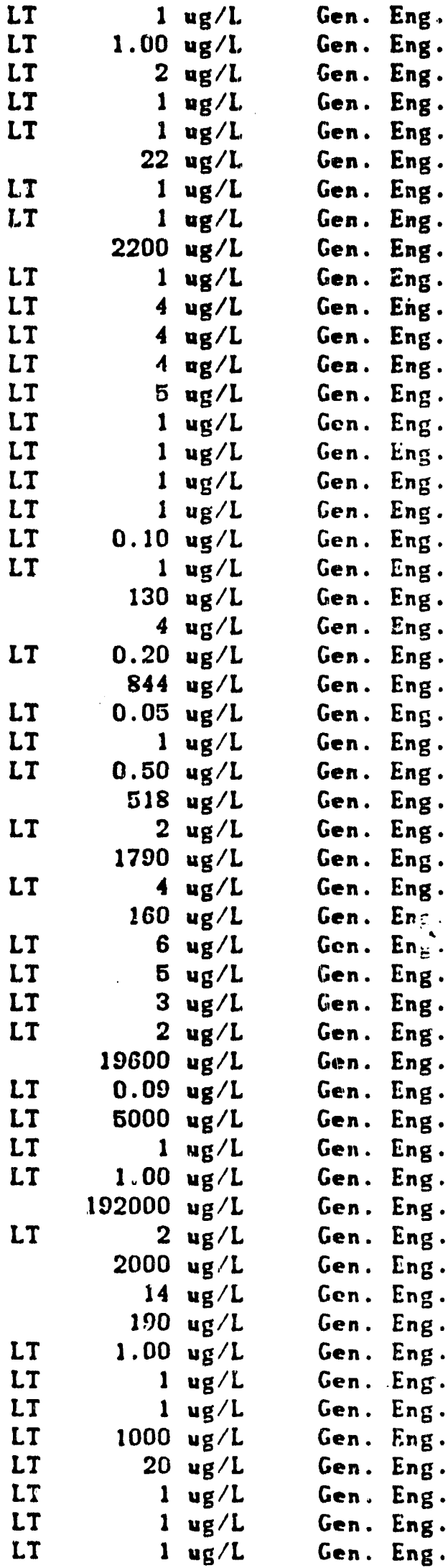


KELL FSB 79A COLLECTED ON 01/09/90 LABORATORY ANALYSES CONTINUED

\begin{tabular}{|c|c|c|c|}
\hline $\begin{array}{l}1,1,2 \text {-TRICILOROETHINE } \\
1,2-D I C H L O R O E T H A N E \\
1,2-D \text { ICILOROPROPANE } \\
\text { CIS-1,3-DICHLOROPROPENE } \\
\text { TRANS-1,3-DICHLOROPROPENE } \\
\text { 2-CHLOROETIYLVINYL ETHER } \\
2,1 \text {-DICILOROPHENOXYACETIC ACID } \\
\text { ZINC } \\
\text { GROSS ALPIIA } \\
\text { NONVOLATILE BETA } \\
\text { TOTAL RADIUM } \\
\text { TRITIIIM }\end{array}$ & $\begin{array}{l}\text { LT } \\
\text { LT } \\
\text { LT } \\
\text { LT } \\
\text { LT } \\
\text { LT } \\
\text { LT } \\
\text { LT } \\
\text { LT } \\
\text { LT } \\
\text { LT } \\
\text { II. }\end{array}$ & $\begin{array}{r}1 \\
1 \\
1 \\
1 \\
1 \\
1 \\
0.30 \\
2 \\
3 \\
2 \\
1 \\
-0.47\end{array}$ & $\begin{array}{l}\mathrm{ug} / \mathrm{L} \\
\mathrm{ug} / \mathrm{L} \\
\mathrm{ug} / \mathrm{L} \\
\mathrm{ug} / \mathrm{L} \\
\mathrm{ug} / \mathrm{L} \\
\mathrm{ug} / \mathrm{L} \\
\mathrm{ug} / \mathrm{L} \\
\mathrm{ug} / \mathrm{L} \\
\mathrm{PCi} / \mathrm{L} \\
\mathrm{PCi} / \mathrm{L} \\
\mathrm{PCi} / \mathrm{L} \\
\mathrm{PCi} / \mathrm{mL}\end{array}$ \\
\hline
\end{tabular}

VELL FSB 79B

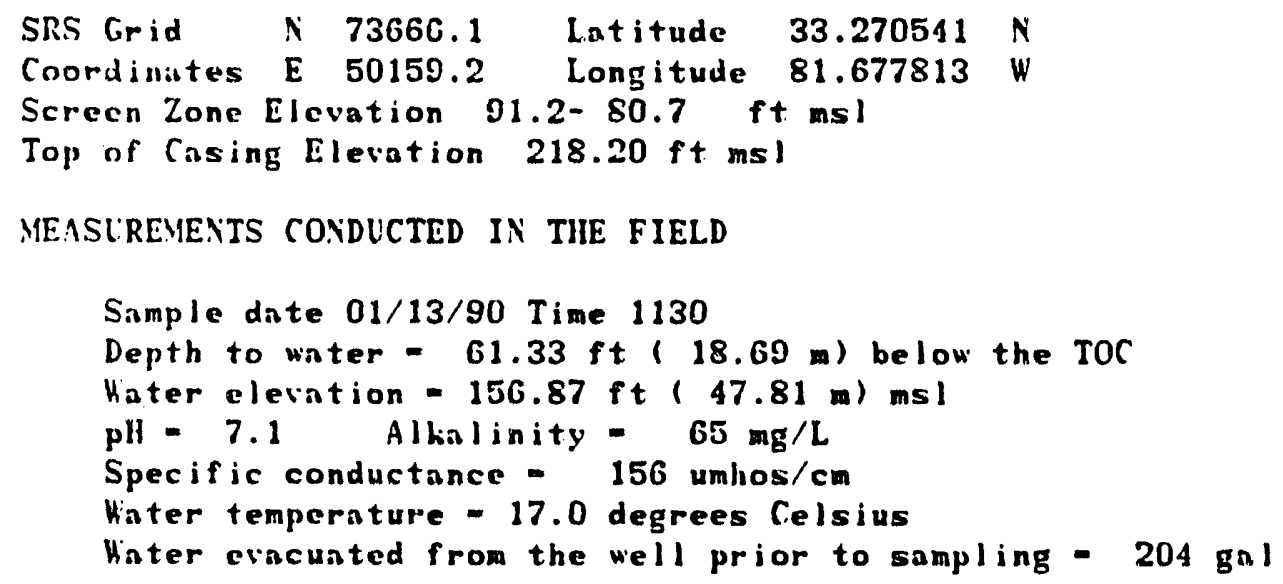

SPECIFIC CONDLCTANCE

SPECIFIC CONDLCTANCE

PII

PH

SILVER

SILVER

ARSE.IIC

ARSEIIC

BARIIM

BARIUM

BROMODICILOROMETHANE

BRONODICILLOROMETIIANE

CALCILM

CALCIUM

TRICILO!OFLUORONETIINE

TRICILOROFLLOROMETIIANE

CARBON TETRACHLORIDE

CARBOX TETRACIILORIDE

CADMILM

CADMILM

CONIIILED

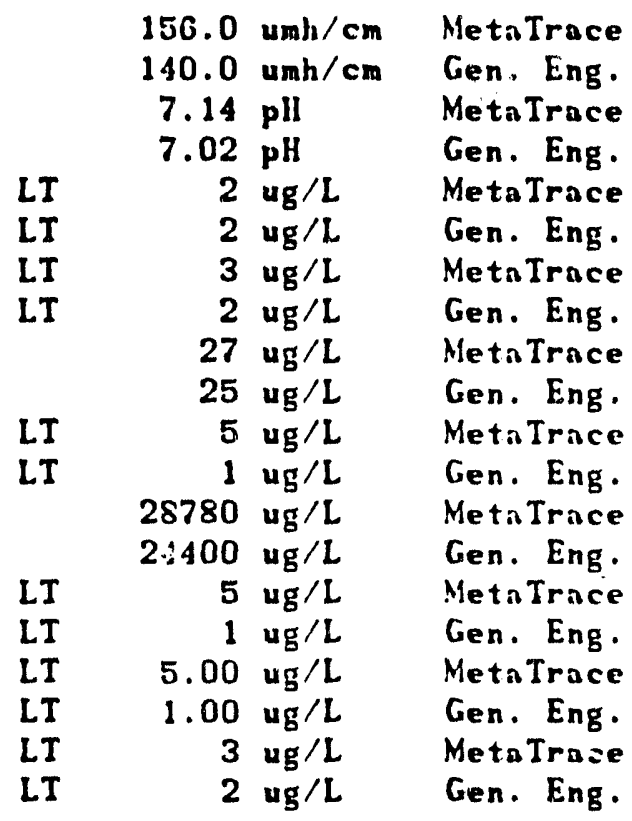


WELL FSB 79B COLLECTED ON 01/13/90 LABORATORY ANALYSES CONTINUED

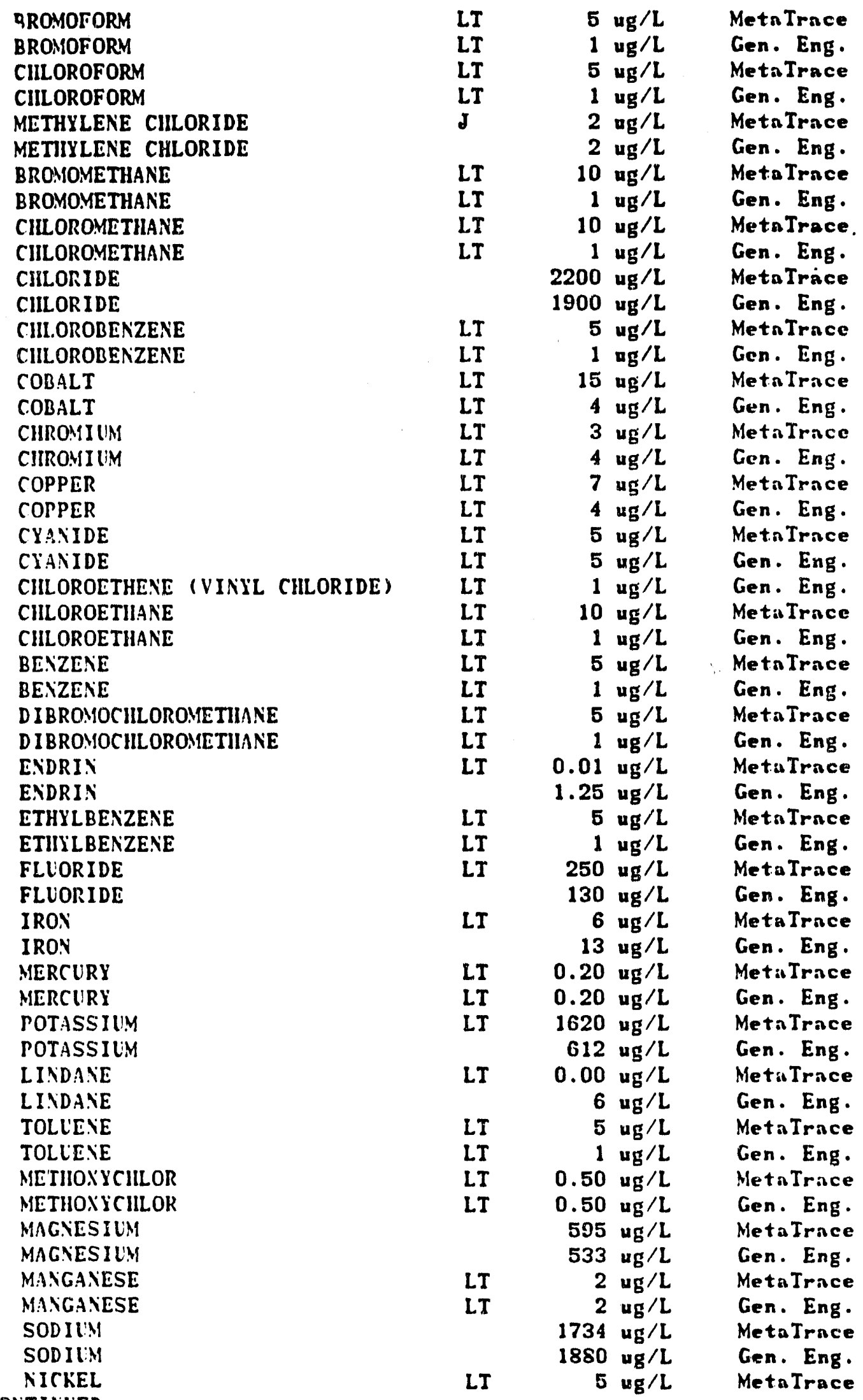


NELL FSB 79B COLLECTED ON 01/13/90 LABORATORY ANALYSES CONTINUED

NICKEL

\section{NITRATE AS NITROGEN \\ NITRATE AS NITROGEN}

LEAD

LEAD

PHENOLS

PIIENOLS

ANTIMONY

ANTIMONY

SELENIUM

SELENIUM

SILICA

SILICA

SILVEX

SILVEX

SILFATE

SLLFATE

$1,1,2,2$-TETRACIILOROETIIANE

$1,1,2,2$-TETRACILLOROETHANE

TETRACILOROETHYLENE

TETRACHLOROETHYLENE

TOTAL DISSOLVED SOLIDS

TOTAL DISSOLVED SOLIDS

THALLIUM

THALLIUM

TOTAL ORGANIC CARBON

TOTAL ORGANIC CARBON

TOTAL ORGANIC HALOGENS

TOTAL ORGANIC HALOGENS

TOTAL PHIOSPHATES

TOTAL PHOSPIIATES

TR I CIILOROETIIILENE

TRICILOROETIILLENE

TOXAPIIENE

TOXAPHENE

TRANS-1, 2-DICHLOROETHENE

IRANS-1,2-DICHLOROETIIENE

IRANIUM

URANILM

VANADILM

VA.TADILY

1,1-DICILOROETHYLENE

1,1-DICILOROETIIYLENE

1,1-DICIILOROETIIANE

1,1 -DICIILOROETHANE

$1,1,1$-TRICHLOROETIHAE

$1,1,1$-TRICHLOROETIIANE

$1,1,2$-TR I CHLOROETHANE

$1,1,2$-TRICILOROETHINE

1,2-DICILLOROETHATE

1,2-DICILOROETHANE

1, 2-DICIILOROPROPANE

1,2-DICILLOROPROPANE

CIS-1, 3-DICILLOROPROPENE

CONIINIED
LT

LT

LT

LT

LT

LI

LT

LI

LT

LT

LT

LT

LI

LI

LI

LT

LT

LT

LI

LT

LI

LT

LI

LT

LT

LI

LT

LT

LT

LI

LT

LT

LT

LT

LI

LT

LT

LT

LI

LT

LI

LI

LT

LT

LT

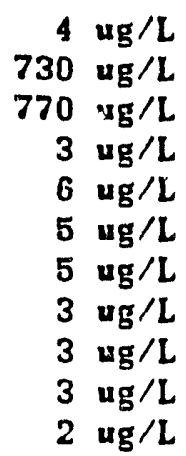

$11480 \mathrm{ug} / \mathrm{L}$

$2580 \mathrm{ug} / \mathrm{L}$

$0.07 \mathrm{ug} / \mathrm{L}$

$0.09 \mathrm{ug} / \mathrm{L}$

$2400 \mathrm{ug} / \mathrm{L}$

$5000 \mathrm{ug} / \mathrm{L}$

$5 \mathrm{ug} / \mathrm{L}$

$1 \mathrm{ug} / \mathrm{L}$

$5.00 \mathrm{ug} / \mathrm{L}$

$1.00 \mathrm{ug} / \mathrm{L}$

$68000 \mathrm{ug} / \mathrm{L}$

$117000 \mathrm{ug} / \mathrm{L}$

$3 \mathrm{ug} / \mathrm{L}$

$2 \mathrm{ug} / \mathrm{L}$

$1000 \mathrm{ug} / \mathrm{L}$

$1000 \mathrm{ug} / \mathrm{L}$

$5 \mathrm{ug} / \mathrm{L}$

$5 \mathrm{ug} / \mathrm{L}$

$509 \mathrm{ug} / \mathrm{L}$

$430 \mathrm{ug} / \mathrm{L}$

$5.00 \mathrm{ug} / \mathrm{L}$

$1.00 \mathrm{ug} / \mathrm{L}$

$0.24 \mathrm{ug} / \mathrm{L}$

$1 \mathrm{ug} / \mathrm{L}$

$5 \mathrm{ug} / \mathrm{L}$

$1 \mathrm{ug} / \mathrm{L}$

$84 \mathrm{ug} / \mathrm{L}$

$1000 \mathrm{ug} / \mathrm{L}$

$3 \mathrm{ug} / \mathrm{L}$

$20 \mathrm{ug} / \mathrm{L}$

$5 \mathrm{ug} / \mathrm{L}$

$1 \mathrm{ug} / \mathrm{L}$

$5 \mathrm{wg} / \mathrm{L}$

$1 \mathrm{ug} / \mathrm{L}$

$5 \mathrm{ug} / \mathrm{L}$

$1 \mathrm{ug} / \mathrm{L}$

$5 \mathrm{ug} / \mathrm{L}$

$1 \mathrm{ug} / \mathrm{L}$

$5 \mathrm{ug} / \mathrm{L}$

$1 \mathrm{ug} / \mathrm{L}$

$5 \mathrm{ug} / \mathrm{L}$

$1 \mathrm{ug} / \mathrm{L}$

$5 \mathrm{ug} / \mathrm{L}$
Gen. Eng. MetaTrace

Gen. Eng.

MetaTrace

Gen. Eng.

MetaTrace

Gen. Eng.

MetaTrace

Gen. Eng.

MetaTrace

Gen. Eng.

MetaTrace

Gen. Eng.

MetaTrace

Gen. Eng.

Metis Trace

Gen. Eng.

MetaTrace

Gen. Eng.

MetaTrace

Gen. Eng.

MetnTrace

Gen. Eng.

MetaTrace

Gen. Eng.

MetaTrace

Gen. Eng.

MetaTrace

Gen. Eng.

MetaTrace

Gen. Eng.

MetaTrace

Gen. Eng.

MetaTrnce

Gen. Eng.

MetaTrace

Gen. Eng.

MetaTrace

Gen. Eng.

MetaTrace

Gen. Eng.

MetaTrace

Gen. Eng.

MetaTrace

Gen. Eng.

MetaTrace

Gen. Eng.

MetaTrace

Gen. Eng.

MetaTrace

Gen. Eng.

MetaTrace

Gen. Eng.

MetaTrace 
VELL FSB 79B COLLECTED ON 01/13/90 LABORATORY ANALXSES CONTINUED

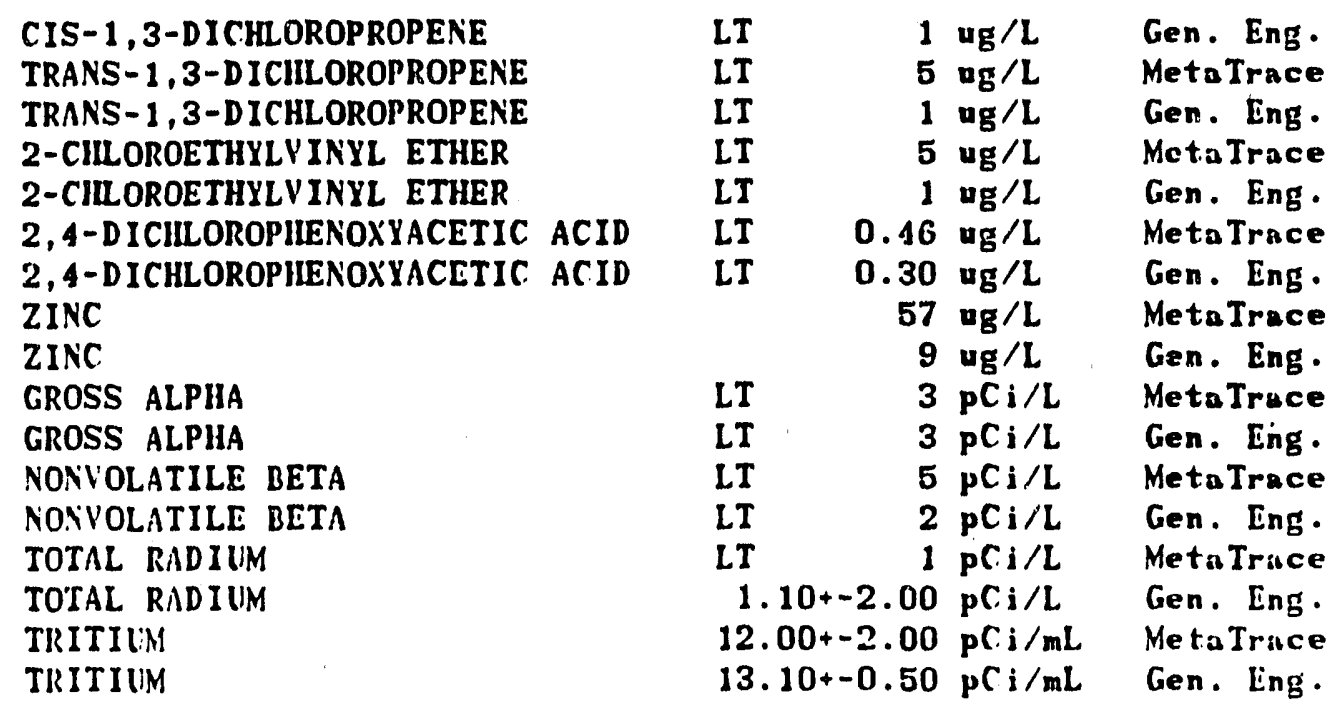

HELL FSB 79B

SRS Grid N 73666.1 Latitude 33.270541 N

Coordinates E 50159.2 Longitude 81.677813 W'

Screen Zone Elevation 91.2- $80.7 \mathrm{ft} m s /$

Top of Casing Elevarion $218.20 \mathrm{ft} \mathrm{ms}$ l

MEASLREMENTS CONDUCTED IN TIE FIELD

Sample date $01 / 13 / 90$ Time 1130

Depth to water - $61.33 \mathrm{ft}(18.69 \mathrm{~m})$ below the TOC

Nater elevation $-156.87 \mathrm{ft}(47.81 \mathrm{~m}) \mathrm{ms}$ l

pH -7.1 Alknlinity $-65 \mathrm{mg} / \mathrm{L}$

Specific conductance - $156 \mathrm{umhos} / \mathrm{cm}$

Water temperature -17.0 degrees Celsius

Vater evacuated from the well prior to sampling - $204 \mathrm{gal}$

LABORATORY ANALYSES

SPECIFIC CONDLCTANCE

SPECIFIC CONDUCTANCE

PII

PH

SILVER

SILIER

SILVER

ARSEYIC

ARSEIIC

BARIUM

BARILM

BROMODICILOROMETIIAE

BRONODICILLOROMETHANE

CALCIIY

CALCIUM

CONTINUED
$159.0 \mathrm{umh} / \mathrm{cm}$ MetaTrace $140.0 \mathrm{umh} / \mathrm{cm}$ Gen. Eng. 7.13 pll 7.27 pll

LT

LI

LT

LT

LT

LT

LT

$2 \mathrm{ug} / \mathrm{L}$

$2 \mathrm{ug} / \mathrm{L}$

$2 \mathrm{ug} / \mathrm{L}$

$3 \mathrm{ug} / \mathrm{L}$

$2 \mathrm{ug} / \mathrm{L}$

$26 \mathrm{ug} / \mathrm{L}$

$24 \mathrm{ug} / \mathrm{L}$

$5 \mathrm{ug} / \mathrm{L}$

$1 \mathrm{ug} / \mathrm{L}$

$28190 \mathrm{ug} / \mathrm{L}$

$23500 \mathrm{ug} / \mathrm{L}$
MetaTrace

Gen. Eng. MetaTrace MetaTrace Gen. Eng. MetaTrace Gen. Eng. MetaTrace Gen. Eng. MetaTrace Cen. Eng. MetaTrace Gen. Eng. 
NELL FSB 79B COLLECTED ON 01/13/90 LABORATORY ANALYSES CONTINUED

TRICHLOROFLUOROMETHIANE TRICHLOROFLUOROMETHANE

CARBON TETRACIILORIDE

CARBON TETRACHLORIDE

CADMIUM

CADMIUM

BROMOFORM

BROMOFORM

CIILOROFORM

CIILOROFORM

METIIXLENE CILORIDE

METHYLENE CIILORIDE

BROMOMETIIANE

BROMOMETIINNE

CIILOROMETIIANE

CHLOROMETHANE

CIILORIDE

CHLORIDE

CIILORIDE

CHLOROBENZENE

CHLOROBENZENE

COBALT

COBALT

CHROMIUM

CHROMIUN

COPPER

COPPER

CIANIDE

CYANIDE

CILLOROETIIENE (VINYL CILLORIDE)

CHLOROETIIANE

CHLOROETIIANE

BEIZENE

BENZENE

DIBROMOCHLOROMETHANE

DIBROMOCILLOROMETHANE

ENDRIN

- ENDRIN

ETHYLBENZENE

ETIILBENZENE

FLIORIDE

FLLORIDE

FLLORIDE

IRON

IRON

MERCIRY

MERCLRY

POTASSIUM

POTASSILM

LINDANE

LINDANE

TOLIENE

TOLUENE

METHOXYCIILOR

CONTINLED
LT

LT

LT

LT

LT

LT

LT

LT

LT

LT

LT

LT

LT

LT

LT

LT

LT

LT

LT

LT

LT

LT

LT

LT

LT

LT

LT

LT

LT

LT

LT

LT

LT

LI

LT

LT

LT

LT

LT

LT

LT

LT

LT

LT
$5 \mathrm{ug} / \mathrm{L}$

$1 \mathrm{ug} / \mathrm{L}$

$5.00 \mathrm{ug} / \mathrm{L}$

$1.00 \mathrm{ug} / \mathrm{L}$

$3 \mathrm{ug} / \mathrm{L}$

$2 \mathrm{ug} / \mathrm{L}$

$5 \mathrm{ug} / \mathrm{L}$

$1 \mathrm{ug} / \mathrm{L}$

$5 \mathrm{ug} / \mathrm{L}$

$1 \mathrm{ug} / \mathrm{L}$

$5 \mathrm{ug} / \mathrm{L}$

$1 \mathrm{ug} / \mathrm{L}$

10 ug/L

$1 u_{6} / \mathrm{L}$

$10 \mathrm{ug} / \mathrm{L}$

$1 \mathrm{ug} / \mathrm{L}$

$2200 \mathrm{ug} / \mathrm{L}$

$2100 \mathrm{ug} / \mathrm{L}$

$2000 \mathrm{ug} / \mathrm{L}$

$5 \mathrm{ug} / \mathrm{L}$

$1 \mathrm{ug} / \mathrm{L}$

$15 \mathrm{ug} / \mathrm{L}$

$4 \mathrm{ug} / \mathrm{L}$

$3 \mathrm{ug} / \mathrm{L}$

$4 \mathrm{ug} / \mathrm{L}$

$7 \mathrm{ug} / \mathrm{L}$

$4 \mathrm{ug} / \mathrm{L}$

$5 \mathrm{ug} / \mathrm{L}$

$5 \mathrm{ug} / \mathrm{L}$

$1 \mathrm{ug} / \mathrm{L}$

$10 \mathrm{ug} / \mathrm{L}$

$1 \mathrm{ug} / \mathrm{L}$

$5 \mathrm{ug} / \mathrm{L}$

$1 \mathrm{ug} / \mathrm{L}$

5 ug/L

$1 \mathrm{ug} / \mathrm{L}$

$0.01 \mathrm{ug} / \mathrm{L}$

$1.85 \mathrm{ug} / \mathrm{L}$

$5 \mathrm{ug} / \mathrm{L}$

$1 \mathrm{ug} / \mathrm{L}$

$250 \mathrm{ug} / \mathrm{L}$

$250 \mathrm{ug} / \mathrm{L}$

$150 \mathrm{ug} / \mathrm{L}$

9 ug/L

15 แg $/ \mathrm{L}$

$0.20 \mathrm{ug} / \mathrm{L}$

$0.20 \mathrm{ug} / \mathrm{L}$

$2288 \mathrm{ug} / \mathrm{L}$

$545 \mathrm{ug} / \mathrm{L}$

$0.00 \mathrm{ug} / \mathrm{L}$

2 ug/L

5 ug/L

$1 \mathrm{ug} / \mathrm{L}$

$0.50 \mathrm{ug} / \mathrm{L}$
MetaTrace

Gen. Eng.

MetaTrace

Gen. Eng.

MetaTrace

Gen. Eng.

MetaTrace

Gen. Eng.

MetaTrace

Gen. Eng.

MetaTrace

Gen. Eng.

MetaTrace

Gen. Eng.

MetaTrace

Gen. Eng.

MetaTrace

MetaTrace

Gen. Eng.

MetaTrace

Gen. Eng.

MetaTrace

Gen. Eng.

MetnTrace

Gen. Eng.

MetaTrace

Gen. Eng.

MetoTrace

Gen. Eng.

Gen. Eng.

MetaTrnce

Gen. Eng.

MetaTrnce

Gen. Eng.

MetaTrace

Gen. Eng.

MetaTrace

Gein. Eng.

MetaTrace

Gen. Eng.

MetiTrace

MetaTrace

Gen. Eng.

MetaTrace

Gen. Eng.

MetaTrace

Gen. Eng.

MetaTrace

Gen. Eng.

MetaTrace

Gen. Eng.

MetaTrace

Gen. Eng.

MetaTrace 
KELL FSB 79B COLLECTED ON 01/13/90 LABORATORY ANALYSES CONTINUED

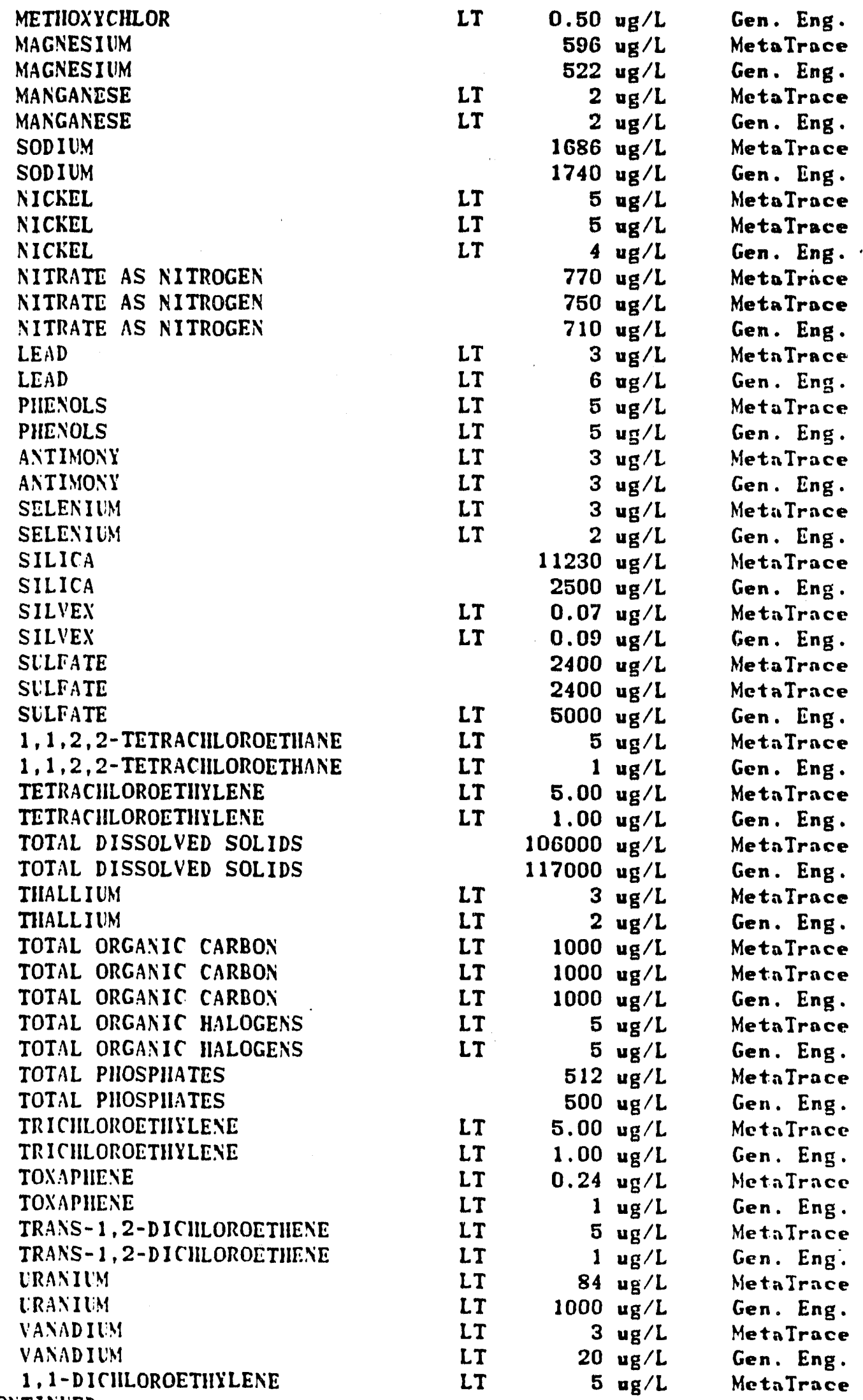


VELL FSB 79B COLLECTED ON 01/13/90 LABORATORY ANALYSES CONTINUED

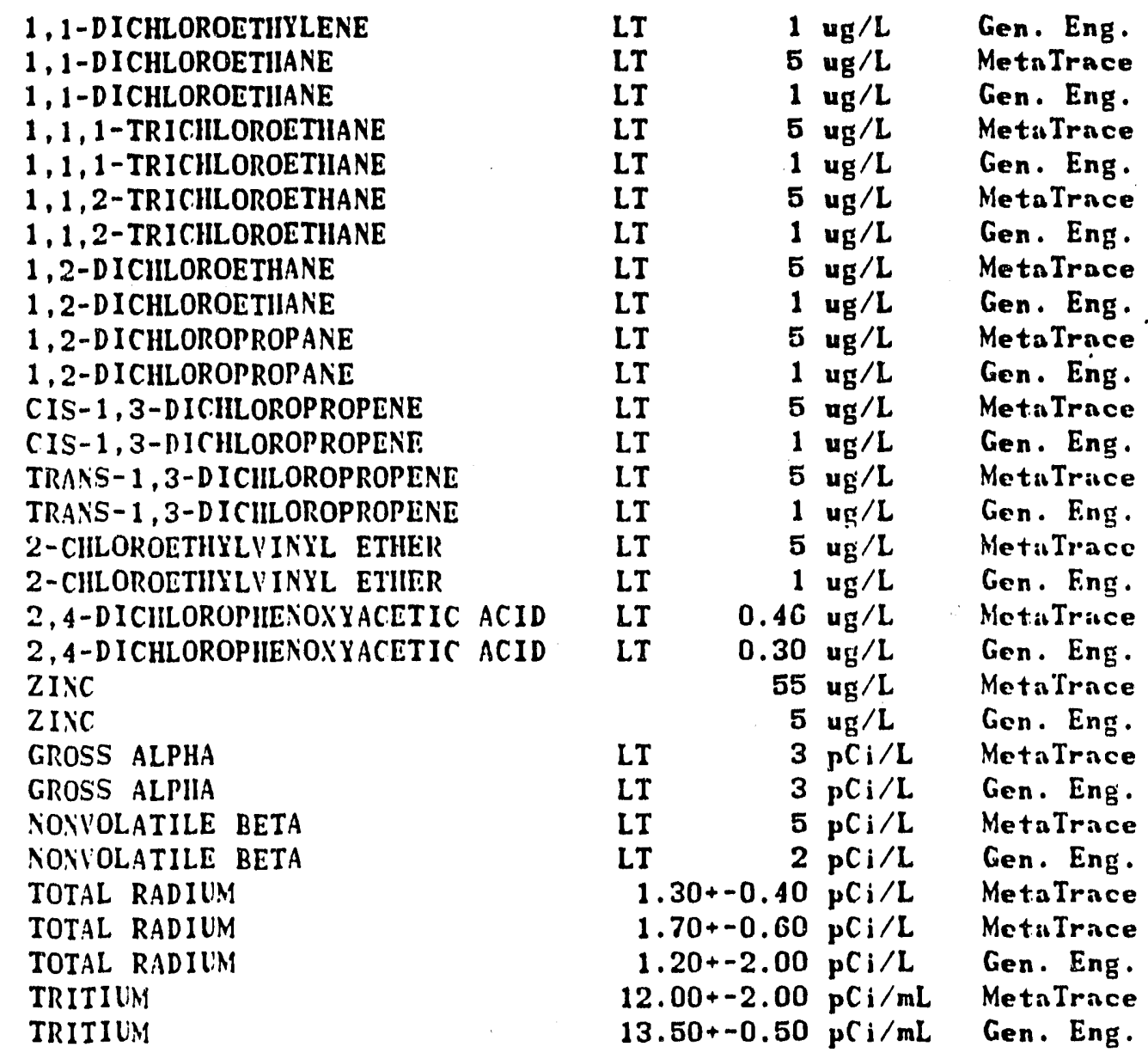

NELL FSB $79 C$

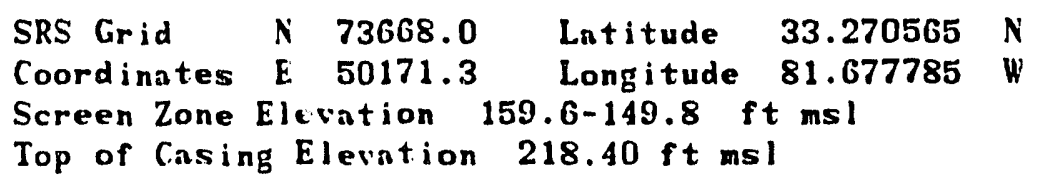

MEASLREMENTS CONDICTED IN TIE FIELD

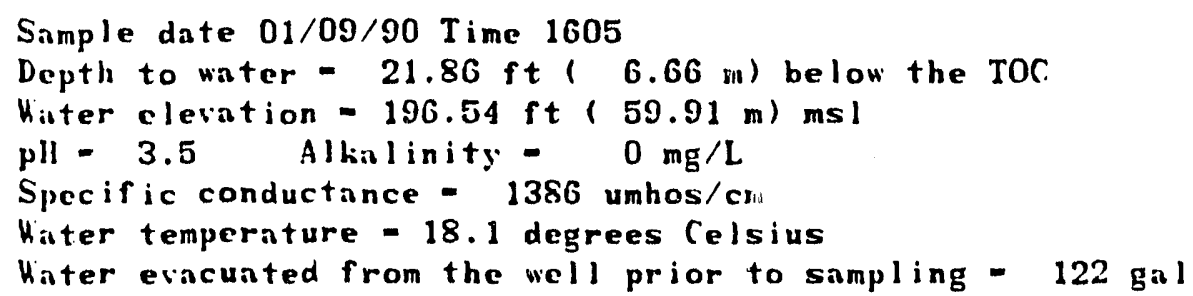


WELL FSB 79C

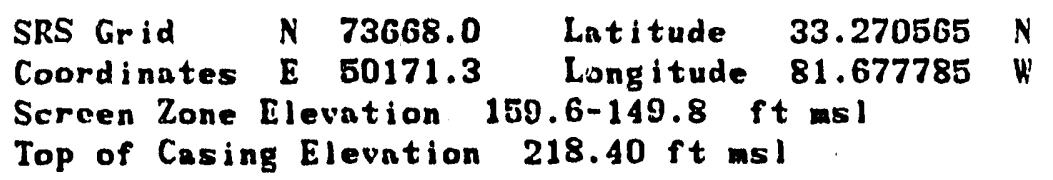

MEASUREMENTS CONDUCTED IN THE FIELD

Sample date 01/09/90 Time 1605

Depth to wnter - $21.86 \mathrm{ft}(6.60 \mathrm{~m})$ below the TOC

Wiater elevation - $196.54 \mathrm{ft}(59.91 \mathrm{~m}) \mathrm{ms}$

pll - 3.5 Alkalinity $=0 \mathrm{mg} / \mathrm{h}$

Specific conductance - 1386 umhos/cm

Winter temperature - 18.1 degrees Celsius

Water evacuated from the well prior to sampling - $122 \mathrm{gal}$

LABORATORY ANALYSES

THESE ANALYSES ARE FROM UNFILTERED SAMPLES

SILICA

$22900 \mathrm{ug} / \mathrm{L}$ Gen. Eng.

HELL FSB 79C

SRS Grid N 73668.0 Lntitude $33.270565 \quad N$

Coordinates E 50171.3 Longitude 81.677785 W'

Screen Zone Elevation 159.6-149.8 $\mathrm{ft} \mathrm{msl}$

Top of Casing Elevation $218.40 \mathrm{ft} \mathrm{ms}$ !

MEASUREMENTS CONDUCTED IN THE FIELD

Sample date $01 / 28 / 90$ Time 1220

Depth to whter - $22.02 \mathrm{ft}(6.71 \mathrm{~m})$ below the TOC

Viater elevation $-196.38 \mathrm{ft}(59.86 \mathrm{~m}) \mathrm{msl}$

pll - $3.3 \quad$ Alkalinity $-0 \mathrm{mg} / \mathrm{L}$

Specific conductance - 1447 umhos $/ \mathrm{cm}$

Water temperature -18.3 degrees Celsius

Hiater evocuated from the well prior to sampling - $121 \mathrm{gal}$

LABORATORY ANALYSES

SPECIFIC CONDUCTANCE

PII

SILVER

ARSENIC

BIRILM

BROMOD ICILLOROMETIIANE

CALCIUM

TRICILLOROFLUOROMETHANE

CARBON TETRACHLORIDE

* CADIIIUM

BROMOFORY

CIILOROFORY

METHILENE CIILORIDE

CONTINUED

$\begin{array}{lrll} & 1370 \mathrm{umh} / \mathrm{cm} & \text { Gen. Eng. } \\ \text { LT } & 3.46 \mathrm{pH} & \text { Gen. Eng. } \\ \text { LT } & 2 \mathrm{ug} / \mathrm{L} & \text { Gen. Eng. } \\ & 2 \mathrm{ug} / \mathrm{L} & \text { Gen. Eng. } \\ \text { LT } & 597 \mathrm{ug} / \mathrm{L} & \text { Gen. Eng. } \\ & 1 \mathrm{ug} / \mathrm{L} & \text { Gen. Eng. } \\ \text { LT } & 15700 \mathrm{ug} / L & \text { Gen. Eng. } \\ \text { LT } & 1 \mathrm{ug} / L & \text { Gen. Eng. } \\ & 1.00 \mathrm{ug} / L & \text { Gen. Eng. } \\ \text { LT } & 35 \mathrm{ug} / L & \text { Gen. Eng. } \\ \text { LT } & 1 \mathrm{ug} / L & \text { Gen. Eng. } \\ \text { LT } & 1 \mathrm{ug} / L & \text { Gen. Eng. } \\ & 1 \mathrm{ug} / \mathrm{L} & \text { Gen. Eng. }\end{array}$


K'ELL FSB 79C COLLECTLD ON 01/28/90 LABORATORY ANALYSES CONTINUED

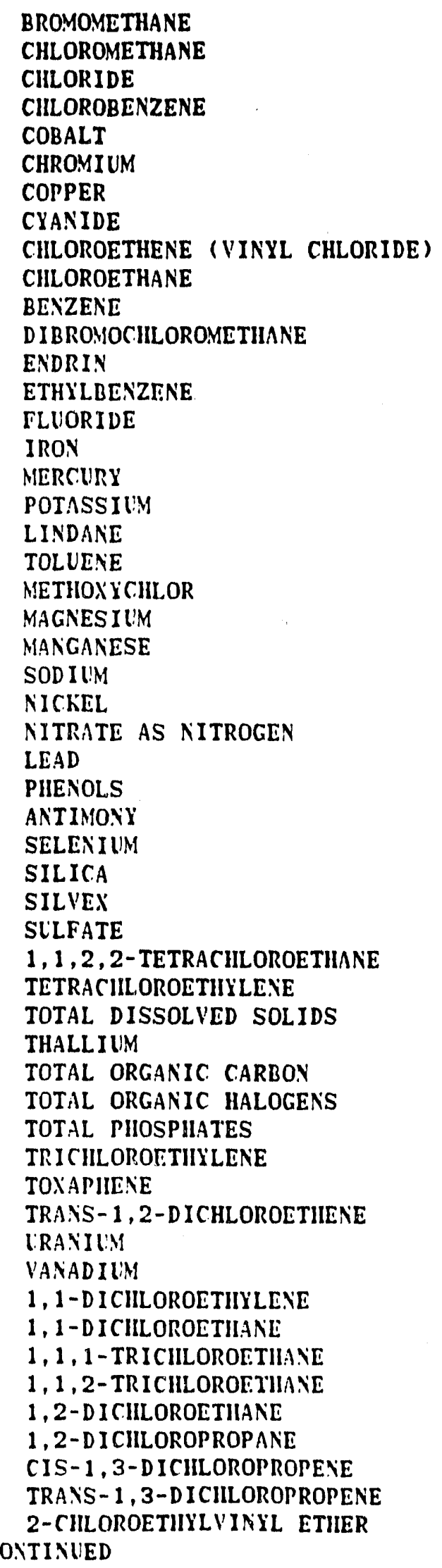

LT
LT

LT

LT

LI

LI

LI

LT

LT

LI

LT

LT

LT

LI

LT

LT

LI

LI

LT

\section{LT}

LT

LT

LT

LI

LT

LT

LT

LT

LT

LT

LT

LT

LT

LI

LI

LT

LI

LT

$$
\begin{aligned}
& 1 \mathrm{ug} / \mathrm{L} \text { Gen. Eng. } \\
& 1 \mathrm{ug} / \mathrm{L} \text { Gen. Eng. } \\
& 2100 \mathrm{ug} / \mathrm{L} \text { Gen. Eng. } \\
& 1 \mathrm{ug} / \mathrm{L} \text { Gen. Eng. } \\
& 83 \mathrm{ug} / \mathrm{L} \text { Gen. Eng. } \\
& 4 \mathrm{ug} / \mathrm{L} \text { Gen. Eng. } \\
& 62 \text { ug/L Gen. Eng. } \\
& 5 \text { ug/L Gen. Eng. } \\
& 1 \text { ug/L Gen. Eng. } \\
& 1 \mathrm{ug} / \mathrm{L} \text { Gen. Eng. } \\
& 1 \mathrm{ug} / \mathrm{L} \text { Gen. Eng. } \\
& 1 \mathrm{ug} / \mathrm{L} \text { Gen. Eng. } \\
& 0.10 \mathrm{ug} / \mathrm{L} \text { Gen. Eng. } \\
& 1 \text { ug/L Gen. Eng. } \\
& 1300 \mathrm{ug} / \mathrm{L} \text { Gen. Eng. } \\
& 26 \mathrm{mg} / \mathrm{L} \text { Gen. Eng. } \\
& 0.20 \mathrm{ug} / \mathrm{L} \text { Gen. Eng. } \\
& 2910 \mathrm{ug} / \mathrm{L} \text { Gen. Eng. } \\
& 0.05 \mathrm{ug} / \mathrm{L} \text { Gen. Eng. } \\
& 1 \mathrm{ug} / \mathrm{L} \text { Gen. Eng. } \\
& 0.50 \mathrm{ug} / \mathrm{L} \text { Gen. Eng. } \\
& 15000 \text { ug } / \mathrm{L} \text { Gen. Eng. } \\
& 2130 \mathrm{ug} / \mathrm{L} \text { Gen. Eng. } \\
& 114000 \mathrm{ug} / \mathrm{L} \text { Gen. Eng. } \\
& 35 \mathrm{ug} / \mathrm{L} \text { Gen. Eng. } \\
& 155000 \mathrm{ug} / \mathrm{L} \text { Gen. Eng. } \\
& 6 \mathrm{ug} / \mathrm{L} \text { Gen. Eng. } \\
& 5 \mathrm{ug} / \mathrm{L} \text { Gen. Eng. } \\
& 3 \text { ug } / \mathrm{L} \text { Gen. Eng. } \\
& 2 \text { ug/L Gen. Eng. } \\
& 16600 \text { ug } / L \text { Gen. Eng. } \\
& 0.09 \text { ug } / \mathrm{L} \text { Gen. Eng. } \\
& 12600 \mathrm{ug} / \mathrm{L} \text { Gen. Eng. } \\
& 1 \mathrm{ug} / \mathrm{L} \text { Gen. Eng. } \\
& 1.00 \mathrm{ug} / \mathrm{L} \text { Gen. Eng. } \\
& 724000 \text { ug/L Gen. Eng. } \\
& 2 \text { ug/L Gen. Eng. } \\
& 1000 \mathrm{ug} / \mathrm{L} \text { Gen. Eng. } \\
& 15 \mathrm{ug} / \mathrm{L} \text { Gen. Eng. } \\
& 50 \mathrm{ug} / \mathrm{L} \text { Gen. Eng. } \\
& 1.00 \mathrm{ug} / \mathrm{L} \text { Gen. Eng. } \\
& 1 \mathrm{ug} / \mathrm{L} \text { Gen. Eng. } \\
& 1 \mathrm{ug} / \mathrm{L} \text { Gen. Eng. } \\
& 1170 \text { ug/L Gen. Eng. } \\
& 20 \mathrm{ug} / \mathrm{L} \text { Gen. Eng. } \\
& 1 \text { ug/L Gen. Eng. } \\
& 1 \mathrm{ug} / \mathrm{L} \text { Gen. Eng. } \\
& 1 \mathrm{ug} / \mathrm{L} \text { Gen. Eng. } \\
& 1 \mathrm{ug} / \mathrm{L} \text { Gen. Eng. } \\
& 1 \mathrm{ug} / \mathrm{L} \text { Gen. Eng. } \\
& 1 \text { ug/L Gen. Eng. } \\
& 1 \mathrm{ug} / \mathrm{L} \text { Gen. Eng. } \\
& 1 \mathrm{ug} / \mathrm{L} \text { Gen. Eng. } \\
& 1 \mathrm{ug} / \mathrm{L} \text { Gen. Eng. }
\end{aligned}
$$


KELL FSB 79C COLLECTED ON 01/28/90 LABORATORY ANALYSES CONTINUED

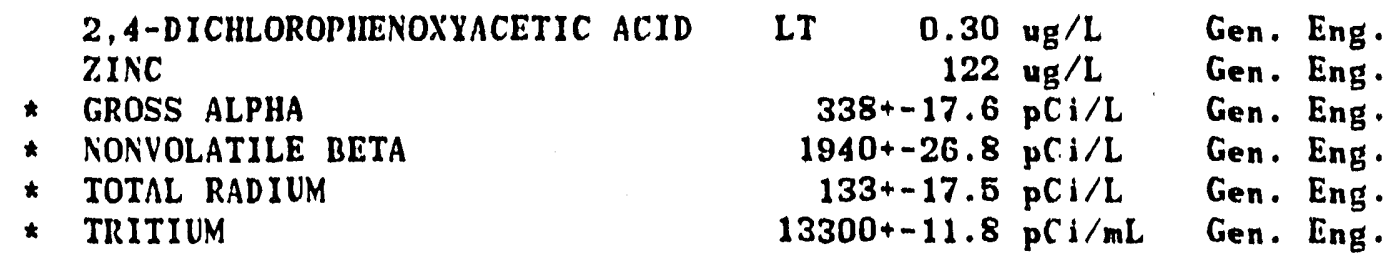

HELL FSB 87A

$\begin{array}{llllll}\text { SRS Grid } & N \quad 75601.7 & \text { Lntitude } 33.274751 & N \\ \text { Coordinates } & E \text { 50115.8 } & \text { Longitude } 81.681688 & W \\ \text { Scrcen Zone Elevation } 43.6-33.1 \quad \text { ft msl } & \end{array}$

Top of Casing Elevation $287.80 \mathrm{ft} \mathrm{msl}$

MEASUREMENTS CONDUCTED IN THE FIELD

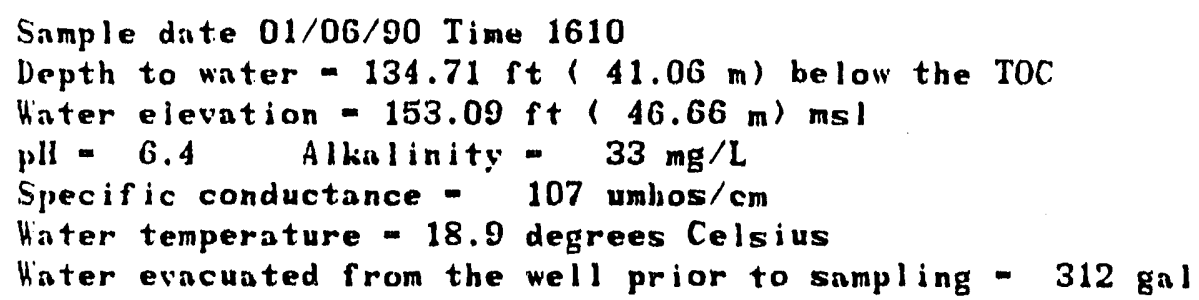

LABORATORI ANALYSES

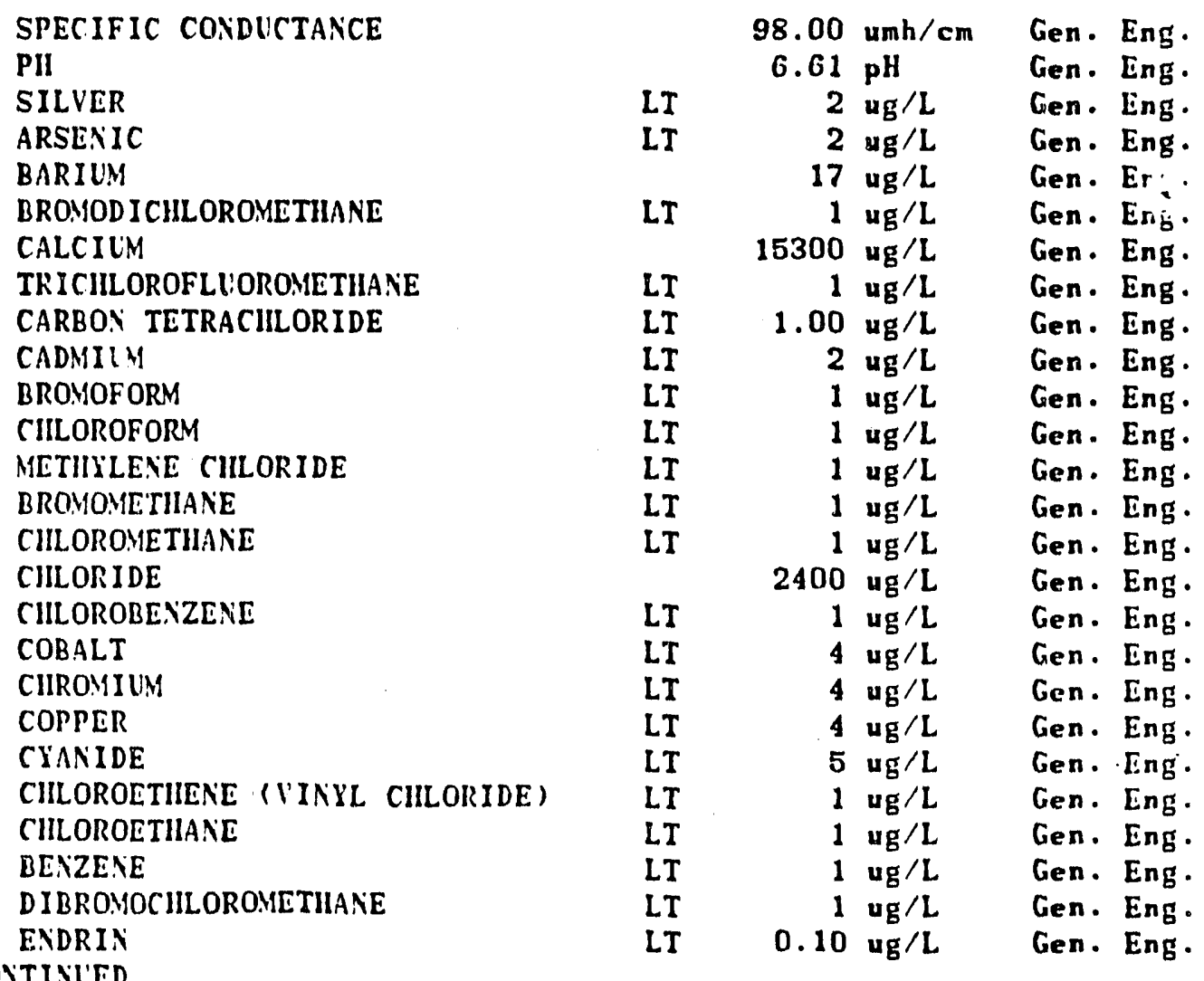


WELL FSB 87A COLLECTED ON 01/06/90 LABORATORY ANALYSES CONTINUED

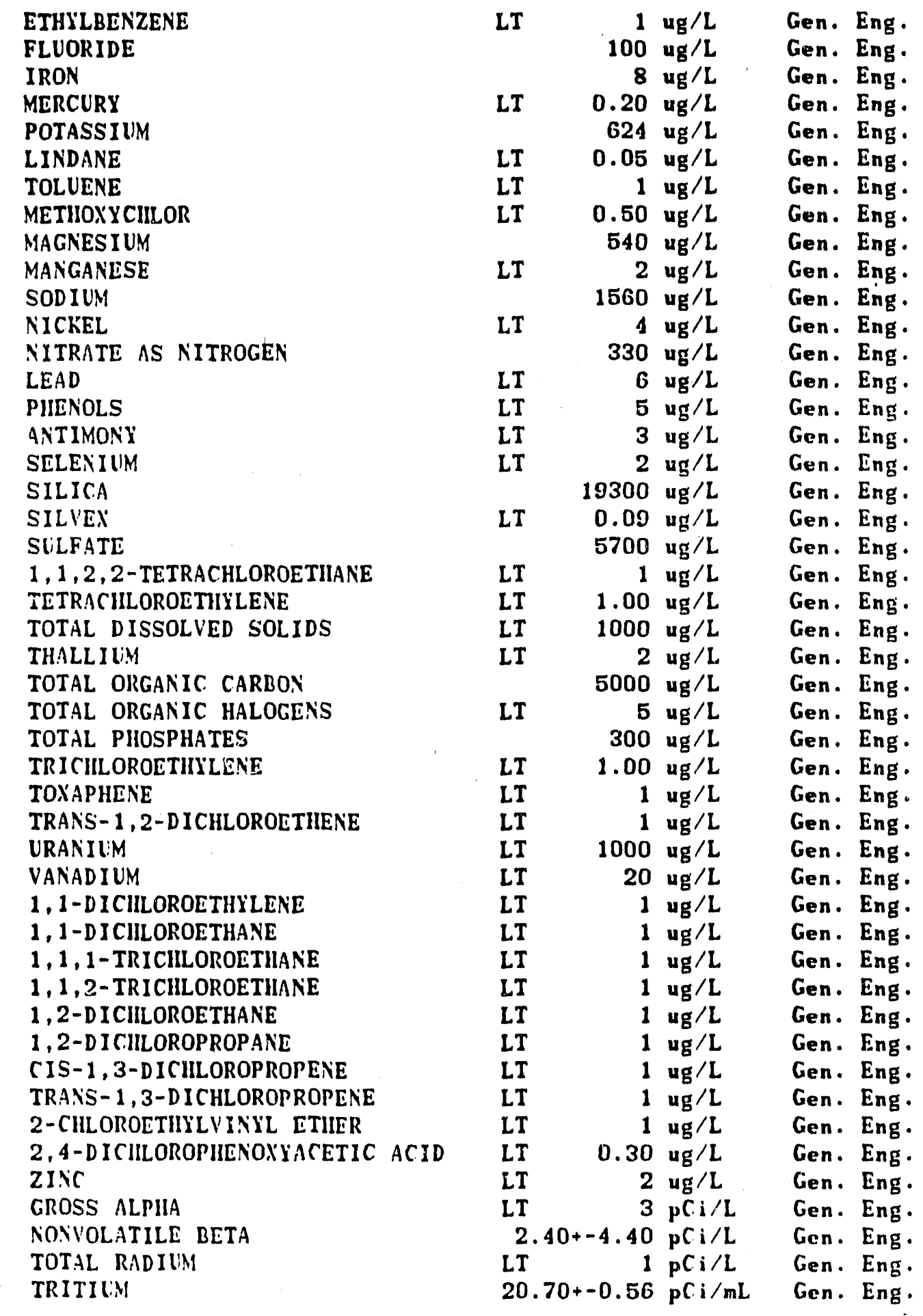


NELL FSB 87B

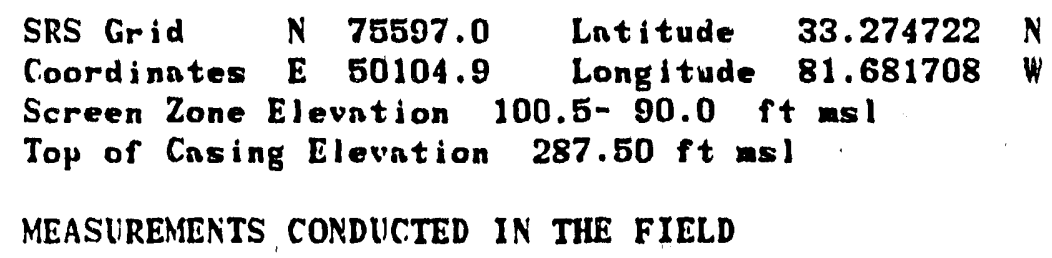

MEASUREMENTS CONDUCTED IN THE FIELD

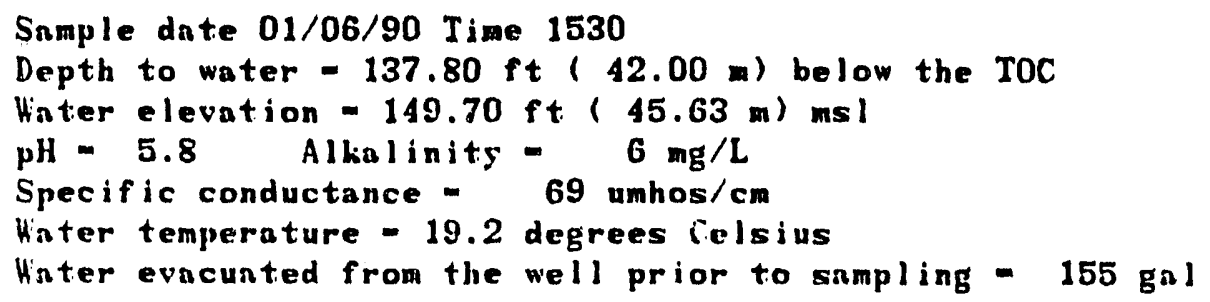

LABORATORY ANALYSES

SPECIFIC CONDUCTANCE
PII
SILUER
ARSENIC
BARIUM
BROMODICIILORONETHANE
CALCIUM
TRICIILOROFLUOROMETHANE
CARBON TETRACIILORIDE
CADNILM
BROMOFORM
CIILOROFORM
METIYLENE CHLORIDE
BROMOMETHANE
CIILORONETIIANE
CIILORIDE
CIILOROBENZENE
COBALT
CIIROMIUM
COPPER
CYANIDE
CIILOROETHENE (VINYL CIILORIDE)
CIILOROETHANE
BENZENE
DIBRONOCILORONITIIANE
ENDRIN
ETIILBENZENE
FLLORIDE
IRON
MERCURY
POTASSILM
LINDANE
TOLLENE
METHOXYCILOR
MAGNESILN
MANGANESE
SODIUM
NTINUED

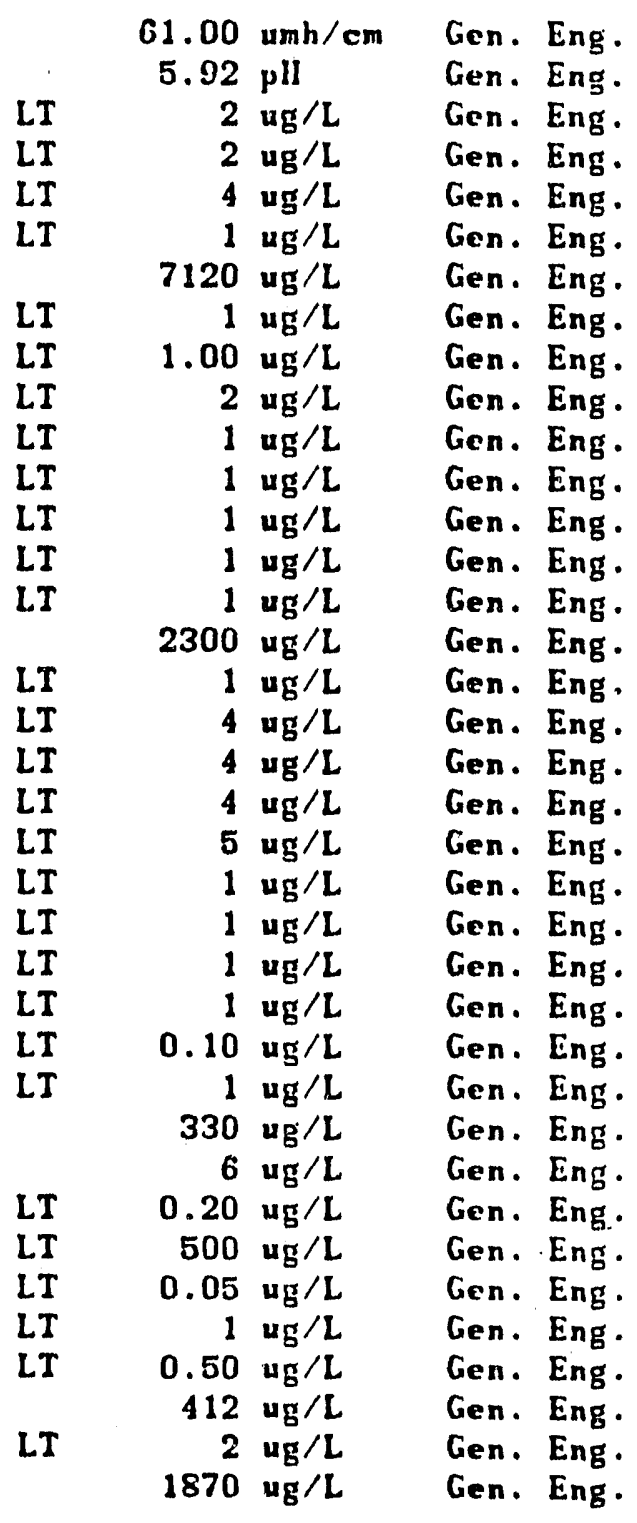


HELL FSB 87B COLLECTED ON 01/06/90 LABORATORY ANALYSES CONTINUED

NICKEL

NITRATE AS NITROGEN

LEAD

PHENOLS

LT

ANTIMONY

SELENIUM

SILICA

SILVEX

SULFATE

1,1,2,2-TETRACILOROETHANE

TETRACHLOROETHYLENE

TOTAL DISSOLVED SOLIDS

TIIALLIUM

TOTAL ORGANIC CARBON

TOTAL ORGANIC IIALOGENS

TOTAL PHOSPIIATES

TRICHLOROETIIYLENE

TOXAPHENE

TRA.SS-1,2-D I CIILOROETIIENE

IRANIUM

VANADIUM

1,1-DICIILOROETHYLENE

1,1-D ICIILOROETHANE

1,1,1-TRICILOROETHANE

1,1,2-TRICHLOROETHANE

1,2-DICIILOROETHANE

1,2-DICILOROPROPANE

CIS-1,3-DICHLOROPROPENE

TRANS-1 , 3-DICILOROPROPENE

2-CIILOROETHYLVINYL ETHER

2,4-DICHLOROPHENOXYACETIC ACID

ZINC

GROSS ALPIIA

NONVOLATILE BETA

TOTAL RADIUM

* TRITIUM

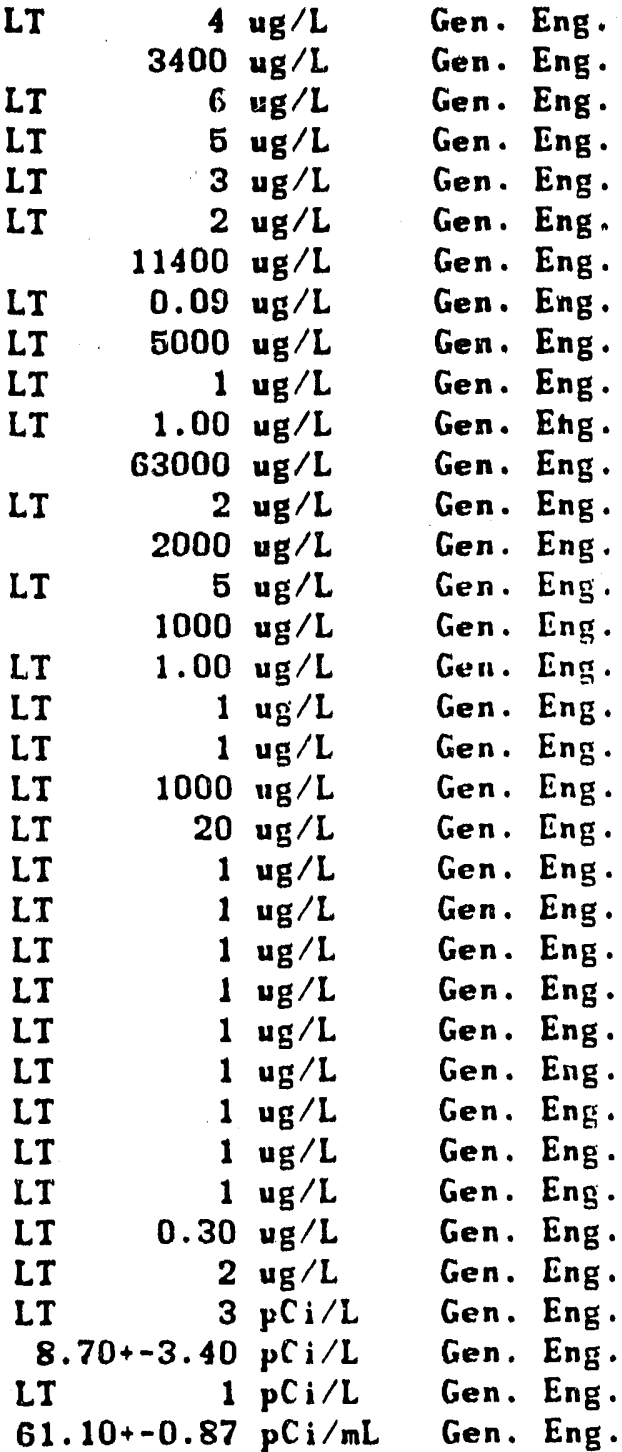


HELL FSB 87C

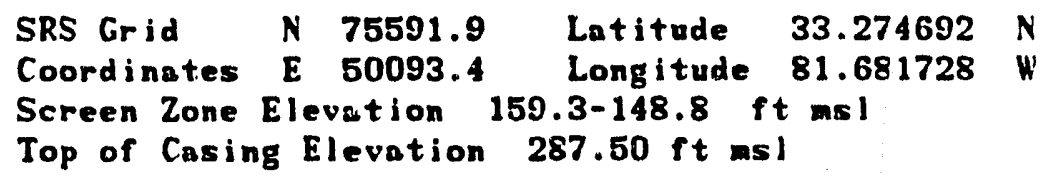

MEASUREMENTS CONDUCTED IN THE FIELD

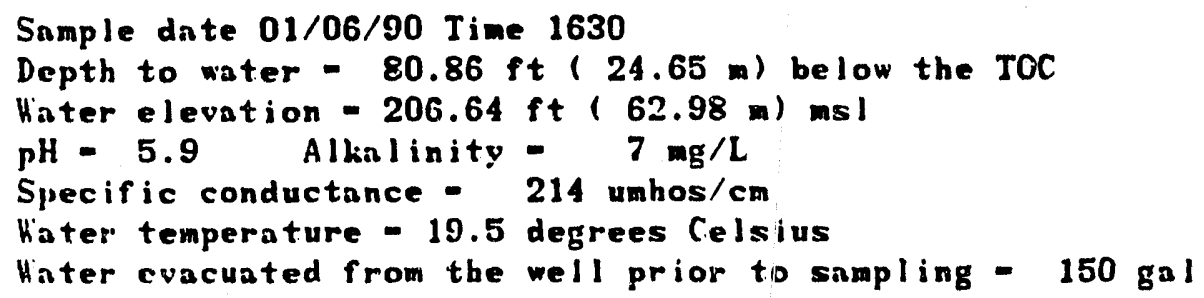

SPECIFIC CONDUCTANCE

PII

SILVER

ARSENIC

BARIUM

BROMOD I CHLOROMETIIANE

CALCIUM

TRICILLOROFLUOROMETHANE

CARBON TETRACHLORIDE

CADMIUM

BROMOTORM

CILOROFORM

METIIILENE CHI.ORIDE

BROMOMETHANE

CIILOROMETHANE

CIILORIDE

CIIL.OROBENZENE

COBALT

CHROMIUM

COPPER

CYANIDE

CILOROETHENE (VINIL CHLORIDE)

CILOROETIIANE

BE.ZZENE

DIBROMOCILLOROMETHINE

ENDRIN

ETIILLENZENE

FLUORIDE

IRON

MERCURY

POTASSIUM

LINDANE

TOLLENE

METIIOXYCHLOR

MAGIESILM

MANGAIESE

SODILY

CONTINLED
$200.0 \mathrm{umh} / \mathrm{cm}$

$$
6.04 \mathrm{pH}
$$

$2 \mathrm{ug} / \mathrm{L}$

$2 \mathrm{ug} / \mathrm{L}$

$33 \mathrm{ug} / \mathrm{L}$

$1 \mathrm{ug} / \mathrm{L}$

17000 ug $/ \mathrm{L}$

$1 \mathrm{ug} / \mathrm{L}$

$1.00 \mathrm{ug} / \mathrm{L}$

2 ug $/ L$

$1 \mathrm{mg} / \mathrm{L}$

$1 \mathrm{ug} / \mathrm{L}$

$1 \mathrm{ug} / \mathrm{L}$

$1 \mathrm{ug} / \mathrm{L}$

$1 \mathrm{ug} / \mathrm{L}$

$2200 \mathrm{ug} / \mathrm{L}$

$1 \mathrm{ug} / \mathrm{L}$

$4 \mathrm{ug} / \mathrm{L}$

$4 \mathrm{ug} / \mathrm{L}$

$4 \mathrm{ug} / \mathrm{L}$

$5 \mathrm{ug} / \mathrm{L}$

$1 \mathrm{ug} / \mathrm{L}$

$1 \mathrm{ug} / \mathrm{L}$

$1 \mathrm{ug} / \mathrm{L}$

$1 \mathrm{ug} / \mathrm{L}$

$0.10 \mathrm{wg} / \mathrm{L}$

$1 \mathrm{~kg} / \mathrm{L}$

$100 \mathrm{ug} / \mathrm{L}$

$7 \mathrm{ug} / \mathrm{L}$

$0.20 \mathrm{\mu g} / \mathrm{L}$

$500 \mathrm{ug} / \mathrm{L}$

$0.05 \mathrm{ug} / \mathrm{L}$

$1 \mathrm{ug} / \mathrm{L}$

$0.50 \mathrm{vg} / \mathrm{L}$

$2660 \mathrm{ug} / \mathrm{L}$

$12 \mathrm{ug} / \mathrm{L}$

$13800 \mathrm{ug} / \mathrm{L}$
Gen. Eng.

Gen. Eng.

Gen. Eng.

Gen. Eng.

Gen. Eng.

Gen. Eng.

Gen. Eng.

Gen. Eng.

Gen. Eng.

Gen. Eng.

Gen. Eng.

Gen. Eng.

Gen. Eng.

Gen. Eng.

Gen. I: $:$.

Gen. Eng.

Gen. Eng.

Gen. Eng.

Gen. Eng.

Gen. Eng.

Gen. Eng.

Gen. Eng.

Gen. Eng.

Gen. Eng.

Gen. Eng.

Gen. En:.

Gen. Est.

Gen. Eng.

Gen. Eng.

Gen. Eng.

Gen. Eng.

Gen. Eng.

Gen. Eng.

Gen. Eng.

Gen. Eng.

Gen. Eng.

Gen. Eng. 
KELL FSB $87 C$ COLLECTED ON 01/06/90 LABORATORY ANALYSES CONTINUED

NICKEL

* NITRATE AS NITROGEN

LEAD

PHENOLS

ANTIMONY

SELENIUM

SILICA

SILVEX

SULFATE

$1,1,2,2$-TETRACHLOROETIIANE

TETRACILOROETHYLENE

TOTAL DISSOL.VED SOLIDS

THALLILM

TOTAL ORGINIC CARBON

TOTAL ORGANIC HALOGENS

TOTAL PHOSPIIATES

TRICILLOROETHILENE

TOXAPIIENE

TRANS-1,2-DICHLOROETHENE

IRANILM

VAMADILM

1,1-D ICIILOROETIIYLENE

1,1-DICHLOROETHANE

$1,1,1$ - TR ICHLOROETHANE

$1,1,2$-TRICILLOROETHANE

1,2-DICIILOROETHANE

1,2-DICIILOROPROPANE

CIS-1,3-DICIILOROPROPENE

TRANS-1,3-DICHLOROPROPENE

2-CILOROETHILVINIL ETHER

2,4-DICHLOROPHENOXYACETIC ACID

ZINC

GROSS ALPIIA

NONI'OLATILE BETA

TOTAL RIDIUM

* TRITIUM
LI

$4 \mathrm{ug} / \mathrm{L}$
$20400 \mathrm{ug} / \mathrm{L}$

$17 \mathrm{ug} / \mathrm{L}$

LT

LT

LT

LT

LI

LT

LT

LIT

LT

LT

LT

LI

LT

LT

LI

LT

LT

LT

LT

LT

LT

LI

LI

LI

LT

LT

LT

$1330+-3.25 \mathrm{pCi} / \mathrm{mL}$
Gen. Eng.

Gen. Frg:

Gen. Eng.

Gen. Titsg.

Gen. Eng.

Gen. Eng.

Gen. Eng.

Gen. Eng.

Gen. Eng.

Gen. Eng.

Gen. Eng.

Gen. Eng.

Gen. Eng.

Gen. Eng.

Gen. Eng.

Gen. Eng.

Gen. Eng.

Gen. Eng.

Gen. Eng.

Gen. Eng.

Gen. Er.g.

Gen. Eng.

Gen. Eng.

Gen. Eng.

Gen. Eng.

Gen. Eng.

Gen. Eng.

Gen. Eng.

Gen. Eng.

Gen. Eng.

Gen. Eng.

Gen. Eng.

Gen. Eng.

Gen. Eng.

Gen. Eng.

Gen. Eng. 
WELL FSB 87D

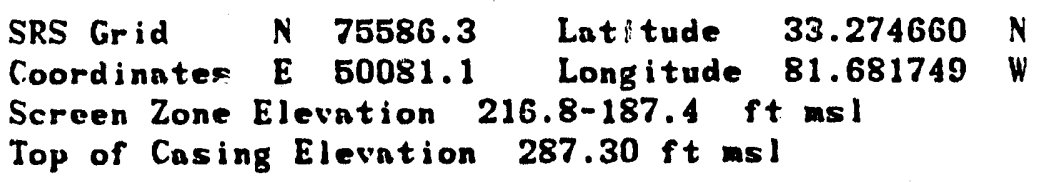

MEASUREMENTS CONDUCTED IN THE FIELD

Sample date 01/02/90 Time 1455

The well was dry.

KELL FSB $8 \delta C$

SRS Grid N 75019.4 Lntitude $33.277078 \mathrm{~N}$

Coordinates E 51518.0 Longitude 81.678031 W'

Screen Zone Elevation 168.4-158.4 ft msl

Top of Casing Elesntion $283.00 \mathrm{ft} \mathrm{ms}$

MEASLREMENTS CONDUCTED IN THE FIELD

Sample date $01 / 16 / 90$ Time 1015

Depth to water - $72.94 \mathrm{ft}(22.23 \mathrm{~m})$ below the TOC

Hate levation $=210.06 \mathrm{ft}(64.03 \mathrm{~m}) \mathrm{msl}$

pH $=5.6 \quad$ Alkalinity $=7 \mathrm{mg} / \mathrm{L}$

Specific sonductance - 59 umhos/cm

Viater temperature -17.3 degrees Celsius

Hater evacuated from the well prior to sampling - $135 \mathrm{gnl}$

LABORATORI ANALYSES

SPFCIFIC CONDUCTANCE

SPECIFIC CONDUCTANCE

PHI

PII

SILVER

SILVER

ARSENIC

ARSENIC

BARIUM

BARILM

BROMOD ICHLORONETHANE

BROMODICHLOROMETHANE

CALCILM

CALCILM

TRICILLOROFLIOROMETHANE

TRICILOROFLIORONETHINE

CARBON TE TKACILORIDE

CARBON TETRACHLORIDE

CADSIIUM

CADMIIIY

BROMOFORM

BRONOFORY
LT

LT

LT

LT

LI

LT

LI

LI

LT

LT

LT

LT

LT

LT
$47.40 \mathrm{umh} / \mathrm{cm}$ $53.00 \mathrm{umh} / \mathrm{cm}$

$5.76 \mathrm{pH}$

$5.73 \mathrm{pll}$

2 ug $/$

$2 \mathrm{ug} / \mathrm{L}$

$3 \mathrm{ug} / \mathrm{L}$

$2 \mathrm{ug} / \mathrm{L}$

$14 \mathrm{ug} / \mathrm{L}$

$12 \mathrm{ug} / \mathrm{L}$

$5 \mathrm{ug} / \mathrm{L}$

$1 \mathrm{ug} / \mathrm{L}$

$3864 \mathrm{ug} / \mathrm{L}$

$4210 \mathrm{ug} / \mathrm{L}$

$5 \mathrm{ug} / \mathrm{L}$

$1 \mathrm{ug} / \mathrm{L}$

$5.00 \mathrm{ug} / \mathrm{L}$

$1.00 \mathrm{ug} / \mathrm{L}$

$3 \mathrm{ug} / \mathrm{L}$

$2 \mathrm{ug} / \mathrm{L}$

$5 \mathrm{ug} / \mathrm{L}$

$1 \mathrm{ug} / \mathrm{L}$
MetaTrace

Gen. Eng.

MetaTrace

Gen. Eng.

MetaTrace

Gen. Eng.

MetaTrnce

Gen. Eng.

MetaTrace

Gen. Eng.

MetaTrace

Gen. Eng.

MetaTrace

Gen. Eng.

MetaTrace

Gen. Eng.

MetaTrace

Gen. Eng.

MetaTrace

Gen. Eng.

MetaTrace

Gen. Eng. 
KELL FSB 88C COLLECTED ON 01/16/90 LABORATORY ANALYSES CONIINUED

\begin{tabular}{|c|c|c|c|c|}
\hline CIILOROFORM & LT & 5 & ug/L & MetaIrnce \\
\hline CIILOROFORM & LT & 1 & $u g / L$ & Gen. Eng. \\
\hline METIYLENE CHLORIDE & LT & 5 & $u g / L$ & MetaTrnce \\
\hline METIIYLENE CIILORIDE & LT & 1 & $u g / L$ & Gen. Eng. \\
\hline BROMOMETHANE & LI & 10 & $u g / L$ & MetaTrace \\
\hline BRONOMETHANE & LT & 1 & $\mathbf{u g} / \mathrm{L}$ & Gen. Eng. \\
\hline CIILOROMETHANE & LT & 10 & $\mathbf{u g} / \mathrm{L}$ & MetaTrace \\
\hline CIILOROMETHANE & LI & 1 & $u_{g} / \mathrm{L}$ & Gen. Eng. \\
\hline CIILORIDE & & 3100 & $u_{g} / L$ & MetaTrace \\
\hline CIILORIDE & & 4100 & $u_{g} / L$ & Gen. Eng. \\
\hline CIILOROBENZENE & LT & 5 & $u_{g} / L$ & MetaTrice \\
\hline CIILOROBENZENE & $\mathrm{LT}$ & 1 & $\mathbf{u g} / \mathrm{L}$ & Gen. Eng. \\
\hline COBALT & LT & 15 & ug/L & MetaTrace \\
\hline COBALT & LT & 4 & $u g / L$ & Gen. Eng. \\
\hline CIIRONIUM & LT & 3 & ug/L & MetaTrace \\
\hline CIIRONIUM & LT & 4 & $\mathbf{u g} / \mathrm{L}$ & Gen. Eng. \\
\hline COPIER & LT & 7 & $\mathbf{u g} / \mathrm{L}$ & MetaTrace \\
\hline COPPER & LT & 4 & $u g / L$ & Gen. Ling. \\
\hline CHANIDE & LI & 5 & $\mathbf{u g} / \mathrm{L}$ & MetaTrace \\
\hline CIANIDE & LI & 5 & $u g / L$ & Gen. Eng. \\
\hline CIILOROETHENE (VINYL CHLORIDE) & LT & 5 & $u g / L$ & MetaTrace \\
\hline CIILOROETUENE (VINYL CHLORIDE) & LT & 1 & $u g / L$ & Gen. Eng. \\
\hline CIILOROETHANE & LI & 10 & $u g / L$ & MetaTrace \\
\hline CILLOROETIIANE & LT & 1 & $w g / L$ & Gen. Eng. \\
\hline BEXZENE & LT & 5 & $u g / L$ & MetaTrace \\
\hline BENZENE & LT & 1 & $u g / L$ & Gen. Eng. \\
\hline DIBROMOCIILOROMETIIANE & LT & 5 & $u g / L$ & MetaTrace \\
\hline DIBROYOCIILOROMETHANE & LT & 1 & $u g / L$ & Gen. Eng. \\
\hline ENDRIN & LT & 0.01 & $u g / L$ & MetaTrace \\
\hline ENDRIX & LT & 0.10 & $u g / L$ & Gen. Eng. \\
\hline ETIILBENZENE & LT & $\mathbf{5}$ & $u g / L$ & MetaTrace \\
\hline ETHXLBE.ZZENE & LT & 1 & $\mathrm{ug} / \mathrm{L}$ & Gen. $I_{i}:$. \\
\hline FLLORIDE & LI & 250 & ug/L & MetaIrice \\
\hline FLLORIDE & LT & 100 & $\mathbf{u g} / \mathrm{L}$ & Gen. Eng. \\
\hline IRON & & 31 & $u g / L$ & MetaTrace \\
\hline IRON & & 14 & $4 g / L$ & Gen. Eng. \\
\hline MERCL'RY & LT & 0.20 & $w g / L$ & MetaTrace \\
\hline MERCLRY & LI & 0.20 & ug $/ L$ & Gen. Eng. \\
\hline POTASSIUM & LT & 1620 & ug $/ L$ & MetaTrace \\
\hline POTISSILN & LI & 500 & $u g / L$ & Gen. Eng. \\
\hline LINDANE & LT & 0.00 & $u g / L$ & MetaTrace \\
\hline LINDANE & LT & 0.05 & $u g / L$ & Gen. Eng. \\
\hline TOLIENE & LT & 5 & ug $/ L$ & MetaTrnce \\
\hline TOLLENE & ZT & 1 & $u g / L$ & Gen. Eng. \\
\hline METIIOXYCIILOR & LT & 0.50 & $u g / L$ & MetaTrnce \\
\hline METHOXYCIILOR & LT & 0.50 & $\mathrm{ug} / \mathrm{L}$ & Gen. Eng. \\
\hline MAG.NES I IM & & 832 & vg $/ L$ & MetaTrace \\
\hline MAGNESILIM & & 474 & $u g / L$ & Gen. Eng. \\
\hline MANGANESE & & 11 & $u g / L$ & MetaTrace \\
\hline MANGANESE & & 9 & $\mathrm{ug} / \mathrm{L}$ & Gen. Eng. \\
\hline SOD I IAI & & 3661 & $u g / L$ & MetaTrace \\
\hline SOD IUY & & 3810 & $u g / L$ & Gen. Eng. \\
\hline NICKEL & LT & 5 & $u g / L$ & MetaTrnce \\
\hline NICKEL & LT & 4 & ug $/ L$ & Gen. Eng. \\
\hline
\end{tabular}


WELL FSB 88C COLLECTED ON 01/16/90 LABORATORY ANALYSES CONTINUED

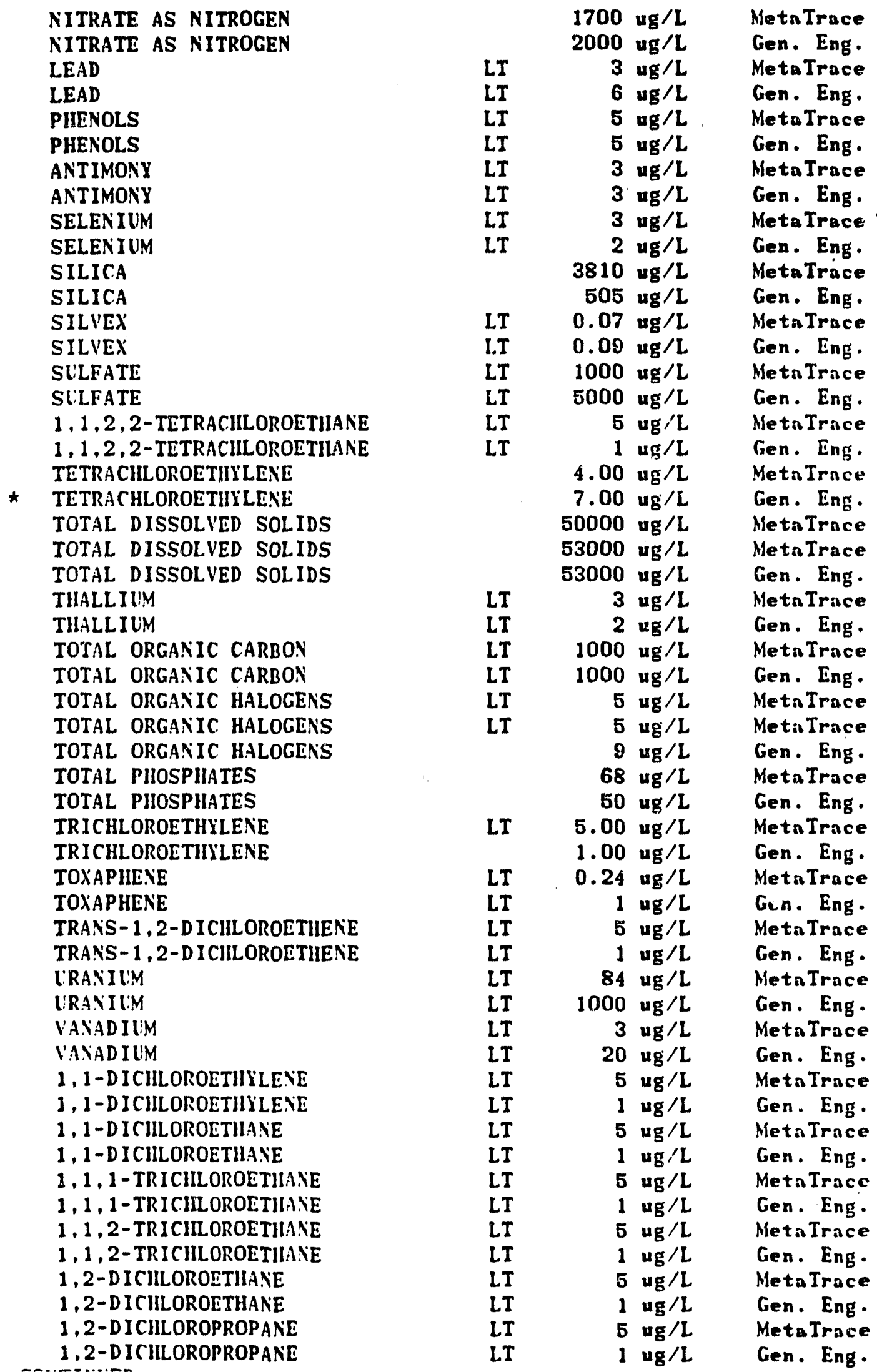


NELL FSB 88C COLLECTED ON 01/16/90 LABORATORY ANALYSES CONTINUED

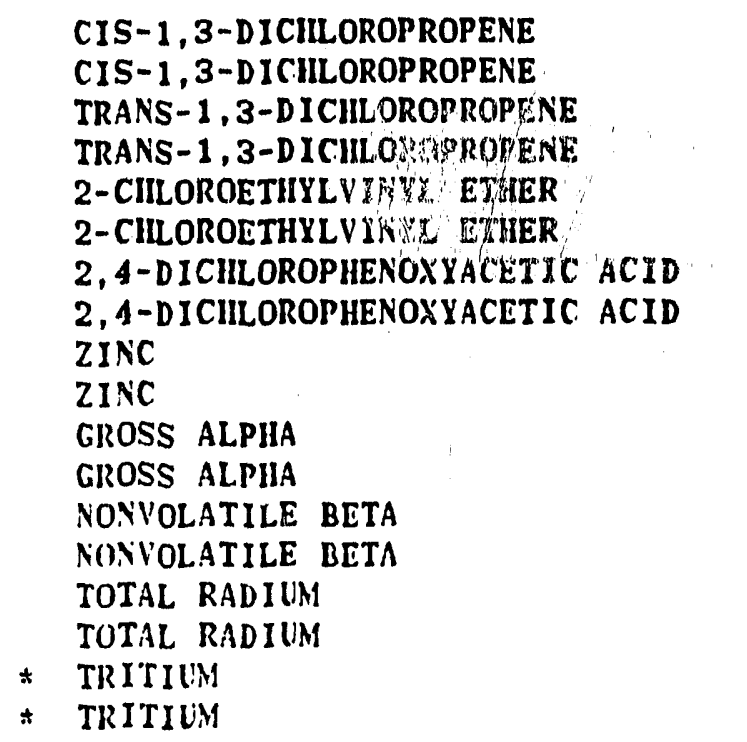

\begin{tabular}{|c|c|c|c|}
\hline LT & & ug $/ \mathrm{L}$ & MetaTrace \\
\hline LT & 1 & ug $/ \mathrm{L}$ & Gen. Eng. \\
\hline LT & 5 & ug $/ \mathrm{L}$ & MetaTrace \\
\hline LT & 1 & ug $/ \mathrm{L}$ & Gen. Eng. \\
\hline LT & 5 & ug $/ L$ & MetaTrace \\
\hline $\mathbf{L T}$ & 1 & ug $/ \mathrm{L}$ & Gen. Eng. \\
\hline LT & 0.16 & $\mathrm{ug} / \mathrm{L}$ & MetaTrace \\
\hline LT & 0.30 & ug $/ \mathrm{L}$ & Gen. Eng. \\
\hline & 42 & ug / L & MetaTrace \\
\hline & 13 & ug / L & Gen. En: \\
\hline LT & 3 & $\mathrm{pCi} / \mathrm{L}$ & MetaTrice \\
\hline LT & 3 & $\mathrm{pC} \mathbf{i} / \mathrm{L}$ & Gen. Eng. \\
\hline LT & 5 & $\mathrm{pCi} / \mathrm{L}$ & MetaTrace \\
\hline LT & 2 & $\mathrm{pCi} / \mathrm{L}$ & Gen. Eng. \\
\hline LI & 1 & $\mathrm{p} C \mathrm{i} / \mathrm{L}$ & MetaTrice \\
\hline LT & 1 & $i \mathrm{i} / \mathrm{L}$ & Gen. Fng \\
\hline 24 & 00 & $\mathrm{pCi} / \mathrm{mL}$ & MetaTrac \\
\hline & 0 & $/ \mathrm{m}$ & Gen. I \\
\hline
\end{tabular}

HELL FSB 88C

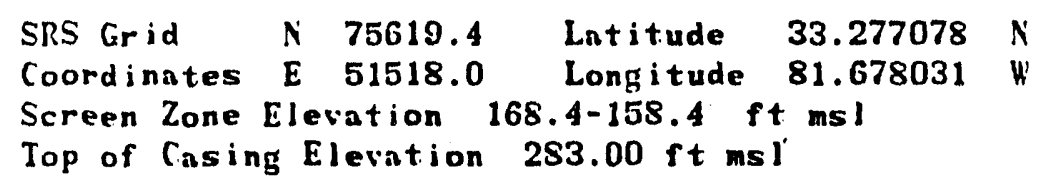

MEASUREMENTS CONDUCTED IN THE FIELD

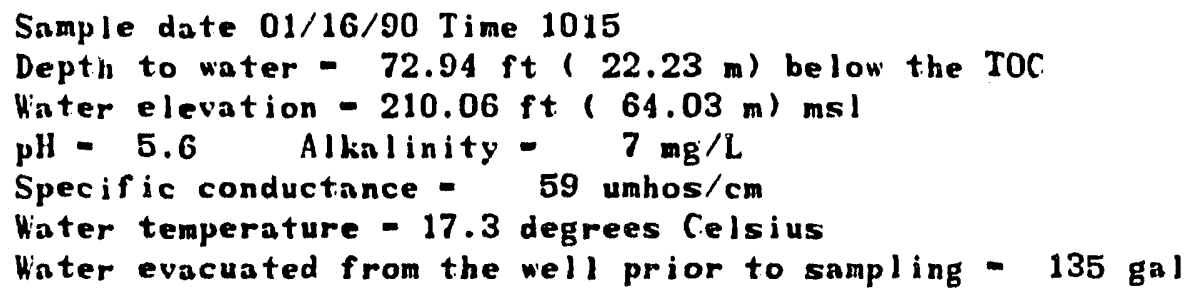

SPECIFIC CONDUCTANCE SPECIFIC CONDLICTANCE SPECIFIC CONDICTANCE

PII

PII

PII

SILVER

SILVER

SILVER

ARSENIC

ARSENIC

ARSEIIC

BAKILIM

BARILM

CONTINIED

$\begin{aligned} 47.50 \mathrm{umh} / \mathrm{cm} & \text { MataTrace } \\ 54.00 \mathrm{umh} / \mathrm{ch} & \text { Gen. Eng. } \\ 54.00 \mathrm{umh} / \mathrm{cm} & \text { Geg. Eng. } \\ 5.74 \mathrm{pll} & \text { MetaTrace } \\ 4.55 \mathrm{pll} & \text { Gen. Eng. } \\ 4.56 \mathrm{pH} & \text { Gen. Eng. } \\ 2 \mathrm{ug} / \mathrm{L} & \text { MetaTrace } \\ 2 \mathrm{ug} / \mathrm{L} & \text { Gen. Eng. } \\ 2 \mathrm{ug} / \mathrm{L} & \text { Gen. Eng. } \\ 3 \mathrm{ug} / \mathrm{L} & \text { MetaTrnce } \\ 2 \mathrm{ug} / \mathrm{L} & \text { Gen. Eng. } \\ 2 \mathrm{ug} / \mathrm{L} & \text { Gen. Eng. } \\ 13 \mathrm{ug} / \mathrm{L} & \text { MetaTrace } \\ 12 \mathrm{ug} / \mathrm{L} & \text { Gen. Eng. }\end{aligned}$


WELL FSB 88C COLLECTED ON 01/16/90 LABORATORY ANALYSES CONTINUED

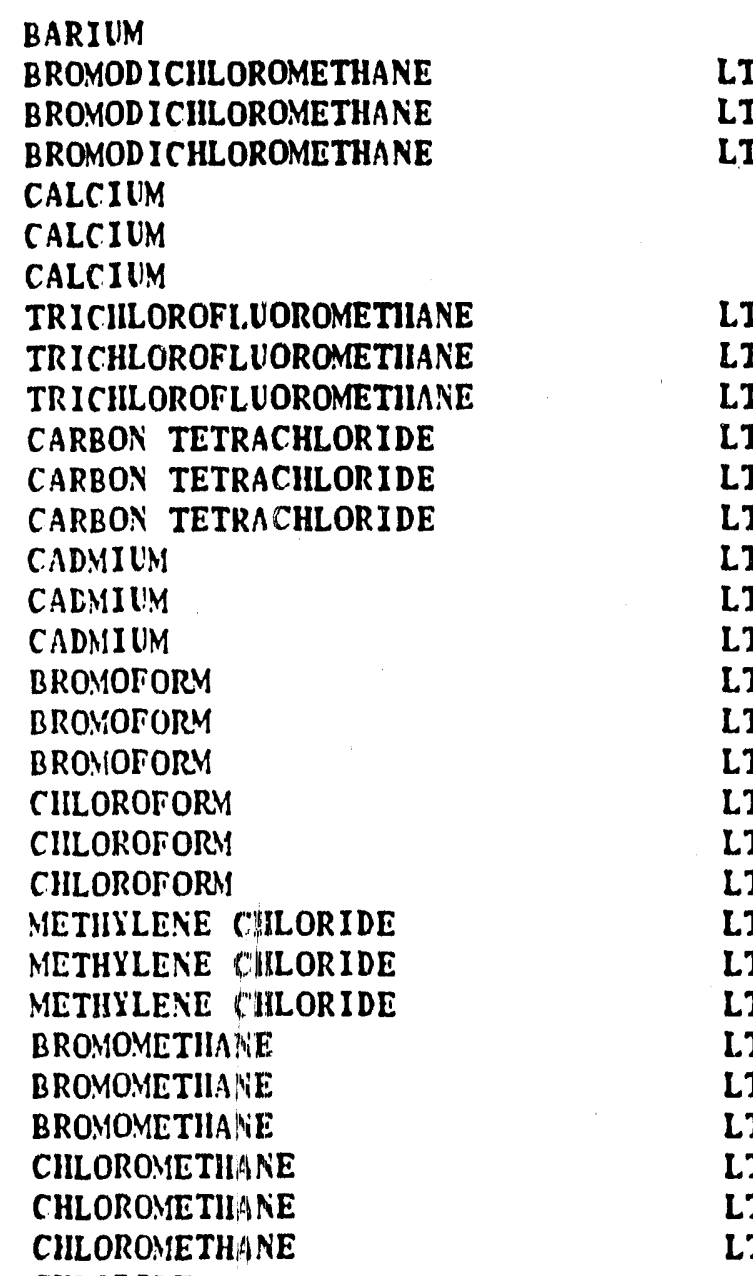

CHLORIDE

CILORIDE

CIILORIDE

CILLOROBENZFENE

CILLOROBENZIENE

CHLOROBENZIENE

COBALT

COBALT

COBALT

CIIROMIUM

CIIROMILM

CHRONIUM

COPPER

COPPER

COPPER

CYANIDE

CYANIDE

CYANIDE

CULOROETHENE (VINYL CILORIDE)

CIILOROETHE.YE (VINYL CILLORIDE)

CHLOROETIIENE (VINYL CILLORIDE)

CHLOROETHANE

CIILOROETHANE

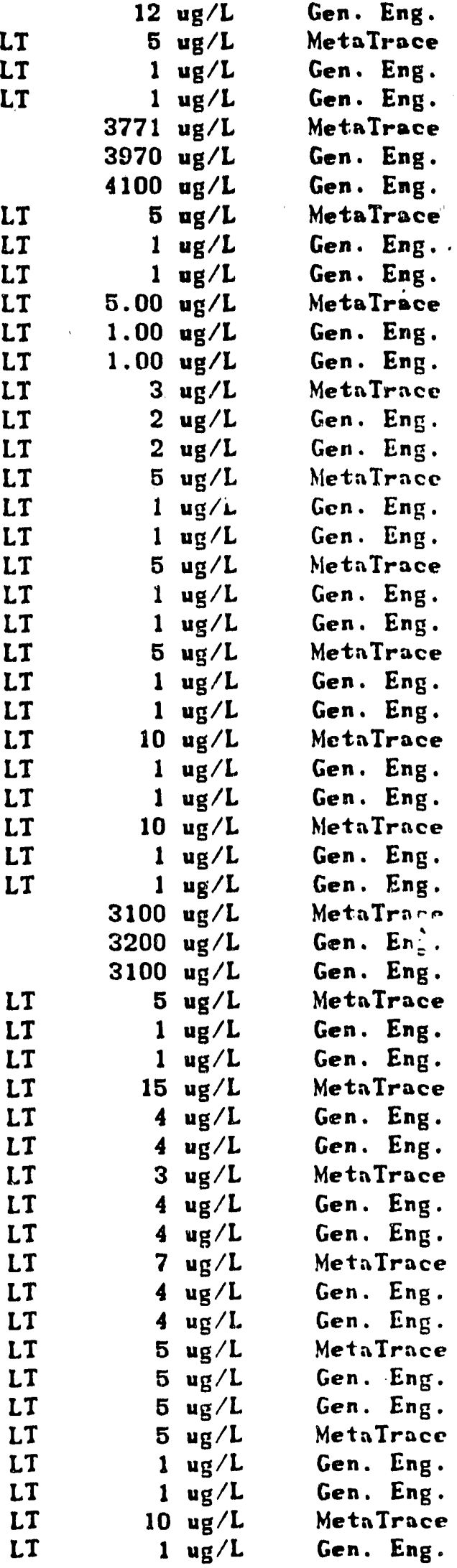




\begin{tabular}{|c|c|c|c|c|}
\hline $\begin{array}{l}\text { CHLOROETHANE } \\
\text { BENZENE }\end{array}$ & LT & 1 & $\mathrm{ug} / \mathrm{L}$ & $\begin{array}{l}\text { Gen. Eng. } \\
\text { MetaTrnce }\end{array}$ \\
\hline $\begin{array}{l}\text { BENZENE } \\
\text { BENZENE }\end{array}$ & LT & $\begin{array}{l}5 \\
1\end{array}$ & $\begin{array}{l}u g / L \\
u g / L\end{array}$ & $\begin{array}{l}\text { MetaTrace } \\
\text { Gen. Eng. }\end{array}$ \\
\hline BENZENE & LT & 1 & ug $/ \mathrm{L}$ & Gen. Eng. \\
\hline DIBROMOCHLOROMETIIANE & LT & 5 & $\operatorname{ug} / L$ & MetaTrace \\
\hline DIBROMOCHLOROMETHANE & LT & 1 & $\mathrm{ug} / \mathrm{L}$ & Gen. Eng. \\
\hline DIBROMOCILLOROMETHANE & LT & 1 & ug/L & Gen. Eng. \\
\hline EXDRIN & LT & 0.01 & $u g / L$ & MetaTrice \\
\hline ENDRIN & LT & 0.10 & $\mathrm{ug} / \mathrm{L}$ & Gen. Eng. \\
\hline ENDRIN & LT & 0.10 & $\operatorname{ug} / \mathrm{L}$ & Gen. Eng. \\
\hline ETIIILBENZENE & LI & 5 & $\mathrm{~kg} / \mathrm{L}$ & MetaTrnce \\
\hline ETIYLBENZENE & LT & 1 & ug $/ \mathrm{L}$ & Gen. Eng. \\
\hline ETIIYLBENZENE & LT & 1 & ug/L & Gen. Eng. \\
\hline FLUIORIDE & LI & 250 & ug $/ \mathrm{L}$ & MetaTrnce \\
\hline FLUORIDE & LT & 100 & ug $/ L$ & Gen. Eng. \\
\hline FLLOORIDE & LT & 100 & ug/L & Gen. Eng. \\
\hline IRON & & 12 & $\mathrm{ug} / \mathrm{L}$ & MetaTrice \\
\hline IRON & & 12 & $\mathrm{ug} / \mathrm{L}$ & Gen. Eng. \\
\hline IRON & & 14 & $\mathrm{ug} / \mathrm{L}$ & Gen. Eng. \\
\hline MERCIRY & LI & 0.20 & ug $/ \mathrm{L}$ & MetaTrace \\
\hline MERCLRY & & 1.08 & ug $/ \mathrm{L}$ & Gen. Eng. \\
\hline MERCLIRY & & 1.22 & ug $/ \mathrm{L}$ & Gen. Eng. \\
\hline POTASSILY! & & 2146 & $u_{g} / L$ & MetaTrace \\
\hline POTASSIUM & L.T & 500 & ug $/ \mathrm{L}$ & Gen. Eng. \\
\hline POTASSILM & LT & 500 & $\operatorname{mg} / L$ & Gen. Eng. \\
\hline LINDANE & LT & 0.00 & ug $/ \mathrm{L}$ & MetaTrace \\
\hline LINDANE & LT & 0.05 & $\mathrm{ug} / \mathrm{L}$ & Gen. Eng. \\
\hline LINDANE & LI & 0.05 & $\mathrm{ug} / \mathrm{L}$ & Gen. Eng. \\
\hline TOLIENE & LT & 5 & ug $/ \mathrm{L}$ & MetaTrace \\
\hline TOLUENE & LI & 1 & $\mathrm{ug} / \mathrm{L}$ & Gen. Eng. \\
\hline TOLIENE & LI & 1 & $u_{g} / L$ & Gen. Eng. \\
\hline METHOXYCIILOR & LT & 0.50 & ug $/ \mathrm{L}$ & MetaTrace \\
\hline METHOXYCHLOR & LT & 0.50 & ug/L & Gen. Eng. \\
\hline METHONYCIILOR & LT & 0.50 & $u g / L$ & Gen. Eng. \\
\hline MAGNESIUM & & 499 & ug $/ L$ & MetaTrace \\
\hline MAGNESIUM & & 449 & $\mathrm{mg} / \mathrm{L}$ & Gen. Eng. \\
\hline MAGNESIUM & & 453 & ug $/ L$ & Gen. Eng. \\
\hline MAYGANESE & & 10 & ug/L & MetaTrace \\
\hline MANGANESE & & 8 & $\operatorname{ug} / \mathrm{L}$ & Gen. Eng. \\
\hline MANGATESE & & 8 & ug $/ \mathrm{L}$ & Gen. Eng. \\
\hline SODILM & & 3576 & $\mathrm{ug} / \mathrm{L}$ & MetaTrace \\
\hline SODILYM & & 3460 & $\operatorname{ug} / \mathrm{L}$ & Gen. Eng. \\
\hline SODILM & & 3650 & $\operatorname{ug} / \mathrm{L}$ & Gen. Eng. \\
\hline NICKEL & LT & 5 & $\operatorname{ug} / \mathrm{L}$ & MetaTrace \\
\hline NICKEL & LT & 4 & ug $/ \mathrm{L}$ & Gen. Eng. \\
\hline NICKFL & LT & 4 & $\mathrm{ug} / \mathrm{L}$ & Gen. Ing. \\
\hline NITRATE AS NITROGEN & & 1700 & ug $/ \mathrm{L}$ & MetaTrace \\
\hline NITRATE AS NITROGEN & & 1800 & ug/L & Gen. Eng: \\
\hline NITRATE AS NITROGEN & & 1800 & ug $/ L$ & Gen. Eng. \\
\hline LEAD & LT & 3 & $\operatorname{ug} / \mathrm{L}$ & MetaTrace \\
\hline LEAD & $\mathrm{LI}$ & 6 & $u g / L$ & Gen. Eng. \\
\hline LEAD & LT & 6 & $\operatorname{\omega g} / \mathrm{L}$. & Gen. Eng \\
\hline PIIEYOLS & LT & 5 & $\operatorname{ug} / \mathrm{L}$ & MetaTrac \\
\hline PIIETOLS & & 22 & ug/L & Gen. Eng \\
\hline
\end{tabular}


NELL FSB 88C COLLECTED ON 01/16/90 LABORATORY ANALYSES CONTINUED

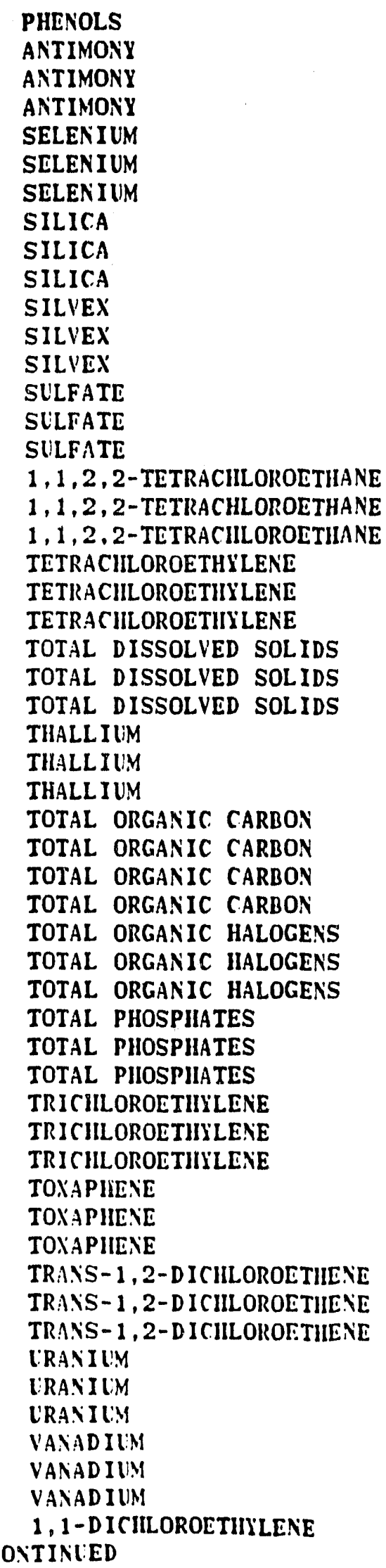

\begin{tabular}{|c|c|c|c|}
\hline & 22 & $\mathrm{ug} / \mathrm{L}$ & Gen. Eng. \\
\hline LT & $\begin{array}{l}3 \\
3\end{array}$ & $\operatorname{mg} / \mathrm{L}$ & MetaTrace \\
\hline $\begin{array}{l}\mathrm{LT} \\
\mathrm{LT}\end{array}$ & $\begin{array}{l}3 \\
3\end{array}$ & $\begin{array}{l}\operatorname{ug} / L \\
\operatorname{ug} / \mathrm{L}\end{array}$ & $\begin{array}{l}\text { Gen. Eng. } \\
\text { Gen. Eng. }\end{array}$ \\
\hline & 3 & $u g / L$ & MetaTrace \\
\hline $\mathbf{T}$ & 2 & $\mathrm{ug} / \mathrm{L}$ & Gen. Eng. \\
\hline & $\begin{array}{r}2 \\
3716\end{array}$ & $\begin{array}{l}u_{g} / L \\
u_{g} / L\end{array}$ & $\begin{array}{l}\text { Gen. Eng. } \\
\text { MetaTrace }\end{array}$ \\
\hline & $780^{1}$ & $\mathrm{ug} / \mathrm{L}$ & Gen. Eng. \\
\hline & 8108 & $u g / L$ & Gen. Eng \\
\hline & 0.07 & $u_{g} / L$ & MetaTrace \\
\hline I & 0.00 & $u_{g} / L$ & Gen. Eng. \\
\hline & 0.09 & $u g / L$ & Gen. Eng. \\
\hline $\mathbf{I}$ & 1000 & $u_{g} / L$ & MetaTrace \\
\hline I & 5000 & $\operatorname{ug} / L$ & Gen. Eng. \\
\hline $\mathbf{T}$ & 5000 & ug/L & Gen. Eng. \\
\hline $\mathbf{I}$ & 5 & $u g / L$ & MetaTrace \\
\hline$L T$ & 1 & $u g / L$ & Gen. Eng. \\
\hline$r$ & 1 & $u g / L$ & Gen. \\
\hline & 4.00 & $u g / L$ & MetaTrace \\
\hline & 6.00 & ug/L & Gen. Eng. \\
\hline & 6.00 & $u_{g} / L$ & Gen. Eng. \\
\hline & 29000 & $\mathrm{ug} / \mathrm{L}$ & MetaTrace \\
\hline & 48000 & ug $/ L$ & Gen. Eng. \\
\hline & 51000 & $u_{g} / L$ & Gen. Eng. \\
\hline$T$ & 3 & $\operatorname{ugg}_{\mathrm{g}} / \mathrm{L}$ & MetaTrace \\
\hline $\mathrm{T}$ & 2 & $u_{g} / L$ & Gen. Eng. \\
\hline I & 2 & ug $/ L$ & Gen. Eng. \\
\hline$T$ & 1000 & ug $/ L$ & MetaTrace \\
\hline.$T$ & 1000 & $u g / L$ & MetaTrace \\
\hline $\mathbf{T}$ & 1000 & $\mathrm{ug} / \mathrm{L}$ & Gen. Eng. \\
\hline & 1000 & $\mathbf{u g} / \mathrm{L}$ & Gen. Eng. \\
\hline & 5 & $\mathrm{ug} / \mathrm{L}$ & MetaTrace \\
\hline & 7 & ug/L & Gen. Eng. \\
\hline & 7 & $\mathrm{ug} / \mathrm{L}$ & Gen. Eng. \\
\hline & 56 & $u_{g} / \mathbb{L}$ & MetaTrace \\
\hline & 70 & $u g / L$ & Gen. Eng. \\
\hline & 70 & $u g / L$ & Gen. Eng. \\
\hline$T$ & 5.00 & $\mathrm{ug} / \mathrm{L}$ & MetaTrace \\
\hline & 1.00 & $\mathrm{ug} / \mathrm{L}$ & Gen. Eng. \\
\hline & 1.00 & $u_{g} / L$ & Gen. Eng. \\
\hline LI & 0.24 & $u_{g} / L$ & MetaTrace \\
\hline LT & 1 & ug $/ L$ & Gen. Eng. \\
\hline LT & 1 & $u_{g} / L$ & Gen. Eng. \\
\hline LT & 5 & $u_{g} / L$ & MetaTrace \\
\hline LI & 1 & $\mathrm{ug} / \mathrm{L}$. & Gen. Eng. \\
\hline LI & 1 & $u_{g} / L$ & Gen. Eng. \\
\hline L' & 84 & ug $/ L$ & MetaTrace \\
\hline LI & 1000 & ug $/ L$ & Gen. Eng. \\
\hline 77 & 1000 & ug/L & Gen. Eng. \\
\hline & 3 & $u_{g} / L$ & MetaTr: \\
\hline & 20 & $\mathrm{ug} / \mathrm{L}$ & Gen. Eng. \\
\hline L' & 20 & $4 \mathrm{~g} / \mathrm{L}$ & Ge \\
\hline & 5 & $\operatorname{sg} / L$ & $n T r$ \\
\hline
\end{tabular}


HELL FSB 88C COLLECTED ON 01/16/90 LABORATORI ANALYSES CONTINUED

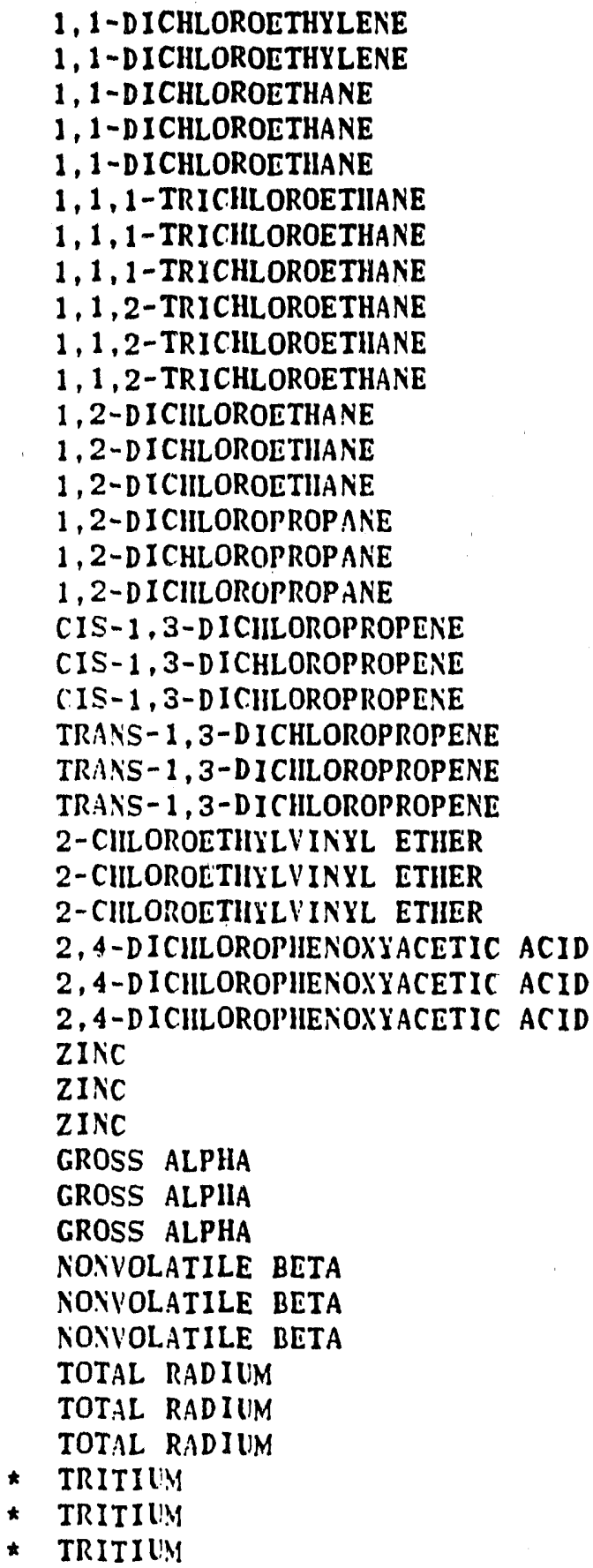

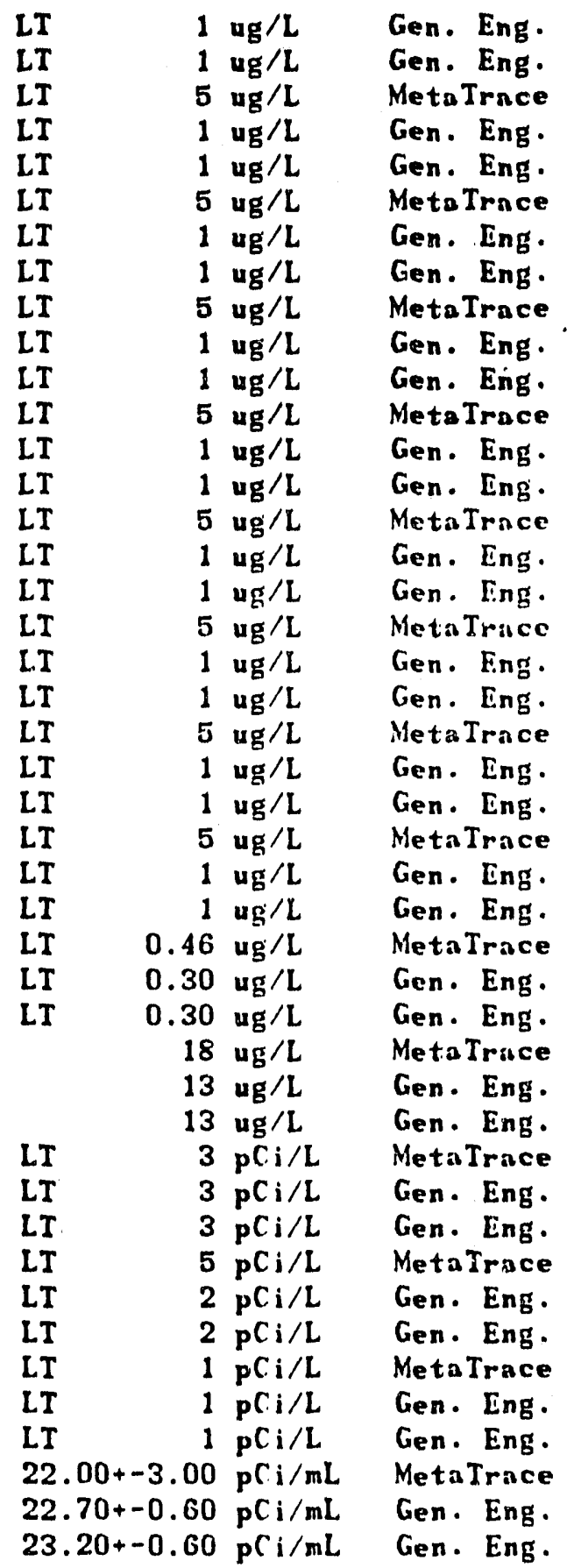


SRS Grid N $75621.8 \quad$ Eatitude $33.277098 \quad N$

Coordinates E 51527.0 Longitude 81.678012 W

Screen Zone Elevation 222.1-202.1 ft msl

Top of Casing Elevation $282.40 \mathrm{ft}$ ms l

MEASUREMENTS CONDUCTED IN THE FIELD

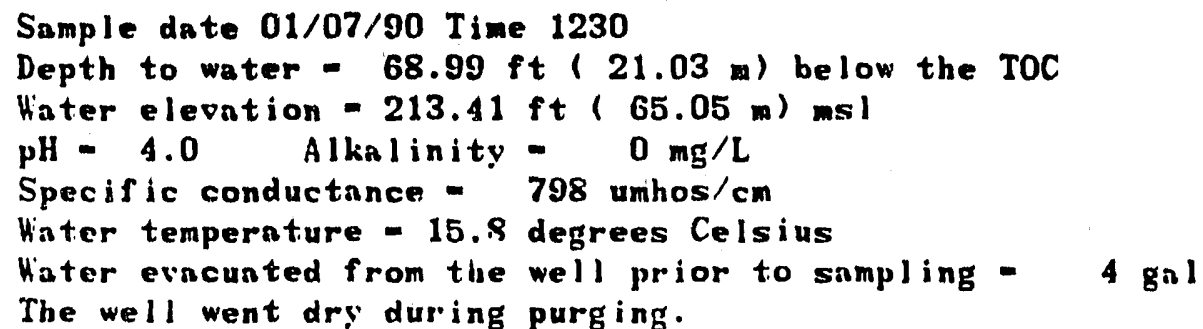

LABORATORY ANALYSES

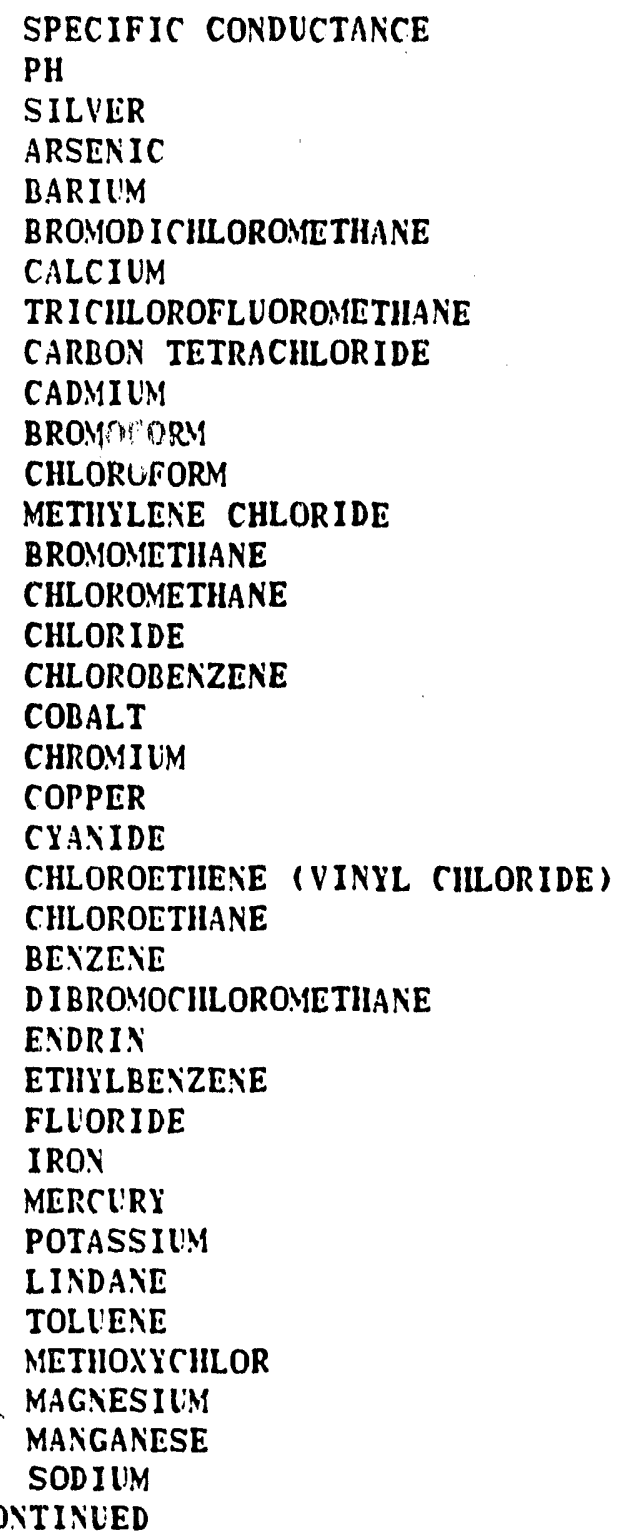

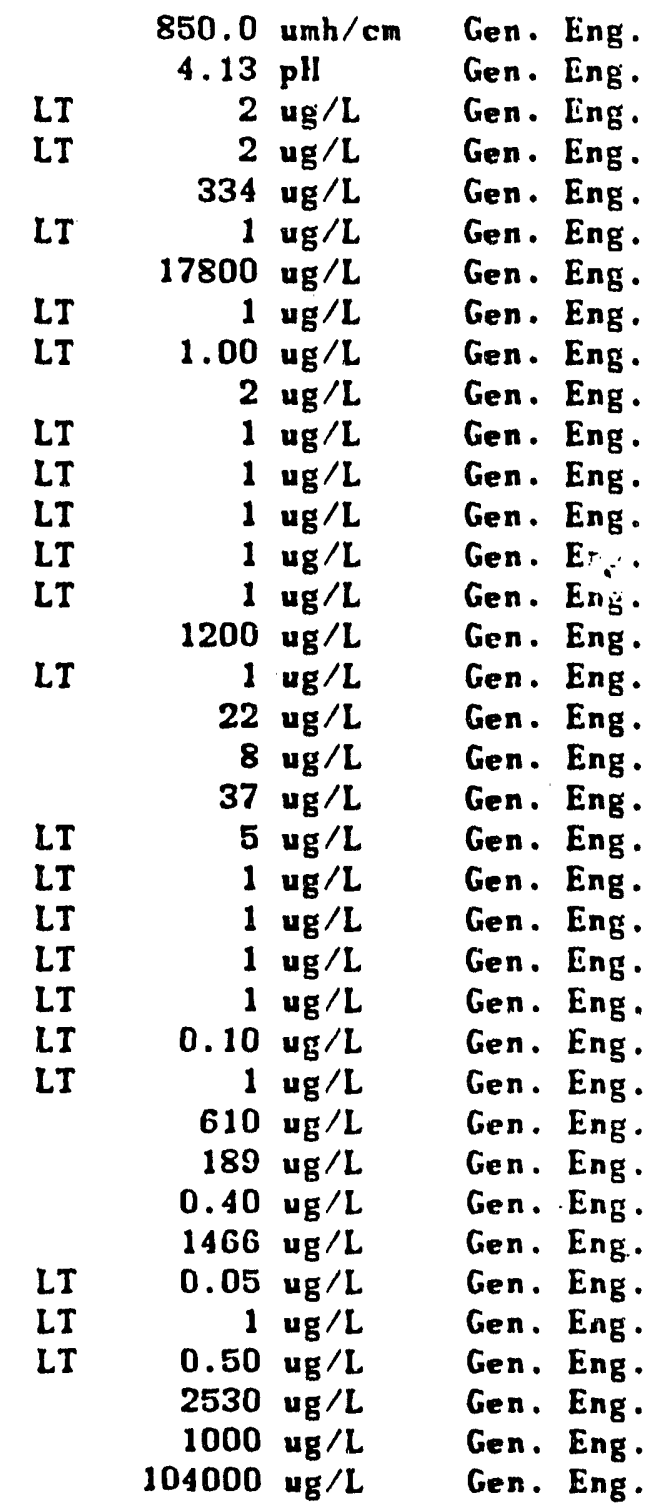


H'ELL FSB 88D COLLECTED ON 01/07/90 LABORATORY ANALYSES CONTINUED

NICKEL

* NITRATE AS NITROGEN

* LEAD

PIIENOLS

ANTIMONY

SELENIUM

SILICA

SILVEX

SULFATE

$1,1,2,2$-TETRACIILOROETHANE

TETRACHLOROETHYLENE

TOTAL DISSOLVED SOLIDS

TIIALLILM

TOTAL ORGANIC CARBON

TOTAL ORGANIC IIALOGENS

TOTAL PHOSPHATES

TRICILOROETIYYLENE

TOXAPHENE

TRANS-1,2-DICHLOROETHENE

IRANIUM

VANADILI

1, 1-D I CHLOROETHYLENE

1, 1-D ICHLOROETHANE

$1,1,1$-TRICILOROETIIANE

$1,1,2$-TRICIILOROETIIANE

1,2-DICHLOROETIIANE

1,2-D ICHLOROPROPANE

CIS-1, 3-DICIILOROPROPENE

TRANS-1,3-DICIILOROPROPENE 2-CHLOROETHYLVINYL ETIIER 2,4-DICHLOROPIIENOXYACETIC ACID ZINC

* Gross alpila

* Nonvolatile beta

TOTAL RADIUM

* TRITILM
27 ug/L Gen. Eng.

$106000 \mathrm{ug} / \mathrm{L}$ Gen. Eng.

$101 \mathrm{mg} / \mathrm{L}$ Gen. Eng.

$5 \mathrm{ug} / \mathrm{L}$ Gen. Eng.

3 ug/L Gen. Eng.

$2 \mathrm{ug} / \mathrm{L}$ Gen. Eng.

$3650 \mathrm{ug} / \mathrm{L}$ Gen. Eng.

$0.09 \mathrm{ug} / \mathrm{L}$ Gen. Eng.

$5000 \mathrm{ug} / \mathrm{L}$ Gen. Eng.

$1 \mathrm{vg} / \mathrm{L}$ Gen. Eng.

$1.00 \mathrm{ug} / \mathrm{L}$ Gen. Eng.

$535000 \mathrm{ug} / \mathrm{L}$ Gen. Eng.

$2 \mathrm{ug} / \mathrm{L}$ Gen. Eng.

$1000 \mathrm{ug} / \mathrm{L}$ Gen. Eng.

$5 \mathrm{ug} / \mathrm{L}$ Gen. Eng.

$50 \mathrm{ug} / \mathrm{L}$ Gen. Eng.

$1.00 \mathrm{ug} / \mathrm{L}$ Gen. Eng.

$1 \mathrm{ug} / \mathrm{L}$ Gen. Eng.

$1 \mathrm{ug} / \mathrm{L}$ Gen. Eng.

$1000 \mathrm{ug} / \mathrm{L}$ Gen. Eng.

$20 \mathrm{ug} / \mathrm{L}$ Gen. Eng.

$1 \mathrm{ug} / \mathrm{L}$ Gen. Eng.

$1 \mathrm{ug} / \mathrm{L}$ Gen. Eng.

$1 \mathrm{ug} / \mathrm{L}$ Gen. Eng.

$1 \mathrm{ug} / \mathrm{L}$ Gen. Eng.

$1 \mathrm{ug} / \mathrm{L}$ Gen. Eng.

$1 \mathrm{ug} / \mathrm{L}$ Gen. Eng.

1 ug/L Gen. Eng.

$1 \mathrm{ug} / \mathrm{L}$ Gen. Eng.

$1 \mathrm{ug} / \mathrm{L}$ Gen. Eng.

$0.30 \mathrm{ug} / \mathrm{L}$ Gen. Eng.

$213 \mathrm{ug} / \mathrm{L}$ Gen. Eng.

$47.30+-6.70 \mu \mathrm{C} i / L$ Gen. Eng.

267+- $12 \mathrm{p} \mathrm{Ci} / \mathrm{L}$ Gen. Eng.

LI $1 \mathrm{pCj} / \mathrm{L}$ Gen. Eng.

$3400+-6.00 \mathrm{pCi} / \mathrm{ml}$ Gen. Eng. 
K'ELL FSB 89C

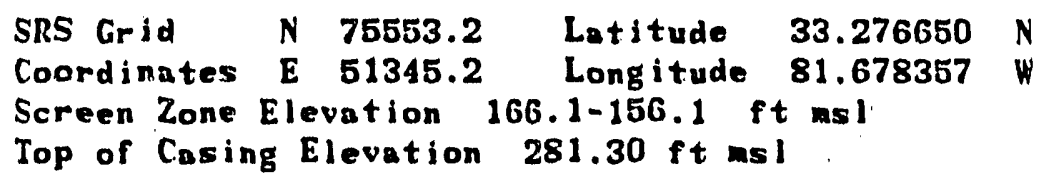

MEASUREMENTS CONDUCTED IN THE FIELD

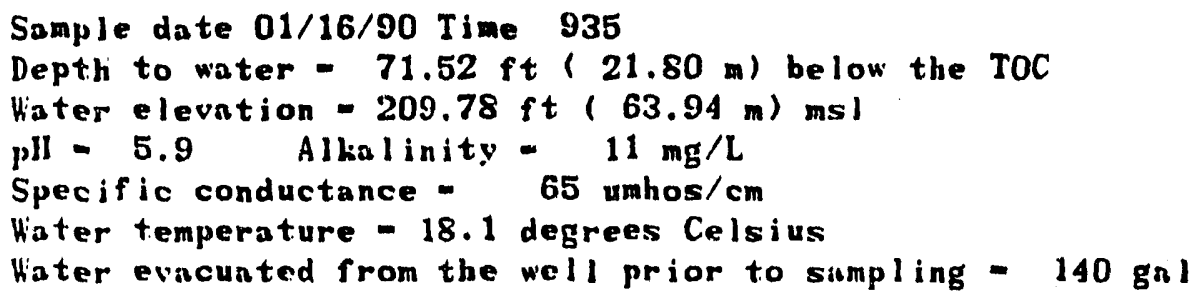

LABORATORY ANALYSES

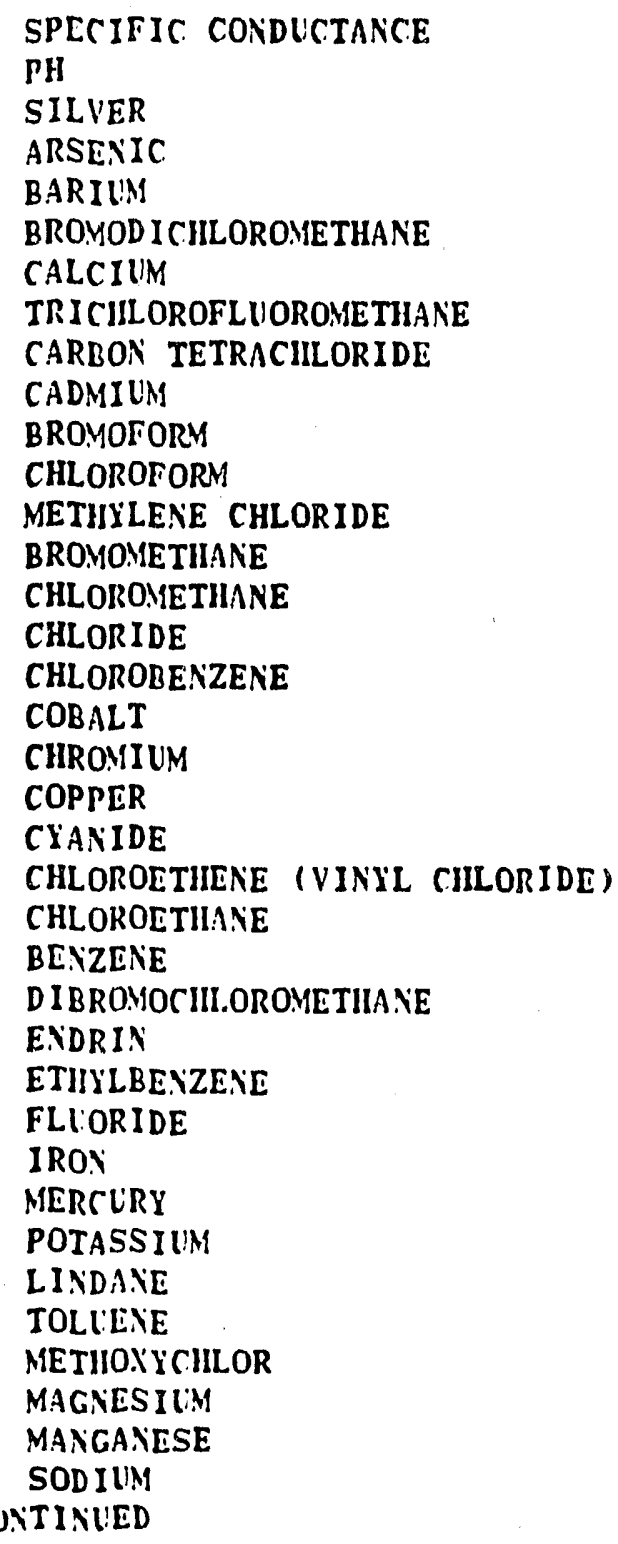

$58.00 \mathrm{umh} / \mathrm{cm}$ Gen. Eng.

LT

LT

LT

LT

L.T

LT

LT

LT

LT

LT

LT

LT

LI

LT

LI

LI

LI

LT

LT

LT

LT

LT

LT

LT

IT

LT

LT

$$
\begin{aligned}
& 5.93 \mathrm{pH} \text { Gen. Eng. } \\
& 2 \mathrm{ag} / \mathrm{L} \text { Gen. Eng. } \\
& 2 \text { ug/L Gen. Eng. } \\
& 13 \mathrm{ug} / \mathrm{L} \text { Gen. Eng. } \\
& 1 \mathrm{ug} / \mathrm{L} \text { Gen. Eng. } \\
& 5350 \mathrm{ug} / \mathrm{L} \text { Gen. Eng. } \\
& 1 \mathrm{ug} / \mathrm{L} \text { Gen. Eng. } \\
& 1.00 \mathrm{ug} / \mathrm{L} \text { Gen. Eng. } \\
& 2 \text { ug/L Gen. Eng. } \\
& 1 \mathrm{ug} / L \text { Gen. Eng. } \\
& 1 \mathrm{ug} / \mathrm{L} \text { Gen. Eng. } \\
& 1 \text { ug/L Gen. Eng. } \\
& 1 \mathrm{ug} / \mathrm{L} \text { Gen. Eng. } \\
& 1 \mathrm{ug} / \mathrm{L} \text { Gen. Eng. } \\
& 2800 \mathrm{ug} / \mathrm{L} \text { Gen. Eng. } \\
& 1 \mathrm{ug} / \mathrm{L} \text { Gen. Eng. } \\
& 4 \mathrm{ug} / \mathrm{L} \text { Gen. Eng. } \\
& 4 \mathrm{ug} / \mathrm{L} \text { Gen. Eng. } \\
& 4 \mathrm{ug} / \mathrm{L} \text { Gen. Eng. } \\
& 5 \mathrm{ug} / \mathrm{L} \text { Gen. Eng. } \\
& 1 \mathrm{ug} / \mathrm{L} \text { Gen. Eng. } \\
& 1 \mathrm{ug} / \mathrm{L} \text { Gen. Eng. } \\
& 1 \mathrm{ug} / \mathrm{L} \text { Gen. Eng. } \\
& 1 \mathrm{ug} / \mathrm{L} \text { Gen. Eng. } \\
& 0.10 \mathrm{ug} / \mathrm{L} \text { Gen. Eng. } \\
& 1 \mathrm{ug} / \mathrm{L} \text { Gen. Eng. } \\
& 100 \mathrm{ug} / \mathrm{L} \text { Gen. Eng. } \\
& 9 \text { ug } / \mathrm{L} \text { Gen. Eng. } \\
& 0.20 \mathrm{ug} / \mathrm{L} \text { Gen. Eng. } \\
& 1010 \mathrm{ug} / \mathrm{L} \text { Gen. Eng. } \\
& 0.05 \mathrm{ug} / \mathrm{L} \text { Gen. Eng. } \\
& 1 \mathrm{ug} / \mathrm{L} \text { Gen. Eng. } \\
& 0.50 \mathrm{ug} / \mathrm{L} \text { Gen. Eng. } \\
& 445 \mathrm{ug} / \mathrm{L} \text { Gen. Eng. } \\
& 7 \mathrm{ug} / \mathrm{L} \text { Gen. Eng. } \\
& 3650 \mathrm{ug} / \mathrm{L} \text { Gen. Eng. }
\end{aligned}
$$


HELL FSB 89C COLLECTED ON 01/16/90 LABORATORY ANALYSES CONTINUED

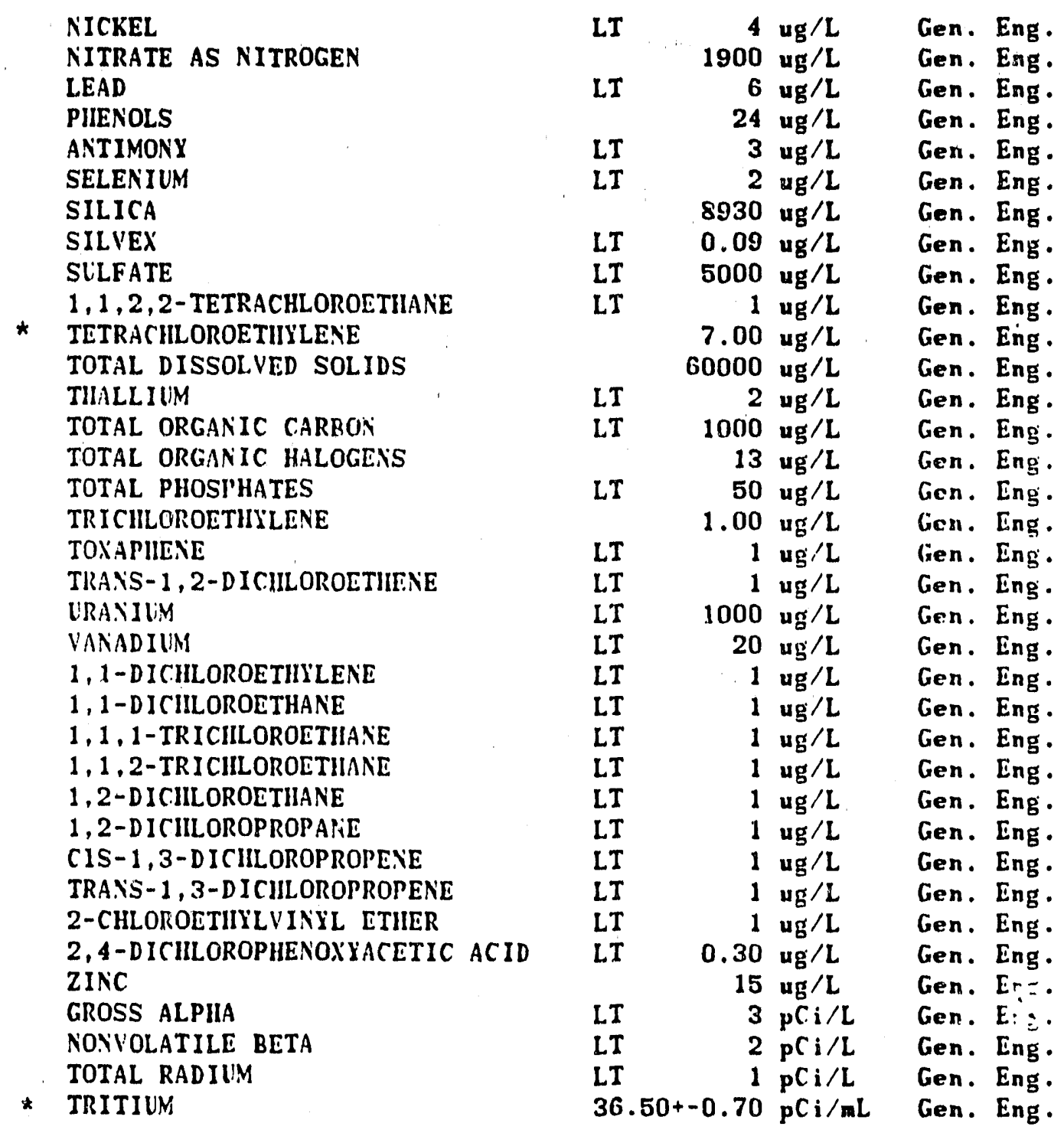


NELL FSB 89D

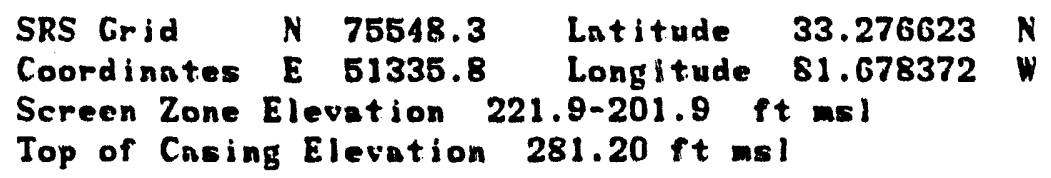

MEASUREMENTS CONDUCTED IN TIE FIELD

Sample date 01/16/90 Time 920

Depth to water - $68.49 \mathrm{ft}(20.88 \mathrm{~m})$ below the TOC

Water elevation - $212.71 \mathrm{ft}(64.83 \mathrm{~m}) \mathrm{ms}$ l

pll - 4.1 Alkalinity $=0 \mathrm{mg} / \mathrm{L}$

Specific conductance - 344 unhos/cm

Water temperature - 17.3 degrees Celsius

Hater evincuated from the well prior to sampling - $28 \mathrm{gal}$

LABORATORY ANALYSES

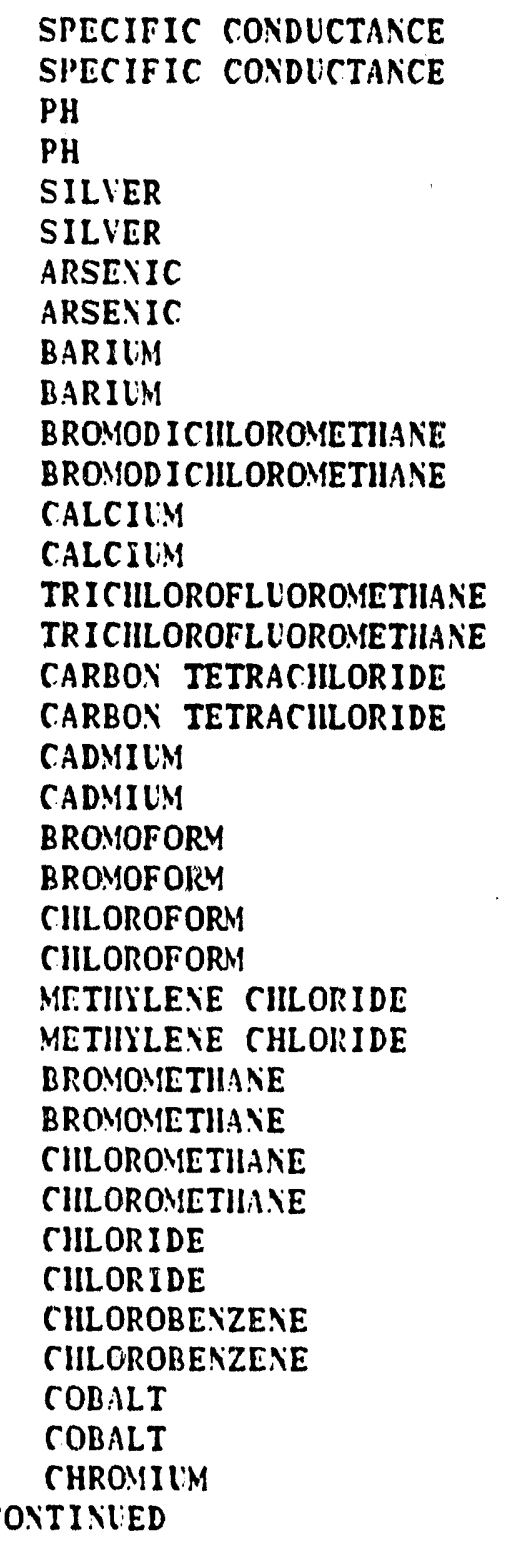

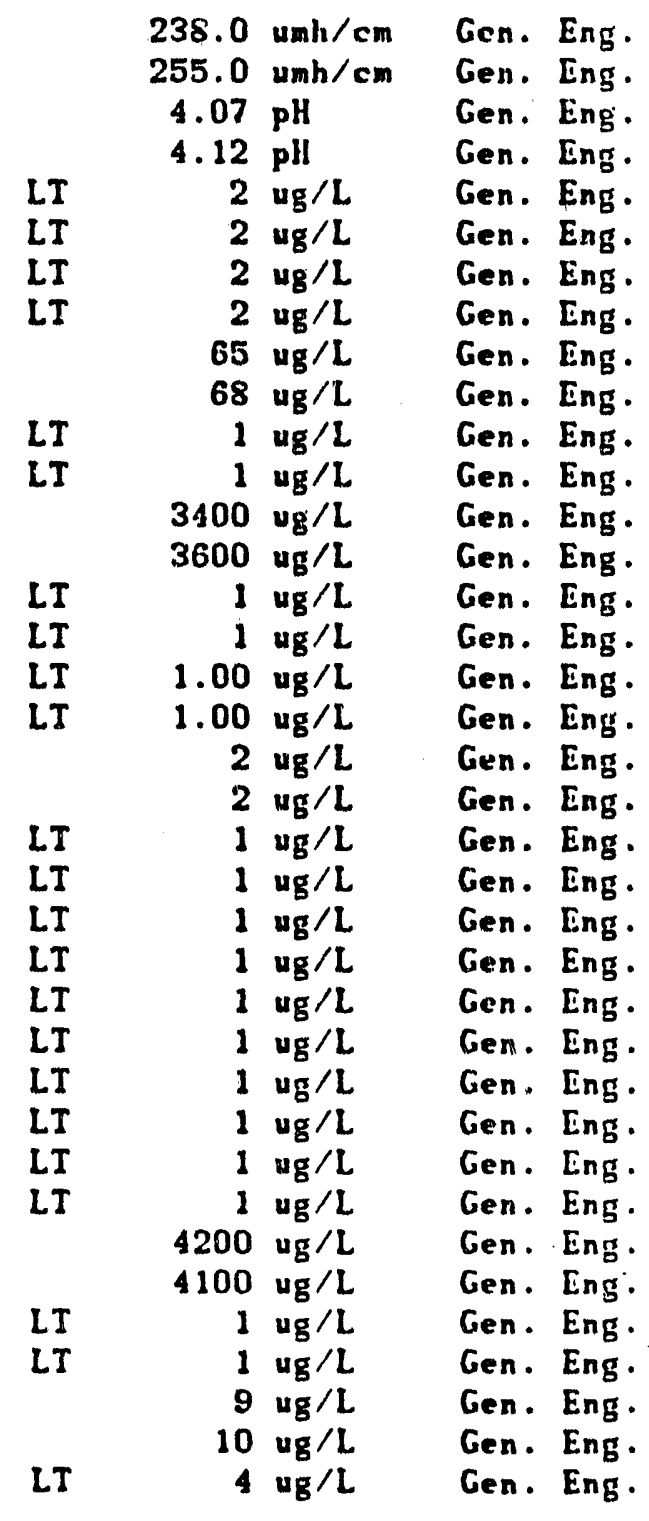


NELL FSB 89D COLLECTED ON 01/16/90 LABORATORY ANALYSKS CONTINUED

CHROMIUM LI

COPPER

COPPER

CYANIDE

CYANIDE

CILLOROETIENE (VINYL CHLORIDE)

CIILOROETHENE (VINYL CILLORIDE)

CIILOROETHANE

CIILOROETHANE

BENZENE

BENZENE

DIBROMOCIILORONETHANE

DIBROMOCIILOROMETHANE

ENDRIN

ENDRIN

ETIYYLENZENE

ETHYLBENZENE

FLIORIDI:

FLIORIDE

IRON

IRON

* mercury

* mercury

POTASSIUM

POTASSIUM

LINDANE

LINDANE

TOLUENE

TOLUENE

METIIOXYCIILOR

METHOXYCHLOR

MAGNESILM

MAGNESIUM

MANGAIESE

MANGANESE

SODILM

SODIUM

NICKEL

NICKEL

* NITRATE AS NITROGEN

* Nitrate as NitrogeN

L.EAD

LEAD

PIIENOLS

PIIENOLS

ANTIMONY

A.ITIMONY

SELENIUM

SELENILM

SILICA

SILICA

SILVEX

SILVEX

SILFATE

CONTINLED
LT

LT

LI

LI

LI

L.T

LT

LT

LI

LI

LT

LI

LI

LT

LT

LI

LI

LT

LI

LT

LT

LT

LI

LT

LI

LT

LI

LI
$4 \mathrm{ug} / \mathrm{L}$

$21 \mathrm{ug} / \mathrm{L}$

$22 \mathrm{ug} / \mathrm{L}$

$5 \mathrm{ug} / \mathrm{L}$

$5 \mathrm{ug} / \mathrm{L}$

$1 \mathrm{ug} / \mathrm{L}$

$1 \mathrm{ug} / \mathrm{L}$

$1 \mathrm{ug} / \mathrm{L}$

$1 \mathrm{ug} / \mathrm{L}$

$1 \mathrm{ug} / \mathrm{L}$

$1 \mathrm{ug} / \mathrm{L}$

$1 \mathrm{ug} / \mathrm{L}$

$1 \mathrm{ug} / \mathrm{L}$

$0.10 \mathrm{ug} / \mathrm{L}$

$0.10 \mathrm{ug} / \mathrm{L}$

$1 \mathrm{ug} / \mathrm{L}$

$1 \mathrm{ug} / \mathrm{L}$

$230 \mathrm{ug} / \mathrm{L}$

$220 \mathrm{ug} / \mathrm{L}$

$42 \mathrm{ug} / \mathrm{L}$

$52 \mathrm{ug} / \mathrm{L}$

$5.83 \mathrm{ug} / \mathrm{L}$

$5.83 \mathrm{ug} / \mathrm{L}$

$500 \mathrm{ug} / \mathrm{L}$

$500 \mathrm{ug} / \mathrm{L}$

$0.28 \mathrm{ug} / \mathrm{L}$

$0.22 \mathrm{ug} / \mathrm{L}$

$1 \mathrm{ug} / \mathrm{L}$

$1 \mathrm{ug} / \mathrm{L}$

$0.50 \mathrm{ug} / \mathrm{L}$

$0.50 \mathrm{ug} / \mathrm{L}$

$1050 \mathrm{ug} / \mathrm{L}$

$1110 \mathrm{ug} / \mathrm{L}$

$346 \mathrm{ug} / \mathrm{L}$

$376 \mathrm{ug} / \mathrm{L}$

$36100 \mathrm{ug} / \mathrm{L}$

$39800 \mathrm{ug} / \mathrm{L}$

$9 \mathrm{ug} / \mathrm{L}$

$10 \mathrm{ug} / \mathrm{L}$

$32300 \mathrm{ug} / \mathrm{L}$

$33600 \mathrm{ug} / \mathrm{L}$

$7 \mathrm{ug} / \mathrm{L}$

$6 \mathrm{ug} / \mathrm{L}$

$5 \mathrm{ug} / \mathrm{L}$

$5 \mathrm{ug} / \mathrm{L}$

$3 \mathrm{ug} / \mathrm{L}$

$3 \mathrm{ug} / \mathrm{L}$

$2 \mathrm{ug} / \mathrm{L}$

$2 \mathrm{ug} / \mathrm{L}$

$10900 \mathrm{ug} / \mathrm{L}$

$11700 \mathrm{ug} / \mathrm{L}$

$0.09 \mathrm{ug} / \mathrm{L}$

$0.09 \mathrm{ug} / \mathrm{L}$

$9900 \mathrm{ug} / \mathrm{L}$
Gen. Eng .

Gen. Eng .

Gen. Eng.

Gen. Eng.

Gen. Eng.

Gen. Eng.

Gen. Eng.

Cen. Eng.

Gen. Eng.

Gen. Eng .

Gen. Eng .

Gen. Eng.

Gen. Eng.

Gen. Eng.

Gen. Eng.

Gen. Eng.

Gen. Enrs.

Gen. Eng.

Gen. Eng.

Gen. Eng.

Gen. Eng.

Gen. Eng.

Gen. Eng.

Gen. Eng.

Gen. Eng .

Gen. Eng.

Gen. Eng.

Gen. Eng.

Gen. Eng.

Gen. Eng.

Gen. Eng.

Gen. Eng.

Gen. Eng.

Gen. Eng.

Gen. Eng.

Gen. Eng.

Gen. Eng.

Gen. Eng.

Gen. Eng.

Gen. Eng.

Gen. Eng.

Gen. Eng.

Gen. Eng.

Gen. Eng.

Gen. Eng.

Gen. Eng.

Gen. Eng.

Gen. Eng.

Gen. Eng .

Gen. Eng .

Gen. Eng.

Gen. Eng.

Gen. Eng.

Gen. Eng. 
WELL FSB 89D COLLECTED ON 01/16/90 LABORATORY ANALYSES CONTINUED

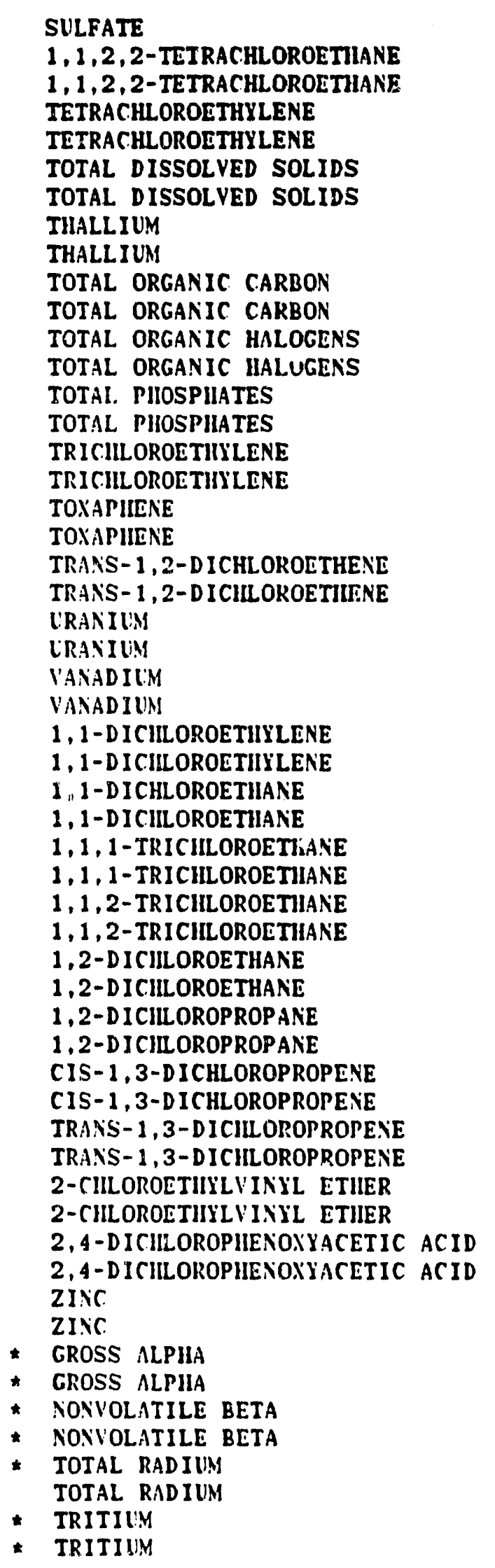

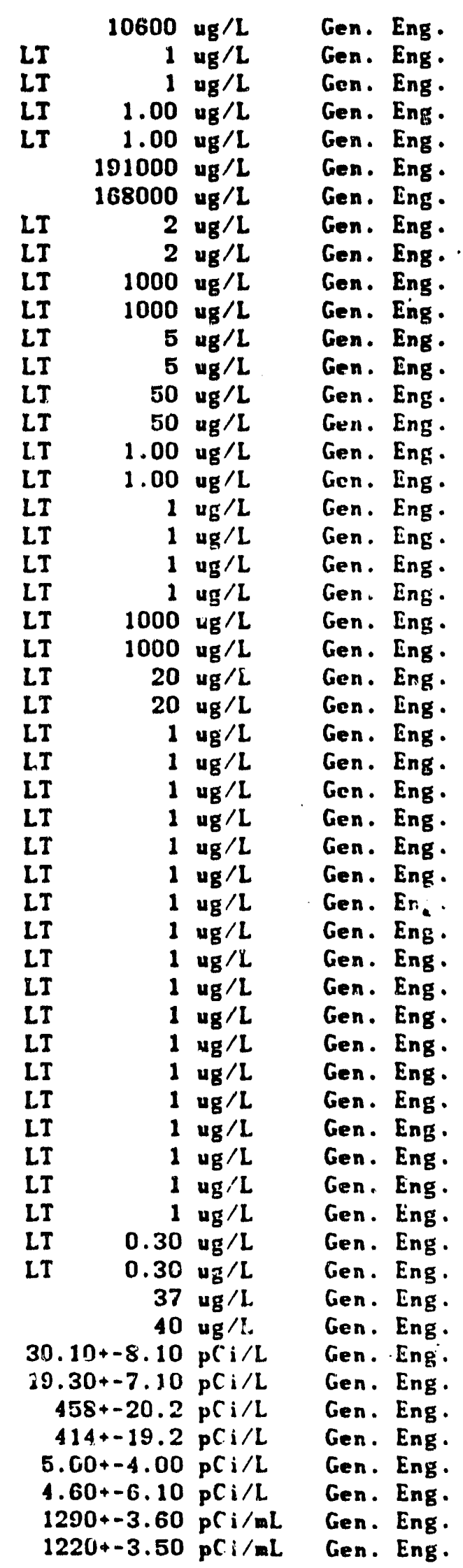


HELL FSB OOC

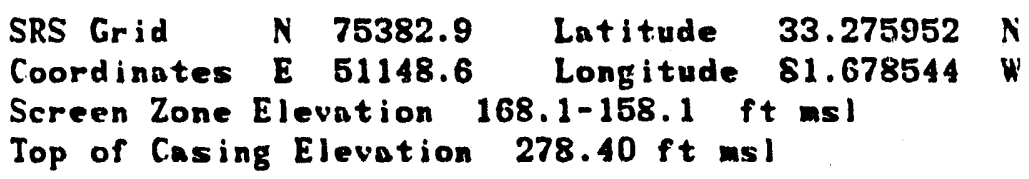

MEASUREMENTS CONDUCTED IN THE FIELD

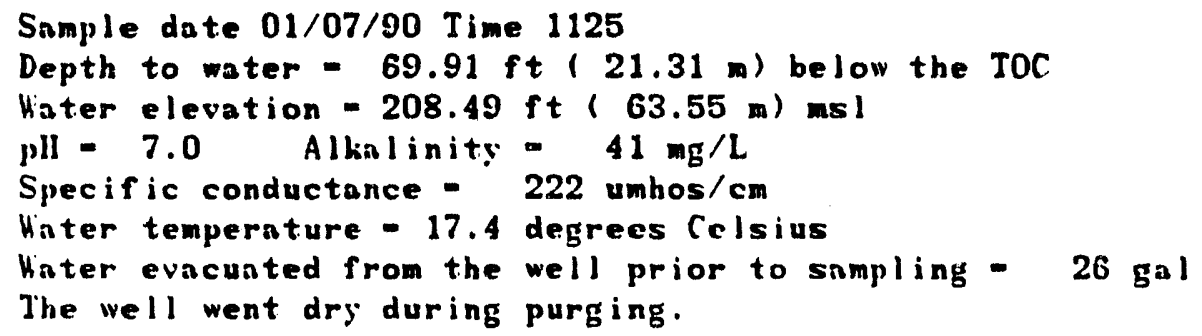

SPECIFIC CONDUCTANCE

PII

SILVER

IRSEIIC

BARILM

BROMODICHLOROMETHANE

CALCIUM

TRICIILOROFLUOROMETHANE

CARBON TETRACILORIDE

CADMILM

BROMOFORM

CILOROFORM

METHILENE CHLORIDE

BROMOMETHANE

CIILOROMETHANE

CIILORIDE

CIILOROBENZENE

COBALT

CHROMILM

COPPER.

CYANIDE

CILLOROETIIENE (VINYL CHLORIDE)

CILLOROETHANE

BENZENYE

DIBROMOCHLOROMETHINE

ENDRIN

ETIILLBENZENE

FLIORIDE

IRON

MERCURY

POTASSICM

LINDANE

TOLLENE

METHOXYCHLOR

MAGNESILM

MANGANESE

SODILM

CONTIAIED

$$
\begin{aligned}
& 200.0 \mathrm{umh} / \mathrm{cm} \text { Geri. Eng. } \\
& 7.57 \mathrm{pH} \text { Gen. Eng. } \\
& \begin{array}{lll}
\text { LI } & 2 \mathrm{ug} / \mathrm{L} & \text { Gen. Eng. } \\
\text { LI } & 2 \mathrm{ug} / \mathrm{L} & \text { Gen. Eng. }
\end{array} \\
& 52 \mathrm{ug} / \mathrm{L} \text { Gen. Eng. } \\
& 1 \mathrm{ug} / \mathrm{L} \text { Gen. Eng. } \\
& 22900 \mathrm{ug} / \mathrm{L} \text { Gen. Eng. } \\
& 1 \mathrm{ug} / \mathrm{L} \text { Gen. Eng. } \\
& 1.00 \mathrm{ug} / \mathrm{L} \text { Gen. Eng. } \\
& 2 \text { ug } / \mathrm{L} \text { Gen. Eng. } \\
& 1 \mathrm{ug} / \mathrm{L} \text { Gen. Eng. } \\
& 1 \text { ug/L Gen. Eng. } \\
& 1 \mathrm{ug} / \mathrm{L} \text { Gen. Eng. } \\
& 1 \text { ug } / \mathrm{L} \text { Gen. Eng. } \\
& 1 \text { ug } / \mathrm{L} \text { Gen. Eng. } \\
& 3200 \mathrm{ug} / \mathrm{L} \text { Gen. Eng. } \\
& 1 \mathrm{ug} / \mathrm{L} \text { Gen. Eng. } \\
& 4 \mathrm{ug} / \mathrm{L} \text { Gen. Eng. } \\
& 4 \mathrm{ug} / \mathrm{L} \text { Gen. Eng. } \\
& 4 \mathrm{ug} / \mathrm{L} \text { Gen. Eng. } \\
& 5 \mathrm{ug} / \mathrm{L} \text { Gen. Eng. } \\
& 1 \mathrm{ug} / \mathrm{L} \text { Gen. Eng. } \\
& 1 \mathrm{ug} / \mathrm{L} \text { Gen. Eng. } \\
& 1 \mathrm{ug} / \mathrm{L} \text { Gen. Eng. } \\
& 1 \mathrm{ug} / \mathrm{L} \text { Gen. Eng. } \\
& 0.10 \mathrm{ug} / \mathrm{L} \text { Gen. Eng. } \\
& 1 \mathrm{ug} / \mathrm{L} \text { Gen. Eng. } \\
& 100 \mathrm{ug} / \mathrm{L} \text { Gen. Eng. } \\
& 15 \mathrm{ug} / \mathrm{L} \text { Gen. Eng. } \\
& 0.20 \mathrm{ug} / \mathrm{L} \text { Gen. Eng: } \\
& 2490 \mathrm{ug} / \mathrm{L} \text { Gen. Eng. } \\
& 0.05 \mathrm{ug} / \mathrm{L} \text { Gen. Eng. } \\
& 1 \text { ug/L Gen. Eng. } \\
& 0.50 \mathrm{ug} / \mathrm{L} \text { Gen. Eng. } \\
& 3040 \mathrm{ug} / \mathrm{L} \text { Gen. Eng. } \\
& 18 \mathrm{ug} / \mathrm{L} \text { Gen. Eng. } \\
& 10800 \mathrm{ug} / \mathrm{L} \text { Gen. Eng. }
\end{aligned}
$$


NELL FSB 90C COLLECTED ON 01/07/90 LABORATORY ANALYSES CONTINUED

NICKEL

* NITRATE AS NITROGEN

LEAD

PIIENOLS

ANTIMONY

SELENIUM

SILICA

SILVEX

SULFATE

$1,1,2,2$-TETRACILLOROETHANE

TETRACHLORDETIIYLENE

TOTAL DISSOLVED SOLIDS

THALLIUM

TOTAL ORGANIC CARBON

TOTAL ORGANIC IIALOGENS

TOTAL PHOSPHATES

TRICHLOROETIIYLENE

TOXAPHENE

TRANS-1,2-DICHLOROETHENE

URANIUM

VANADILM

1,1-DICHLOROETHYLENE

1,1-DICHLOROETHA.NE

$1,1,1$-TRICHLOROETHANE

1,1,2-TRICIILOROETIIANE

1, 2-DICILLOROETIIANE

1,2-DICHLOROPROPANE

CIS-1,3-DICIILOROPROPENE

TRANS-1, 3-DICILLOROPROPENE

2-CILLOROETHYLVINYL ETIER

2,4-DICILOROPHENOXYACETIC ACID

ZINC

GROSS ALPHA

NONVOLATILE BETA

TOTAL RADIUM

* TRITILM
LT

LT

LT

LT

LT

LT

\section{LT}

LT

LI

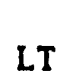

LT

LI

LT

LT

LT

LI

LT

LT

LT

LI

LT

LT

LT

LT

LT

LT

$2.53+-2.20 \mathrm{pCi} / \mathrm{L}$ LT

$1040+-3.30 \mathrm{pCi} / \mathrm{mL}$
Gen. Eng.

Gen. Eng.

Gen. Eng.

Gen. Eng.

Gen. Eng.

Gen. Eng.

Cen. Eng.

Gen. Eng.

Gen. Eng.

Gen. Eng.

Gen. Eng.

Gen. Eng.

Gen. Eng.

Gen. Eng.

Gen. Eng.

Gen. Eng.

Gen. Eng.

Gen. Eng.

Gen. Eng.

Gen. Eng.

Gen. Eng.

Gen. Eng.

Gen. Eng.

Gen. Eng.

Gen. Eng.

Gen. Eng.

Gen. Eng.

Gen. Eng.

Gen. Eng.

Gen. Eng.

Gen. Eng.

Gen. Eng.

Gen. Eng.

Gen. Eng.

Gen. Eng.

Gen. Eng. 
NELL FSB 90D

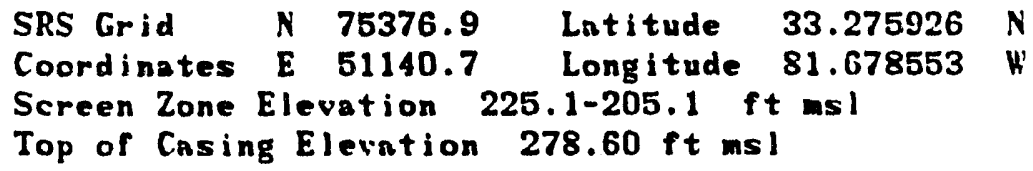

MEASUREMENTS CONDUCTED IN THE FIELD

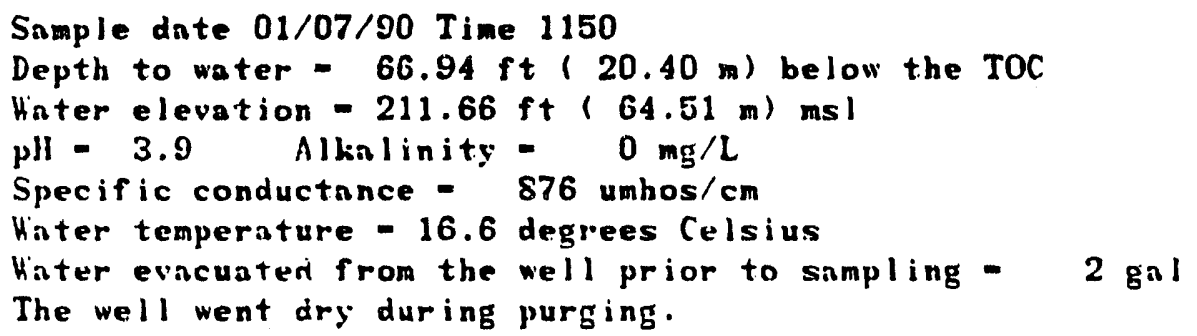

SPECIFIC CONDLCTANCE

PII

SILVER

ARSENIC

BARIUN

BROMOD ICILOROMETHANE

CALCIUM

TRICIILOROFLUOROMETHANE

CARBOI TETRACILLORIDE

CADYIIM

BROYOFORY

CIILOROFORM

METIIILENE CHLORIDE

BROYONETHANE

CHLOROMETHANE

CIILORIDE

CIILOROBENZENE

COBILT

CIIROMIUM

COPPER

CYAIIDE

CIILOROETIIENE (WINYL CIILORIDE)

CHLOROETIIANE

BEIZENE

DIBROMOCILLRONETHANE

ENDRIN

ETHILBENZENE

FLIORIDE

IRON

MERCURY

POTASSILY

LINDANE

TOLIENE

METIIOXYCILOR

MAGIESILYY

MANGANESE

SODIUN

CONTINIED
$820.0 \mathrm{umh} / \mathrm{cm}$ Gen. Eng. $4.20 \mathrm{pH}$ Gen. Eng.

2 ug/L Gen. Eng.

LI

$2 \mathrm{ug} / \mathrm{L}$

$563 \mathrm{ug} / \mathrm{L}$

$1 \mathrm{ug} / \mathrm{L}$

$14500 \mathrm{ug} / \mathrm{L}$

LT 1 ug $/ L$

LI $\quad 1.00 \mathrm{ug} / \mathrm{L}$

$9 \mathrm{ug} / \mathrm{L}$

LT

LI

LT

LI

LT

LI

LI

LI

LT

LT

LT

LT

LT

$1 \mathrm{ug} / \mathrm{L}$

$1 \mathrm{ug} / \mathrm{L}$

$1 \mathrm{ug} / \mathrm{L}$

$1 \mathrm{ug} / \mathrm{L}$

$1 \mathrm{ug} / \mathrm{L}$

$3800 \mathrm{ug} / \mathrm{L}$

$1 \mathrm{ug} / \mathrm{L}$.

$22 \mathrm{ug} / \mathrm{L}$

$8 \mathrm{ug} / \mathrm{L}$

$8 \mathrm{ug} / \mathrm{L}$

$5 \mathrm{ug} / \mathrm{L}$

$1 \mathrm{ug} / \mathrm{L}$

$1 \mathrm{ug} / \mathrm{L}$

$1 \mathrm{ug} / \mathrm{L}$

$1 \mathrm{ug} / \mathrm{L}$

$0.10 \mathrm{ug} / \mathrm{L}$

$1 \mathrm{ug} / \mathrm{L}$

$1850 \mathrm{ug} / \mathrm{L}$

$200 \mathrm{ug} / \mathrm{L}$

LT

$0.20 \mathrm{ug} / \mathrm{L}$

$1840 \mathrm{ug} / \mathrm{L}$

$0.34 \mathrm{ug} / \mathrm{L}$

$2 \mathrm{ug} / \mathrm{L}$

LT

$0.50 \mathrm{ug} / \mathrm{L}$

$4510 \mathrm{ug} / \mathrm{L}$

$1480 \mathrm{ug} / \mathrm{L}$

$71300 \mathrm{ug} / \mathrm{L}$
Gen. Eng.

Gen. Eng.

Gen. Eng.

Gen. Eng.

Gen. Eng.

Gen. Eng.

Gen. Eng.

Gen. Eng.

Gen. Ing.

Gen. Eng.

Gen. Eng.

Gen. Eng.

Gen. Eng.

Gen. Eng.

Gen. Eng.

Gen. Eng.

Gen. Eng.

Gen. Eng.

Gen. Eng.

Gen. Eng.

Gen. Eng.

Gen. Eng.

Gen. Eng.

Gen. Eng.

Gen. Eng.

Gen. Eng:

Gen. Eng.

Gen. Eng.

Gen. Eng.

Gen. Eng.

Gen. Eng.

Gen. Eng.

Gen. Eng.
Gen. Eng. 
NELL FSB 9OD COLLECTED ON 01/07/00 LABORATORY ANALYSES CONTINUED

NICKEL

* NITRATE AS NITROGEN

* LEAD

PHENOLS

ANTIMONY

SELENIUM

SILICA

SLLFATE

$1,1,2,2$-TETRACHLOROETHANE

* TETRACHLOROETHYLENE

TOTAL DISSOLVED SOLIDS

THALLIUM

TOTAL ORGANIC CARBON

TOTAL ORGANIC HILOGENS

TOTAL PIIOSPHATES

TRICILOROETHYLENE

TOXAPIIENE

TRANS-1,2-DICHLOROETHENE

IRAKIUM

VANADILM

1.1-DICHLOROETHYLENE

1,1-DICILLOROETHANE

$1,1,1$ - TRICIILOROETHIANE

1,1,2-TRICILLOROETHANE

1,2-DICILLOROETHANE

1, 2-DICIILOROPROPANE

CIS-1,3-DICHLOROPROPENE

TRANS-1,3-DICILOROPROPENE

2-CHLOROETHYLVINYL ETIER

ZINC

GROSS ALPHA

- NoNiolatile beTa

TOTAL RADIUM

* TRITIUM

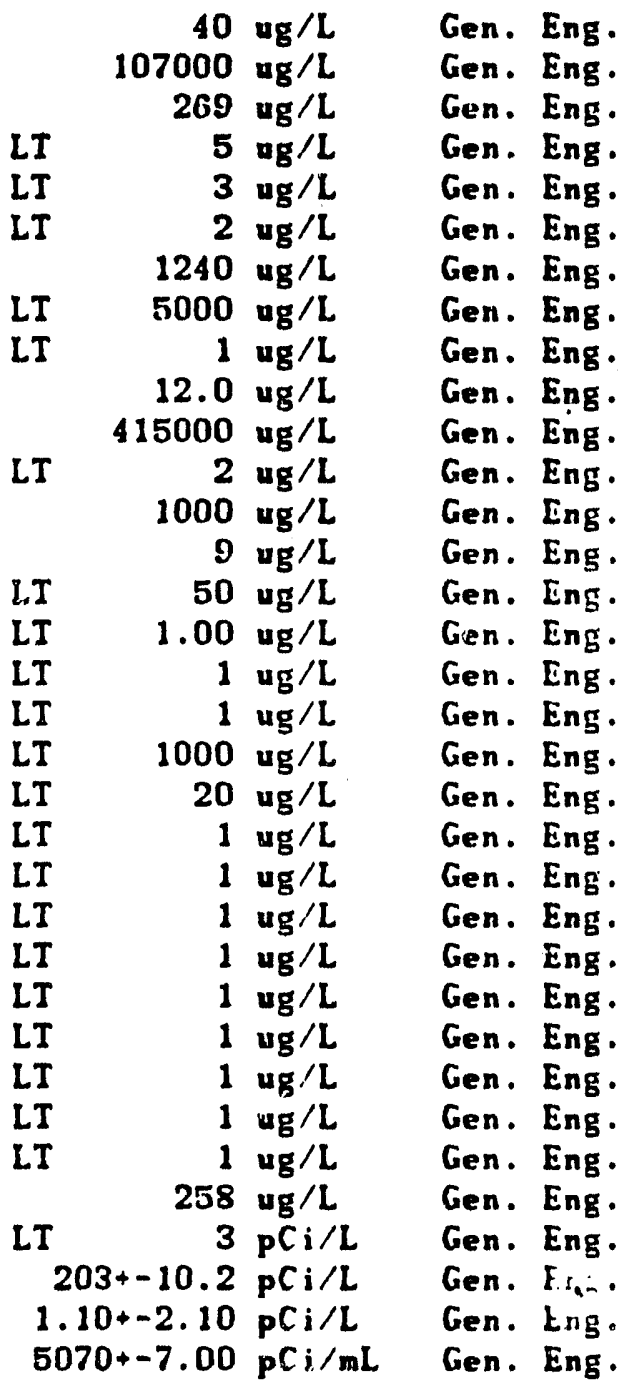


NELL FSB 91C

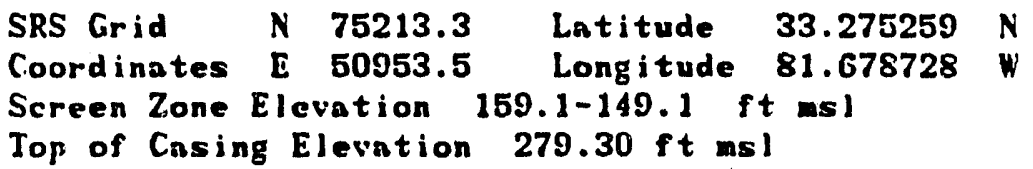

MEASUREMENTS CONDLICTED IN THE FIELD

Sample date 01/07/90 Time 1055

Depth to water - $70.54 \mathrm{ft}(21.50 \mathrm{~m})$ below the TOC

Water elevation $=208.76 \mathrm{ft}(63.63 \mathrm{~m}) \mathrm{msl}$

$\mathrm{pH}=6.5$ Alkalinity $-31 \mathrm{mg} / \mathrm{L}$

Specific conductance - 418 umhos/cm

Winter temperature -17.8 degrees Celsius

Katcr evacunted from the well prior to sampling - $21 \mathrm{gal}$

The well went dry during purging.

LABORITORY ANALYSES

\begin{tabular}{|c|c|c|c|c|c|}
\hline $\begin{array}{l}\text { SPECIFIC CONDUCTANCE } \\
\text { PII }\end{array}$ & & $\begin{array}{r}440.0 \\
7.34\end{array}$ & $\mathrm{umh} / \mathrm{cm}$ & Gen. & Eng. \\
\hline SILVER & LT & $\begin{array}{r}.34 \\
2\end{array}$ & $\mathrm{pH} / \mathrm{L}$ & Gen. & $\begin{array}{l}\text { Lng. } \\
\text { Eng. }\end{array}$ \\
\hline ARSENIC & LI & 2 & ug /L & Gen. & Eng. \\
\hline BARIUM & & 252 & $u g / L$ & Gen. & Eng. \\
\hline BRONOD I CHLORONETHIANE & LI & 1 & $u g / L$ & Gen. & Eng. \\
\hline CALIILM & & 31500 & $\mathrm{ug} / \mathrm{L}$ & Gen. & Eng. \\
\hline TRICHLOROFLUORONETHANE & LT & 1 & $u g / L$ & Gen. & Eng. \\
\hline CARBON TETRACIILORIDE & LT & 1.00 & $\mathrm{ug} / \mathrm{L}$ & Gen. & Eng. \\
\hline CADMIUM & & 7 & $\mathbf{u g} / L$ & Gen. & Eng. \\
\hline BROMOFORM & LT & 1 & $\operatorname{ug} / \mathrm{L}$ & Gen. & Eng. \\
\hline CIILOROFORM & LT & 1 & ug $/ L$ & Gen. & Eng. \\
\hline METIILENE CHLORIDE & LT & 1 & ug $/ L$ & Gen. & Eng. \\
\hline BROMOMETIIANE & LI & 1 & ug $/ L$ & Gen. & Eng. \\
\hline CHLOROMETHANE & LT & 1 & $\mathbf{u g} / \mathrm{L}$ & Gen. & Eng. \\
\hline CIILORIDE & & 1700 & ug/L & Gen. & Eng. \\
\hline CIILOROBENZENE & LT & 1 & $\mathbf{u g} / \mathrm{L}$ & Gen. & Eng . \\
\hline COBALT & & 16 & ug $/ L$ & Gen. & Eng. \\
\hline CIIROMILM & LT & 4 & $u_{g} / L$ & Gen. & Eng. \\
\hline COPPER & LT & 4 & ug $/ \mathrm{L}$ & Gen. & Eng. \\
\hline CYAIIDE & LT & 5 & ug $/ L$ & Gen. & Eng. \\
\hline CIILOROETHENE (VINYL CILORIDE) & LT & 1 & $u g / L$ & Gen. & Eng. \\
\hline CHLOROETHANE & LT & 1 & ug $/ \mathrm{L}$ & Gen. & Eng. \\
\hline BENZENE & LT & 1 & ug $/ \mathrm{L}$ & Gen. & Eng. \\
\hline DIBROMOCIILOROMIETIHNE & LT & 1 & $u g / L$ & Gen. & Eng. \\
\hline ENDRIN & LT & 0.10 & $\mathrm{ug} / \mathrm{L}$ & Gen. & Eng. \\
\hline ETIILBENZENE & LT & 1 & ag $/ L$ & Gen. & Eng. \\
\hline ILLORIDE & & 580 & $\mathbf{u g} / \mathrm{L}$ & Gen. & Eng. \\
\hline IRON & & 42 & $\operatorname{ug} / L$ & Gen. & Eng. \\
\hline MERCIRY & LT & 0.20 & $\mathbf{u g} / \mathrm{L}$ & Gen. & Eng. \\
\hline POTASSILM & & 5.370 & ugil & Gen. & Eng. \\
\hline LINDINE & LT & 0.05 & ug $/ L$ & Gen. & Eng. \\
\hline TOLLEYE & $L I$ & 1 & ug $/ \mathrm{L}$ & Gen. & Eng. \\
\hline METHOXYCHLOR & LT & 0.50 & $u_{g} / L$ & Gen. & Eng. \\
\hline MAGNESILM & & 3610 & $u_{g} / L$ & Gen. & \\
\hline MANGANESE & & 535 & $u g / L$ & Gen. & Eng. \\
\hline SODIIM & & 37200 & $\mathrm{ug} / \mathrm{L}$ & Gen. & \\
\hline
\end{tabular}


WELL FSB 91C COLLECTED ON 01/07/90 LABORATORY ANALYSES CONTINUED

NICKEL

* NITRATE AS NITROGEN

LEAD

PIIENOLS

A.TIMONY

SELENIUM

SILICA

SILVEX

SULTATE

$1,1,2,2$-TETRACHLOROETHIANE

TETRACIILOROETHYLENE

TOTAL DISSOLVED SOLIDS

THALLIUM

TOTAL ORGANIC CARBON

TOTAL ORGANIC HALOGENS

TOTAL PIOSPHATES

TRICILLOROETHYLENE

TOXAPIIENE

TRANS-1,2-DICIILOROETHENE

IRANIUM

VANADIUM

1,1-DICILLOROETHYLENE

1,1-DICHLOROETHANE

$1,1,1$-TRICHLOROETHINE

1,1,2-TRICILOROETIIANE

1,2-DICIILOROETHANE

1,2-DICIILOROPROPANE

CIS-1,3-DICHLOROPROPENE

TRANS-1, 3-D ICILLOROPROPENE

2-CILLOROETHYLVINIL ETIIER

2,4-DICILOROPIENOXYACETIC ACID

ZINC

GROSS ALPIIA

* NONIOLATILE beTa

TOTAL RADIUM

* TRITIUM

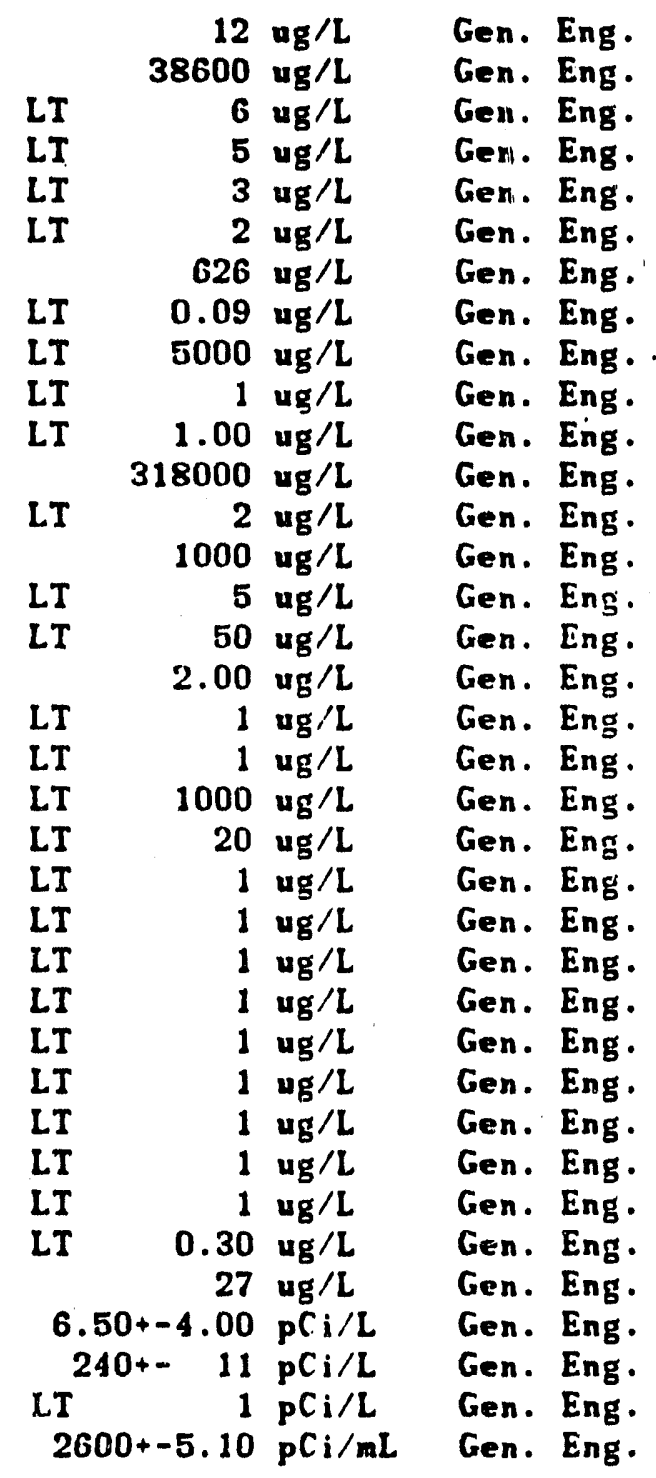


WELL FSB 91D

SRS Grid N 75207.6 Lntitude 33.275235 N

Coordinates E 50946.6 Longitude 81.678735 W

Screen Zone Elevation 220.9-200.9 ft msl

Top of Casing Elevation $279.20 \mathrm{ft} \mathrm{ms}$ l

MEASUREMENTS CONDUCTED IN THE FIELD

Snmple date 01/06/90 Time 1300

Depth to water $=68.35 \mathrm{ft}(20.83 \mathrm{~m})$ below the TOC

Winter elevation $=210.85 \mathrm{ft}(64.27 \mathrm{~m}) \mathrm{msl}$

pll - 3.2 Alknlinity - $0 \mathrm{mg} / \mathrm{L}$

Specific conductance - 2060 umhos $/ \mathrm{cm}$

Wiater temperature $=18.5$ degrees Celsius

Hiater evacuated from the well prior to simpling - 26 gal

LABORATORY ANALYSES

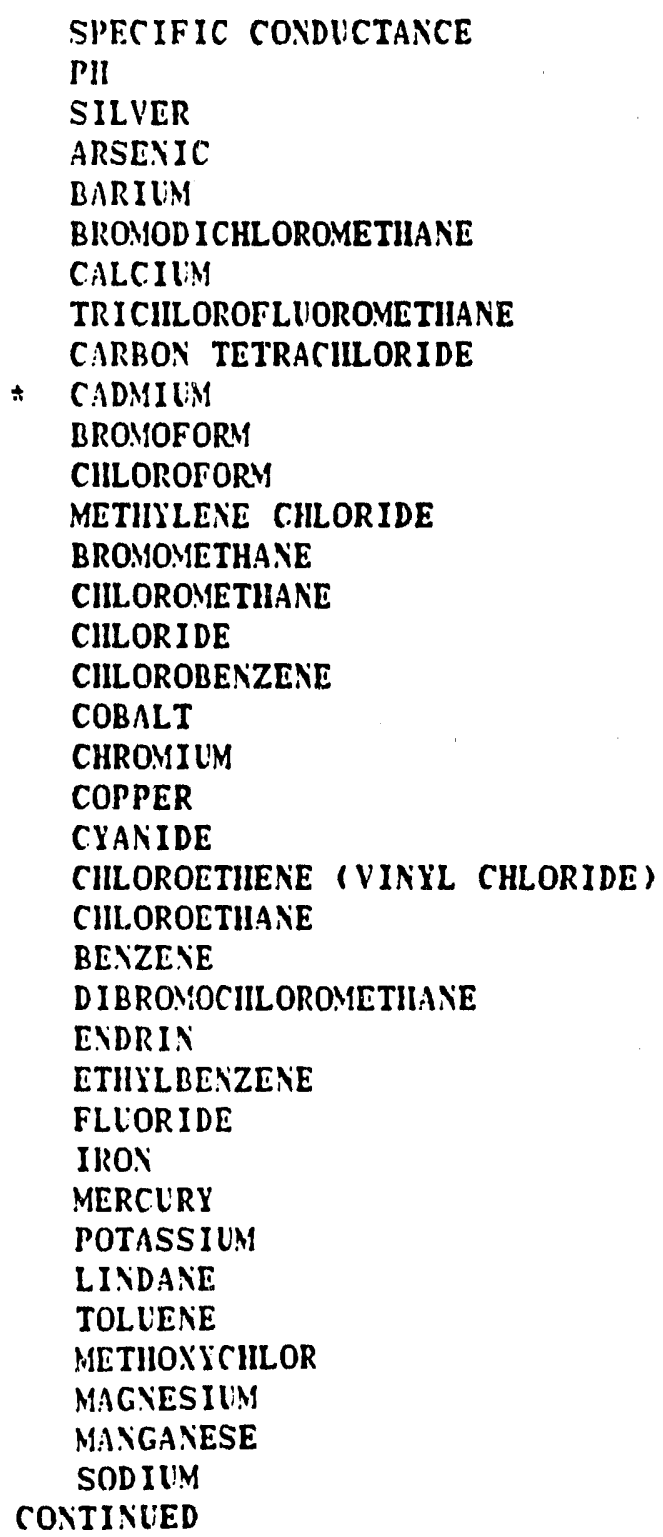

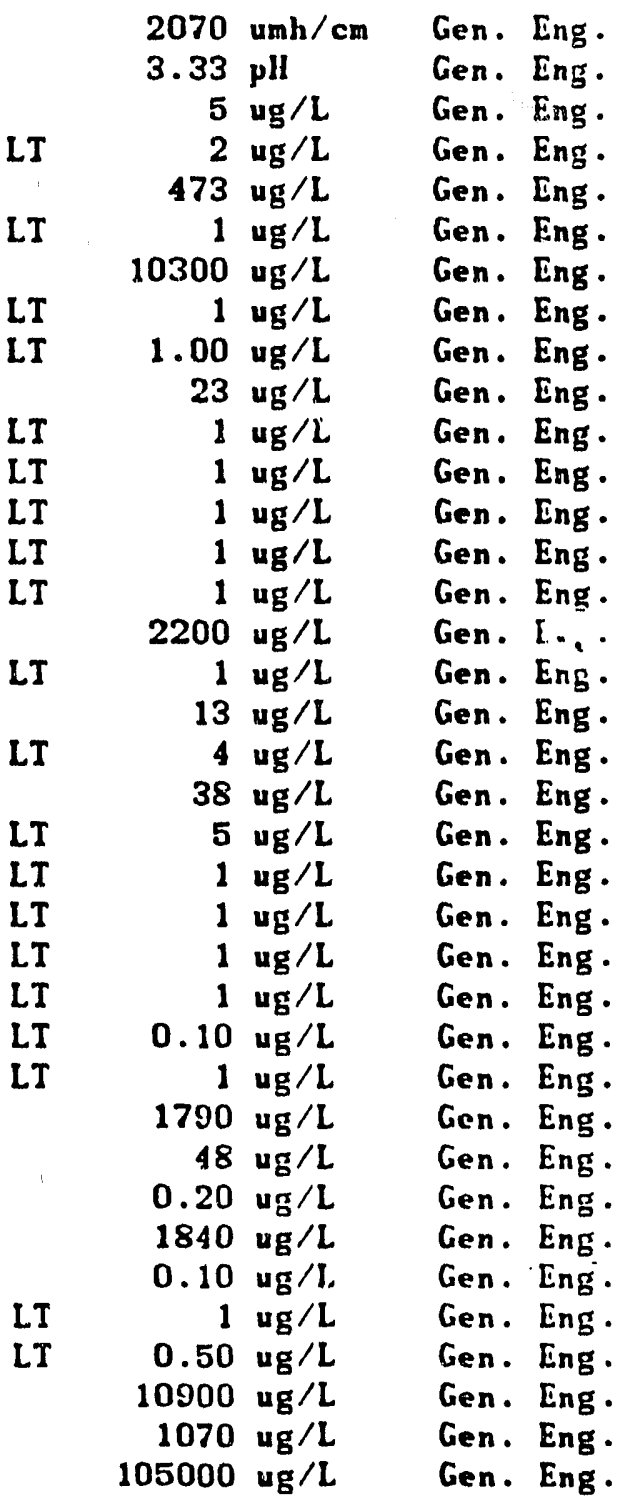


NELL FSB 91D COLLECTED ON 01/06/90 LABORATORY ANALYSES CONTINUED

NICKEL

* NITRATE AS NITROGEN

LEAD

PIIENOLS

ANTIMONY

SELENIUM

SILICA

SILVEX

SULFATE

$1,1,2,2$-TETRACHLOROETHANE

TETRACHLOROETIIYLENE

TOTAL DISSOLVED SOLIDS

THALLIUM

TOTAL ORGANIC CARBON

TOTAL ORGANIC MALOGENS

TOTAL PHOSPHATES

TRICHLOROETHYI.ENE

TOXAPIIENE

TRANS-1,2-DICILOROETIIENE

URANILM

VANADIUM

1,1-DICHLOROETHYLENE

1,1-DICHLOROETHANE

$1,1,1$-TRICHLOROETHANE

1, 1,2-TRICHLOROETHANE

1,2-DICIILOROETIIANE

1,2-DICIILOROPROPANE

CIS-1,3-DICHLOROPROPENE

TRANS-1,3-DICHLOROPROPENE

2-CILOROETIIYLVINYL ETHER

2,4-DICILLOROPLENOXYACETIC ACID

ZINC

GROSS ALPHA

* NoNvolatile beta

* total Radilim

* tritilu

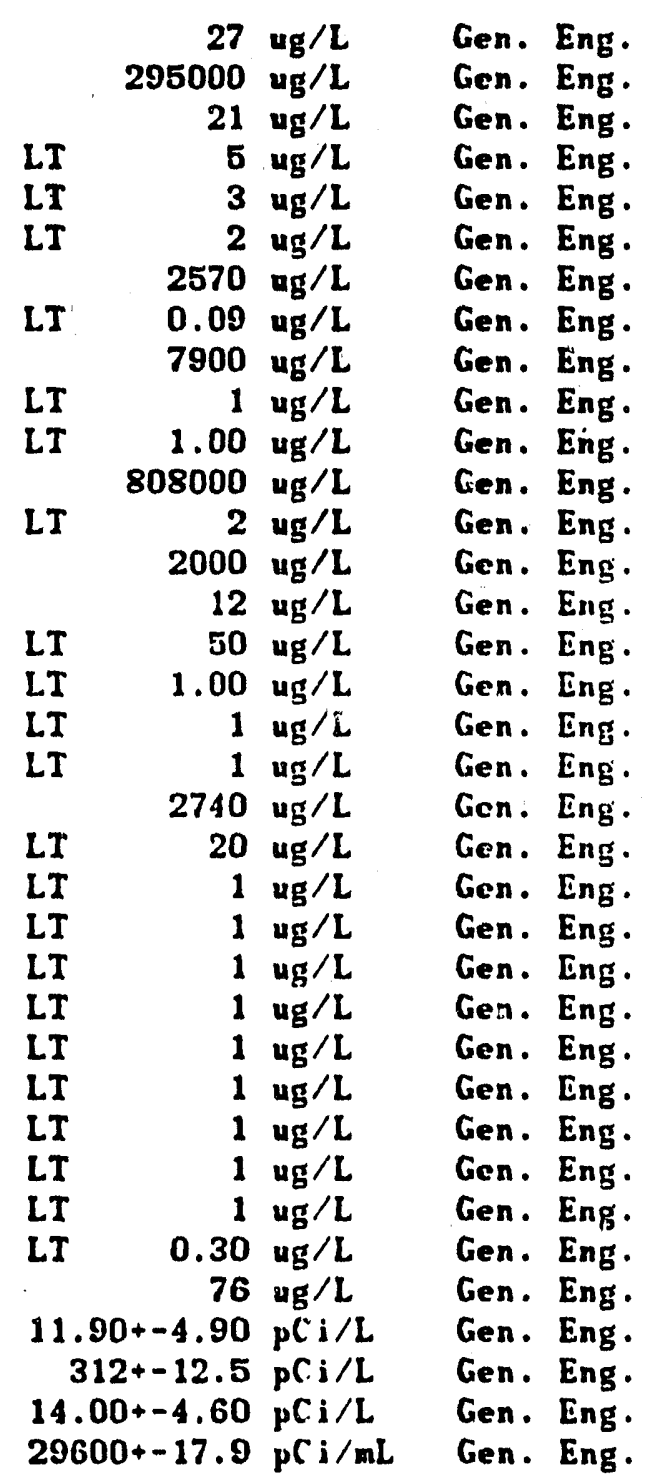


KELL FSB 91D

SRS Grid N 75207.6 Latitude $33.275235 N$

Coordinates E 50946.6 Longitude 81.678735 W

Screen Zone Elevation 220.9-200.9 ft ms

Top of Casing Elevation $279.20 \mathrm{ft} \mathrm{ms}$

MEASUREMENTS CONDUCTED IN THE FIELD

Sample date 01/06/90 Time 1300

Depth to water $=68.35 \mathrm{ft}(20.83 \mathrm{~m})$ below the TOC

Hater elevation $=210.85 \mathrm{ft}(64.27 \mathrm{~m}) \mathrm{msl}$

$\mathrm{pH}=3.2$ Alkalinity $=0 \mathrm{mg} / \mathrm{L}$

Specific conductince - 2060 umhos/cm

Viater temperature - 18.5 degrees Celsius

Hater evacunted from the well prior to sampling - $26 \mathrm{gal}$

LABORATORY ANALYSES

THISE ANALYSES ARE FROM UNILTERED SAMPLES

SILICA

488 ug/L Gen. Eng.

HILL FSB 92D

SRS Grid N 75045.8 Latitude $33.274242 N$

Coordinates E 50557.6 Longitude 81.679445 W

Screen Zone Elevation 221.7-201.7 ft ms I

Top of Casing Elevation $275.90 \mathrm{ft} \mathrm{ms}$ !

MEASUREMENTS CONDLCTED IN THE FIELD

Sample date 01/06/90 Time 1705

Depth to water - $66.31 \mathrm{ft}(20.21 \mathrm{~m})$ below the TOC

Hater elevation $=209.59 \mathrm{ft}(63.88 \mathrm{~m}) \mathrm{ms}$

pH - 3.5 Alkalinity $=0 \mathrm{mg} / \mathrm{L}$

Specific conductance - 1264 umhos/cm

Water temperature - 18.8 degrees Celsius

Water evacuated from the well prior to sampling - $20 \mathrm{gal}$

LARORATORY ANALYSES

SPECIFIC CONDLCTANCE

$\mathrm{PH}$

SILVER

ARSENIC

BARILIM

BROMODICILLOROMETILANE

CALCILM

TRICILOROFLUOROMETIIANE

CARBON TETRACILORIDE

* CADMIIIN

BROMOFORY

CHLOROTORY

METHILENE CILLORIDE

CONTINIED

\begin{tabular}{|c|c|c|c|}
\hline & $\begin{array}{l}1300 \\
353\end{array}$ & $\mathrm{umh} / \mathrm{cm}$ & Gen. \\
\hline L.T & 2 & urd & Gen \\
\hline LT & $\begin{array}{r}2 \\
2 \\
252\end{array}$ & ugg $/ 2$ & \\
\hline LT & $\begin{array}{r}353 \\
1 \\
9090\end{array}$ & $\begin{array}{l}\text { ug/L } \\
\text { ug/L }\end{array}$ & $\begin{array}{l}\text { Gen. } \\
\text { Gen. }\end{array}$ \\
\hline LT & 1 & $\begin{array}{l}\text { ug } / L \\
\text { ug } / L\end{array}$ & Gen. \\
\hline LT & $\begin{array}{r}1.00 \\
15\end{array}$ & $\begin{array}{l}u g / L \\
u g / L\end{array}$ & $\begin{array}{l}\text { Gen. } \\
\text { Gen. }\end{array}$ \\
\hline LI & 1 & $v g / L$ & Gen. \\
\hline LT & 1 & ug $/ L$ & Gen. \\
\hline $1 T$ & 1 & $u g / l$ & Gen. \\
\hline
\end{tabular}


VELL FSB 92D COI,LECTED ON 01/06/90 LABORATORY ANALYSES CONTINUED

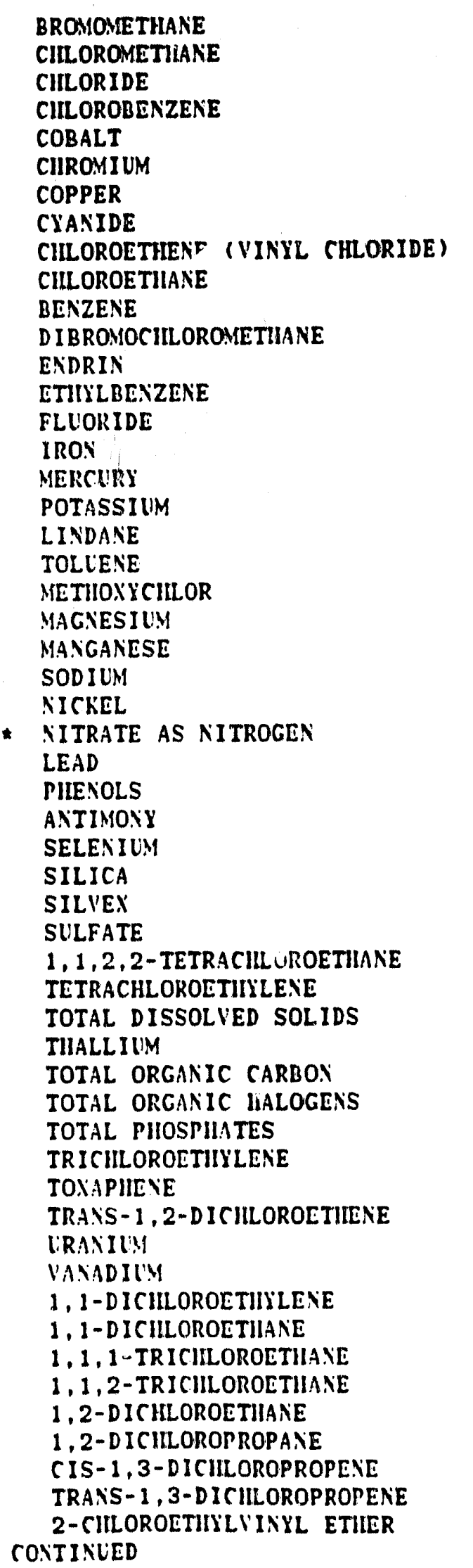

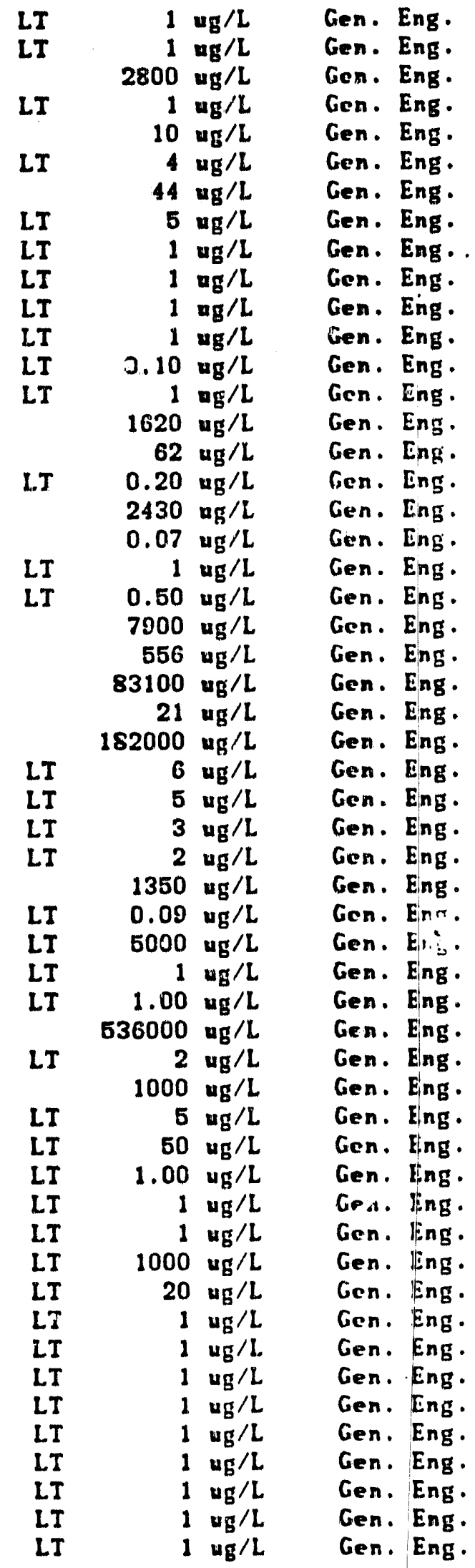


NELL FSB 92D COLLECTED ON 01/06/90 LABORATORY ANALYSES CONTINUED

\begin{tabular}{|c|c|c|}
\hline $\begin{array}{l}\text { 2,4-DICHLOROPIIENOXYACETIC ACID } \\
\text { ZINC } \\
\text { GROSS ALPHA } \\
\text { NONVOLATILE BETA } \\
\text { TOTAL RADIUM } \\
\text { TRITIUM }\end{array}$ & $\begin{array}{r}0.30 \\
66 \\
50.10+-7.30 \\
403+-14.5 \\
9.20+-3.80 \\
14500+-13.7\end{array}$ & $\begin{array}{l}u g / L \\
\operatorname{ug} / L \\
\mathrm{pC} i / L \\
\mathrm{pCi} / \mathrm{L} \\
\mathrm{pC} \mathbf{i} / \mathrm{L} \\
\mathrm{pC} i / \mathrm{mL}\end{array}$ \\
\hline
\end{tabular}

WELL FSB 92D

SRS Grid N 75045.8 Latitude 33.271242 N

Coordinntes E 50557.6 Longitude 81.679445 W'

Screen Zone Elevation 221.7-201.7 ft msl

Top of Casing Elevation $275.90 \mathrm{ft} \mathrm{msl}$

AFASUREMENTS CONDUCTED IN THE FIELD

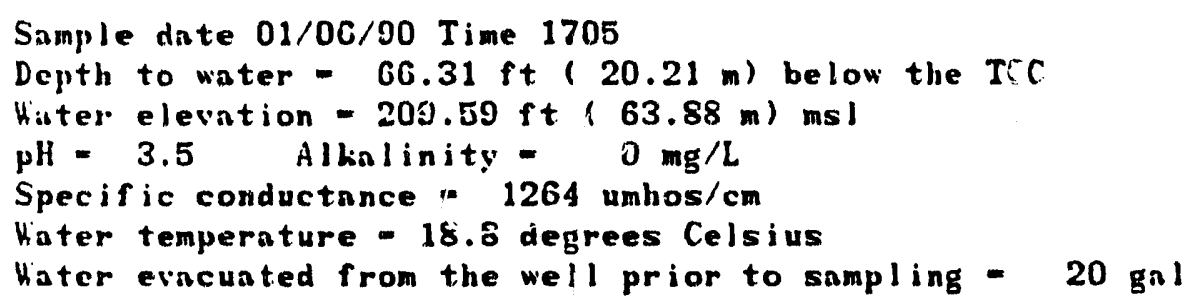

HELL FSB 93C

SRS Grid $N 74897.3$ Latitude 33.273752 N

Coordinates E 50.158 .3 Longitude 81.679418 Hi

Screen Zone Elevation 152.0-142.0 ft $\mathrm{msl}$

Top of Casing Elevation $276.20 \mathrm{ft} \mathrm{msl}$

MEASUREMENTS CONDUCTED IN TIE FIELD

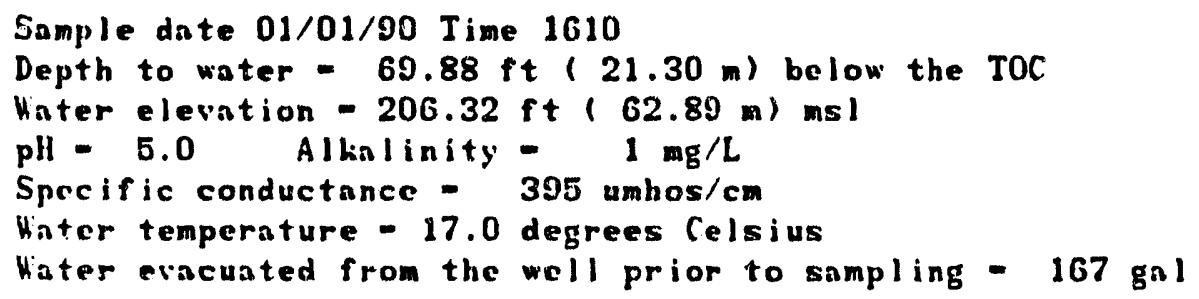

LABORATORY AIALYSES

SPECIFIC CONDLCTANCE

$\mathrm{PH}$

SILVER

ARSENIC

BARILY

BROMODICHLORONETIIANE

CONTINIED

$\begin{array}{rrll} & 345.0 \mathrm{umh} / \mathrm{cm} & \text { Gen. Eng. } \\ & 5.56 \mathrm{pH} & \text { Gen. Eng. } \\ \text { LT } & 2 \mathrm{ug} / \mathrm{L} & \text { Gen. Eng. } \\ \text { LT } & 2 \mathrm{ug} / \mathrm{L} & \text { Gen. Eng. } \\ & 76 \mathrm{ug} / \mathrm{L} & \text { Gen. Eng. } \\ \text { LT } & : \mathrm{ug} / \mathrm{L} & \text { Gen. Eng. }\end{array}$


WELL FSB 93C COLLECTED ON 01/01/90 LABORATORY ANALYSES CONTINUED

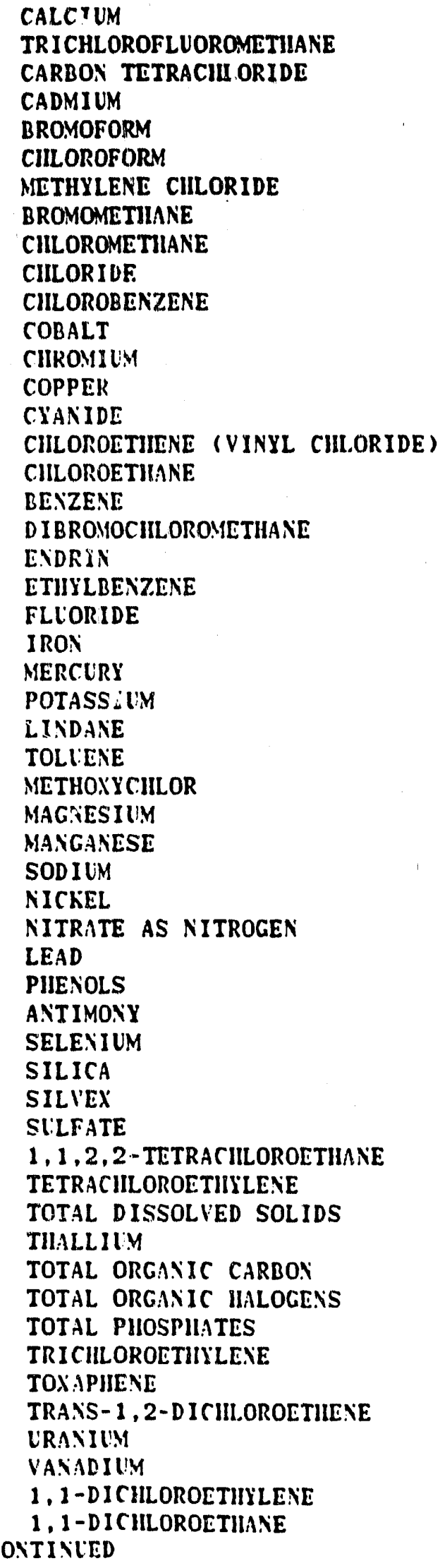

$31700 \mathrm{ug} / \mathrm{L}$ Gen. Eng.

LT

LI

LI

LI

LI

LI

LI

LI

I.T

LT

LT

S.T

LT

LT

LT

LI

LI

LI

LT

LI

LT

LT

L.T

L.T

I.T

LT

LI

LT

LI

LT

LI

LI

LI

LI

LT

LT

LI

LT

LT

LI

LI

LI

$$
1 \mathrm{ug} / \mathrm{L} \text { Gen. Eng. }
$$$$
1.00 \mathrm{ug} / \mathrm{L} \text { Gen. Eng. }
$$$$
2 \text { ug/L Gen. Eng. }
$$$$
1 \mathrm{ug} / \mathrm{L} \text { Gen. Eng. }
$$$$
1 \mathrm{ug} / \mathrm{L} \text { Gen. Eng. }
$$$$
1 \mathrm{ug} / \mathrm{L} \text { Gen. Eng. }
$$$$
1 \mathrm{mg} / \mathrm{L} \text { Gen. Eng. }
$$$$
1 \mathrm{ug} / \mathrm{L} \text { Gen. Eng. }
$$

Gen. Eng.

Gen. Eng .

Gen. Eng.

Gen. Eng.

Gen. Eng.

Gen. Eng.

Gen. Eng.

Gen. Engr.

Gen. Eng.

Gen. Eng.

Gen. Eng.

Gen. Eng.

Gen. Eng .

Gen. Eng.

Gen. Eng.

Gen. Eng.

Gen. Eng.

Gen. Eng.

Gen. Eng.

Gen. Eng.

Gen. Eng .

Gen. Ing .

Gen. Eng.

Gen. Eng.

Gen. Eng.

Gen. Eng .

Gen. Eng.

Gen. Eng.

Gen. Eng.

Gen. Eng.

Gen. Eng .

Gen. Eng.

Gen. Eng.

Gen. Eng .

Gen. Eng.

Gen. Eng.

Gen. Eng.

Gen. Eng.

Gen. Ing.

Gen. Eng.

Gen. Eng.

Gen. Eng.

Gen. Eng.

Gen. Eng.

Gen. Eng. 
NELL FSB 93C COLLECTED ON 01/01/90 LABORATORY ANALYSES CONTINUED

\author{
1,1,1-TRICIILOROETHANE \\ $1,1,2$-TRICHLOROETHANE \\ 1.2-DICILLOROETIAANE \\ 1,2-DICHLOROPROPANE \\ CIS-1,3-DICHLOROPROPENE \\ TRANS-1, 3-D ICIILOROPROPENE \\ 2-CILOROETHXLVINYL ETHER \\ 2,4-DICHLOROPHENOXYACETIC ACID \\ ZINC \\ GROSS ALPHA \\ NONVOLATILE BETA \\ TOTAL RADIUM \\ * TRITIUM
}

$\begin{array}{lrl}\mathrm{LT} & 1 \mathrm{ug} / \mathrm{L} \\ \mathrm{LT} & 1 \mathrm{ug} / \mathrm{L} \\ \mathrm{LT} & 1 \mathrm{ug} / \mathrm{L} \\ \mathrm{LT} & 1 \mathrm{ug} / \mathrm{L} \\ \mathrm{LT} & 1 \mathrm{ug} / \mathrm{L} \\ \mathrm{LT} & 1 \mathrm{ug} / \mathrm{L} \\ \mathrm{LT} & 1 \mathrm{ug} / \mathrm{L} \\ \mathrm{LT} & 0.30 \mathrm{ug} / \mathrm{L} \\ & 93 \mathrm{ug} / \mathrm{L} \\ \mathrm{LT} & 3 \mathrm{pCi} / \mathrm{L} \\ 5.30+-2.50 \mathrm{pCi} / \mathrm{L} \\ 4.20+-2.80 \mathrm{pCi} / \mathrm{L} \\ 2050+-4.60 \mathrm{pCi} / \mathrm{mL}\end{array}$

Gen. Eng.

Gen. Eng.

Gen. Eng.

Gen. Eng.

Gen. Eng.

Gen. Eng.

Gen. Eng.

Gen. Eng.

Gen. Eng.

Ger. Eng.

Gen. Eng.

Gen. Eng.

Gen. Eng.

\section{HELL TSB 93D \\ SRS Grid N 74888.5 Latitude $33.273723 \quad N$ \\ Coordinates E 50452.4 Longitude 81.679 .16 W' \\ Screen Zone Elevation 217.9-197.9 ft msl \\ Top of Casing Elevation $276.10 \mathrm{ft} \mathrm{ms}$ l}

MEASIREMENTS CONDUCTED IN TIIE FIELD

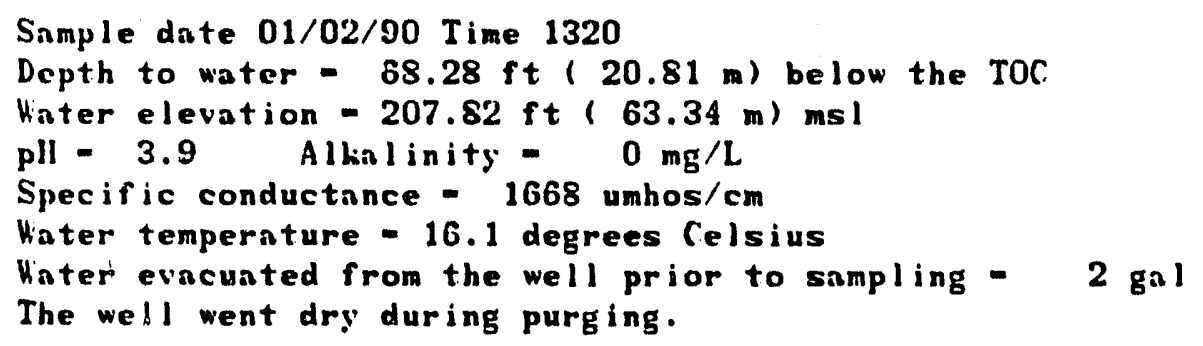

LABURATORY ANALYSES

\begin{tabular}{|c|}
\hline 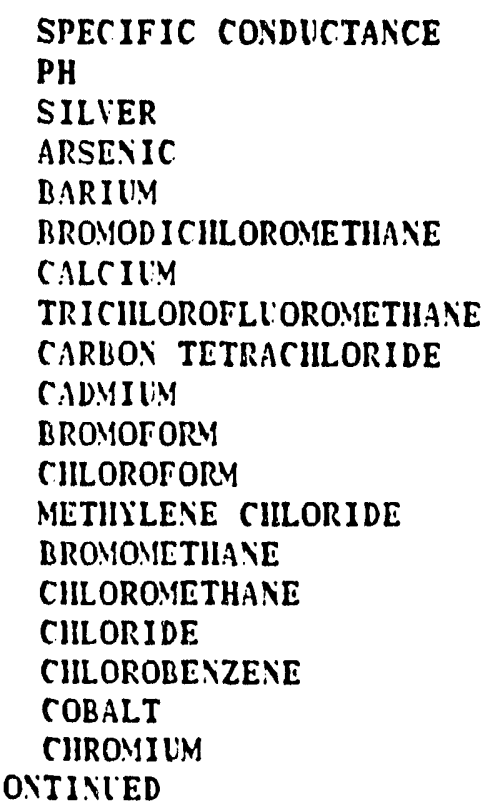 \\
\hline
\end{tabular}

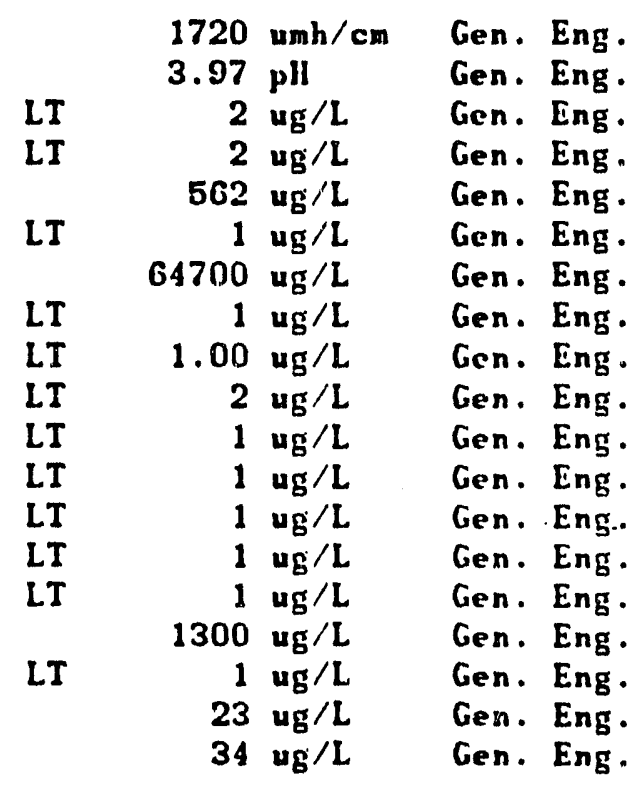


NELL FSB 93D COLLECTED ON 01/02/90 LABORATORY ANALYSES CONTINUED

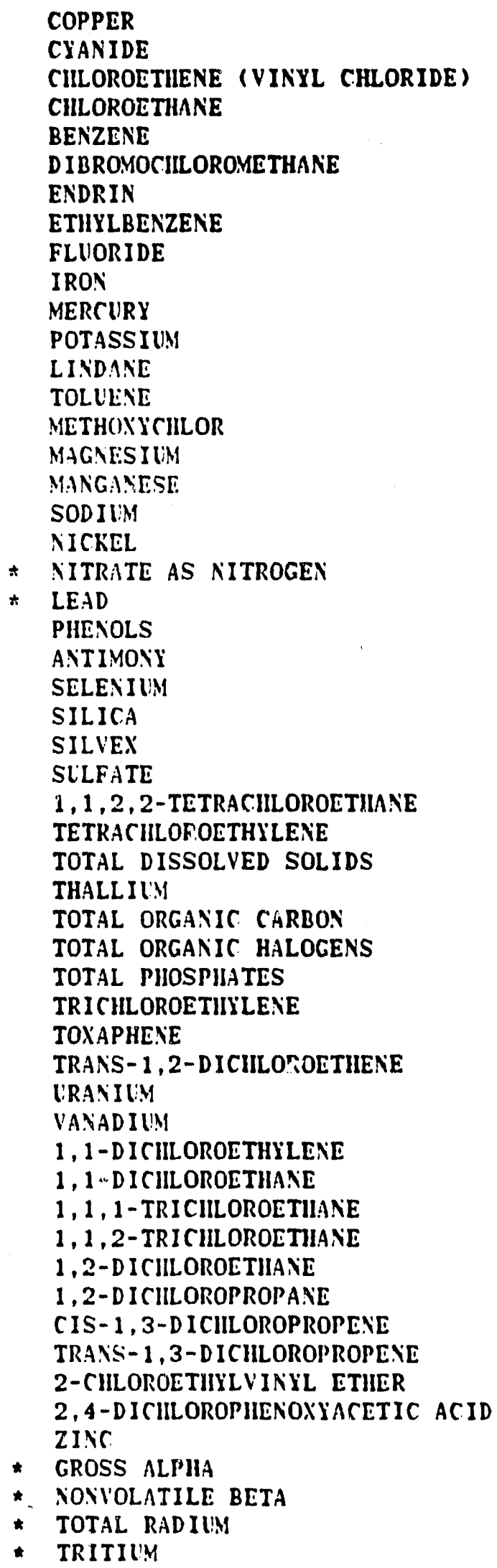

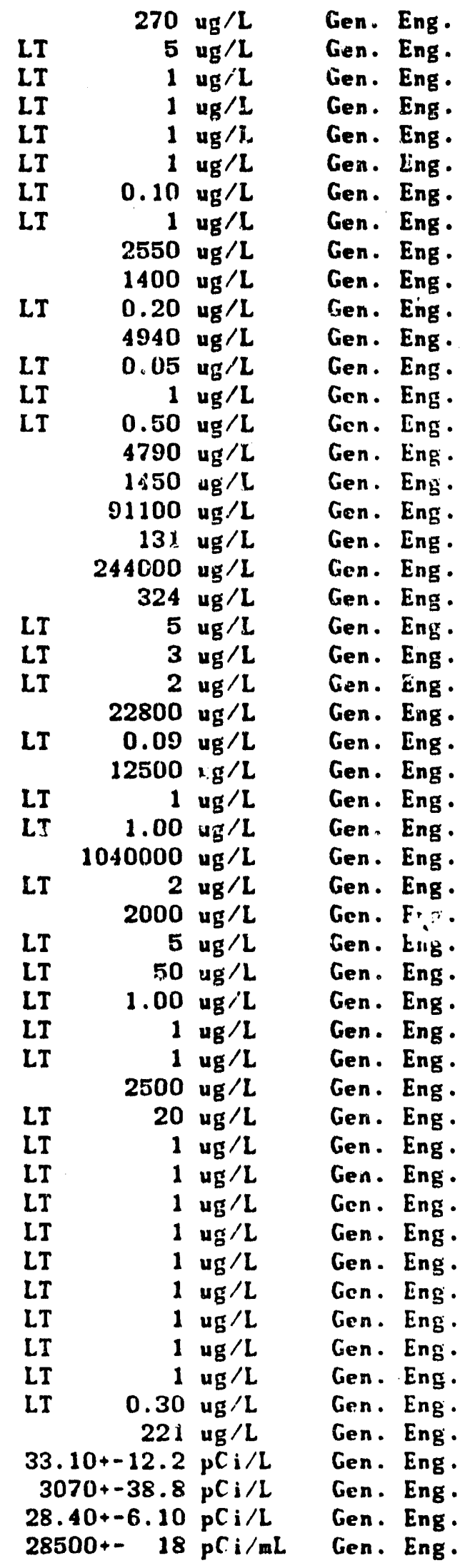


KELL FSB 94C

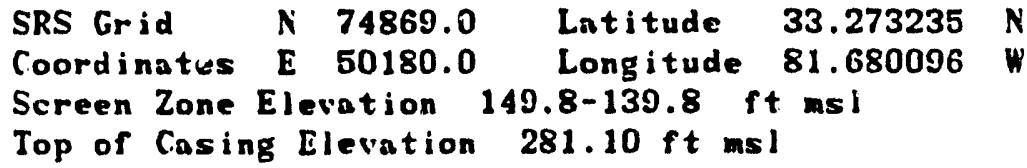

MEASUREMENTS CONDUCTED IN THE FIELD

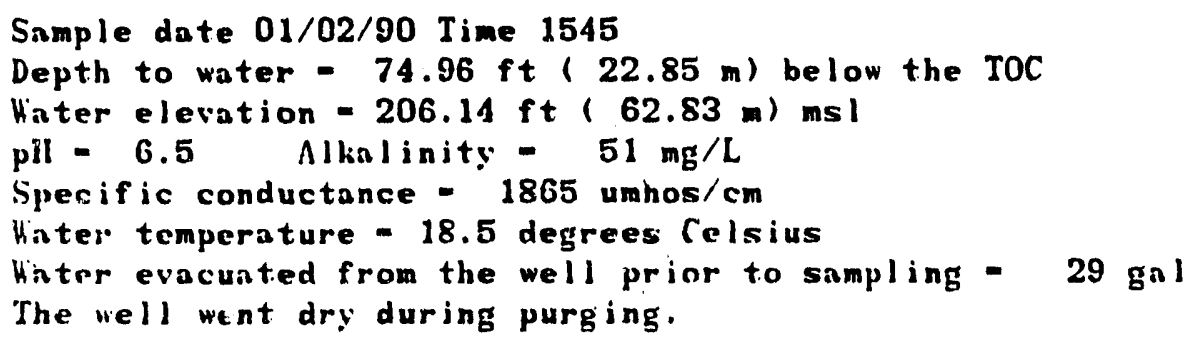

LABORATORY ANALISES

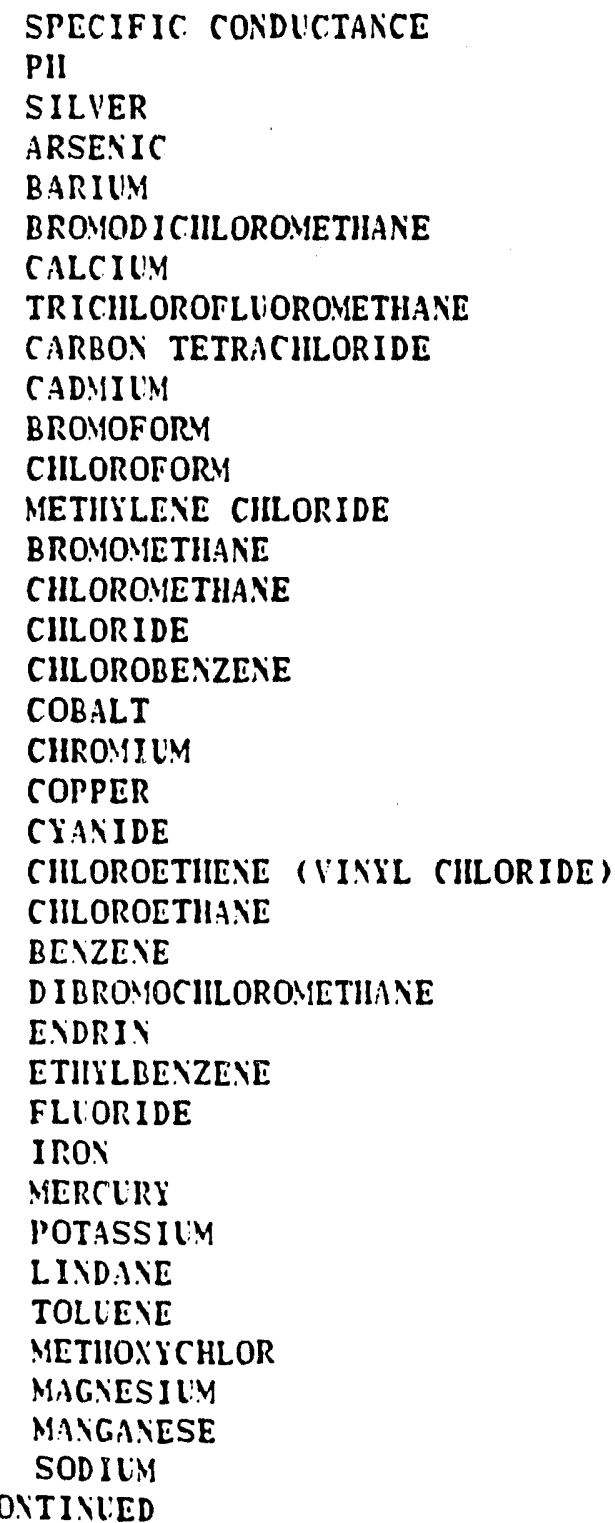

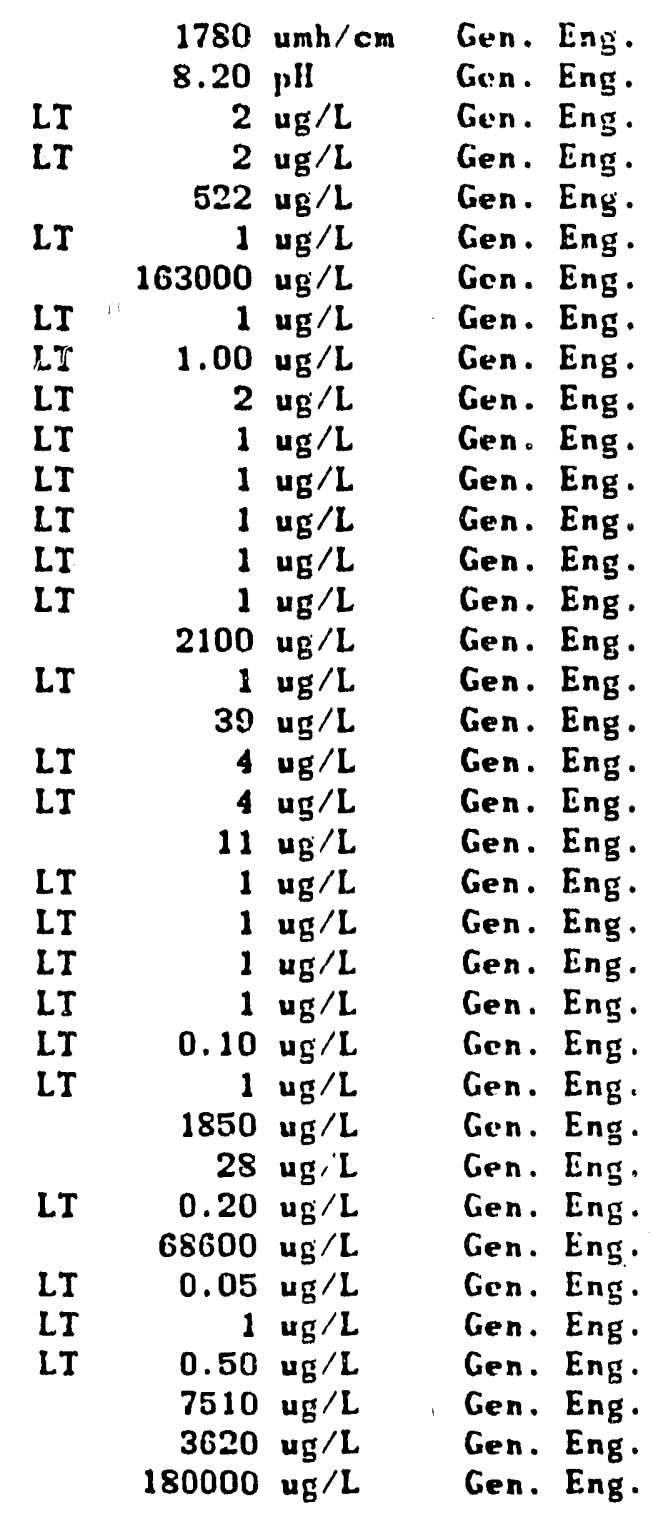


WELL FSB 94C COLLECTED ON 01/02/90 LABORATORY ANALYSES CONTINUED

NICKEL

* NITRATE as NITROGEN

LEAD

PHENOLS

ANTIMONY

SELENIUM

SILICA

SILVEX

SILLFATE

$1,1,2,2$-TETRACIILOROETHANE

TETRACILOROETIYLENE

TOTAL. DISSOLIED SOLIDS

TIIALLIUM

TOTAL ORGANIC CARBON

TOTAL ORGANIC HALOGENS

TOTAL PIIOSPHATES

TRICILOROETHYLENE

TONAPIIENE

TRANS-1, 2-D ICHLOROETHENE

LRANIUM

VANADIUM

1,1-DICHLOROETHILENE

1,1-DICIILOROETHANE

1,1,1-TRICIILOROETILANE

$1,1,2$-TRICHLOROLTHANE

1,2-D ICILLOROETIINE

1,2-DICILLOROPROPANE

CIS-1,3-DICIILOROPROPENE

TRANS-1,3-DICHLOROPROPENE

2-CIILOROETHYLVINYL ETHER

2,4-DICILOROPHENOXYACETIC ACID ZINC.

GROSS ALPHA

* Nonvolatile beta

TOTAL RADILM

* TRITIUM

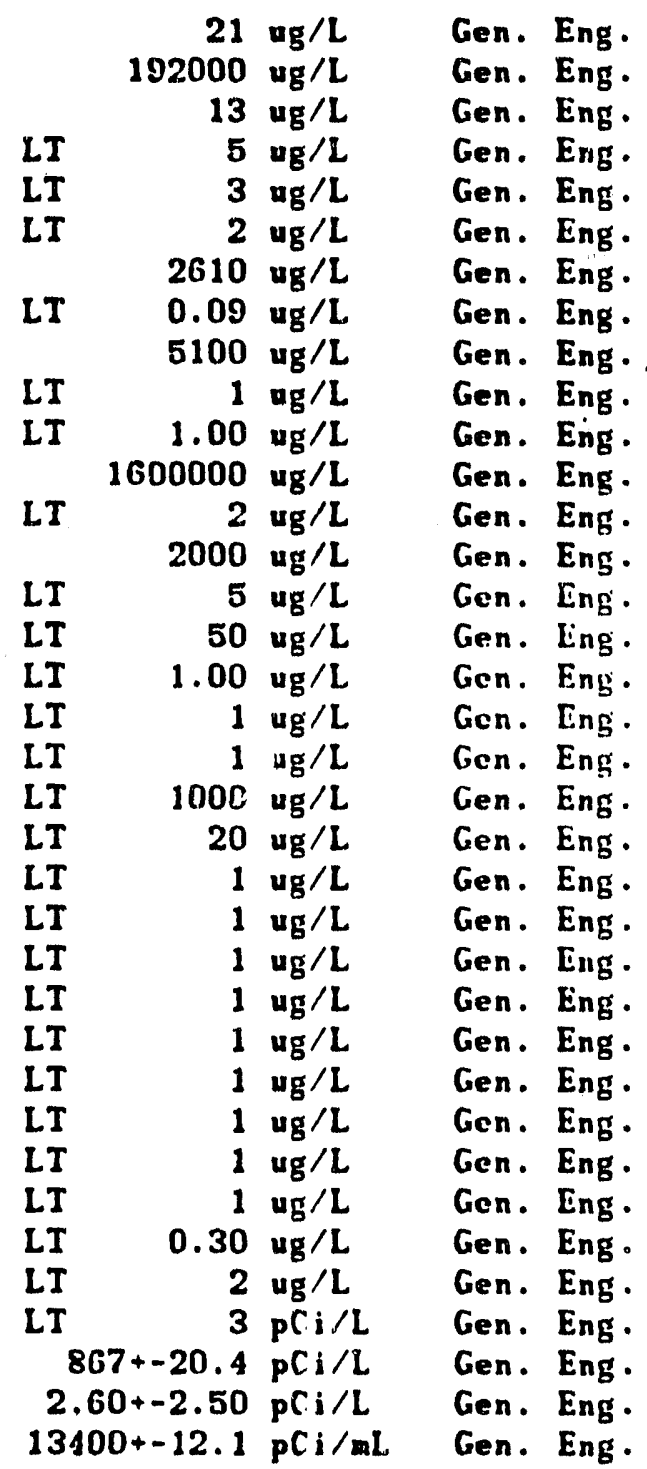


HELI. FSB 94D

SRS Grid N 74863.4 Latitude $33.273237 N$

Coordinates E 50188.6 Longitude 81.680062 W

Screen Zone Elevation 221.8-201.8 ft ms

Top of Casing Elevation $281.10 \mathrm{ft} \mathrm{msl}$

MEASUREMENTS CONDUCTED IN THE FIELD

Sample date 01/01/90 Time 1730

The well was dry.

NELL FSB 95C

SRS Grid N 74971.7 Lititude $33.273196 \quad N$

Conrdinates E 50016.7 Longitude 81.680725 V

Screen Zone Elevation 155.8-145.8 ft msl

Top of Casing Elevation $284.00 \mathrm{ft} \mathrm{msl}$

MEISIREMENTS CONDUCTED IN TIIE FIELD

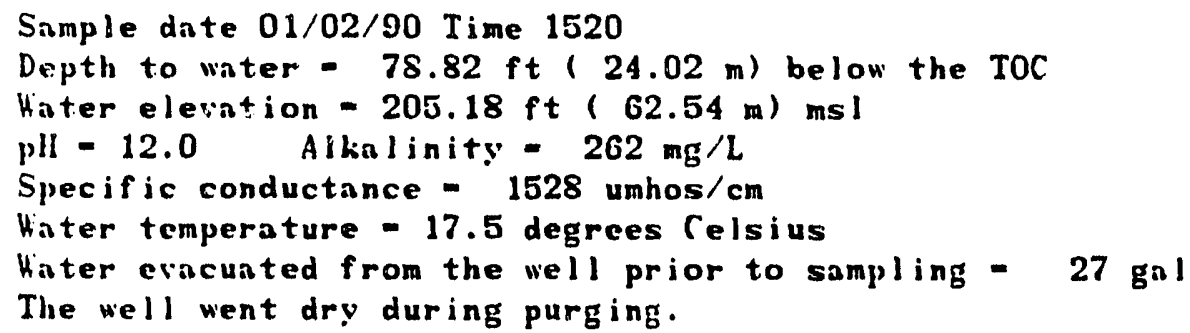

LABORATORY ANALYSES

\begin{tabular}{|c|c|c|c|c|}
\hline $\begin{array}{l}\text { SPECIFIC CONDUCTANCE } \\
\text { PII }\end{array}$ & & $\begin{array}{l}1520 \\
11.7\end{array}$ & $\begin{array}{l}u m h / c m \\
p H\end{array}$ & $\begin{array}{l}\text { Gen. } \\
\text { Gen. }\end{array}$ \\
\hline SILIER & LT & 2 & $\operatorname{ug} / \mathrm{L}$ & Gen. \\
\hline ARSENIC & LT & 2 & $u_{g} / L$ & Gen. \\
\hline BARIUM & & 202 & $u_{g} / L$ & Gen. \\
\hline BROMOD ICIILOROMETHANE & LT & 1 & $u g / L$ & Gen. \\
\hline CALCILM & & 160000 & $\operatorname{ug} / \mathrm{L}$ & Gen. \\
\hline TRICHLOROFLUOROMETHANE & LT & 1 & $u_{g} / L$ & Gen. \\
\hline CARBOS TETRACILLORIDE & LT & 1.00 & ug/L & Gen. \\
\hline CADMILYY & LT & 2 & $u g / L$ & Gen. \\
\hline BROMOFORY & LT & 1 & ug $/ 2$ & Gen. \\
\hline CIILOROFORY & LI & 1 & ug/L & Gen. \\
\hline METIIYLENE CIILORIDE & LT & 1 & $\operatorname{ug} / \mathrm{L}$ & Gen. \\
\hline BROMONETIINE & LT & 1 & $u g / L$ & Gen. \\
\hline CIILOROMETIIANE & LT & 1 & $u g / L$ & Gen. \\
\hline CIILORIDE & & 2400 & $\operatorname{ug} / \mathrm{L}$ & Gen. \\
\hline CIILOROBE.IZENE & LI & 1 & ug $/ \mathrm{L}$ & Gen. \\
\hline COBALT & & 10 & ug $/ \mathrm{L}$ & Gen. \\
\hline CIIROMILM & LT & 4 & ug $i^{\prime} L$ & lien. \\
\hline COPPER & LT & 4 & $\operatorname{ug} / \mathrm{L}$ & Gen. \\
\hline CIAIIDE & & 6 & ug $/ \mathrm{L}$ & Gen. \\
\hline CHLOROETIIENE (VINYL CIILORIDE) & LT & 1 & $u g / L$ & Gen. \\
\hline
\end{tabular}


HELL FSB 95C COLLECTED ON 01/02/90 LABORATORY ANALYSES CONTINUED

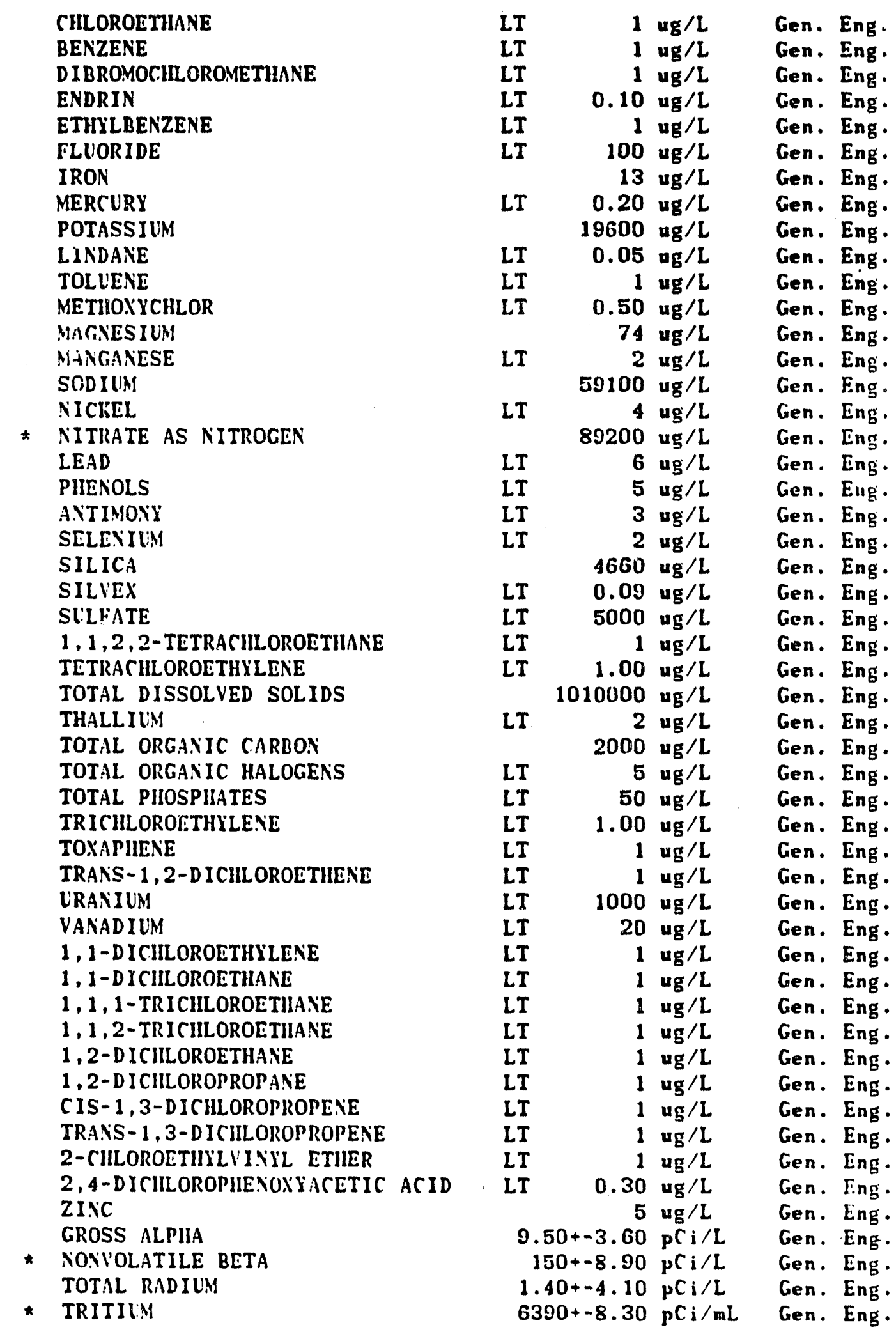


VELL FSB 95D

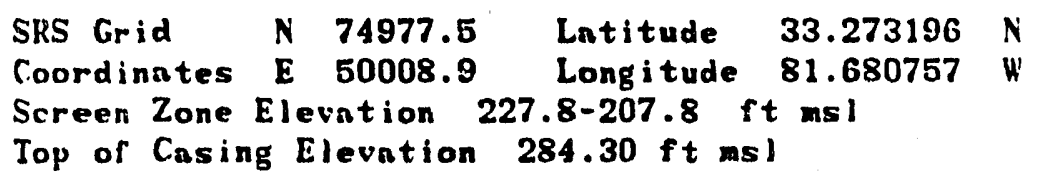

MEASUREMENTS CONDUCTED IN THE FIELD

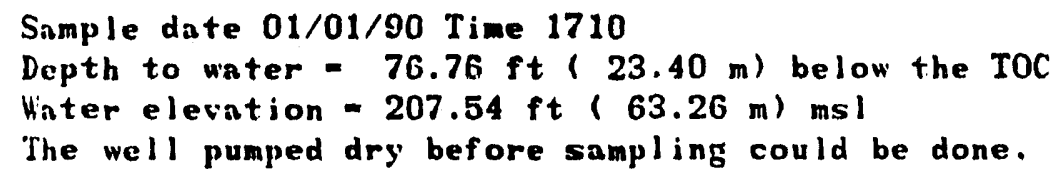

HELL FSB $96 A$

SRS Grid $N 74882.2$ Latitude $33.272609 N$

Coordinates E 49778.7 Longitude 81.681178 "'

Screen Zone Elevation 95.7-85.7 ft $\mathrm{msl}$

Top of Casing Elevation $279.80 \mathrm{ft} \mathrm{ms}$ l

MEASURTMETTS CONDUCTED IN TIE FIELD

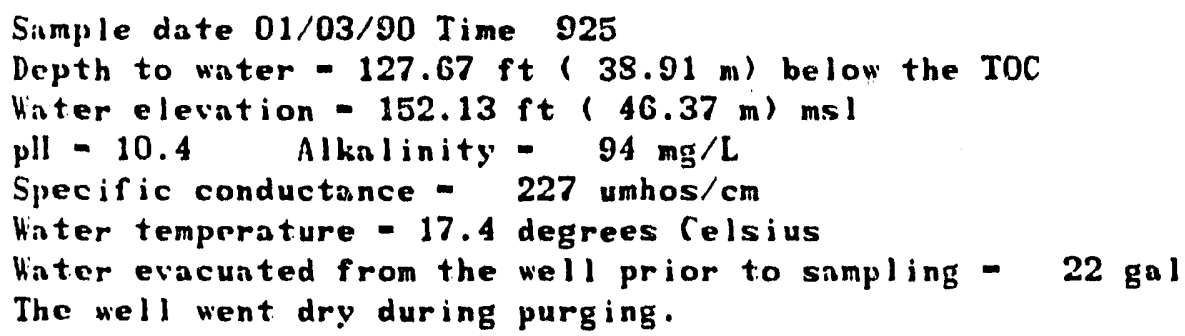

\section{LABORATORY ANALYSES}

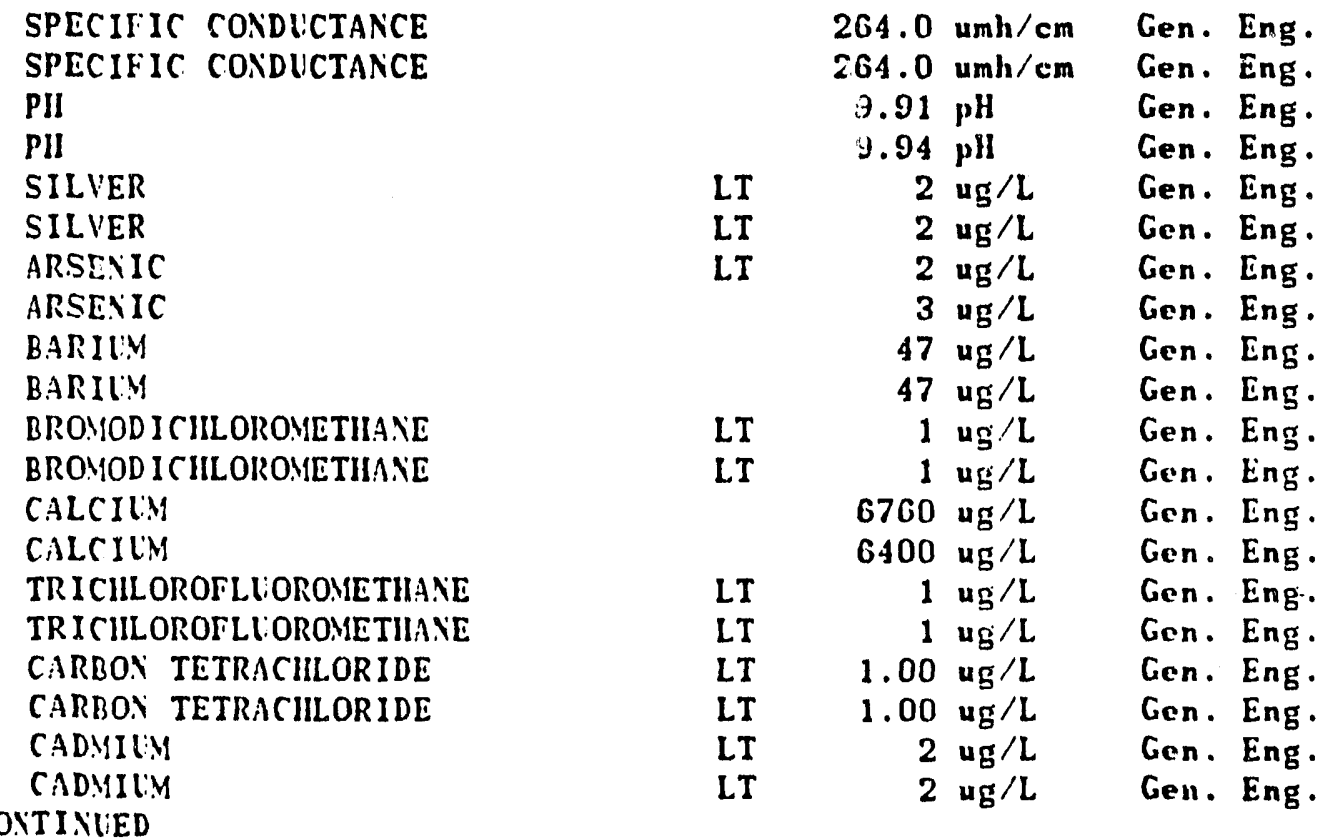


NELL FSB 96A COLLECTED ON 01/03/90 LABORATORY ANALYSES CONTINUED

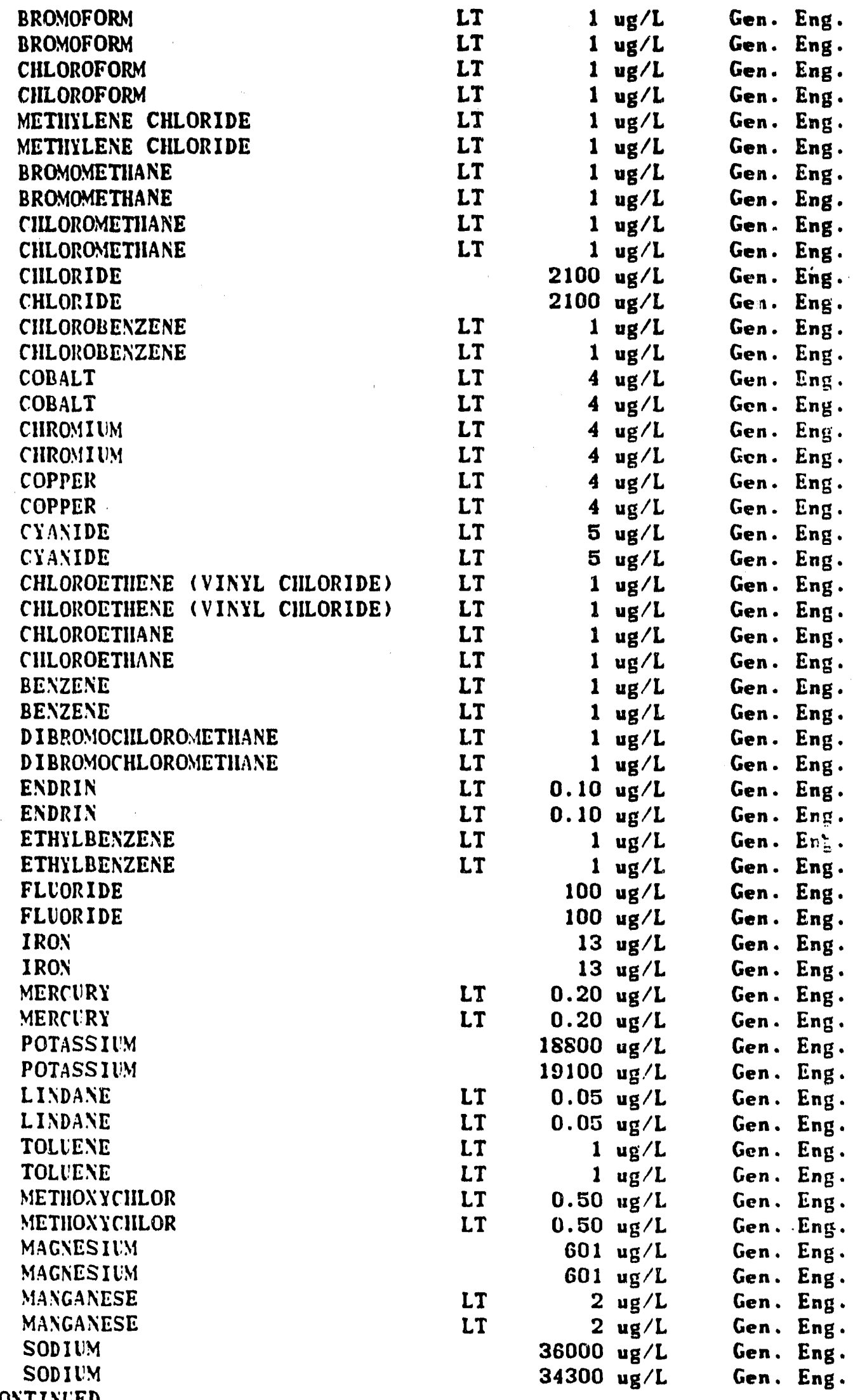


WELI FSB 96A COLLECTED ON 01/03/90 LABORATORY ANALYSES CONTINUED

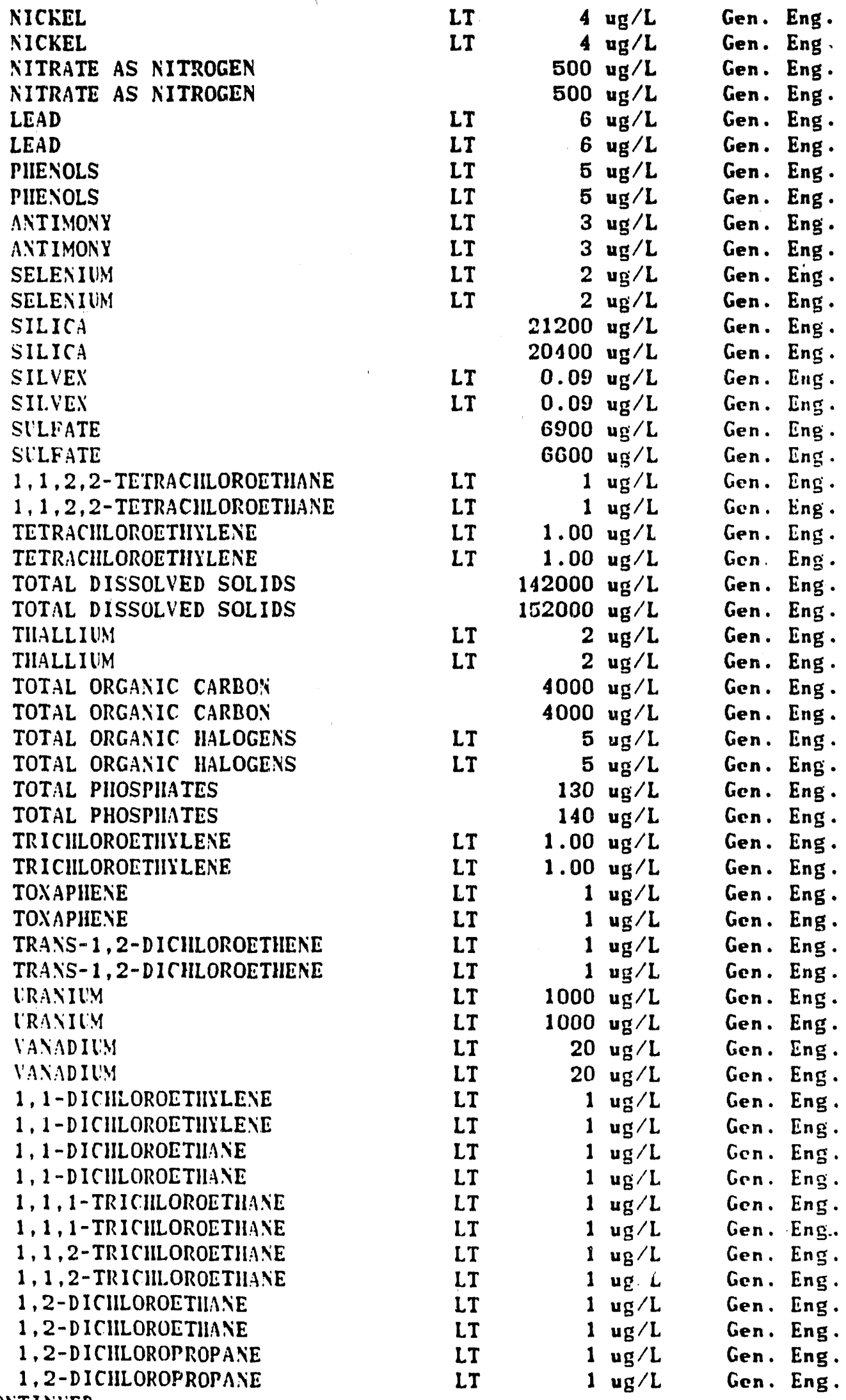


WELL FSB 96A COLLECTED ON 01/03/90 LABORATORY ANALYSES CONTINUED

\begin{tabular}{|c|c|c|c|}
\hline 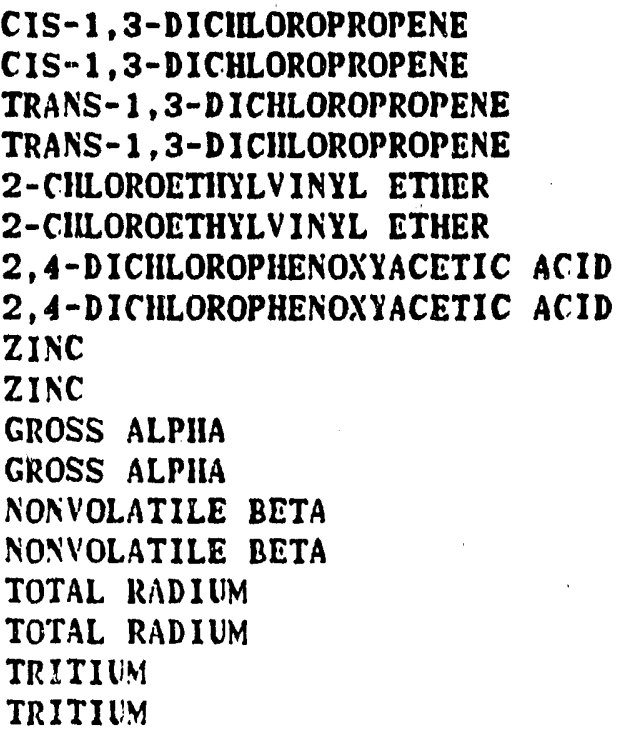 & $\begin{array}{l}\text { LT } \\
\text { LT } \\
\text { LT } \\
\text { LT } \\
\text { LT } \\
\text { LT } \\
\text { LT } \\
\text { LT } \\
\text { LT } \\
\text { LT } \\
\text { LT } \\
26.2 \\
29.8 \\
2.1 \\
3.2 \\
12.5 \\
12.2\end{array}$ & $\begin{array}{r}1 \\
1 \\
1 \\
1 \\
1 \\
1 \\
0.30 \\
0.30 \\
2 \\
2 \\
3 \\
3 \\
-2.30 \\
-2.40 \\
-1.70 \\
-1.50 \\
-0.49 \\
-0.47\end{array}$ & $\begin{array}{l}\mathrm{ug} / \mathrm{L} \\
\mathrm{ug} / \mathrm{L} \\
\mathrm{ug} / \mathrm{L} \\
\mathrm{ug} / \mathrm{L} \\
\mathrm{ug} / \mathrm{L} \\
\mathrm{ug} / \mathrm{L} \\
\mathrm{ug} / \mathrm{L} \\
\mathrm{ug} / \mathrm{L} \\
\mathrm{ug} / \mathrm{L} \\
\mathrm{ug} / \mathrm{L} \\
\mathrm{pCi} / \mathrm{L} \\
\mathrm{pCi} / \mathrm{L} \\
\mathrm{pCi} / \mathrm{L} \\
\mathrm{pCi} / \mathrm{L} \\
\mathrm{pCi} / \mathrm{L} \\
\mathrm{pCi} / \mathrm{L} \\
\mathrm{pCi} / \mathrm{mL} \\
\mathrm{pCi} / \mathrm{mL}\end{array}$ \\
\hline
\end{tabular}

NELL FSB 97A

SRS Grid N 75171.2 Latitude $33.273554 \quad N$

Coordinates E 49065.7 Longitude 81.681247 W

Screen Zone Elevation 95.8- 85.8 ft msl

Top of Casing Elevation $286.10 \mathrm{ft} \mathrm{msl}$

MEASUREMENTS CONDUCTED IN THE FIELD

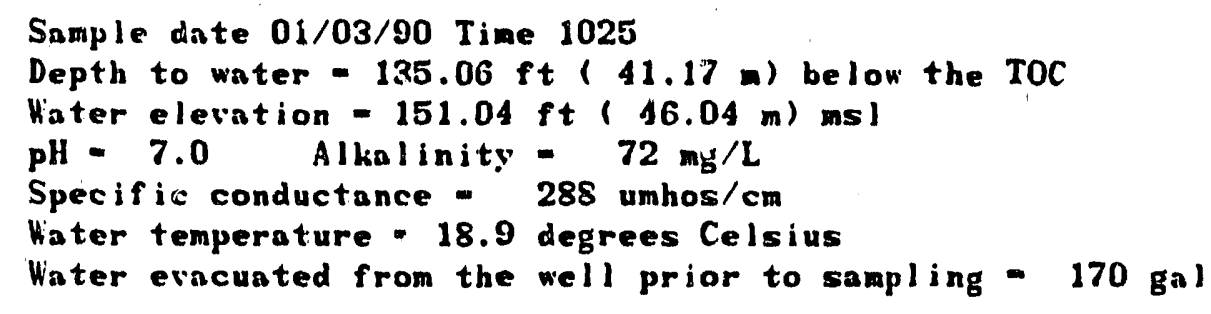

LABORATORY ANALYSES

SPECIFIC CONDUCTANCE
PII

SILVER

ARSENIC

BARILM

BROMOD ICIILOROMETHANE

CALCIINY

TRICHLOROFLUOROMETIIANE

CARBON TETRACHLORIDE

CADMILM

BROMOTORY

CIILOROFORY

METIILENE CILORIDE

BROMOMETHANE

CONTINLED

\begin{tabular}{|c|c|c|c|c|}
\hline & $\begin{array}{r}270.0 \\
7.34\end{array}$ & $\begin{array}{l}\operatorname{umh}_{\text {pll }} / \mathrm{cm} \\
\text { la }\end{array}$ & $\begin{array}{l}\text { Gen. } \\
\text { Gen. }\end{array}$ & Er \\
\hline $\begin{array}{l}\text { LI } \\
\text { LT }\end{array}$ & & & $\begin{array}{l}\text { Gen. } \\
\text { Gen. }\end{array}$ & $\begin{array}{l}E_{I} \\
E_{1}\end{array}$ \\
\hline LT & $\begin{array}{r}42 \\
1 \\
43200\end{array}$ & $\begin{array}{l}\text { ug } / L \\
\text { ug } / L \\
\text { ug } / L\end{array}$ & $\begin{array}{l}\text { Gen. } \\
\text { Gen. } \\
\text { Gen. }\end{array}$ & 1 \\
\hline $\begin{array}{l}\text { LT } \\
\text { LT }\end{array}$ & $\begin{array}{r}1 \\
1.00\end{array}$ & $\begin{array}{l}u g / L \\
u g / L\end{array}$ & $\begin{array}{l}\text { Gen. } \\
\text { Gen. }\end{array}$ & \\
\hline LT & 2 & ug $/ \mathrm{L}$ & Gen. & \\
\hline LT & 1 & ug $/ \mathrm{L}$ & Gen. & \\
\hline LT & 1 & ug $/ \mathrm{L}$ & Gen. & \\
\hline LT & 1 & $\mu g / L$ & Gen. & \\
\hline LT & 1 & $\mathrm{ug} / \mathrm{L}$ & Gen. & \\
\hline
\end{tabular}


HELL FSB 97A COLLECTED ON 01/03/90 LABORATORY ANALYSES CONTINUED

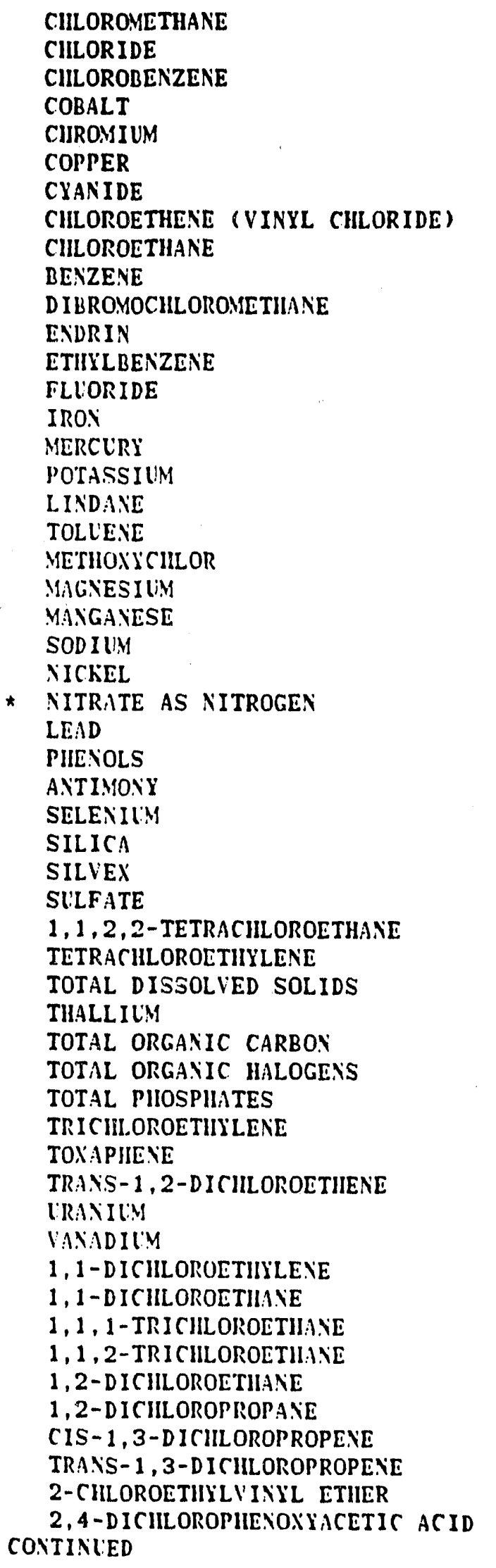

LT

LT

LT

LT

LT

LT

LT

LT

LT

LT

LT

LT

LT

LT

LT

LT

LT

LT

LI

LIT

LT

LT

LT

LT

LT

LT

LT

LT

LT

LT

LT

LT

LT

LT

LT

LT

LT

LT

LT

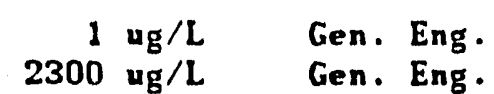

$1 \mathrm{ug} / \mathrm{L}$ Gen. Eng.

$4 \mathrm{ug} / \mathrm{L}$ Gen. Eng.

$4 \mathrm{ug} / \mathrm{L}$ Gen. Eng.

$4 \mathrm{ug} / \mathrm{L}$ Gen. Eng.

5 ug $/ \mathrm{L}$ Gen. Eng.

$1 \mathrm{ug} / \mathrm{L}$ Gen. Eng.

$1 \mathrm{ug} / \mathrm{L}$ Gen. Eng.

$1 \mathrm{ug} / \mathrm{L}$ Gen. Eng.

$1 \mathrm{ug} / \mathrm{L}$ Gen. Eng.

$0.10 \mathrm{ug} / \mathrm{L}$ Gen. Eng.

$1 \mathrm{ug} / \mathrm{L}$ Gen. Eng.

$100 \mathrm{ug} / \mathrm{L}$. Gen. Eng.

9 ug/L Gen. Eng.

$0.20 \mathrm{ug} / \mathrm{L}$ Gen. Eng.

$640 \mathrm{ug} / \mathrm{L}$ Gen. Eng.

$0.05 \mathrm{ug} / \mathrm{L}$ Gen. Eng.

$1 \mathrm{ug} / \mathrm{L}$ Gen. Ing.

$0.50 \mathrm{ug} / \mathrm{L}$ Gen. Ing.

$1110 \mathrm{ug} / \mathrm{L}$ Gen. Eng.

$18 \mathrm{ug} / \mathrm{L}$ Gen. Eng.

$8900 \mathrm{ug} / \mathrm{L}$ Gen. Eng.

$4 \mathrm{ug} / \mathrm{L}$ Gen. Eng.

$14500 \mathrm{ug} / \mathrm{L}$ Gen. Eng.

$6 \mathrm{ug} / \mathrm{L}$ Gen. Eng.

$5 \mathrm{ug} / \mathrm{L}$ Gen. Eng.

$3 \mathrm{ug} / \mathrm{L}$ Gen. Eng.

2 ug/L Gen. Eng.

$19900 \mathrm{ug} / \mathrm{L}$ Gen. Eng.

$0.09 \mathrm{ug} / \mathrm{L}$ Gen. Eng.

$5000 \mathrm{ug} / \mathrm{L}$ Gen. Eng.

1 ug/L Gen. Eng.

$1.00 \mathrm{ug} / \mathrm{L}$ Gen. Eng.

70000 ug $/ \mathrm{L}$ Gen. Eng.

$2 \mathrm{ug} / \mathrm{L}$ Gen. Eng.

$2000 \mathrm{ug} / \mathrm{L}$ Gen. Eng.

5 ug/L Gen. Eng.

$120 \mathrm{ug} / \mathrm{L}$ Gen. Eng.

$1.00 \mathrm{ug} / \mathrm{L}$ Gen. Eng.

1 ug $/ \mathrm{L}$ Gen. Eng.

1 ug/L Gen. Eng.

$1000 \mathrm{ug} / \mathrm{L}$ Gen. Eng.

$20 \mathrm{ug} / \mathrm{L}$ Gen. Eng.

$1 \mathrm{ug} / \mathrm{L}$ Gen. Eng.

$1 \mathrm{ug} / \mathrm{L}$ Gen. Eng.

$1 \mathrm{ug} / \mathrm{L}$ Gen. Eng.

$1 \mathrm{ug} / \mathrm{L}$ Gen. Eng.

1 ug $/ \mathrm{L}$ Gen. Eng.

$1 \mathrm{ug} / \mathrm{L}$ Gen. Eng.

1 ug/L Gen. Eng.

$1 \mathrm{ug} / \mathrm{L}$ Gen. Eng.

$1 \mathrm{ug} / \mathrm{L}$ Gen. Eng.

$0.30 \mathrm{ug} / \mathrm{L}$ Gen. Eng. 
NELL FSB 97A COLLECTED ON U1/03/90 LABORATORY ANALYSES CONTINLED

$\begin{array}{llrl}\text { ZINC } & \mathrm{LT} & 2 \mathrm{ug} / \mathrm{L} & \text { Gen. Eng. } \\ \text { GROSS ALPHA } & \text { LT } & 3 \mathrm{pCi} / \mathrm{L} & \text { Gen. Eng. } \\ \text { NONVOLATILE BETA } & 4.58+-2.40 \mathrm{pCi} / \mathrm{L} & \text { Gen. Eng. } \\ \text { TOTAL RADIUM } & L T & 1 \mathrm{pCi} / \mathrm{L} & \text { Gen. Eng. } \\ \text { *RITIUM } & 552+-2.43 \mathrm{pCi} / \mathrm{mL} & \text { Gen. Eng. }\end{array}$

HELL FSB 97A

SRS Grid N 75171.2 Latitude 33.273554 N

Conrdinates $\mathbb{E}$ 49965.7 Longitude 81.681247 W'

Sereen Zone Elevation $05.8-85.8 \mathrm{ft} \mathrm{msl}$

iop of Casing Elevation $286.10 \mathrm{ft} \mathrm{msl}$

BTASIREMENTS CONDUCTED IN THE FIELD

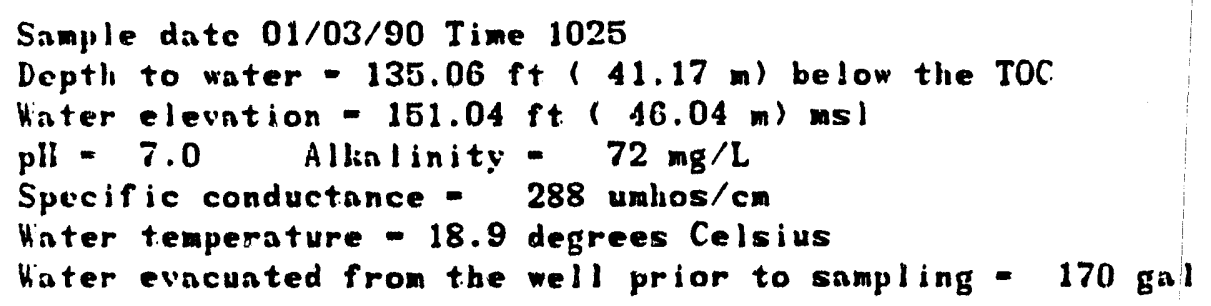

\section{VELL FSB 97C}

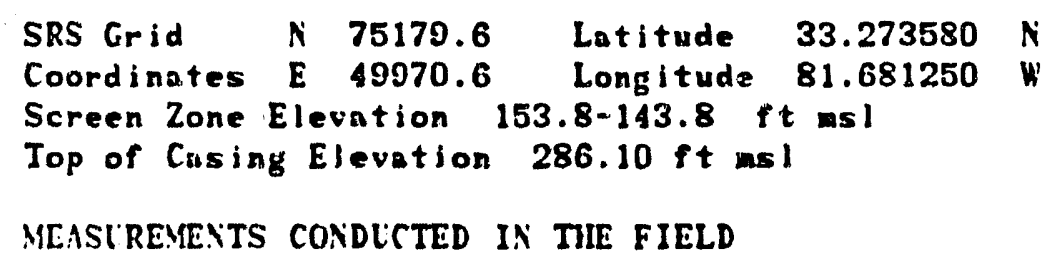

MEASIREMENTS CONDLCTED IN TIE FIELD

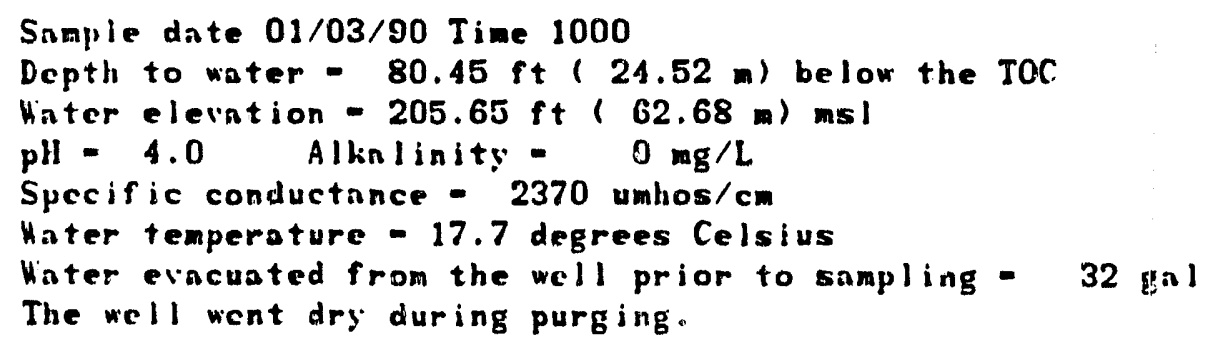




\section{ARSENIC}

* BARIUM

BROMODICIILOROATETHANE

CALCIUM

TRICIILOROFLUOROMETHANE

CARBON TETRACIILORIDE

CADMIUM

BROMOFORM

CIILORGFORM

METIIYLENE CIILORIDE

BROMONETHANE

CILLRONETIIANE

CIII.ORIDE

CIILOROBENZENE

COBALT

CIIROMIIUM

COPPER

CIANIDE

CILLOROETIENE (IINYI. CHLORIDE)

CHLOROETIIANE

BE.IZENE

DIBROYOCHLORONETIIANE

ENDRIN

ETIILBENZENE

* FLlOORIDE

IRON

MERCIERY

POTASSILM

LINDANE

TOLLENE

METIOXYCIILOR

MAGNESIIYY

MANGANESE

SODILM

NICKEL

* NITRate as NItrogeN

* lead

PIIENOLS

ANT IMONY

SELEXILY

SILICA

SILIEX

SILFATE

$1,1,2,2$-TETRACHI.OROETHANE

TETRACIILOROETIILENE

TOTIL DISSOLVED SOLIDS

TIIILLILN

TOTAL ORGANIC CARBOX

TOTAL ORGANIC IIII.OGENS

TOTAL PIIOSPIIITES

TRICIILOROETIIYLENE

TOXAPIIENE

TRANS-1,2-DICILLOROF:THENE

URAKIUM

CONTINIED
LT

LT

LI

LI

LT

LT

LT

LI

LT

LT

LT

LT

LT

LT

LT

LT

LI

LI

LT

LT

LT

LT

LT

LT

LI

LT

LT

L"

LT

LT

LT

LI

LI

LT

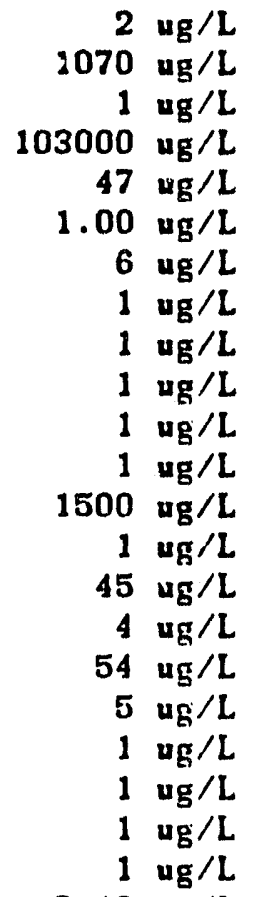

$0.10 \mathrm{ug} / \mathrm{L}$

$1 \mathrm{ug} / \mathrm{L}$

$4400 \mathrm{ug} / \mathrm{L}$

$846 \mathrm{ug} / \mathrm{L}$

$0.20 \mathrm{ug} / \mathrm{L}$

$14600 \mathrm{ug} / \mathrm{L}$

$0.05 \mathrm{ug} / \mathrm{L}$

$1 \mathrm{ug} / \mathrm{L}$

$0.50 \mathrm{ug} / \mathrm{L}$

$12500 \mathrm{ug} / \mathrm{L}$

$6810 \mathrm{ug} / \mathrm{L}$

$138000 \mathrm{ug} / \mathrm{L}$

$113 \mathrm{ug} / \mathrm{L}$

$317000 \mathrm{ug} / \mathrm{L}$

$76 \mathrm{ug} / \mathrm{L}$

$5 \mathrm{ug} / \mathrm{L}$

$3 \mathrm{ug} / \mathrm{L}$

$2 \mathrm{ug} / \mathrm{L}$

$39300 \mathrm{ug} / \mathrm{L}$

$0.09 \mathrm{ug} / \mathrm{L}$

5000 ug $/ L$

$1 \mathrm{ug} / \mathrm{L}$

$1.00 \mathrm{ug} / \mathrm{L}$

$1110000 \mathrm{ug} / \mathrm{L}$

$2 \mathrm{ug} / \mathrm{L}$

3000 ug $/ L$

$5 \mathrm{ug} / \mathrm{L}$

$50 \mathrm{ug} / \mathrm{L}$

$1.00 \mathrm{ug} / \mathrm{L}$

$1 \mathrm{ug} / \mathrm{L}$

$1 \mathrm{ug} / \mathrm{L}$

$1270 \mathrm{ug} / \mathrm{L}$
Gen. Eng.

Gen. Eng.

Gen. Eng.

Gen. Eng.

Gen. Eng.

Gen. Eng.

Gen. Eng.

Gen. Eng.

Gen. Eng.

Gen. Eng.

Gen. Eng.

Gen. Eng.

Gen. Eng.

Gen. Eng.

Gen. Eng.

Gen. Eng.

Gen. Eng.

Gen. Eng.

Gen. Eng.

Gen. Eng.

Gen. Eng.

Gen. Eng.

Gen. Eng.

Gen. Eng.

Gen. Eng.

Gen. Eng.

Gen. Eng.

Gen. Eng.

Gen. Eng.

Gen. Eng.

Gen. Eng.

Gen. Eng.

Gen. Eng.

Gen. Eng.

Gen. Eng.

Gen. Eng.

Gen. Eng.

Gen. Eng.

Gen. Eng.

Gen. Ing.

Gen. Eng.

Gen. Eng.

Gen. Eng.

Gen. Eng.

Gen. Eng.

Gen. Eng.

Gen. Eng.

Gen. Eng.

Gen. Eng.

Gen. Eng.

Gen. Eng.

Gen. Eng.

Gen. Eng.

Gen. Eng. 
RELL FSB 97C COLLECTED ON 01/03/90 LABORATORY ANALYSES CONTINUED

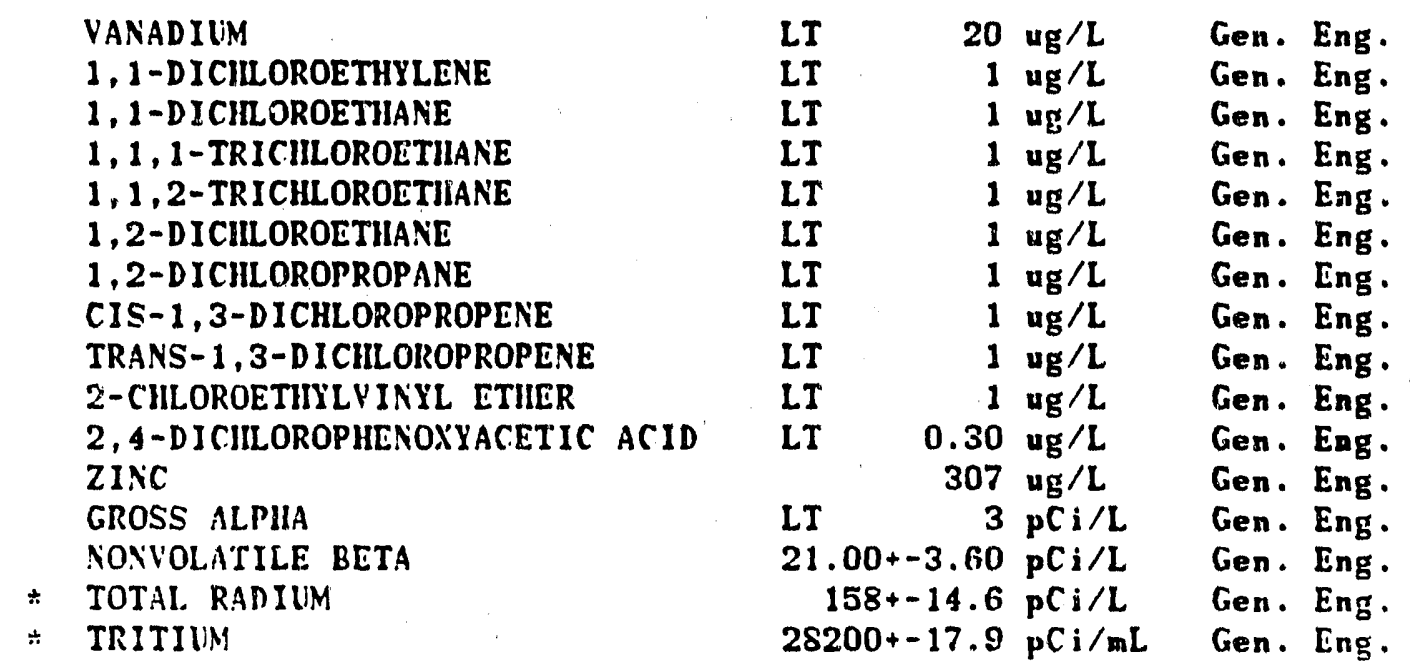

HLLL FSB 97C

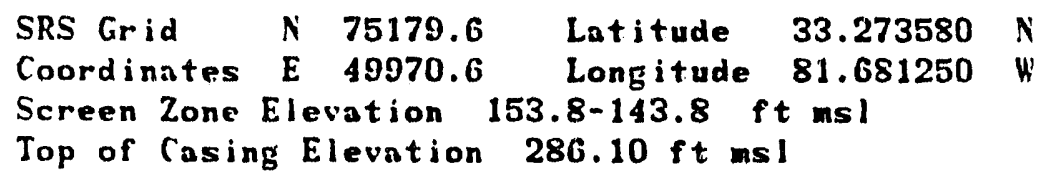

MEASLREMENTS CONDUCTED IN THE FIEL?

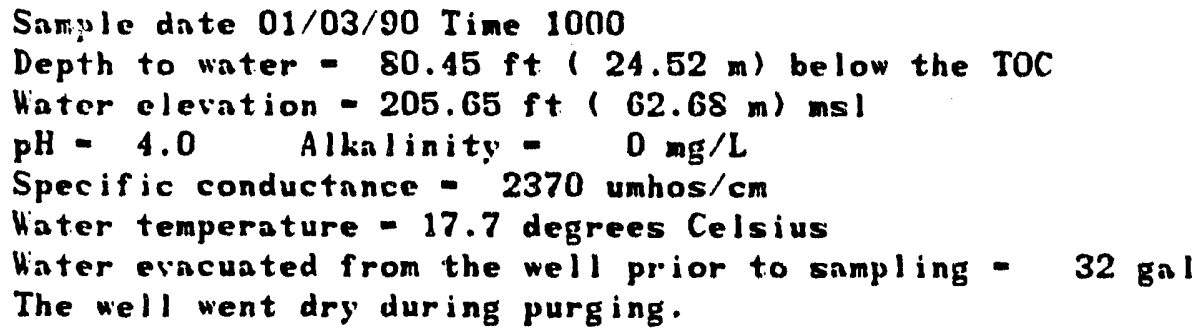

$47000 \mathrm{ug} / \mathrm{L}$ Gen. Eng. 
HELL FSB 97D

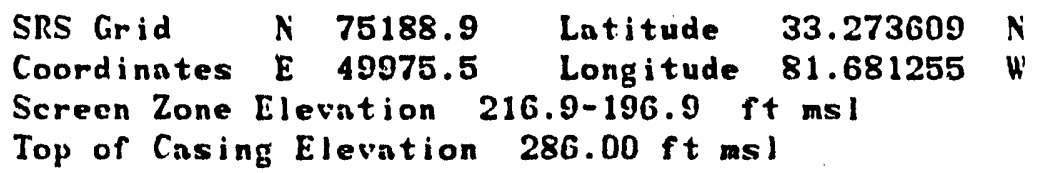

MEASLIREMENTS CONDVICTED IN THE FIELD

Sample date 01/03/90 Time 1040

Depth to water = $77.74 \mathrm{ft}(23.70 \mathrm{~m})$ below the TOC

Hater elevation - $208.26 \mathrm{ft}(63.48 \mathrm{~m}) \mathrm{msl}$

pll = 3.0 Alkalinity $=0 \mathrm{mg} / \mathrm{L}$

Specific conductance - 2040 umhos/cm

Hiater temperature $=17.4$ degrees Celsius

Hater evacunted from the well prior to sampling - $2 \mathrm{gal}$

The well went dry during purging.

LABOMTORY ANALYSES

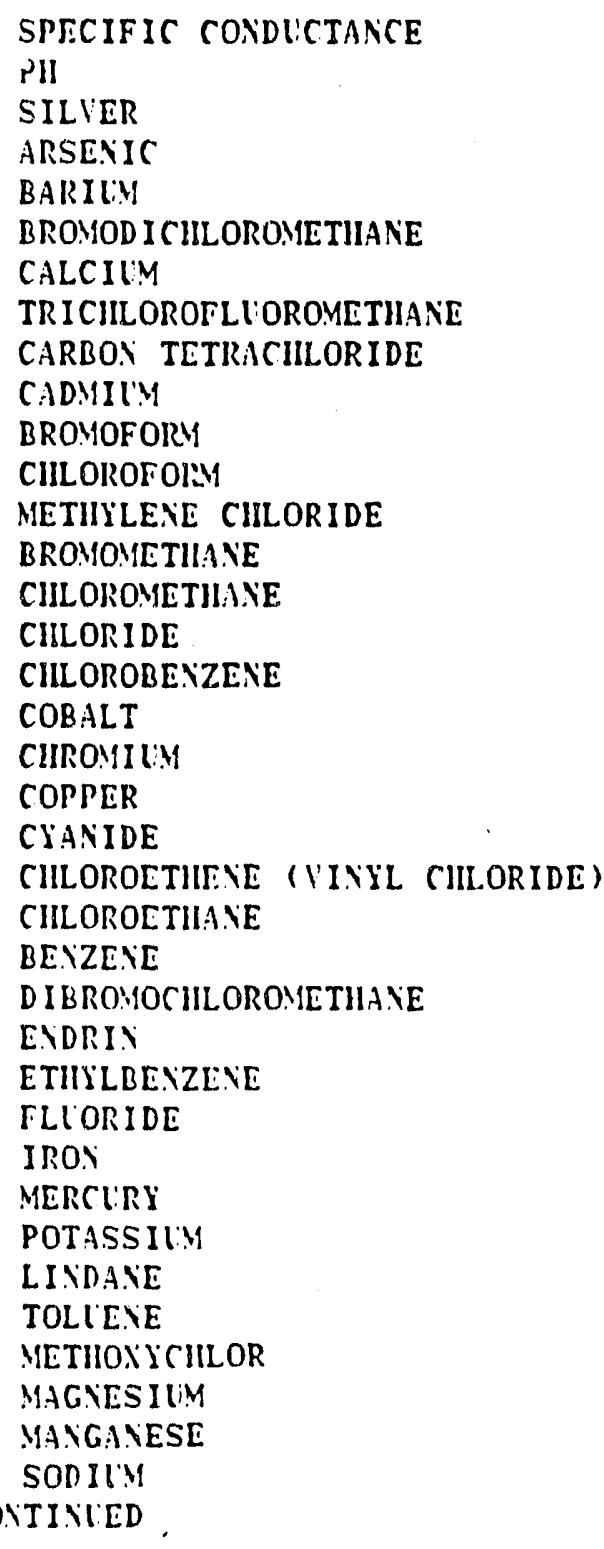

\begin{tabular}{|c|c|c|c|c|}
\hline & $\begin{array}{l}1620 \\
4.28\end{array}$ & $\mathrm{umh}_{\mathrm{h}} / \mathrm{cm}$ & $\begin{array}{l}\text { Gen. } \\
\text { Gen. }\end{array}$ & Eng \\
\hline LT & 2 & $4 \mathrm{~L} / \mathrm{L}$ & Gen. & Eng. \\
\hline $\mathrm{LT}$ & 2 & ug $/ \mathrm{L}$ & Gen. & Eng \\
\hline LT & 1 & ug $/ \mathrm{L}$ & Gen. & Eng \\
\hline & 157000 & ug $/ L$ & Gen. & Eng \\
\hline LT & 1 & ug $/ L$ & Gen. & Eng \\
\hline LI & $\begin{array}{r}1.00 \\
7\end{array}$ & $\begin{array}{l}u g / L \\
u g / L\end{array}$ & $\begin{array}{l}\text { Gen. } \\
\text { Gen. }\end{array}$ & $\begin{array}{l}\text { Eng } \\
\text { Eng }\end{array}$ \\
\hline LT & 1 & ug $/ \mathrm{L}$ & Gen. & Eng \\
\hline LT & 1 & ug /L & Gen. & Eng \\
\hline LT & 1 & ug $/ \mathrm{L}$ & Gen. & Eng \\
\hline LT & 1 & ug $/ \mathrm{L}$ & Gen. & Er \\
\hline LT & 1 & ug / L & Gen. & {$["$} \\
\hline & 2700 & $\mathrm{\mu g} / \mathrm{L}$ & Gen. & Eng \\
\hline LT & 1 & $\mathrm{Mg} / \mathrm{L}$ & Gen. & Eng \\
\hline & 27 & $\mathrm{ug} / \mathrm{L}$ & Gen. & Eng \\
\hline & 18 & ug $/ \mathrm{L}$ & Gen. & Eng \\
\hline & 10 & ug $/ \mathrm{L}$ & Gen. & Eng \\
\hline & 0 & ug $/ \mathrm{L}$ & Gen. & Eng \\
\hline$L T$ & 1 & $u_{G} / L$ & Gen. & Ling \\
\hline & $\begin{array}{l}1 \\
2\end{array}$ & $\begin{array}{l}u g / L \\
u g / L\end{array}$ & $\begin{array}{l}\text { Gen. } \\
\text { Gen. }\end{array}$ & $\begin{array}{l}\text { Eng } \\
\text { Eng }\end{array}$ \\
\hline LT & 1 & ug $/ \mathrm{L}$ & Gen. & Eng \\
\hline LI & 0.10 & ug/L & Gen. & Eng \\
\hline LT & 1 & ug $/ \mathrm{L}$ & Gen. & Eng \\
\hline & 1180 & $u_{G} / L$ & Gen. & \\
\hline & 3850 & ug $/ \mathrm{L}$ & Gen & \\
\hline LT & 0.20 & ug $/ \mathrm{L}$ & Gen. & Eng \\
\hline & 4070 & ug $/ \mathrm{L}$ & Gen. & \\
\hline LT & 0.05 & $u g / L$ & Gen. & \\
\hline LT & 1 & ug/L & Gen & \\
\hline L.T & 0.50 & $\mathrm{ug} / \mathrm{L}$ & Gen. & \\
\hline & 5130 & ug $/ L$ & Gen. & \\
\hline & 2630 & $\mathrm{ug} / \mathrm{L}$ & Gen. & \\
\hline & 1000 & ug $/ \mathrm{L}$ & Gen & \\
\hline
\end{tabular}


WELL FSB 97D COLLECTED ON 01/03/90 LABORATORY ANALYSES CONTINUED

NICKEL

* NITRATE AS NITROGEN

* LEAD

PHIENOLS

ANTIMONY

SELENIUM

SILICA

SILVEX

SI:LFATE

$1,1,2,2$-TETRACHLOROETHANE

TETRACHLOROETIYLENE

TOTAL DISSOLVED SOLIDS

TIIILLILY

TOTAL ORGANIC CARBON

TOTAL ORGANIC HILOGENS

TOTAL PIIOSPHATES

TRICHLOROETHYLENE

TOXAPIIENE

TRANS-1,2-DICHLOROETHENE

LRANIUM

VANADILM

1,1-DICHLOROETHYLENE

$1,1-D$ ICILLOROETHANE

$1,1,1$-TRI CILOROETHANE

$1,1,2$-TRICILOROETHANE

1,2-D ICIILOROETHINE

1,2-D ICILLOROPROPANE

CIS-1,3-DICIILOROPROPENE

TRANS - 1,3-DICILLOROPROPENE

2-CHLOROETHILVINIL ETIER

2,4-DICILOROPHENOXYACETIC ACID

ZINC

GROSS ALPHA

* NoNíolatile beta

* total radium

* tritilim

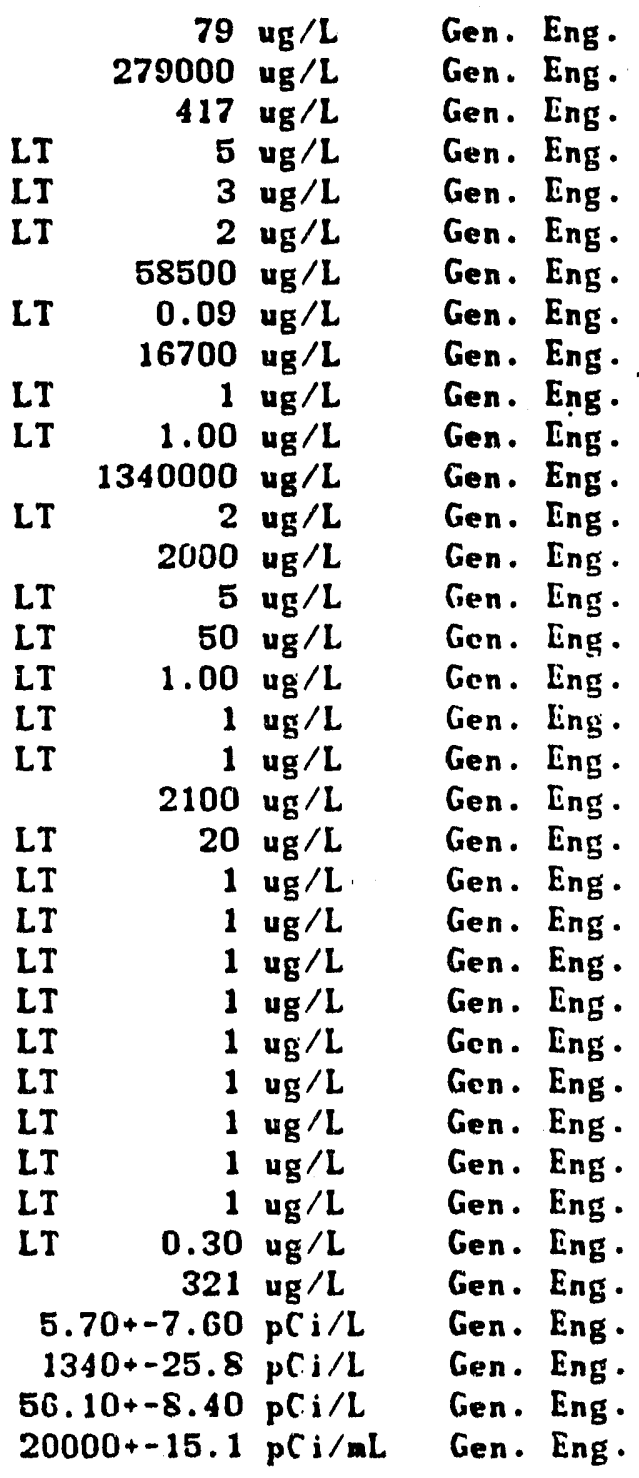


NELL FSB 98A

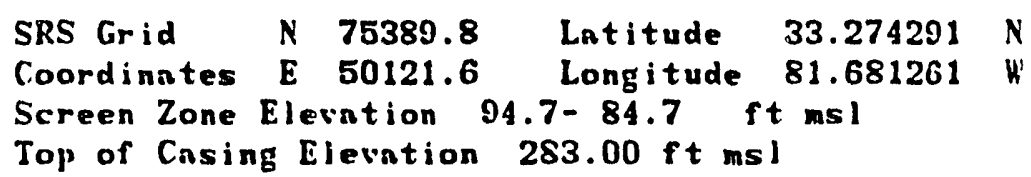

MEASLREMENTS CONDUCTED IN THE FIELD

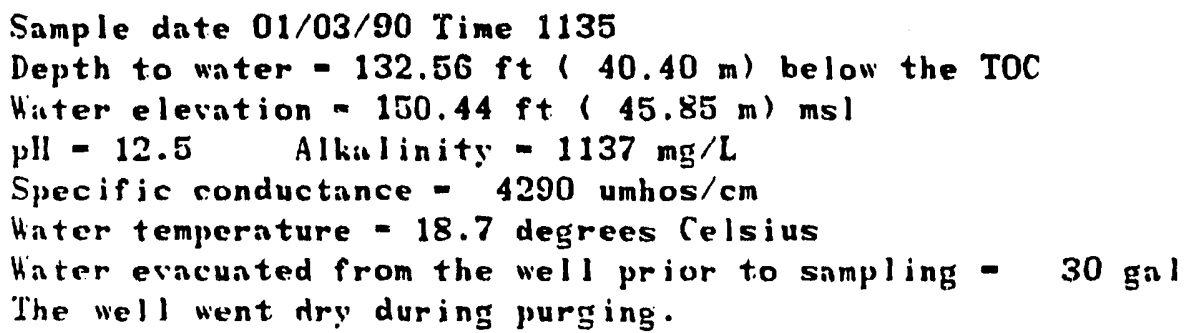

\section{LABORITORY ANALYSES}

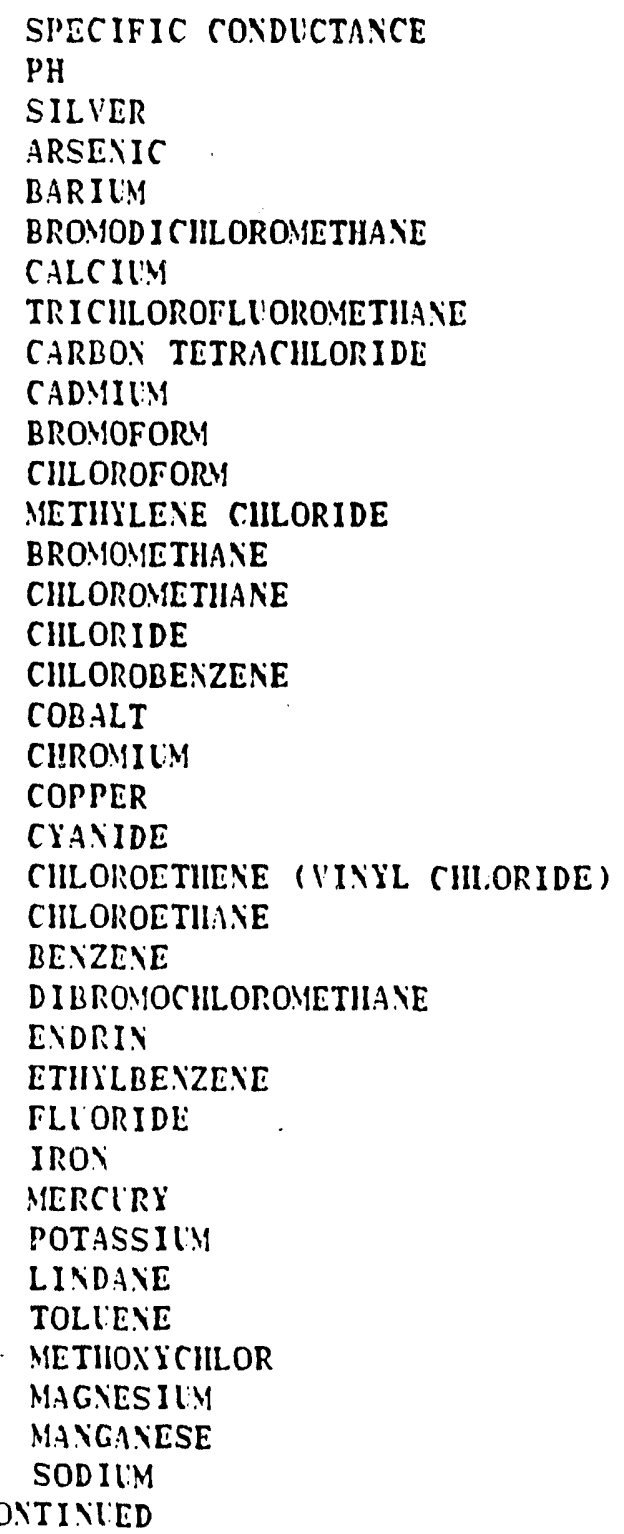

\begin{tabular}{|c|c|c|c|c|}
\hline & 5500 & $\mathrm{umh} / \mathrm{cm}$ & Gen. & Eng. \\
\hline & 12.4 & pll & Gen. & Eng. \\
\hline $\mathrm{LT}$ & 2 & ug /L & Gen. & Eng \\
\hline LT & 2 & ug/L & Gen. & Eng \\
\hline & 573 & $u g / L$ & Gen. & Eng. \\
\hline LT & 1 & $u g / L$ & Gen. & Eng \\
\hline & 442000 & $u g / L$ & Gen. & Eng. \\
\hline LT & 1 & $u g / L$ & Gen. & Eng. \\
\hline LT & 1.00 & ug/L & Gen. & Eng \\
\hline LT & 2 & ug/L & Gen. & Eng \\
\hline L.T & 1 & ug/z & Gen. & Er \\
\hline LT & 1 & $u g / L$ & Gen. & \\
\hline $\mathrm{LT}$ & 1 & ug $/ L$ & Gen. & Eng \\
\hline$L T$ & 1 & $\mathrm{ug} / \mathrm{L}$ & Gen. & Eng \\
\hline LT & 1 & $u g / L$ & Gen. & Eng \\
\hline LT & 1000 & $u g / L$ & Gen. & Eng \\
\hline LT & 1 & $u g / L$ & Gen. & Eng \\
\hline LT & 4 & ug $/ \mathrm{L}$ & Gen. & Eng \\
\hline LT & 4 & $u g / L$ & Gen. & En \\
\hline$L T$ & 4 & ug $/ L$ & Gen. & Eng \\
\hline LI & 5 & ug $/$ L & Gen. & Er \\
\hline LT & 1 & $u g / L$ & Gen. & En \\
\hline LT & 1 & $u_{g} / L$ & Gen. & \\
\hline$L T$ & 1 & $u g / L$ & Gen. & \\
\hline LT & 1 & $u g / L$ & Gen. & \\
\hline LT & 0.10 & ug $/ \mathrm{L}$ & Gen. & $E_{n}$ \\
\hline LT & 1 & ug/L & Cen. & En \\
\hline & 120 & ug / L & Gen. & \\
\hline & 7 & ug/L & Gen. & \\
\hline & 0.70 & $u_{g} / L$ & Gen. & E \\
\hline & 13700 & $u_{g} / L$ & $\operatorname{Gen}$. & \\
\hline LT & 0.05 & ug $/ L$ & Gen. & Er \\
\hline$L T$ & 1 & ug $/ \mathrm{L}$ & Gen. & En \\
\hline L.T & 0.50 & ug / & Gen & En \\
\hline & 10 & $u g / L$ & & En \\
\hline $\mathrm{T}$ & $\begin{array}{r}2 \\
11300\end{array}$ & $\begin{array}{l}u g / L \\
u g / L\end{array}$ & Ger & En \\
\hline
\end{tabular}


WELL FSB 98A COLLECTED ON 01/03/90 LABORATORY ANALYSES CONTINUED

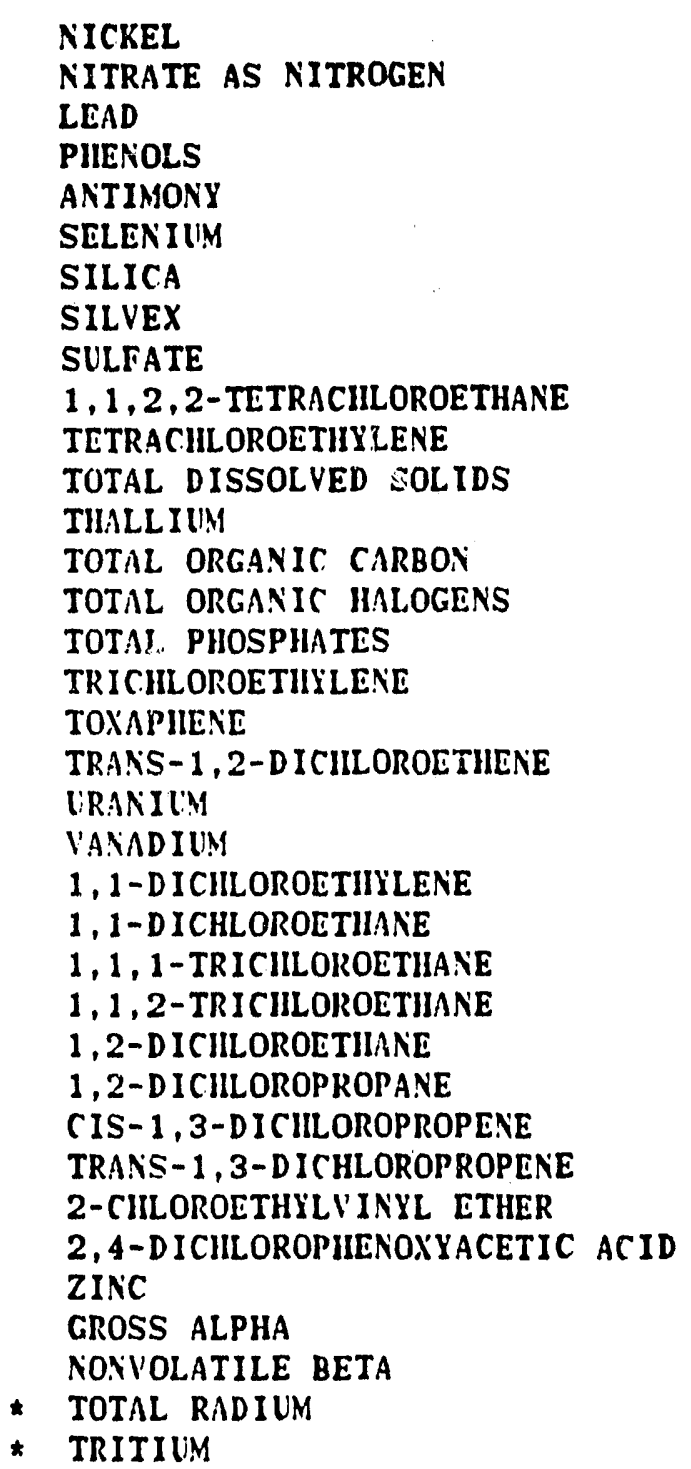

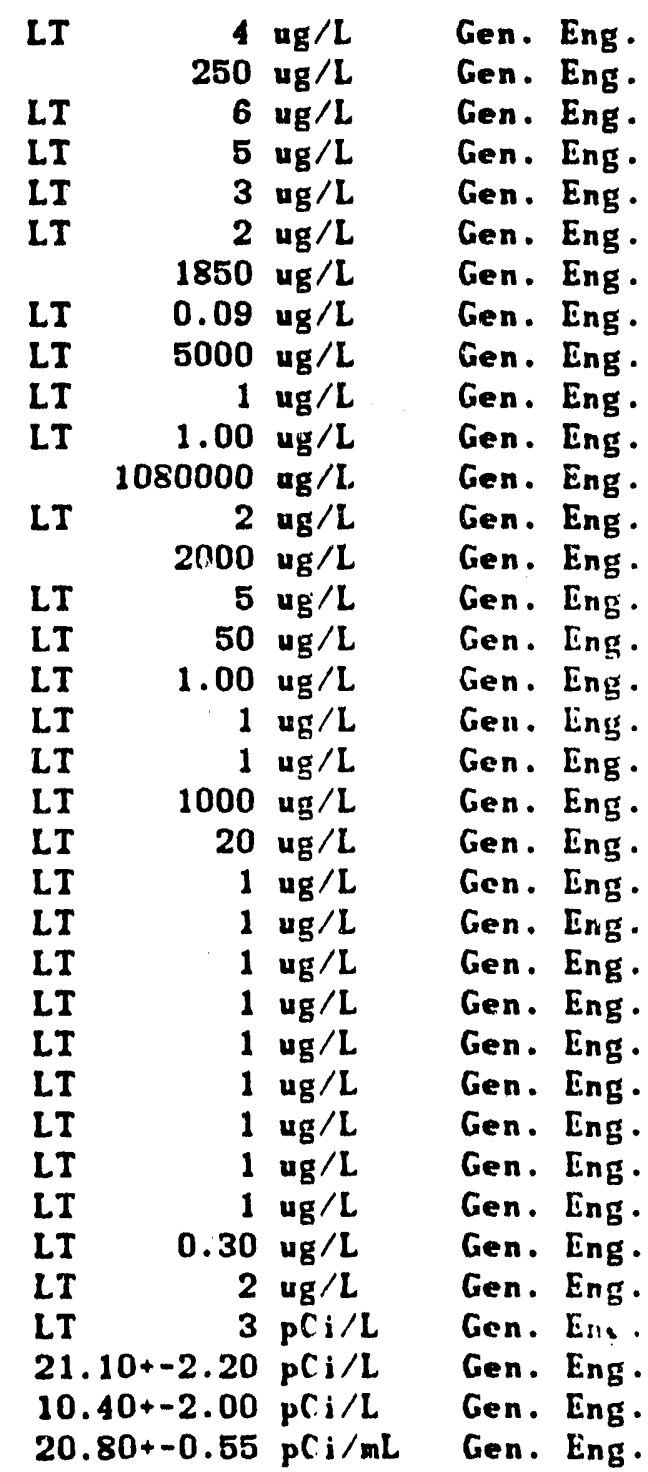


W'ELL FSB 98C

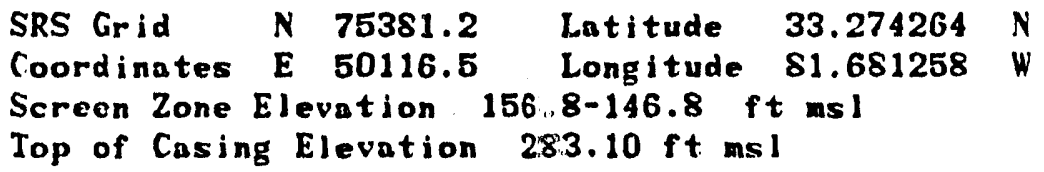

MEASUREMENTS C.ONDUCTED IN THE FIELD

Sample date 01/03/90 Time 1200

Depth to water $=76.45 \mathrm{ft}(23.30 \mathrm{~m})$ below the TOC

Hater elevation $=206.65 \mathrm{ft}(62.99 \mathrm{~m}) \mathrm{ms}$ )

pH - $3.1 \quad$ Alknlinity $=0 \mathrm{mg} / \mathrm{L}$

Specific conductance - $1943 \mathrm{umhos} / \mathrm{cm}$

Hater temperature $=18.7$ degrees Celsius

Hater evacunted from the well prior to sampling - $156 \mathrm{gal}$

LABORATORI ANALYSES

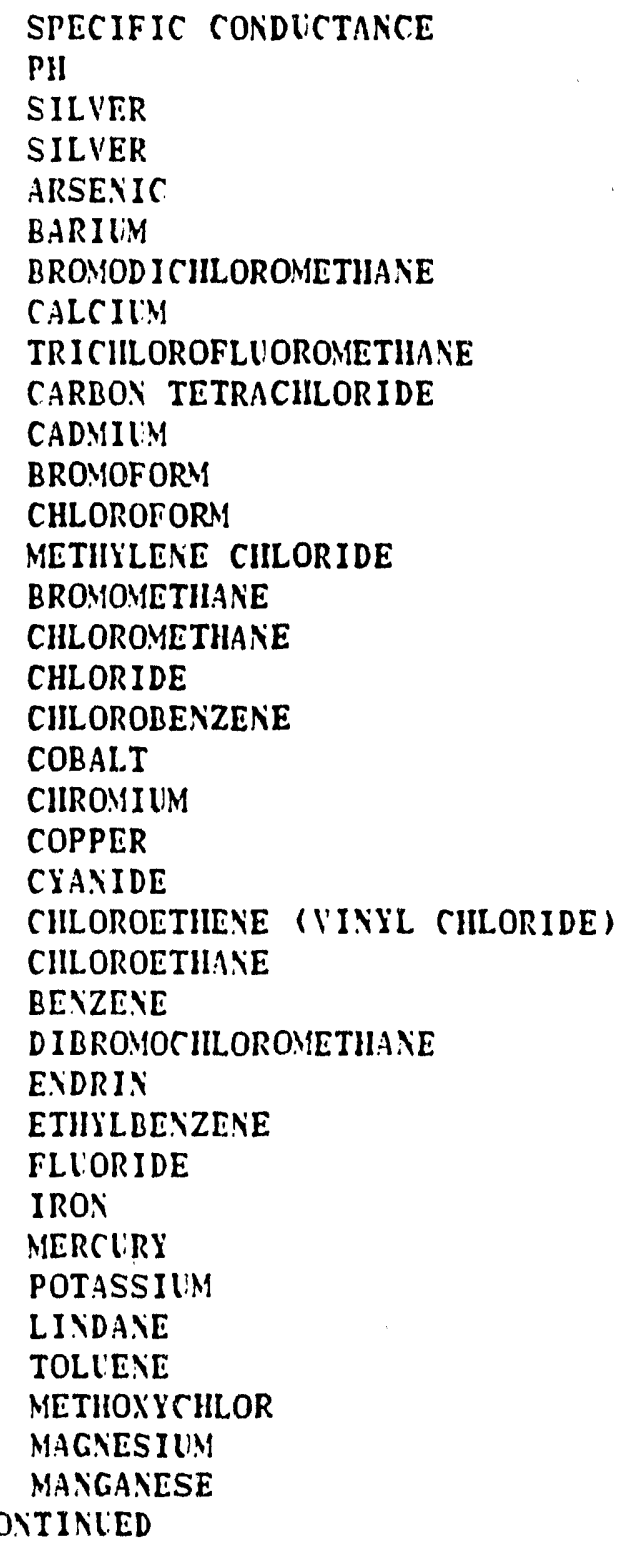

\begin{tabular}{|c|c|c|c|c|}
\hline & $\begin{array}{l}1620 \\
3.35\end{array}$ & $\begin{array}{l}\mathrm{umh} / \mathrm{cm} \\
\mathrm{pH}\end{array}$ & $\begin{array}{l}\text { Gen } \\
\text { Gen. }\end{array}$ & Eng. \\
\hline$L T$ & 2 & ug $/ L$ & Gen. & Eng \\
\hline LT & 2 & $u_{g} / L$ & Gen. & Eng \\
\hline LT & 2 & ug/L & Gen & Eng \\
\hline & 508 & ug / L & Gen & Eng \\
\hline LT & 1 & $\mathbf{u g} / \mathrm{L}$ & Gen & Eng \\
\hline & 10200 & ug/L & Gen & Eng \\
\hline LT & 1 & $u g / L$ & Gen. & Eng \\
\hline LT & $\begin{array}{r}1.00 \\
6\end{array}$ & $\begin{array}{l}u g / L \\
u g / L\end{array}$ & $\begin{array}{l}\text { Gen } \\
\text { Gen }\end{array}$ & $\begin{array}{l}\text { Eng } \\
\text { Eng }\end{array}$ \\
\hline LT & 1 & $u g / L$ & Gen & Eng \\
\hline LT & 1 & ug/L & Gen. & Eng \\
\hline & 4 & $u_{g} / L$ & Gen & En \\
\hline LT & 1 & $u g / L$ & Gen & $E_{n}$ \\
\hline LT & 1 & ug/L & Gen & $\therefore$ \\
\hline & 1900 & $u g / L$ & Gen & Eng \\
\hline LT & 1 & $u_{g} / L$ & Gen & Eng \\
\hline & 281 & ug/L & Gen & Eng \\
\hline LI & 4 & $u_{g} / L$ & Gen & Eng \\
\hline & 88 & $u_{g} / L$ & Gen & Eng \\
\hline LI & 5 & $u_{g} / L$ & Gen & Eng \\
\hline LT & 1 & $u_{g} / L$ & Gen & En \\
\hline$L T$ & 1 & ug $/ L$ & Gen & En \\
\hline $\mathrm{LT}$ & 1 & $u g / L$ & Gen & $E_{n}$ \\
\hline LT & 1 & $u g / L$ & Gen & En \\
\hline LT & 0.10 & $\mathrm{ug} / \mathrm{L}$ & Gen & Er \\
\hline LT & 1 & ug/L & Gen & Er \\
\hline & 1160 & $\mathbf{u g} / \mathrm{L}$ & Ger & \\
\hline & 47 & $\mathrm{ug} / \mathrm{L}$ & Gen & En \\
\hline & 0.20 & ug/L & Ger & $E_{n}$ \\
\hline & 32360 & $u g / L$ & Ger & \\
\hline LT & 0.05 & $\mathrm{ug} / \mathrm{L}$ & Gen & En \\
\hline LT & 1 & $\mathbf{u g} / \mathrm{L}$ & Ge & $E_{r}$ \\
\hline LT & 0.50 & ug $/ L$ & & En \\
\hline & 3700 & ug / I & 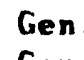 & \\
\hline & 4390 & $\mathbf{\mu g} / L$ & & \\
\hline
\end{tabular}


RELL FSB 98C COLLECTED ON 01/03/90 LABORATORY ANALYSES CONTINUED

SODIUM

NICKEL

* NITRATE AS NITROGEN

LEAD

PIIENOLS

ANTIMONY

SELENIUM

SILICA

SILVEX

SULFATE

1,1,2,2-TETRACIILOROETHANE

TETRACIILOROETHYLENE

TOTAL DISSOLVED SOLIDS

THALLIUM

TOTAL ORGANIC CARBON

TOTAL ORGANIC IIALOGENS

TOTAL PHOSPHATES

TR I CIILOROETIILENE

TOXAPIIENE

TRANS-1,2-DICHLOROETHENE

IRANILM

VANADIUM

1,1-DICHLOROETHYLENE

1,1 -D ICHLOROETIIANE

$1,1,1$-TRICILLOROETHANE

$1,1,2$-TRICIILOROETHANE

1,2-DICHLOROETHANE

1,2-DICHLOROPROPANE

CIS-1,3-DICHLOROPROPENE

TRANS-1, 3-DICIILOROPROPEAE

2-CILOROETHILVINYL ETHER

2,4-DICILOROPHENOXYACETIC ACID ZINC

* GROSS ALPIIA

* Nonvolatile beta

* total radily

* trITIUM

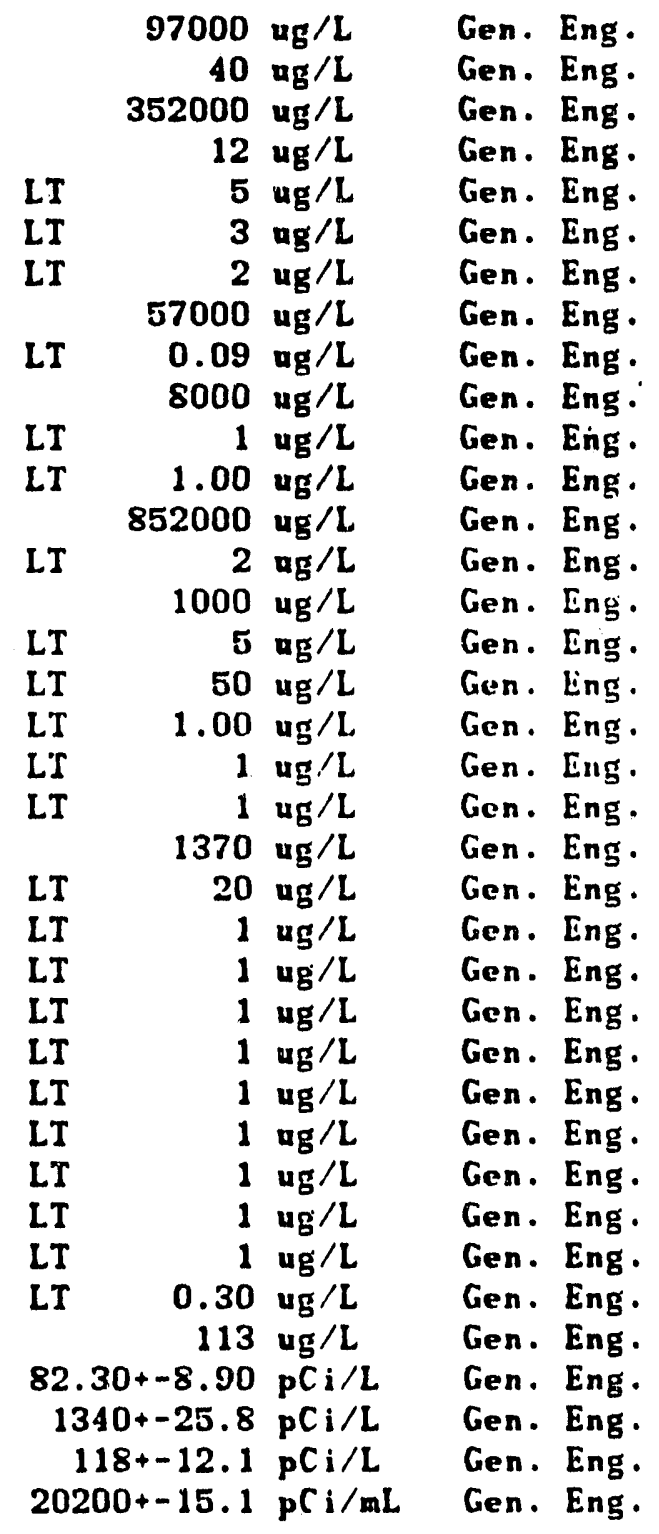


HELL FSB 98D

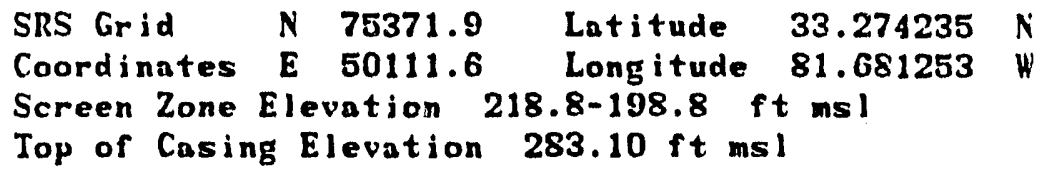

\section{MEASUREMENTS CONDUCTED IN THE FIELD}

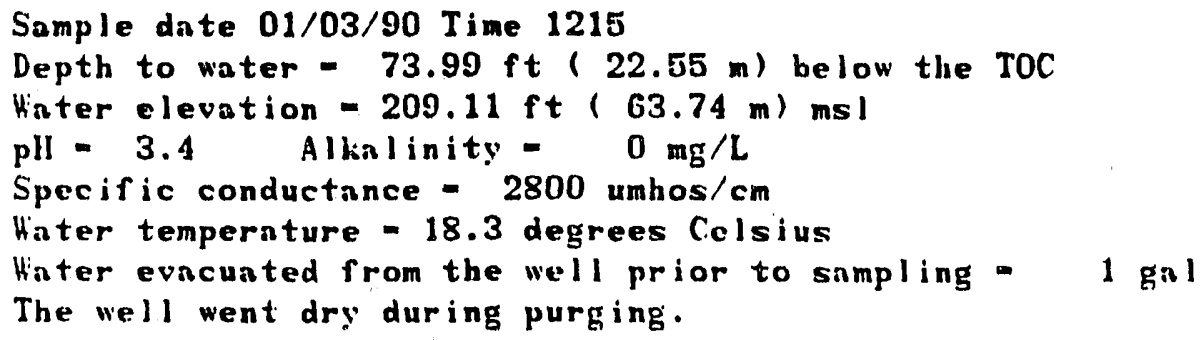

LABORATORY ANALYSES

SPECIFIC CONDUCTANCE

PH

SILVER

ARSEYIC

* baRIUM

BRONODICHLOROMETIIANE

CALCIUM

TRICILLOROFLUOROMETIIANE

CARBOX TETRACILORIDE

CADMIUM

BROMOFORM

CIILOROFORM

METIIYLENE CHLORIDE

BROMONETHANE

CIILORONETHPNE

CILLORIDE

CHLOROBENZENE

COBALT

CHROMIUM

COPPER

CYANIDE

CILOROETIENE (VINIL CILORIDE)

CIILOROETILANE

BE.YZENE

DIBRONOCILLOROMETIIANE

ENDRIN

ETIYLBE.YZENE

FLIORIDE

IRON

MERCURY

POTASSIUM

LINDANE

TOLLENE

METHOXYCIILOR

MAGNESIIM

MANGANESE

SODIUM

CONTINLED

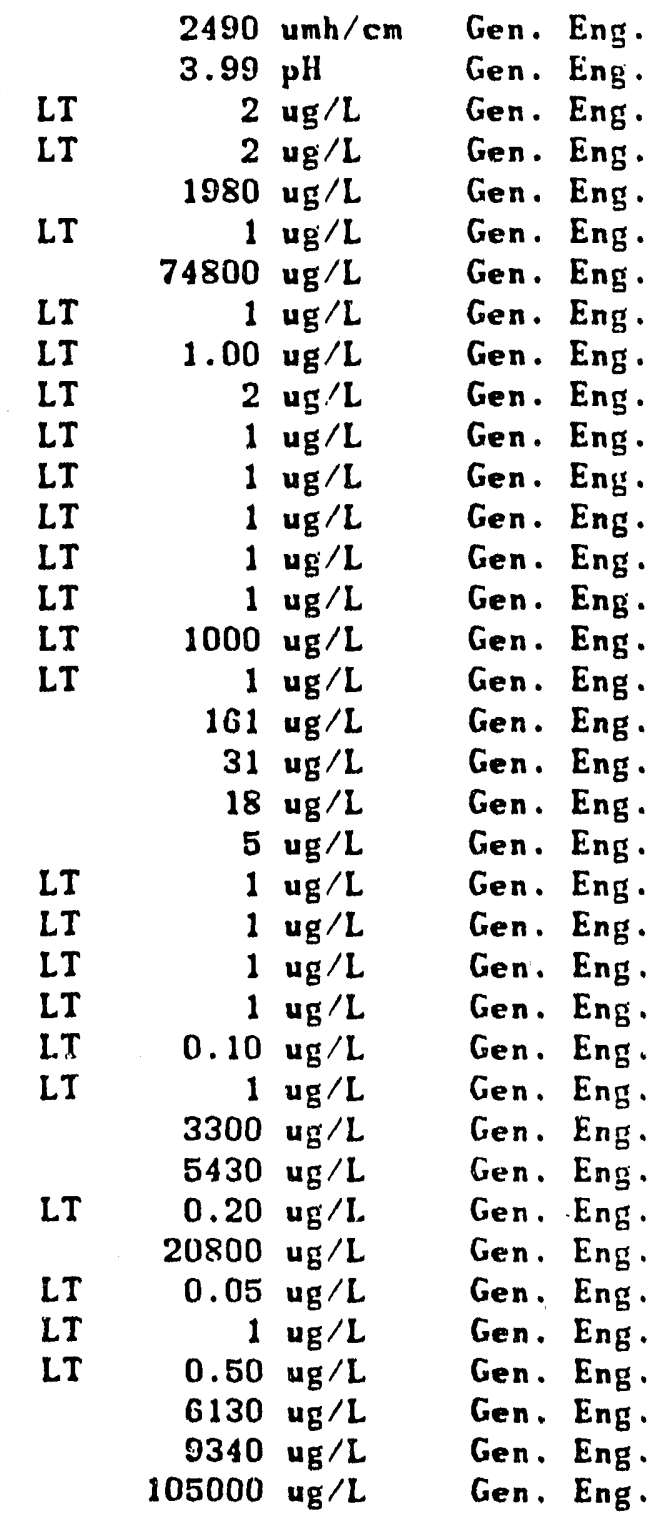


NELL FSB 98D COLLECTED ON 01/03/90 LABORATORY ANALYSES CONTINUED

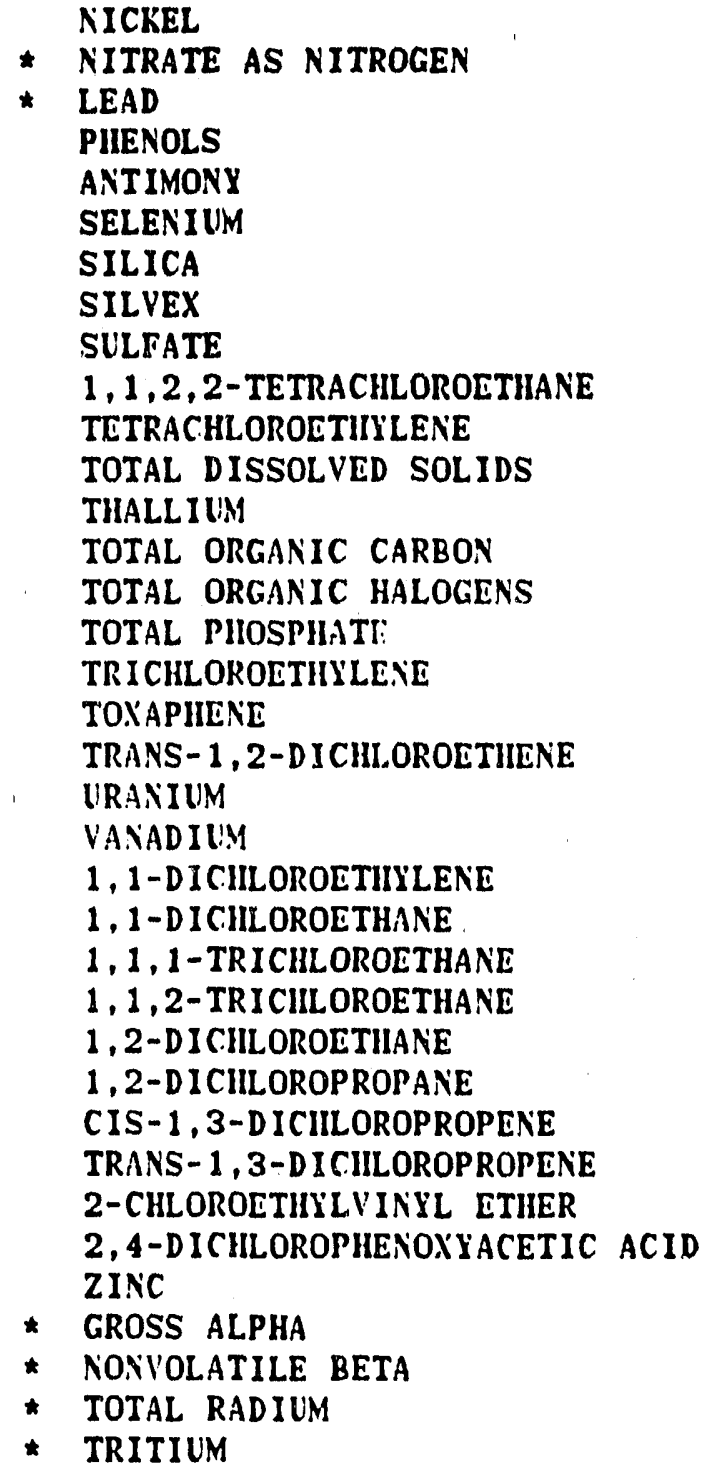

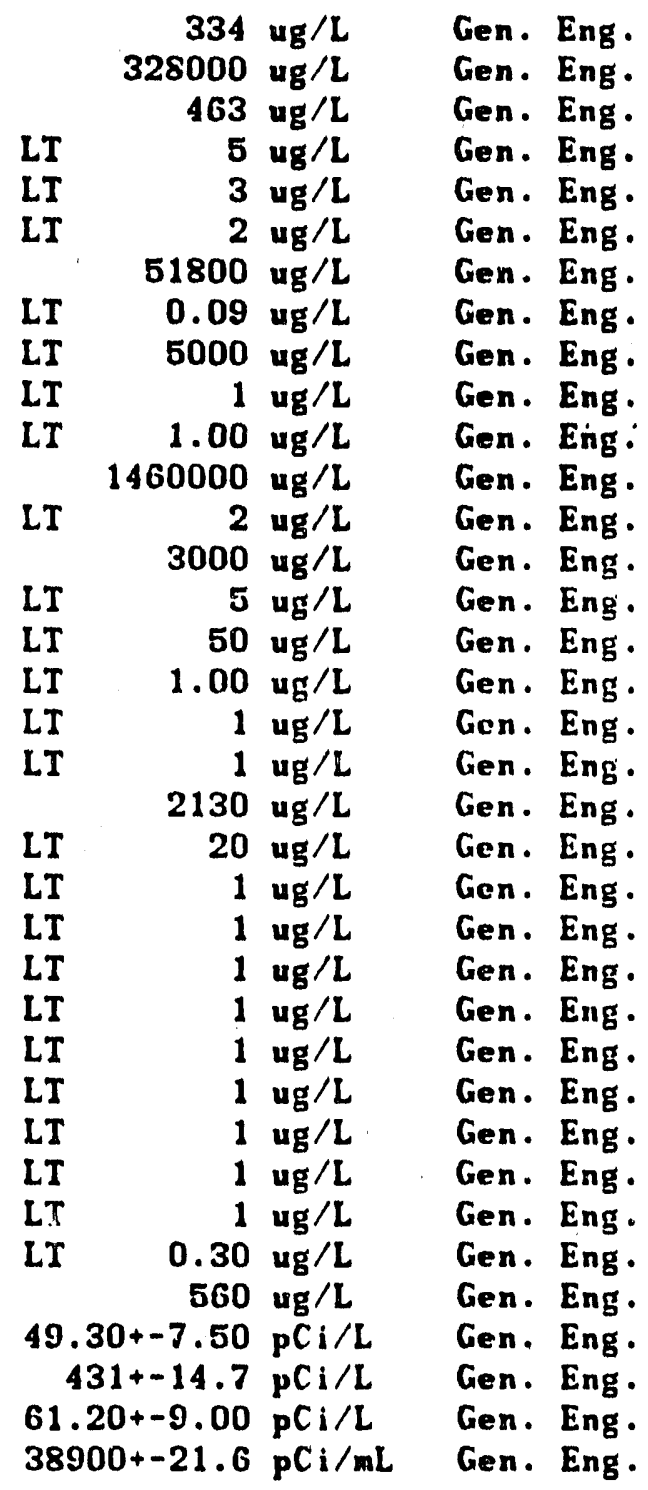


NELL FSB 99A

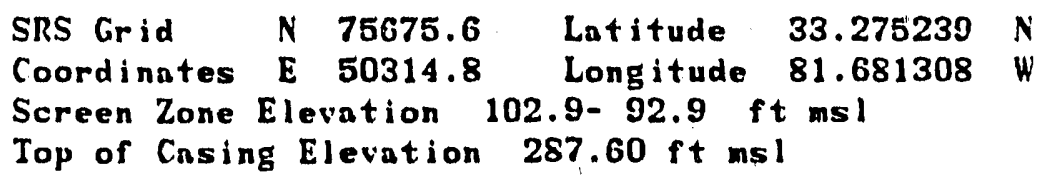

\section{MEASUREMENTS CONDUCTED IN THE FIELD}

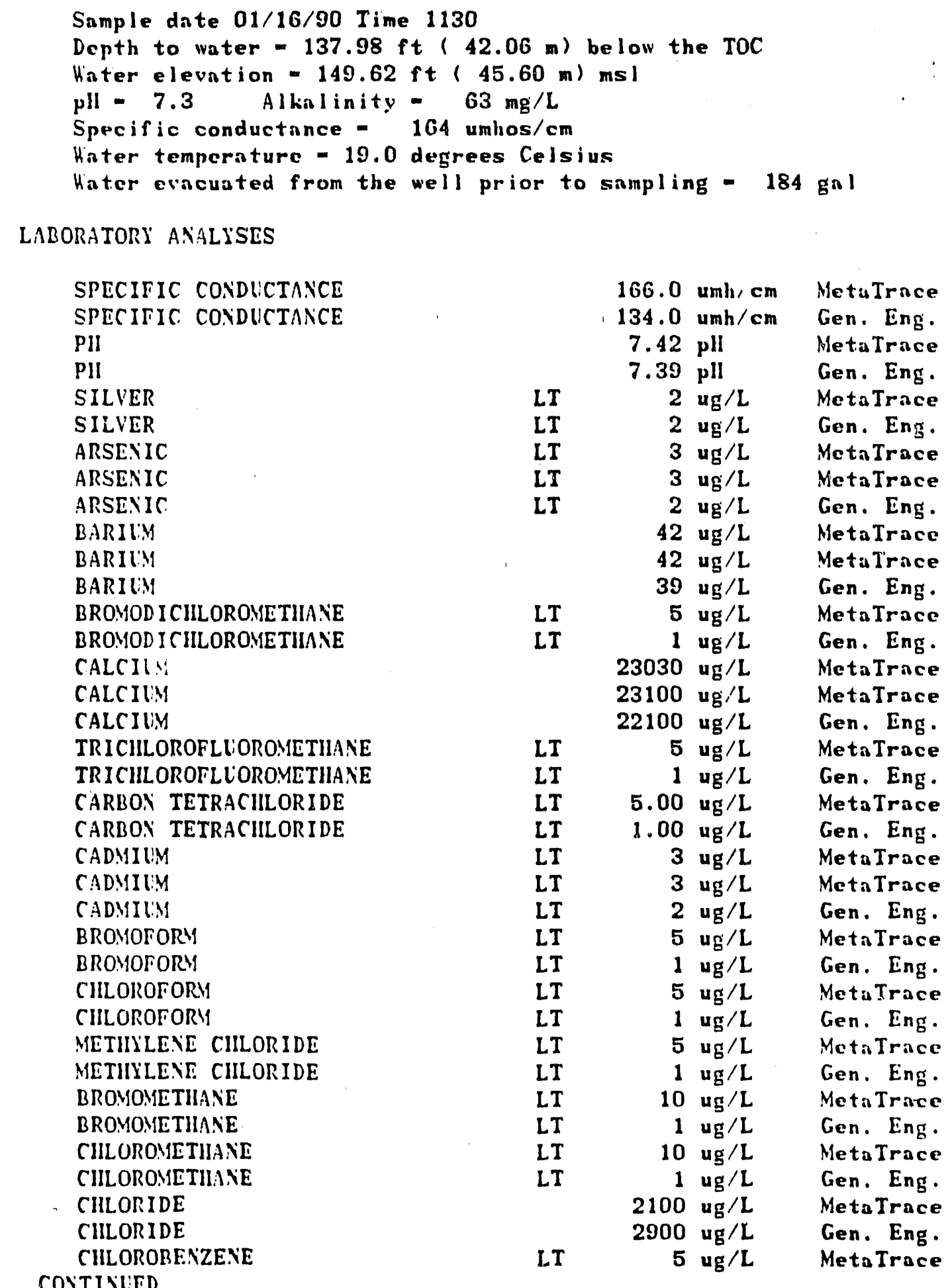


K'ELL FSB 99A COLLECTED ON 01/16/90 LABORATORY ANALYSES CONTINUED

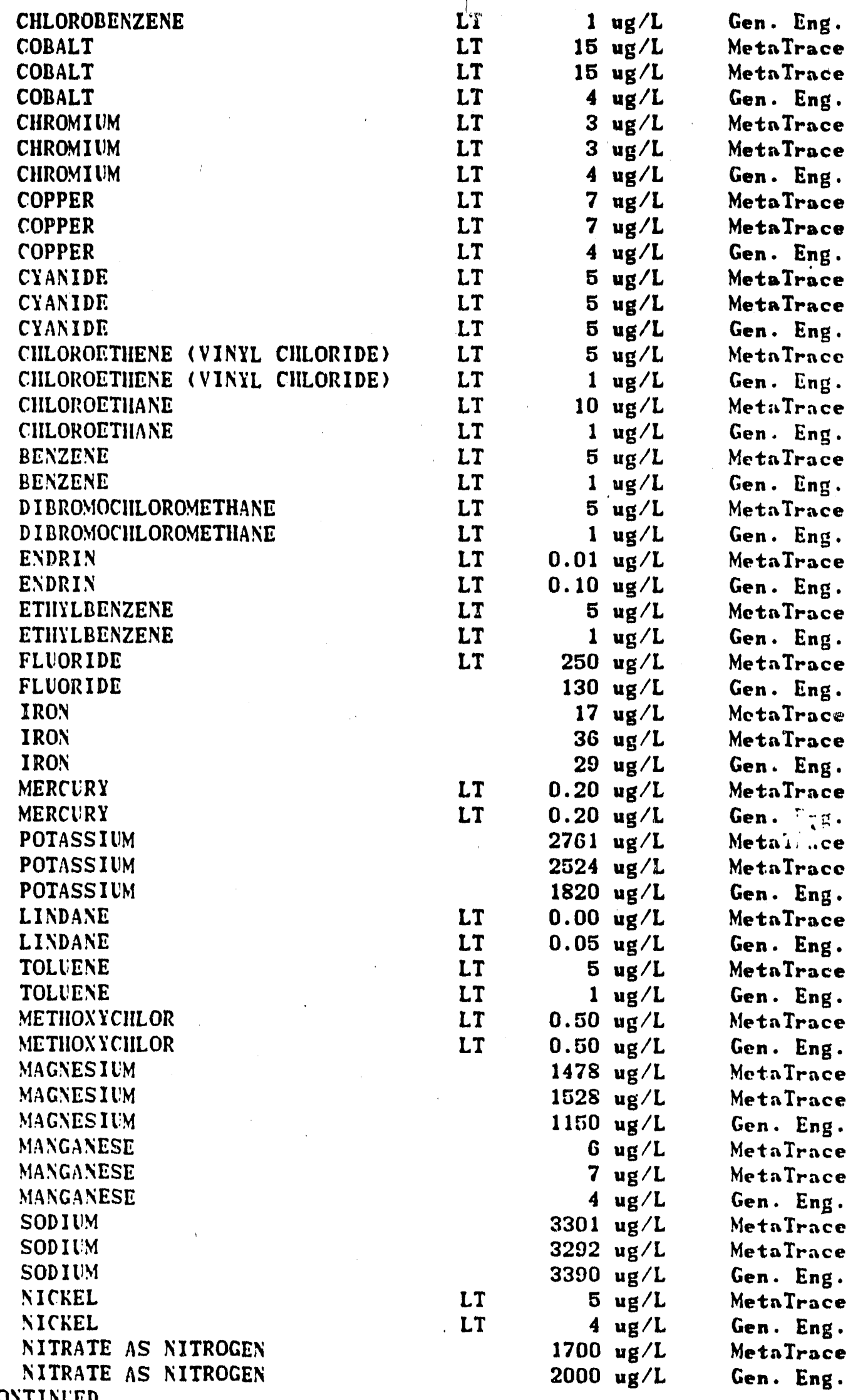


VELL FSB 99A COLLECTED ON 01/16/90 LABORATORY ANALYSES CONTINUED

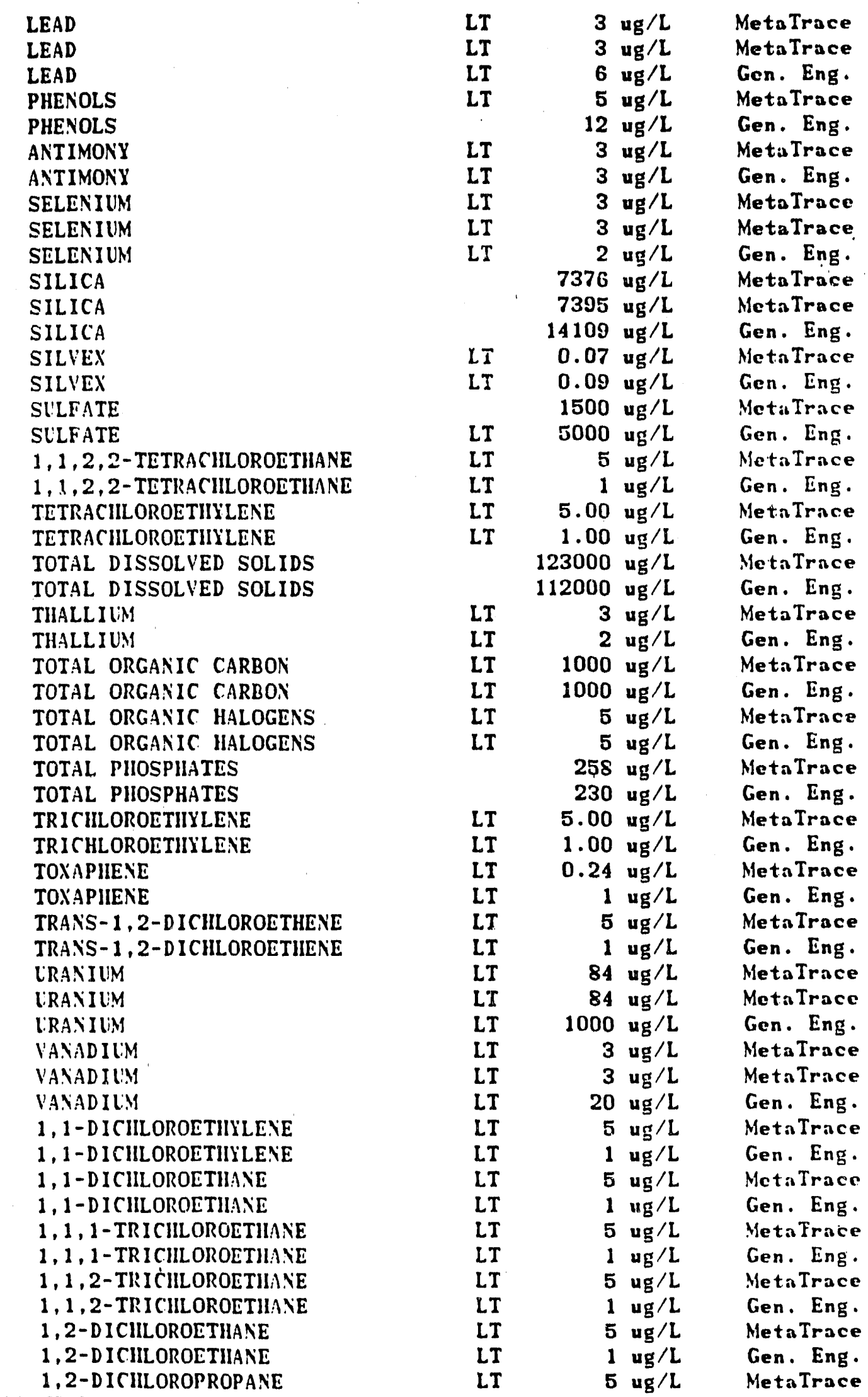


VELL FSB 99A COLLECTED ON 01/16/90 LABORATORY ANALYSES CONTINUED

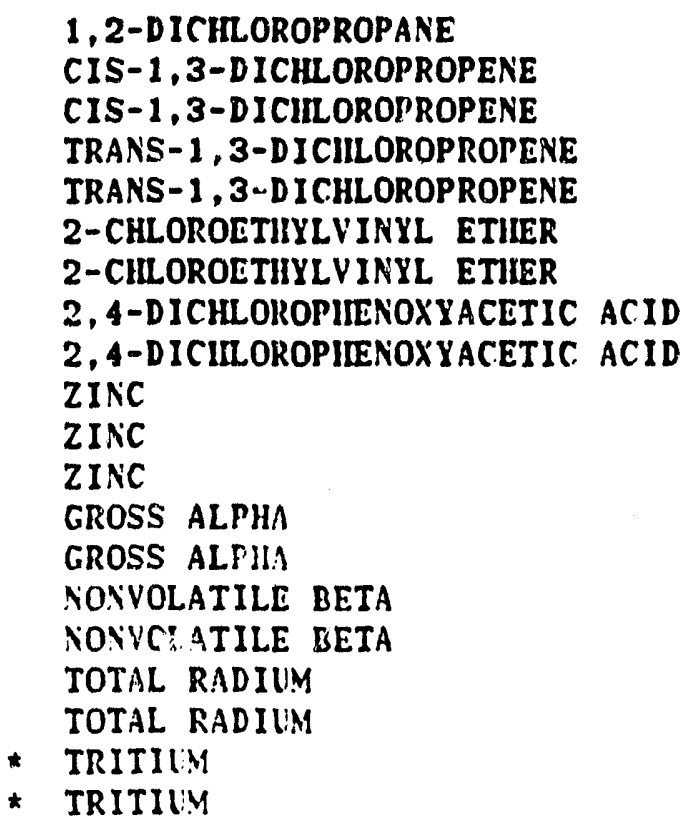

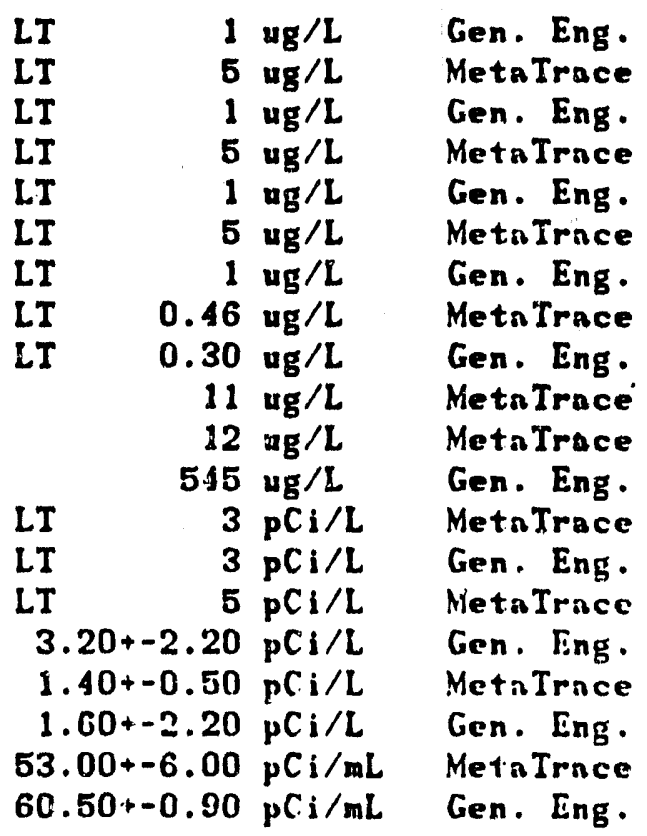

\section{KELL FSB 99A}

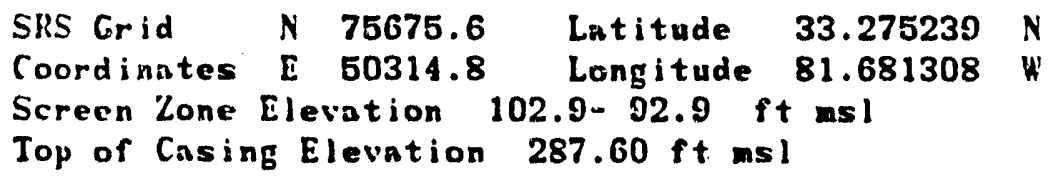

MEASUREMENTS CONDUCTED IN TUE FIELD

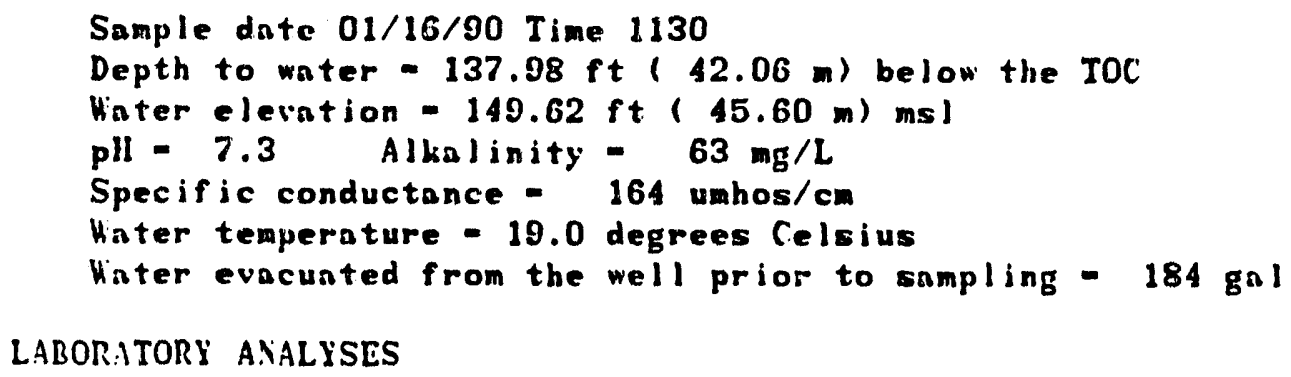

SPECIFIC CONDLCTANCE

SPECIFIC CONDLCTANCE

PII

PII

SILIER

SILVER

ARSENIC

ARSENIC

BARIIIM

BARILIM

BROMOD I CILLOROMETIANE BROMOD ICILLOROMETHANE COITIINED

$\begin{aligned} 145.0 \mathrm{umh} / \mathrm{cm} & \text { MetaTrace } \\ 150.0 \mathrm{umh} / \mathrm{cm} & \text { Gen. Eng. } \\ 7.49 \mathrm{pll} & \text { MetaTrnce } \\ 5.87 \mathrm{pll} & \text { Gen. Eng. } \\ 2 \mathrm{ug} / \mathrm{L} & \text { MetaTrace } \\ 2 \mathrm{ug} / \mathrm{L} & \text { Gen. Eng. } \\ 3 \mathrm{ug} / \mathrm{L} & \text { MetnTrace } \\ 2 \mathrm{ug} / \mathrm{L} & \text { Gen. Eng. } \\ 42 \mathrm{ug} / \mathrm{L} & \text { MetaTrnce } \\ 38 \mathrm{ug} / \mathrm{L} & \text { Gen. Eng. } \\ 5 \mathrm{ug} / \mathrm{L} & \text { MetaTrace } \\ 1 \mathrm{ug} / \mathrm{L} & \text { Gen. Eng. }\end{aligned}$




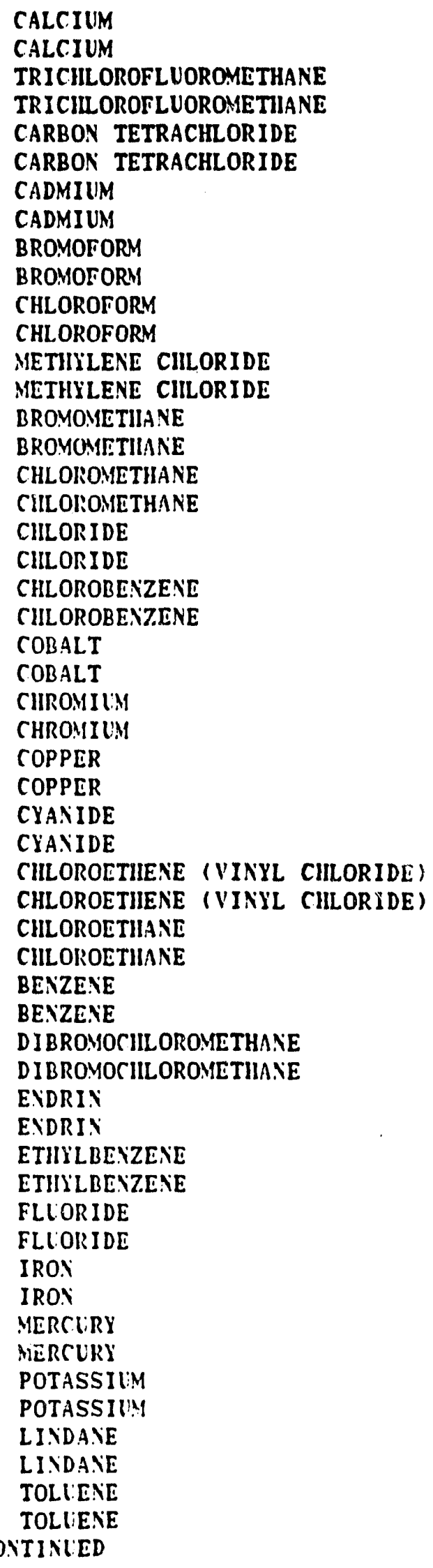

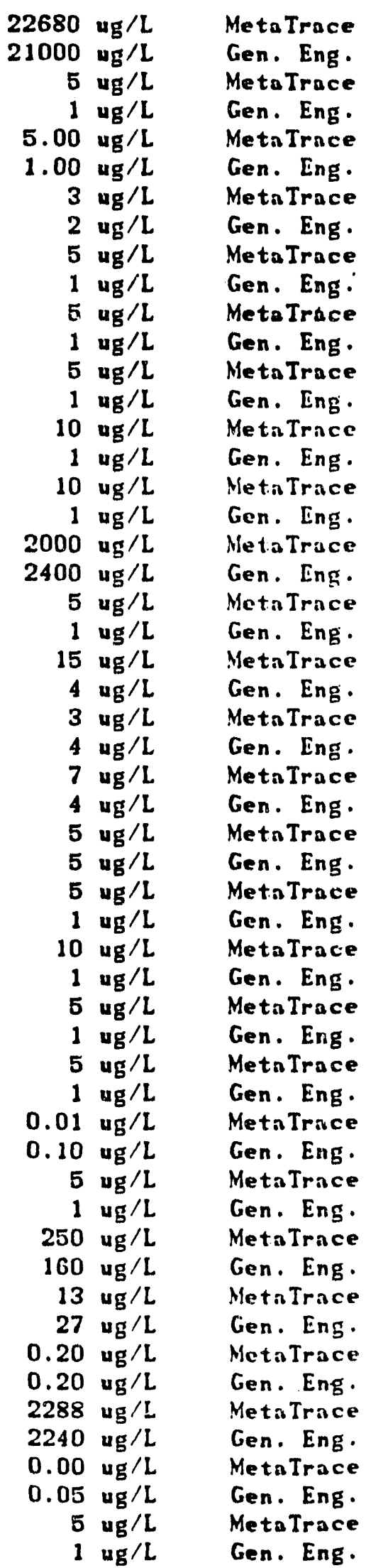


HELL FSB 99A COLLECTED ON 01/16/90 LABORATORY ANALYSES CONTINLED

\begin{tabular}{|c|c|c|c|c|}
\hline METHOXYCILLOR & LI & 0.50 & $\operatorname{ug} / \mathrm{L}$ & MetaTrace \\
\hline METHOXYCILLOR & LT & 0.50 & ug/L & Gen. Eng. \\
\hline MAGNESIUM & & 1588 & ug $/ L$ & MetaTrace \\
\hline MAGNESIUM & & 1320 & $\operatorname{ug} / \mathrm{L}$ & Gen. Eng. \\
\hline MANGANESE & & 6 & ug $/ \mathrm{L}$ & MetaTrnce \\
\hline MANGANESE & & 4 & ug/L & Gen. Eng. \\
\hline SODIUM & & 3527 & ug $/ L$ & MetaTrace \\
\hline SODIUM & & 4010 & $\operatorname{ug} / \mathrm{L}$ & Gen. Eng. \\
\hline NICKEL & LT & 5 & ug/L & MetaTrace \\
\hline NICKEL & LT & 4 & $\operatorname{lgg} / L$ & Gen. Eng: \\
\hline NITRATE AS NITROGEN & & 1740 & ug $/ \mathrm{L}$ & MetaTrace \\
\hline NITRATE AS NITROGEN & & 2200 & $\mathrm{ug} / \mathrm{L}$ & Gen. Eng. \\
\hline L.EAD & LT & 3 & $\mathrm{ug} / \mathrm{L}$ & MetaTrace \\
\hline LRAD & LT & 6 & ug $/ L$ & Gen. Eng. \\
\hline PILENOLS & LT & $\mathbf{5}$ & ug $/ \mathrm{L}$ & MetaTrace \\
\hline PHENOLS & LT & $\mathbf{5}$ & ug $/ \mathrm{L}$ & Gen. Eng. \\
\hline ANTIMONY & LT & 3 & ug $/ \mathrm{L}$ & MetaTrace \\
\hline ANTIMONY & LT & 3 & ug $/ \mathrm{L}$ & Gen. Eng. \\
\hline SEI.ENIUM & LT & 3 & ug $/ \mathrm{L}$ & MetaTrace \\
\hline SELENIUM & LT & 2 & ug $/ \mathrm{L}$ & Gen. Eng. \\
\hline SILICA & & 7450 & ug $/ \mathfrak{L}$ & MetaTrace \\
\hline SILICA & & 16015 & $\operatorname{ug} / \mathrm{L}$ & Gen. Eng. \\
\hline SILVEX & LT & 0.07 & $\operatorname{ug} / \mathrm{L}$ & MetaTrace \\
\hline SILIEX & LI & 0.09 & $\operatorname{ug} / \mathrm{L}$ & Gen. Eng. \\
\hline SI:LFATE & & 1500 & ug $/ \mathrm{L}$ & MetaTrace \\
\hline SILLFATE & LT & 5000 & $\operatorname{ug} / \mathrm{L}$ & Gen. Eng. \\
\hline $1,1,2,2$-TETRACILOROETIIANE & LT & $\mathbf{5}$ & ug $/ \mathrm{L}$ & MetaTrace \\
\hline $1,1,2,2$-TETRACHLOROETHANE & LT & 1 & ug $/ \mathrm{L}$ & Gen. Eng. \\
\hline TETRACHLOROETIYLENE & LT & 5.00 & $\operatorname{ug} / \mathrm{L}$ & MetaTrace \\
\hline TETRACILLOROETHYLENE & LI & 1.00 & ug $/ \mathrm{L}$ & Gen. Eng. \\
\hline TOTAL DISSOLVED SOLIDS & & 108000 & $4 \mathrm{~g} / \mathrm{L}$ & MetaTrace \\
\hline TOTAL DISSOLVED SOLIDS & & 115000 & $\mathrm{ug} / \mathrm{L}$ & Gen. Eng. \\
\hline THALLIVM & LT & 3 & $\operatorname{ug} / \mathrm{L}$ & MetaTrace \\
\hline TIIALLIUM & LT & 2 & ug $/ \mathrm{L}$ & Gen. Eng. \\
\hline TOTAL ORGANIC CARBON & LT & 1000 & $u g / L$ & MetaTrace \\
\hline TOTAL ORGANIC CARBON & LT & 1000 & ug $/ \mathrm{L}$ & Gen. Eng. \\
\hline TOTAL ORGANIC HALOGENS & LT & 5 & $4 \mathrm{~g} / \mathrm{L}$ & MetaTrace \\
\hline TOTAL ORGANIC HALOGENS & LI & 5 & $\mu g / L$ & Gen. Eng. \\
\hline TOTAL PHOSPHIATES & & 262 & $u g / L$ & MetaTrace \\
\hline TOTAL PHOSPIIATES & & 247 & ug/L & MetaTrace \\
\hline TOTAL PIIOSPIIATES & & 210 & ug $/ \mathrm{L}$ & Gen. Eng. \\
\hline TRICIILOROETIIILENE & LT & 5.00 & ug $/ \mathrm{L}$ & MetaTrace \\
\hline TRICHLOROETIILLENE & LT & 1.00 & $\mathrm{ug} / \mathrm{L}$ & Gen. Eng. \\
\hline TOXAPIIENE & LT & 0.24 & ug/L & MetaTrace \\
\hline TOX:APIIENE & LT & 1 & ug $/ \mathrm{L}$ & Gen. Eng. \\
\hline TRANS-1,2-DICHLOROETUENE & LT & $\mathbf{5}$ & $\mathrm{ug} / \mathrm{L}$ & MetaTrace \\
\hline TRATS-1,2-DICHLOROETIIENE & LT & 1 & ug $/ \mathrm{L}$ & Gen. Eng. \\
\hline LRANILM & LIT & 84 & ug $/ \mathrm{L}$ & MetaTrice \\
\hline IRANIUM & LT & 1000 & $\mathrm{ug} / \mathrm{L}$ & Gen. Eng. \\
\hline VANADILY & LT & 3 & $\mathrm{ug} / \mathrm{L}$ & MetaTrace \\
\hline V'ANADILM & LT & 20 & ug $/ \mathrm{I}$. & Gen. Eng. \\
\hline 1,1-DICIILOROETIILLENE & LT & 5 & $\operatorname{ugg} / \mathrm{L}$ & MetaTrnce \\
\hline 1,1-DICILLROETIILENE & LT & 1 & ug $/ \mathrm{L}$ & Gen. Eng. \\
\hline 1,1-DICHLOROETHANE & LT & 5 & $u g / L$ & MetaTrice \\
\hline
\end{tabular}


HELL FSB 99A COLLECTED ON 01/16/90 LABORATORY ANALYSES CONTINUED

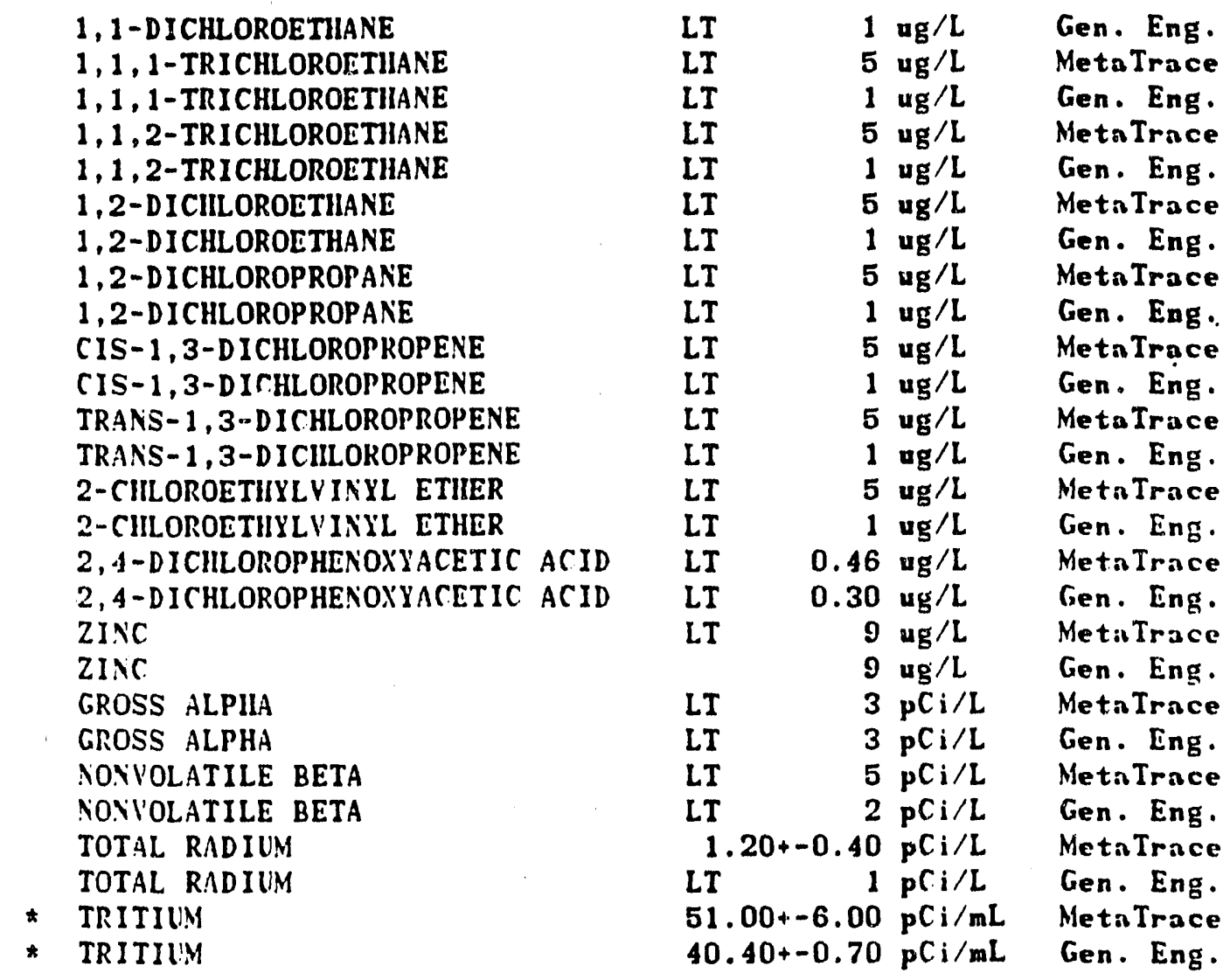

HELL FSB 99C

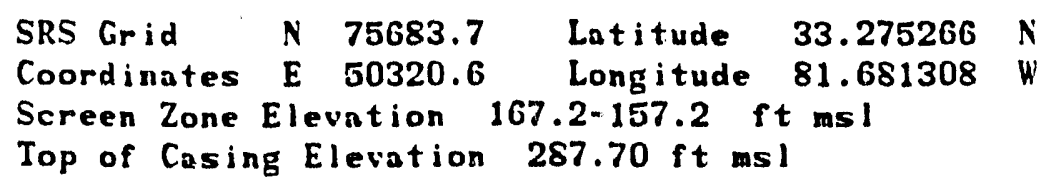

LABORATORY ANMLYSES

SPECIFIC CONDLCTANCE

$\mathrm{PH}$

SILIER

ARSENIC

BARIUM

CONTINIED

$\begin{array}{rrll} & 288.0 \mathrm{umh} / \mathrm{cm} & \text { Gen. Eng. } \\ & 5.81 \mathrm{pH} & \text { Gen. Eng. } \\ \text { LT } & 2 \mathrm{ug} / \mathrm{L} & \text { Gen. Eng. } \\ \text { LT } & 2 \mathrm{ug} / \mathrm{L} & \text { Gen. Eng. } \\ & 84 & \mathrm{ug} / \mathrm{L} & \text { Gen. Eng. }\end{array}$


KELL FSB 99C COLLECTED ON 01/09/90 LABORATORY ANALYSES CONTINUED

\begin{tabular}{|c|c|c|c|c|c|}
\hline $\begin{array}{l}\text { BROMODICHLOROMETHANE } \\
\text { CALCIUM }\end{array}$ & LT & $\begin{array}{r}1 \\
14600\end{array}$ & $\begin{array}{l}u g / L \\
\text { ug } / \mathrm{L}\end{array}$ & $\begin{array}{l}\text { Gen. } \\
\text { Gen. }\end{array}$ & Eng. \\
\hline TRICHLOROFLUOROMETUANE & LT & 1 & $\mathrm{ug} / \mathrm{L}$ & Gen. & Eng. \\
\hline CARBON TETRACILORIDE & LI & 1.00 & ug $/ \mathrm{L}$ & Gen. & Eng. \\
\hline CADMIUM & LT & 2 & $\mathrm{ug} / \mathrm{L}$ & Gen. & Eng. \\
\hline BROMOFORM & LT & 1 & $u g / L$ & Gen. & Eng. \\
\hline CIILOROFORM & LT & 1 & ug $/ \mathrm{L}$ & Gen. & Eng. \\
\hline METIIYLENE CHLORIDE & & 6 & ug $/ \mathrm{L}$ & Gen. & Eng. \\
\hline BROMOMETHANE & LT & 1 & ug $/ L$ & Gen. & Eng. \\
\hline CHLORONETHIANE & LT & 1 & $u g / L$ & Gen. & Eng. \\
\hline CILLORIDE & & 3300 & $\mathrm{ug} / \mathrm{L}$ & Gen. & Eng. \\
\hline CIIL.OROBENZENE & LT & 1 & ug/L & Gen. & Eng. \\
\hline COBALT & & 4 & ug/L & Gen. & Eng. \\
\hline CIIROMIUM & LT & 4 & ug $/ \mathrm{L}$ & Gen. & Eng. \\
\hline COPPER & LT & 4 & ug/L & Gen. & Eng. \\
\hline CYANIDE & LT & 5 & ug/L & Gen. & Eng. \\
\hline CIII.OROETHENE (VINYL CILLORIDE) & LT & 1 & ug $/$ L & Gen. & Eng. \\
\hline CIILOROETHANE & LT & 1 & $\mathrm{ug} / \mathrm{L}$ & Gen. & Eng. \\
\hline BENZENE & LT & 1 & ug/L & Gen. & Eng. \\
\hline DIBROMOCHLOROMETHANE & LT & 1 & ug/L & Gen. & Eng. \\
\hline ENDRIN & LT & 0.10 & ug/L & Gen. & Eng. \\
\hline ETHYLBENZENE & LT & 1 & ug $/ \mathrm{L}$ & Gen. & Eng. \\
\hline FLUORIDE & $\mathbf{L T}$ & 100 & ug $/ L$ & Gen. & Eng. \\
\hline IRON & & 15 & ug $/ \mathrm{L}$ & Gen. & Eng. \\
\hline MERCURY & LT & 0.20 & ug/L & Gen. & Eng. \\
\hline POTASSIUM & & 1600 & ug/L & Gen. & Eng. \\
\hline LINDANE & LI & 0.05 & ug $/ \mathrm{L}$ & Gen。 & Eng. \\
\hline TOLLENE & LT & 1 & ug $/ L$ & Gen. & Eng. \\
\hline METHOXYCHLOR & LT & 0.50 & ug/L & Gen. & Eng. \\
\hline MAGNESIUM & & 8740 & $u g / L$ & Gen. & Eng. \\
\hline MANGANESE & & 82 & $\mathrm{ug} / \mathrm{L}$ & Gen. & Eng. \\
\hline SODIUM & & 23400 & ug $/ L$ & Gen. & Eng. \\
\hline NICKEL & LI & 4 & $u g / L$ & Gen. & Eng. \\
\hline NITRATE AS NITROGEN & & 29100 & ug $/ L$ & Gen. & Eng. \\
\hline LEAD & LT & 6 & $\mathrm{ug} / \mathrm{L}$ & Gen. & Eng. \\
\hline PHENOLS & LI & 5 & $\mathrm{ug} / \mathrm{L}$ & Gen. & Eng. \\
\hline ANTIMONY & LT & 3 & $\mathrm{ug} / \mathrm{L}$ & Gen. & Eng. \\
\hline SELENIUM & LT & 2 & ug $/ \mathrm{L}$ & Gen. & Eng. \\
\hline SILICA & & 9260 & $u g / L$ & Gen. & Eng. \\
\hline SILVEX & LT & 0.09 & ug $/ L$ & Gen. & Eng. \\
\hline SI'LFATE & LT & 5000 & $\mathrm{ug} / \mathrm{L}$ & Gen. & Eng. \\
\hline $1,1,2,2$-TETRACHLOROETHANE & LT & 1 & $\mathrm{ug} / \mathrm{L}$ & Gen. & Eng. \\
\hline TETRACHLOROETHILENE & LT & 1.00 & $u g / L$ & Gen. & Eng. \\
\hline TOTAL DISSOLVED SOLIDS & & 217000 & $\operatorname{ug} / \mathrm{L}$ & Gen. & Eng. \\
\hline THILLIIM & LT & 2 & ug $/ L$ & Gen. & Eng. \\
\hline TOTAL ORGANIC CARBON & & 2000 & ug/L & Gen. & En \\
\hline TOTAL ORGANIC IIALOGENS & LT & 5 & $u g / L$ & Gen. & Eng. \\
\hline TOTAL PHOSPHATES & LT & 50 & ug $/ \mathrm{L}$ & Gen. & Eng. \\
\hline TRICHLOROETHLLENE & & 2.00 & $\operatorname{ug} / \mathrm{L}$ & Gen. & Fnn \\
\hline TOXAPHENE & LT & 1 & $-g / L$ & Gen. & En \\
\hline TRANS-1,2-DICHLOROETIENE & LI & 1 & $u_{g} / L$ & Gen. & \\
\hline liRANILM & LT & 1000 & $\operatorname{ug} / \mathrm{L}$ & Gen. & Eng. \\
\hline VANADIUM & LT & 20 & $\operatorname{ug} / \mathrm{L}$ & Gen. & En \\
\hline 1,1-DICHLOROETHILENE & LT & 1 & $u g / L$ & Gen. & En \\
\hline
\end{tabular}


HELL FSB 99C COLLECTED ON 01/09/90 LABORATORY ANALYSES CONTINUED

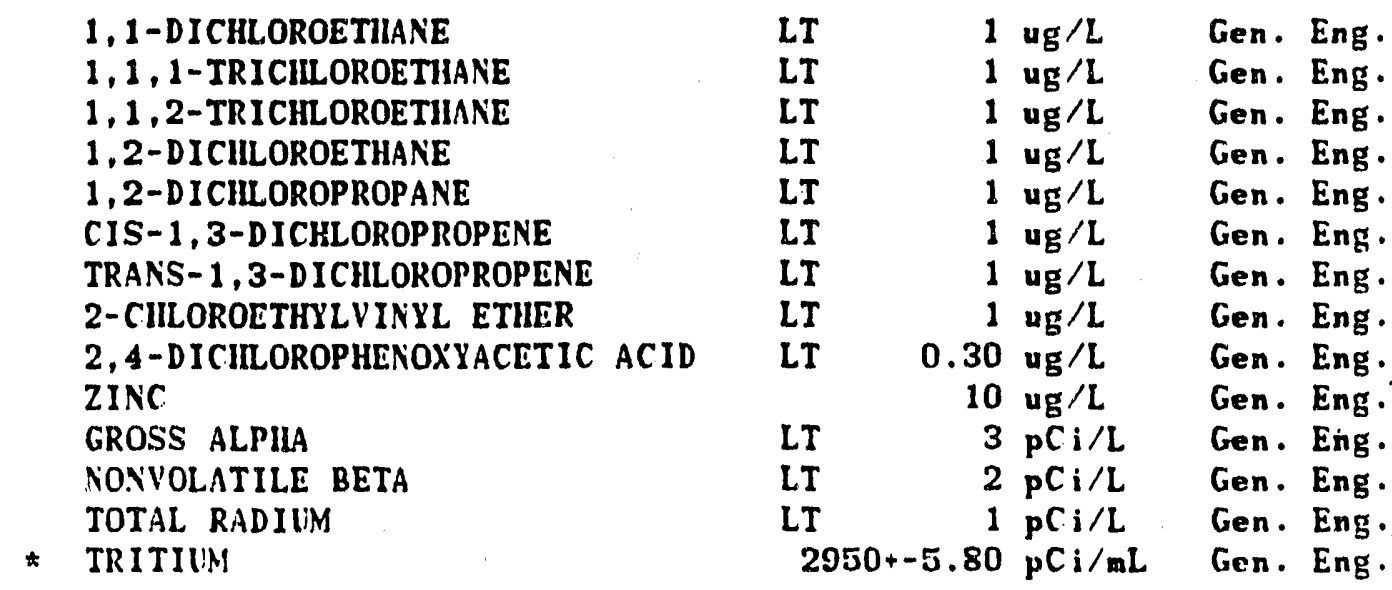

HFI.L FSB 99D

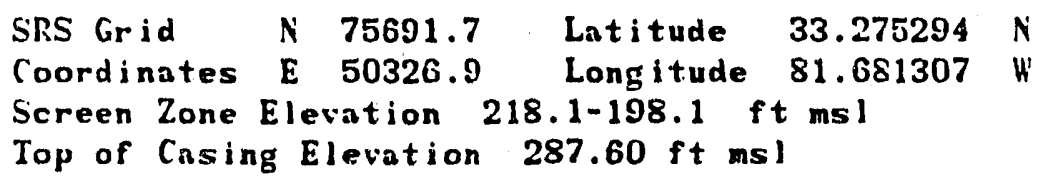

MEASUREMENTS CONDUCTED IN THE FIELD

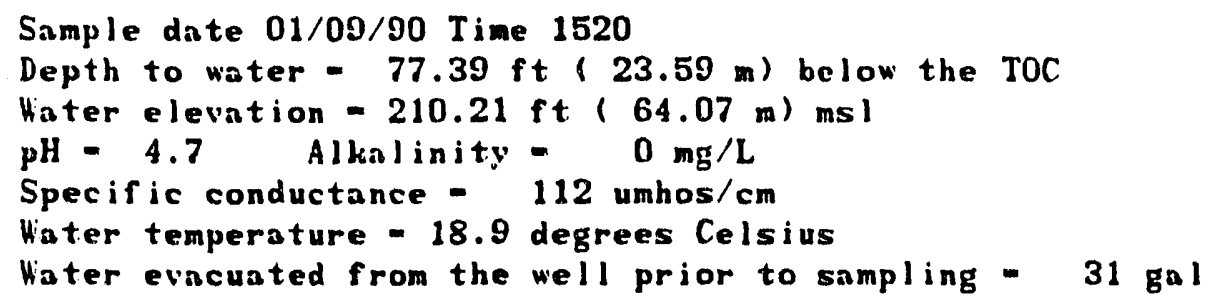

SPECIFIC CONDUCTANCE

$\mathrm{PH}$

SILVER

ARSENIC

BARIUM

BROMOD ICILLOROMETHANE

CALCIIM

TRICIILOROFLLOROMETIIANE

CARBON TETRACILORIDE

CADMILM

BROMOFORY

CHLOROFORM

METIILENE CHLORIDE

BROMOMETIIANE

CIILOROMETHANE

CIILORIDE

CIILOROBENZENE

COBALT

CONTINLED

$\begin{array}{lrll} & 47.00 \mathrm{umh} / \mathrm{cm} & \text { Gen. Eng. } \\ \text { LT } & 4.51 \mathrm{pH} & \text { Gen. Eng. } \\ \text { LT } & 2 \mathrm{ug} / \mathrm{L} & \text { Gen. Eng. } \\ & 2 \mathrm{ug} / \mathrm{L} & \text { Gen. Eng. } \\ \text { LT } & 12 \mathrm{ug} / \mathrm{L} & \text { Gen. Eng. } \\ & 1 \mathrm{ug} / \mathrm{L} & \text { Gen. Eng. } \\ \text { LT } & 1750 \mathrm{ug} / \mathrm{L} & \text { Gen. Eng. } \\ \text { LT } & 1 \mathrm{ug} / \mathrm{L} & \text { Gen. Eng. } \\ \text { LT } & 1.00 \mathrm{ug} / \mathrm{L} & \text { Gen. Eng. } \\ \text { LT } & 2 \mathrm{ug} / \mathrm{L} & \text { Gen. Eng. } \\ \text { LT } & 1 \mathrm{ug} / \mathrm{L} & \text { Gen. Eng. } \\ \text { LT } & 1 \mathrm{ug} / \mathrm{L} & \text { Gen. Eng. } \\ \text { LT } & 1 \mathrm{ug} / \mathrm{L} & \text { Gen. Eng. } \\ \text { LT } & 1 \mathrm{ug} / \mathrm{L} & \text { Gen. Eng. } \\ & 1 \mathrm{ug} / \mathrm{L} & \text { Gen. Eng. } \\ \text { LT } & 1800 \mathrm{ug} / \mathrm{L} & \text { Gen. Eng. } \\ \text { LT } & 1 \mathrm{ug} / \mathrm{L} & \text { Gen. Eng. } \\ & 4 \mathrm{ug} / \mathrm{L} & \text { Gen. Eng. }\end{array}$


WELL FSB 99D COLLECTED ON 01/09/90 LABORATORY ANALYSES CONTINUED

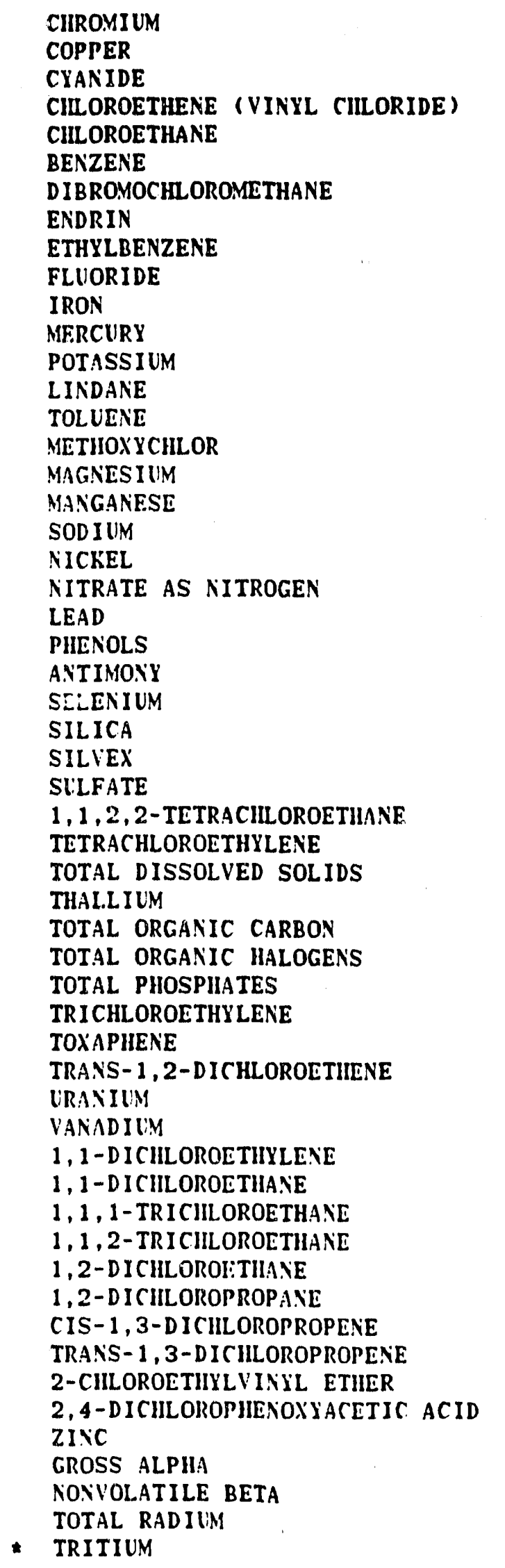

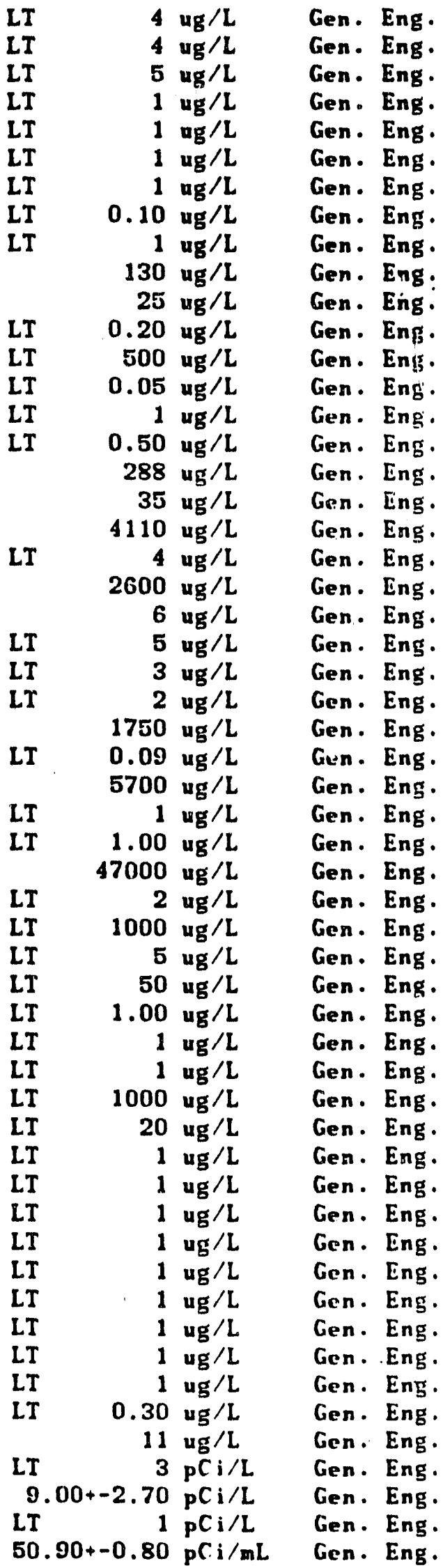




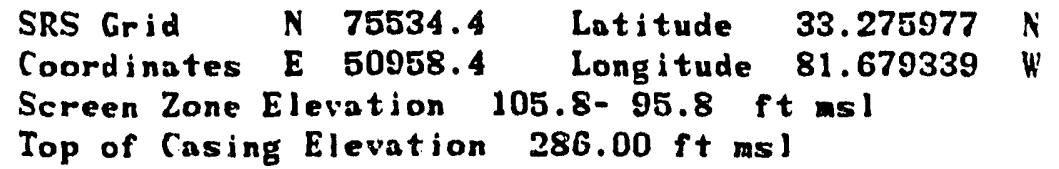

MEASUREMENTS CONDUCTED IN THE FIELD

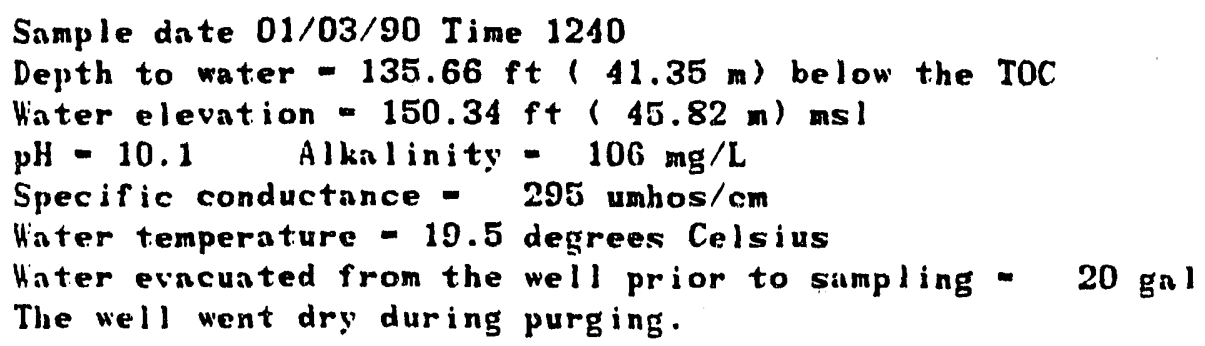

LABORATORY ANALYSES

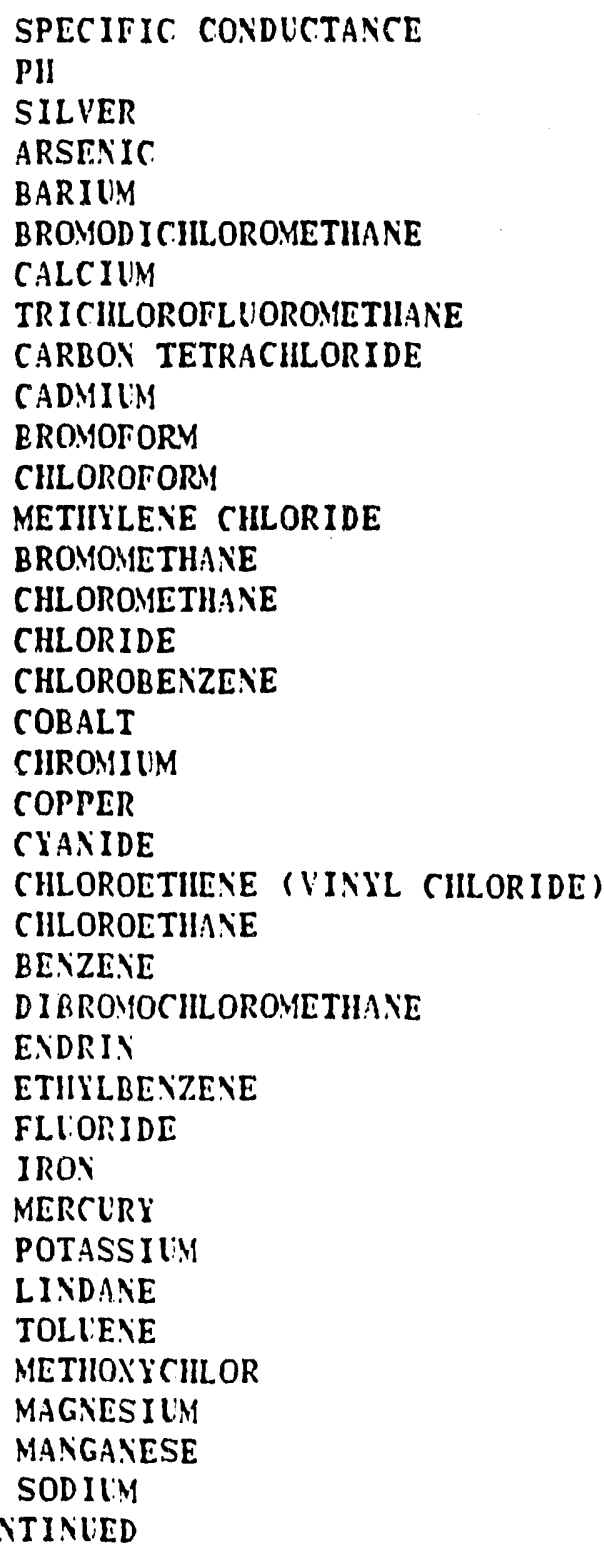

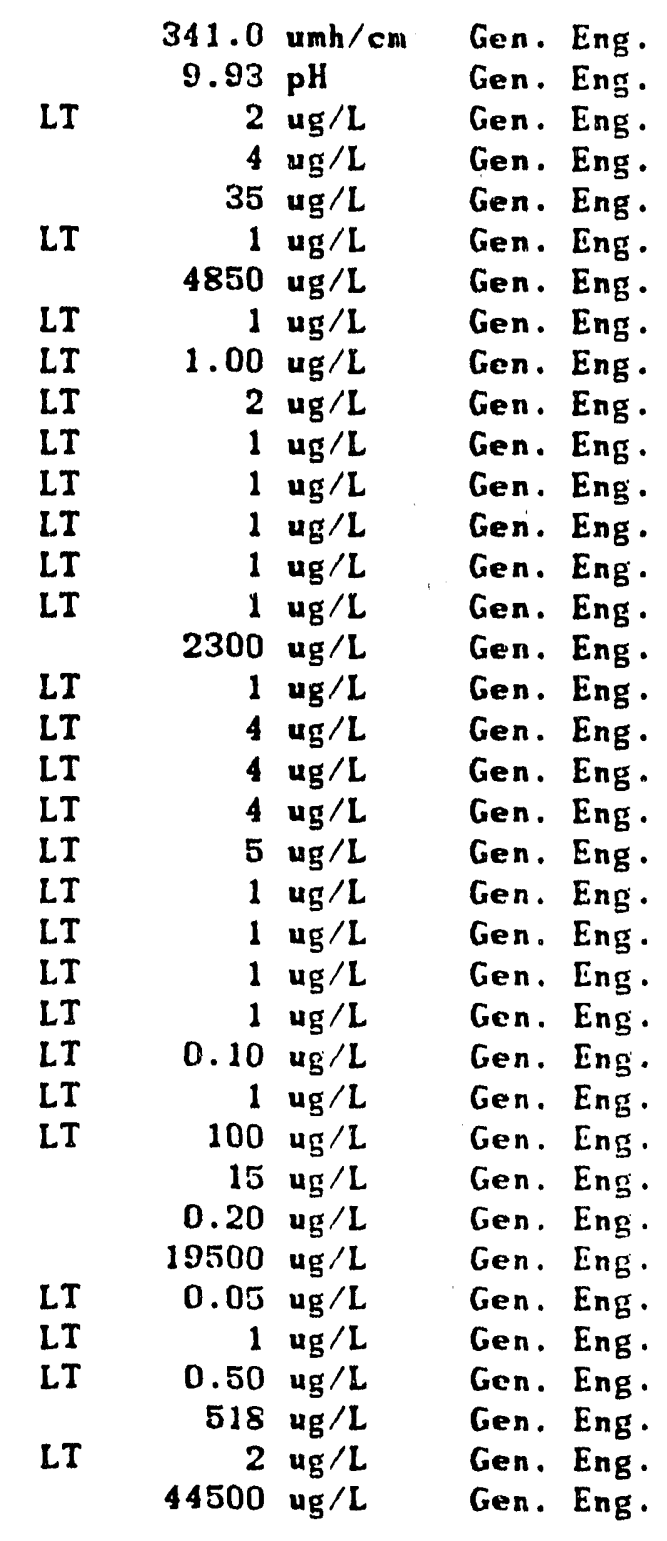


VELL ISB 100A COLLECTED ON 01/03/90 LABORATORY ANALYSES CONTINUED

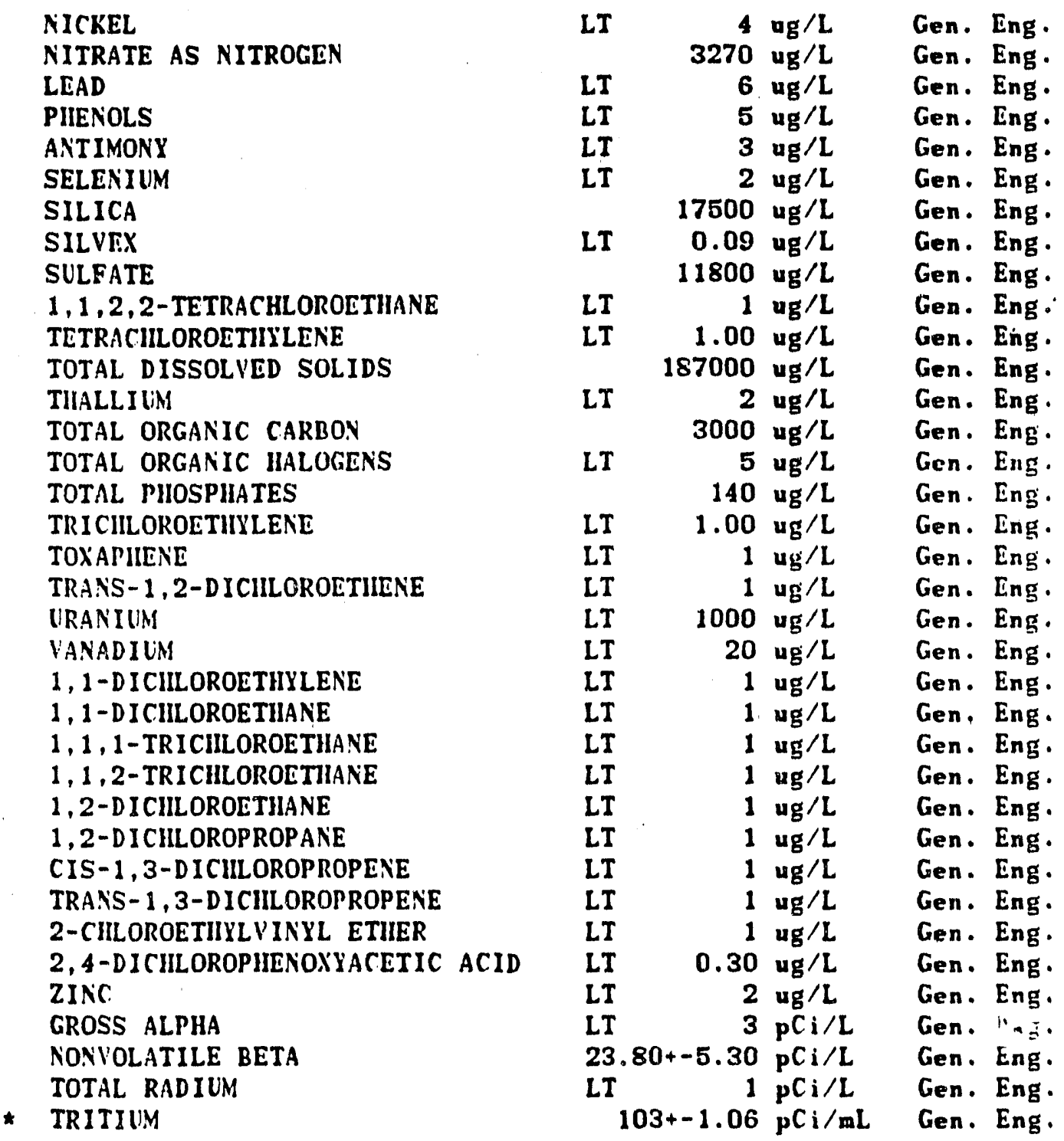




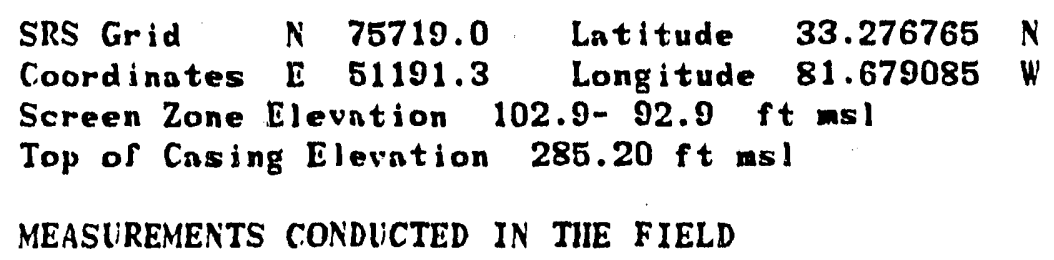

MEASUREMENTS CONDUCTED IN THE FIELD

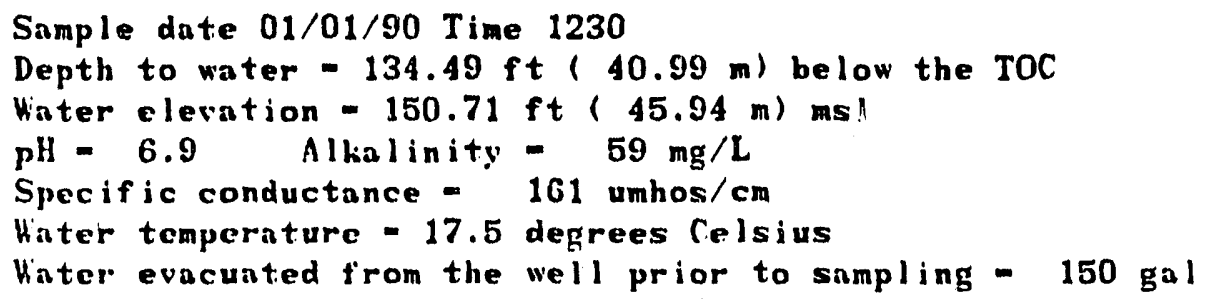

SPECIFIC CONDUCTANCE

PII

SILVER

ARSENIC

BARIUM

BROYOD ICHLOROMETHANE

CALCIUM

TRICILOROFLUOROMETHANE

CARBOX TETRACHLORIDE

CADMIUM

BROMOFORM

CIILOROFORM

METIIILENE CIILORIDE

BROMOMETHANE

CHLOROMETHANE

CHLORIDE

CIILOROBENZENE

COBALT

CHROMIUM

COPPER

CYANIDE

CIILOROETIENE (NINYL CILORIDE)

CIILOROETIIANE

BENZENE

DIBROMOCHLOROMETHANE

ENDRIN

ETHYLEYZENE

FLI'ORIDE

IRON

MERCURI

POTASSIL'M

LINDANE

TOLLENE

METHOXYCILLOR

MAGIESILM

MANGANESE

SOD IUM

CONTINLED

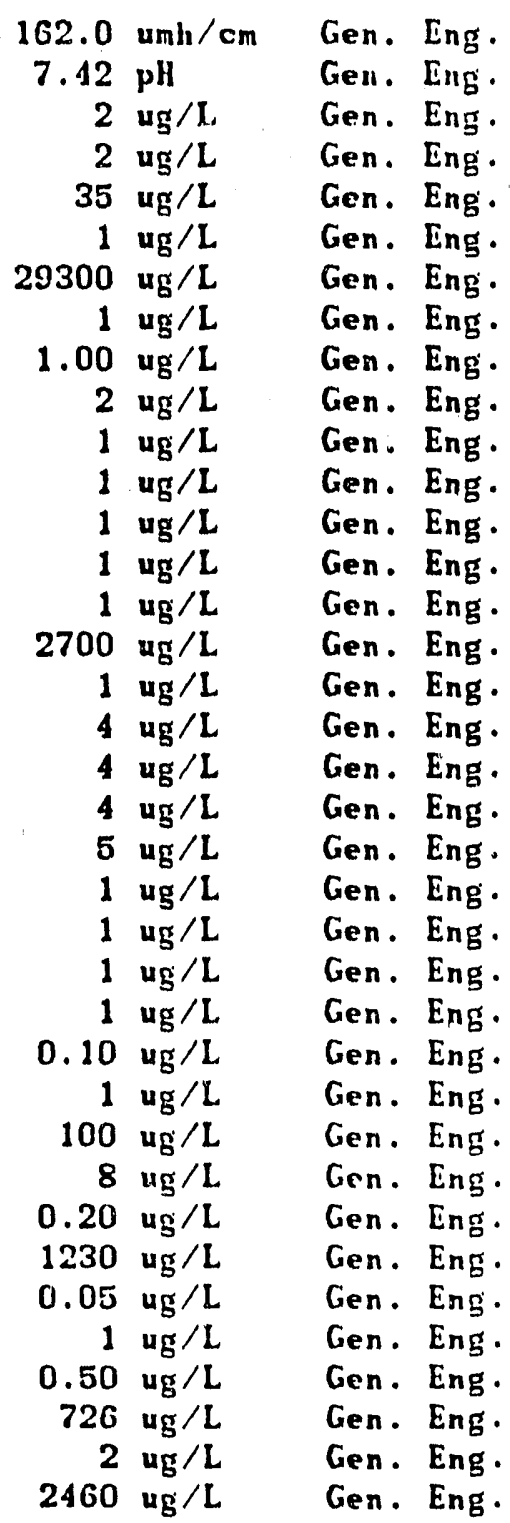


HELL FSB1O1A COLLECTED ON 01/01/90 LABORATORY ANALYSES CONTINUED

NICKEL

NITRATE AS NITROGEN

LEAD

PHIENOLS

ANTIMONY

SELENIUM

SILICA

SILVEX

SULFATE

$1,1,2,2$-TETRACIILOROETIIANE

TETRACHLOROETIIYLENE

TOTAL DISSOLVED SOLIDS

THALLIUM

TOTAL ORGANIC CARBON

TOTAL ORGANIC HALOGENS

TOTAL PHOSPIIATES

TRICIILOROETHILENE

TOXAPHENE

TRANS-1,2-DICHLOROETHENE

IRANIIM

VANADIUM

1,1-DICILOROETHYLENE

1,1-DICHLOROETHANE

$1,1,1$-TR ICHLOROETHANE

$1,1,2$-TR ICHLOROETHANE

1,2-DICIILOROETIIANE

1,2-DICIILOROPROPANE

CIS-1,3-DICILLOROPROPENE

TRANS-1,3-DICHLOROPROPENE

2-CIILOROETIIYLVINYL ETHER

2,4-DICILLOROPIIENOXYACETIC ACID

ZINC

GROSS ALPHA

NONVOLATILE BETA

TOTAL RADILM

TRITIIM
LI

LT

LT

LT

LT

LT

LT

LT

LT

LT

LT

LI

LI

LT

LT

LT

LT

LT

LT

LT

LI

LT

LT

LT

LT

LT

LT

ZT

8

LI

LT
$4 \mathrm{ug} / \mathrm{L}$ Gen. Eng.

$1640 \mathrm{ug} / \mathrm{L}$ Gen. Eng.

$6 \mathrm{ug} / \mathrm{L}$ Gen. Eng.

5 ug/L Gen. Eng.

3 ug/L Gen. Eng.

$2 \mathrm{ug} / \mathrm{L}$ Gen. Eng.

$17000 \mathrm{ug} / \mathrm{L}$ Gen. Eng.

$0.09 \mathrm{ug} / \mathrm{L}$ Gen. Eng.

$5000 \mathrm{ug} / \mathrm{L}$ Gen. Eng.

$1 \mathrm{ug} / \mathrm{L}$ Gen. Eng.

$1.00 \mathrm{ug} / \mathrm{L}$ Gen. Eng.

$113000 \mathrm{ug} / \mathrm{L}$ Gen. Eng.

2 ug/L Gen. Eng.

$10000 \mathrm{ug} / \mathrm{L}$

$5 \mathrm{ug} / \mathrm{L}$

$200 \mathrm{ug} / \mathrm{L}$

$1.00 \mathrm{ug} / \mathrm{L}$

$1 \mathrm{ug} / \mathrm{L}$

$1 \mathrm{ug} / \mathrm{L}$

$1000 \mathrm{ug} / \mathrm{L}$

$20 \mathrm{ug} / \mathrm{L}$

$1 \mathrm{ug} / \mathrm{L}$

$1 \mathrm{ug} / \mathrm{L}$

$1 \mathrm{ug} / \mathrm{L}$

$1 \mathrm{ug} / \mathrm{L}$

$1 \mathrm{ug} / \mathrm{L}$

$1 \mathrm{ug} / \mathrm{L}$

$1 \mathrm{ug} / \mathrm{L}$

$1 \mathrm{ug} / \mathrm{L}$

$1 \mathrm{ug} / \mathrm{L}$

$0.30 \mathrm{ug} / \mathrm{L}$

$2 \mathrm{ug} / \mathrm{L}$

$3 \mathrm{pCi} / \mathrm{L}$

$.10+-2.60 \mathrm{pCi} / \mathrm{L}$

$1 \mathrm{pCi} / \mathrm{L}$

$0.70 \mathrm{pCi} / \mathrm{mL}$

Gen. Eng.

Gen. Eng.

Gen. Eng.

Gen. Eng.

Gen. Eng.

Gen. Eng.

Gen. Eng.

Gen. Eng.

Gen. Eng.

Gen. Eng.

Gen. Eng.

Gen. Eng.

Gen. Eng.

Gen. Eng.

Gen. Eng.

Gen. Eng.

Gen. Eng.

Gen. Eng.

Gen. Eng.

Gen. Eng.

Gen. Eng.

Gen. Eng.

Gen. Eng. 
K'ELL FSB $102 C$

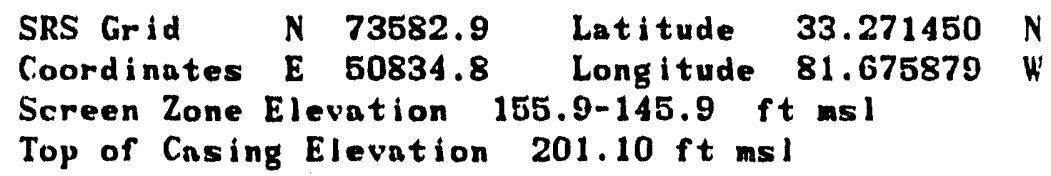

MEASUREMENTS CONDUCTED IN THE FIELD

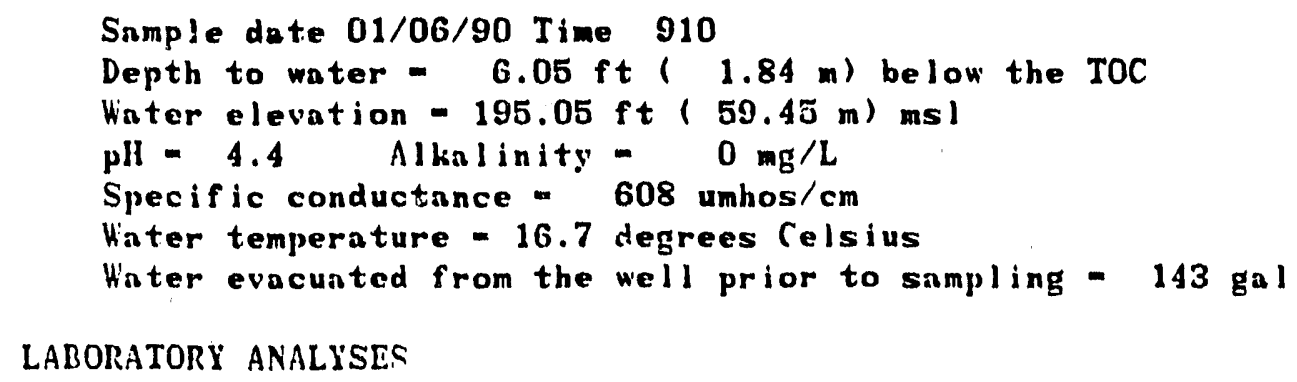

SPECIFIC CONDUCTANCE

PII

SILVER

ARSENIC

BARIUM

BROMODICHLOROMETHANE

CALCILM

TRICILOROFLUOROMETHANE

CARBON TETRACILLORIDE

CADMIUM

BROMOFORM

CHLOROFORM

METHLLENE CILLORIDE

BROMONETUANE

CHLOROMETHANE

CIILORIDE

CIILOROBENZENE

COBALT

CHRONIUM

COPPER

CXANIDE

CILOROETIIENE (VINYL CILORIDE)

CIILOROETHANE

BE.ZENE

DIBROMOCILLOROMETIIANE

ENDRIN

ETIILBENZENE

FLIOORIDE

IRON

MERCLRY

POTASSIUM

LINDANE

TOLLENE

METIIOXYCIILOR

MAG.YESIIM

MATGANESE

SODIUM

CONTINLED

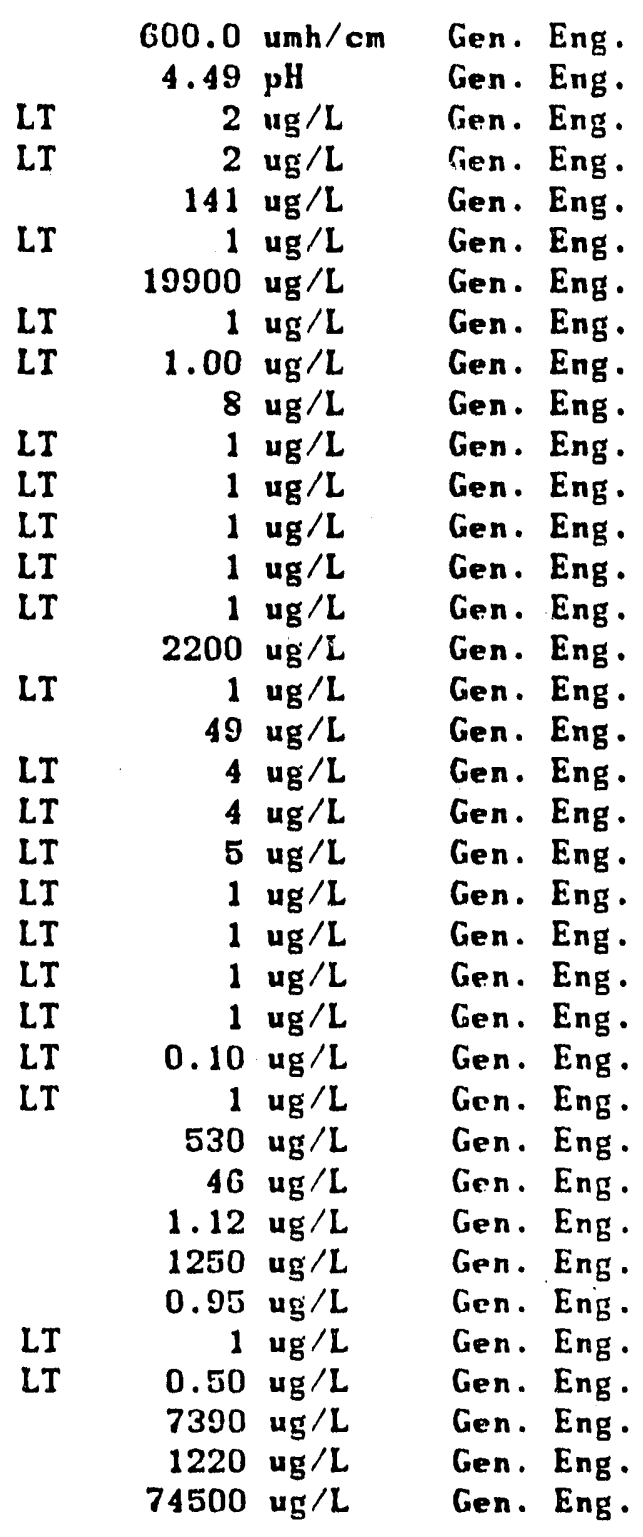


HELL FSB102C COLLECTED ON 01/06/90 LABORATORY ANALYSES CONTINUED

NICKEL

* NITRATE AS NITROGEN

LEAD

PIIENOLS

ANTIMONY

SELENIUM

SILICA

SILVEX

SULFATE

1,1,2,2-TETRACILOROETHANE

TETRACHLOROETIIILENE

TOTAL DISSOLVED SOLIDS

TIIALLIUM

TOTAL ORGANIC CARBON

TOTAL ORGANIC HALOGENS

TOTAL PIIOSPIIATES

TRICILOROETIIYLENE

TOXAPIIENE

TRANS-1, 2-D ICHLOROETHENE

LRANIUM

VANADIUM

1, 1-DICHLOROETHYLENE

1,1-D I CILLOROETHANE

$1,1,1$-TRICHLOROETHANE

$1,1,2$-TRICHLOROETHANE

1,2-DICHLOROETHANE

1,2-DICILLOROPROPANE

CIS-1,3-DICHLOROPROPENE

TRANS-1 , 3-DICILLOROPROPENE

2-CIILOROETIIYLVINYL ETHER

2,4-DICHLOROPHENOXIACETIC ACID

ZINC

* Gross alpha

NONYOLATILE BETA

TOTAL RADIUM

* tritium

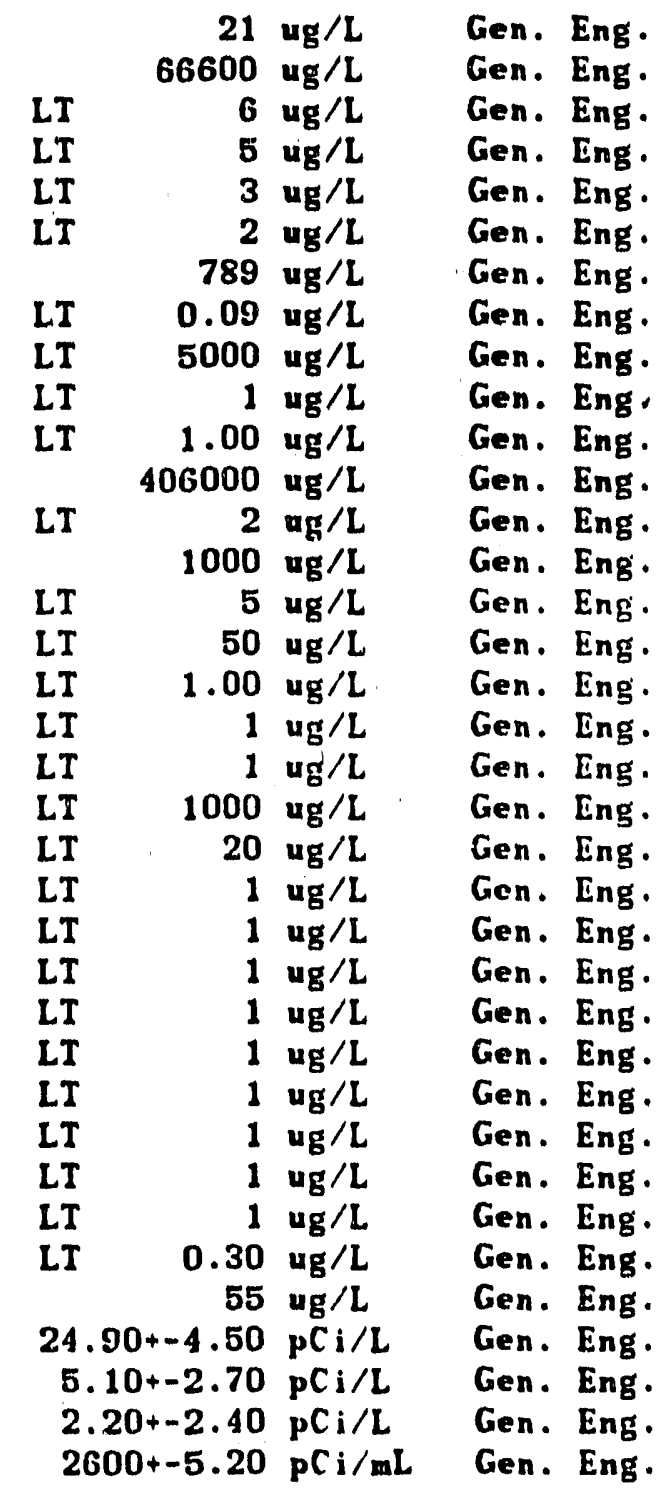




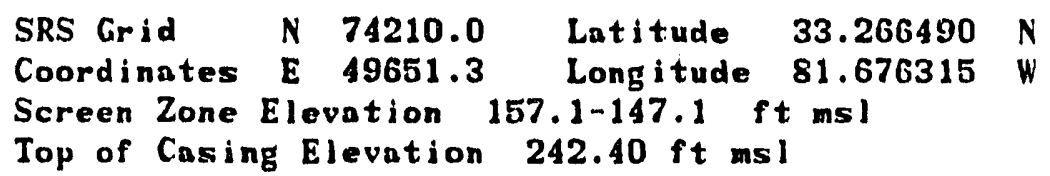

MEASLREMENTS CONDUCTED IN THE FIELD

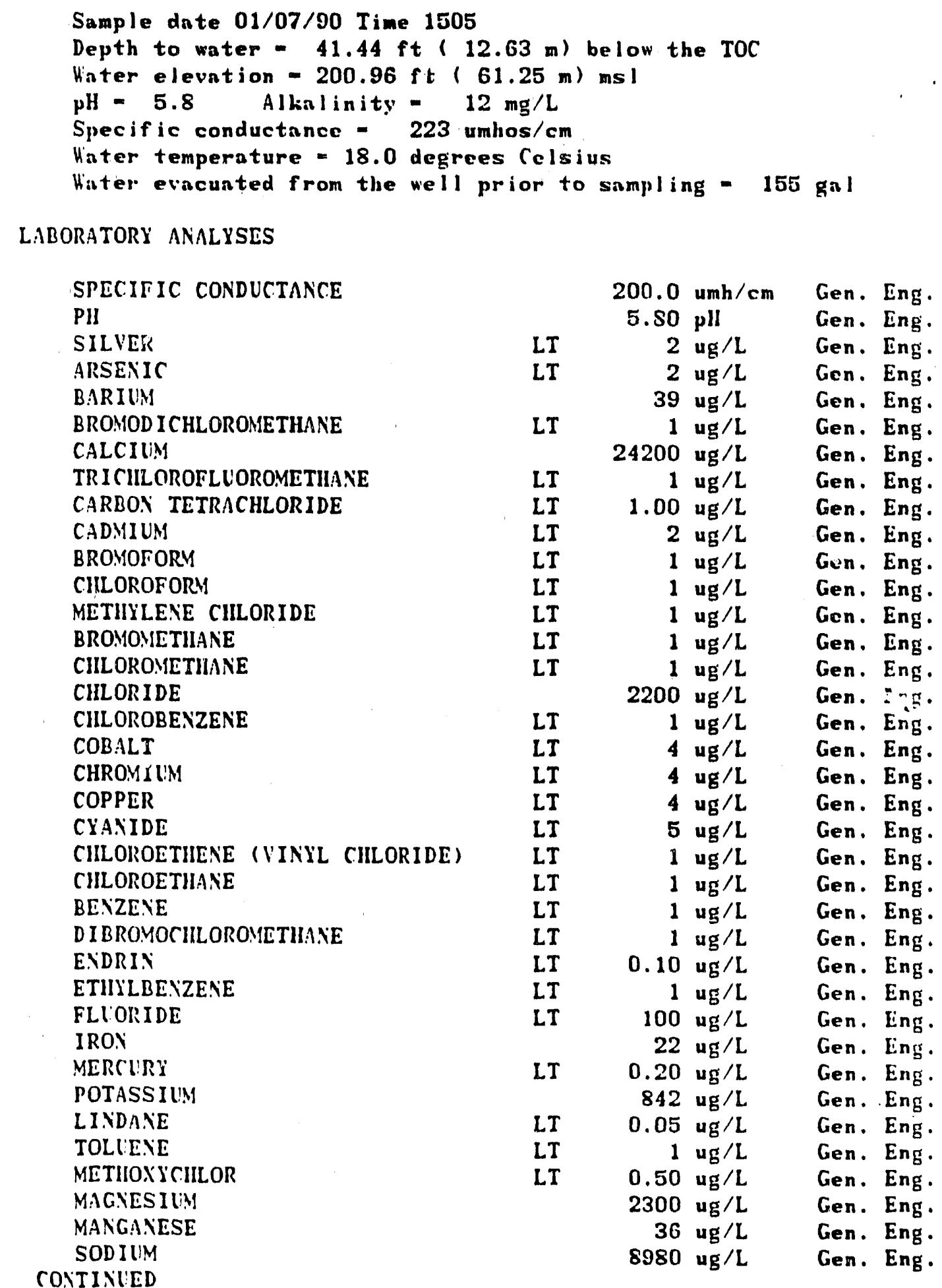


WELL FSB103C COLLECTED ON 01/07/90 LABORATORY ANALYSES CONTINUED

NICKEL

* NITRATE as NITROGEN

LEAD

PHENOLS

ANTIMONY

SELENIUM

SILICA

SILVEX

SULFATE

$1,1,2,2$-TETRACHLOROETHLNE

TETRACHL.OROETHILENE

TOTAL DISSOLVED SOLIDS

THALLIUM

TOTAL ORGANIC CARBON

TOTAL ORGANIC IIALOGENS

TOTAL PIIOSPIIATES

TRICIILOROETIIYLENE

TOXAPHENE

TRANS-1,2-DICIILOROETIIENE

IRANIUM

VANADIUM

1, 1-D I CIILOROETIIYLENE

1,1-D I CILLOROETHANE

$1,1,1$-TRICILLOROETHANE

1,1,2-TRICIILOROETIIANE

1, 2-D I CILLOROETHANE

1,2-D ICIILOROPROPANE

CIS-1, 3-DICIILOROPROPENE

TRANS-1, 3-DICIILOROPROPENE

2-CHLOROETUYLYINYL ETIIER

2,1-DICILLOROPIENOXYACETIC ACID

ZINC

GROSS ALPIIA

NONVOLATILE BETA

TOTAL RADIUM

* TRITIUM
LT

LI

LI

LI

LI

LT

LT

LT

LT

LT

LT

LT

LT

LT

LT

LT

LT

LT

LT

LT

LT

LT

LT

LT

LT

LT

LT

LT

$3.92+-2.40 \mathrm{pCi} / \mathrm{L}$ LT

$714+-2.71 \mathrm{pCi} / \mathrm{mL}$
Gen. Eng.

Gen. Eng.

Gen. Eng.

Gen. Eng.

Gen. Eng.

Gen. Eng.

Gen. Eng.

Gea. Eng.

Gen. Eng.

Gen. Eng:

Gen. Eng .

Gen. Eng.

Gen. Eng.

Gen. Eng.

Gen. Eng.

Gen. Eng.

Gen. Eng.

Gen. Eng.

Gen. Eng.

Gen. Eng.

Gen. Eng.

Gen. Eng.

Gen. Eng.

Gen. Eng.

Gen. Eng.

Cen. Eng.

Gen. Eng.

Gen. Eng.

Gen. Eng.

Gen. Eng.

Gen. Eng.

Gen. Eng.

Gen. Eng.

Gen. Eng.

Gen. Eng.

Gen. Eng. 


\section{KELL FSB104C}

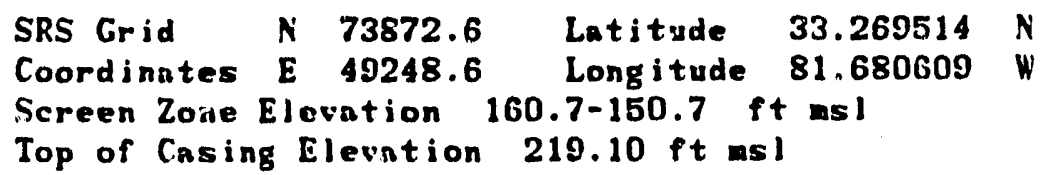

MEASUREMENTS CONDUCTED IN THE FIELD

Sample date 01/09/90 Time 1125

Depth to water $-19.46 \mathrm{ft}(5.93 \mathrm{~m})$ below the TOC

Vinter elevation $-199.64 \mathrm{ft}(60.85 \mathrm{~m}) \mathrm{msl}$

pll $=5.2 \quad$ Alkalinity $=1 \mathrm{mg} / \mathrm{L}$

Specific conductance = 324 umhos/cm

Hinter temperature -16.7 degrees Celsius

Hater evacuated from the well prior to sampling - $141 \mathrm{gal}$

\section{LABORITORY ANALYSES}

SPICIFIC CONDUCTANCE

PII

SILIER

ARSENIC

BARILYY

BROMOD ICHLOROMETHANE

CALCILM

TRICILOROFLUORONETIIANE

CARBOI TETRACHLORIDE

CADMILM

BROMOFORM

CIILOROTORM

METIYLENE. CHLORIDE

BROMOMETHANE

CIILOROMETHANE

CIILORIDE

CIILOROBENZENE

COBALT

CHIONIUM

COPPER

CYANIDE

CIILOROETHENE (VINYL CIILORIDE)

CILLOROETHANE

BEIZENE

DIBROMOCHLORONETHANE

ENDRIN

ETIIYLBENZENE

FLIORIDE

IRON

MERCURY

POTASSILY

LINDANE

TOLCENE

METIIOXYCIILOR

MAGNESILM

MATGANESE

SODIIM

COITINIED

\section{LI}

LT

LT

LI

LT

LT

LT

LT

LT

LT

LT

LT

LI

LT

LIT

LT

LI

LI

LT

LT

LT

LT

LT

LT

LT

LT

LI
$300.0 \mathrm{umh} / \mathrm{cm}$

$$
5.52 \mathrm{pll}
$$

$2 \mathrm{ug} / \mathrm{L}$

$2 \mathrm{ug} / \mathrm{L}$

58 ug/L

$1 \mathrm{ug} / \mathrm{L}$

$25900 \mathrm{ug} / \mathrm{L}$

$1 \mathrm{ug} / \mathrm{L}$

$1.00 \mathrm{ug} / \mathrm{L}$

2 ug/L

$1 \mathrm{ug} / \mathrm{L}$

$1 \mathrm{ug} / \mathrm{L}$

$1 \mathrm{ug} / \mathrm{L}$

$1 \mathrm{ug} / \mathrm{L}$

$1 \mathrm{ug} / \mathrm{L}$

$2400 \mathrm{ug} / \mathrm{L}$

$1 \mathrm{ug} / \mathrm{L}$

$4 \mathrm{ug} / \mathrm{L}$

$4 \mathrm{ug} / \mathrm{L}$

$4 \mathrm{ug} / \mathrm{L}$

$5 \mathrm{ug} / \mathrm{L}$

$1 \mathrm{ug} / \mathrm{L}$

$1 \mathrm{ug} / \mathrm{L}$

$1 \mathrm{ug} / \mathrm{L}$

$1 \mathrm{ug} / \mathrm{L}$

$0.10 \mathrm{ug} / \mathrm{L}$

$1 \mathrm{ug} / \mathrm{L}$

$100 \mathrm{ug} / \mathrm{L}$

$22 \mathrm{ug} / \mathrm{L}$

$0.20 \mathrm{ug} / \mathrm{L}$

$1480 \mathrm{ug} / \mathrm{L}$

$0.05 \mathrm{ug} / \mathrm{L}$

$1 \mathrm{ug} / \mathrm{L}$

$0.50 \mathrm{ug} / \mathrm{L}$

$4580 \mathrm{ug} / \mathrm{L}$

$21 \mathrm{ug} / \mathrm{L}$

$13700 \mathrm{ug} / \mathrm{L}$
Gen. Eng.

Gen. Eng.

Gen. Eng.

Gen. Eng.

Gen. Eng.

Gen. Eng.

Gen. Eng.

Gen. Eng.

Gen. Eng.

Gen. Eng.

Gen. Eng.

Gen. Eng.

Gen. Eng.

Gen. Eng.

Gen. Eng.

Gen. Eng.

Gen. Eng.

Gen. Eng.

Gen. Eng.

Gen. Eng.

Gen. Eng.

Gen. Eng.

Gen. Eng.

Gen. Eng.

Gen. Eng.

Gen. Eng.

Gen. Eng.

Gen. Eng.

Gen. Eng.

Gen. Eng.

Gen. Eng.

Gen. Eng.

Gen. Eng.

Gen. Eng.

Gen. Eng.

Gen. Eng.

Gen. Eng. 
WELL FSB104C COLLECTED ON 01/09/90 LABORATORY ANALYSES CONTINUED

NICKEL

* NITRATE AS NITROGEN

LEAD

PIIENOLS

ANTIMONY

SELENIUM

SILICA

SILVEX

SULFATE

$1,1,2,2$-TETRACHLOROETIIANE

TETRACIILOROETHYLENE

TOTAL DISSOLVED SOLIDS

THALLIUM

TOTAL ORGANIC CARBON

TOTAL ORGANIC IIALOGENS

TOTAL PIIOSPHATES

TRICHLOROETIYLENE

TOXAPIIENE

TRANS-1, 2-DICILOROETHENE

URANIUM

VANADIUM

1,1-DICHLOROETHYLENE

1,1 -DICHLOROETHANE

$1,1,1$-TRICILLOROETHANE

$1,1,2$-TRICILOROETHANE

1,2-DICILLOROETHANE

1,2-DICILLOROPROPANE

CIS-1,3-DICIILOROPROPENE

TRANS-1,3-DICHLOROPROPENE

2-CILOROETHYLVINYL ETHER

2,4-DICHLOROPHEKOXYACETIC ACID ZINC

- gross alpha

NONVOLATILE BETA

TOTAL RADIUM

- TRITIUM

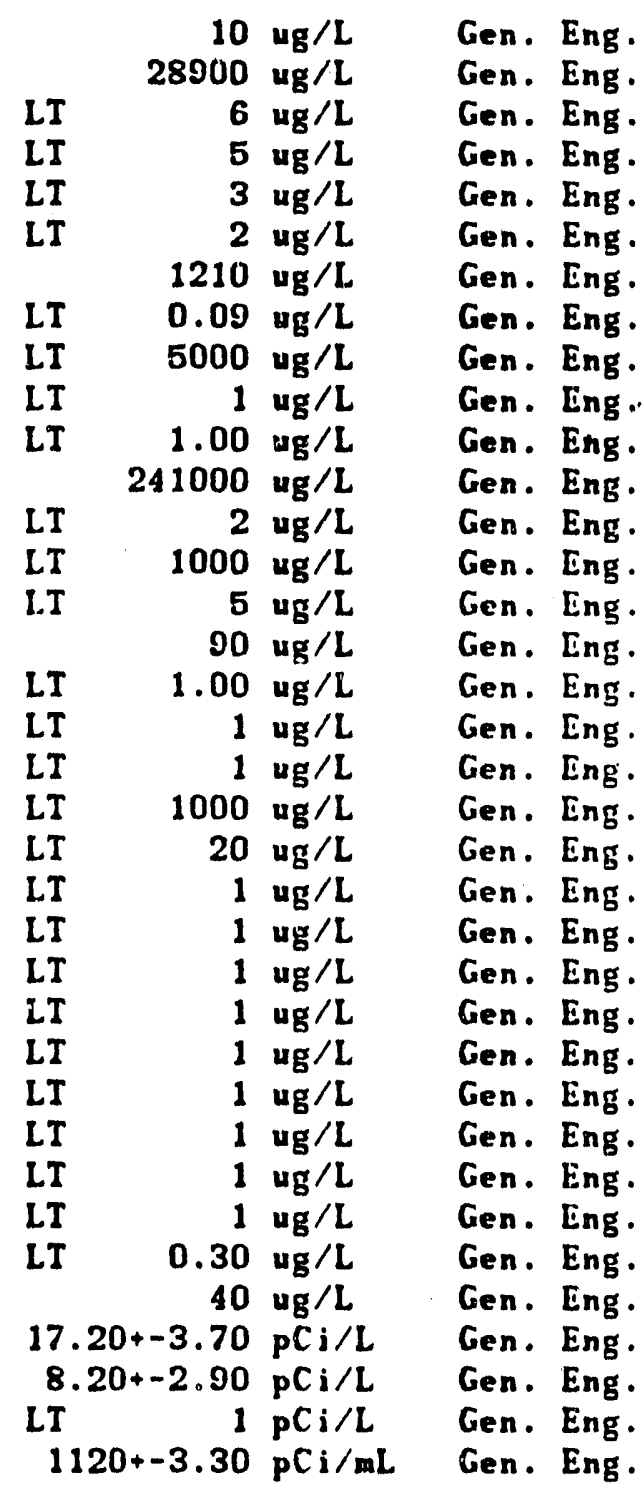




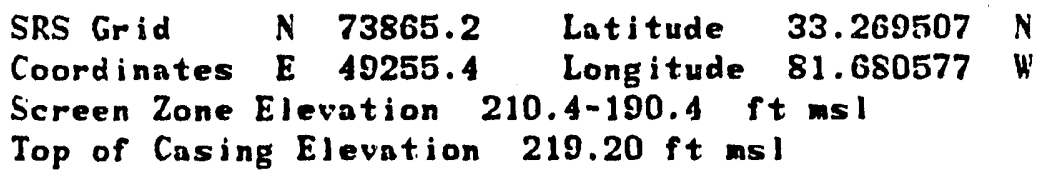

MEASLREMENTS CONDUCTED IN THE FIELD

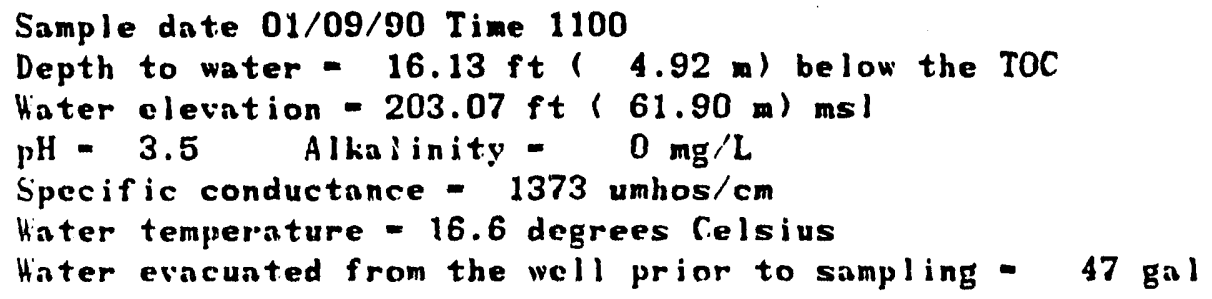

SPECIFIC. C.ONDLCTANCE

PH

SILVER

ARSENIC

BARIUM

BROMODICILOROMETHANE

CALCIUM

TRICIILOROFLUOROMETHANE

CARBON TETRACIILORIDE

CADMIUM

BROMOFORY

CHLOROFORM

METHILENE CHLORIDE

BROMONETHANE

CIILOROMETHANE

CIILORIDE

CHLOROBENZENE

COBALT

CHROYIUM

COPPER

CYANIDE

CIILOROETIIENE (VINYL CIILORIDE)

CIILOROETIIANE

BEIZENE

DIBROMOCILLOROMETHANE

ENDRIN

ETIYLBEYZENE

FLIORIDE

IRON

MERCLRY

POTASSIUM

LINDANE

TOLIENE

METHOAYCHLOR

MAGNESILM

MANGANESE

SODIUM

CONIINLED

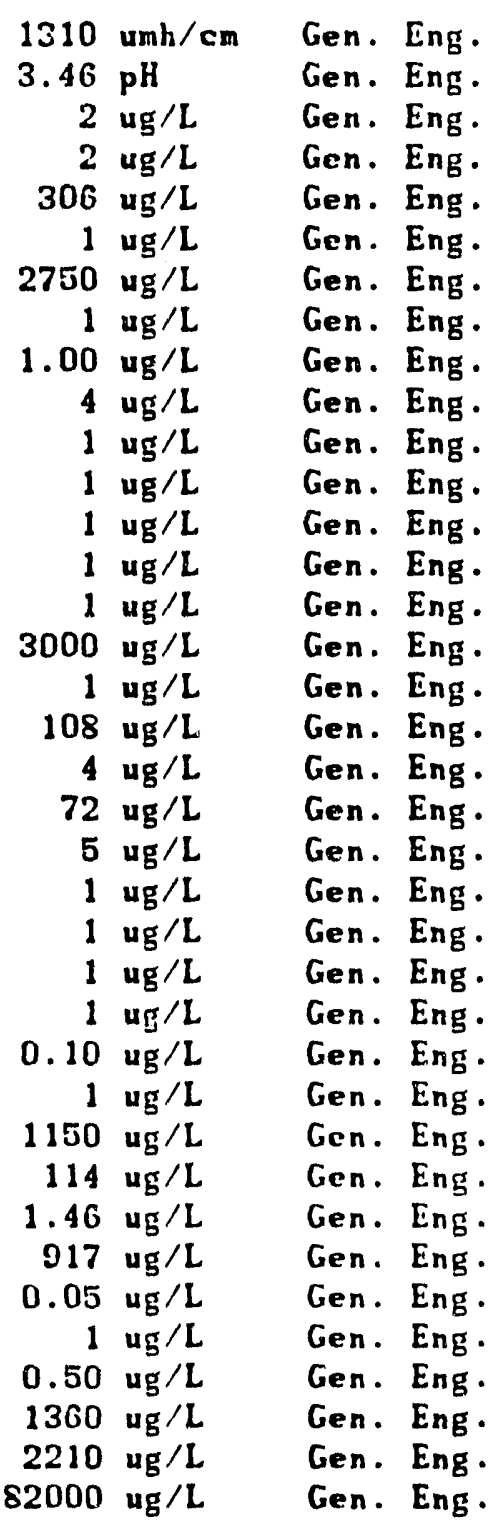


HELL FSB 104D COLLECTED ON 01/09/90 LABORATORY ANALYSES CONTINUED

NICKEL

* NITRATE AS NITROGEN

LEAD

PIIENOLS

ANTIMONY

SELENIUM

SILICA

SILVEX

SULFATE

$1,1,2,2$-TETRACIILOROETHANE

TETRACILOROETIILLENE

TOTAL DISSOLVED SOLIDS

THALLIUM

TOTAL ORGANIC CARBON

TOTAL ORGANIC HALOGENS

TOTAL PIIOSPIIATES

TRICIILOROETIIXLENE

TOXAPIENE

TRANS-1 , 2-DICILOROETHENE

URANIUM

VANADIUM

1,1-DICHLOROETIILENE

1,1-DICHLOROETHANE

$1,1,1$-TRICIILOROETHANE

1,1,2-TRICIILOROETIIANE

1,2-DICHLOROETHANE

1,2-DICHLOROPROPANE

CIS-1,3-DICHLOROPROPENE

TRANS-1, 3-DICHLOROPROPENE

2-CILOROETIIYLVINYL ETHER

2,4-DICHLOROPHENOXYACETIC ACID

ZINC

* gross alpha

* NoNvolatile beta

TOTAL RADILM

- TRITIUM

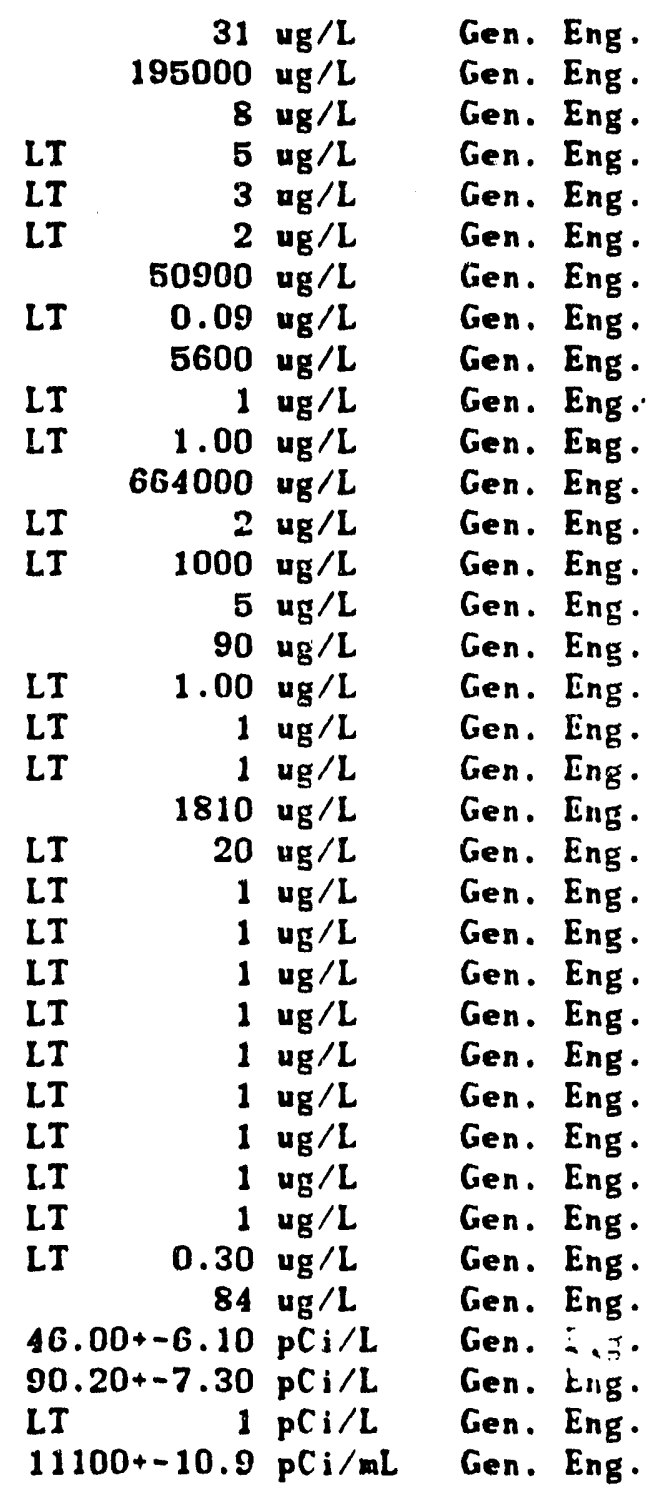


VELL FSB105C

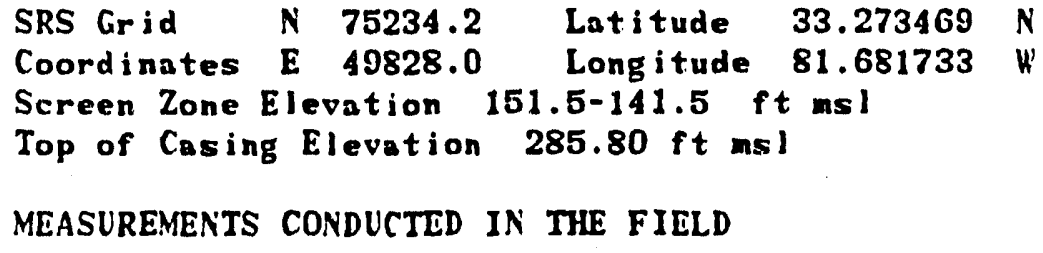

MEASUREMENTS CONDUCTED IN THE FIELD

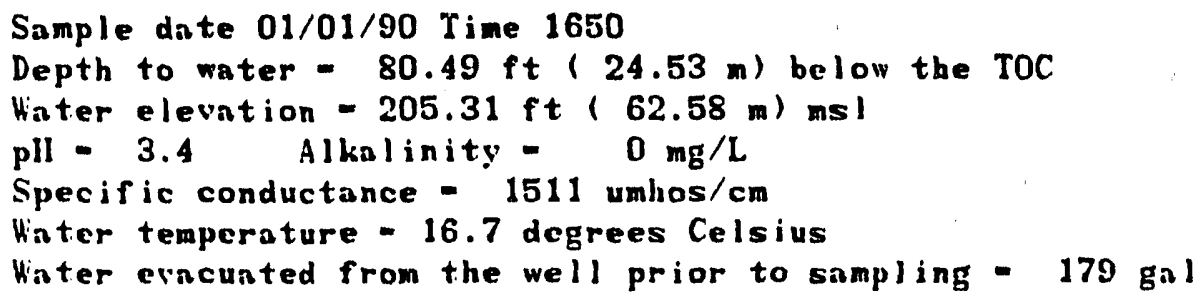

LABORATORY ANALYSES

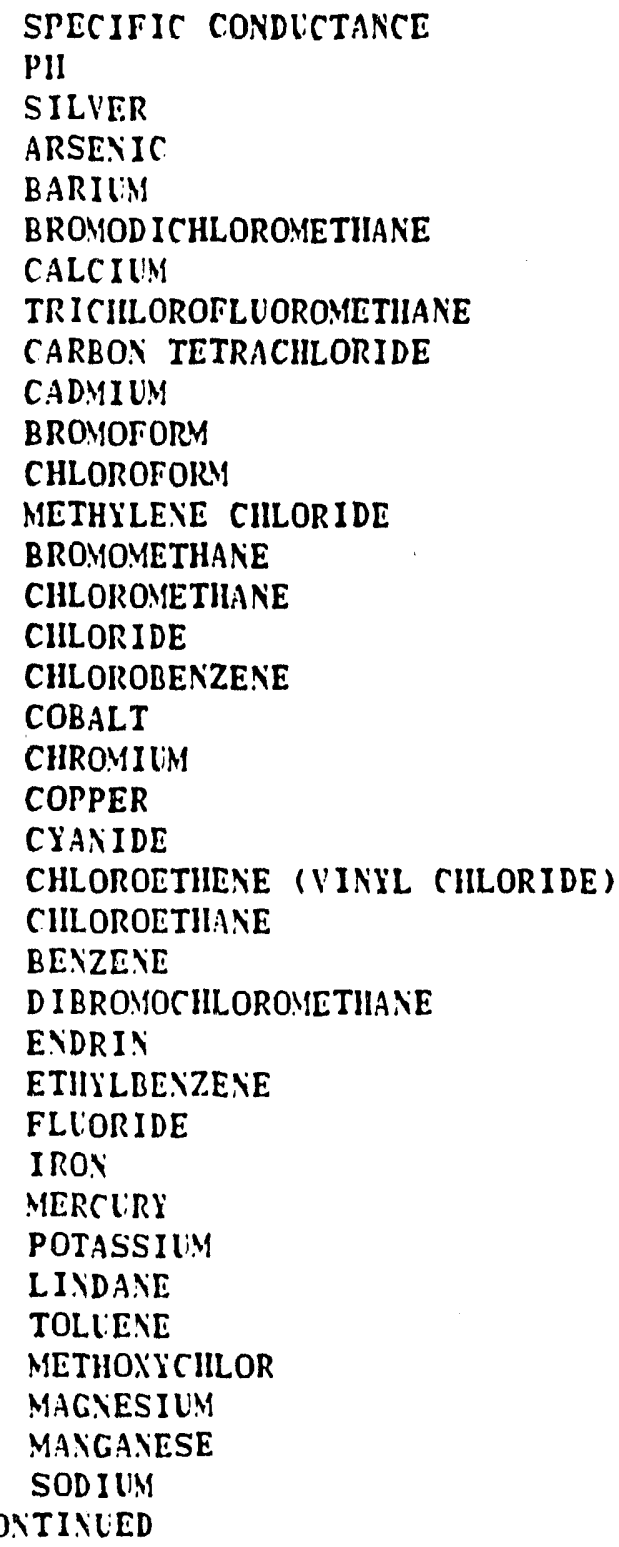

\begin{tabular}{|c|c|c|c|c|}
\hline & $\begin{array}{l}1850 \\
3.56\end{array}$ & $\begin{array}{l}u m h / c m \\
p H\end{array}$ & $\begin{array}{l}\text { Gen. } \\
\text { Gen. }\end{array}$ & $\begin{array}{l}\text { Eng. } \\
\text { Eng. }\end{array}$ \\
\hline LT & 2 & $u_{\mathfrak{g}} / L$ & Gen. & Eng. \\
\hline LT & 2 & ug $/ L$ & Gen. & Eng. \\
\hline & 559 & ug $/ \mathrm{L}$ & Gen. & Eng \\
\hline LT & $\begin{array}{r}1 \\
180 n n\end{array}$ & ug/L & Gen. & Eng \\
\hline LT & 1 & $u_{g} / L$ & Gen. & Eng \\
\hline $\mathrm{LT}$ & 1.00 & ug $/ \mathrm{L}$ & lien. & Eng \\
\hline & 17 & $\mathbf{u g} / \mathrm{L}$ & Gen. & Eng \\
\hline LT & 1 & $u_{g} / L$ & Gen. & Eng \\
\hline LT & 1 & $u_{g} / L$ & Gen. & Eng \\
\hline $\mathrm{LI}$ & 1 & ug/L & Gen. & Eng. \\
\hline LT & 1 & $u_{g} / L$ & Gen. & Eng. \\
\hline LT & 1 & $u_{g} / L$ & Gen. & Eng \\
\hline & 2600 & $u_{g} / L$ & $\mathrm{Ge}$ & En \\
\hline LT & 1 & ug $/ \mathrm{L}$ & Gen. & Eng \\
\hline & 348 & $u_{g} / L$ & Gen. & En \\
\hline LT & 4 & ug/L & & \\
\hline & 62 & $u_{g} / L$ & & • \\
\hline LI & 5 & $u_{g} / L$ & Gen. & Eng. \\
\hline LI & 1 & $u_{g} / L$ & Gen. & Eng. \\
\hline LT & 1 & $\mathrm{ug} / \mathrm{L}$ & Gen. & Eng \\
\hline LT & 1 & ug $/ L$ & Gen. & Eng. \\
\hline LT & 1 & $u_{g} / L$ & Gen. & Eng. \\
\hline LT & 0.10 & $\mathbf{u g}_{\mathrm{g}} / \mathrm{L}$ & Gen. & Eng. \\
\hline LT & 1 & ug $/ \mathrm{L}$ & Gen. & Eng. \\
\hline & 2500 & ug $/ L$ & Gen. & Eng. \\
\hline & 40 & $u_{g} / L$ & Gen. & Eng \\
\hline & 0.20 & ug/L & Gen. & Eng. \\
\hline & 2320 & ug / L & Ge & \\
\hline LT & 0.05 & $u g / L$ & Gen. & Eng. \\
\hline LT & 1 & ug $/ L$ & Gen. & En \\
\hline LT & 0.50 & $u_{g} / L$ & Gen. & Eng. \\
\hline & 7480 & ug / L & & \\
\hline & $\begin{array}{r}4400 \\
94800\end{array}$ & $\begin{array}{l}u g / L \\
u g / L\end{array}$ & $\begin{array}{l}\text { Gen. } \\
\text { Gen. }\end{array}$ & $E_{1}$ \\
\hline
\end{tabular}


KELL FSB105C COLLECTED ON 01/01/90 LABORATORY ANALYSES CONTINUED

NICKEL

* NITRATE AS NITROGEN

LEAD

PIIENOLS

ANTIMONY

SELENIUM

SILICA

SILVEX

SLLFATE

$1,1,2,2$-TETRACHLOROETHANE

IETRACHLOROETIIYLENE

TOTAL DISSOLVED SOLIDS

TILALLIUM

TOTAL ORGANIC CARBON

TOTAL ORGANIC HALOGENS

TOTAL PHOSPIIATES

TRICIIL OROETHYLENE

TOXAPUENE

TRAYS - 1, 2-DICILOROETHENE

URANIUM

VANADIUM

1,1 -DICIILOROETHYLENE

1,1-D ICILOROETHANE

$1,1,1$-TRICIILOROETHANE

$1,1,2$-TRICIILOROETHANE

1,2-D I CIILOROETHANE

1,2-DICHLOROPROPANE

CIS-1,3-DICIIL OROPROPENE

TRANS-1,3-DICHLOROPROPENE

2-CIILOROETHYLVINYL ETHER

2,4-DICHLOROPHENOXYACETIC ACID ZINC

GROSS ALPIIA

* NoNílatile beta

- total Radium

* tritium

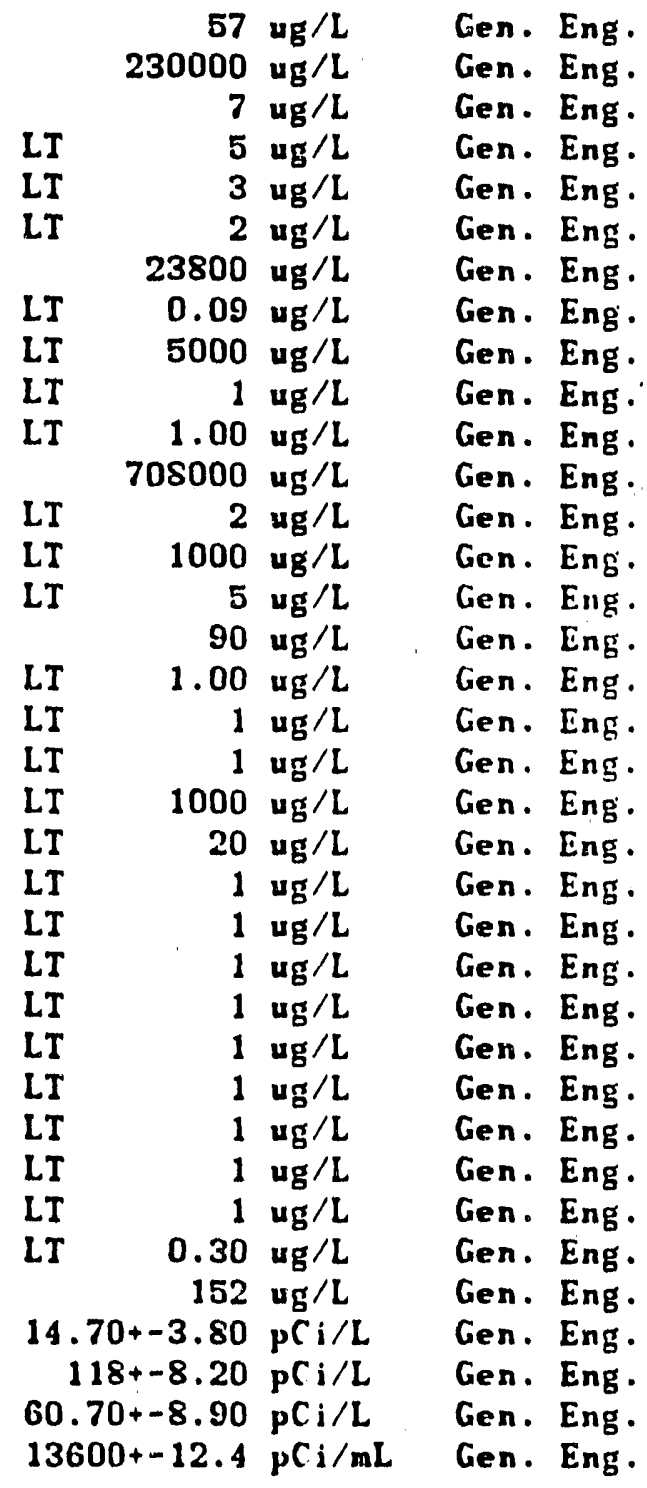


KELL FSB 105D

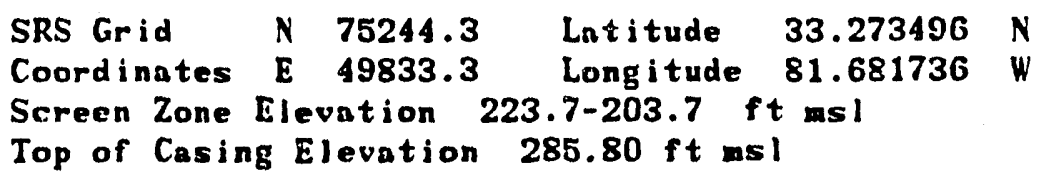

\section{MEASUREMENTS CONDUCTED IN THE FIELD}

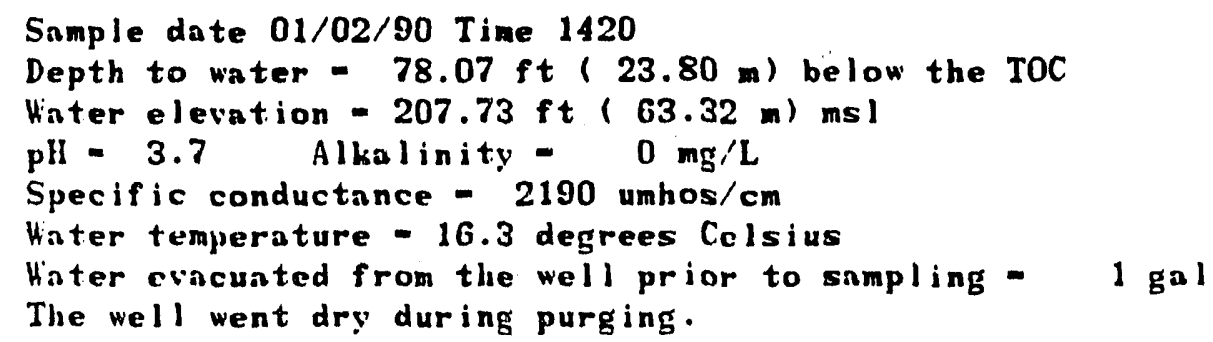

LABORATORY ANALYSES

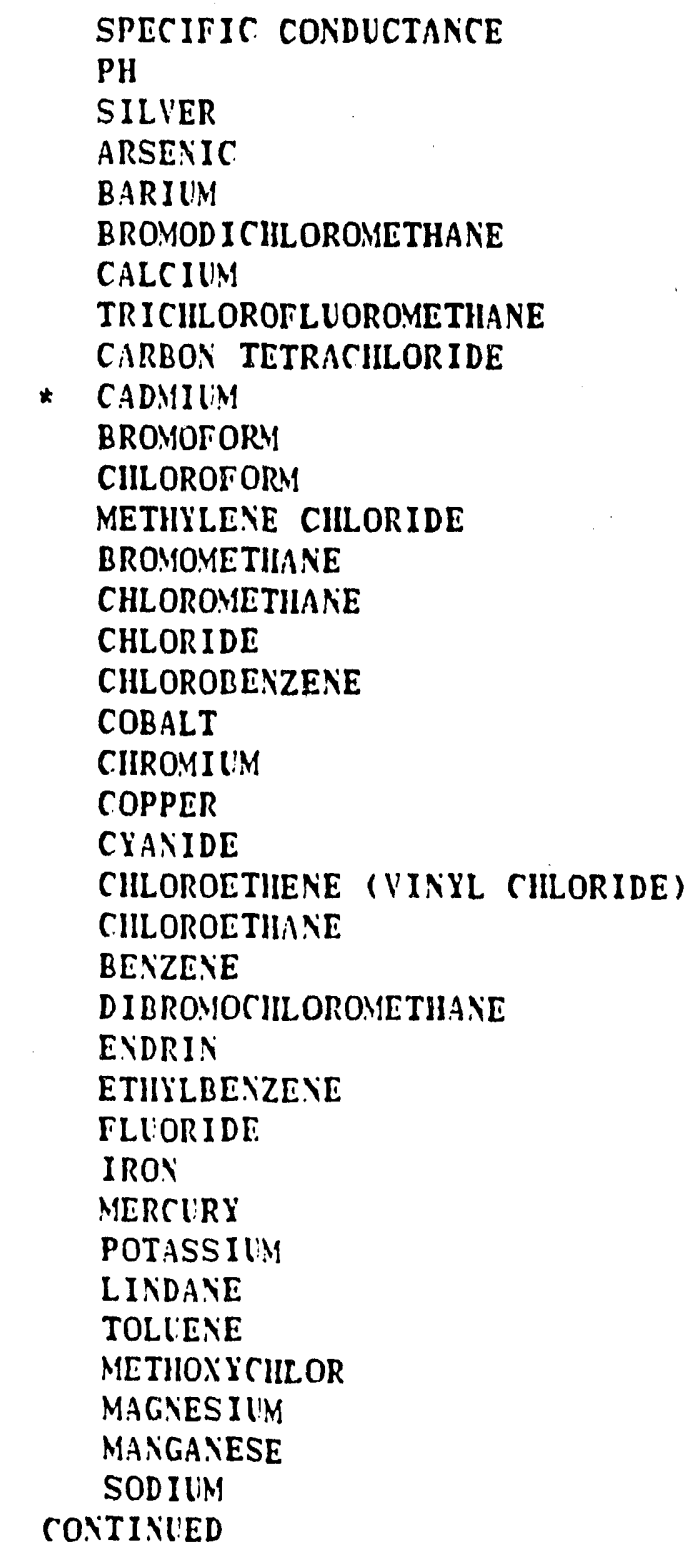

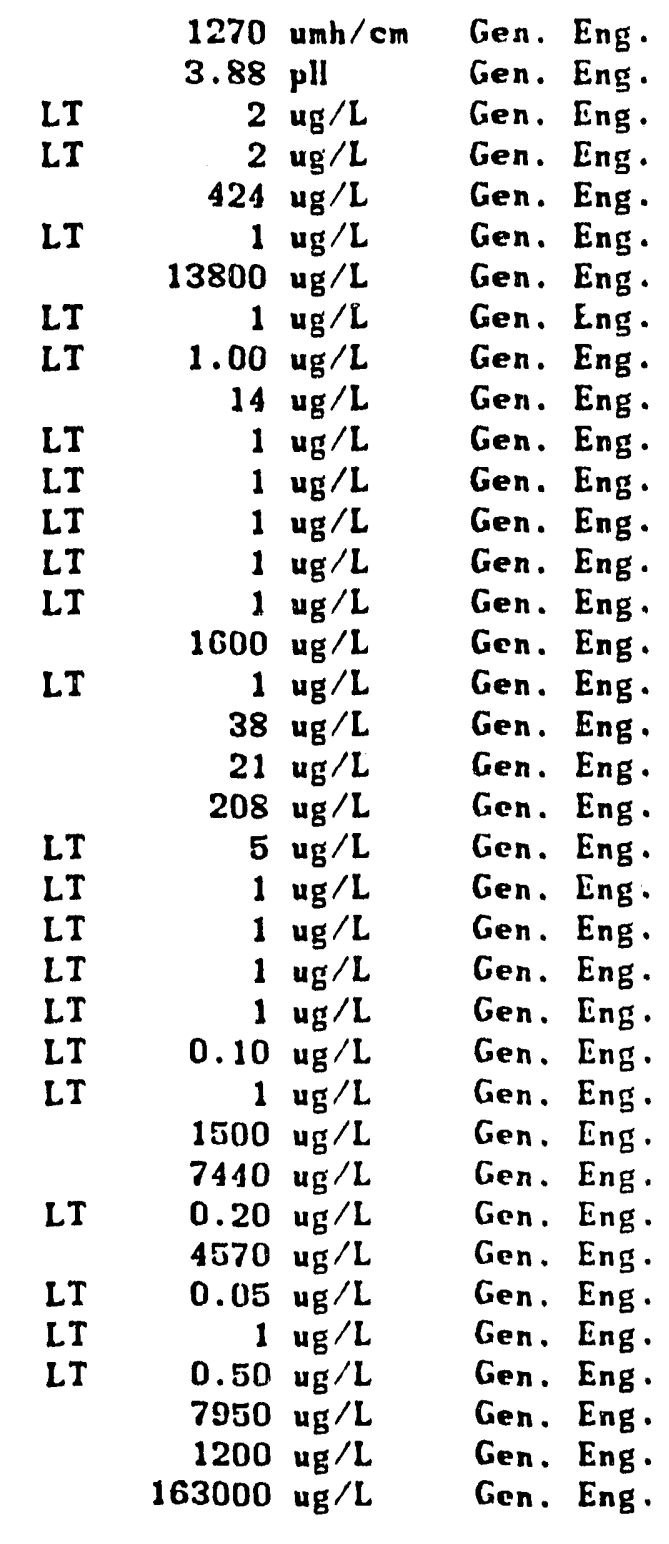


NICKEL

* NITRATE AS NITROGEN

* LEAD

PIIENOLS

ANTIMONY

SELENIUM

SILICA

SILVFX

SULF CE

$1,1, \therefore, 2$-TETRACHLOROETHANE

TETRACHLOROETIIYLENE

TOTAL DISSOLVED SOLIDS

THILLIUM

TOTAL ORGANIC CARBON

TOTAL ORGANIC HALOGENS

TOTAL PHOSPHATES

TRICIILOROETHYLENE

TOXAPHENE

TRANS-1,2-DICHLOROETIENE

liRANILIM

I'ANADILM

1,1-DICHLOROETHYLENE

1,1-DICHLOROETHANE

$1,1,1$-TR I CIILOROETHANE

$1,1,2$-TRICHLOROETHANE

1,2-DICHLOROETHANE

1,2-DICHLOROPROPANE

CIS-1,3-DICHLOROPROPENE

TRANS-1,3-DICHLOROPROPENE

2-CILOROETIILVINYL ETUER

2,4-DICIILOROPHENOXYACETIC ACID

ZINC

* Gross alpha

* Noniolatile beta

* total radium

* tritilum

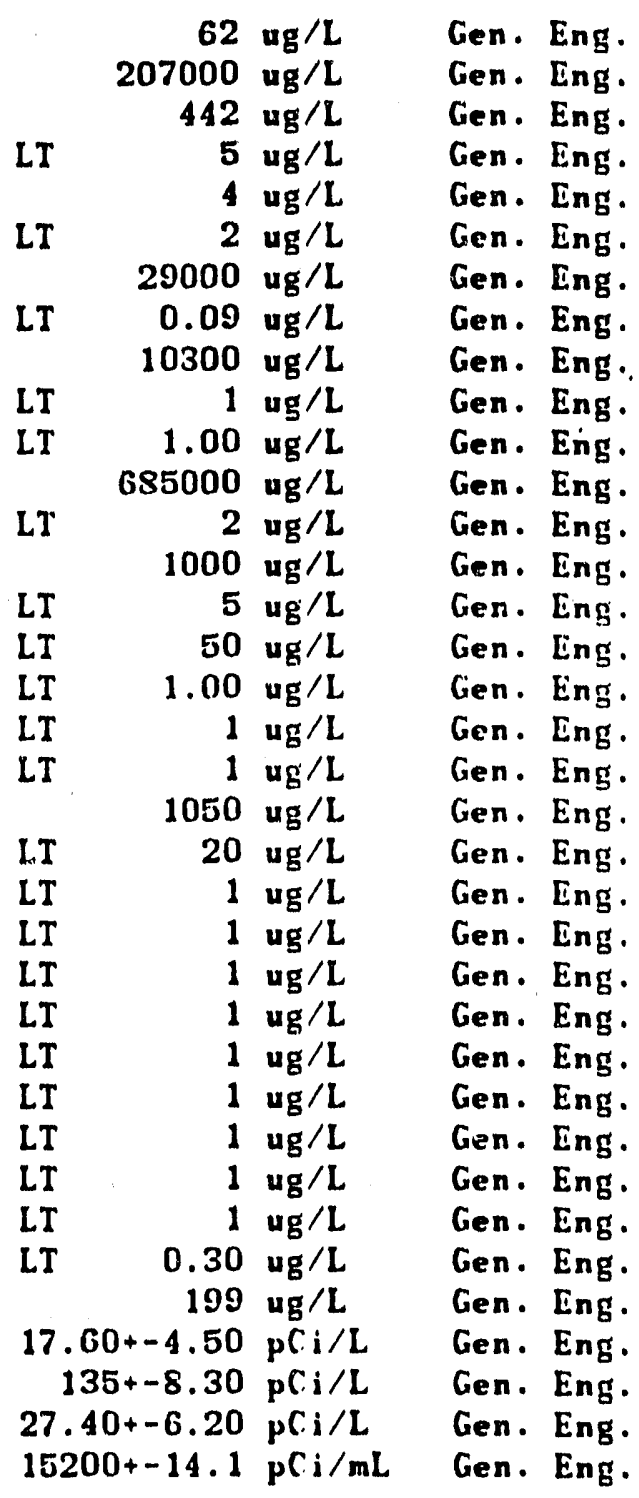


NELL FSB10GC

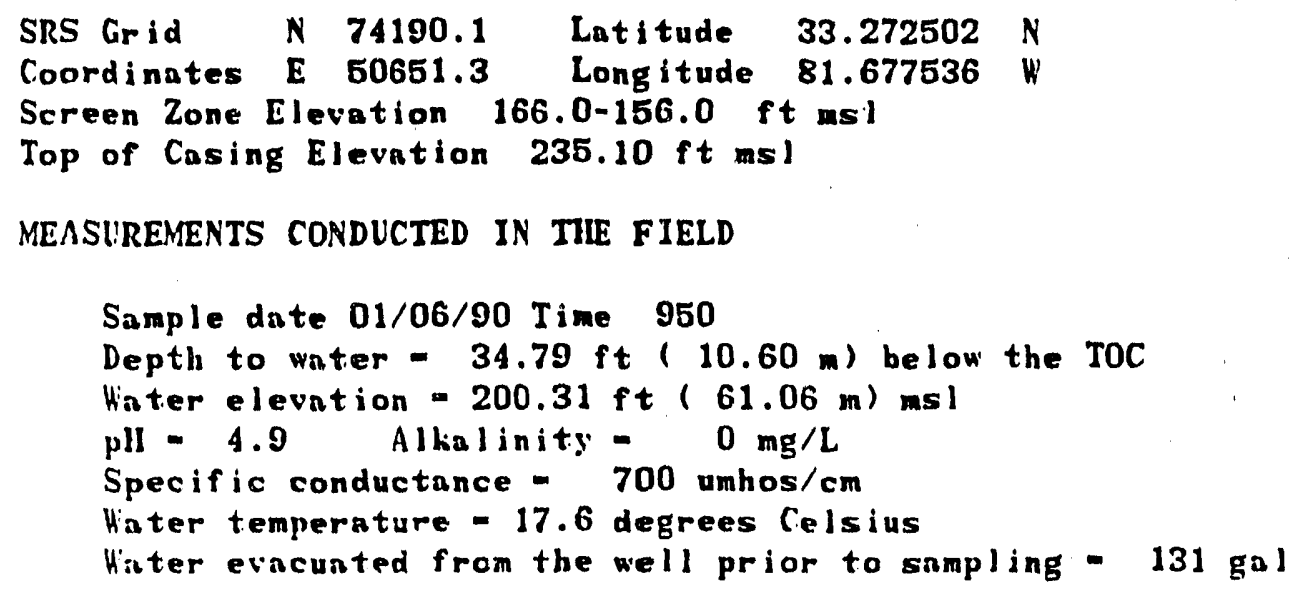

LABORATORY ANALYSES

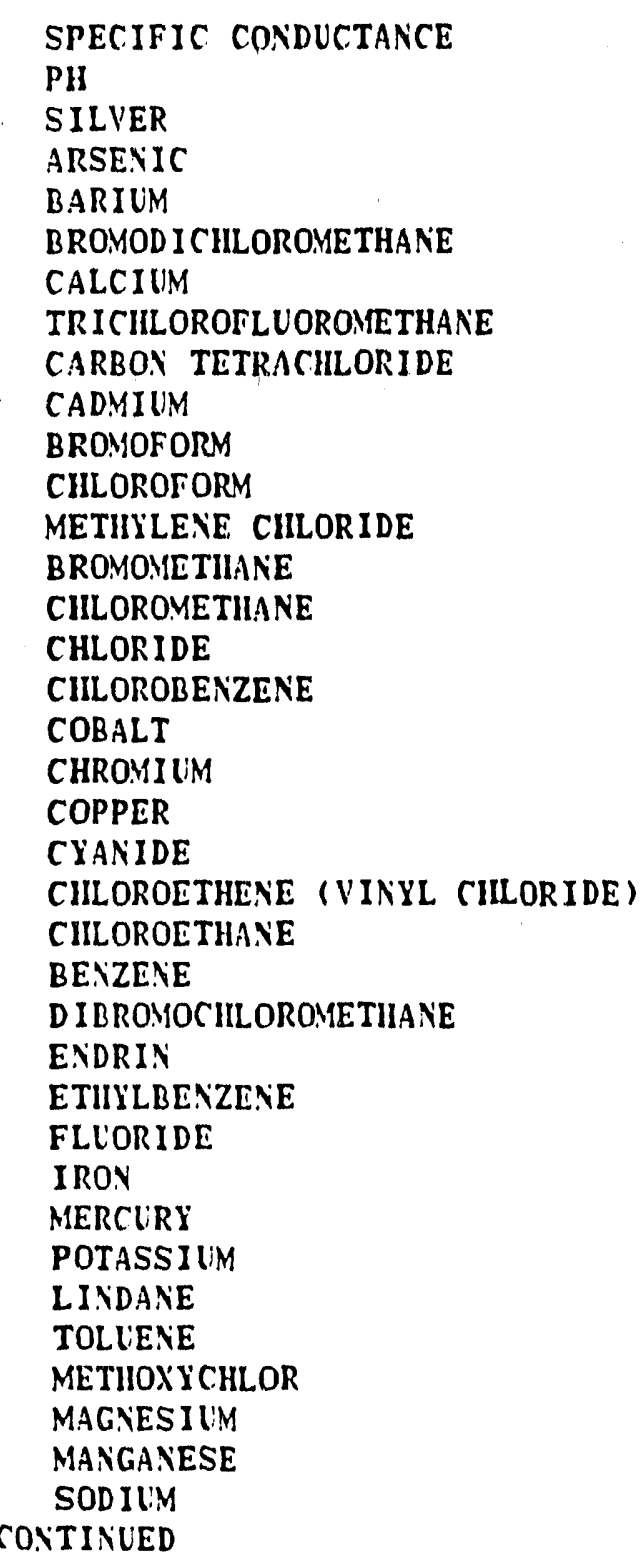

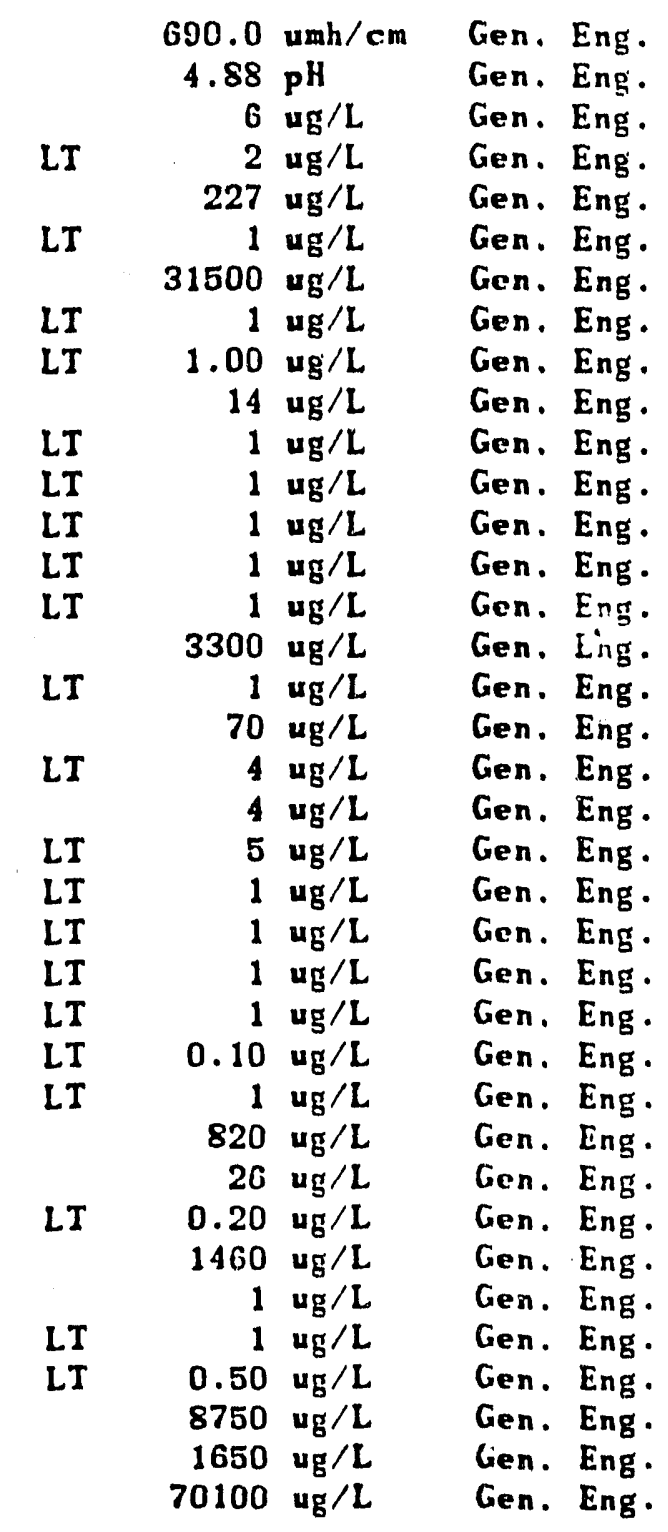


WELL FSB 106C COLLECTED ON 01/06/90 LABORATORX ANALYSES CONTINUED

NICKEL

* NITRATE AS NITROGEN

LEAD

PHENOLS

ANTIMONY

SELENIUM

SILICA

SILVEX

SLLFATE

$1,1,2,2$-TETRACILLROETHANE

TETRACIILOROETIYLENE

TOTAL DISSOLVED SOLIDS

THALL IUM

TOTAL ORGANIC CARBON

TOTAL ORGANIC IIALOGENS

TOTAL PHOSPHATES

TRICIILOROFTHYLENE

TOXAPIIENE

TRANS-1,2-DICILLOROETIENE

URANIUM

VANADIVM

1,1-D ICHLOROETHYLENE

1, 1-D ICILLOROETIIANE

$1,1,1$-TR I CHLOROETHANE

1,1,2-TRICILLOROETIIANE

1,2-D ICIILOROETHANE

1,2-DICIILOROPROPANE

CIS-1,3-DICILOROPROPENE

TRANS-1,3-DICHLOROPROPENE

2-CHLOROETIIYLVINYL ETHER

2,4-DICILOROPHENOXYACETIC ACID

ZINC

GROSS ALPIIA

NOIVOLA'ILE BETA

TOTAL RADIUM

* TRITIUM
$19 \mathrm{ug} / \mathrm{L}$

$81800 \mathrm{ug} / \mathrm{L}$

$6 \mathrm{ug} / \mathrm{L}$

$5 \mathrm{ug} / \mathrm{L}$

3 ug/L

$2 \mathrm{ug} / \mathrm{L}$

$864 \mathrm{ug} / \mathrm{L}$

$0.09 \mathrm{ug} / \mathrm{L}$

$5000 \mathrm{ug} / \mathrm{L}$

$1 \mathrm{ug} / \mathrm{L}$

$1.00 \mathrm{ug} / \mathrm{L}$

$458000 \mathrm{ug} / \mathrm{L}$

$2 \mathrm{ug} / \mathrm{L}$

$1000 \mathrm{ug} / \mathrm{L}$

$5 \mathrm{ug} / \mathrm{L}$

$50 \mathrm{ug} / \mathrm{L}$

$1.00 \mathrm{ug} / \mathrm{L}$

$1 \mathrm{ug} / \mathrm{L}$

$1 \mathrm{ug} / \mathrm{L}$

$1000 \mathrm{ug} / \mathrm{L}$

$20 \mathrm{ug} / \mathrm{L}$

$1 \mathrm{ug} / \mathrm{L}$

$1 \mathrm{ug} / \mathrm{L}$

$1 \mathrm{ug} / \mathrm{L}$

$1 \mathrm{ug} / \mathrm{L}$

$1 \mathrm{ug} / \mathrm{L}$

$1 \mathrm{ug} / \mathrm{L}$

$1 \mathrm{ug} / \mathrm{L}$

$1 \mathrm{ug} / \mathrm{L}$

$1 \mathrm{ug} / \mathrm{L}$

$0.30 \mathrm{ug} / \mathrm{L}$

$49 \mathrm{ug} / \mathrm{L}$

$3 \mathrm{pCi} / \mathrm{L}$

$2 \mathrm{pCi} / \mathrm{L}$

$\begin{array}{lll}\mathrm{LT} & 1 \mathrm{pCi} / \mathrm{L} \\ 3200+-5.80 & \mathrm{pCi} / \mathrm{mL}\end{array}$
Gen. Eng.

Gen. Eng.

Gen. Eng.

Gen. Eng.

Gen. Eng.

Gen. Eng.

Gen. Eng.

Gen. Eng.

Gen. Eng.

Gen. Ing.

Gen. Eng.

Gen. Eng.

Gen. Eng.

Gen. Eng.

Gen. Eng.

Gen. Eng.

Gen. Eng.

Gen. Eng.

Gen. Eng.

Gen. Eng.

Gen. Eng.

Gen. Eng.

Gen. Eng.

Gen. Eng.

Gen. Eng.

Gen. Eng.

Gen. Eng.

Gen. Eng.

Gen. Eng.

Gen. Eng.

Gen. Eng.

Gen. Eng.

Gen. Eng.

Gen. Eng.

Gen. Eng.

Gen. Eng. 
NELL FSB $107 C$

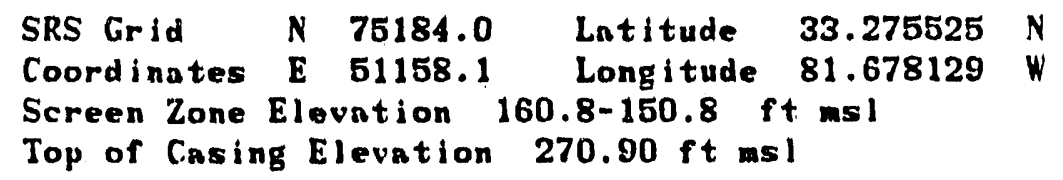

\section{LABORATORY ANALYSES}

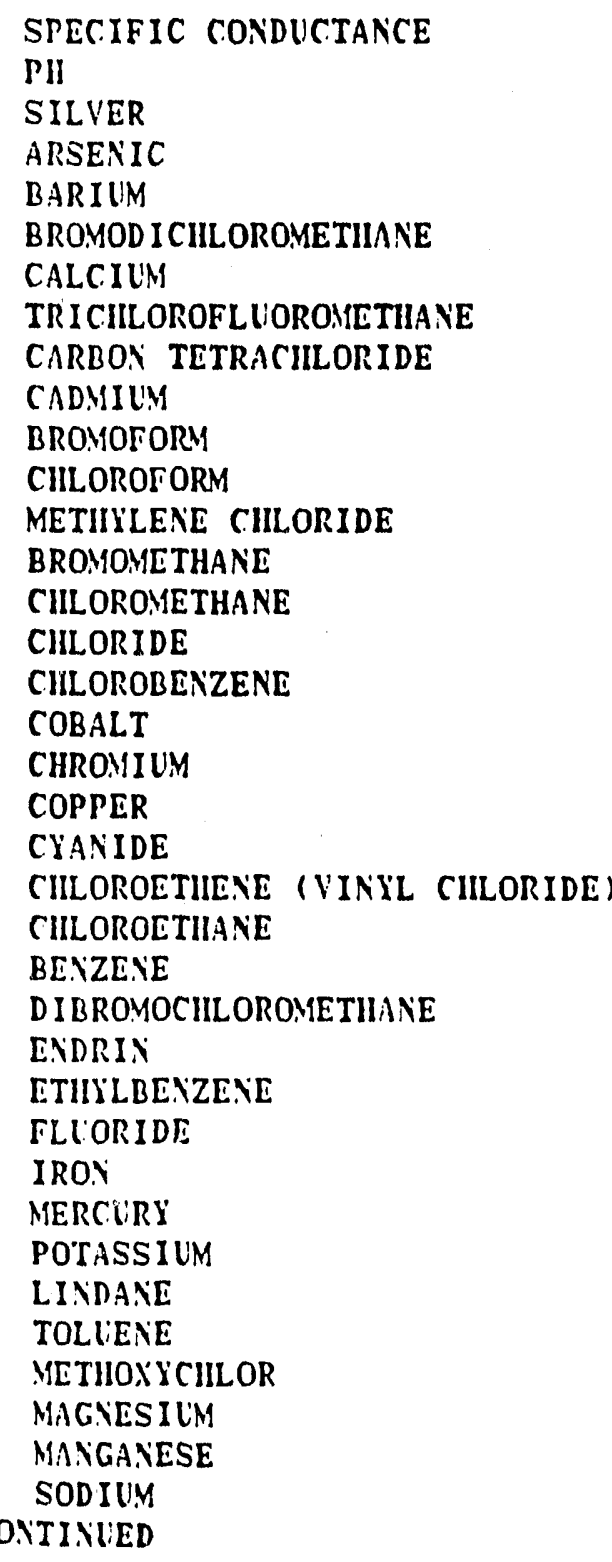

$\begin{array}{lrll} & 296.0 \mathrm{umh} / \mathrm{cm} & \text { Gen. Eng. } \\ \text { LT } & 7.02 \mathrm{ull} & \text { Gen. Eng. } \\ \text { LT } & 2 \mathrm{ug} / \mathrm{L} & \text { Gen. Eng. } \\ & 2 \mathrm{ug} / \mathrm{L} & \text { Gen. Eng. } \\ \text { LT } & 43 \mathrm{ug} / \mathrm{L} & \text { Gen. Eng. } \\ & 1 \mathrm{ug} / \mathrm{L} & \text { Gen. Eng. } \\ \text { LT } & 37800 \mathrm{ug} / \mathrm{L} & \text { Gen. Eng. } \\ \text { LT } & 1.00 \mathrm{ug} / \mathrm{L} & \text { Gen. Eng. } \\ \text { LT } & 2 \mathrm{ug} / \mathrm{L} & \text { Gen. Eng. } \\ \text { LT } & 1 \mathrm{ug} / \mathrm{L} & \text { Gen. Eng. Eng. } \\ \text { LT } & 1 \mathrm{ug} / \mathrm{L} & \text { Gen. Eng. } \\ \text { LT } & 1 \mathrm{ug} / \mathrm{L} & \text { Gen. Eng. } \\ \text { LT } & 1 \mathrm{ug} / \mathrm{L} & \text { Gen. Eng. } \\ \text { LT } & 1 \mathrm{ug} / \mathrm{L} & \text { Gen. Eng. } \\ & 2500 \mathrm{ug} / \mathrm{L} & \text { Gen. Eng. } \\ \text { LT } & 1 \mathrm{ug} / \mathrm{L} & \text { Gen. Eng. } \\ \text { LT } & 4 \mathrm{ug} / \mathrm{L} & \text { Gen. Eng. } \\ \text { LT } & 4 \mathrm{ug} / \mathrm{L} & \text { Gen. Eng. } \\ \text { LT } & 4 \mathrm{ug} / \mathrm{L} & \text { Gen. Eng. } \\ \text { LT } & 5 \mathrm{ug} / \mathrm{L} & \text { Gen. Eng. } \\ \text { LT } & 1 \mathrm{ug} / \mathrm{L} & \text { Gen. Eng. } \\ \text { LT } & 1 \mathrm{ug} / \mathrm{L} & \text { Gen. Eng. } \\ \text { LT } & 1 \mathrm{ug} / \mathrm{L} & \text { Gen. Eng. } \\ \text { LT } & 1 \mathrm{ug} / \mathrm{L} & \text { Gen. Eng. } \\ \text { LT } & 0.10 \mathrm{ug} / \mathrm{L} & \text { Gen. Eng. } \\ \text { LT } & 1 \mathrm{ug} / \mathrm{L} & \text { Gen. Eng. } \\ \text { LT } & 100 \mathrm{ug} / \mathrm{L} & \text { Gen. Eng. } \\ \text { LT } & 4 \mathrm{ug} / \mathrm{L} & \text { Gen. Eng. } \\ \text { LT } & 0.20 \mathrm{ug} / \mathrm{L} & \text { Gen. Eng. } \\ & 761 \mathrm{ug} / \mathrm{L} & \text { Gen. Eng. } \\ \text { LT } & 0.05 \mathrm{ug} / \mathrm{L} & \text { Gen. Eng. } \\ \text { LT } & 1 \mathrm{ug} / \mathrm{L} & \text { Gen. Eng. } \\ \text { LT } & 0.50 \mathrm{ug} / \mathrm{L} & \text { Gen. Eng. } \\ & 3740 \mathrm{ug} / \mathrm{L} & \text { Gen. Eng. } \\ & 93 \mathrm{ug} / \mathrm{L} & \text { Gen. Eng. } \\ & 1500 \mathrm{ug} / \mathrm{L} & \text { Gen. Eng. } & \\ & & & \end{array}$




\begin{tabular}{|c|c|c|c|c|c|}
\hline $\begin{array}{l}\text { NICKEL } \\
\text { NITRATE AS NITROGEN }\end{array}$ & LT & $\begin{array}{r}4 \\
15700^{4}\end{array}$ & $\begin{array}{l}u g / L \\
u g / L\end{array}$ & $\begin{array}{l}\text { Gen. } \\
\text { Gen. }\end{array}$ & $\begin{array}{l}\text { Eng } \\
\text { Eng }\end{array}$ \\
\hline LEAD & LT & 6 & $\mathrm{ug} / \bar{L}$ & Gen. & Eng \\
\hline PIIENOLS & LT & 5 & $4 \mathrm{~g} / \mathrm{L}$ & Gen. & Eng \\
\hline ANTIMONY & LI & 3 & ug/L & Gen. & Eng \\
\hline SELENIUM & LT & 2 & ug/L & Gen. & Eng \\
\hline SILICA & & $\mathbf{8 2 3 0}$ & $\mathbf{u g} / \mathrm{L}$ & Gen. & - Eng \\
\hline SILVEX & LT & 0.09 & ug/L & Gen. & Eng \\
\hline SULFATE & LT & 5000 & $\mathrm{ug} / \mathrm{L}$ & Gen: & - Eng \\
\hline $1,1,2,2$-TETKACHLOROETIIANE & LT & 1 & $\mathbf{u g} / \mathrm{L}$ & Gen. & Eng \\
\hline TETRACIILOROETIIYLENE & & 2.00 & $\mathbf{u g} / \mathrm{L}$ & Gen. & Eng \\
\hline TOTAL DISSOLVED SOLIDS & & 210000 & $w_{t} / L$ & Gen. & - Eng \\
\hline THALLIUM & LT & 2 & ug $/ \mathrm{L}$ & Gen. & Eng \\
\hline TOTAL ORGANTC CARBON & & 4000 & $\operatorname{ug} / \mathrm{L}$ & Gen. & - Eng \\
\hline TOTAL ORGANIC HALOGENS & LT & 5 & $\mathrm{ug} / \mathrm{L}$ & Gen. & Eng \\
\hline TOTAL PIIOSPIIATES & & 80 & $\mathbf{u g} / \mathrm{L}$ & Gen. & Eng \\
\hline TRICHLOROETIIYLENE & & 1.00 & $\mathbf{u g} / \mathrm{L}$ & Gen. & Eng \\
\hline TOXAPHERE & LT & 1 & $\mathrm{ug} / \mathrm{L}$ & Gen. & - Eng \\
\hline TRANS-1,2-DICILLOROETIIENE & LT & 1 & $\mathbf{u g} / \mathrm{L}$ & Gen. & - Eng \\
\hline URANIUM & LT & 1000 & $u g / L$ & Gen. & . Eng \\
\hline VANADIUM & LT & 20 & $\mathrm{ug} / \mathrm{L}$ & Gen. & - Ens \\
\hline 1,1-DICHLOROETHYLENE & LT & 1 & ug $/ \mathrm{L}$ & Gen. & . En \\
\hline 1,1-DICHLOROETHANE & LT & 1 & $\mathrm{ug} / \mathrm{L}$ & Gen. & . En \\
\hline $1,1,1$-TRICHLOROETHANE & LT & 1 & $\mathbf{u g} / \mathrm{L}$ & Gen. & - $\mathbf{E}$ \\
\hline $1,1,2$-TRI CILLOROETHANE & LT & 1 & ug $/ \mathrm{L}$ & Gen. & . Eng̨ \\
\hline 1,2-DICILLOROETHIANE & LT & 1 & $\mathbf{u g} / \mathrm{L}$ & Gen. & - En \\
\hline 1,2-DICIILOROPROPANE & LT & 1 & ug $/ \mathrm{L}$ & Gen. & . \\
\hline CIS-1,3-DICIILOROPROPENE & LT & 1 & ug $/ L$ & Gen. & . Eng \\
\hline TRANS-1,3-DICHLOROPROPENE & LT & 1 & ug $/ \mathrm{L}$ & Gen. & . Eng \\
\hline 2-CHLOROETHYLVINYL ETIIER & LI & 1 & $\mathrm{ug} / \mathrm{L}$ & Gen. & - Eng \\
\hline $\begin{array}{l}\text { 2,4-DICILLROPHENOXYACETIC ACID } \\
\text { ZINC }\end{array}$ & LT & $\begin{array}{l}0.30 \\
18\end{array}$ & $\mathrm{ug} / \mathrm{L}$ & $\begin{array}{l}\text { Gen. } \\
\text { Gen. }\end{array}$ & Eng \\
\hline GROSS ALPIIA & LT & 3 & $\mathrm{pC}_{\mathrm{i}} / \mathrm{L}$ & Gen. & \\
\hline NONVOLATILE BETA & & 9.50 & $\mathrm{Ci} / \mathrm{L}$ & Ger & \\
\hline TOTAL RADIUM & LT & 1 & $\mathrm{PCi} / \mathrm{L}$ & Gen. & \\
\hline TRITIUM & & -2.07 & $\mathrm{pCi} / \mathrm{mL}$ & Gen. & \\
\hline
\end{tabular}


NELL FSB107D

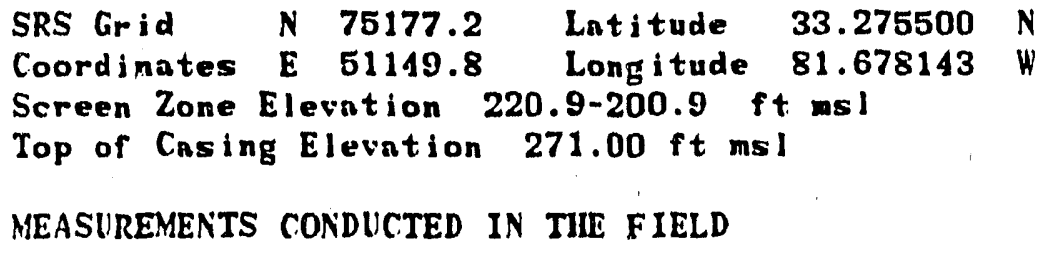

Sample date 01/01/90 Time 1500

Depth to water - $59.77 \mathrm{ft}(18.22 \mathrm{~m})$ below the TOC

Niater elevation $=211.23 \mathrm{ft}(64.38 \mathrm{~m}) \mathrm{ms}$ l

pll = $3.8 \quad$ Alkalinity $=0 \mathrm{mg} / \mathrm{L}$

Specific conductance - 297 umhos/cm

Water tempernture - 17.0 degrees Celsius

Hater evacuated from the well prior to sumpling - $42 \mathrm{gal}$

LABORATORY ANALYSES

\begin{tabular}{|c|c|c|c|c|}
\hline $\begin{array}{l}\text { SPECIFIC CONDUCTANCE } \\
\text { PII }\end{array}$ & & $\begin{array}{r}289.0 \\
3.91\end{array}$ & $\begin{array}{l}\mathrm{umh} / \mathrm{cm} \\
\mathrm{pH}\end{array}$ & $\begin{array}{l}\text { Gen. } \\
\text { Gen. }\end{array}$ \\
\hline SILVER & LT & 2 & ug/L & Gen. \\
\hline ARSENIC & LT & 2 & ug $/ L$ & Gen. \\
\hline BARIUM & & 72 & ug $/ \mathrm{L}$ & Gen. \\
\hline BROMOD I CHLOROMETHUNE & LT & 1 & ug $/ \mathrm{L}$ & Gen. \\
\hline CALCIIM & & 1530 & $u g / L$ & Gen. \\
\hline TRICILLOROFLUOROMETIIANE & LT & 1 & ug/L & Gen. \\
\hline CARBON TETRACILORIDE & LT & 1.00 & ug $/ \mathrm{L}$ & Gen. \\
\hline CADMIUM & LT & 2 & ug $/ L$ & Gen. \\
\hline BROMOFORM & LT & 1 & ug $/ \mathrm{L}$ & Gen. \\
\hline CHLOROFORM & LI & 1 & ug $/ \mathrm{L}$ & Gen. \\
\hline METHILENE CILLORIDE & LT & 1 & $u g / L$ & Gen. \\
\hline BROMOMETHANE & LT & 1 & $u g / L$ & Gen. \\
\hline CILLOROMETHANE & LT & 1 & ug $/ \mathrm{L}$ & Gen. \\
\hline CIILORIDE & & 2400 & $\mathrm{ug} / \mathrm{L}$ & Gen. \\
\hline CHLOROBENZENE & LT & 1 & ug $/ \mathfrak{L}$ & Gen. \\
\hline COBALT & LT & 4 & ug $/ \mathrm{L}$ & Gen. \\
\hline CIIROMIUM & LI & 4 & ug/L & Gen. \\
\hline COPPER & & 16 & ug $/ L$ & Gen. \\
\hline CYANIDE & LT & 5 & $\operatorname{ug} / \mathrm{L}$ & Gen. \\
\hline CIILOROETHENE (VINYL CHLORIDE) & $L T$ & 1 & $u g / L$ & Gen. \\
\hline CIILOROETHANE & LI & 1 & ug $/ \mathfrak{L}$ & Gen. \\
\hline BENZENE & LI & 1 & $\operatorname{ug} / \mathrm{L}$ & Gen. \\
\hline DIBROMOCIILOROMETIIANE & LI & 1 & ug $/ L$ & Gen. \\
\hline ENDRIN & LI & 0.10 & ug $/ L$ & Gen. \\
\hline ETIILBEYZENE & $L T$ & 1 & $u g / L$ & Gen. \\
\hline FLUORIDE & & 410 & ug / L & Gen. \\
\hline IRON & & 102 & ug $/ L$ & Gen. \\
\hline MLRCURY & & 0.30 & ug $/ L$ & Gen. \\
\hline POTASSILM & LT & 500 & ug $/ L$ & Gen. \\
\hline LINDANE & IT & 0.05 & ug $/ L$ & Gen. \\
\hline TOLLENE & LT & 1 & ug $/ \mathrm{L}$ & Gen. \\
\hline METHOXYCHLOR & LT & 0.50 & ug $/ L$ & Gen. \\
\hline MAGNESIUM & & 907 & ug $/ \mathrm{L}$ & Gen. \\
\hline MANGANESE & & 397 & ug $/ L$ & Gen. \\
\hline SODIUM & & 30800 & $\mathrm{ug} / \mathrm{L}$ & Gen. \\
\hline
\end{tabular}


NICXEL

NITRATE AS NITROGEN

LEAD

PIIENOLS

ANTIMONY

SELENIUM

SILICA

SILVEX

SULFATE

$1,1,2,2$-TETRACIILOROETIMNE

TE, IRACIILOROETHYLENE

TOTAL DISSOLVED SOLIDS

TIIALLIUN

TOTAL ORGANIC CARBON

IOTAL ORGANIC HALOGENS

TOTAL PIIOSPIIATES

TRICIILOROETIIYLENE

TOKAPHENE

TRANS-1, 2-DICILOROETHENE

URANIUM

VAKADIUM

1,1-DICIILOROETIYLENE

1,1-DICIILOROETHANE

$1,1,1$-TRICHLOROETHANE

$1,1,2$-TRICIILOROETHANE

1,2-DICIILOROETIIANE

1,2-DICIILOROPROPANE

CIS-1,3-DICIILOROPROPENE

TRANS-1 , 3-D ICILLOROPROPENE

2-CIILOROETIILL VINIL ETHER

2,4-DICHLOROPHENOXYACETIC ACIL

ZINC

CROSS ALPHA

NONVOLATILE BETA

TOTAL RADILM

* TRITIUM
LI

LT
LI

LT

LI

LT

LT

T

LT

$\mathrm{T}$

T

I

T

\section{LT}

LT

LI

LT

LT

LT

LI

LT

LT

LT

LT

LT

LT

LT

LI

LT

LT

LT

LI

LT

$1200+-2.70 \mathrm{pCi} / \mathrm{mL}$
Gen. Eng.

Gen. Eng.

Gen. Eng.

Gen. Eng.

Gen. Eng.

Gen. Eng.

Gen. Eng.

Gen. Eng.

Gen. Eng.

Gen. Eng.

Gen. Eng.

Gen. Eng.

Gen. Eng.

Gen. Eng.

Gen. Eng.

Gen. Eng.

Gen. Eng.

Gen. Eng.

Gen. Eng.

Gen. Eng.

Gen. Eng.

Gen. Eng.

Gen. Eng.

Gen. Eng.

Gen. Eng.

Gen. Eng.

Gen. Eng.

Gen. Eng.

Gen. Eng.

Gen. Eng.

Gen. Eng.

Gen. Eng.

Gen. :,

Gen. kng.

Gen. Eng.

Gen. Eng. 
MEASUREMENTS CONDUCTED IN THE FIELD

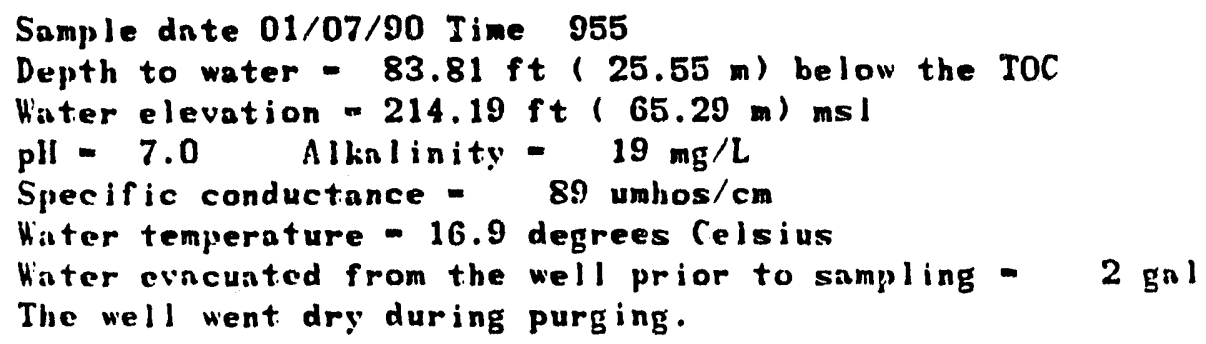

LABORATORY ANALYSES

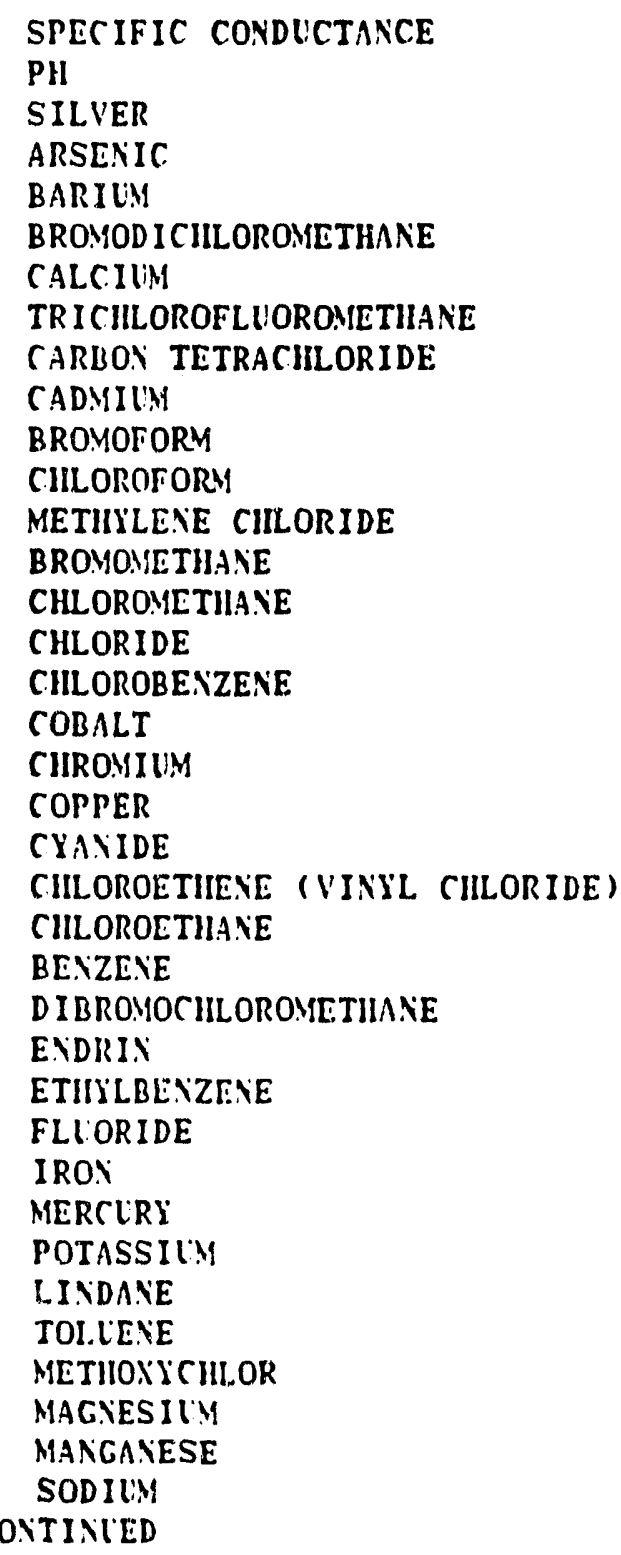

\begin{tabular}{|c|c|c|c|c|}
\hline & $\begin{array}{r}73.00 \\
6.81\end{array}$ & $\begin{array}{l}\mathrm{umh} / \mathrm{cm} \\
\mathrm{pH}\end{array}$ & $\begin{array}{l}\text { Gen. } \\
\text { Gen. }\end{array}$ & $\begin{array}{l}\text { Eng. } \\
\text { Eng. }\end{array}$ \\
\hline LT & 2 & $\operatorname{ug} / \mathrm{L}$ & Gen. & Eng. \\
\hline LT & 2 & ug $/ L$ & Gen. & Eng. \\
\hline & 15 & $\operatorname{ug} / \mathrm{L}$ & Gen. & Eng. \\
\hline LT & 1 & $u_{\mathrm{g}} / \mathrm{L}$ & Gen. & Eng. \\
\hline & 7880 & ug $/ L$ & Gen. & Eng. \\
\hline LI & 1 & ug $/ \mathfrak{L}$ & Gen. & Eng. \\
\hline LT & 1.00 & ug $/ \mathrm{L}$ & Gen. & Eng. \\
\hline LT & 2 & $u g / L$ & Gen. & Eng. \\
\hline LT & 1 & ug $/ \mathrm{L}$ & Gen. & Eng. \\
\hline LI & 1 & $4 \mathrm{~g} / \mathrm{L}$ & Gen. & Eng. \\
\hline LT & 1 & ug $/ \mathrm{L}$ & Gen. & Eng. \\
\hline LT & 1 & $u_{\mathrm{g}} / \mathrm{L}$ & Gen. & Eng. \\
\hline LT & 1 & $\mathrm{ug} / \mathrm{L}$ & Gen. & Eng. \\
\hline & 3100 & ug $/ L$ & & \\
\hline LI & 1 & ug $/ \mathrm{L}$ & Gen. & Eng. \\
\hline LI & 4 & $u_{E} / L$ & Gen. & $\mathrm{g}$. \\
\hline LI & 4 & ug / L & Gen. & Eng. \\
\hline LI & 4 & ug $/ L$ & Gen. & Eng \\
\hline LT & 5 & ug $/ \mathrm{L}$ & Gen. & Eng \\
\hline LT & 1 & $\mathrm{ug} / \mathrm{L}$ & Gen. & $\operatorname{Eng}$ \\
\hline LT & 1 & ug $/ \mathrm{L}$ & Gen. & Eng \\
\hline LT & 1 & $u_{g} / L$ & Gen. & Eng \\
\hline LT & 1 & ug $/ \mathrm{L}$ & Gen. & Int \\
\hline LT & 0.10 & $u_{\mathrm{g}} / \mathrm{L}$ & Gen. & En \\
\hline LT & 1 & $u g / L$ & Gen. & En \\
\hline $\mathrm{LT}$ & 100 & ug $/ \mathrm{L}$ & Gen. & En \\
\hline & 7 & $\mathrm{ug}_{\mathrm{g}} / \mathrm{L}$ & Gen. & En \\
\hline LI & 0.20 & $u_{g} / L$ & Gen. & En! \\
\hline LT & 500 & $u_{g} / L$ & Gen. & En \\
\hline LT & 0.05 & $u_{g} / L$ & Gen. & En \\
\hline LT & 1 & ug $/ \mathrm{L}$ & Gen. & En \\
\hline LT & 0.50 & $u_{g} / L$ & Gen. & En \\
\hline & 482 & ug $/ \mathrm{L}$ & Gen. & \\
\hline & $\begin{array}{r}21 \\
3720\end{array}$ & $\begin{array}{l}\text { ug } / L \\
\text { ug } / L\end{array}$ & $\begin{array}{l}\text { Gen. } \\
\text { Gen. }\end{array}$ & Eng \\
\hline
\end{tabular}


NELL FSB108D COLLECTED ON 01/07/90 LABORATORY ANALYSES CONTINLED

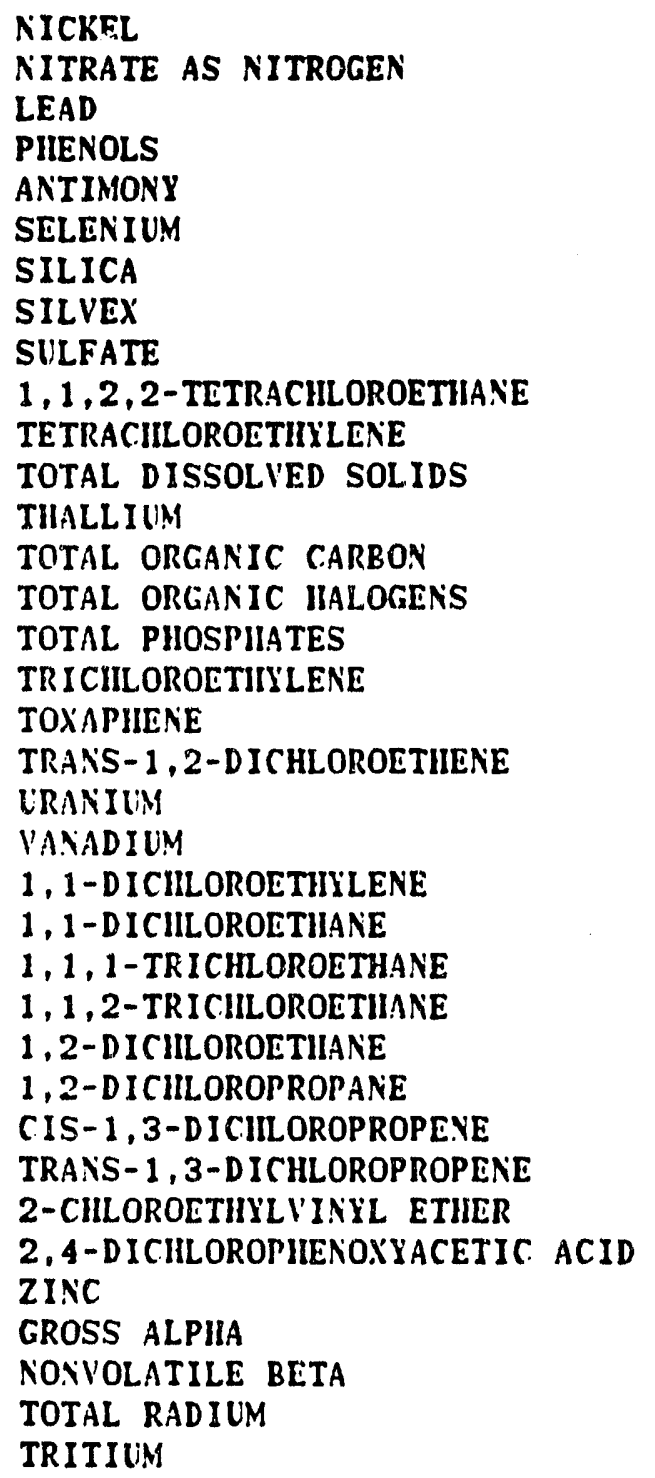

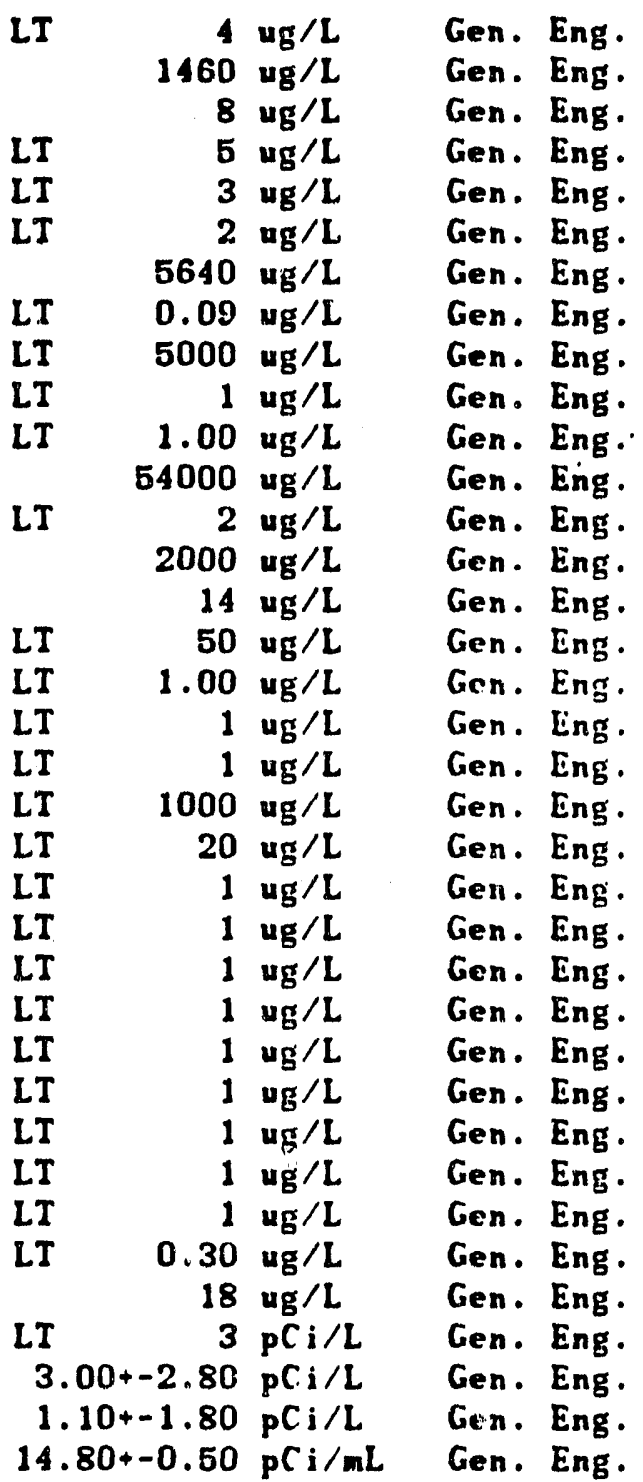




\section{K'ELL FSB109D}

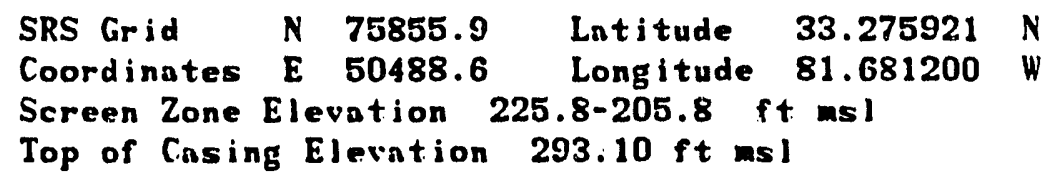

MEASUREMENTS CONDUCTED IN THE FIELD

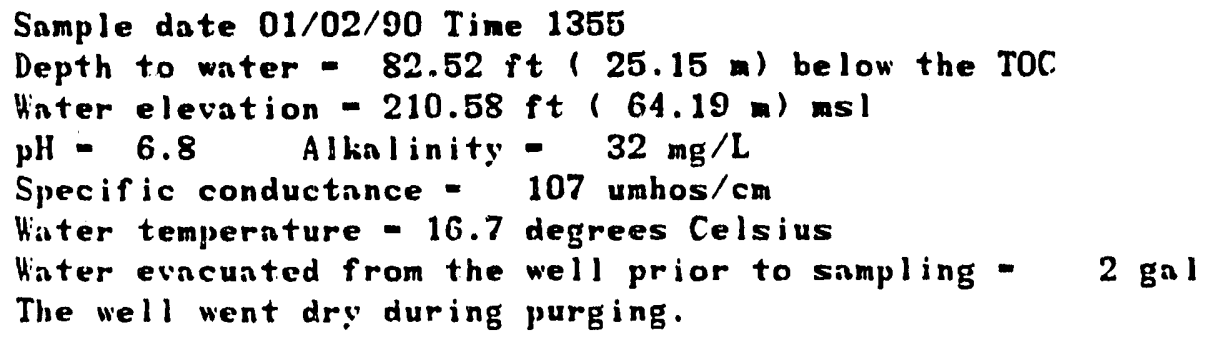

LABOPATORY ANALYSES

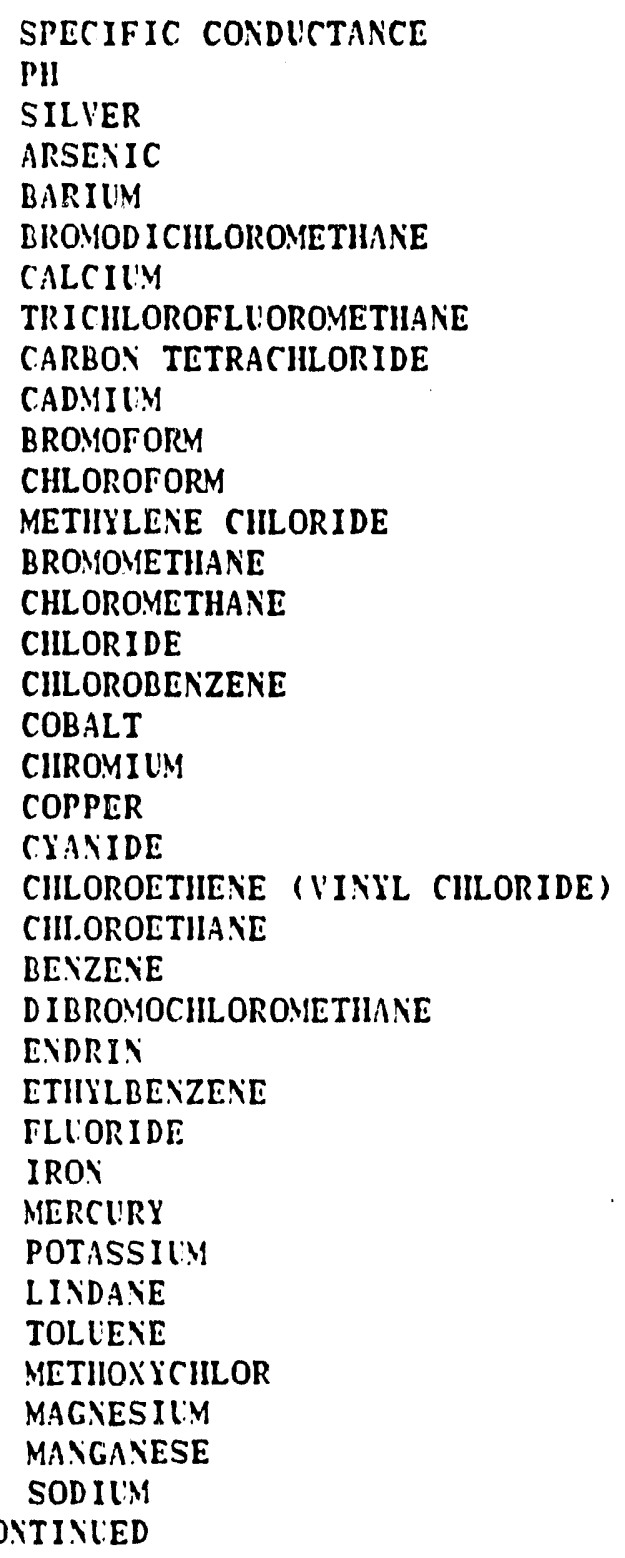

$78.00 \mathrm{umh} / \mathrm{cm}$ Gen. Eng. $7.00 \mathrm{pH}$ Gen. Eng. LT 2 ug/L Gen. Eng. LI $2 \mathrm{ug} / \mathrm{L}$ Gen. Eng. 12 ug $/ L$ Gen. Eng. LI $1 \mathrm{ug} / \mathrm{L}$ Gen. Eng. $4230 \mathrm{ug} / \mathrm{L}$ Gen. Eng. $\begin{array}{rrr}\text { LT } & 1 \mathrm{ug} / \mathrm{L} & \text { Gen. Eng. } \\ \text { LT } & 1.00 \mathrm{ug} / \mathrm{L} & \text { Gen. Eng. }\end{array}$ LT 2 ug/L Gen. Eng. LT 1 ug/L Gen. Eng. LT 1 ug/L Gen. Eng. LT 1 ug/L Gen. Eng. IT $1 \mathrm{ug} / \mathrm{L}$. Gen. Eng. LI $1 \mathrm{ug} / \mathrm{L}$ Gen. Eng. $1200 \mathrm{ug} / \mathrm{L}$ Gen. Eng. 1 ug/L Gen. Eng. $4 \mathrm{ug} / \mathrm{L}$ Gen. Eng. $4 \mathrm{ug} / \mathrm{L}$ Gen. Eng. $4 \mathrm{ug} / \mathrm{L}$ Gen. Eng. 5 ug/L Gen. Eng. $1 \mathrm{ug} / \mathrm{L}$ Gen. Eng. 1 ug/L Gen. Eng. 1 ug $/ \mathrm{L}$ Gen. Eng. $1 \mathrm{ug} / \mathrm{L}$ Gen. Eng. $0.10 \mathrm{ug} / \mathrm{L}$ Gen. Eng. $1 \mathrm{ug} / \mathrm{L}$ Gen. Eng. $100 \mathrm{ug} / \mathrm{L}$ Gen. Eng. $18 \mathrm{ug} / \mathrm{L}$ Gen. Eng. $0.20 \mathrm{ug} / \mathrm{L}$ Gen. Eng. $1500 \mathrm{ug} / \mathrm{L}$ Gen. Eng. $0.05 \mathrm{ug} / \mathrm{L}$ Gen. Eng. 1 ug/L Gen. Eng. $0.50 \mathrm{ug} / \mathrm{L}$ Gen. Eng. $1280 \mathrm{ug} / \mathrm{L}$ Gen. Eng. $10 \mathrm{ug} / \mathrm{L}$ Gen. Eng. $10000 \mathrm{ug} / \mathrm{L}$ Gen. Eng. 
RELL PSB109D COLLECTED ON 01/02/90 LABORATORY ANALYSES CONTINLED

NICKEL

NITRATE AS NITROGEN

LEAD

PHENOLS

A.TIMONY

SELENIUM

SILICA

SILVEX

SULFATE

$1,1,2,2$-TETRACHLOROETIIANE TETRACILOROETHILENE TOTAL DISSOLVED SOLIDS

THALLIG'M

TOTAL ORGANIC CAREON

TOTAL ORGANIC HALOGENS

TOTAL PHOSPIIATES

TRICIILOROETIYLENE

TOXAPHE.VE

IRANS-1, 2-DICILOROETIIENE

URANIUM

VANADILN

1,1-DICILOROETIILENE

1, 1-DICIILOROETHANE

$1,1,1$-TRICILOROETHANE

$1,1,2$-TRICILOROETHANE

1,2-DICIILOROETHANE

1,2-DICHLOROPROPANE

CIS-1,3-DICIILOROPROPENE

TRANS-1,3-DICHLOROPROPENE

2-CHLOROETIYLVINYL ETIIER

2,4-DICHLOROPHENOXYACETIC ACID

ZINC

GROSS ALPIIA

NONVOLATILE BETA

TOTAL RADILIM

- TRITIUM
LT

LT

LT

LT

LT

LT

LT

LT

LT

LT

2 ug/L

$2000 \mathrm{ug} / \mathrm{L}$

$11 \mathrm{wg} / \mathrm{L}$

$50 \mathrm{ug} / \mathrm{L}$

$4.00 \mathrm{ug} / \mathrm{L}$

$1 \mathrm{ug} / \mathrm{L}$

$1 \mathrm{ug} / \mathrm{L}$

LT

LI

LT

LT

LT

LT

LT

LT

LT

LT

LT

LT

LT

LT

LT

$1.10+-1.90 \mathrm{pCi} / \mathrm{L}$ $150+-1.33 \mathrm{pCi} / \mathrm{mL}$
Gen. Eng.

Gen. Eng.

Gen. Eng.

Gen. Eng.

Gen. Eng.

Gen. Eng.

Gen. Eng.

Gen. Eng.

Gen. Eng.

Gen. Eng.

Gen. Eng.

Gen. Eng.

Gen. Eng.

Gen. Eng.

Gen. Eng.

Gen. Eng.

Gen. Eng.

Gen. Eng.

Gen. Eng.

Gen. Eng.

Gen. Eng.

Gen. Eng.

Gen. Eng.

Gen. Eng.

Gen. Eng.

Gen. Eng.

Gen. Eng.

Gen. Eng.

Gen. Eng.

Gen. Eng.

Gen. Eng.

Gen. Eng.

Gen. Eng.

Gen. Eng.

Gen. Eng.

Gen. Eng. 


\section{HELL FSB $110 C$}

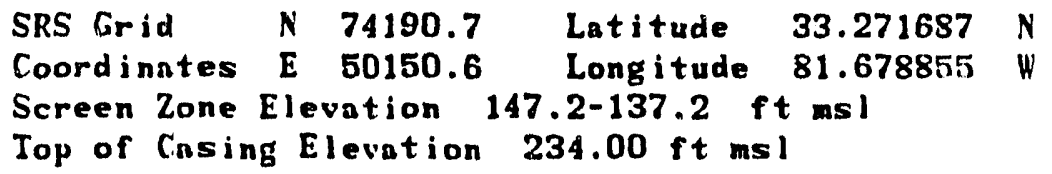

SPECIFIC CONDUCTANCE

PII

SILIER

ARSEAIC

BARILN

BROMODICILLOROMETHANE

CALCIVIM

TRICIILOROFLUOROMETHANE

CARBON TETRACHLORIDE

CADMILM

BROMOFORM

CHILOROTORM

METIILENE CILLORIDE

BROMOMETHANE

CILLORONETHANE

CHILORIDE

CHLOROBENZENE

COBALT

CIIROMILM

COPPER

CYAIIDE

CILOROETHENE (VINIL CILORIDE)

CILOROETHINE

BENZENE

DIBROYOCILOROMETIINE

ENDRIN

ETIILBENZENE

FLLORIDE

IROX

MERCLRY

POTASSIUM

LINDANE

TOLLENE

METHOXYCIILOR

MAGNESILM

MANGANESE

SODILM

CONTINIED

\begin{tabular}{|c|c|c|c|c|}
\hline & $\begin{array}{r}269.0 \\
6.34\end{array}$ & $\begin{array}{l}\text { umh } / \mathrm{cm} \\
\mathrm{pII}\end{array}$ & $\begin{array}{l}\text { Gen. } \\
\text { Gen. }\end{array}$ & $\begin{array}{l}\text { Eng. } \\
\text { Eng. }\end{array}$ \\
\hline LI & 2 & ug $/ \lambda$ & Gen. & Eng. \\
\hline \multirow[t]{2}{*}{ LI } & 2 & ug $/ L$ & Gen. & Eng. \\
\hline & 71 & ug $/ \mathrm{L}$ & Gen. & Eng. \\
\hline LT & $\begin{array}{r}1 \\
35078\end{array}$ & ug $/ L$ & $\begin{array}{l}\text { Gen. } \\
\text { Gen. }\end{array}$ & $\begin{array}{l}\text { Eng. } \\
\text { Eng. }\end{array}$ \\
\hline$L T$ & 1 & ug $/ L$ & Gen. & Eng. \\
\hline LT & 1.00 & $\mathrm{ug} / \mathrm{L}$ & Gen. & Eng. \\
\hline LT & 2 & ug $/ L$ & Gen. & Eng. \\
\hline LI & 1 & ug $/ \mathrm{L}$ & Gen. & Eng. \\
\hline LT & 1 & $4 \mathrm{~g} / \mathrm{L}$ & Gen. & Eng. \\
\hline LT & 1 & ug $/ L$ & Gen. & Eng. \\
\hline LT & 1 & ug $/ \mathrm{L}$ & Gen. & Eng. \\
\hline LT & 1 & ug $/ L$ & Gen. & Eng. \\
\hline & 3000 & ug /L & Gen. & irig. \\
\hline LT & 1 & ug $/ L$ & Gen. & Eng. \\
\hline LT & 4 & ug $/ \mathrm{L}$ & Gen. & Eng. \\
\hline LT & 4 & ug $/ L$ & Gen. & Eng - \\
\hline & 6 & ug $/ \mathrm{L}$ & & \\
\hline LT & 5 & ug $/ L$ & Gen. & Eng. \\
\hline LI & 1 & ug $/ \mathrm{L}$ & Gen. & Eng. \\
\hline LT & 1 & wg $/ L$ & Gen. & Eng. \\
\hline LT & 1 & ug $/ L$ & Gen. & Eng. \\
\hline LT & 1 & ug $/ L$ & Gen. & Eng. \\
\hline LT & 0.10 & ug $/ \mathrm{L}$ & Gen. & Eng. \\
\hline LT & 1 & ug $/ \mathrm{L}$ & Gen. & Eng. \\
\hline LT & 100 & $\mu \mathrm{g} / \mathrm{L}$ & Gen. & Eng. \\
\hline & 20 & $u_{g} / L$ & Gen. & Eng. \\
\hline LT & 0.20 & ug $/ \mathrm{L}$ & Gen. & Eng. \\
\hline & 3770 & ug $/ \mathrm{L}$ & Gen. & $\mathrm{En}_{\mathrm{E}}$ \\
\hline LT & 0.05 & ug $/ L$ & Gen. & Eng. \\
\hline IT & 1 & ug $/ \mathrm{L}$ & Gen & Eng \\
\hline LT & 0.50 & ug $/ L$ & Gen. & Eng. \\
\hline & 3460 & $\mathrm{ug} / \mathrm{L}$ & Gen. & \\
\hline & 12 & ug $/ L$ & Gen. & Eng. \\
\hline & 14600 & ug $/ L$ & Gen. & Eng. \\
\hline
\end{tabular}


WELL FSB110C COLLECTED ON 01/16/90 LABORATORY ANALYSES CONTINUED

NICKEL

* NITRATE AS NITROGEN

LEAD

PILENOLS

ANTIMONY

SELENIUM

SILICA

SILVEX

SILFATE

$1,1,2,2$-TETRACHLOROETHANE

TETRACHLOROETHYLENE

TOTAL DISSOLVED SOLIDS

TIIALLIUM

TOTAL ORGANIC CARBON

TOTAL ORGANIC HALOGENS

TOTAL PHOSPIIAT:

TRICHLOROETIYYLENE

TOXAPHENE

TRANS-1,2-D ICIILOROETIIENE

URANIUM

VANADILM

1,1-DICILOROETHYLENE

1,1-DICILLOROETHANE

$1,1,1$-TRICHLOROETHANE

1,1,2-TRICIILOROETHANE

1,2-D I CILLOROETIIANE

1,2-DICILOROPROPANE

CIS-1,3-DICIILOROPROPENE

TRANS-1,3-D ICILLOROPROPENE

2-CHLOROETIIYLVINYL ETHER

2,4-DICILLOROPIIENOXYACETIC ACID

ZINC

GROSS ALPIIA

NONVOLATILE BETA

TOTAL RADIUM

* TRITIUM
LT

LT

LT

LT

LT

LT

LT

LT

LT

LT

LT

LT

LT

LT

LT

LT

LT

LT

LT

LT

LT

LT

LT

LT

LT

LT

LT

22

$$
2 .
$$
$.30+-2.50 \mathrm{pCi} / \mathrm{L}$ $734+-2.80 \mathrm{pCi} / \mathrm{mL}$
Gen. Eng.

Gen. Eng.

Gen. Eng.

Gen. Eng.

Gen. Eng.

Gen. Eng.

Gen. Eng.

Gen. Eng.

Gen. Eng.

Gen. Eng.

Gen. Eng.

Gen. Eng.

Gen. Eng.

Gen. Eng.

Gen. Eng.

Gen. Eng.

Gen. Eng.

Gen. Eng.

Gen. Eng.

Gen. Eng.

Gen. Eng.

Gen. Eng.

Gen. Eng.

Gen. Eng.

Gen. Eng.

Gen. Eng.

Gen. Eng.

Gen. Eng.

Gen. Eng.

Gen. Eng.

Gen. Eng.

Gen. Eng.

Gen. Eng.

Gen. Eng.

Gen. Eng.

Gen. Eng. 
KELL FSB11OD

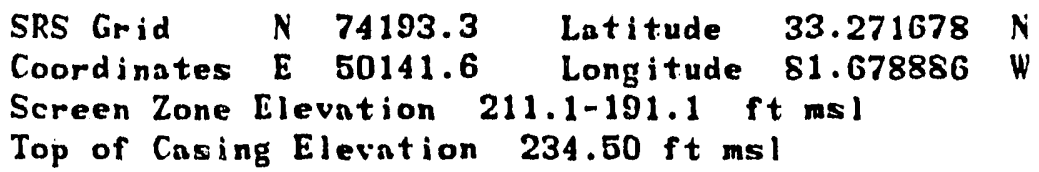

MEASUREMENTS CONDUCTED IN THE FIELD

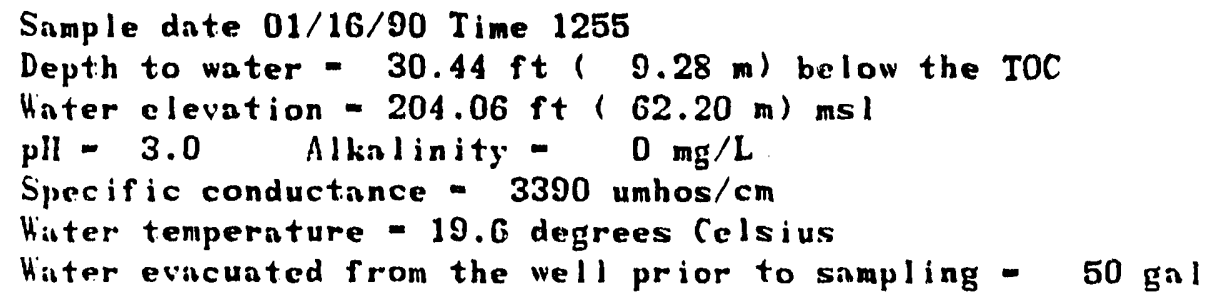

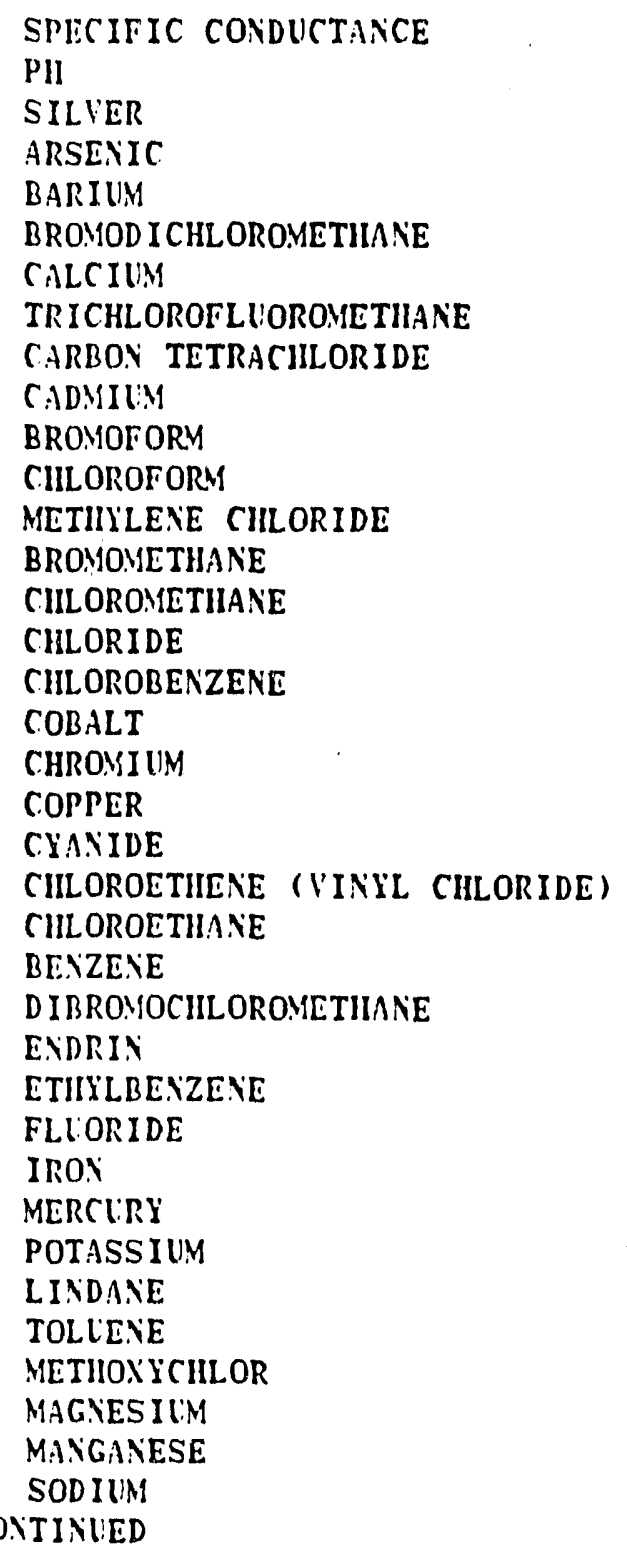

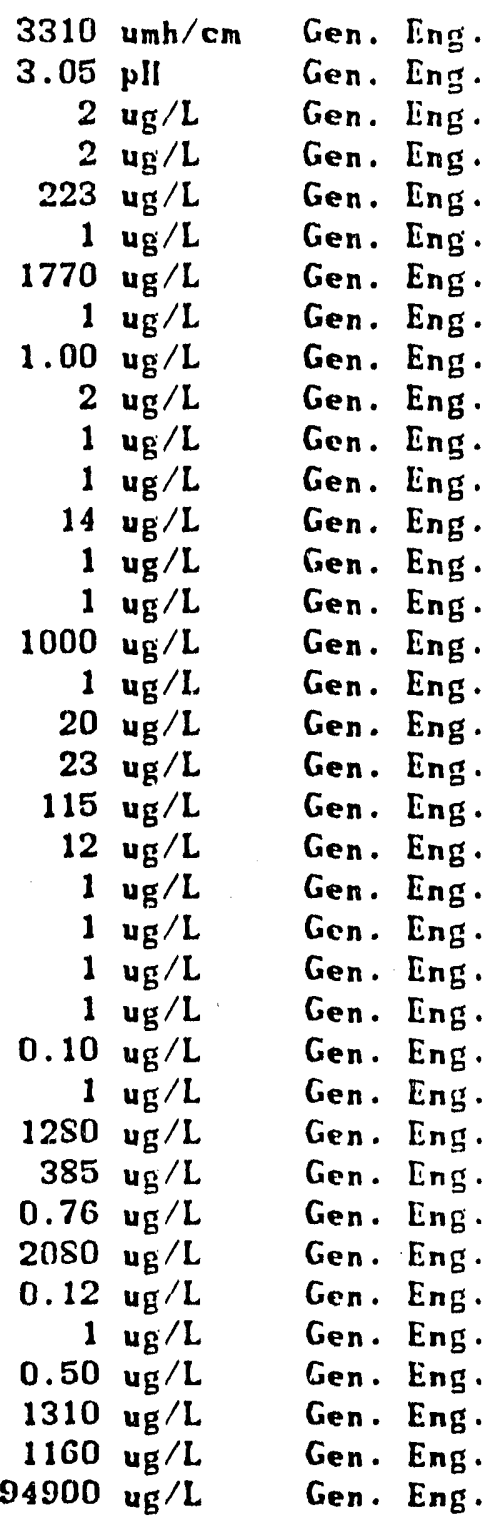


NELL FSB110D COLLECTED ON 01/16/90 LABORATORY ANALYSES CONTINUED

NICKEL

* NITRATE AS NITROGEN

LEAD

PIIENOLS

ANTIMONY

SELENIUM

SILICA

SILVEX

SULFATE

1,1,2,2-TETRACHLOROETHANE

TETRACHLOROETIIYLENE

TOTAL DISSOLVED SOLIDS

THALLYUM

TOTAL ORGANIC CARBON

TOTAL ORGANIC HALOGENS

TOTAL PIIOSPIIATES

TRICIILOROETIMYLENE

TOXAPIENE

TRANS-1,2-D ICIILOROETHENE

URANIUM

VANADIUM

1,1 -D I CIILOROETHYLENE

1,1-DICILLOROETHANE

$1,1,1$-TRICIILOROETHANE

$1,1,2$-TRICHLOROETIIANE

1,2-DICHLOROETHIANE

1,2-DICIILOROPROPANE

CIS-1,3-DICILLOROPROPENE

TRANS-1,3-DICHLOROPROPENE

2-CHLOROETIIYLVINYL ETIIER

2,4-DICHLOROPIIENOXYACETIC ACID ZINC

* gross alpila

* NoNvolatile beta

* total radium

* tRItIlim

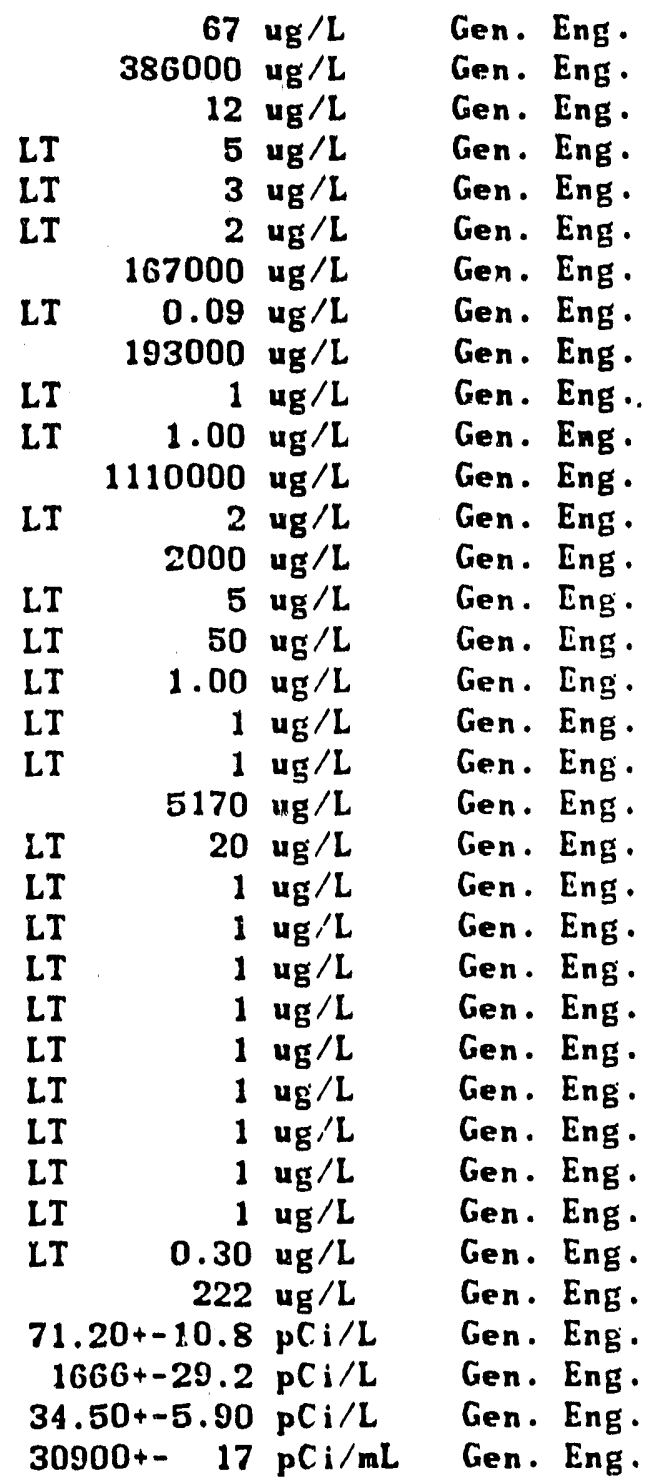


H'ELL FSB $110 D$

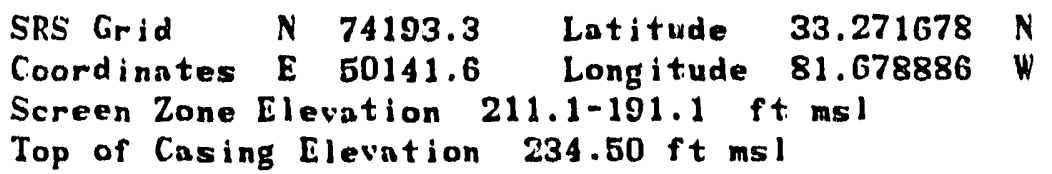

MEASLREMENTS CONDUCTED IN TILE FIELD

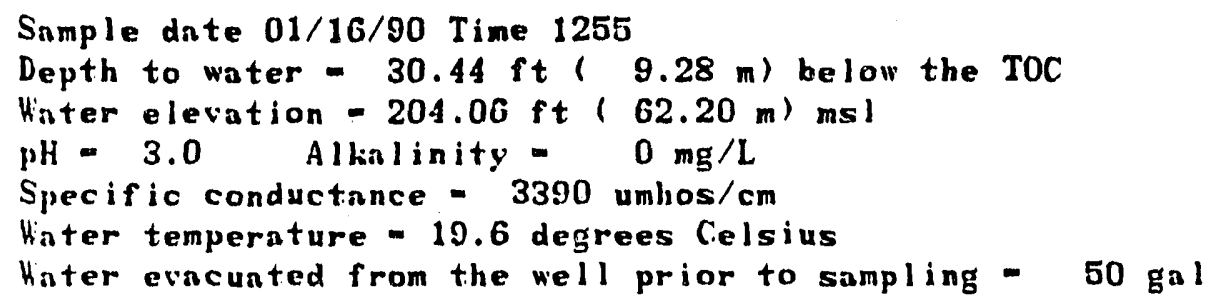

HELL TSS 1100

SRS Grid N 74193.3 Latitude 33.271678 N

Coordinates E 50141.6 Longitude 81.678886

Screen Zone Elevation 211.1-191.1 ft msl

Top of Casing Elevation $234.50 \mathrm{ft} \mathrm{msl}$

MEASLIREMENTS CONDUCTED IN THE FIELD

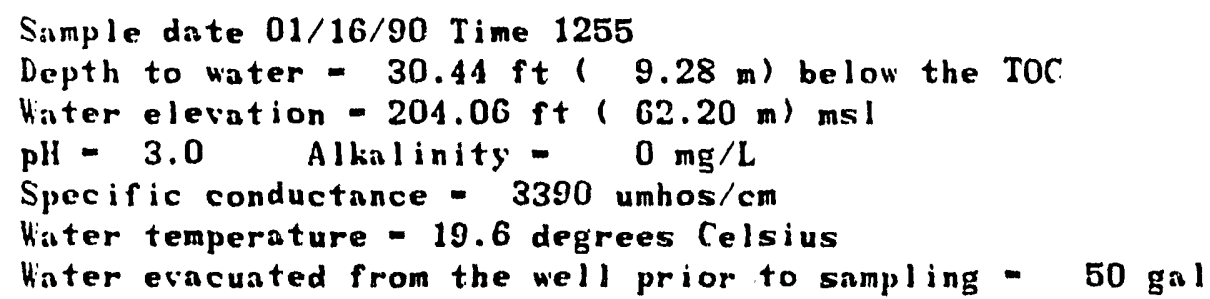

LABORATORY ANALYSES

THESE ANALYSES ARE FROM UNFILTERED SAMPLES

SILICA

$156000 \mathrm{ug} / \mathrm{L}$ Gen. Eng. 
WELL FSB $111 \mathrm{C}$

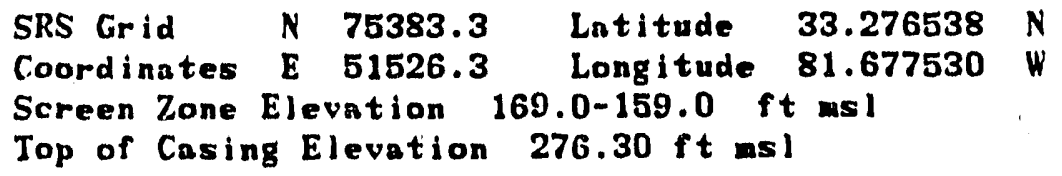

MEASUREMENTS CONDUCTED IN THE FIELD

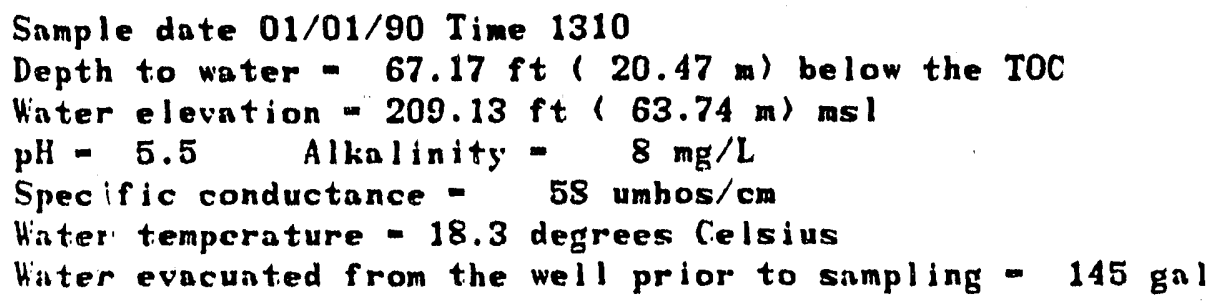

LARORATORY ANALISES

\begin{tabular}{|c|c|c|c|c|c|}
\hline $\begin{array}{l}\text { SPECIFIC CONDUCTANCE } \\
\text { PII }\end{array}$ & & $\begin{array}{r}58.00 \\
5.74\end{array}$ & $\begin{array}{l}\operatorname{umh}_{\mathrm{pH}} / \mathrm{cm} \\
\end{array}$ & $\begin{array}{l}\text { Gen. } \\
\text { Gen. }\end{array}$ & $\begin{array}{l}\text { Eng. } \\
\text { Eng. }\end{array}$ \\
\hline SILVER & LT & 2 & Lg $/ L$ & Gen. & Eng. \\
\hline ARSENIC & LT & 2 & ug $/ \mathrm{L}$ & Gen. & Eng. \\
\hline BARIUM & & 7 & $\operatorname{ug} / \mathrm{L}$ & Gen. & Eng. \\
\hline BROMOD I CHLOROMETHANE & LT & 1 & ug $/ \mathrm{L}$ & Gen. & Eng. \\
\hline CALCIUM & & 6540 & $\operatorname{vg} / L$ & Gen. & Eng. \\
\hline TRICIILOROFLUOROMETHANE & LT & 1 & ug/L & Gen. & Eng. \\
\hline CARBON TETRACILLORIDE & LT & 1.00 & ug $/ \mathrm{L}$ & Gen. & Eng. \\
\hline CADMIUM & LT & 2 & ug $/ \mathrm{L}$ & Gen. & g. \\
\hline BROMOFORY & LT & 1 & $u g / L$ & Gen. & ng. \\
\hline CIILOROFORM & LT & 1 & ug $/ \mathrm{L}$ & Gen. & Eng. \\
\hline METHYLENE CHLORIDE & LT & 1 & $\operatorname{ug} / \mathrm{L}$ & Gen. & Eng. \\
\hline BROMOMETHANE & LT & 1 & ug $/ L$ & Gen. & Eng. \\
\hline CHLOROMETHANE & LT & 1 & $u g / L$ & Gen. & Eng. \\
\hline CILLORIDE & & 3500 & ug/L & Gen. & $\therefore$ \\
\hline CIILOROBENZENE & LT & 1 & ug $/ \mathrm{L}$ & Gen. & Eng. \\
\hline COBALT & LT & 4 & ug $/ \mathrm{L}$ & Gen. & Eng. \\
\hline CHROMIUM & LT & 4 & ug/L & Gen. & Eng. \\
\hline COPPER & LT & 4 & $\operatorname{ug} / \mathrm{L}$ & Gen. & Eng. \\
\hline CYANIDE & & 9 & ug $/ \mathrm{L}$ & Gen. & Eng. \\
\hline CILLOROETHENE (VINIL CILLORIDE) & LT & 1 & ug $/ \mathrm{L}$ & Gen. & Eng. \\
\hline CILLOROETHANE & LT & 1 & ug $/ \mathrm{L}$ & Gen. & ng. \\
\hline BENZENE & LT & 1 & $\mathrm{ug} / \mathrm{L}$ & Ger & Eng. \\
\hline DIBROMOCILOROMETHANE & LT & 1 & $\operatorname{ug} / L$ & Gen. & Enq \\
\hline ENDRIN & LT & 0.10 & ug $/ L$ & Gen. & - En \\
\hline ETIYLBENZENE & LT & 1 & ug $/ \mathrm{L}$ & Gen. & - Eng. \\
\hline FLLORIDE & LT & 100 & ug $/ \mathrm{L}$ & Gen. & - En \\
\hline IRON & & 9 & ug $/ \mathrm{L}$ & Gen. & - En \\
\hline MERCLRY & LI & 0.20 & ug $/ \mathrm{L}$ & Gen. & En \\
\hline POTASSIUM & LT & 500 & $\operatorname{ug} / \mathrm{L}$ & Gen. & Eng. \\
\hline LINDANE & LT & 0.05 & ug 1 & Gen. & En \\
\hline TOLLENE & L.T & 1 & ug $/ \mathrm{L}$ & Gen. & En \\
\hline METHONYCILOR & LT & 0.50 & ug $/ \mathrm{L}$ & Ger & $\pi$ \\
\hline MAGNESIUM & & 437 & $u g / L$ & Gen. & II. \\
\hline MANGANESE & & 6 & ug/L & Gen. & Eng. \\
\hline SODIUM & & 4050 & $u g / L$ & Gen. & - Eng. \\
\hline
\end{tabular}


WELL FSB111C COLLECTED ON 01/01/90 LABORATORY ANALYSES CONTINUED

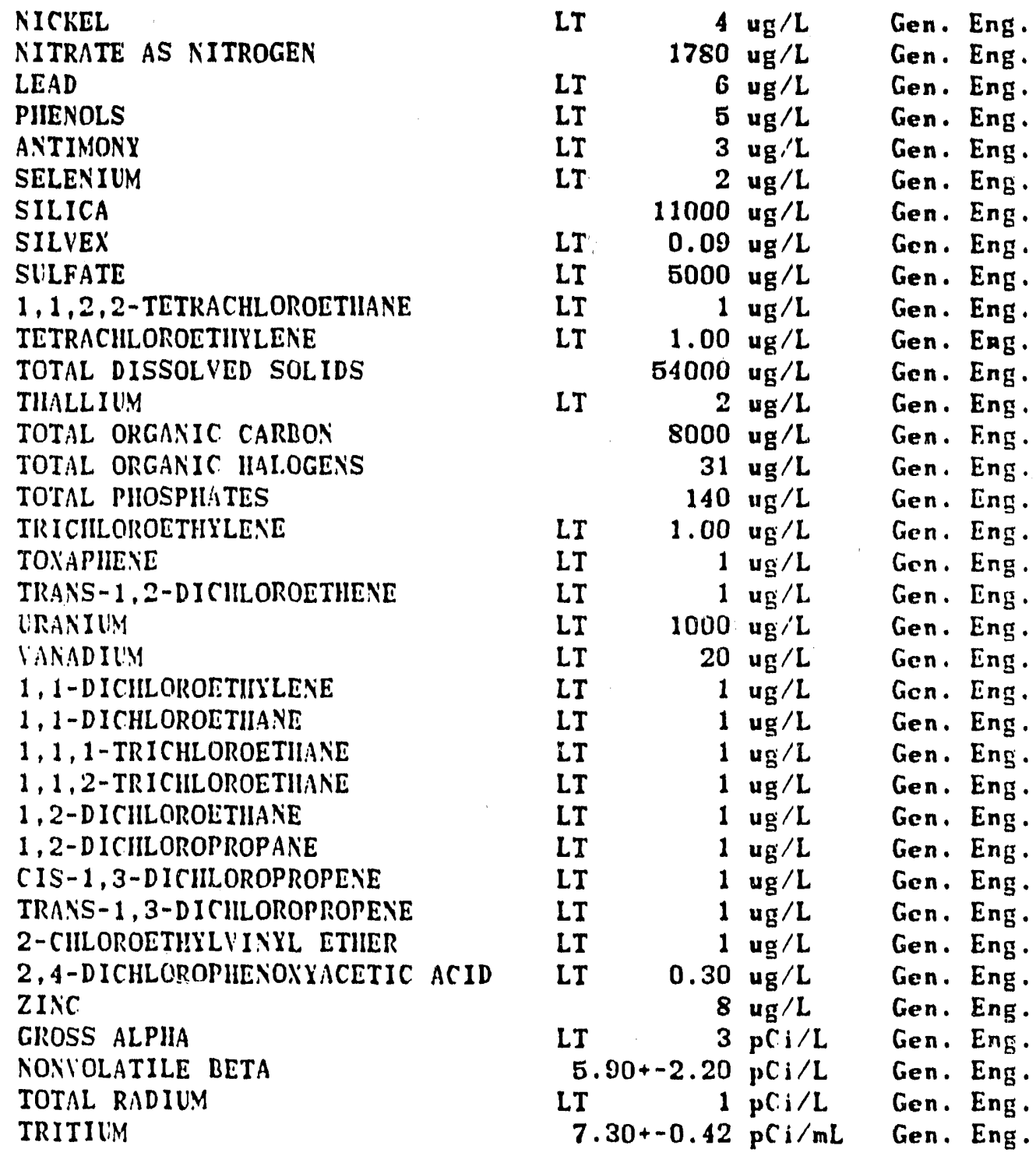


HELL FSB111D

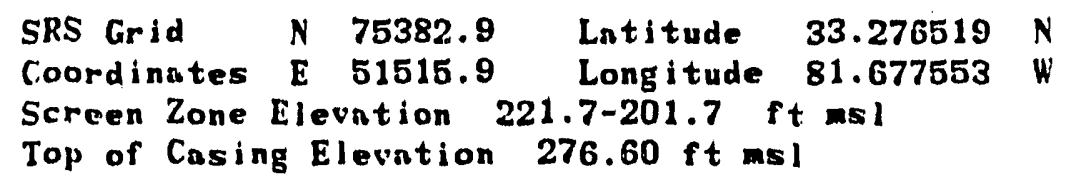

MEASUREMENTS CONDUCTED IN TIE FIELD

Snmple date 01/01/90 Time 1345

Depth to water - $65.18 \mathrm{ft}(19.87 \mathrm{~m})$ below the TOC

Winter elevation - $211.42 \mathrm{ft}(64.44 \mathrm{~m}) \mathrm{ms}$ l

$\mathrm{pH}=4.5$ Alkalinity $=0 \mathrm{mg} / \mathrm{L}$

Specific conductance - 52 umhos/cm

Hater tempernture - 18.3 degrees Celsius

Hinter evacuated from the well prior to sampling - $40 \mathrm{gal}$

LABORATORY ANALYSES

\begin{tabular}{|c|c|c|c|c|}
\hline $\begin{array}{l}\text { SPECIFIC CONDLICTANCE } \\
\text { PII }\end{array}$ & & $\begin{array}{r}40.00 \\
5.02\end{array}$ & $\begin{array}{l}\text { umbircm } \\
\text { pll }\end{array}$ & $\begin{array}{l}\text { Gen. } \\
\text { Gen. }\end{array}$ \\
\hline SILVER & LT & 2 & ug $/ \mathrm{L}$ & Gen. \\
\hline ARSENIC & LT & 2 & ug $/ \mathrm{L}$ & Gen. \\
\hline BARYUM & & 13 & ug $/ \mathrm{L}$ & Gen. \\
\hline BROMOD I CUL OROMETHANE & LT & 1 & $\operatorname{ug} / \mathrm{L}$ & Gen. \\
\hline CALCIUM & & 1150 & ug $/ L$ & Gen. \\
\hline TRICIILOROFLUOROMETHANE & & 2 & ug $/ \mathrm{L}$ & Gen. \\
\hline CARBON TETRACHLORIDE & LT & 1.00 & ug $/ \mathrm{L}$ & Gen. \\
\hline CADMILM & LT & 2 & $\mathrm{ug} / \mathrm{L}$ & Gen. \\
\hline BROMOFORM & LT & 1 & ug /L & Gen. \\
\hline CIILOROFORM & LT & 1 & ug/L & Gen. \\
\hline METHILENE CILLORIDE & & 6 & $u_{g} / L$ & Gen. \\
\hline BROMOMETHANE & LT & 1 & $\operatorname{ug} / \mathrm{L}$ & Gen. \\
\hline CIILOROMETHANE & LT & 1 & $\operatorname{ugg}_{\mathrm{g}} / \mathrm{L}$ & Gen. \\
\hline CIILORIDE & & 2700 & $u_{g} / L$ & Gen. \\
\hline CIILOROBENZENE & LI & 1 & ug/L & Gen. \\
\hline COBALT & LT & 4 & ug/L & Gen. \\
\hline CHRONI UM & LT & 4 & ug $/ \mathrm{L}$ & Gen. \\
\hline COPPER & LT & 4 & ug/L & Gen. \\
\hline CYANIDE & LT & 5 & ug/L & Gen. \\
\hline CIILOROETIENE (VINYL CIILORIDE) & LT & 1 & ug $/ L$ & Gen. \\
\hline CIILOROETHANE & LT & 1 & ug $/ L$ & Gen. \\
\hline BEIZENE & LT & 1 & ug $/ L$ & Gen. \\
\hline DIBROMOCILORONETIIANE & LT & 1 & ug $/ L$ & Gen. \\
\hline ENDRIN & LT & 0.10 & $u g / L$ & Gen. \\
\hline ETHLLBENZENE & LT & 1 & ug $/ \mathrm{L}$ & Gen. \\
\hline FLIORIDE & LT & 100 & $u g / L$ & Gen. \\
\hline IRON & & 6 & ug $/ \mathrm{L}$ & Gen. \\
\hline MERCL'RY & LT & 0.20 & ug/L & Gen. \\
\hline POTASSIUM & $L T$ & 500 & $u_{g} / L$ & Gen. \\
\hline LINDANE & LT & 0.05 & ug/L & Gen. \\
\hline TOLLENE & LI & 1 & ug/L & Gen. \\
\hline METHOXYCILLOR & LT & 0.50 & ug/L & Gen. \\
\hline MAGNESILY & & 634 & ug $/ \mathrm{L}$ & Gen. \\
\hline MANGANESE & & 19 & ug/L & Gen. \\
\hline SODIUM & & 3090 & ug/L & Gen. \\
\hline
\end{tabular}


HELL FSB111D COLLECTED ON 01/01/90 LABORATORY ANALYSES CONIINUED

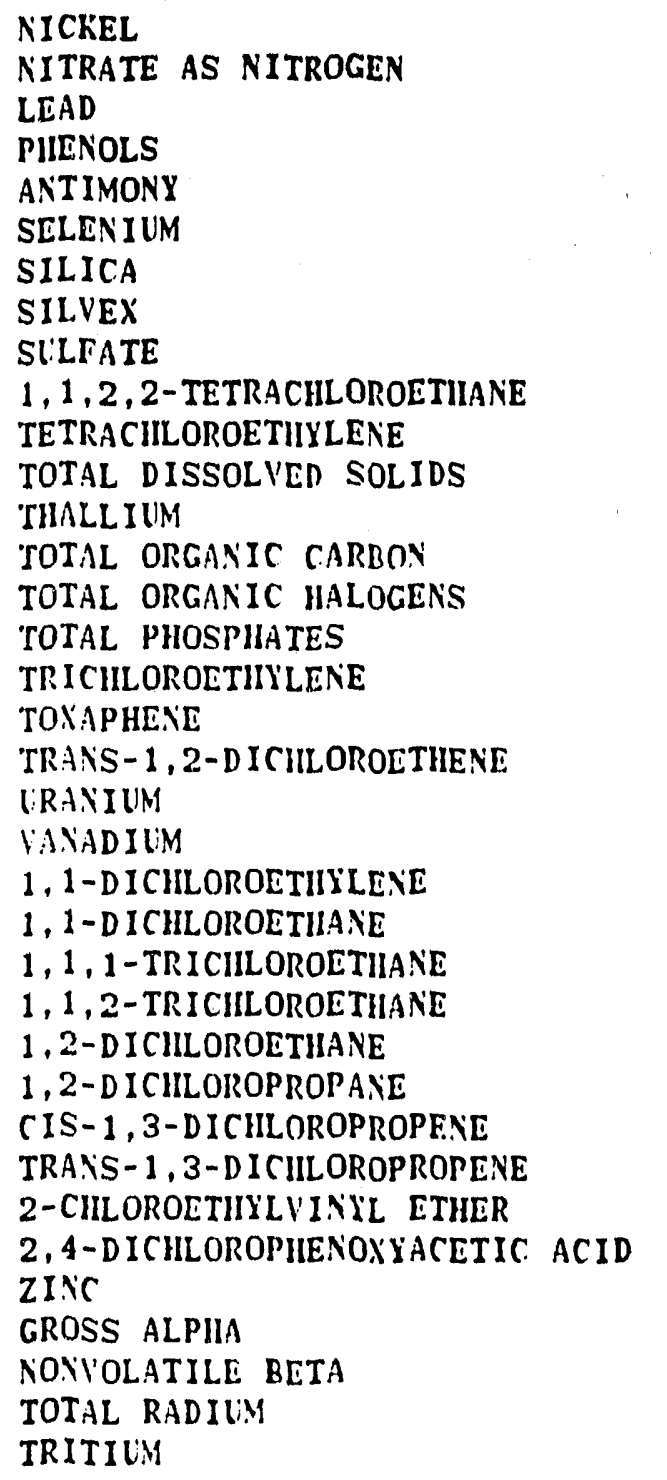

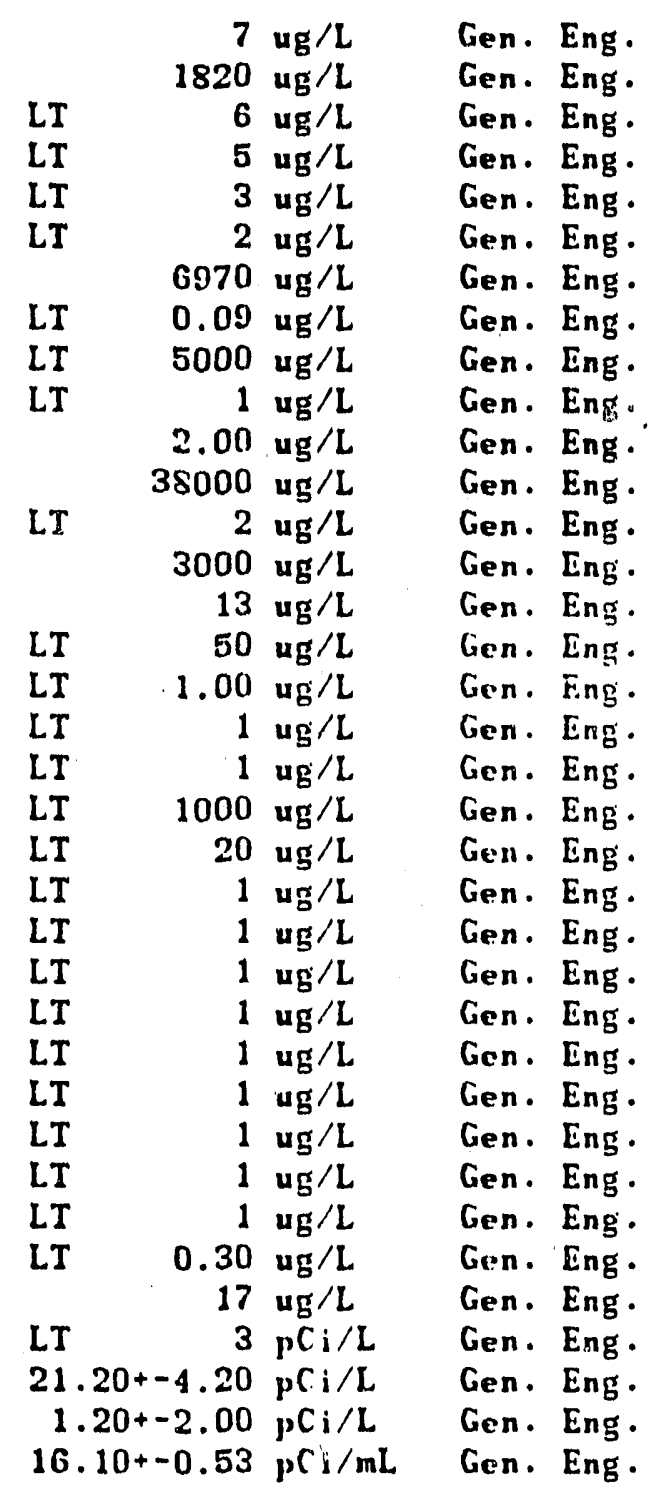


Maps of results of analyseis for selected parameters at the $F$ Area Seepage Basin monitoring wells in each hydrologic unit.

WATER TABIE

$\begin{array}{lll}\text { FIGURE } & \text { B-WT1: } & \text { TMRITIUN } \\ \text { FIGURE } & \text { B-WT2: } & \text { WITRATE } \\ \text { FIGURE } & \text { B-WT3: } & \text { OADMIUN } \\ \text { FIGURE B-WT4: } & \text { WEAD } \\ \text { FIGURE B-WT5: } & \text { PH } \\ \text { FIGURE } & \text { B-WT6: } & \text { CONDUCTIVITY }\end{array}$

YCBEAN
TRITIUM

NITRATE

CADMIUM

IEAD

PH

CONDUCTIVITY

\section{PAGE}

B-1

B-2

B-3

B-4

B-5

B-6

$\begin{array}{lll}\text { FIGURE B-MCB2: } & \text { NITRATE } & B-7 \\ \text { FIGURE B-MCB3: } & \text { CADMIUM } & B-8 \\ \text { FIGURE B-MCB4: } & \text { LEAD } & B-9 \\ \text { FIGURE B-MCB5: } & \text { PH } & B-10 \\ \text { FIGURE B-MCB6: } & \text { CONDUCTIVITY } & B-11 \\ & & B-12\end{array}$

\section{UPPER CONGAREE}

$\begin{array}{lll}\text { FIGURE B-UPCONG1: } & \text { TRITIUM } & \text { B-13 } \\ \text { FIGURE B-UPCONG2: } & \text { NITRATE } & \text { B-14 } \\ \text { FIGURE B-UPCONG3: } & \text { CADMIUM } & \text { B-15 } \\ \text { FIGURE B-UPCONG4: } & \text { IEAD } & \text { B-16 } \\ \text { FIGURE B-UPCONG5: } & \text { PH } & \text { B-17 } \\ \text { FIGURE B-UPCONG6: } & \text { CONDUCTIVITY } & \text { B-18 }\end{array}$

\section{IOWER CONGAREE}

$\begin{array}{lll}\text { FIGURE B-IOCONG1: } & \text { TRITIUM } & \text { B-19 } \\ \text { FIGURE B-IOCONG2: } & \text { NITRATE } & \text { B-20 } \\ \text { FIGURE B-IOCONG3: } & \text { CADMIUN } & \text { B-21 } \\ \text { FIGURE B-IOCONG4: } & \text { IEAD } & \text { B-22 } \\ \text { FIGURE B-IOCONG5: } & \text { PH } & \text { B-23 } \\ \text { FIGURE B-LOCONG6: } & \text { CONDUCTIVITY } & \text { B-24 }\end{array}$

* The numbers posted on the maps in this section represent the maximum values reported by Metatrace Engineering Analytical Laboratory for the various analytical tests. A complete listing of all analytical results including those from the QA labs is contained in Appendix $A$. 


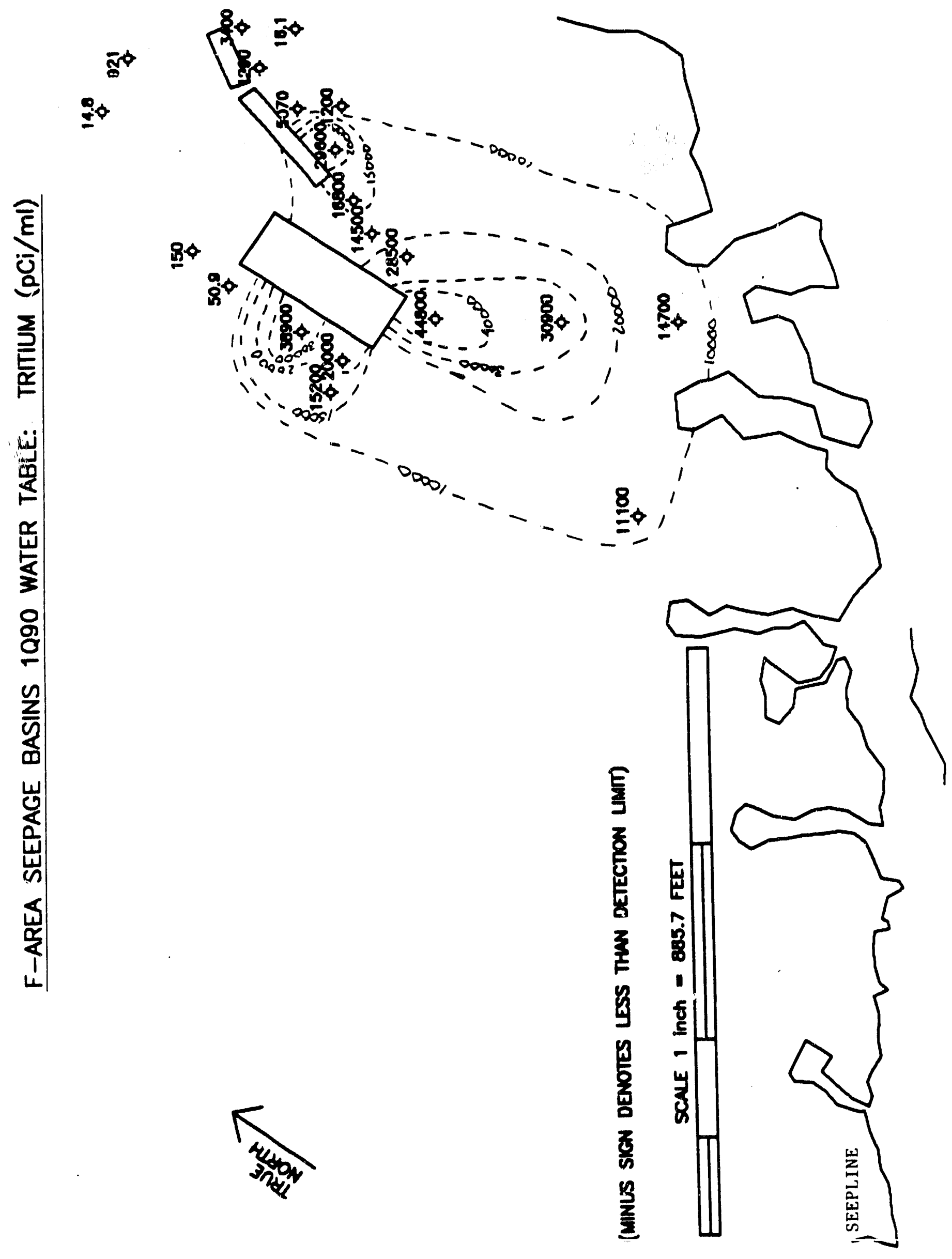




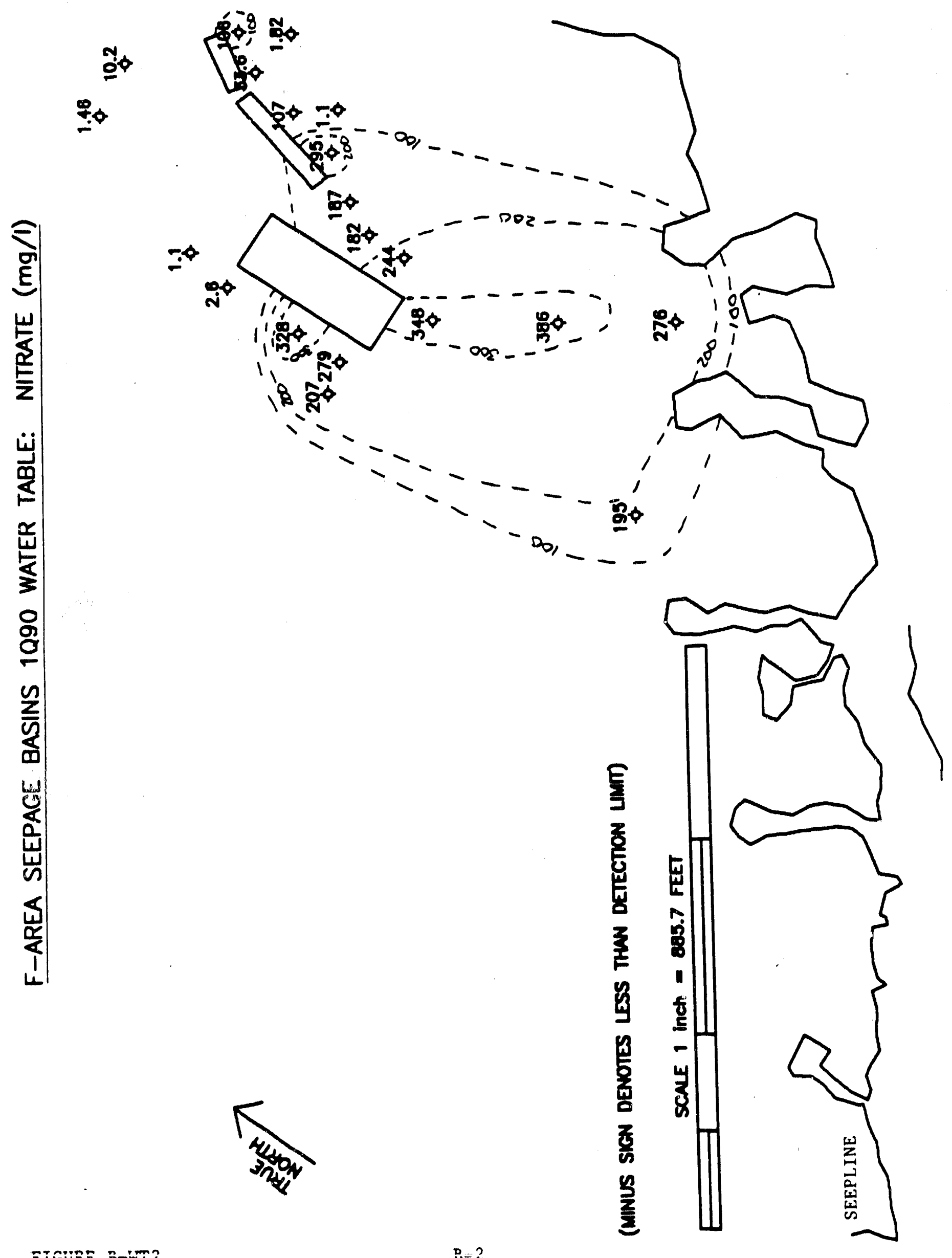



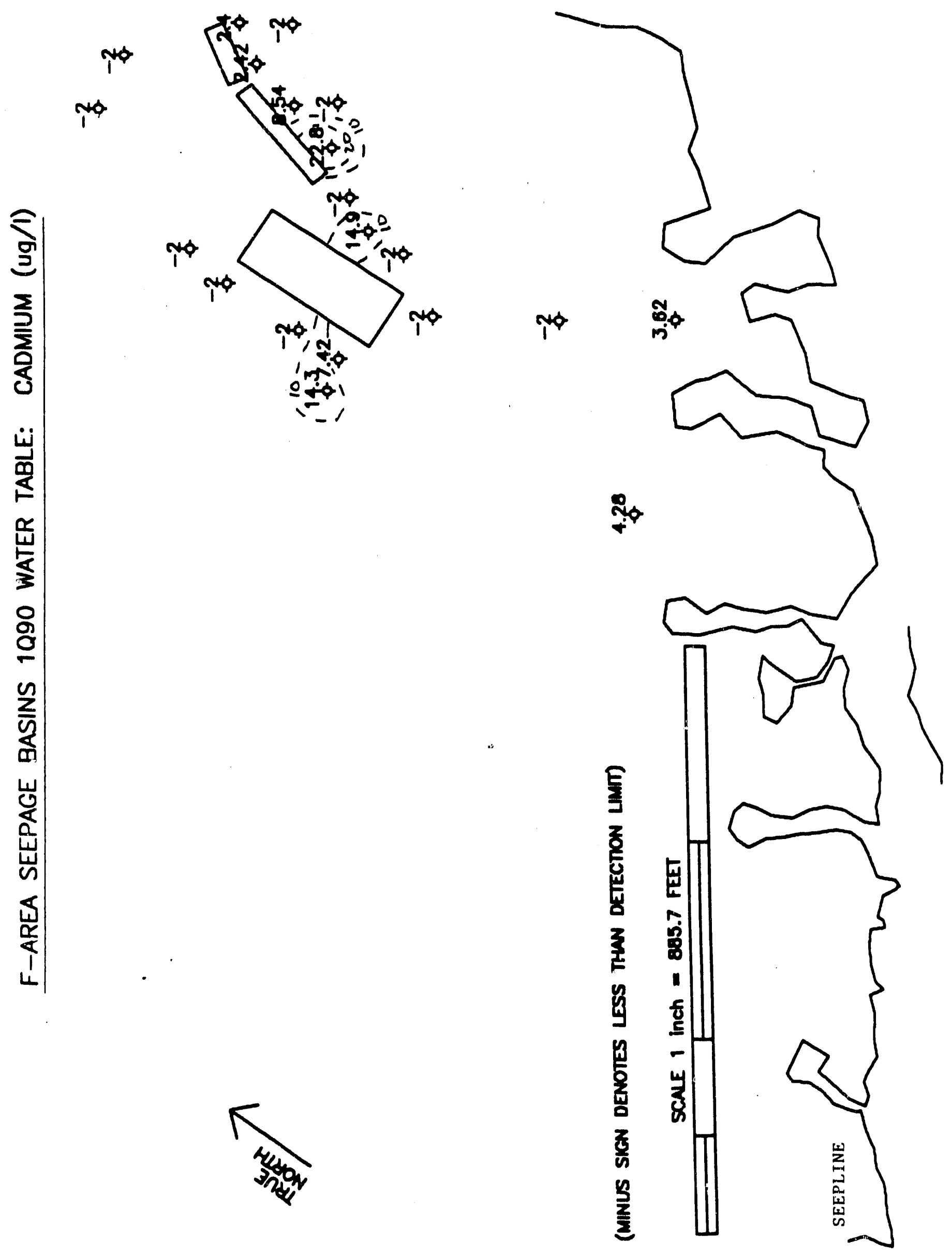

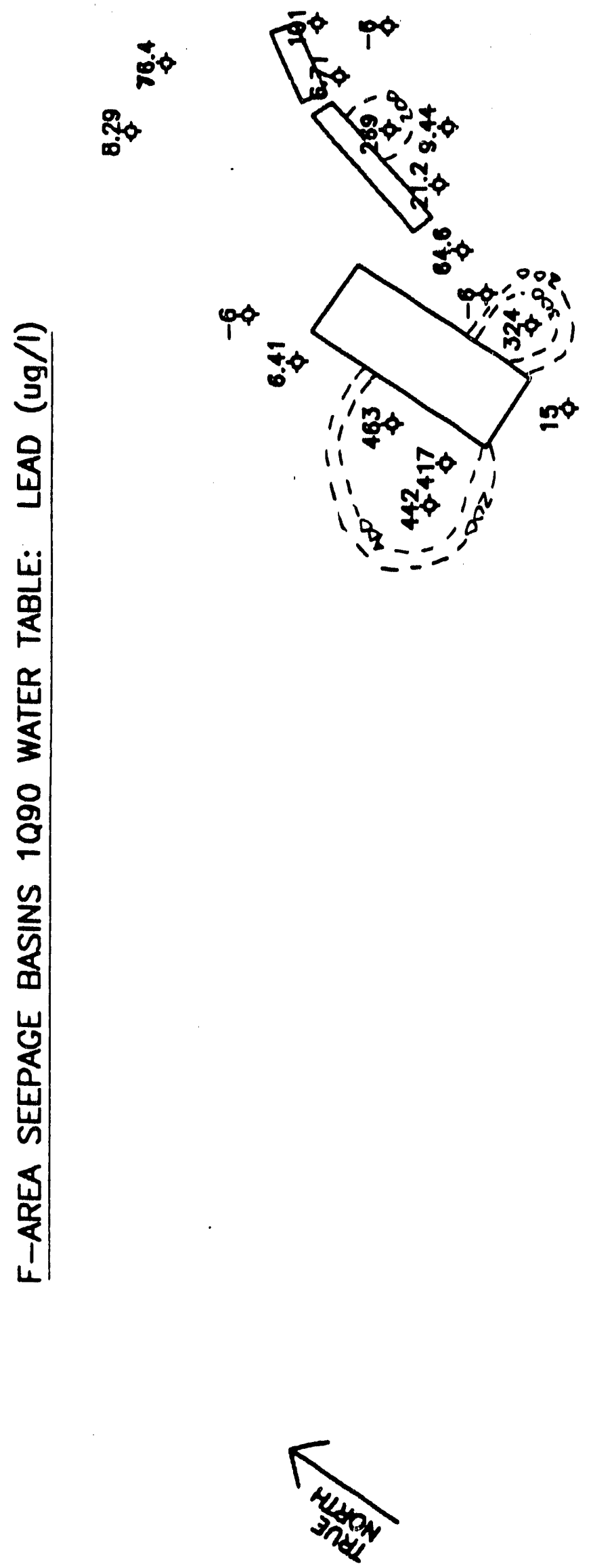

趣

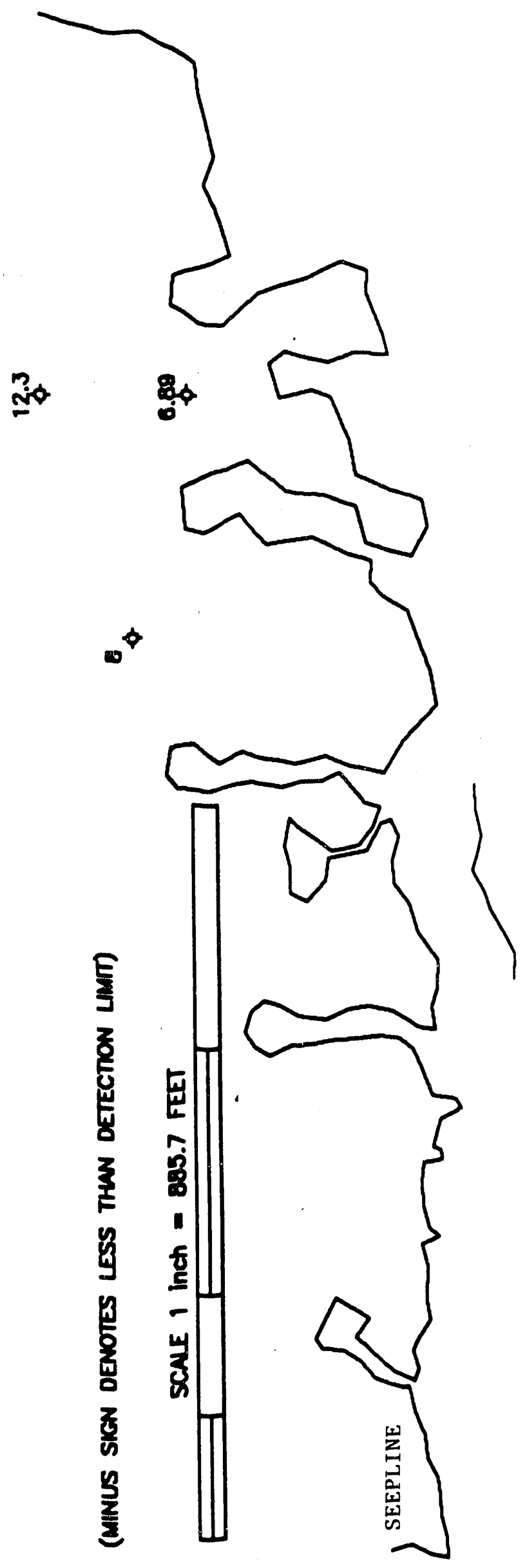




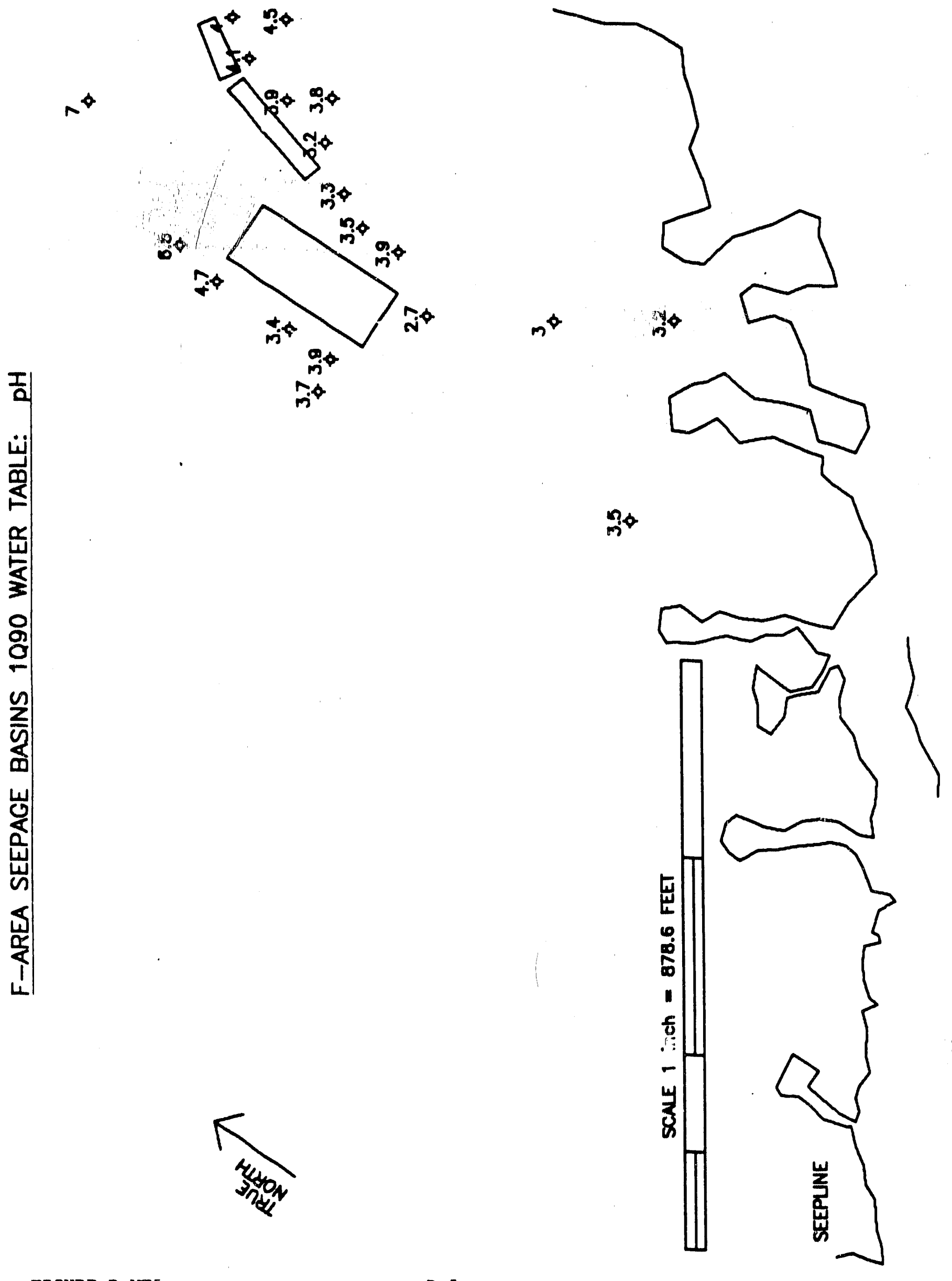



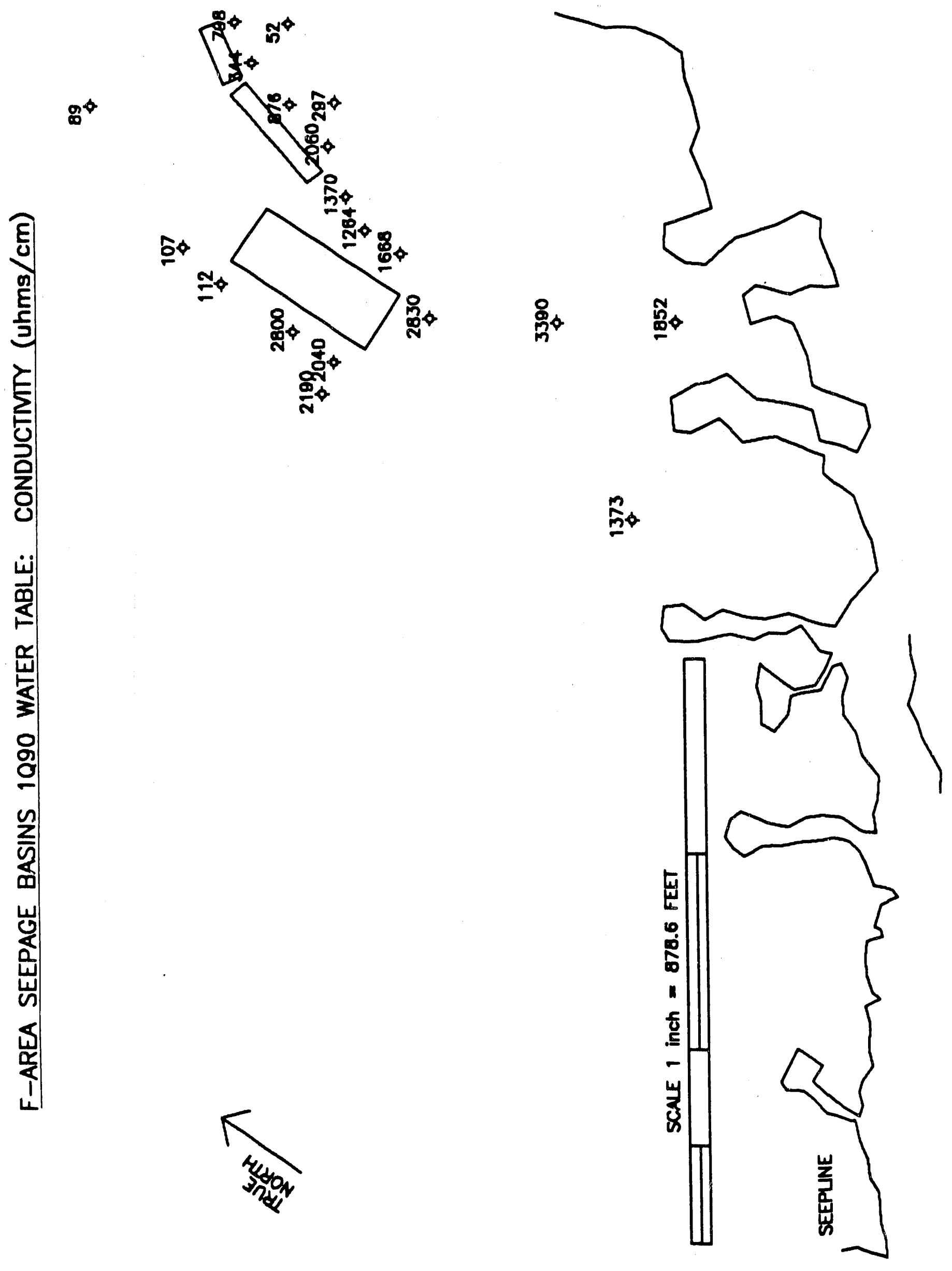


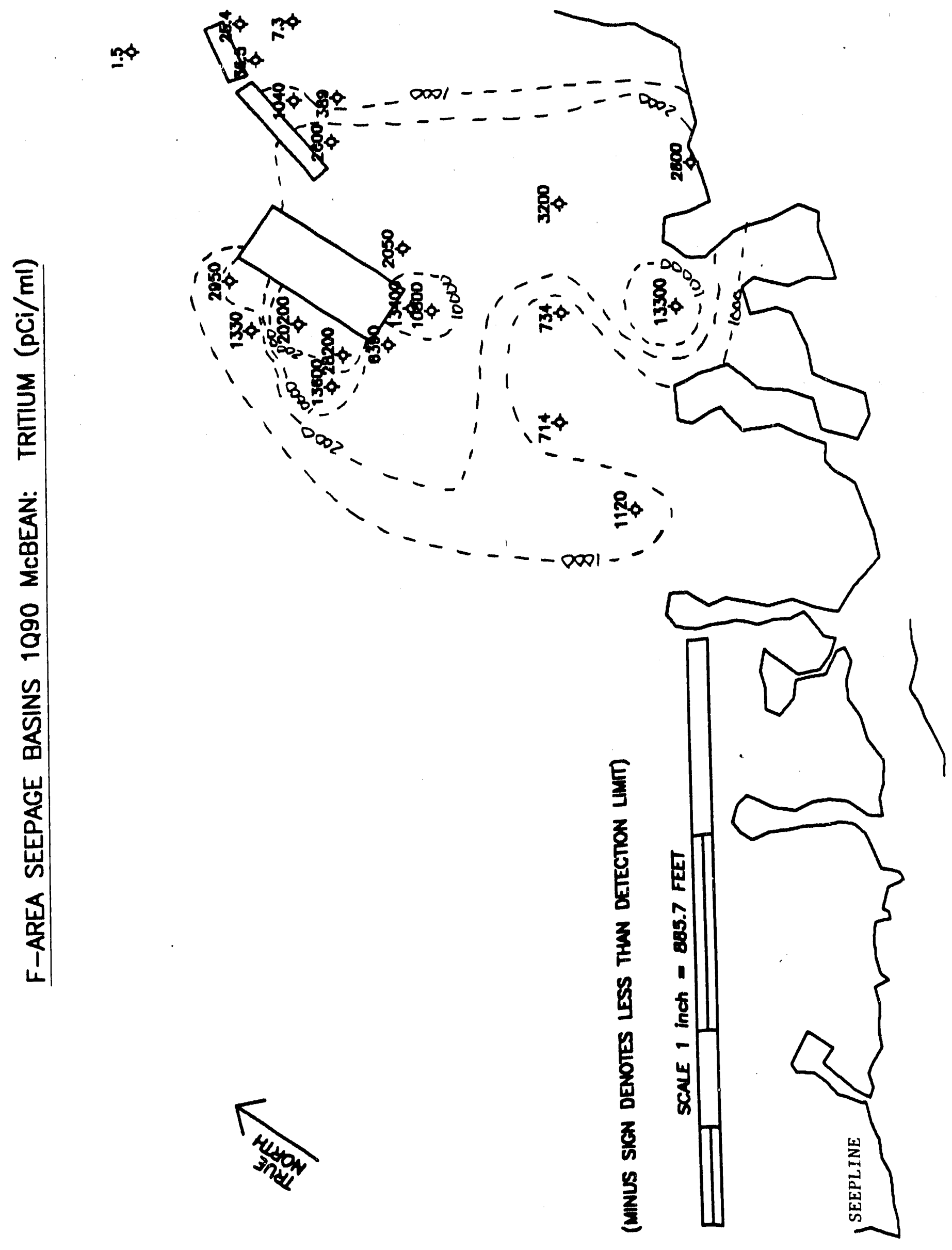




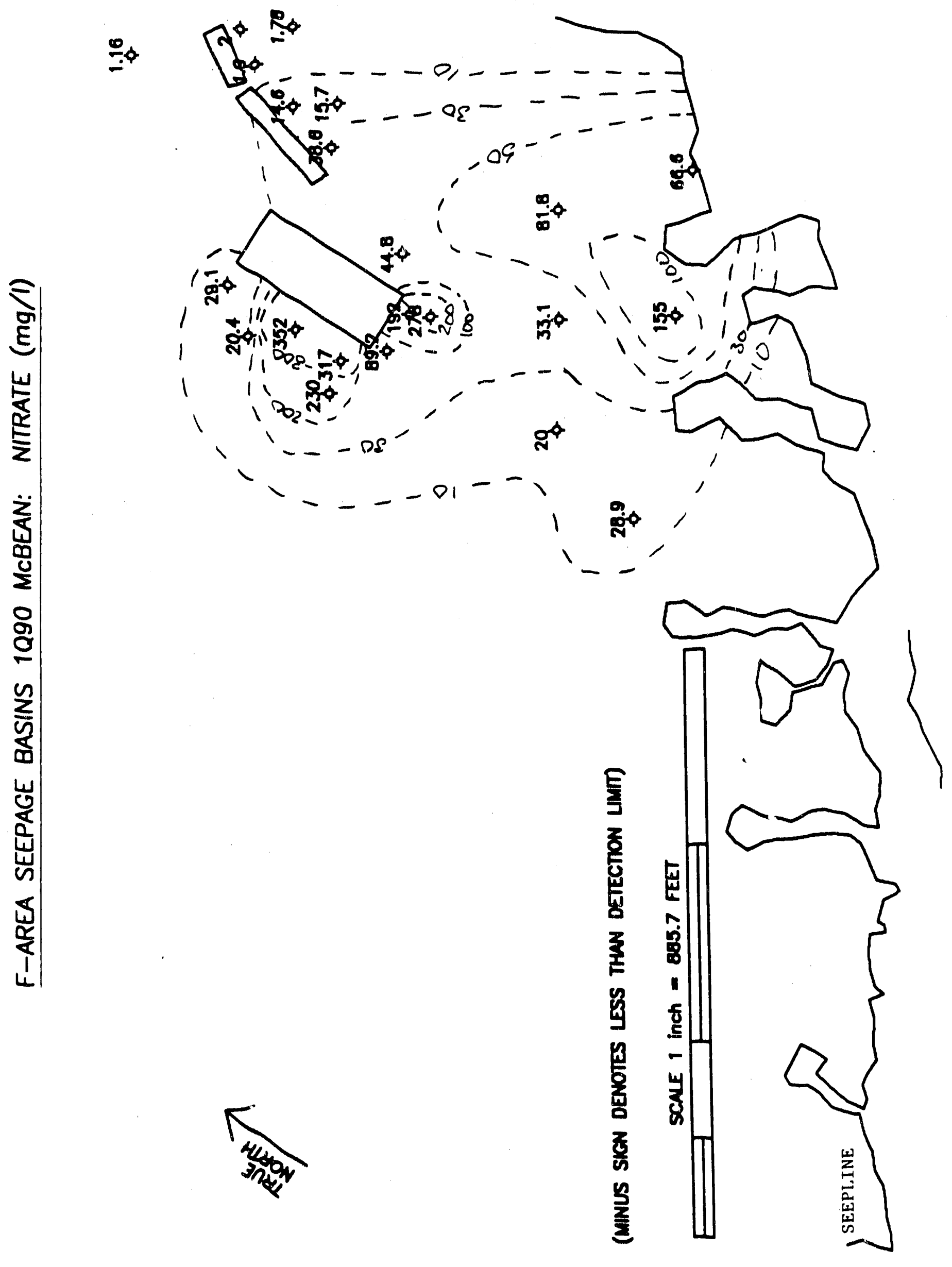




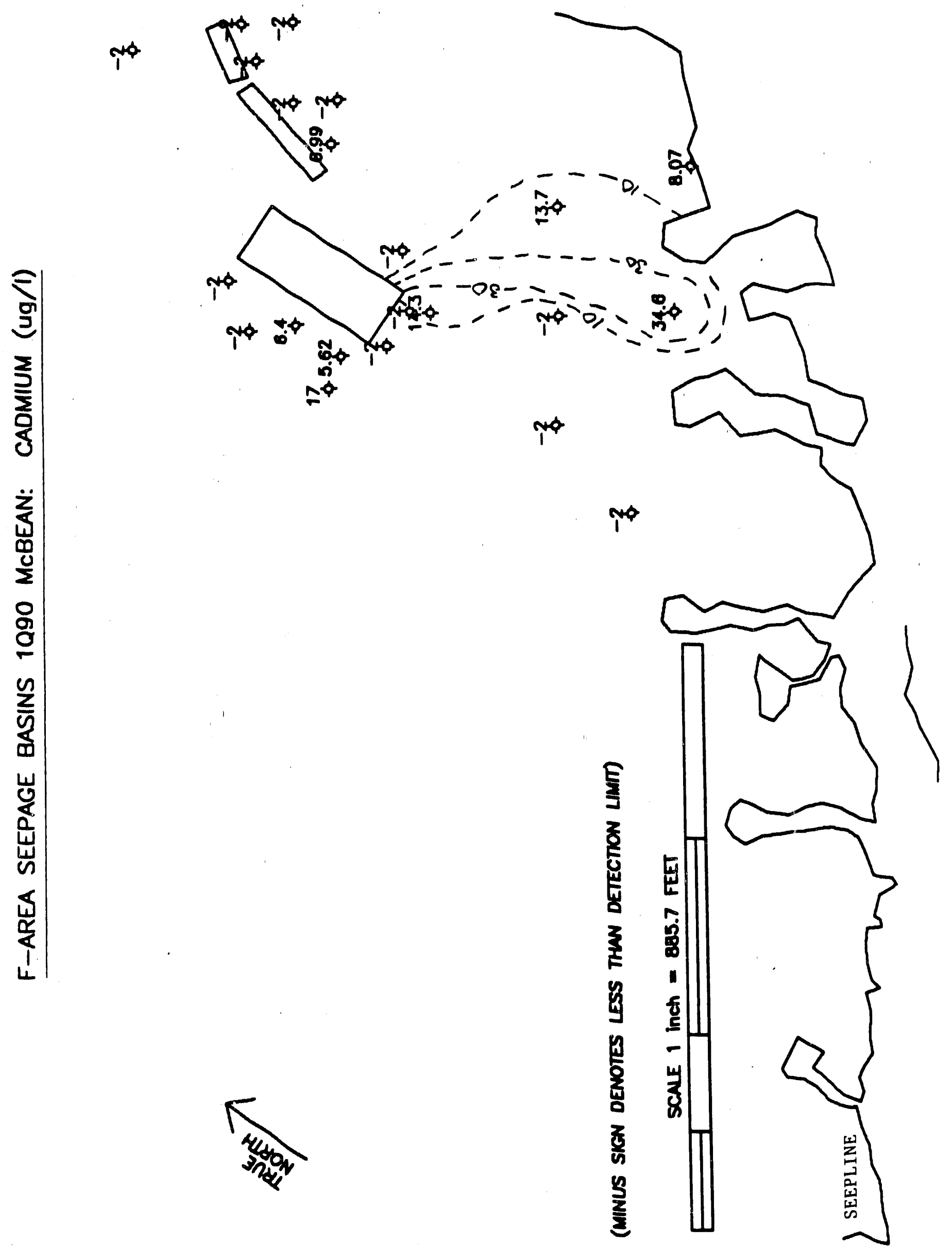




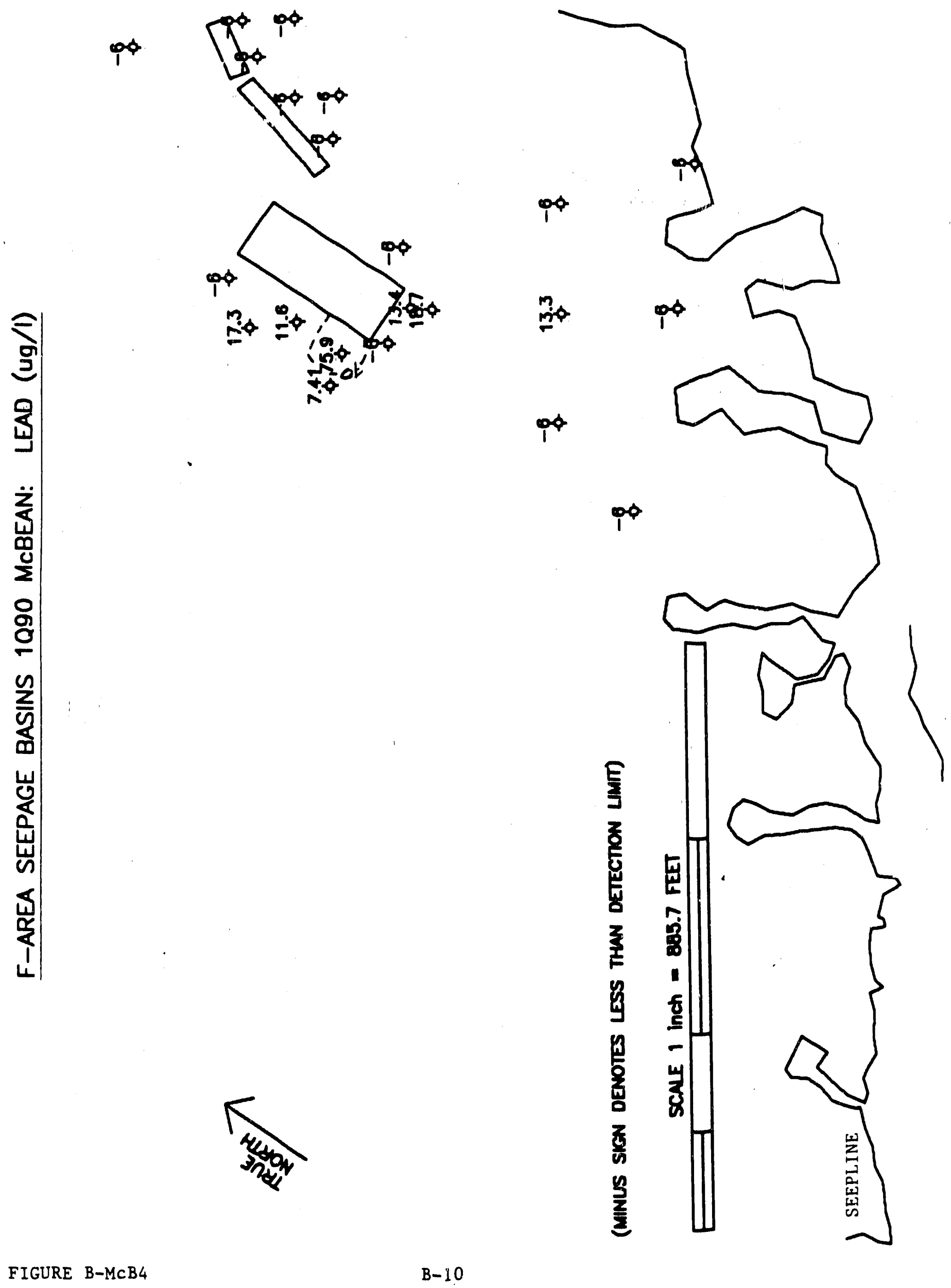




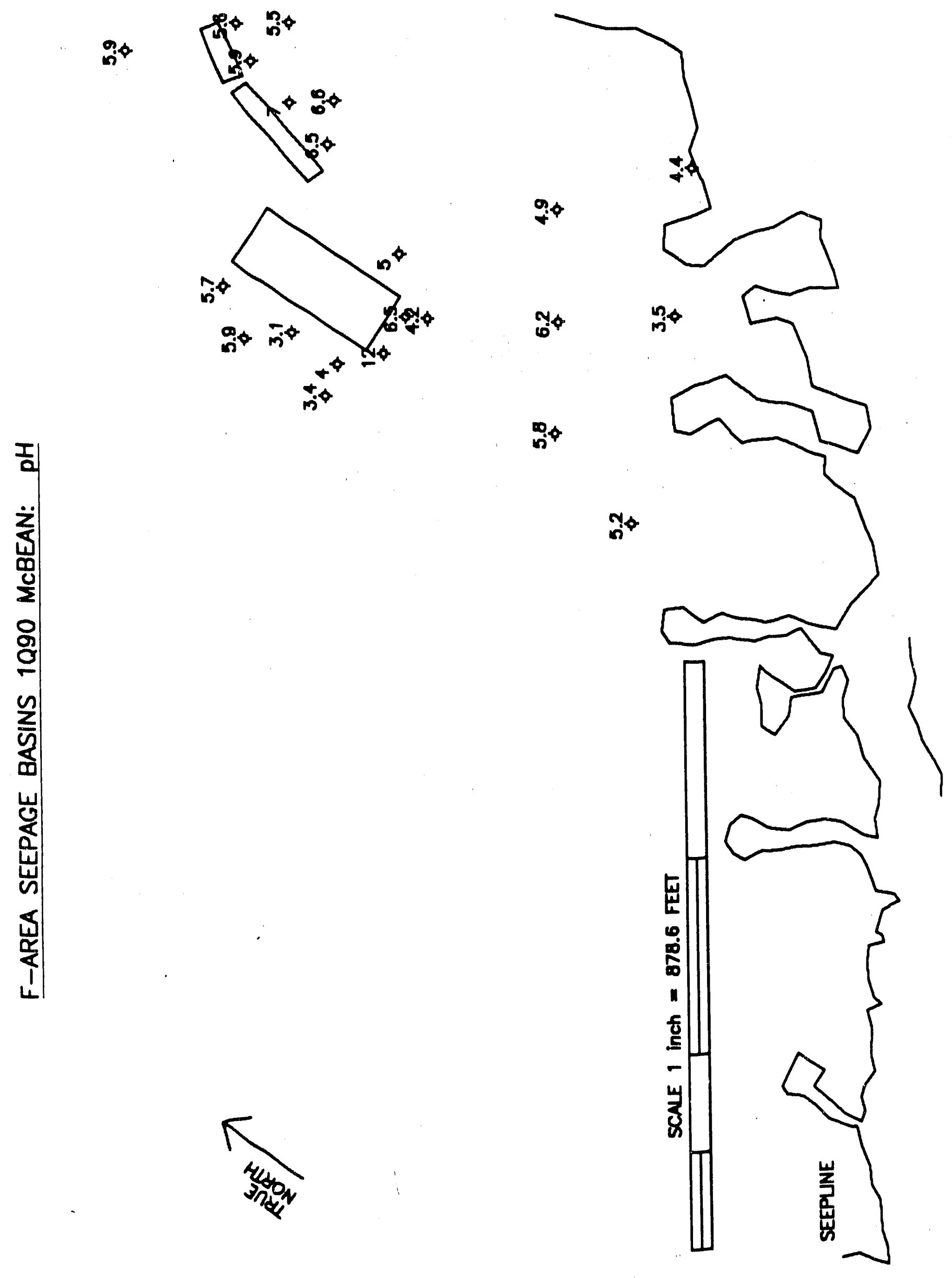



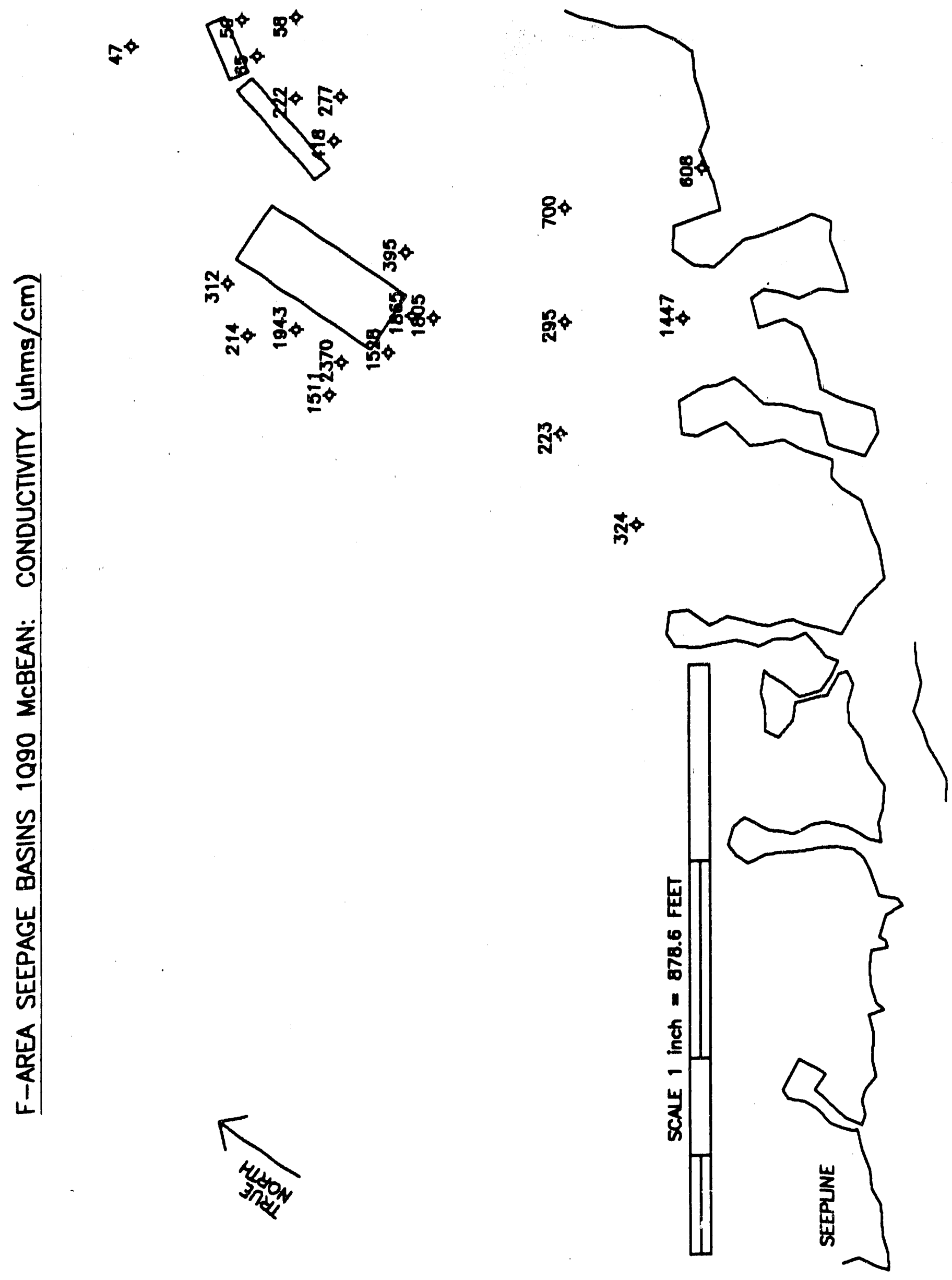

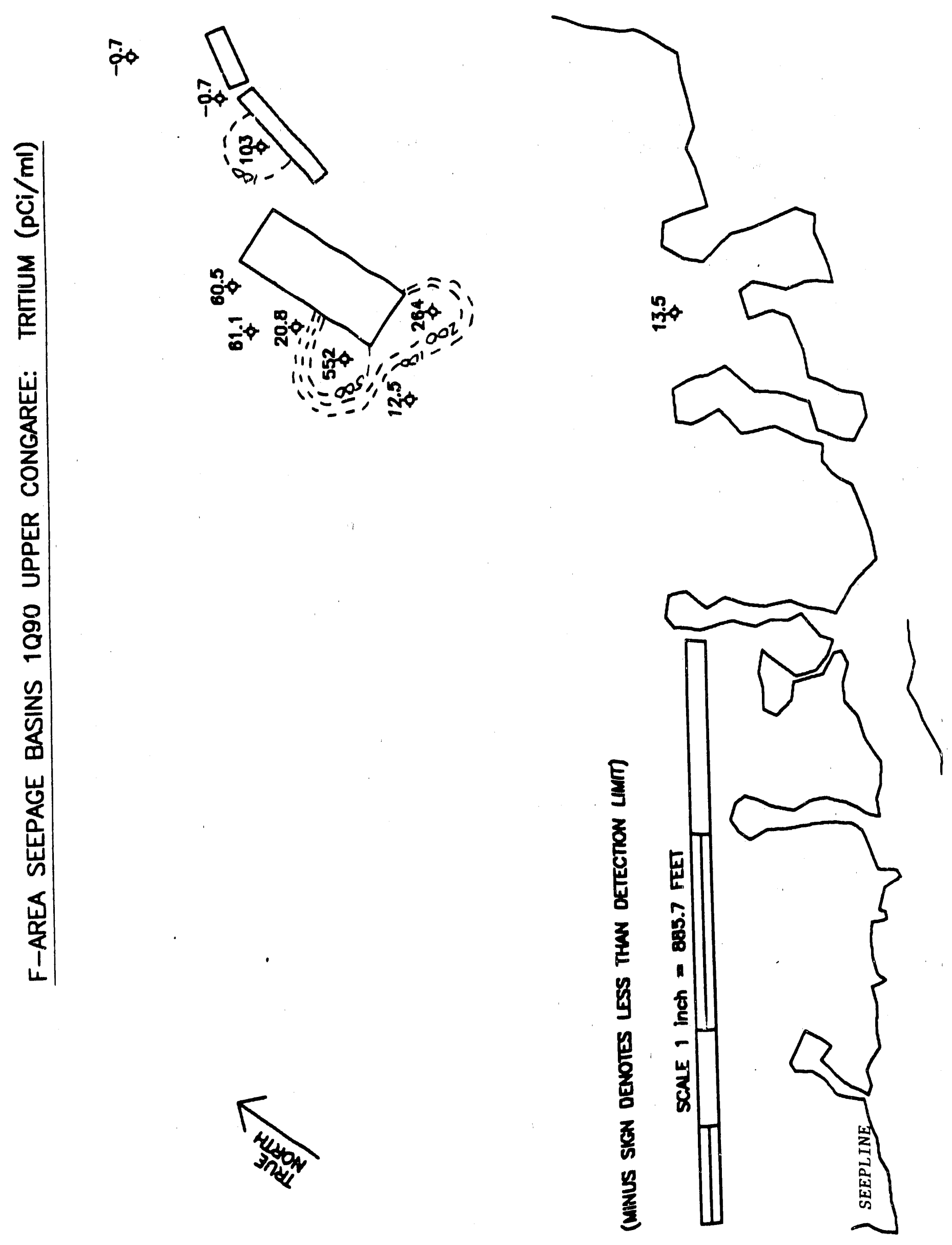

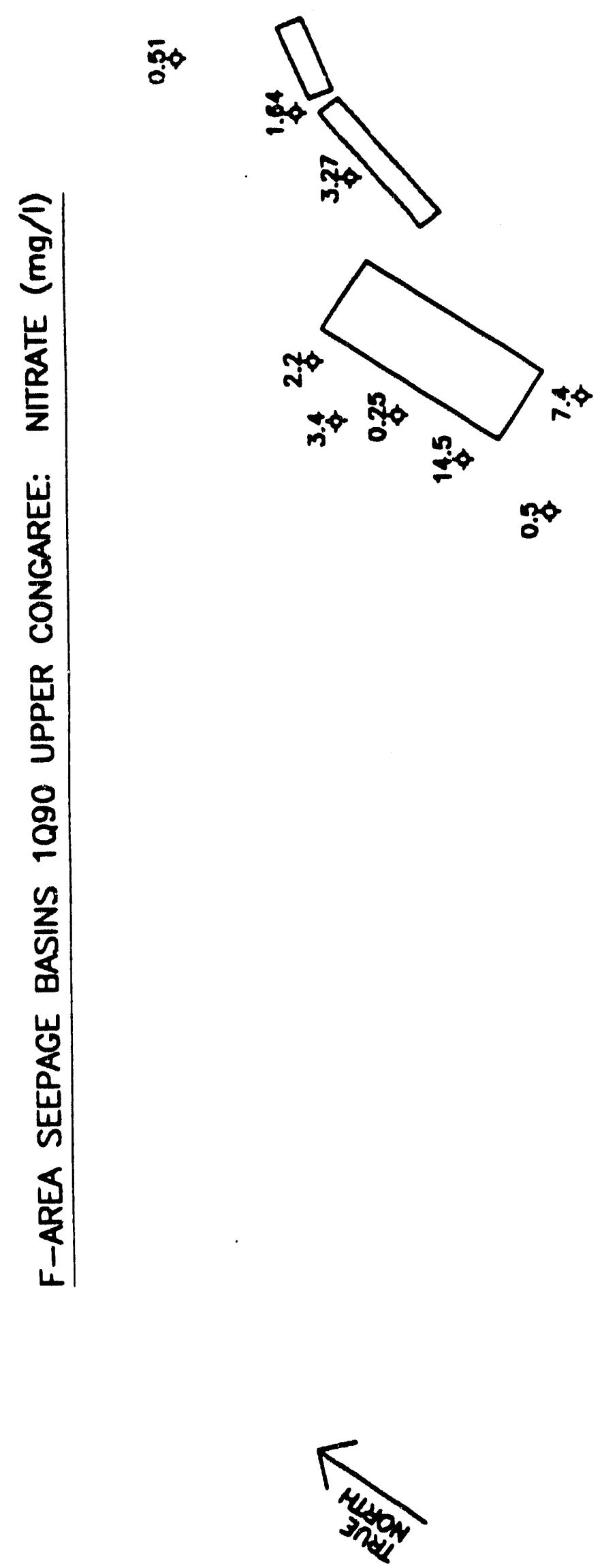

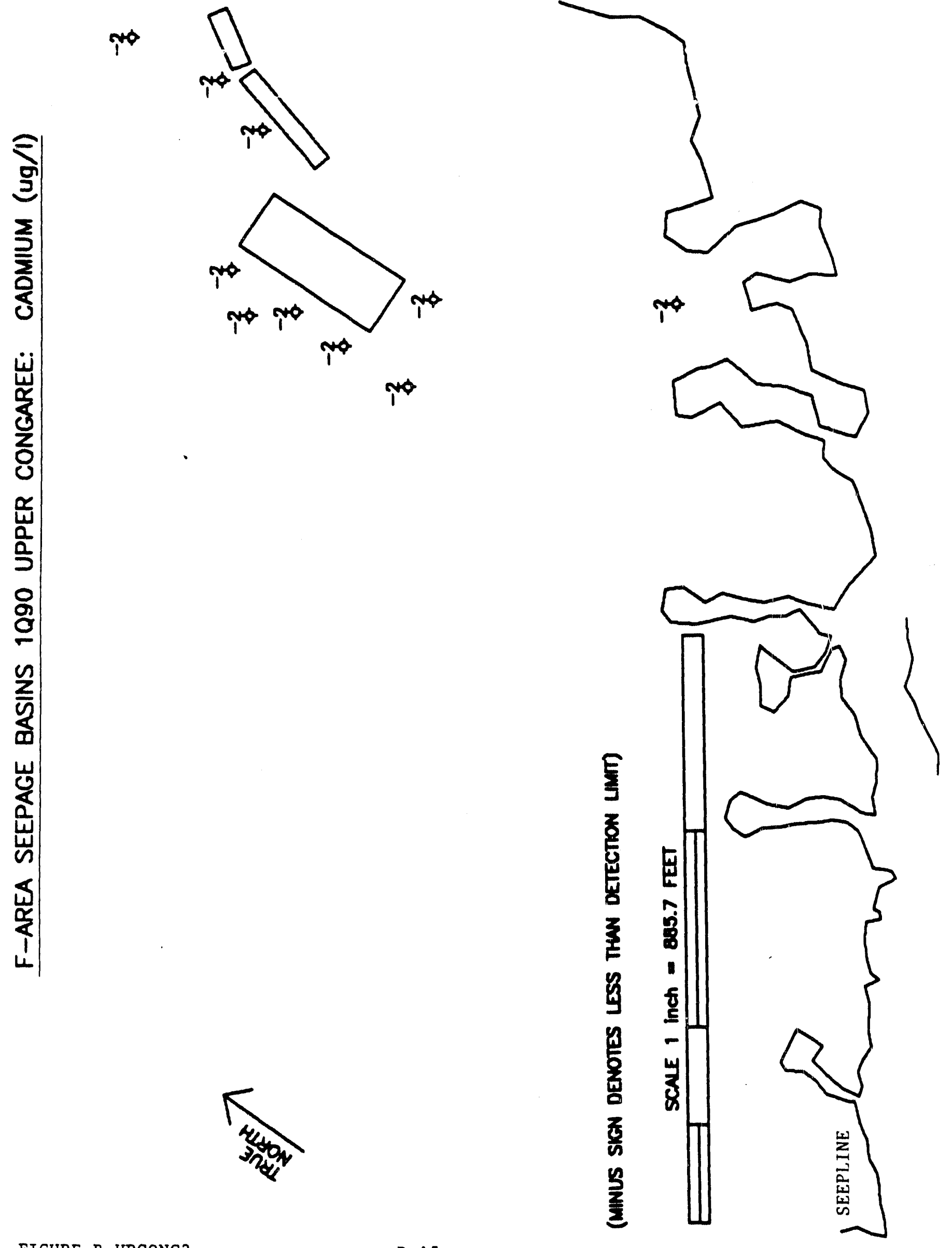


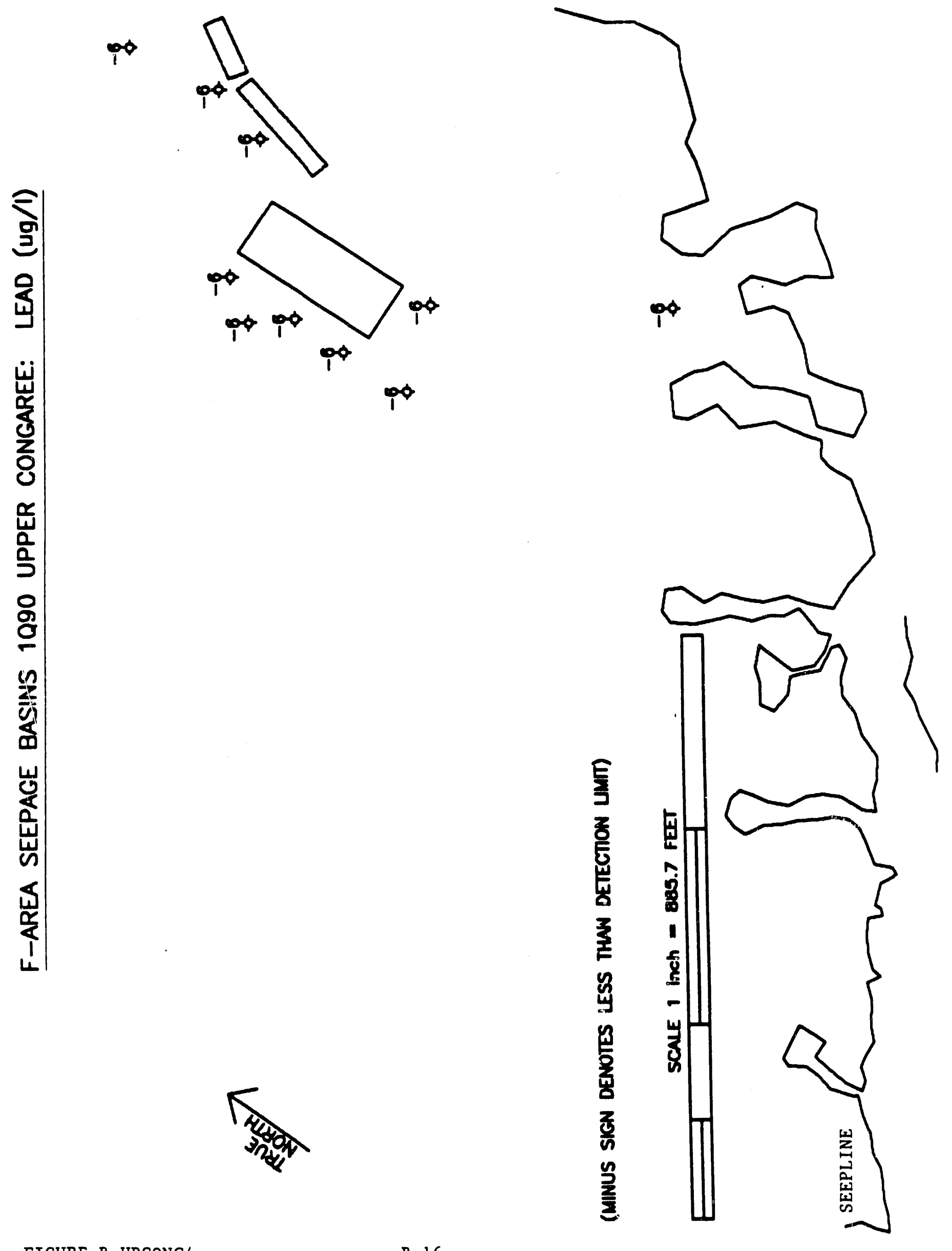




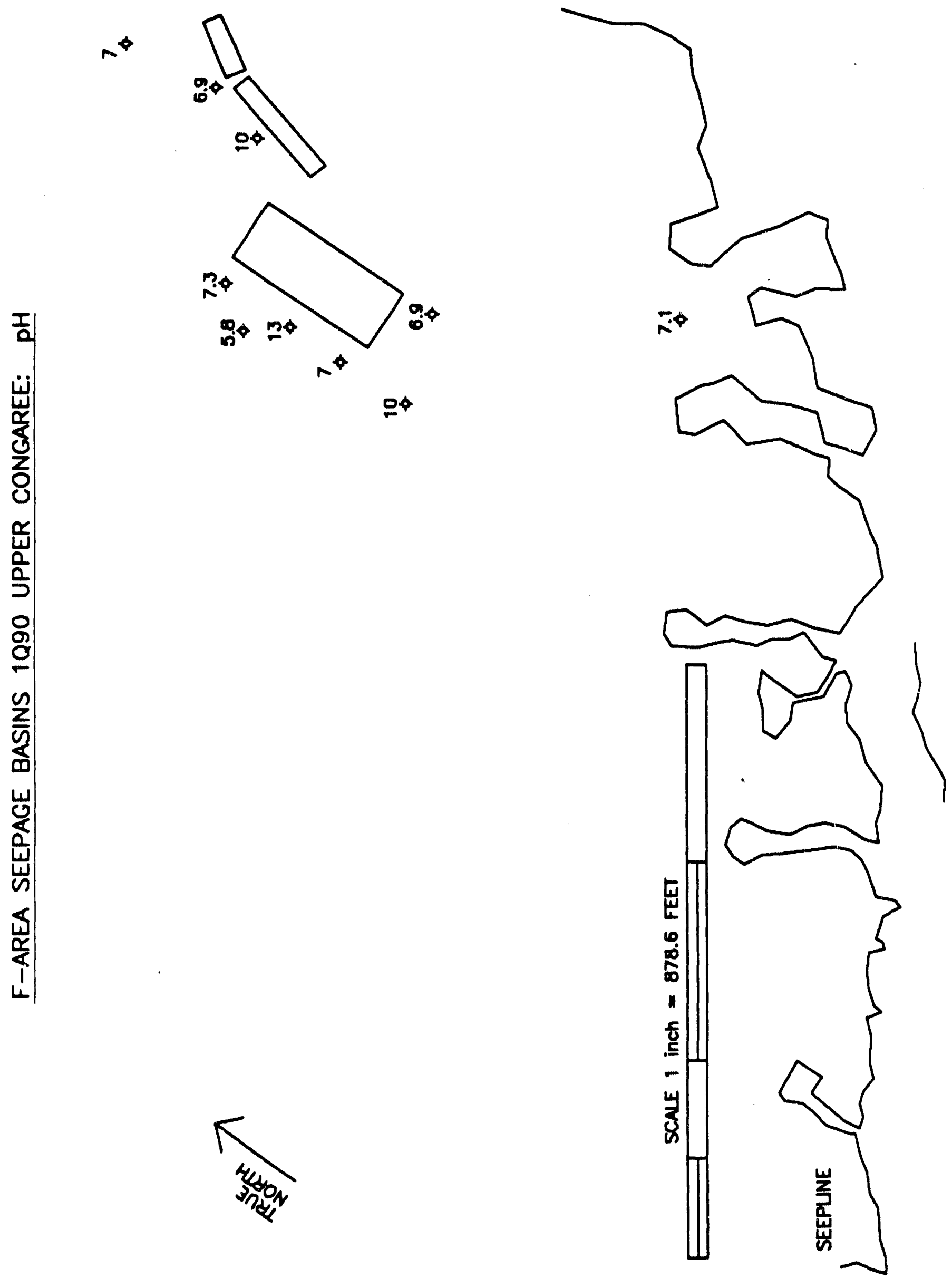



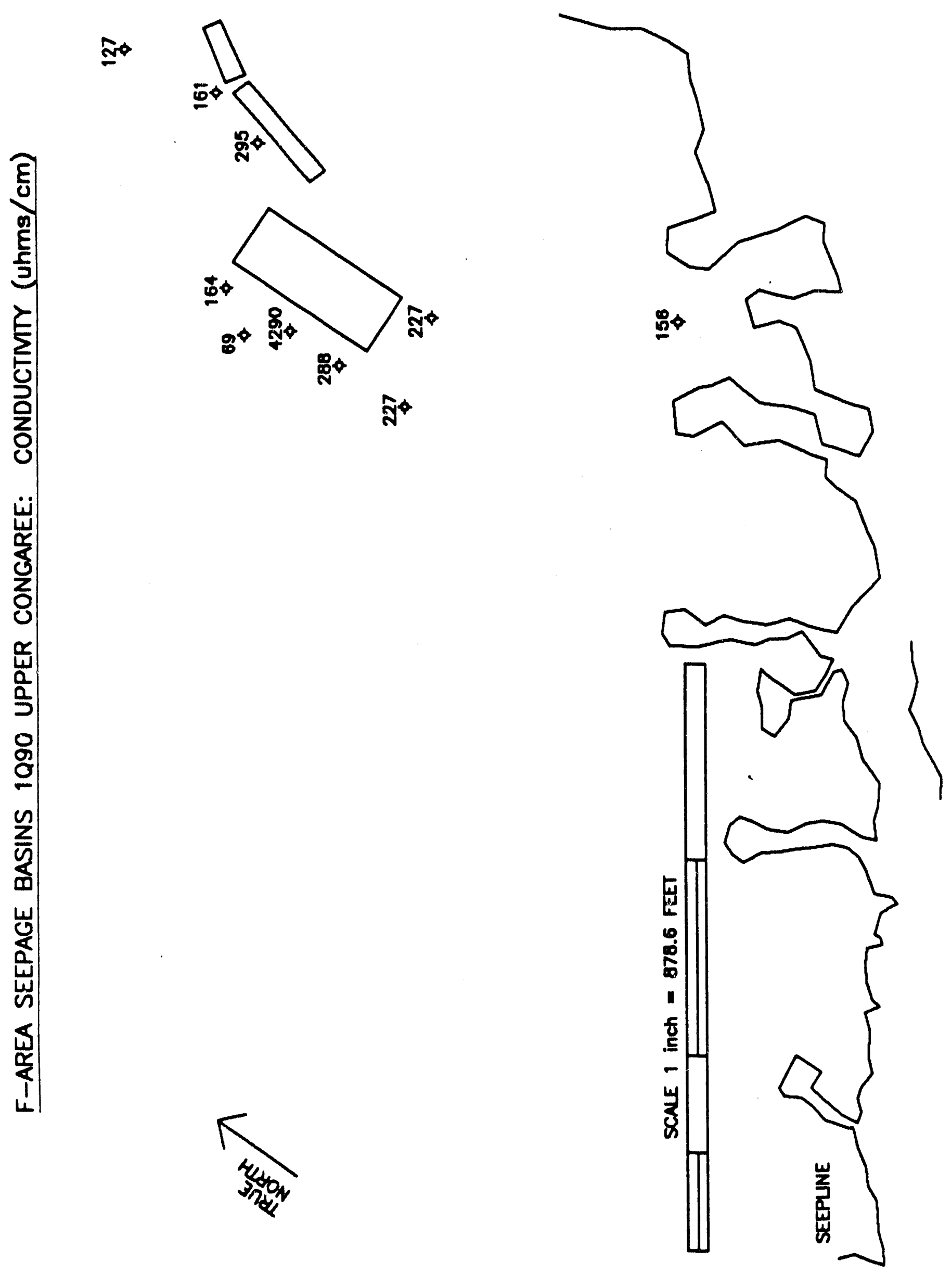

FIGURE B-UPCONG6 

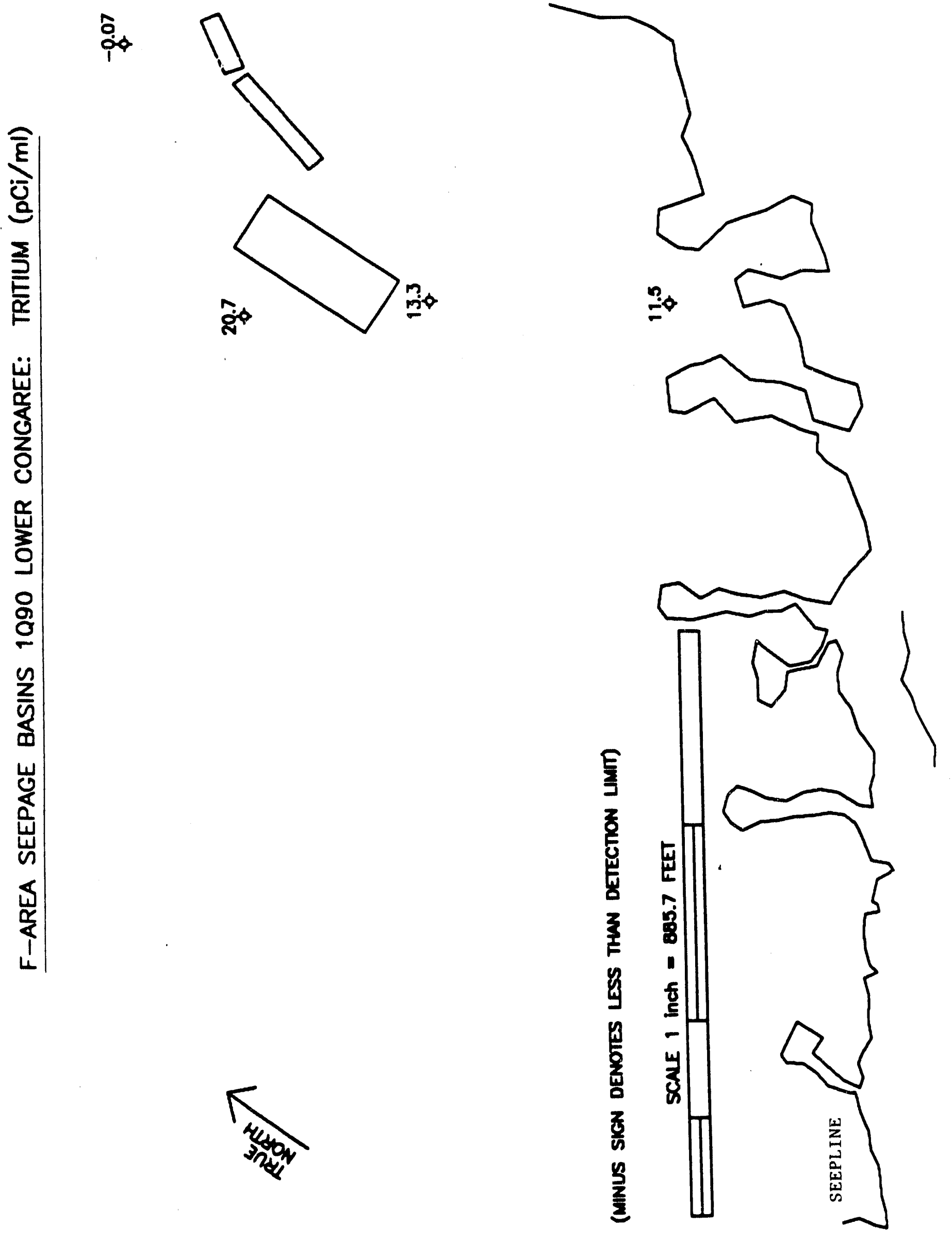

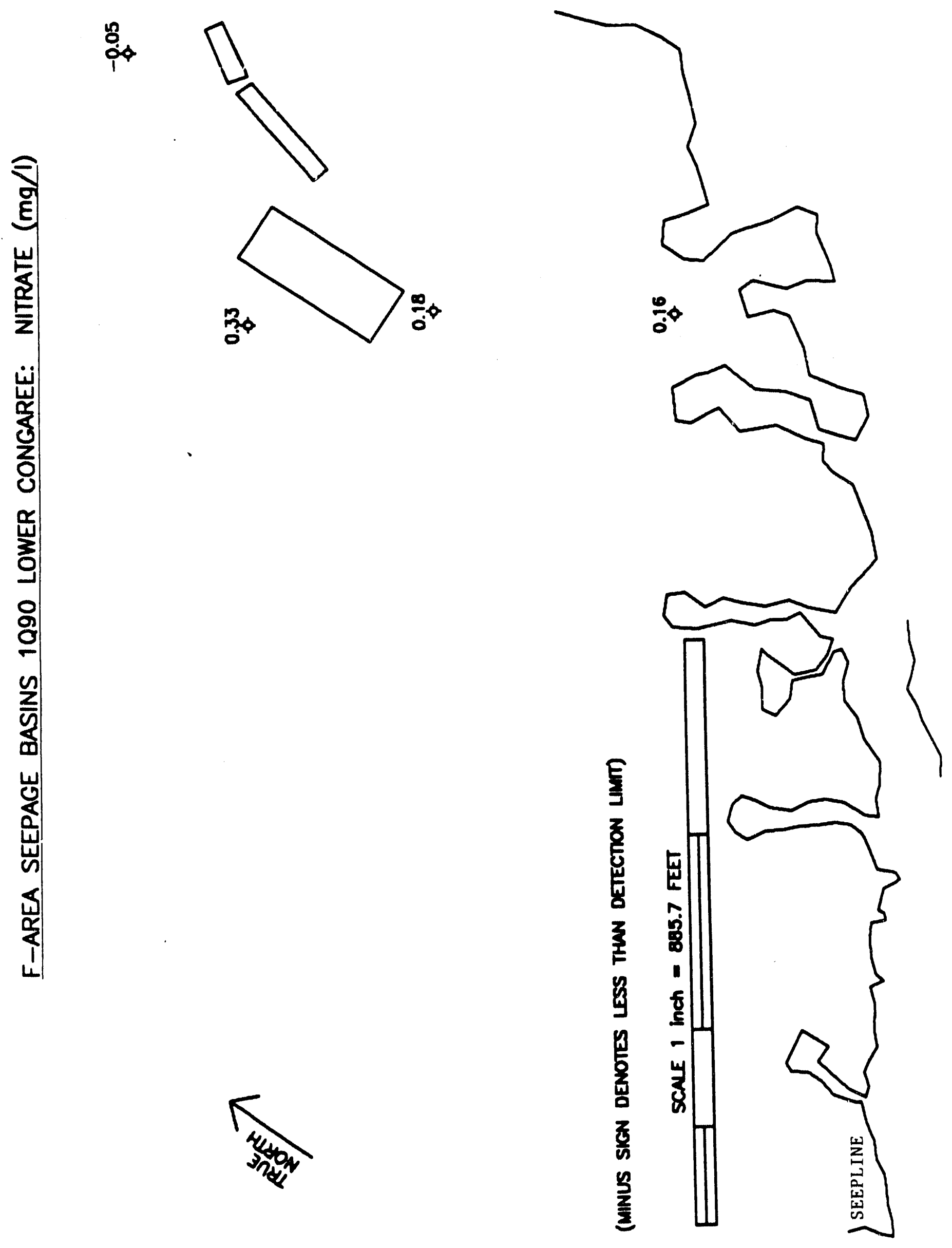

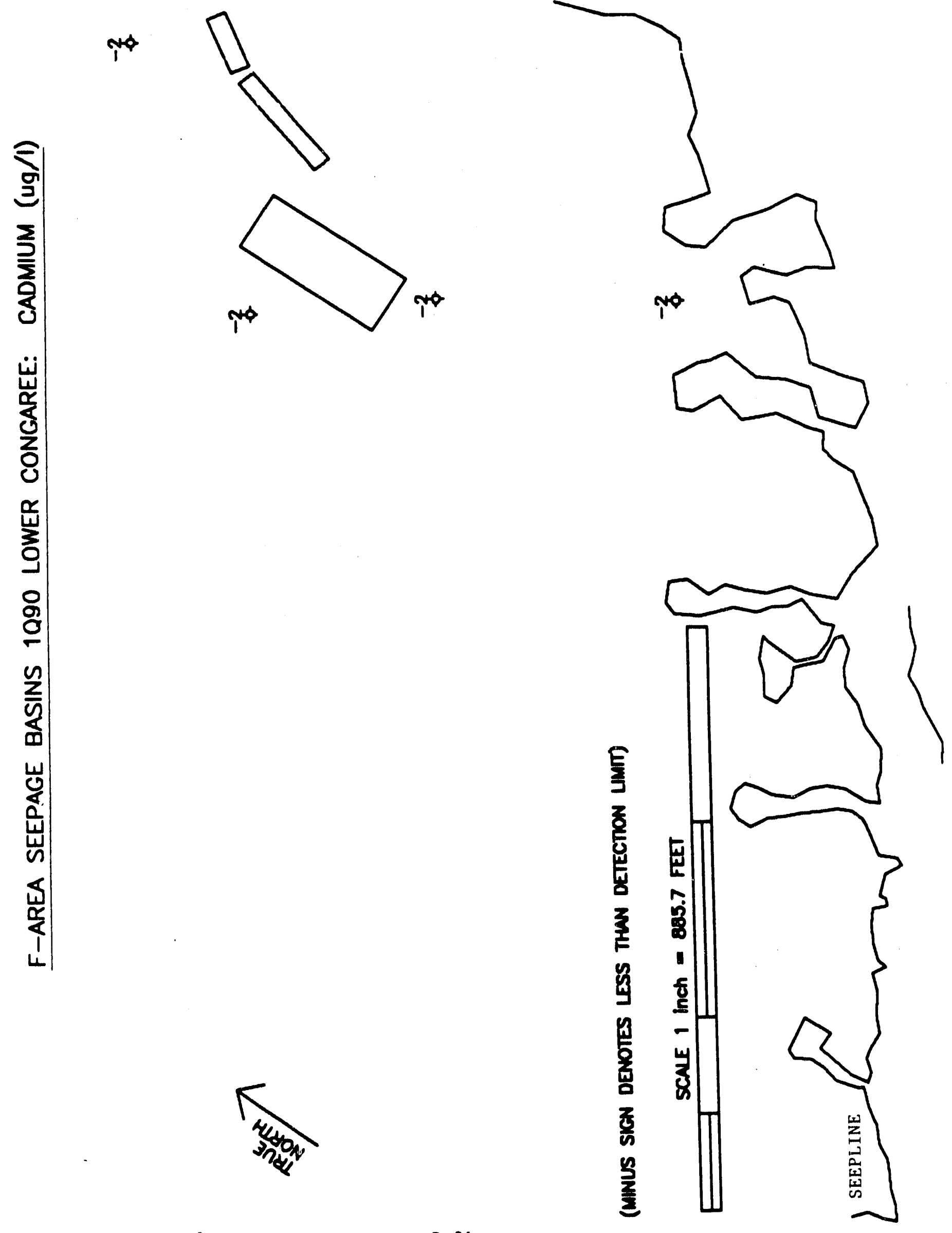


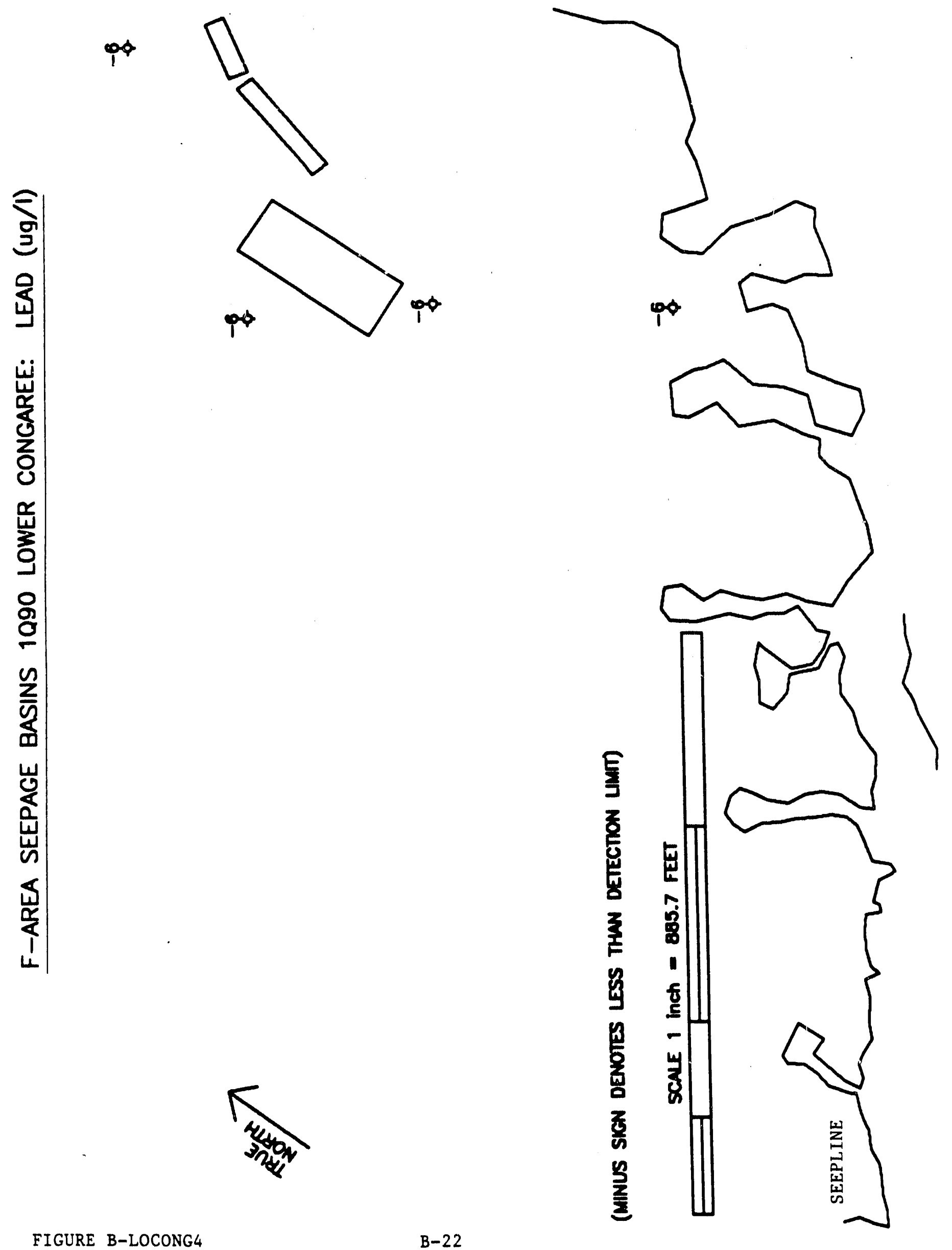



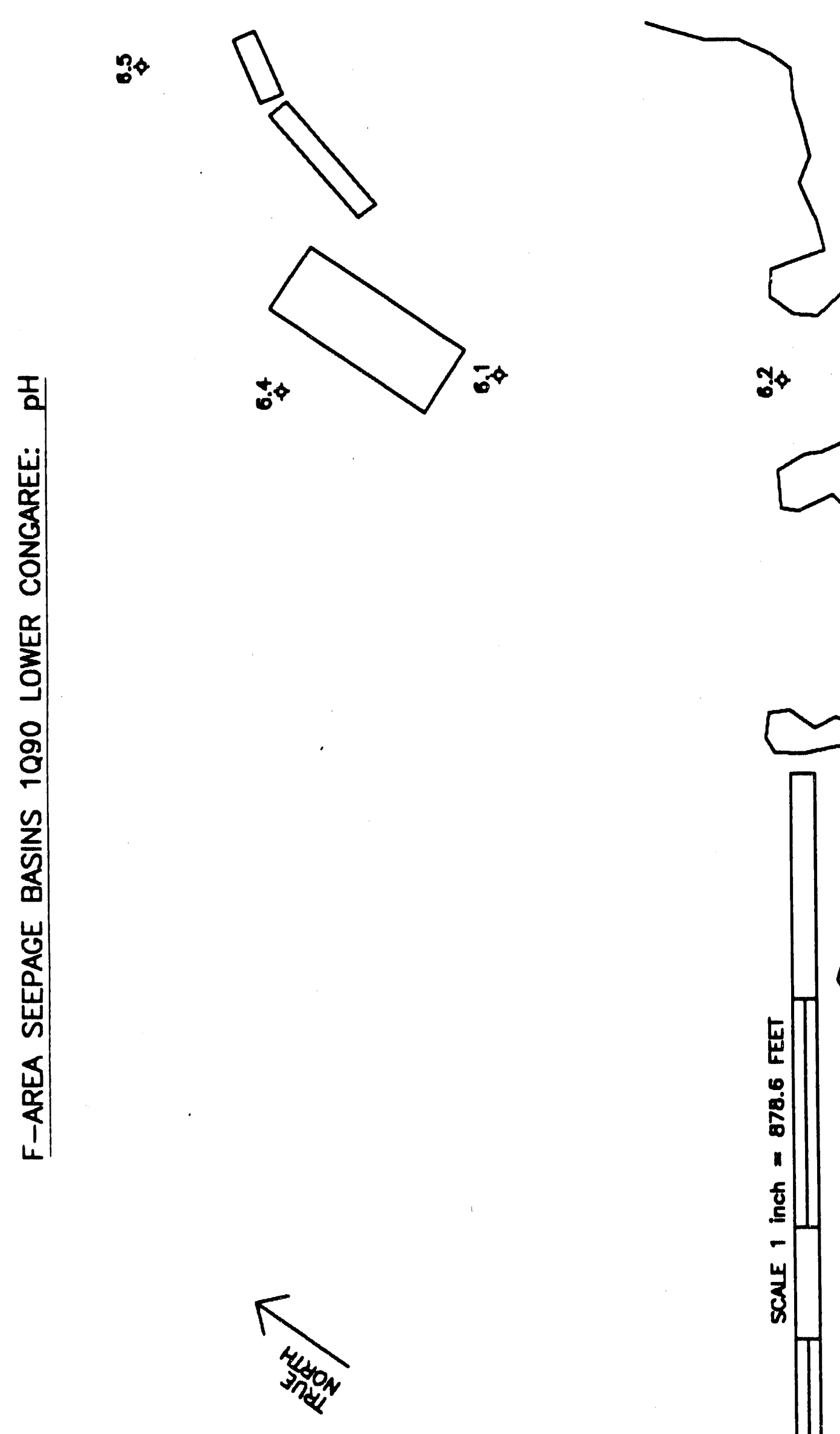

I
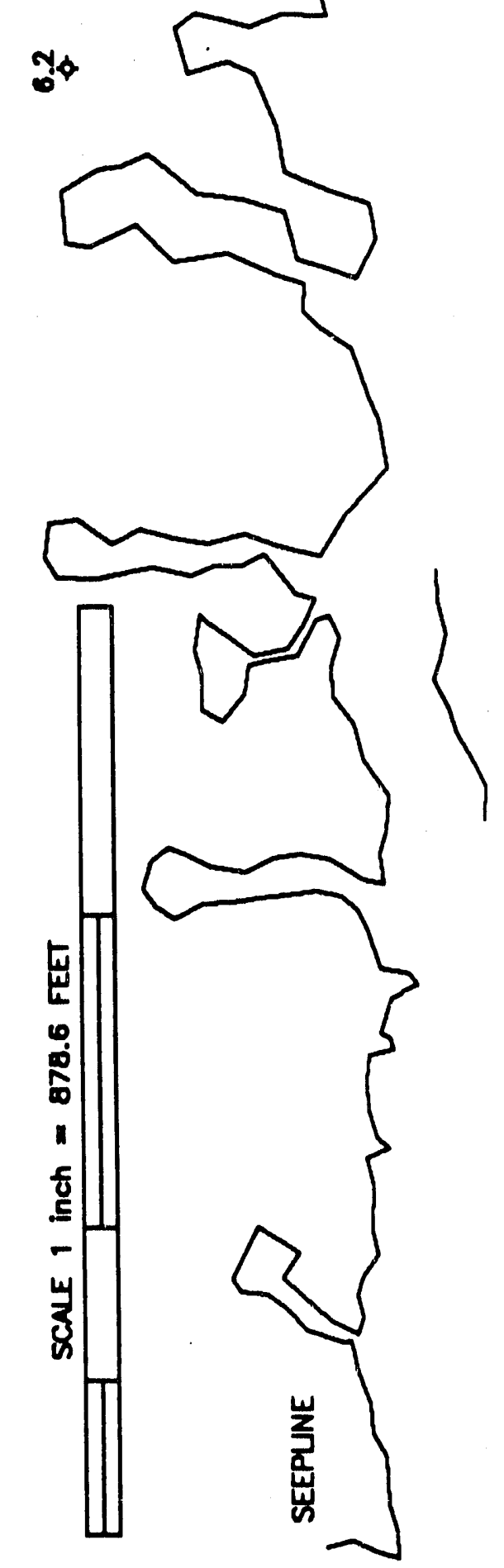

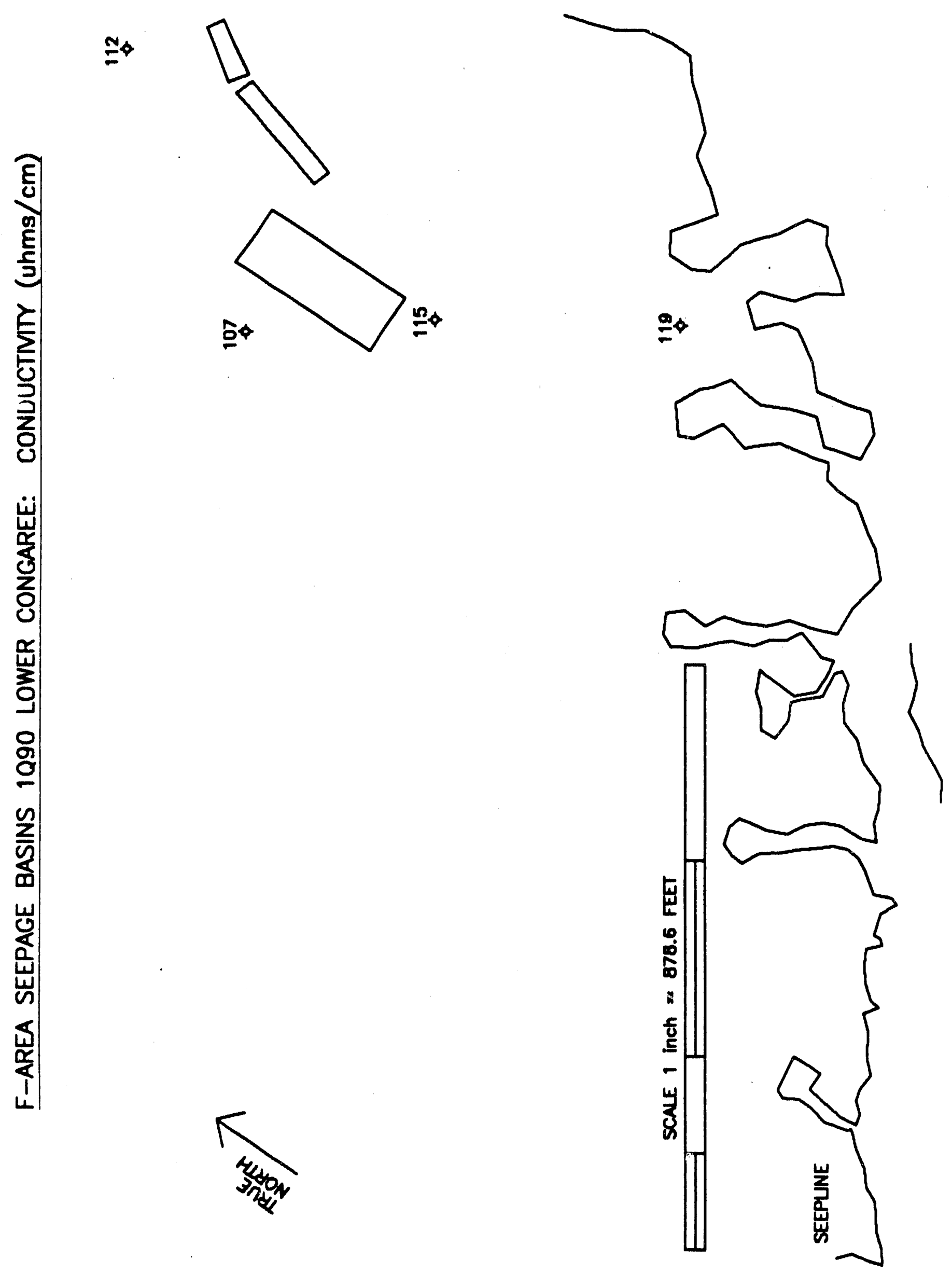


\section{APREADIX C}

\section{HYDROGRAPHS}

Figures C1-C29 display the water levels in each hydrologic unit in every well cluster as a function of time. The hydrographs demonstrate the consistent vertical decline in water levels experienced since closure operations began. 


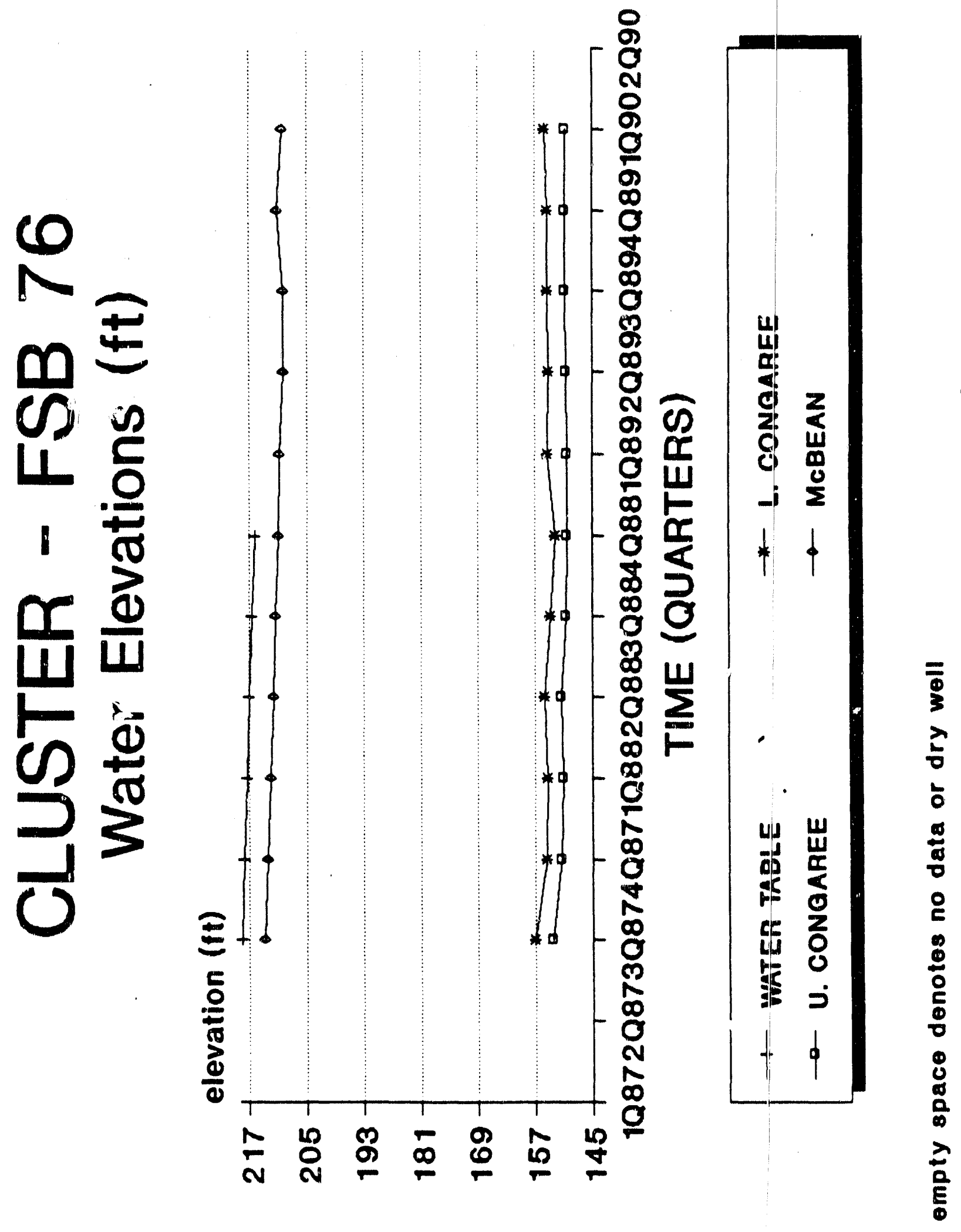




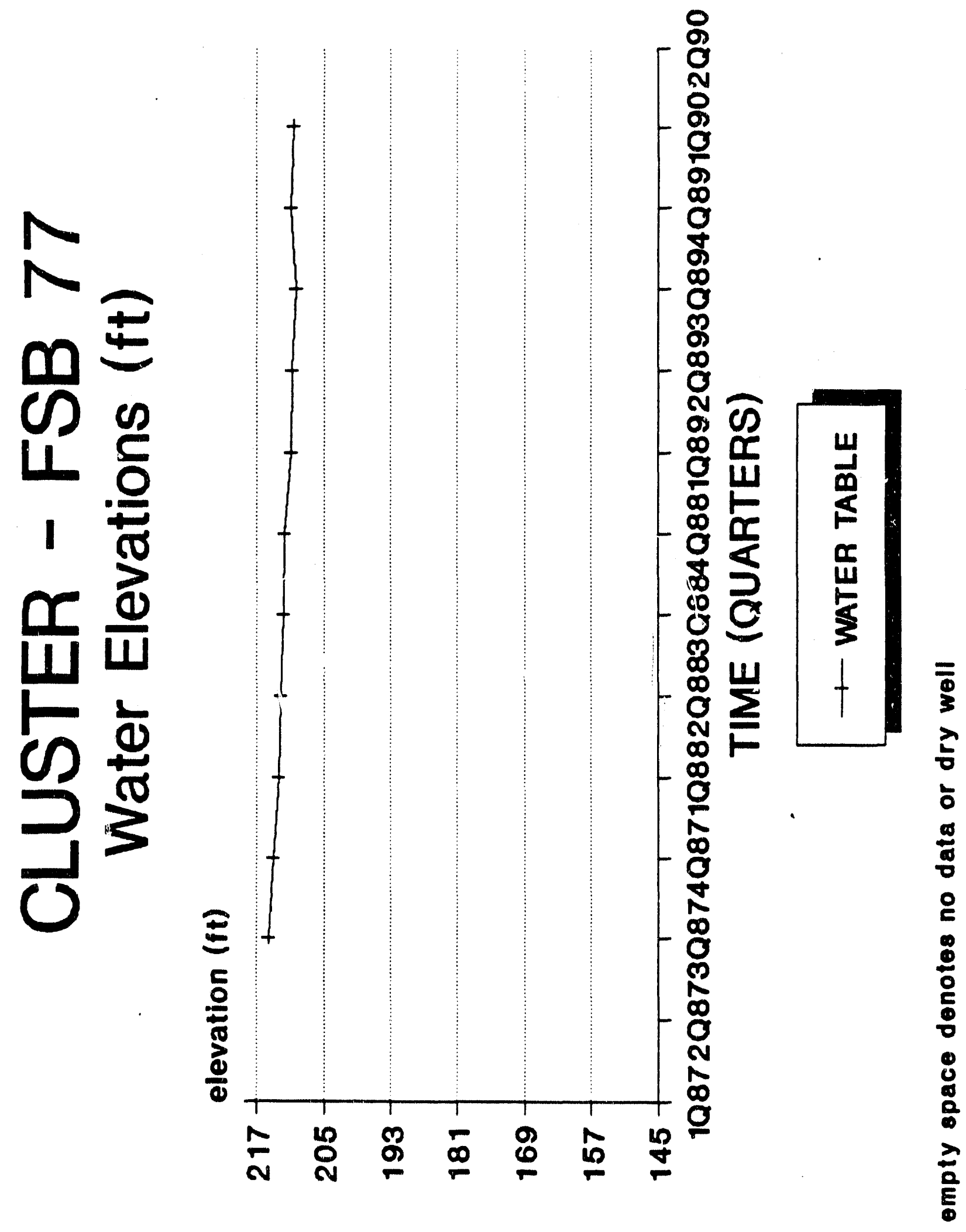




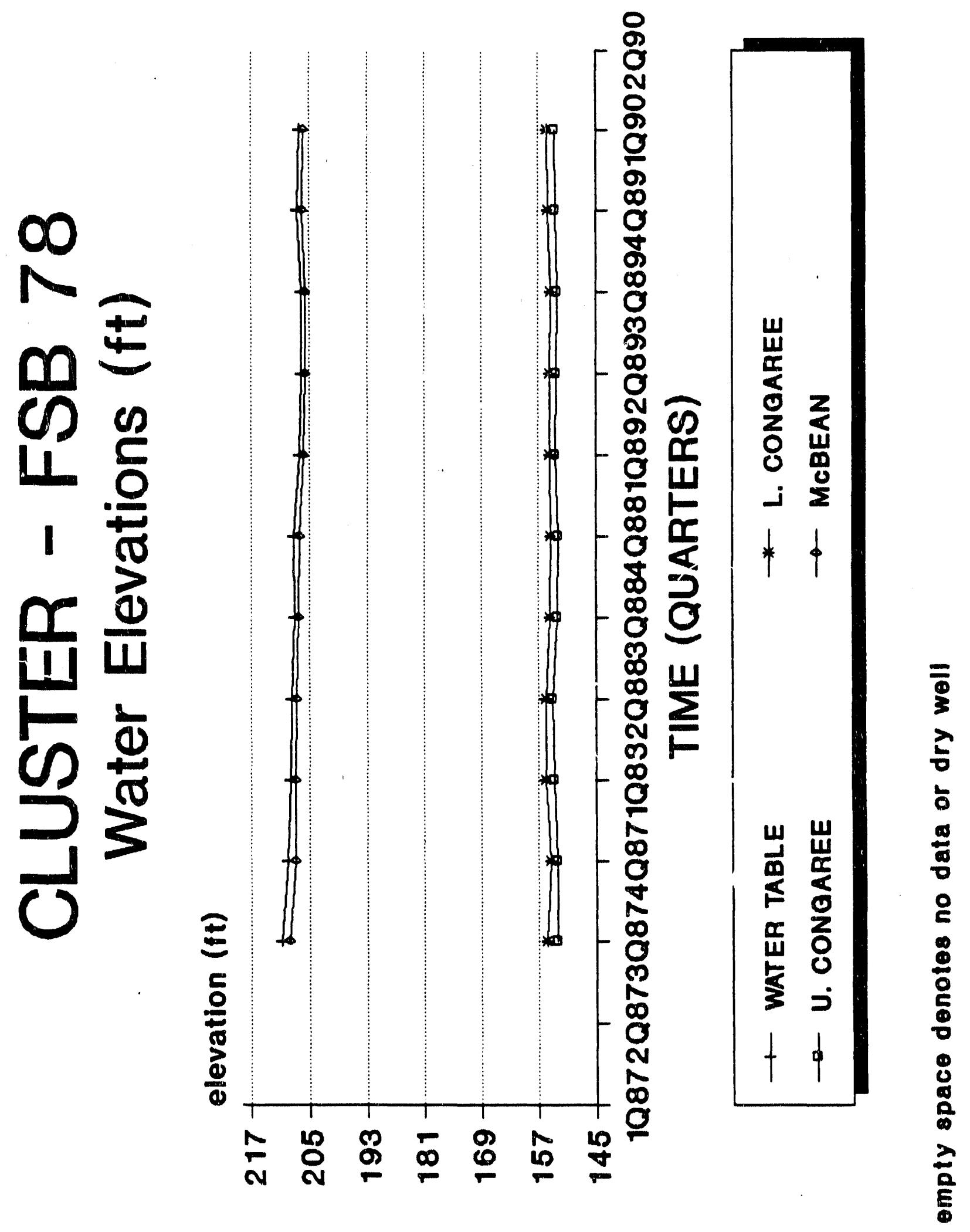




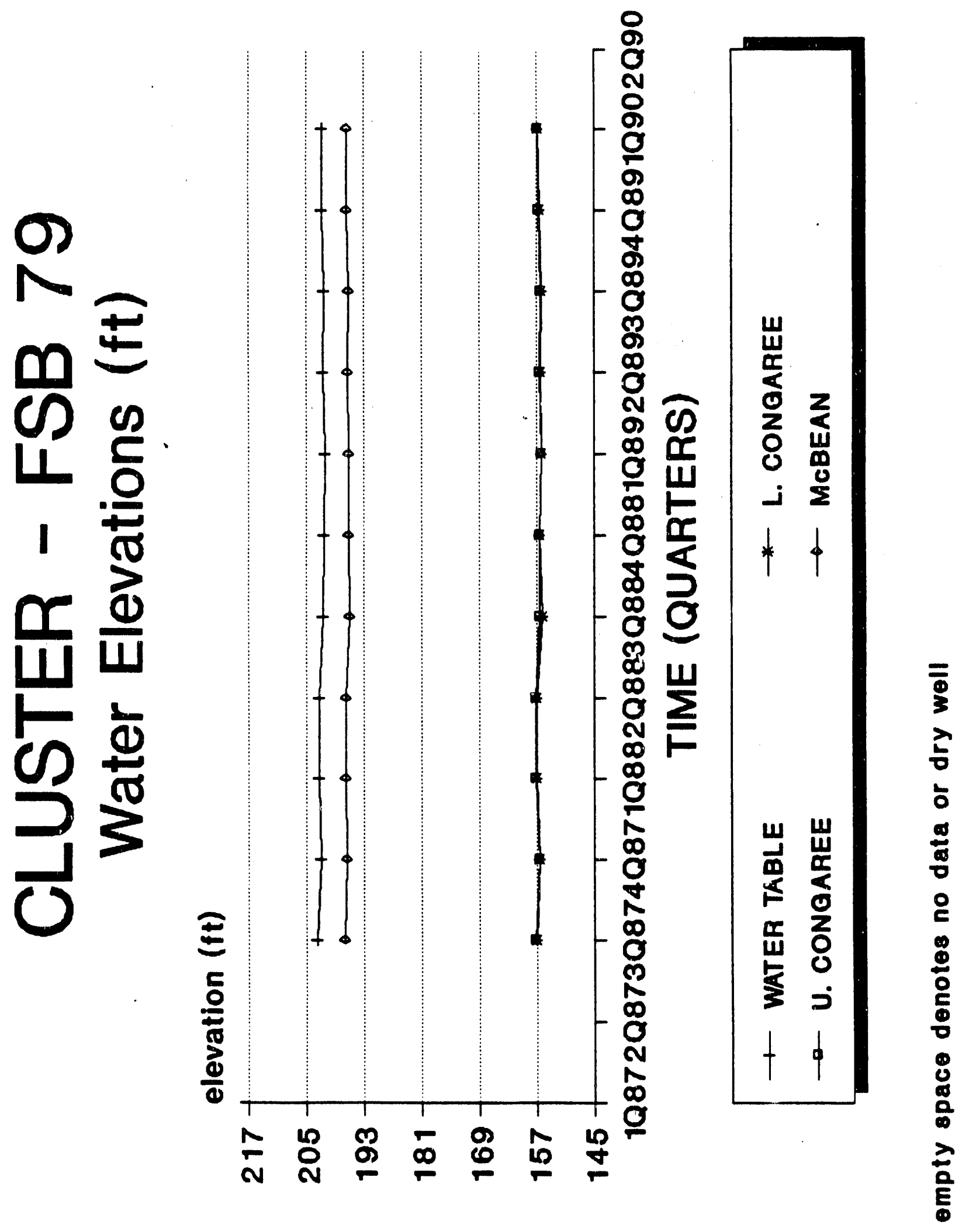




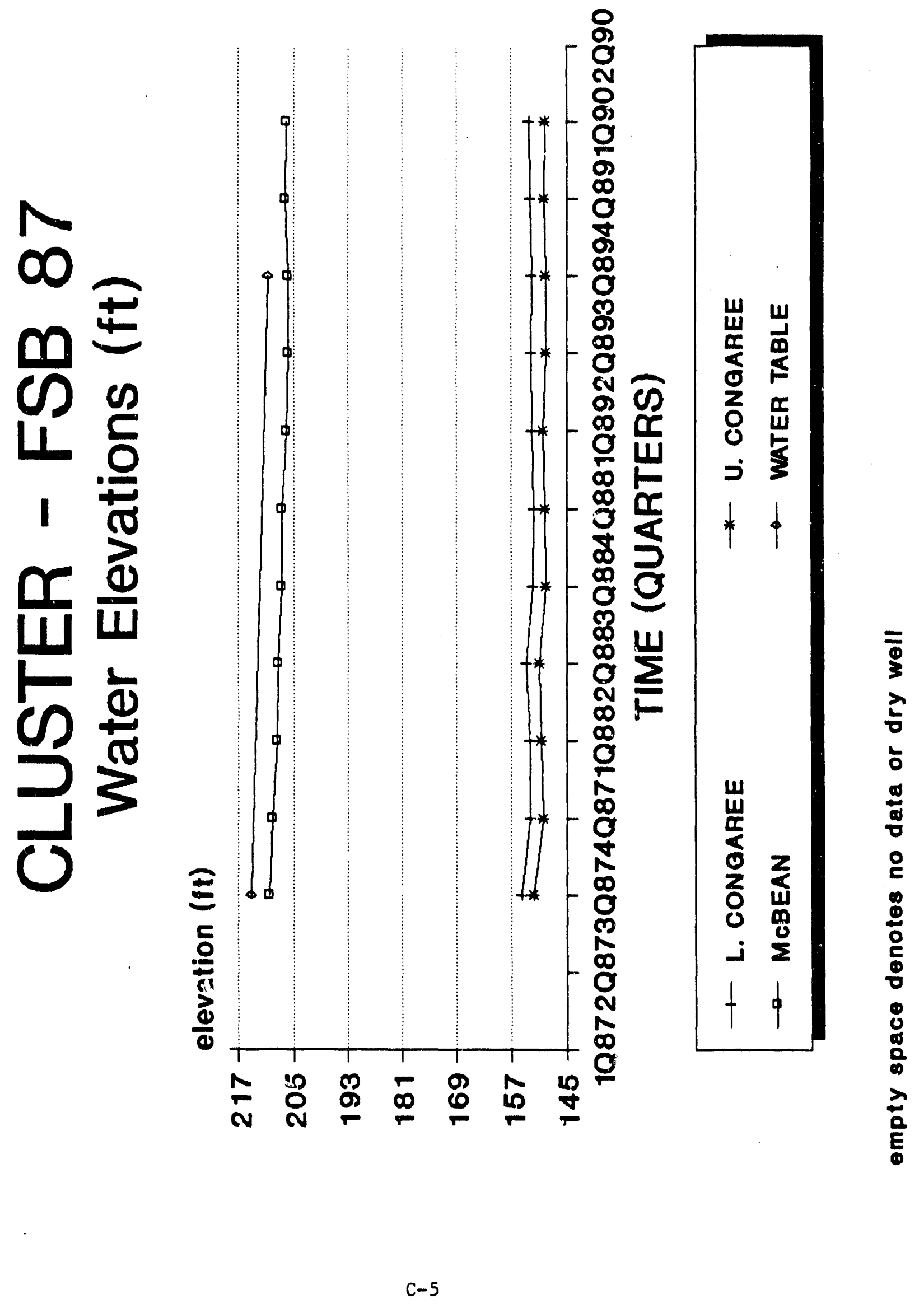




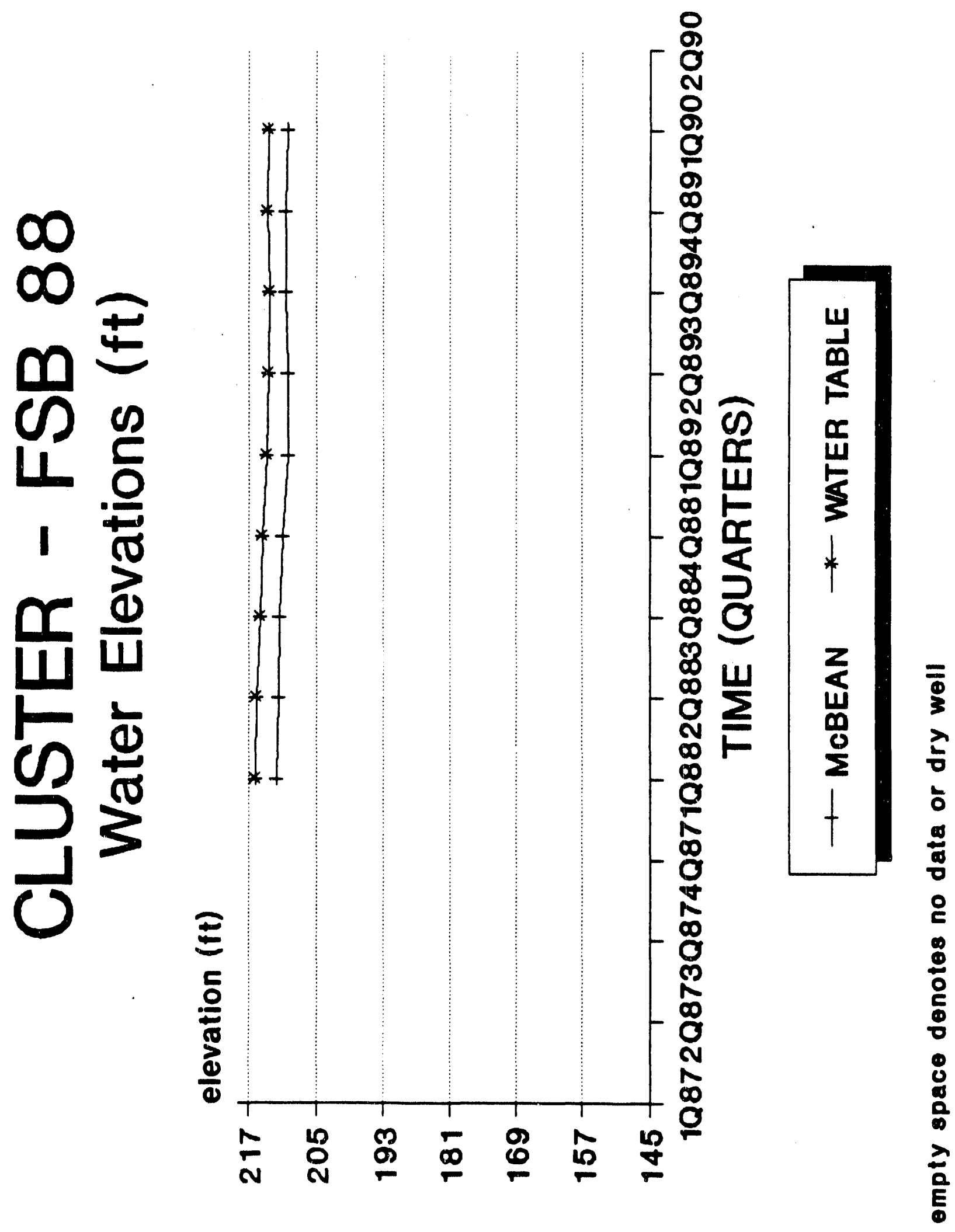




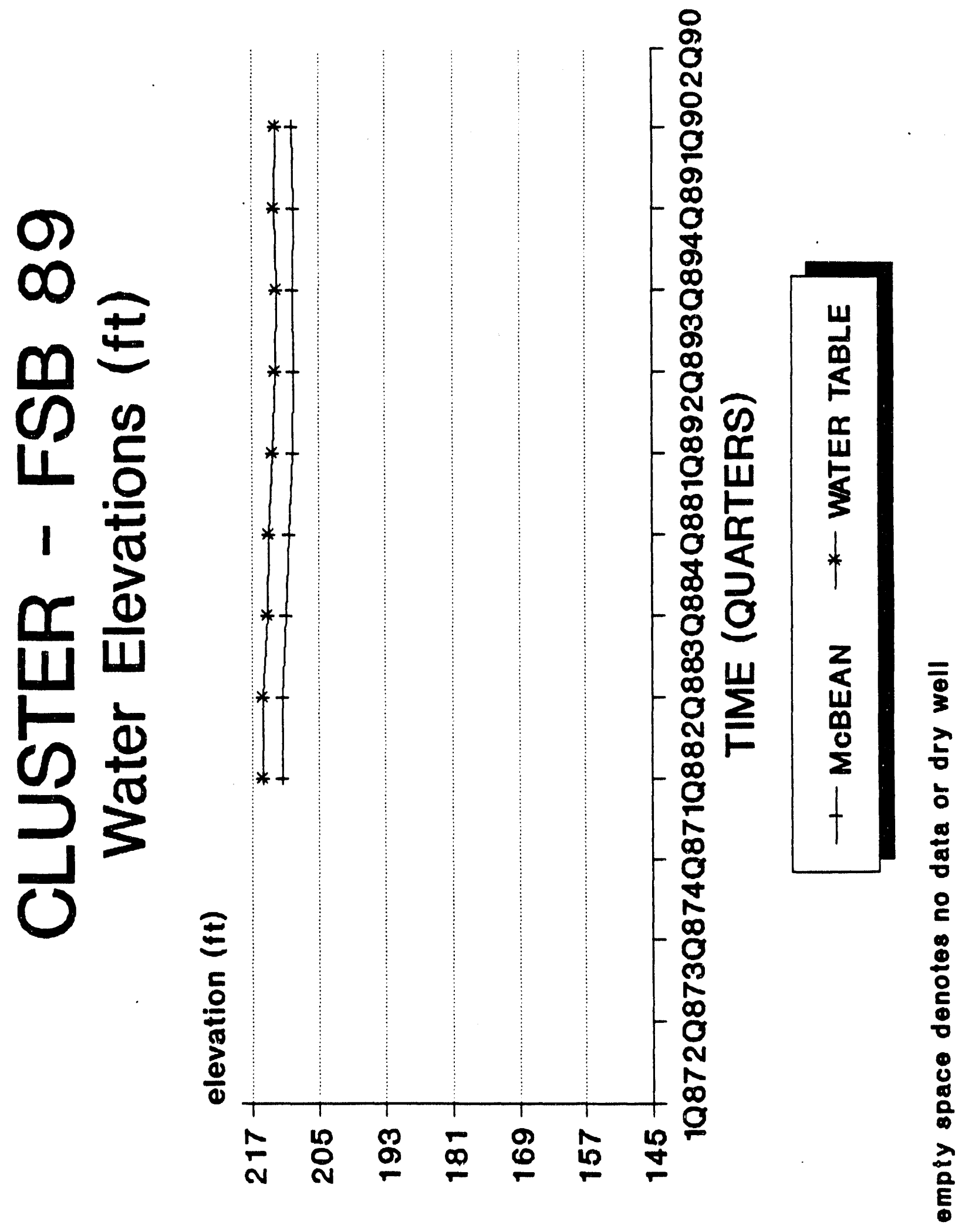




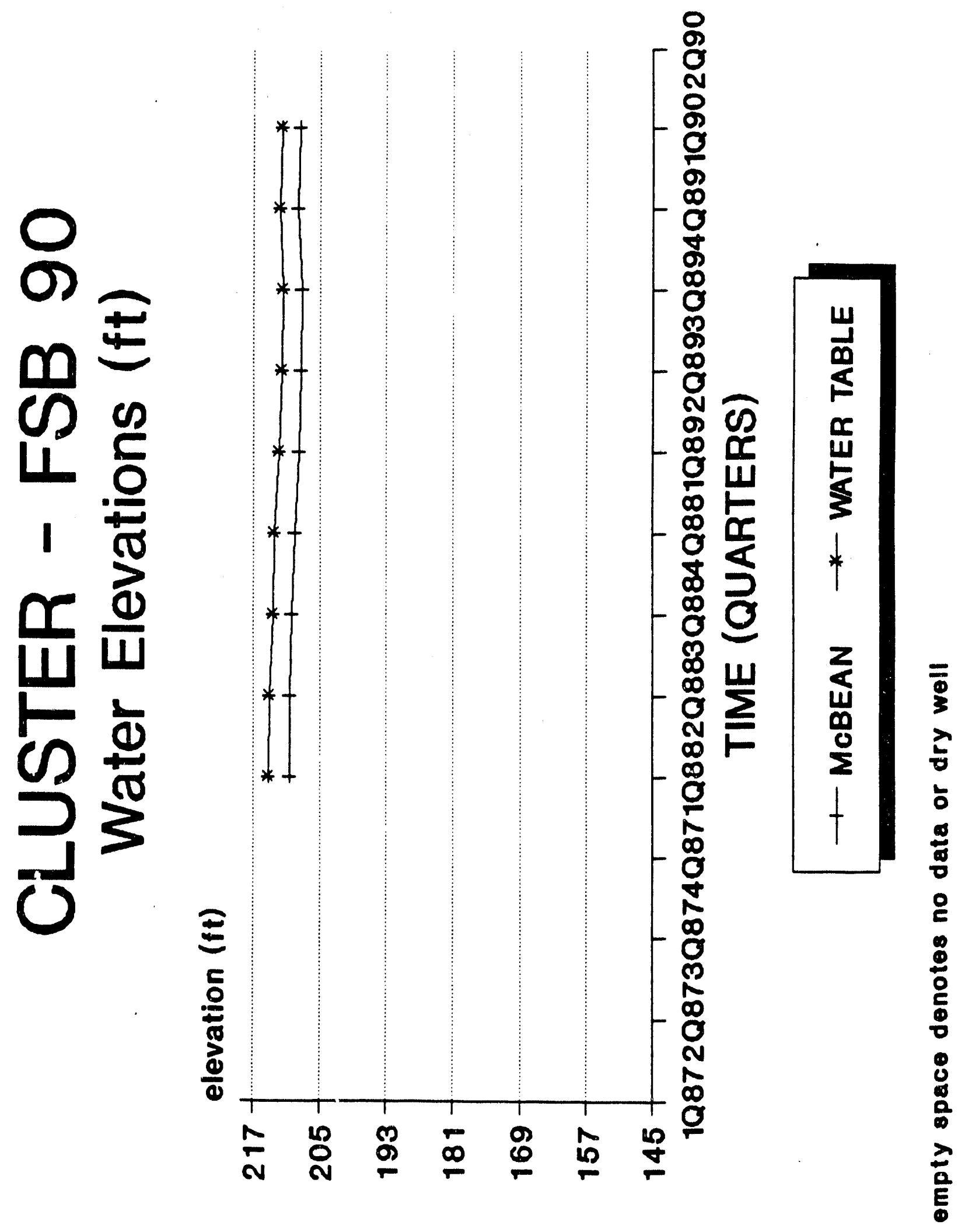




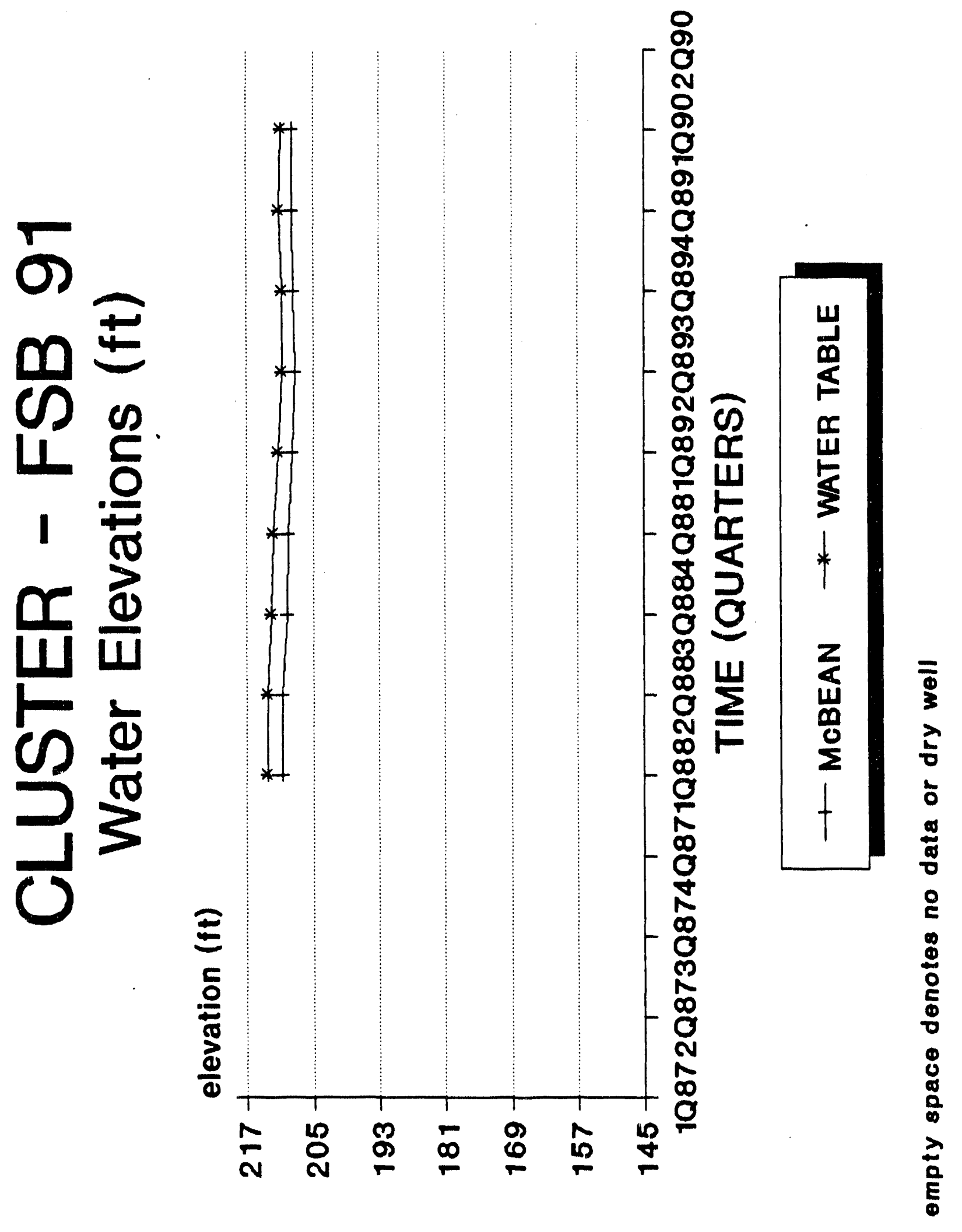




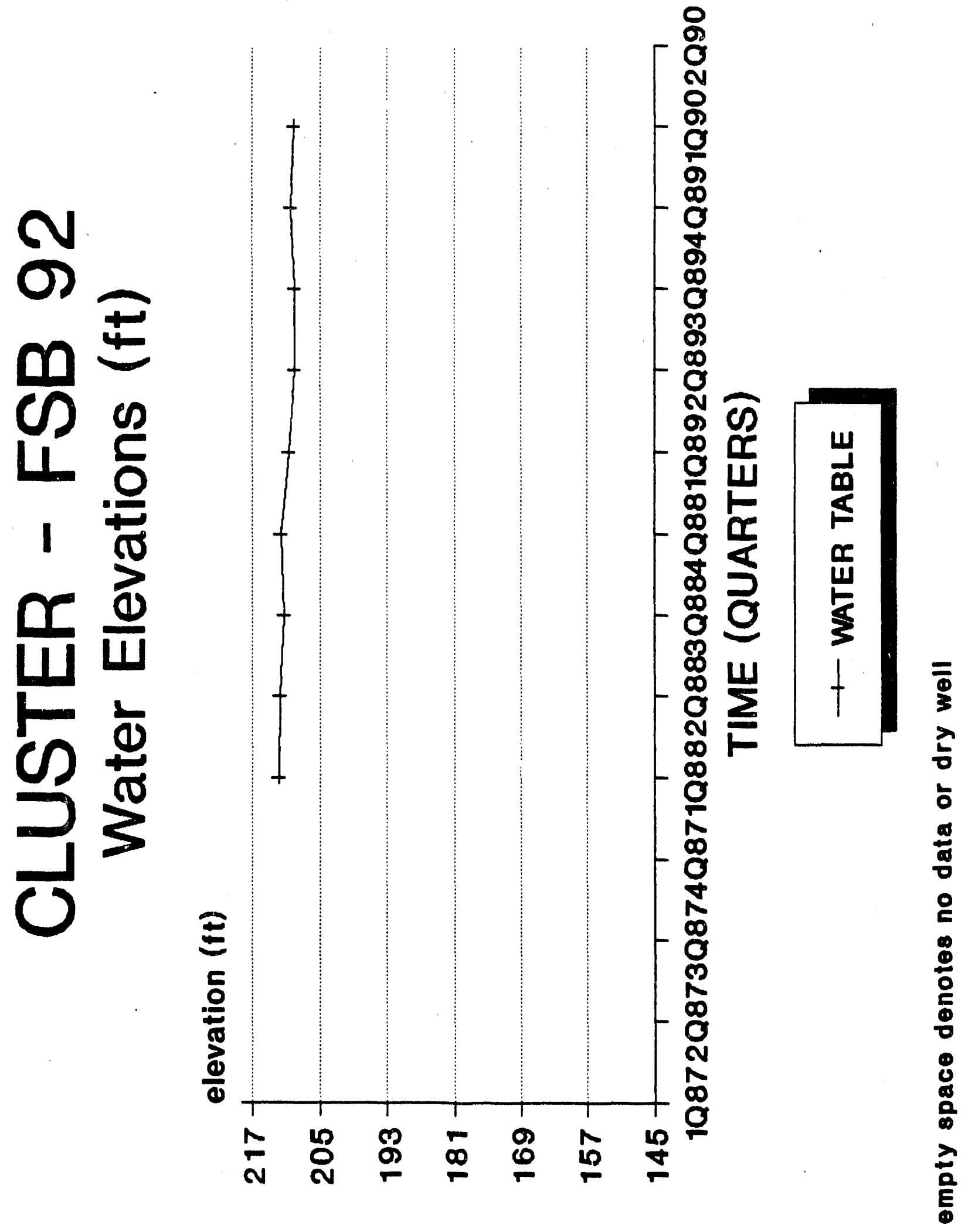




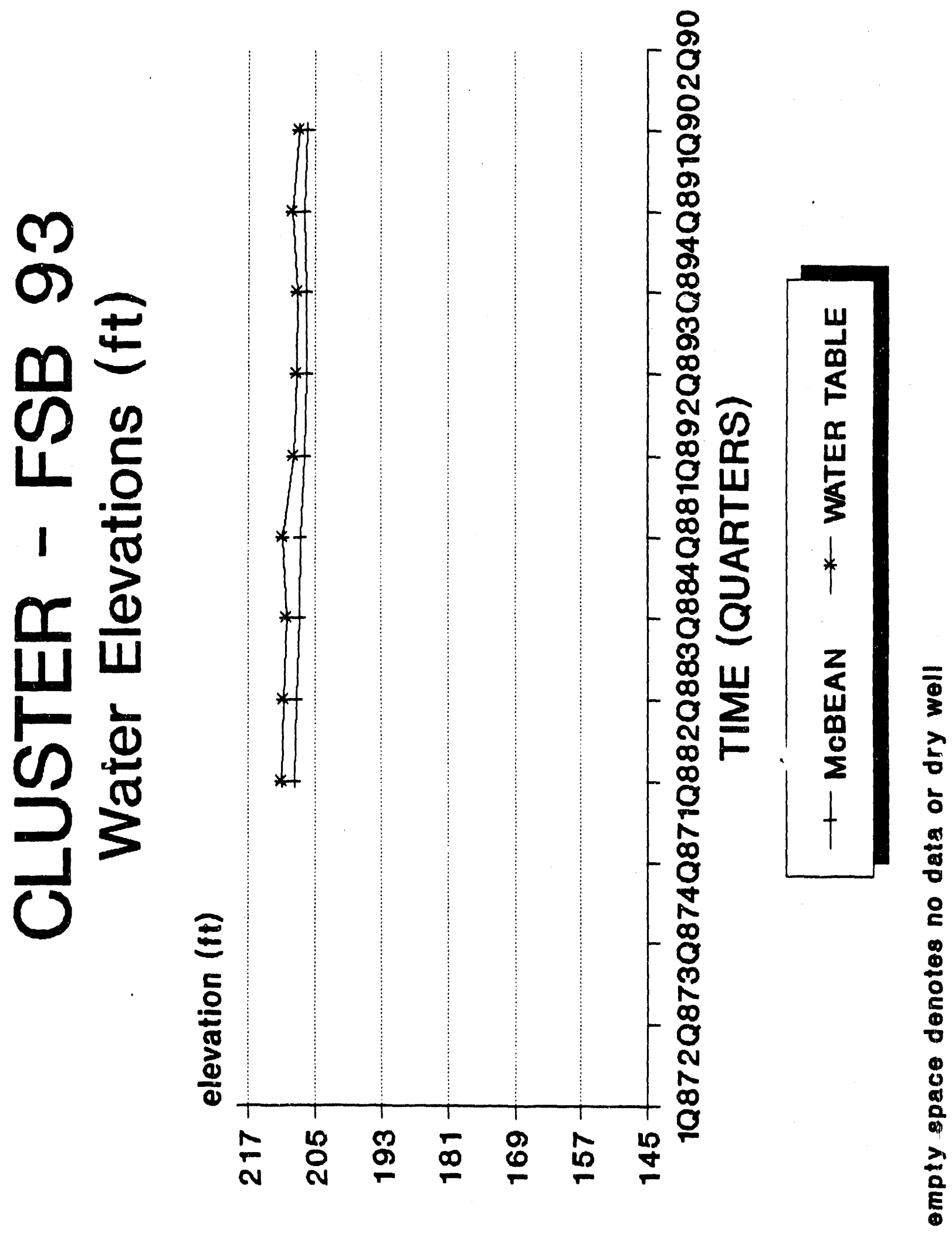




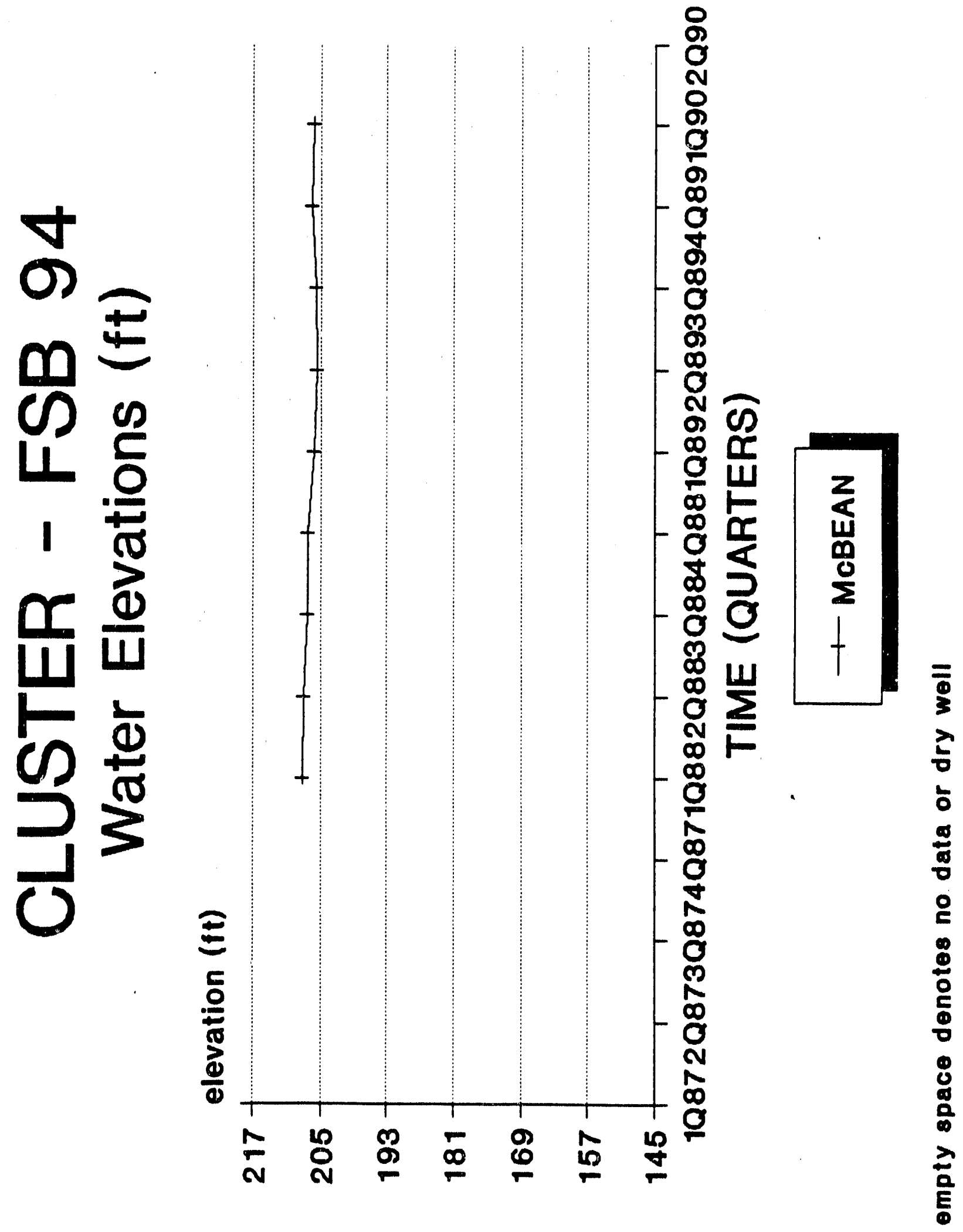




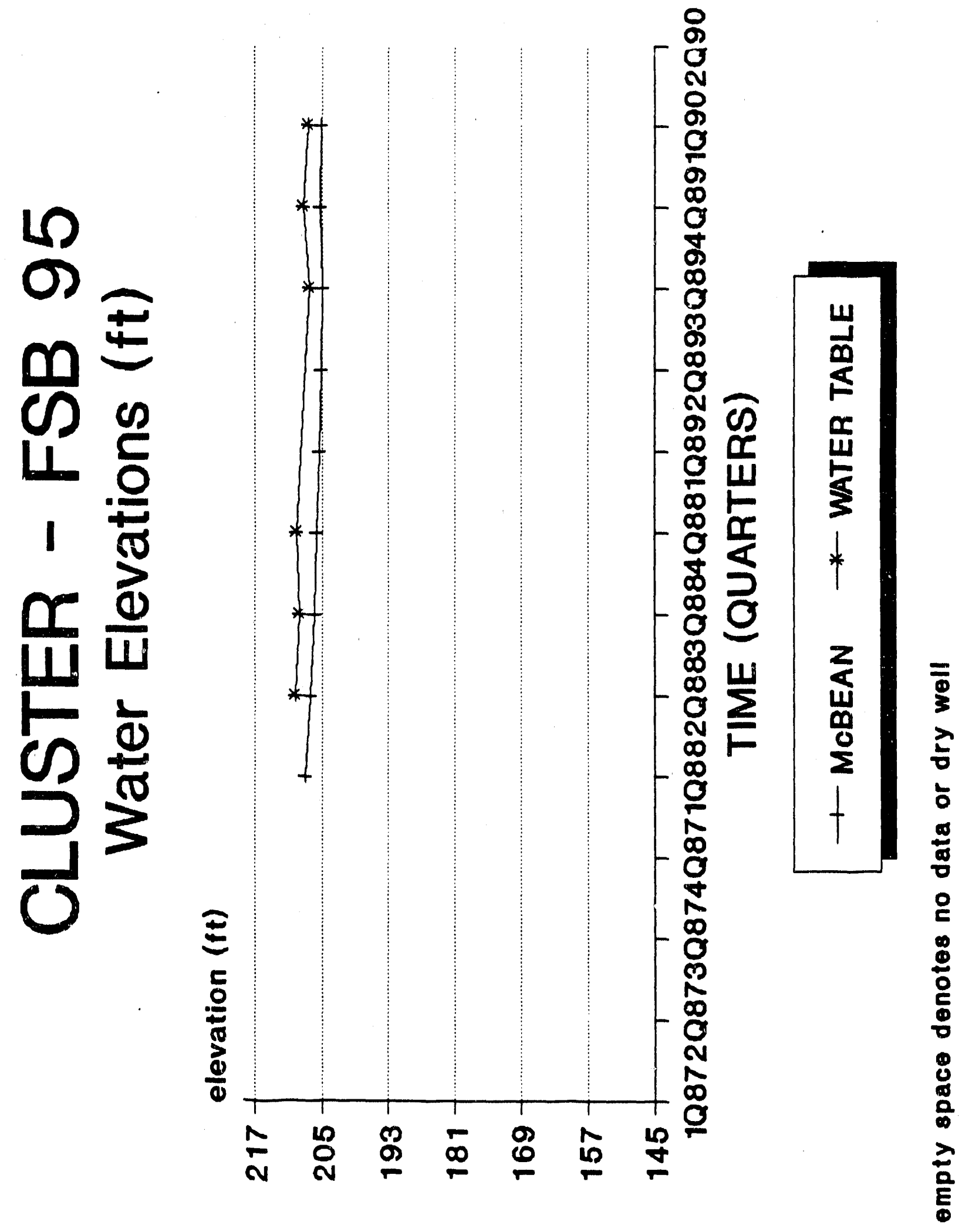




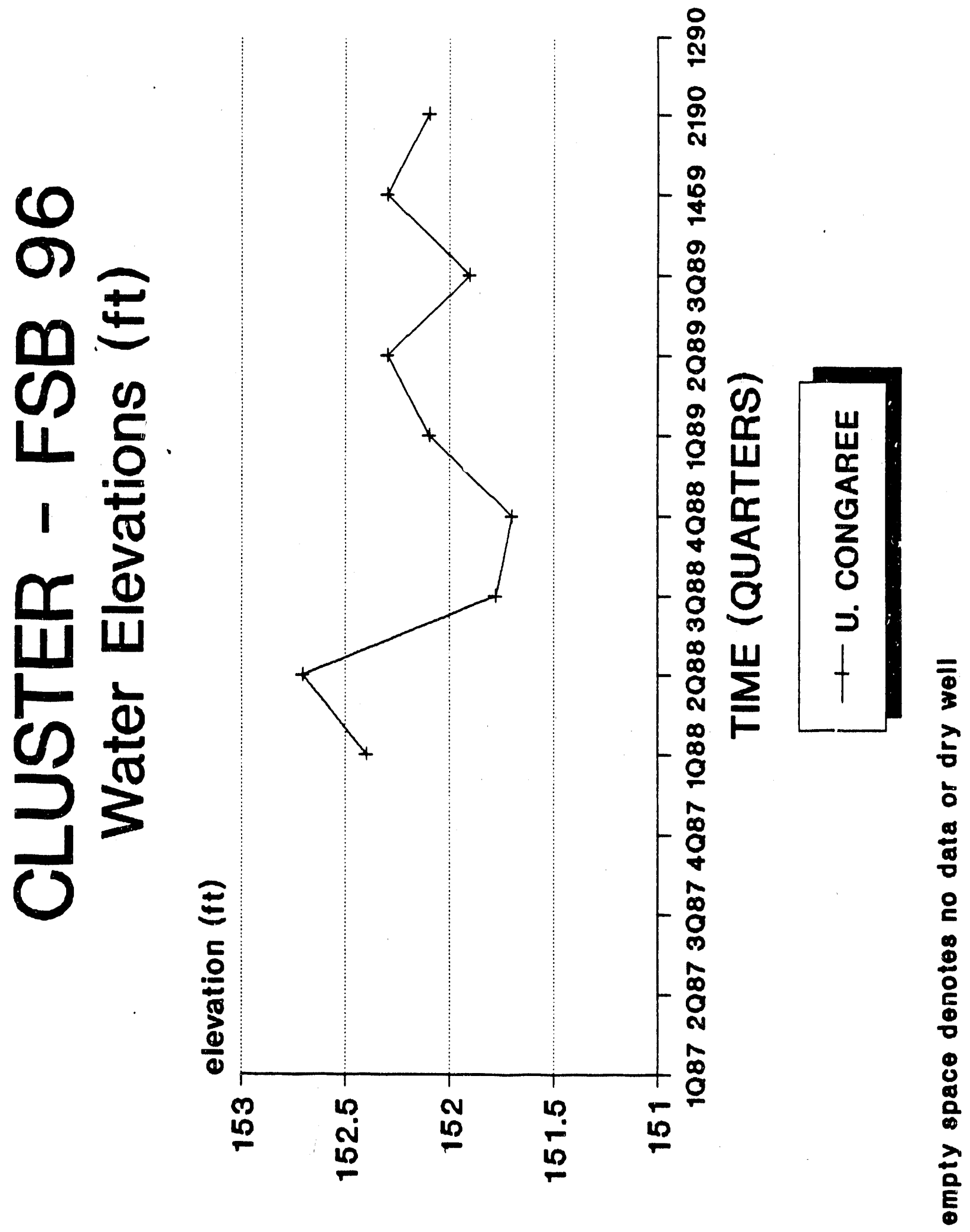




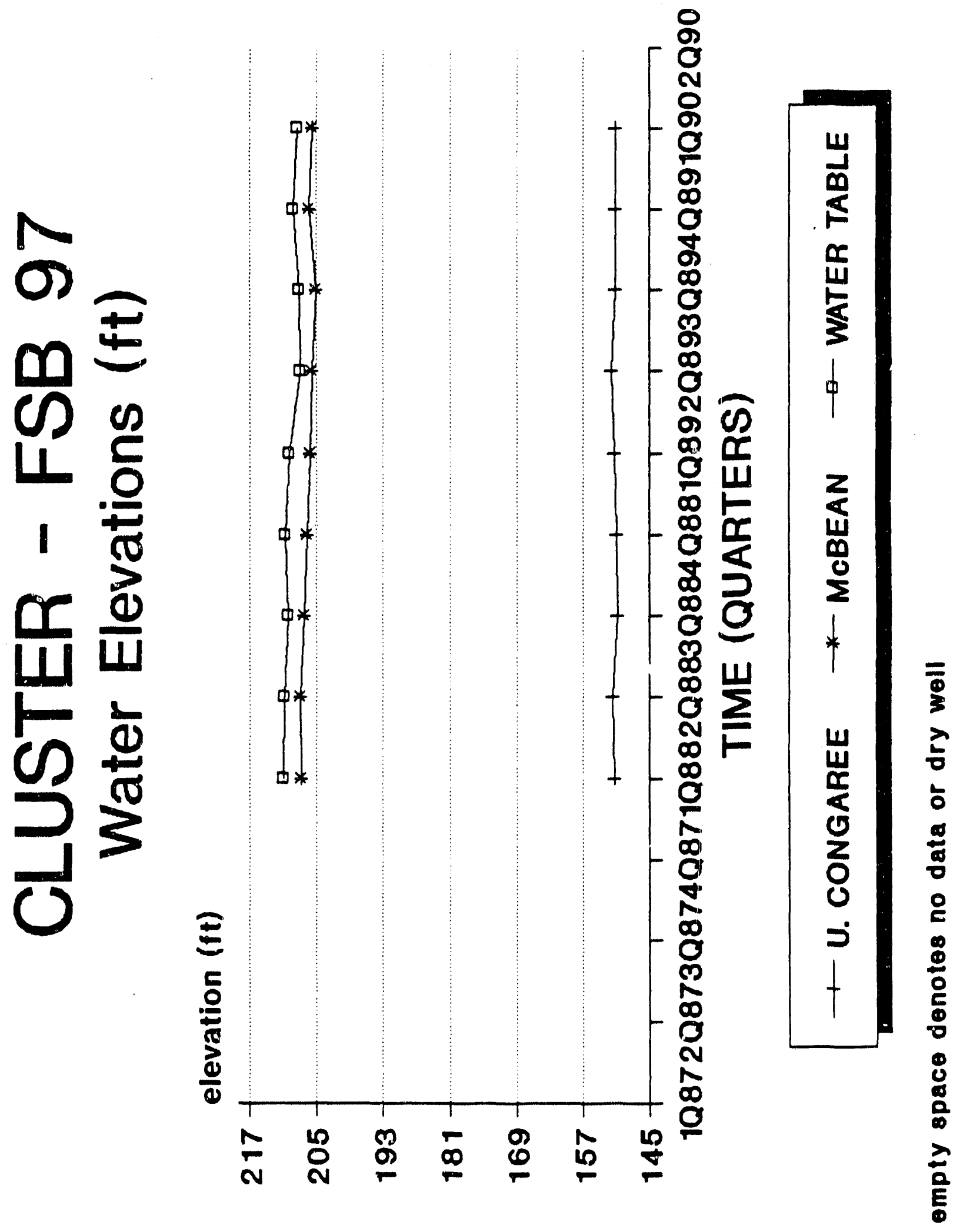




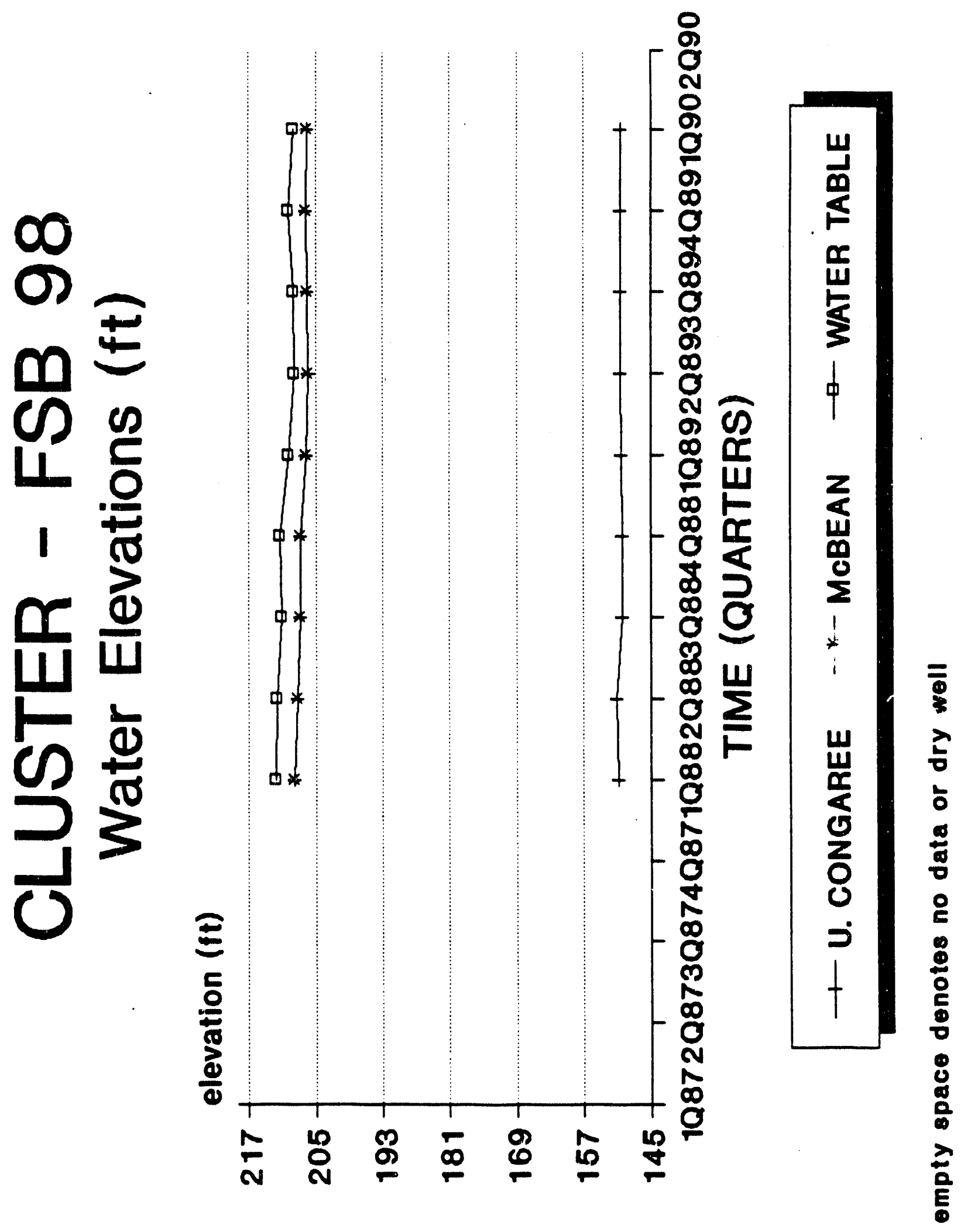




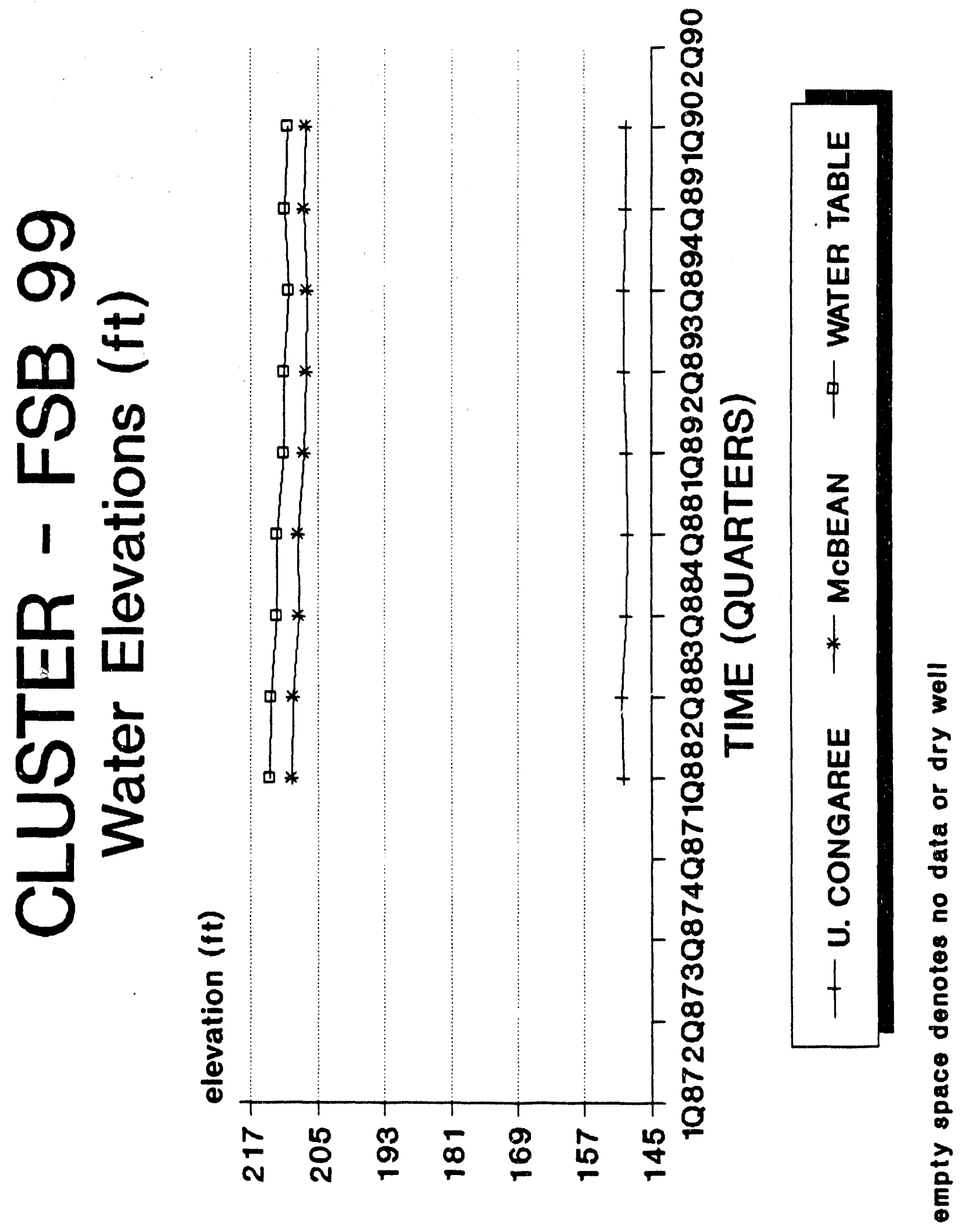




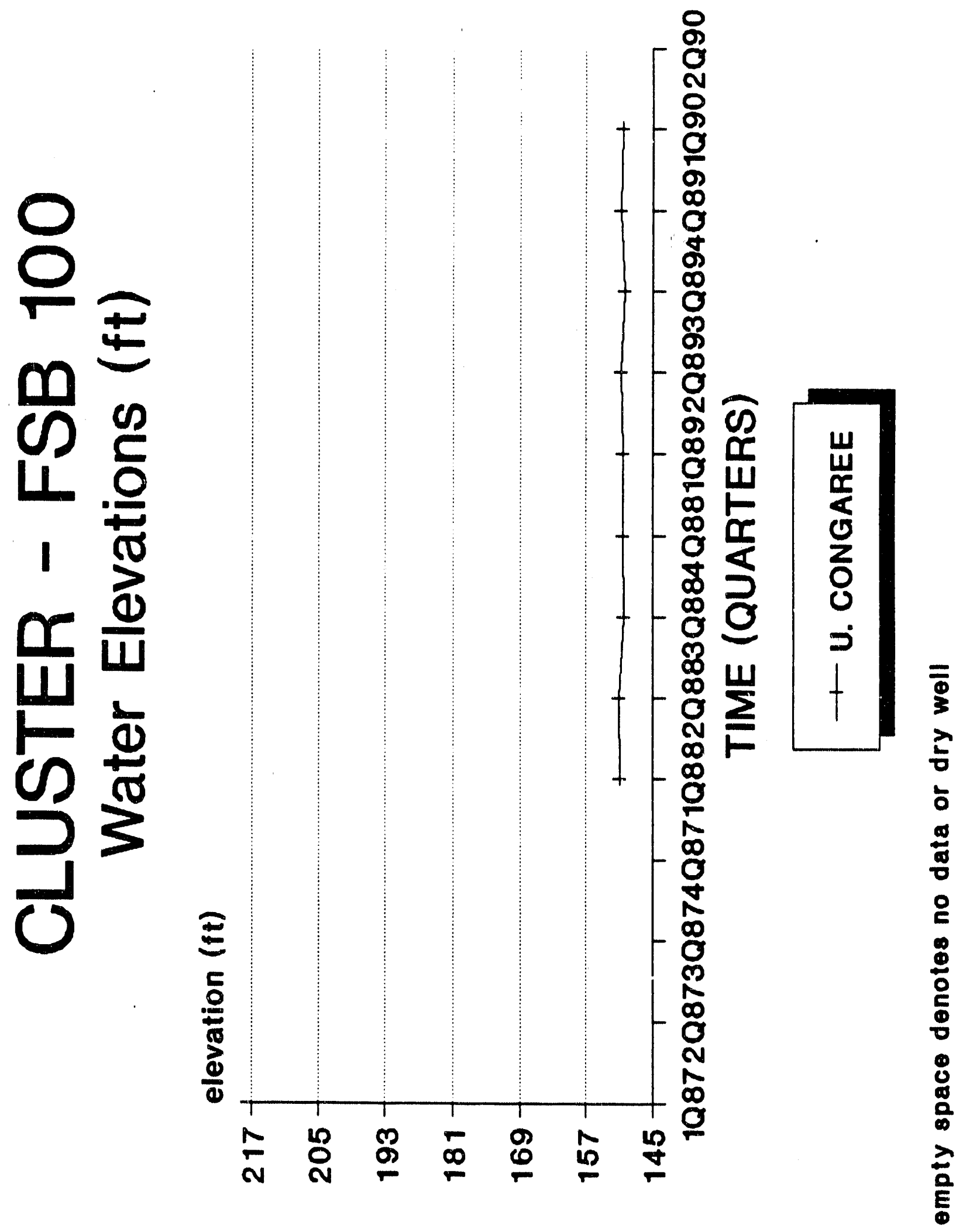




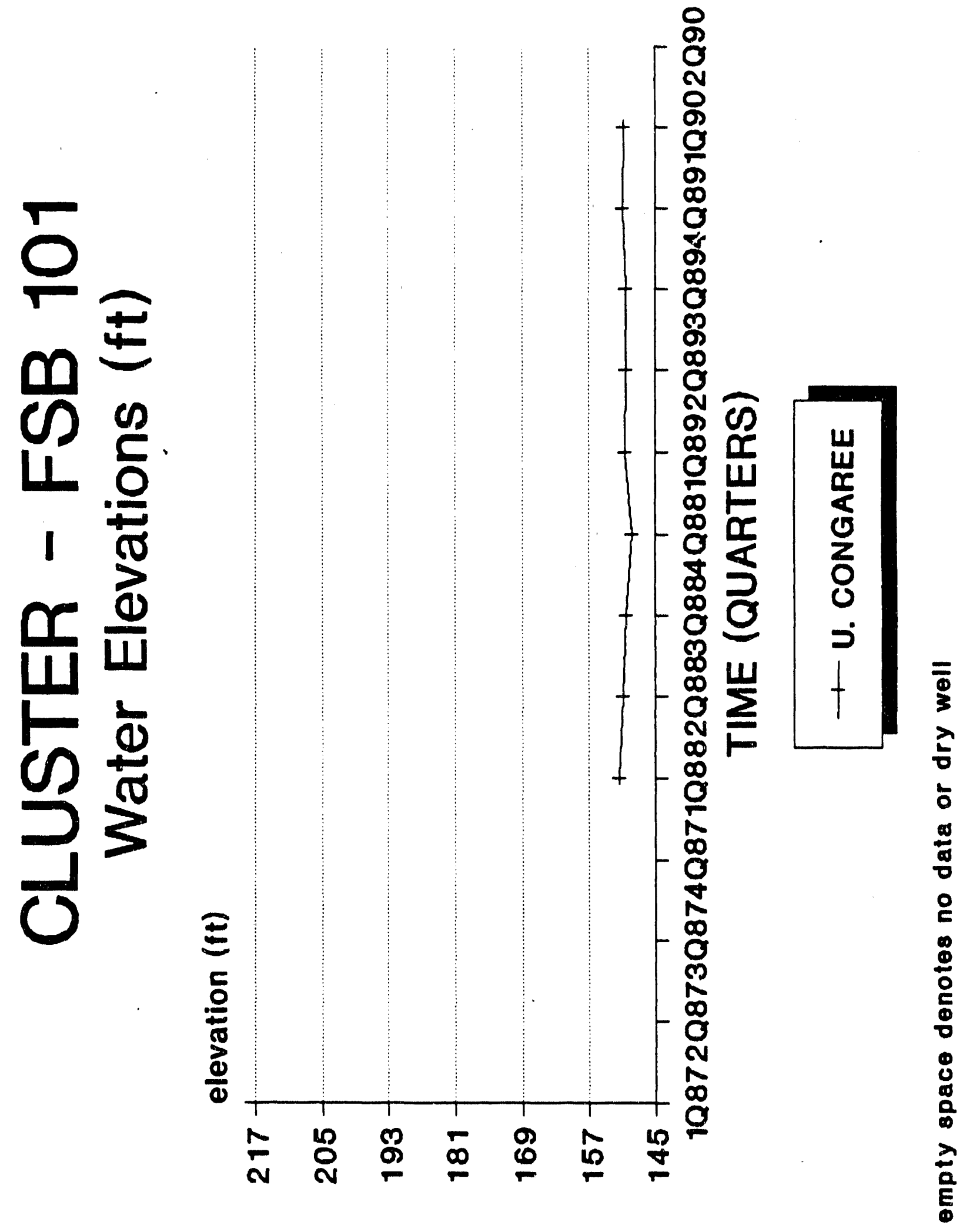




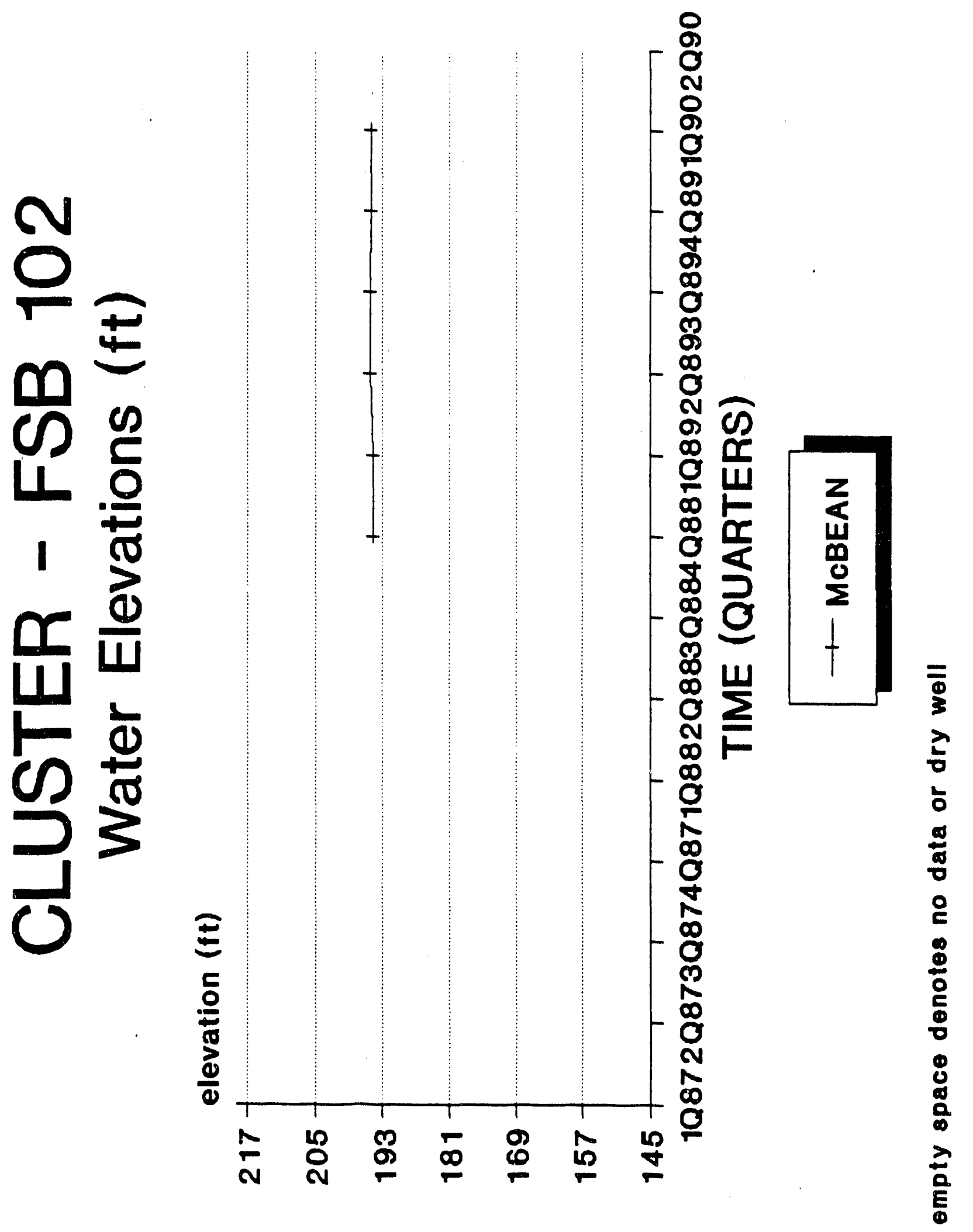




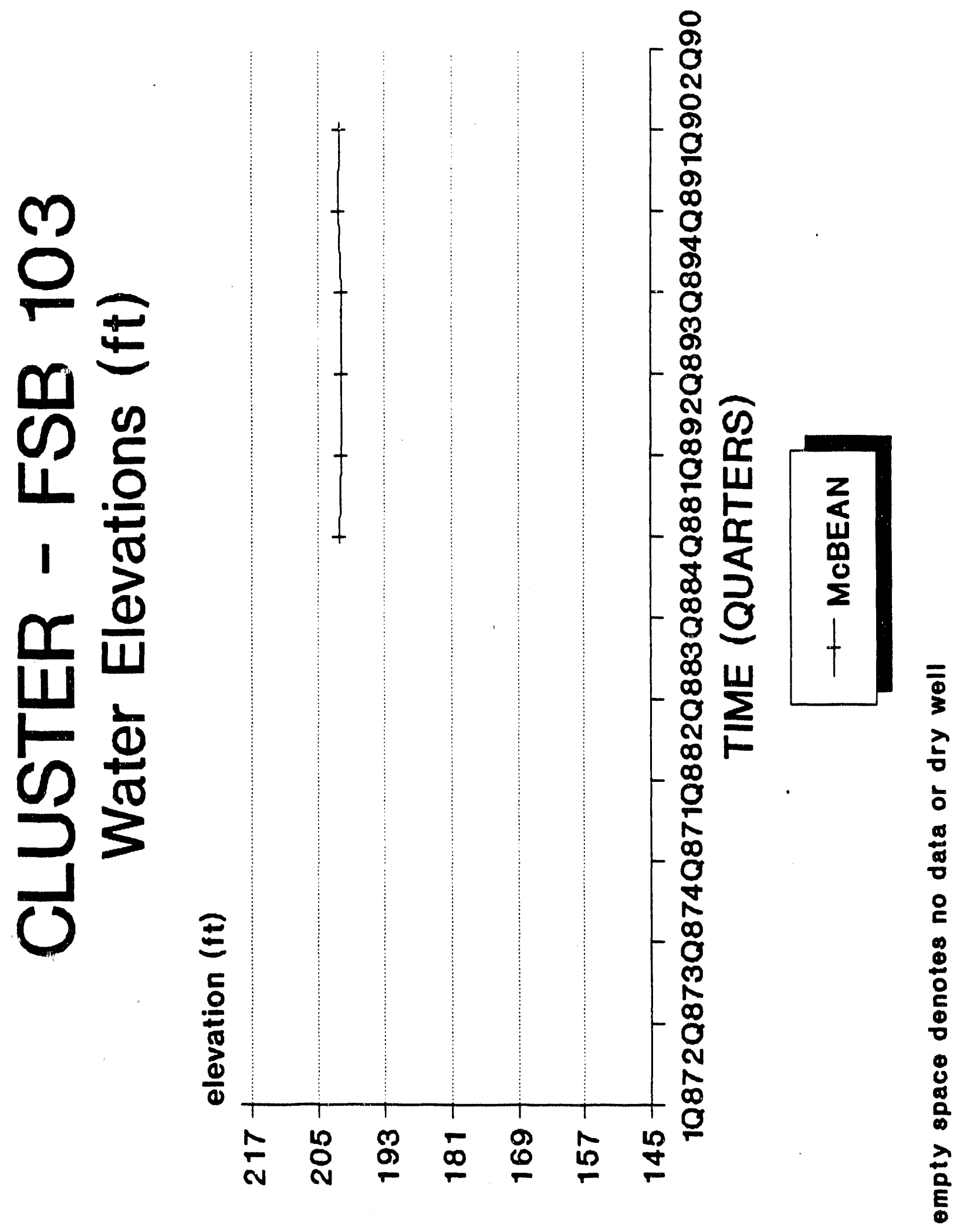




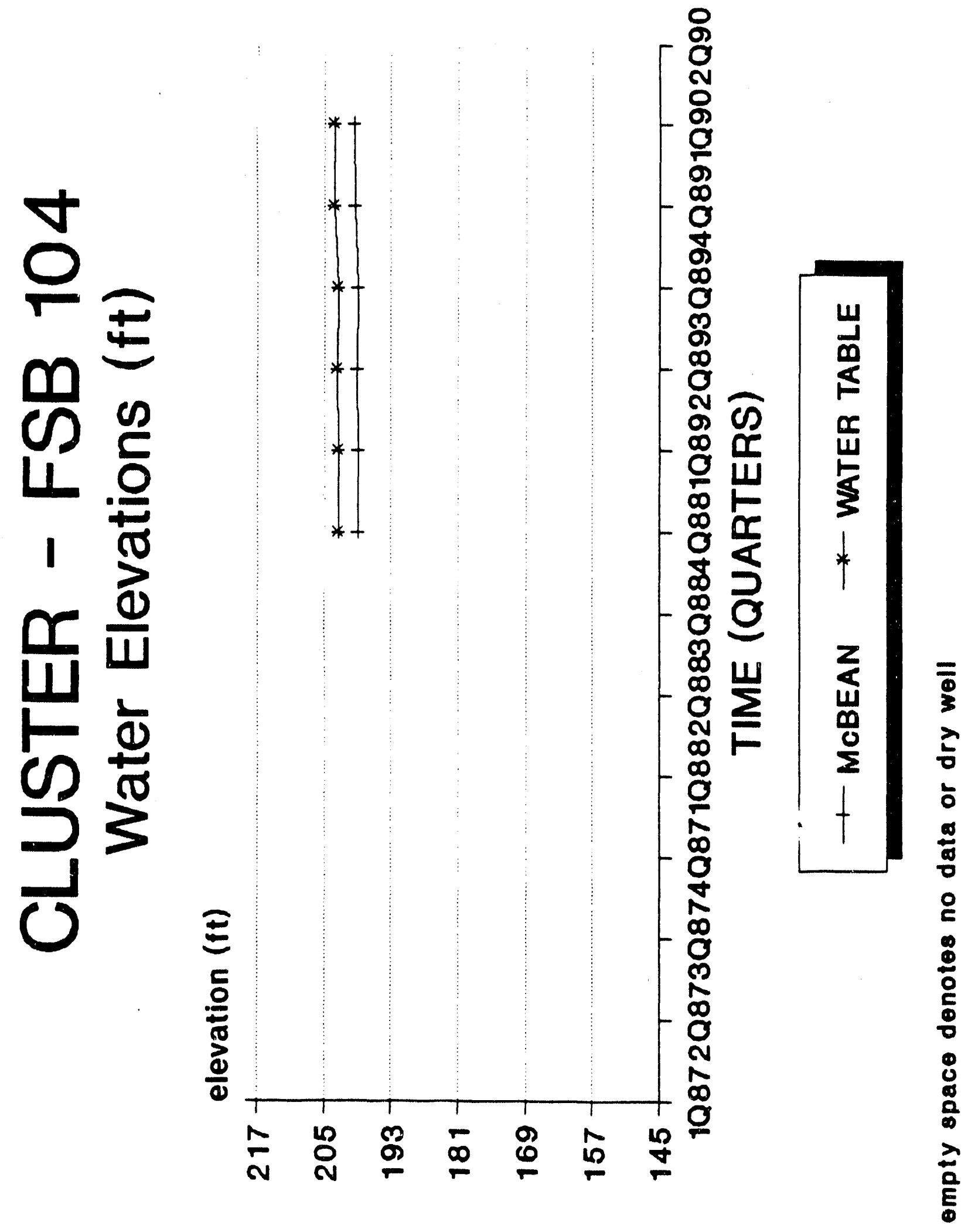




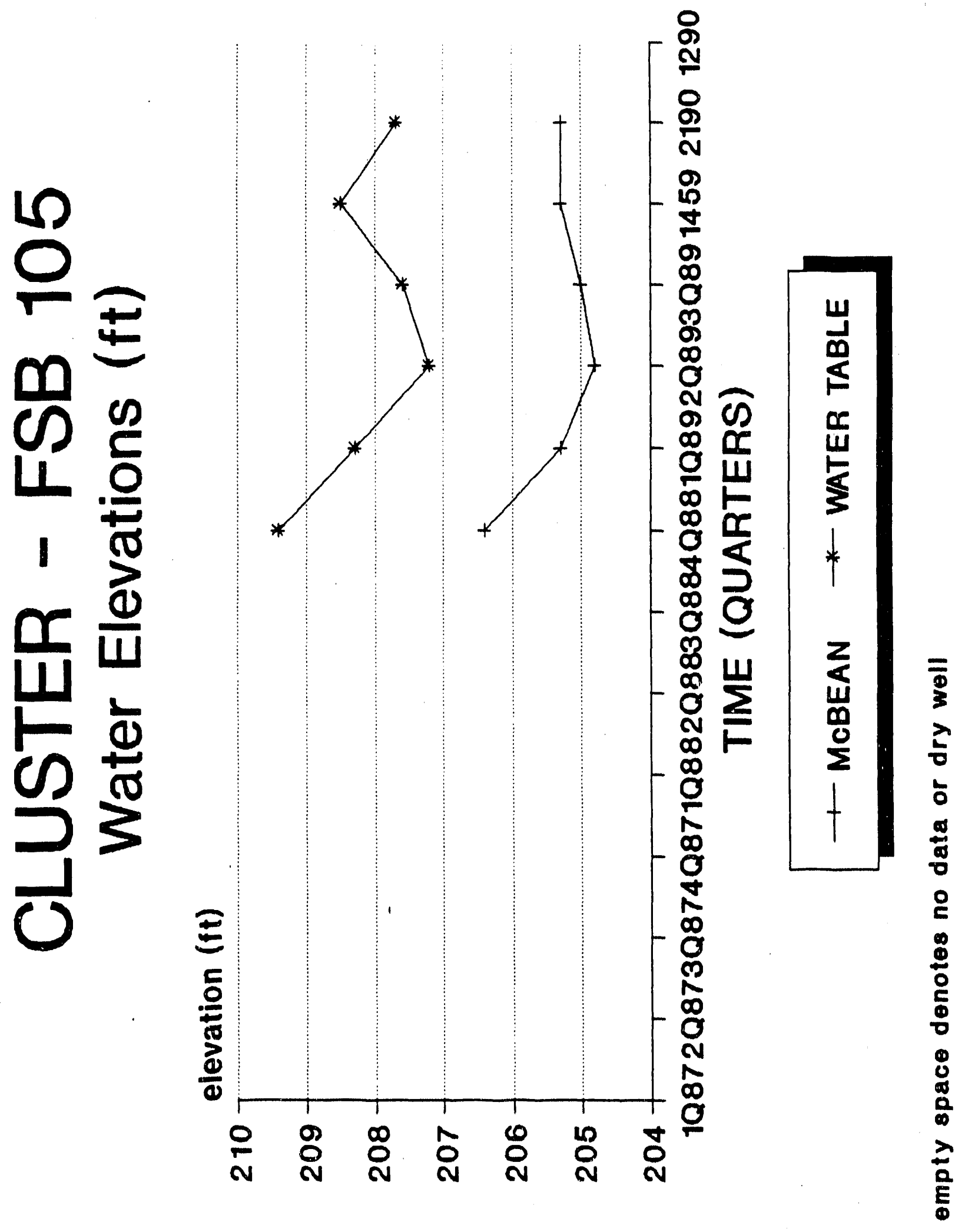




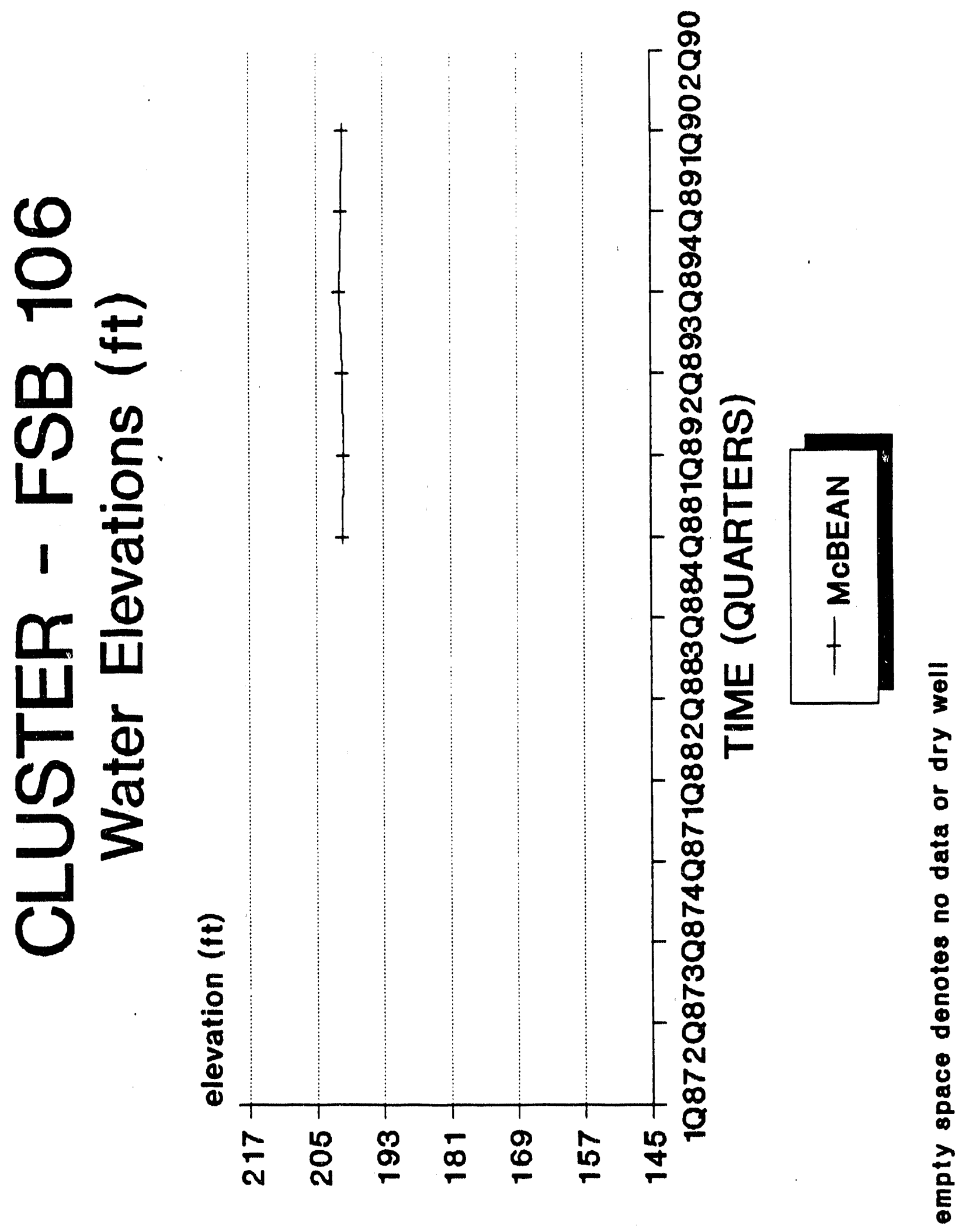




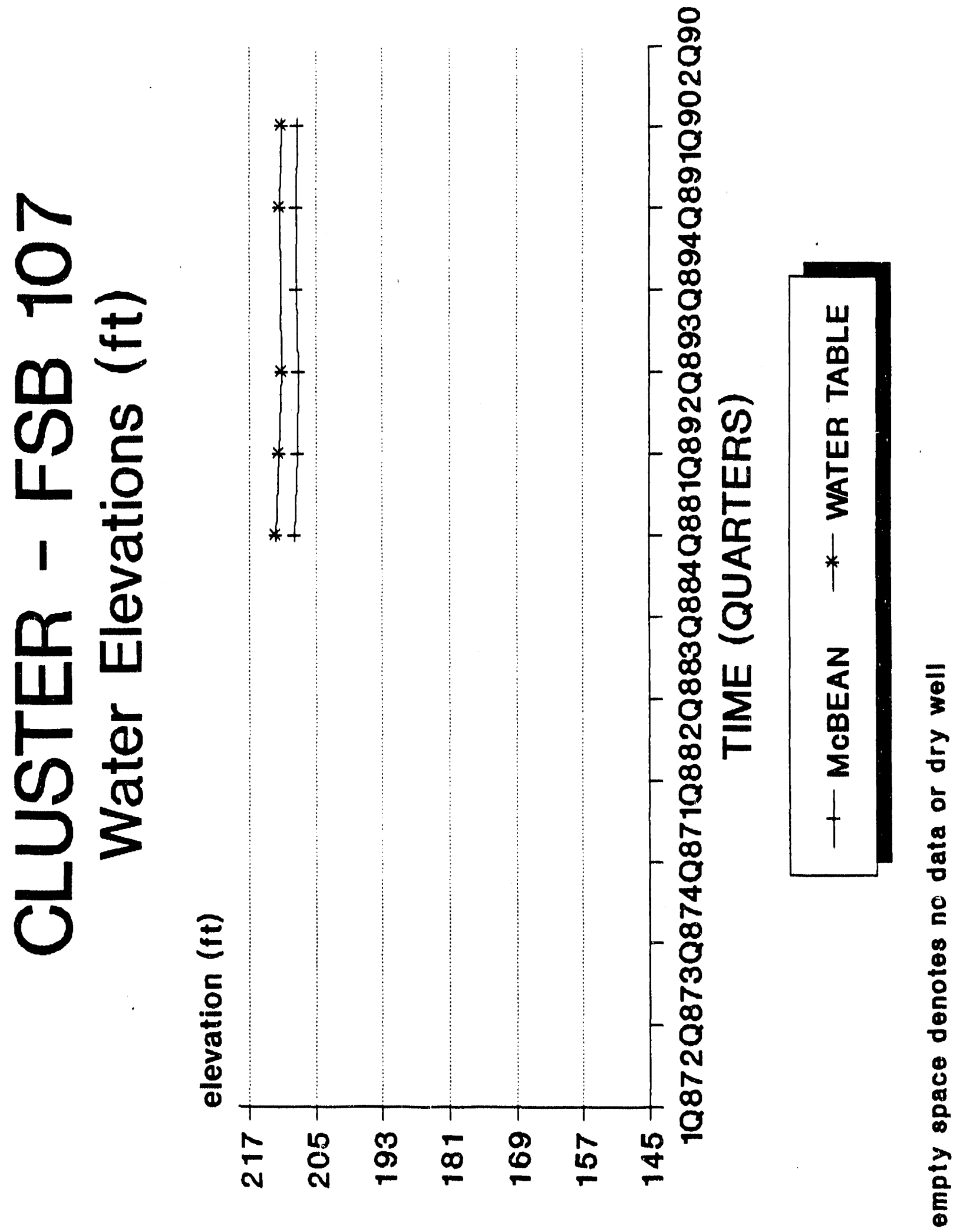




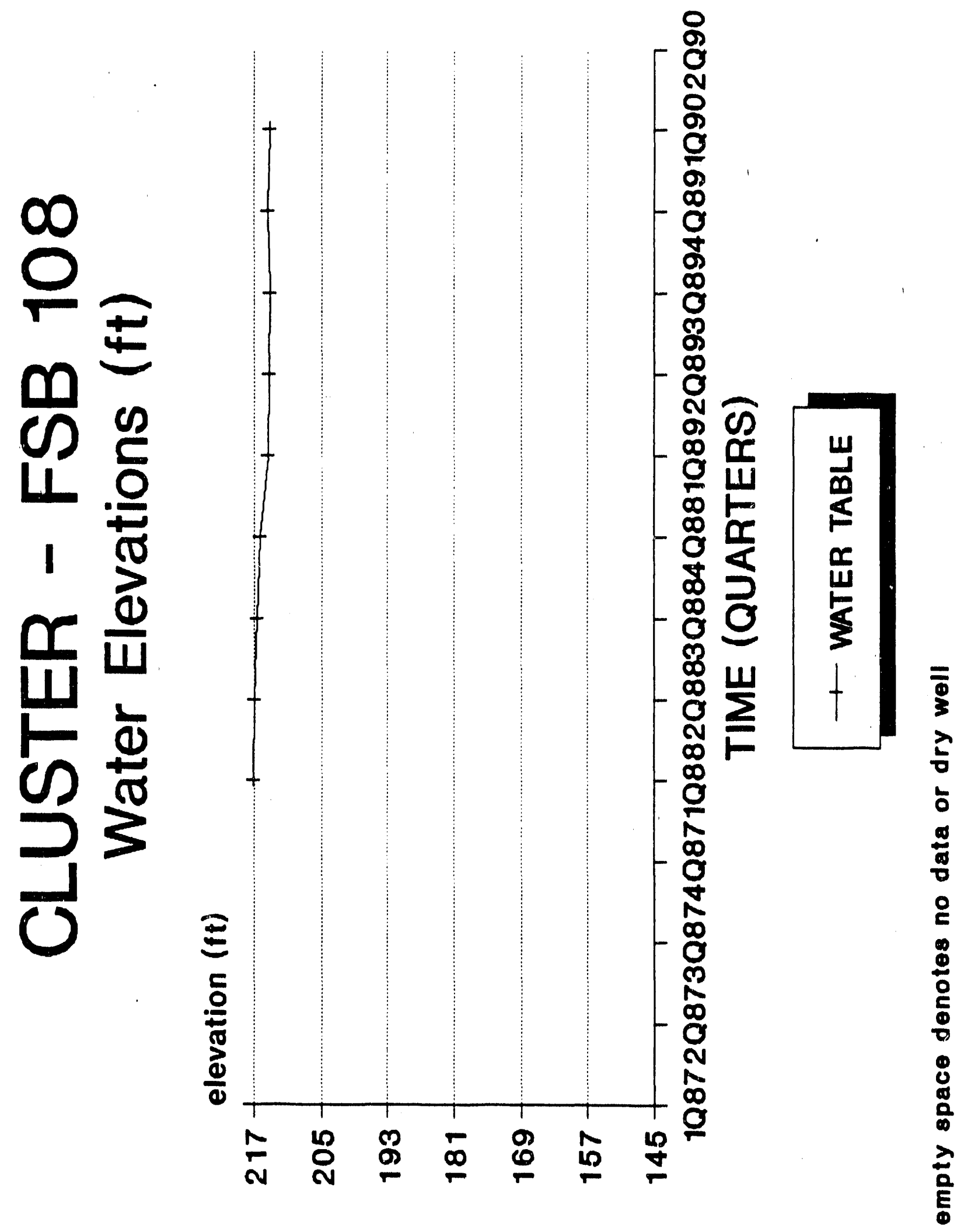




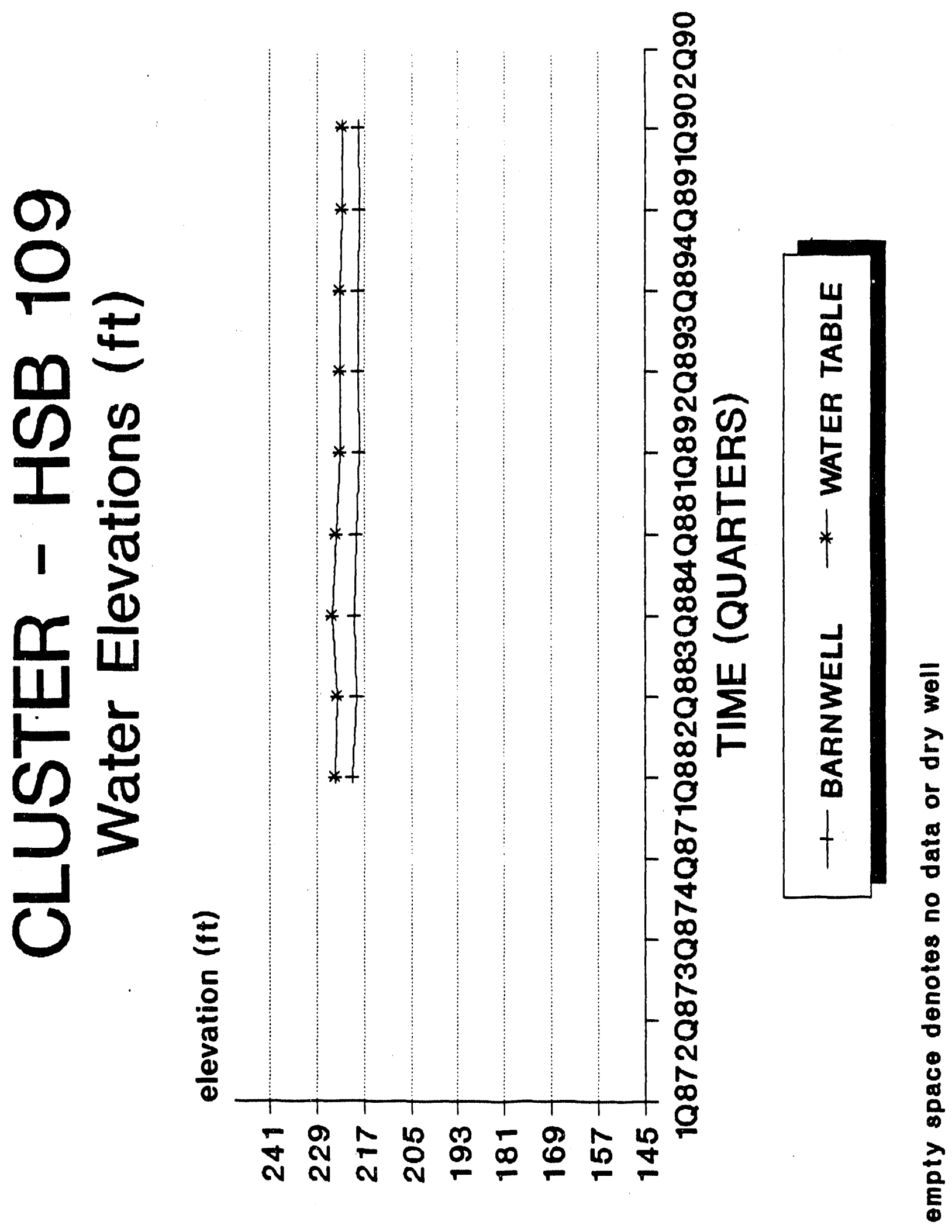




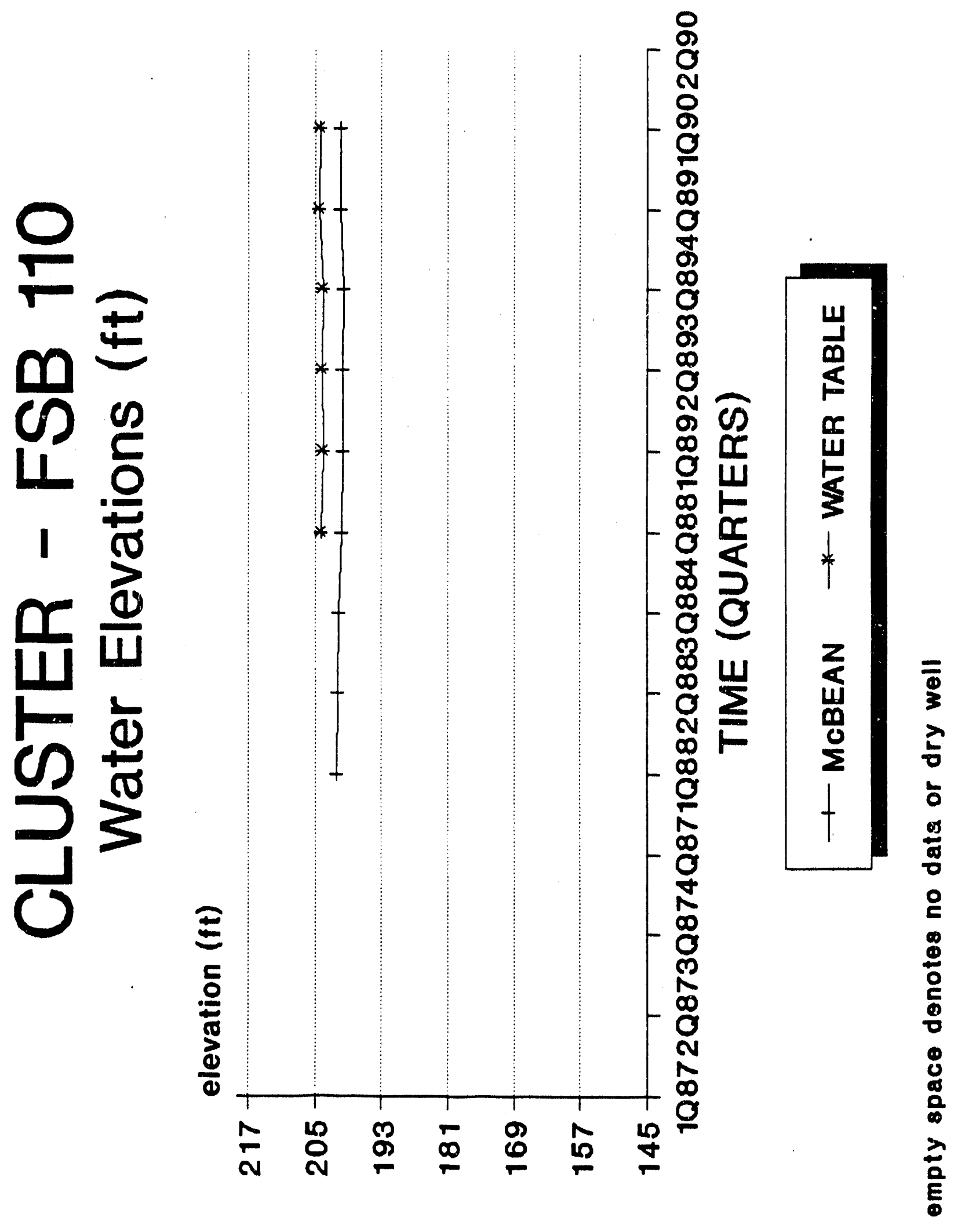




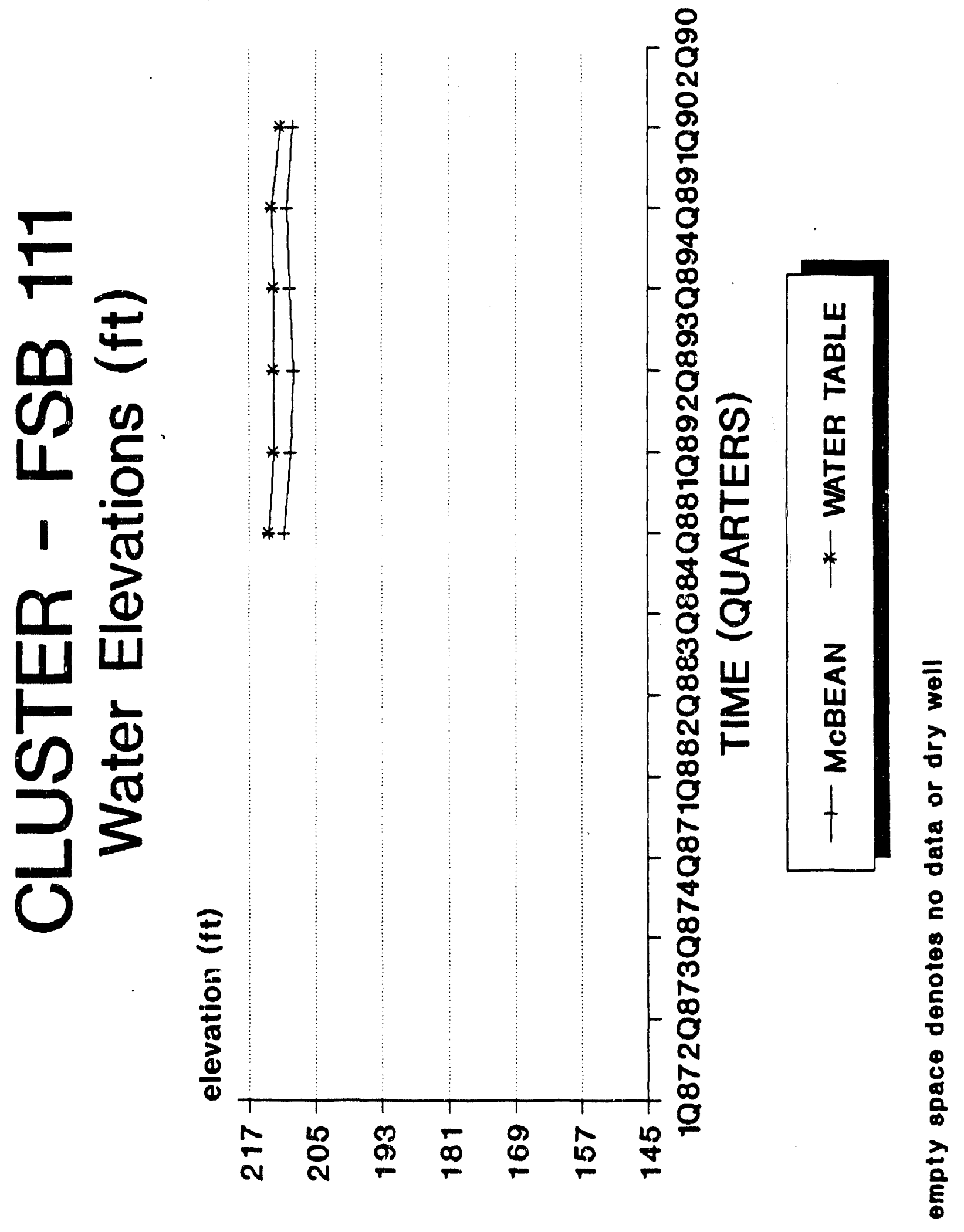


APPEIDIX D

NITRATE-TTYTE TRENDS

Figures D1-D29 display the levels of nitrate measured at each unit in every well cluster as a function of time. 

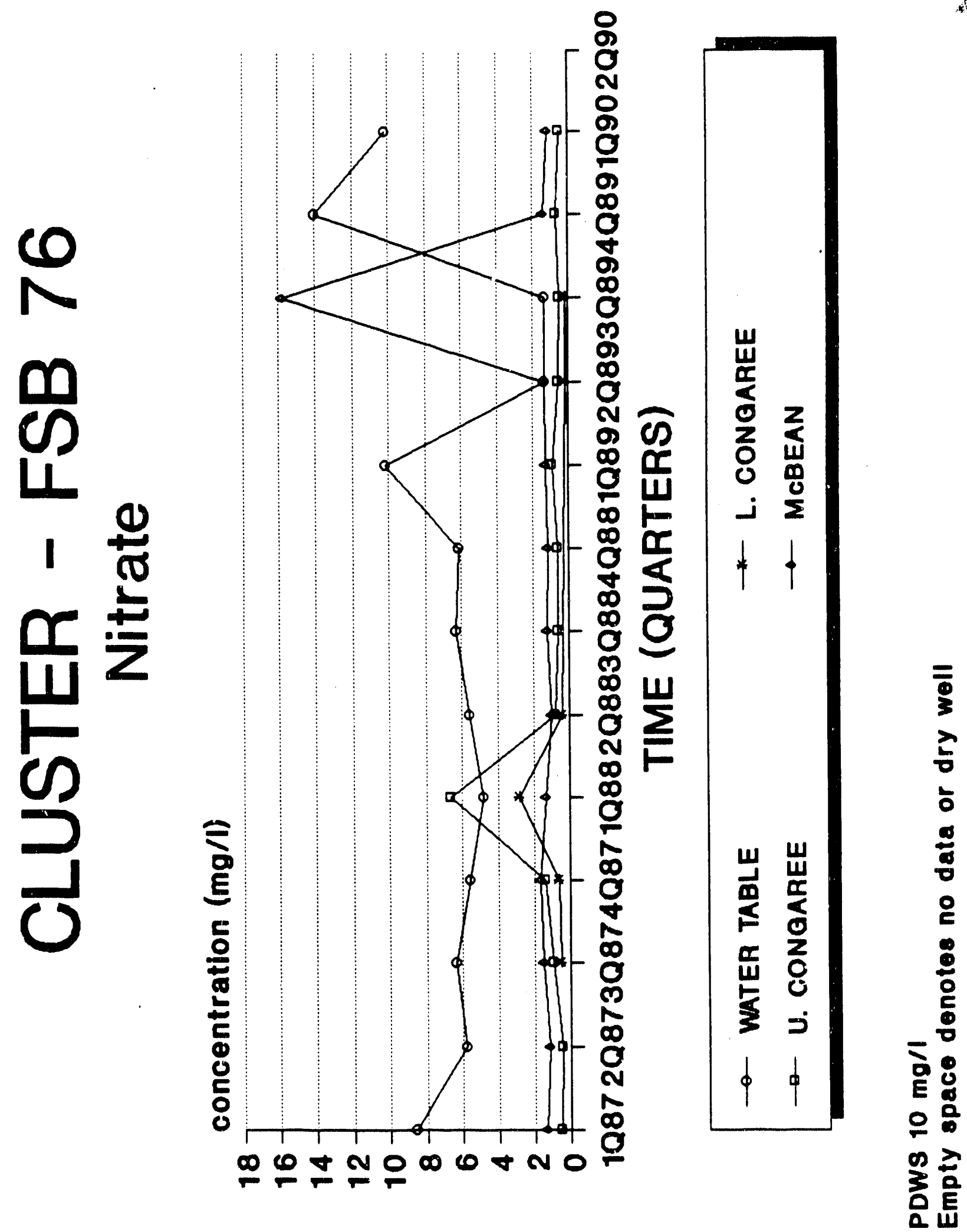
*.

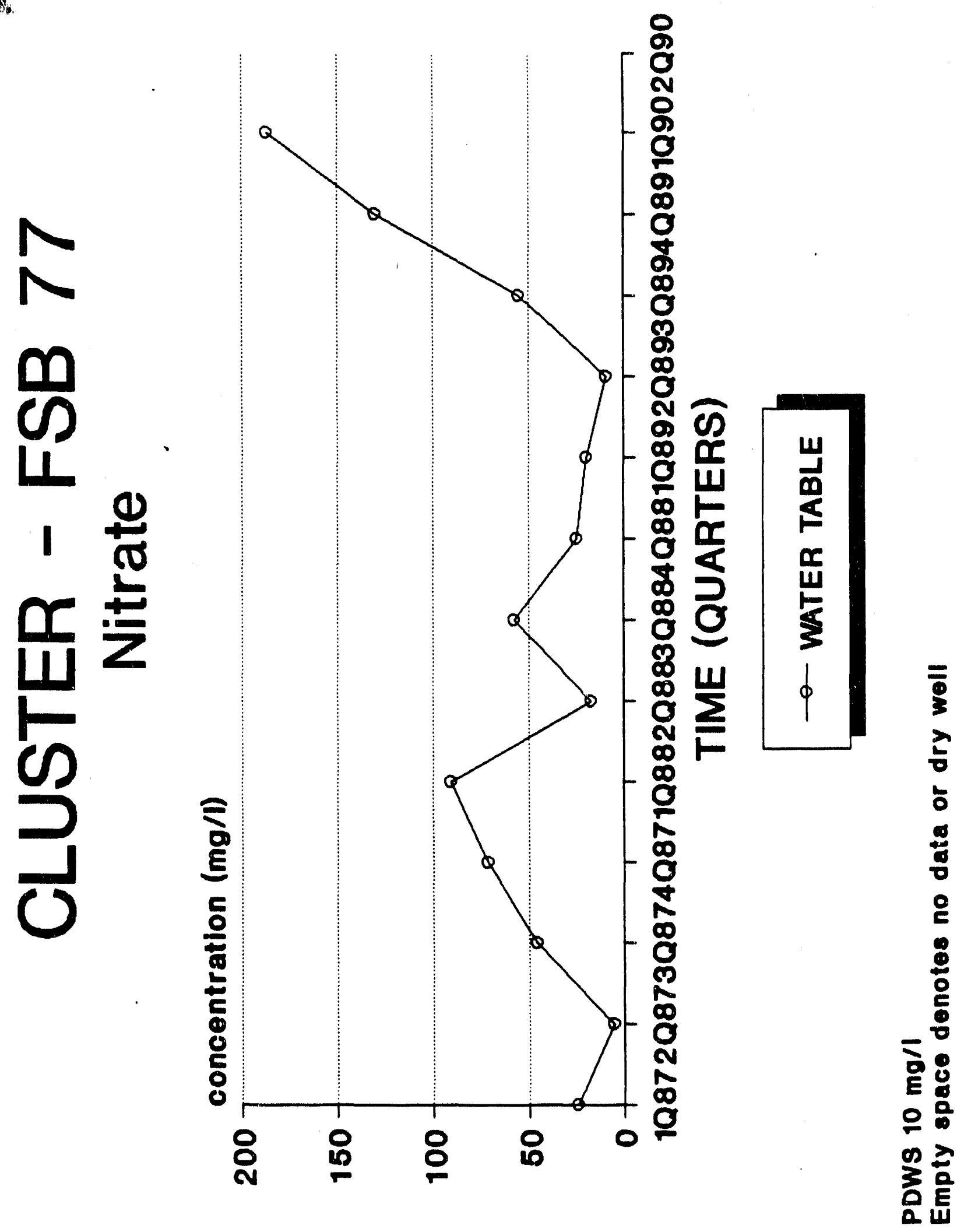




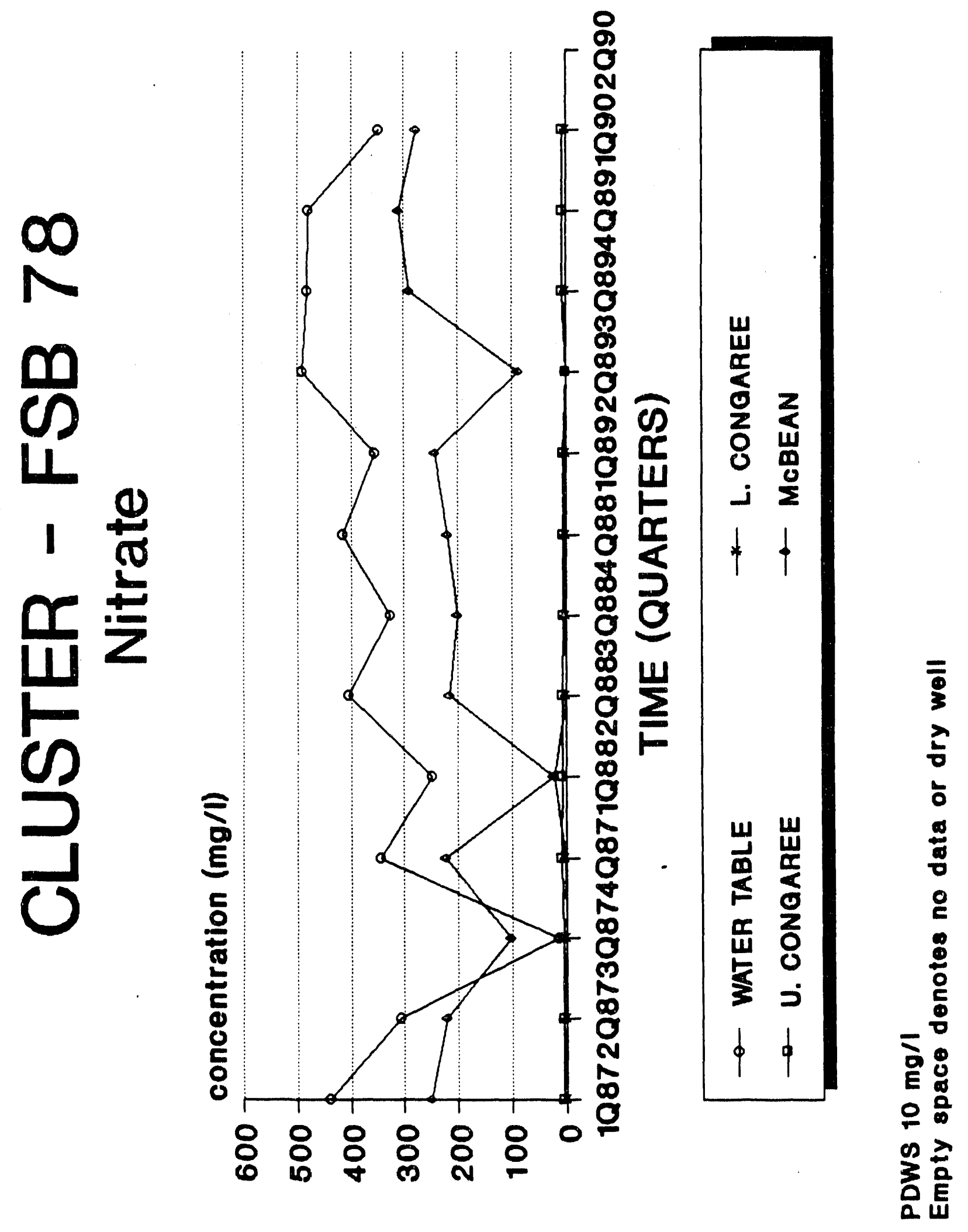




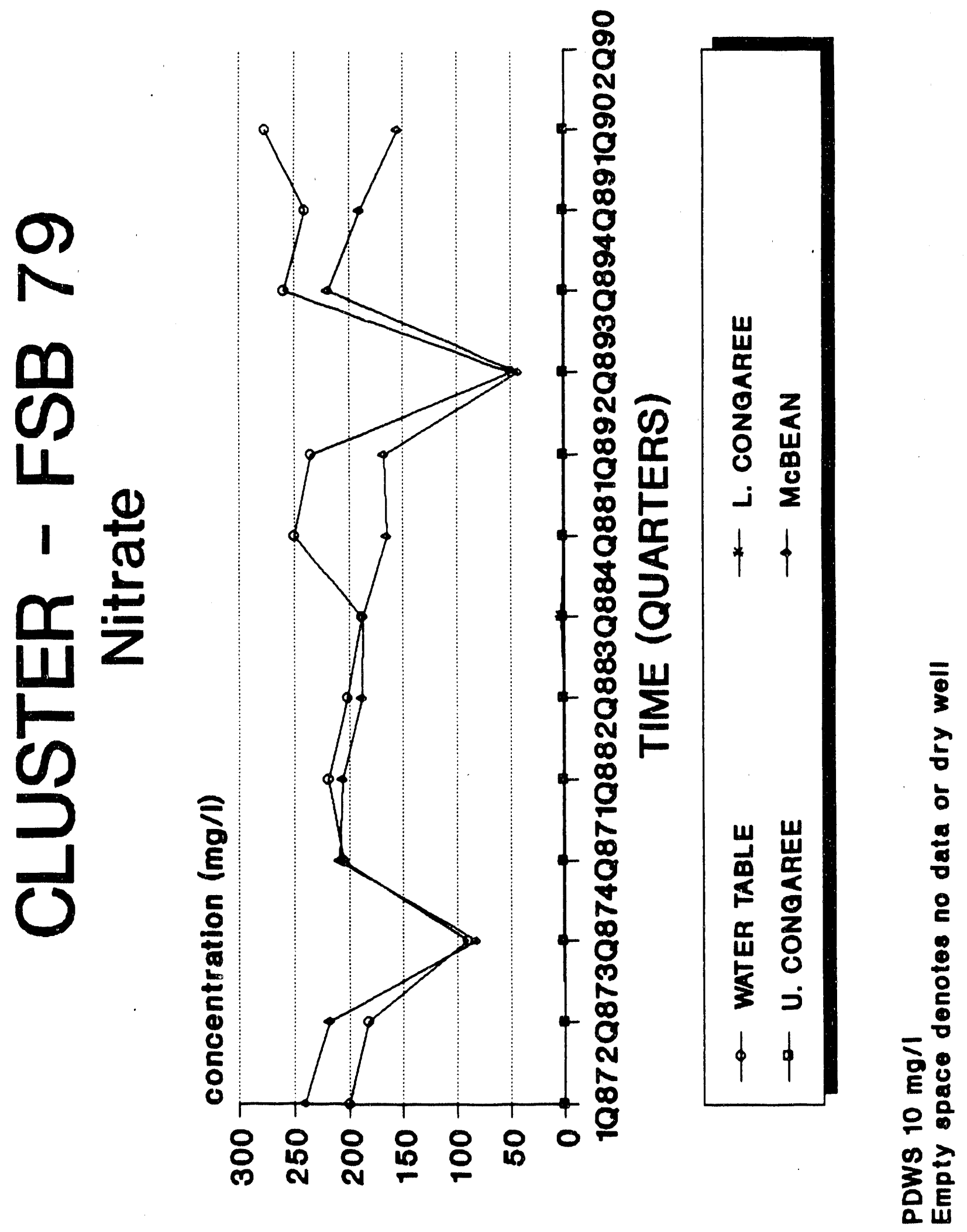




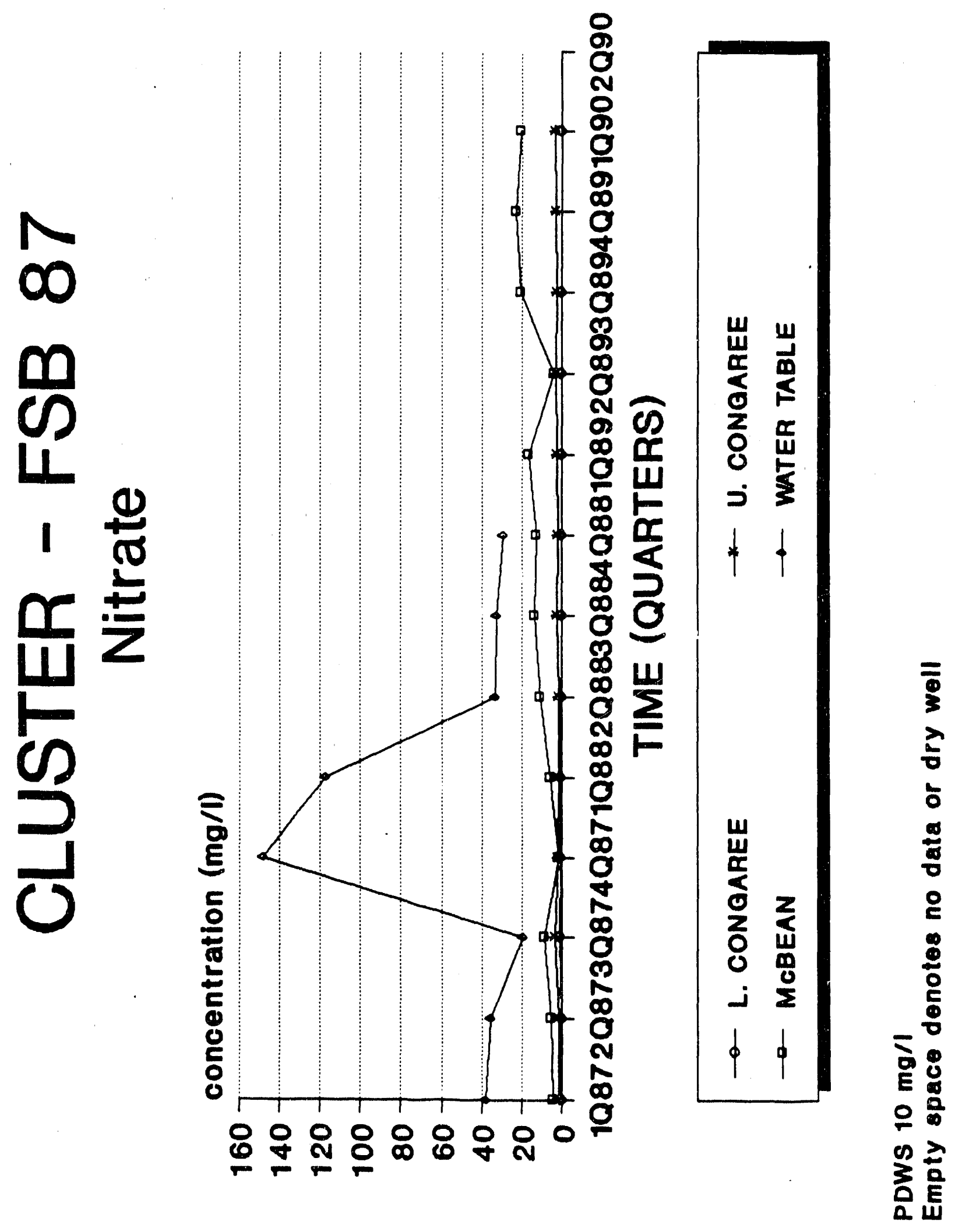




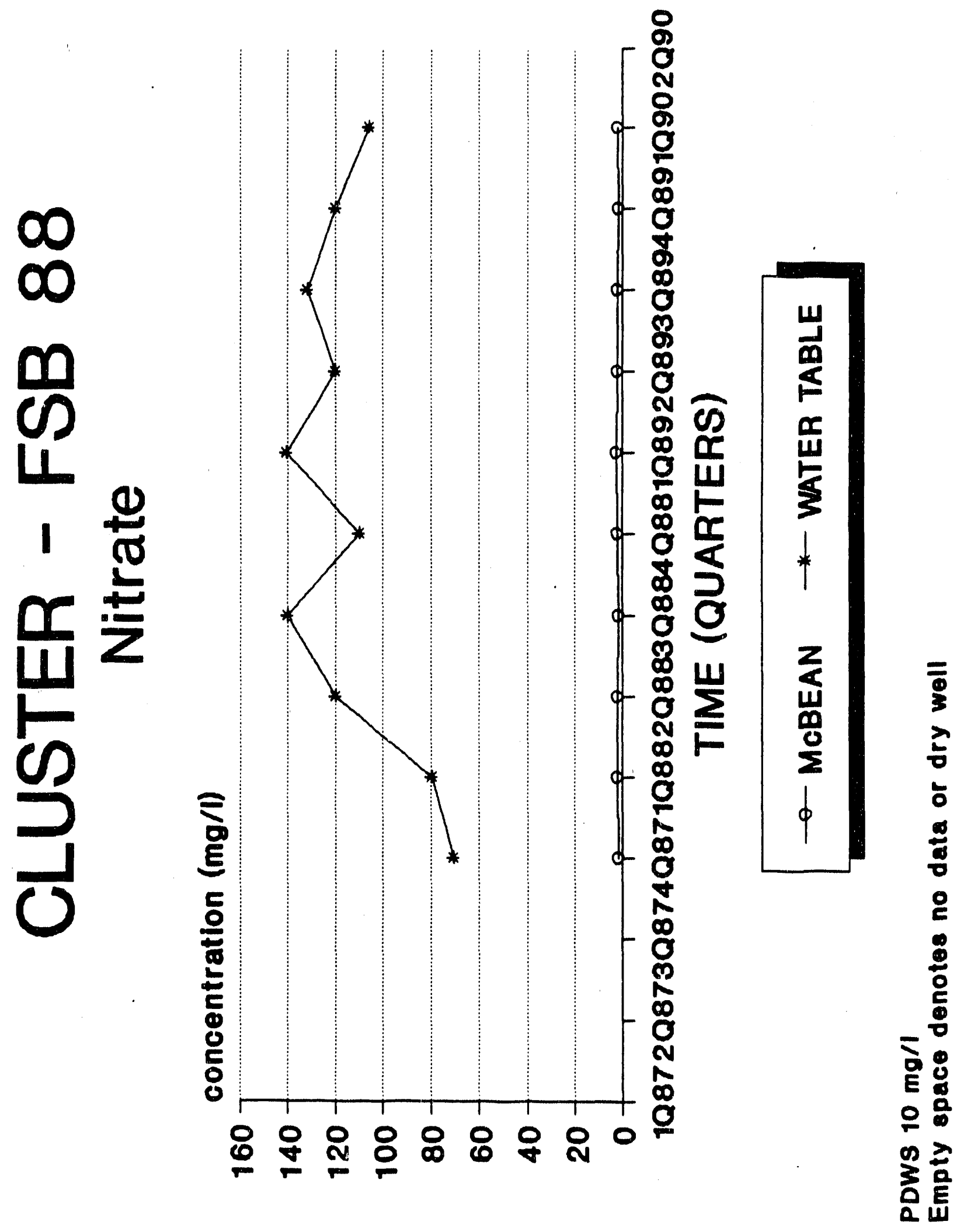




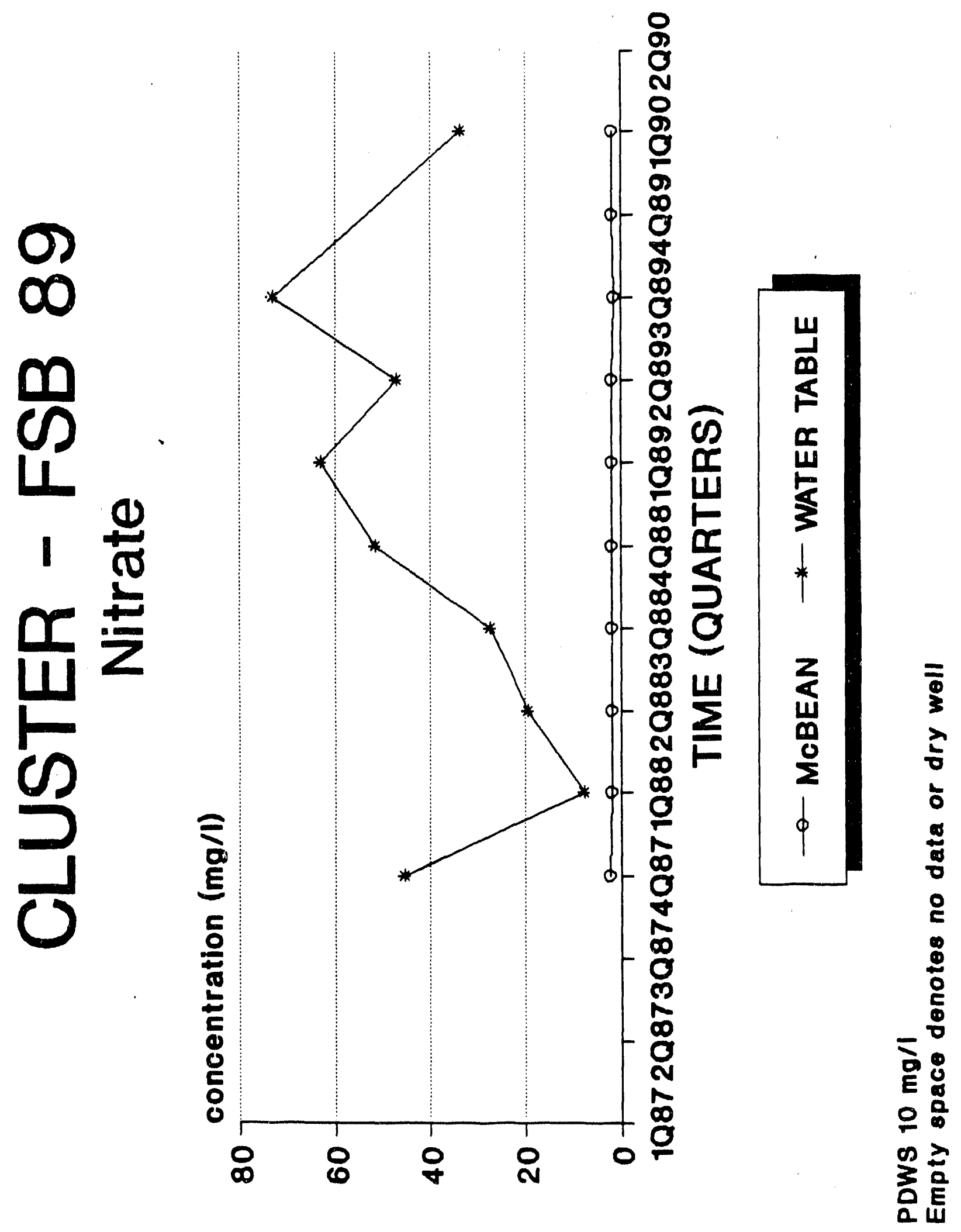




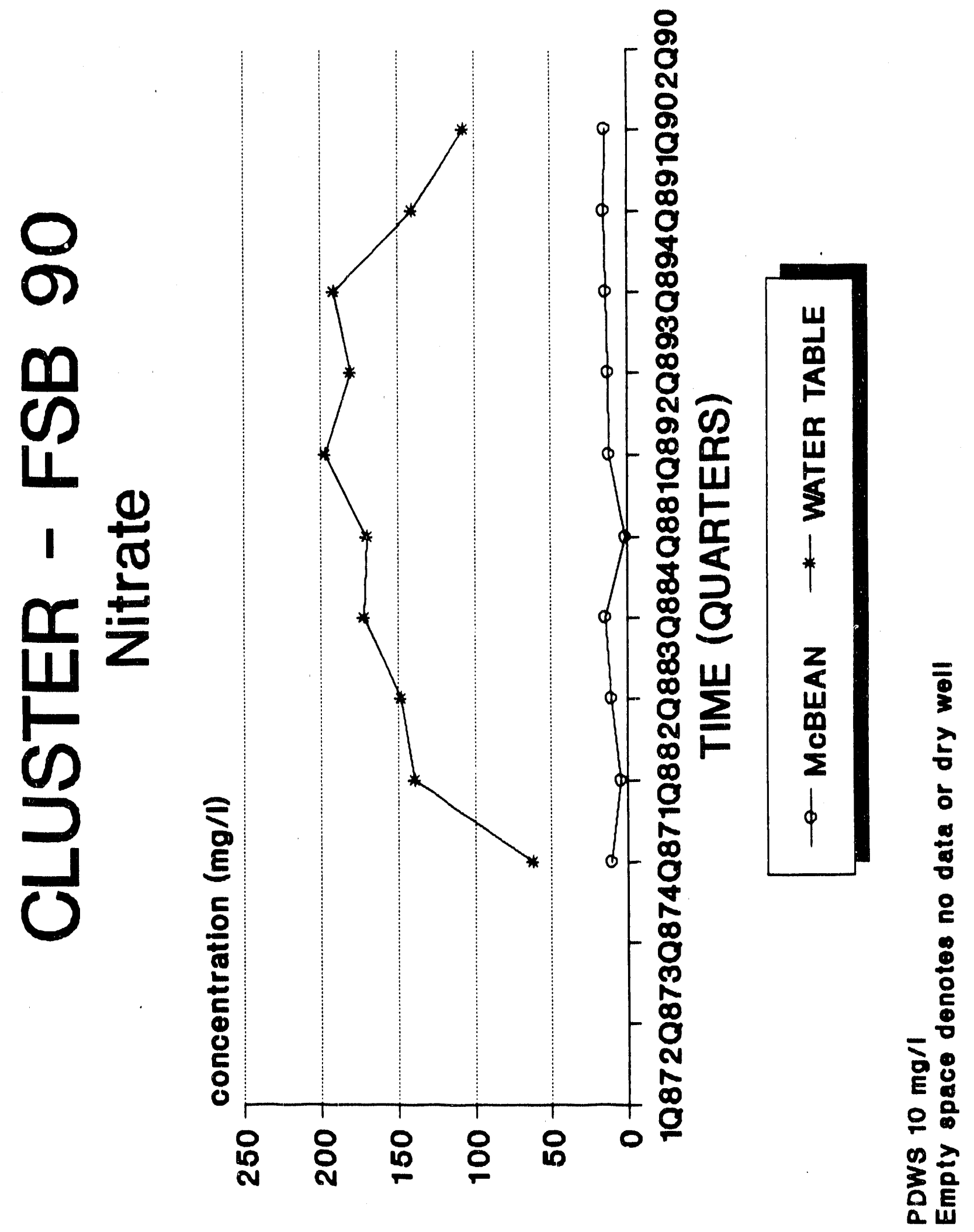




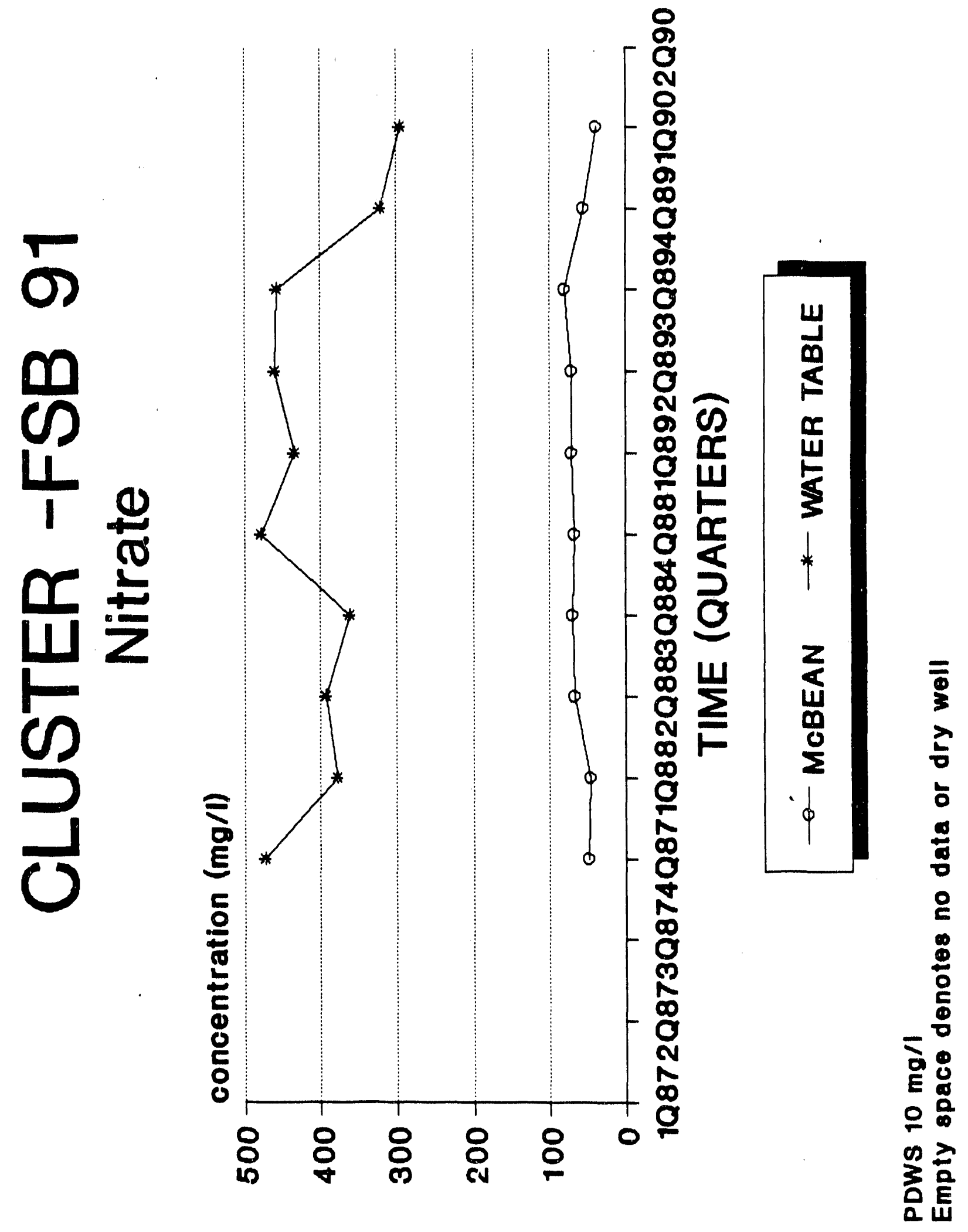




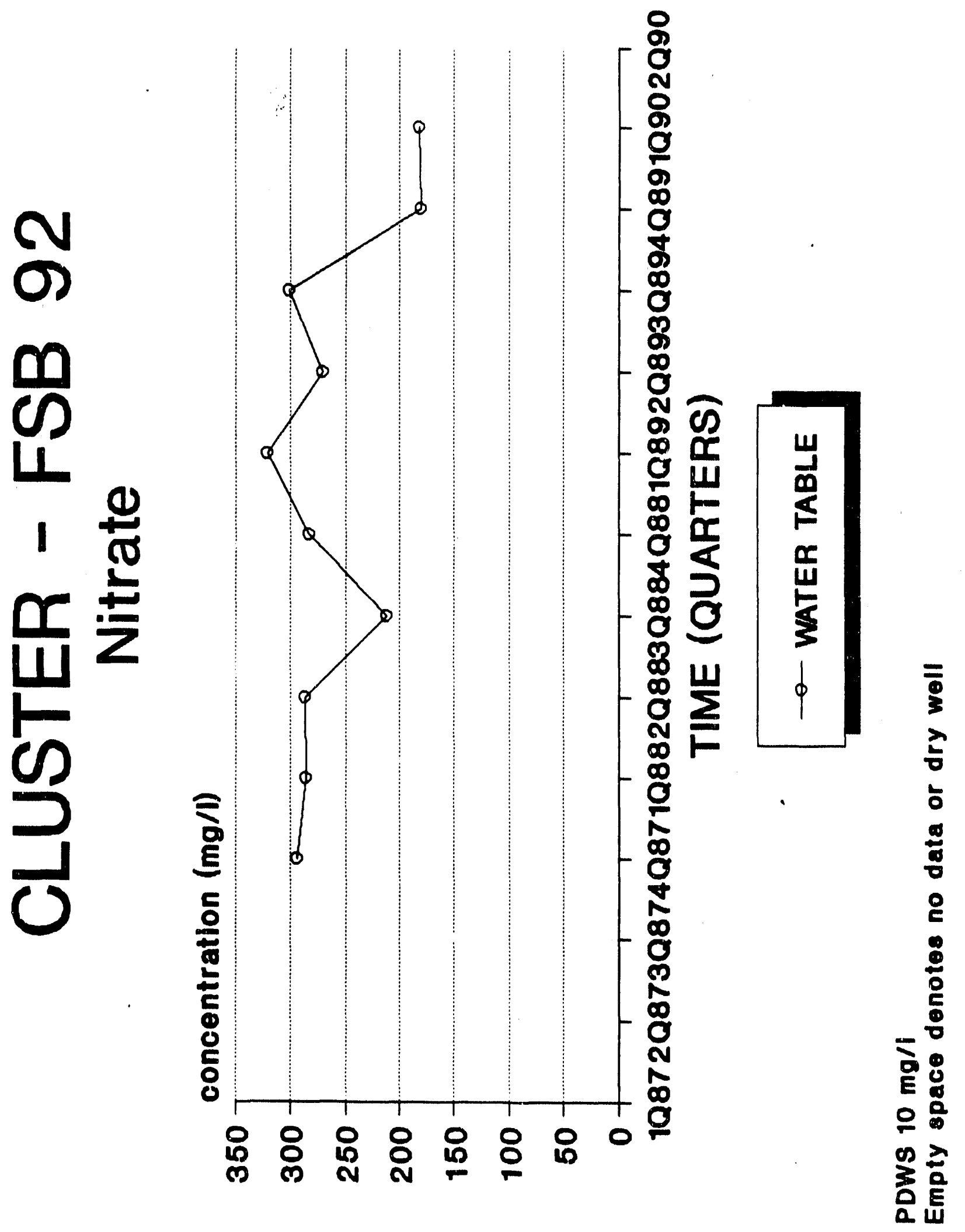




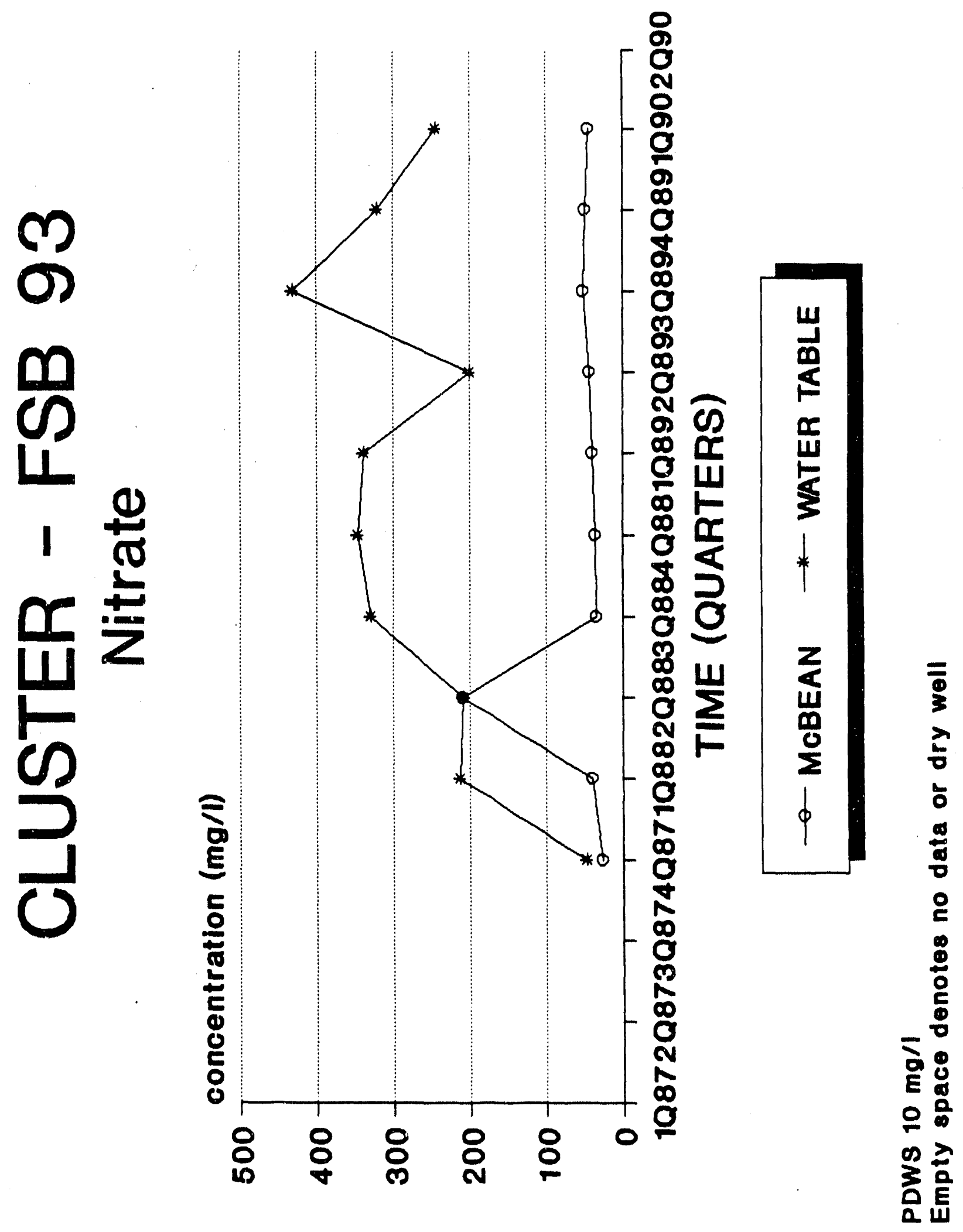




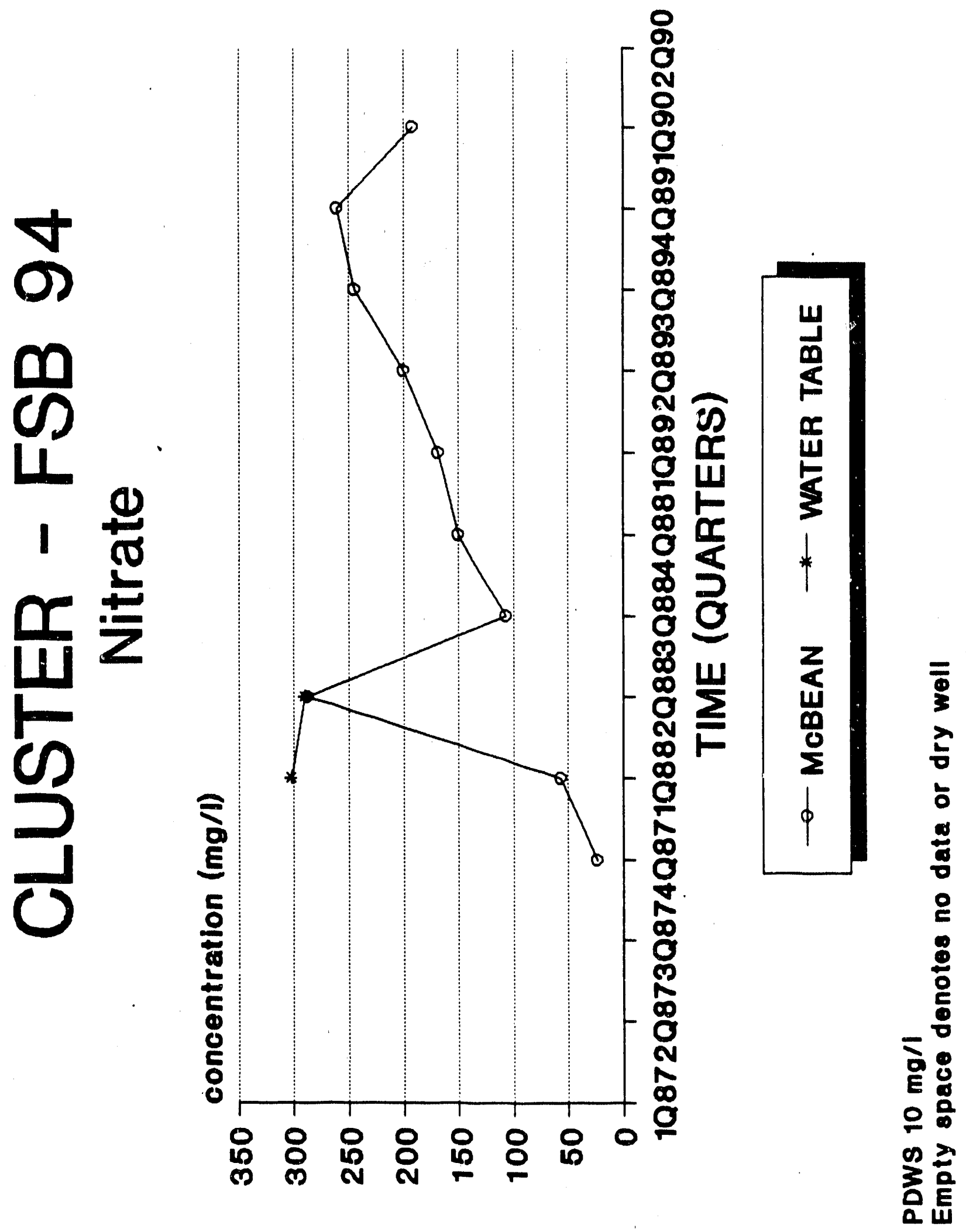




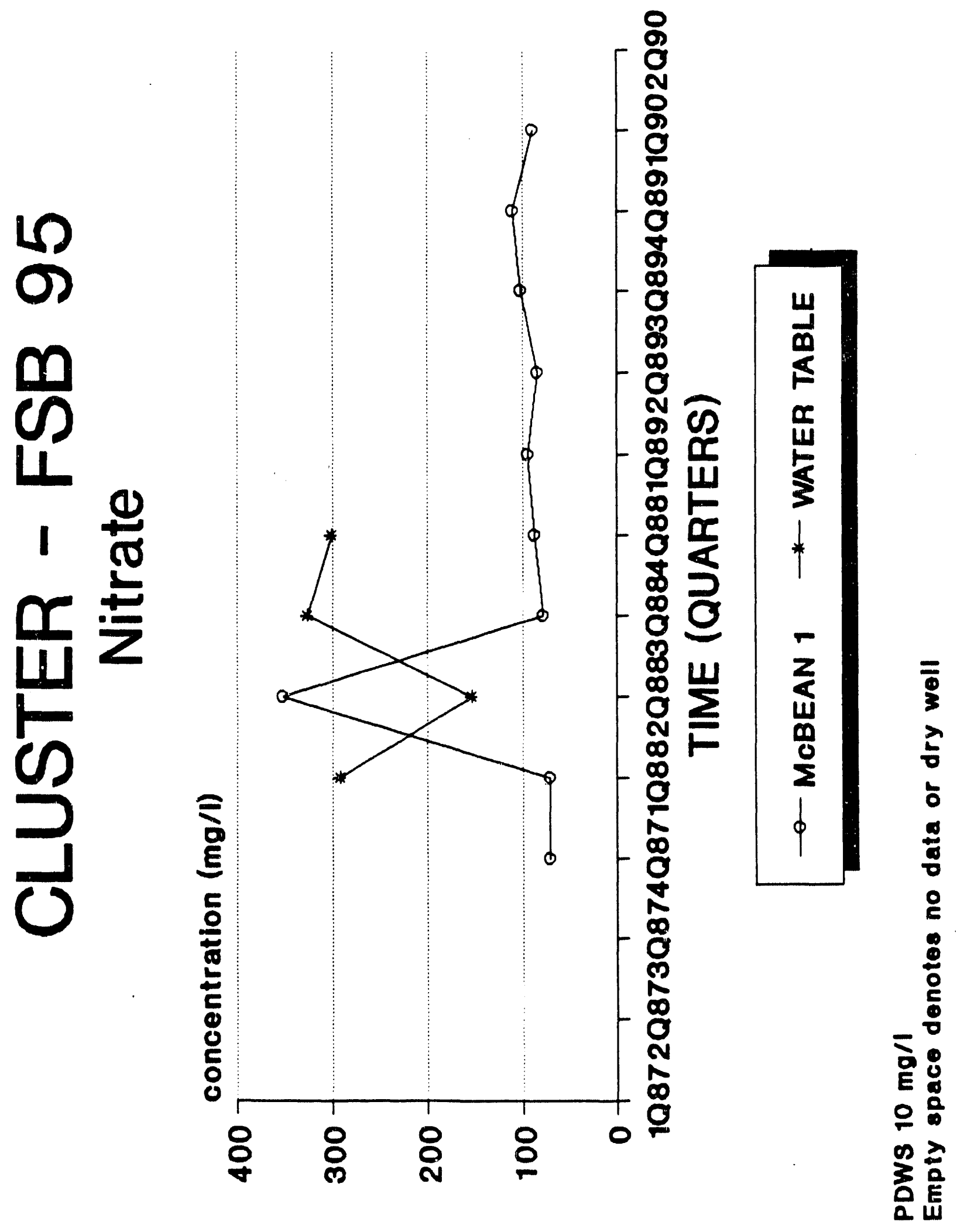




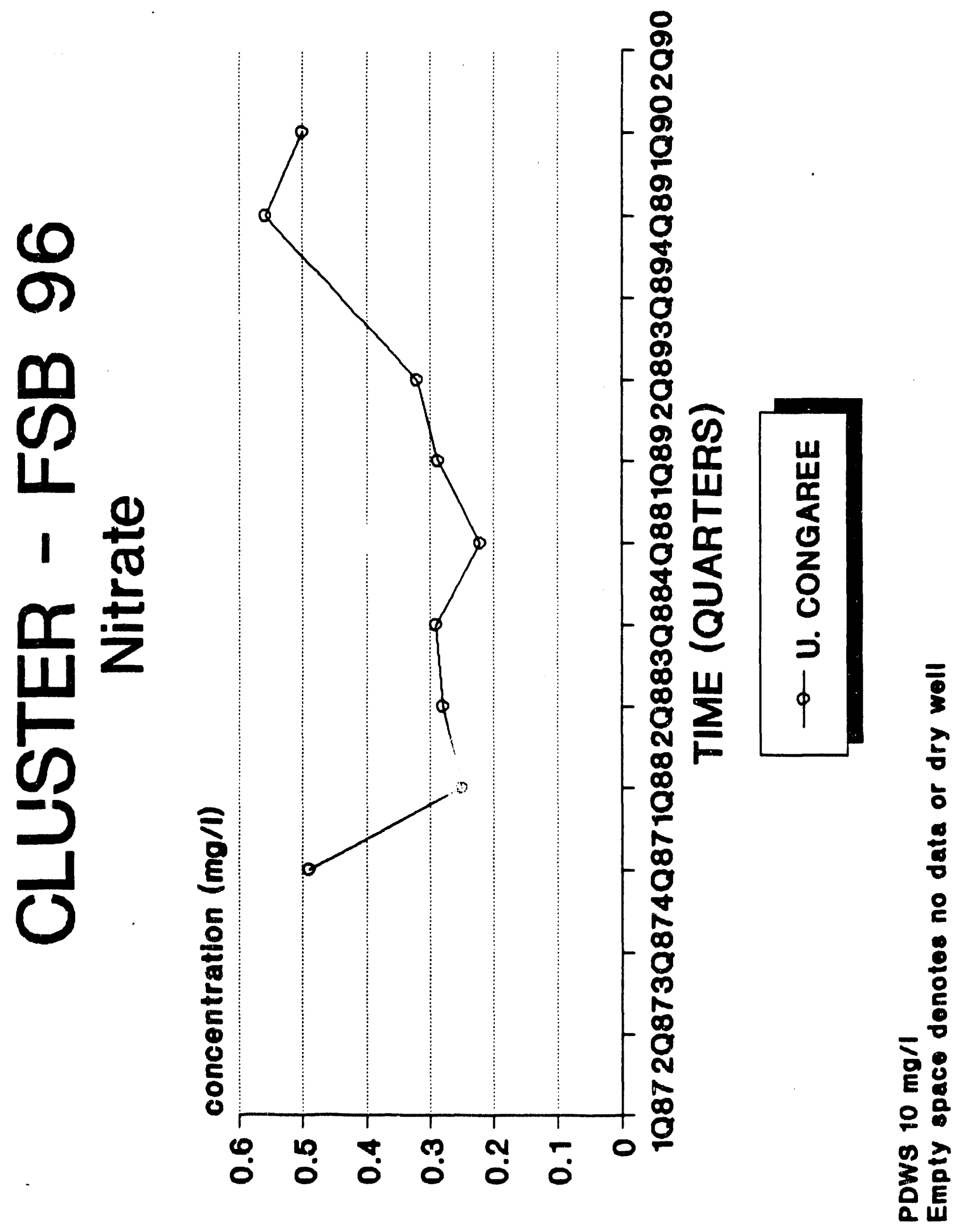



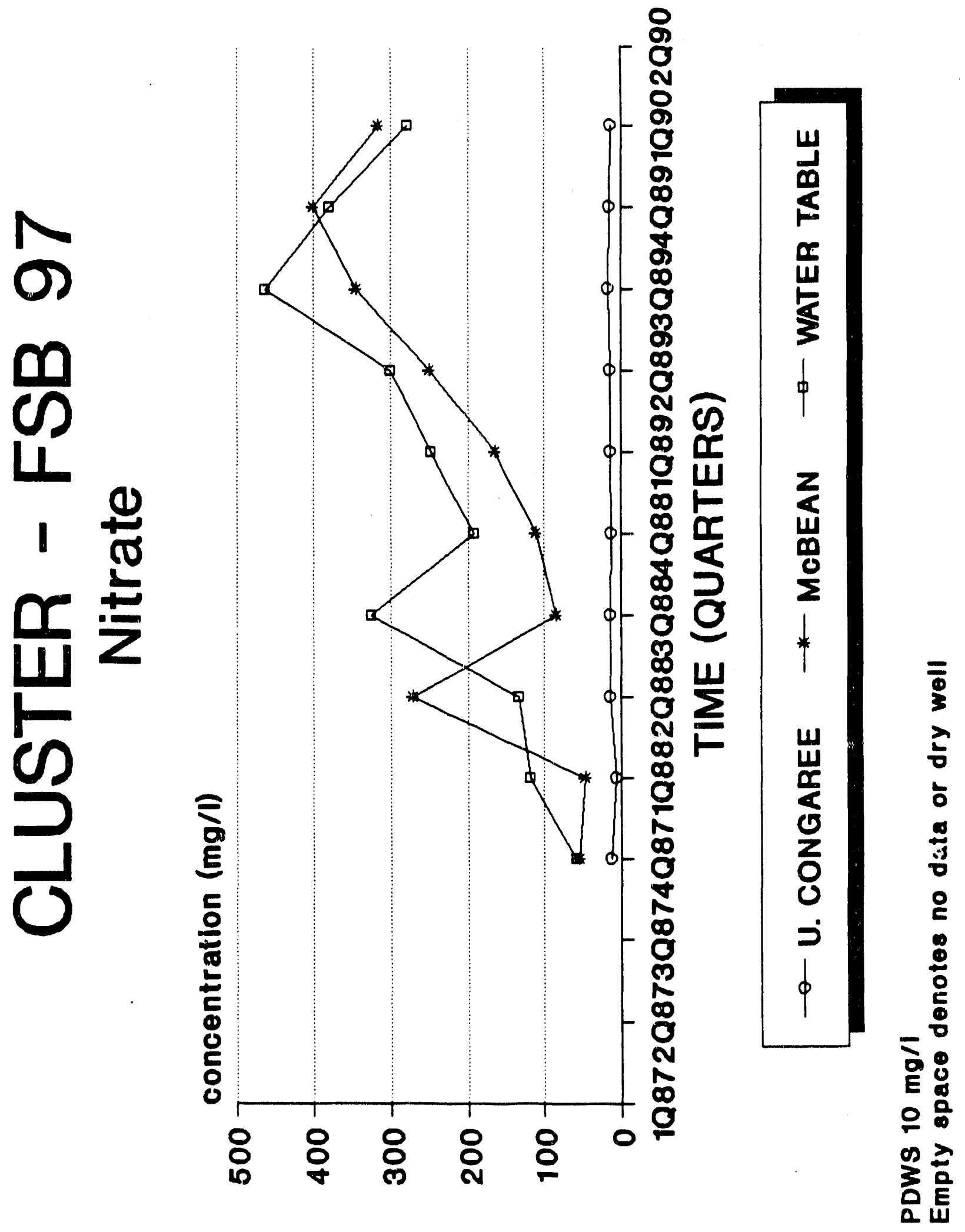


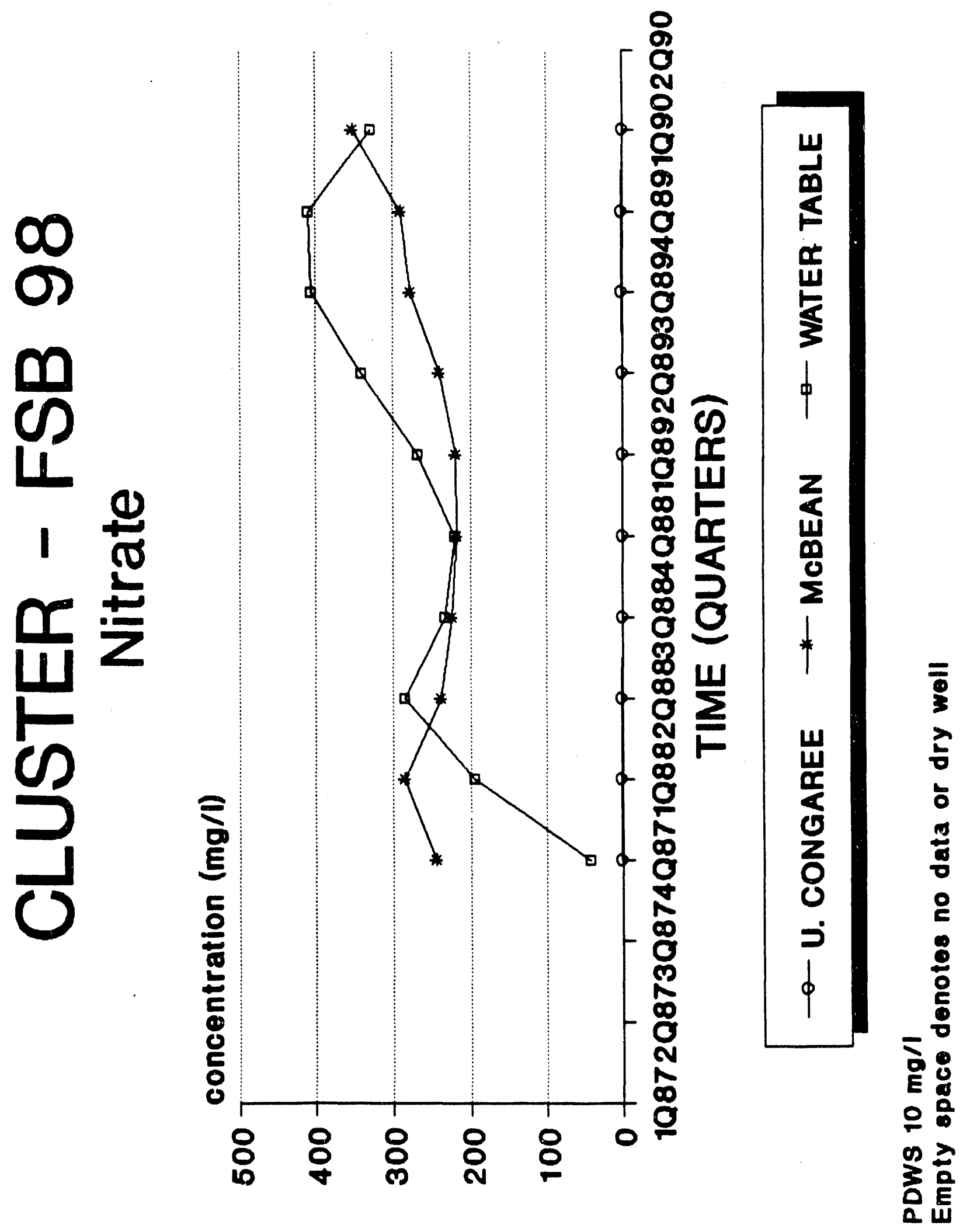




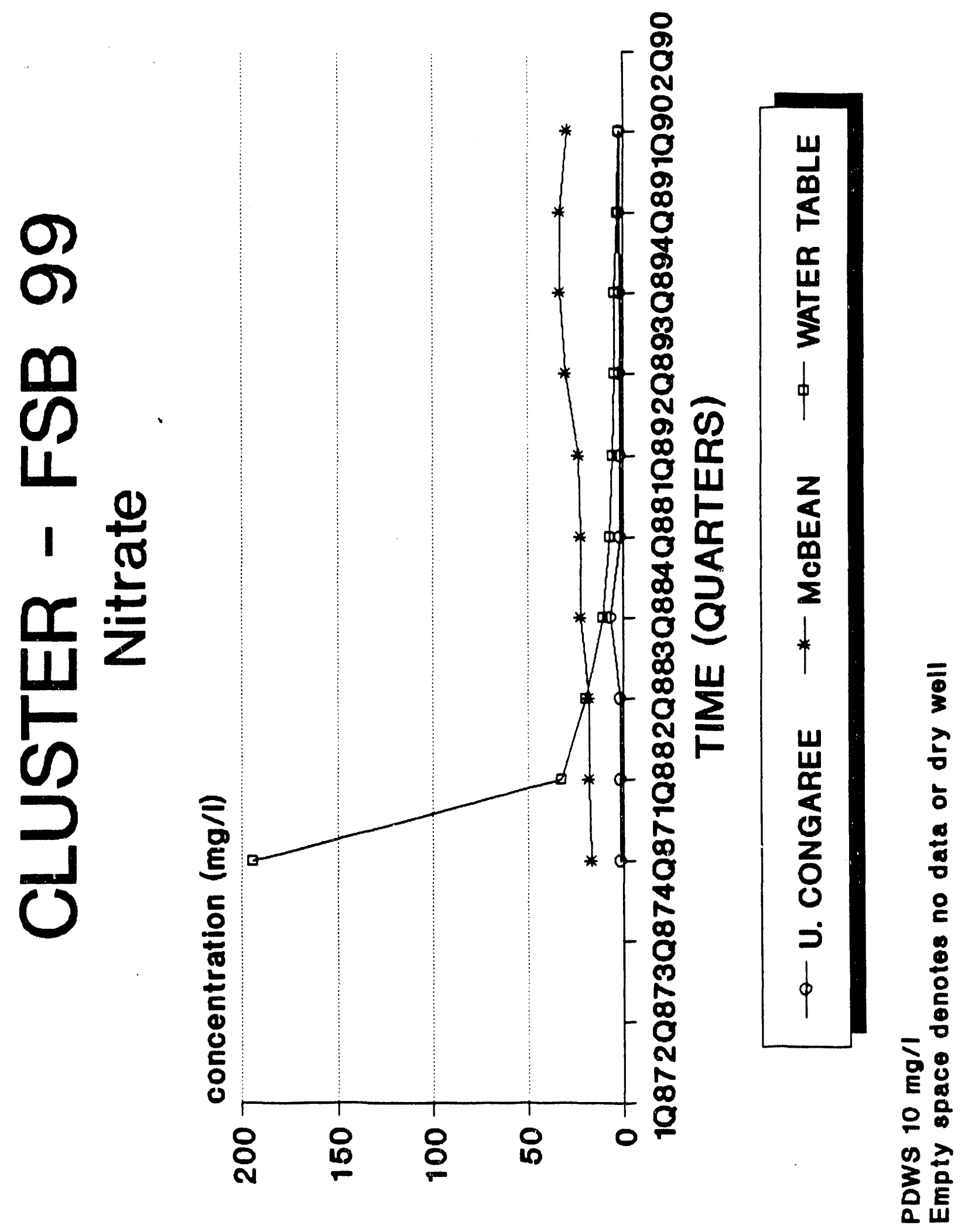




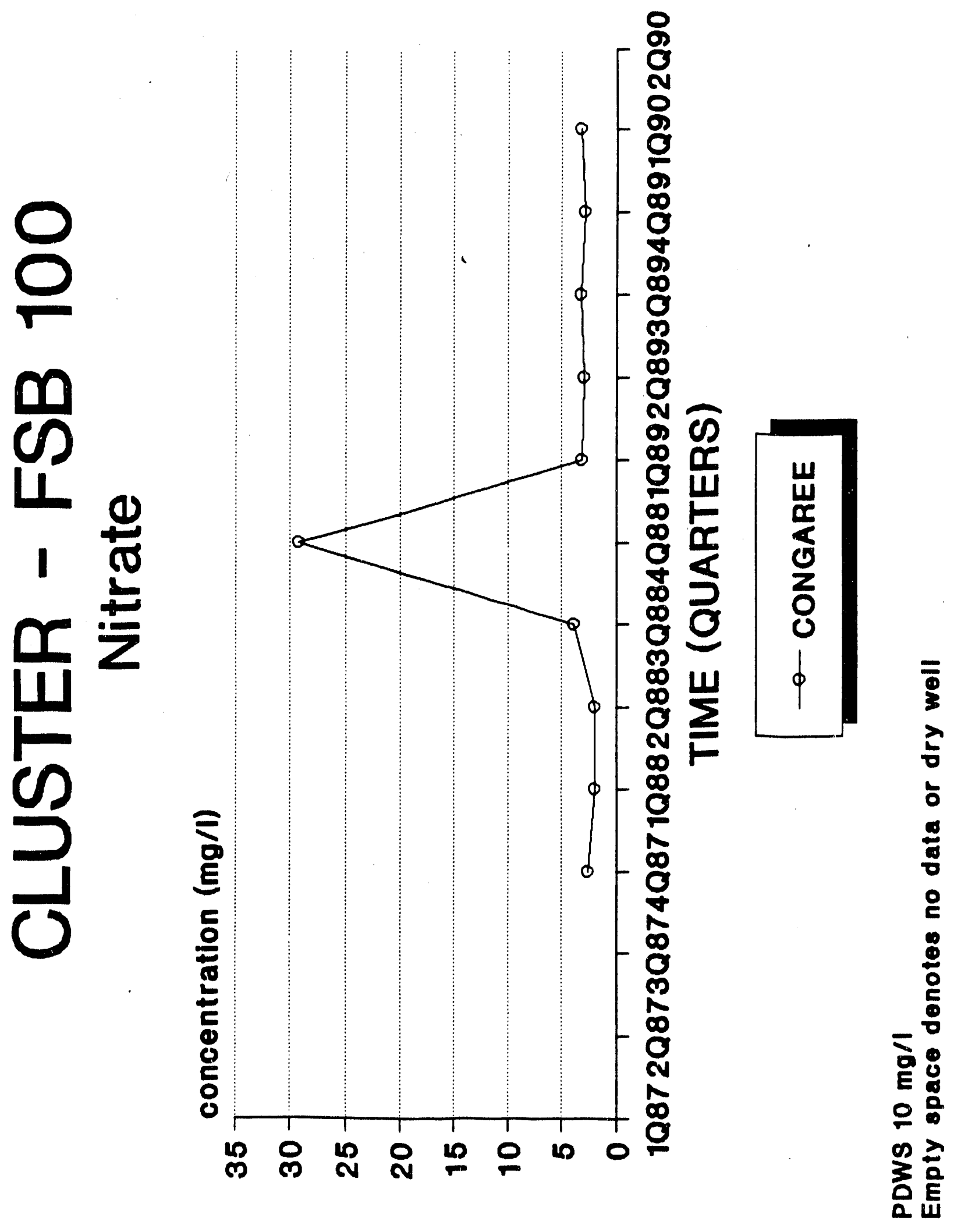




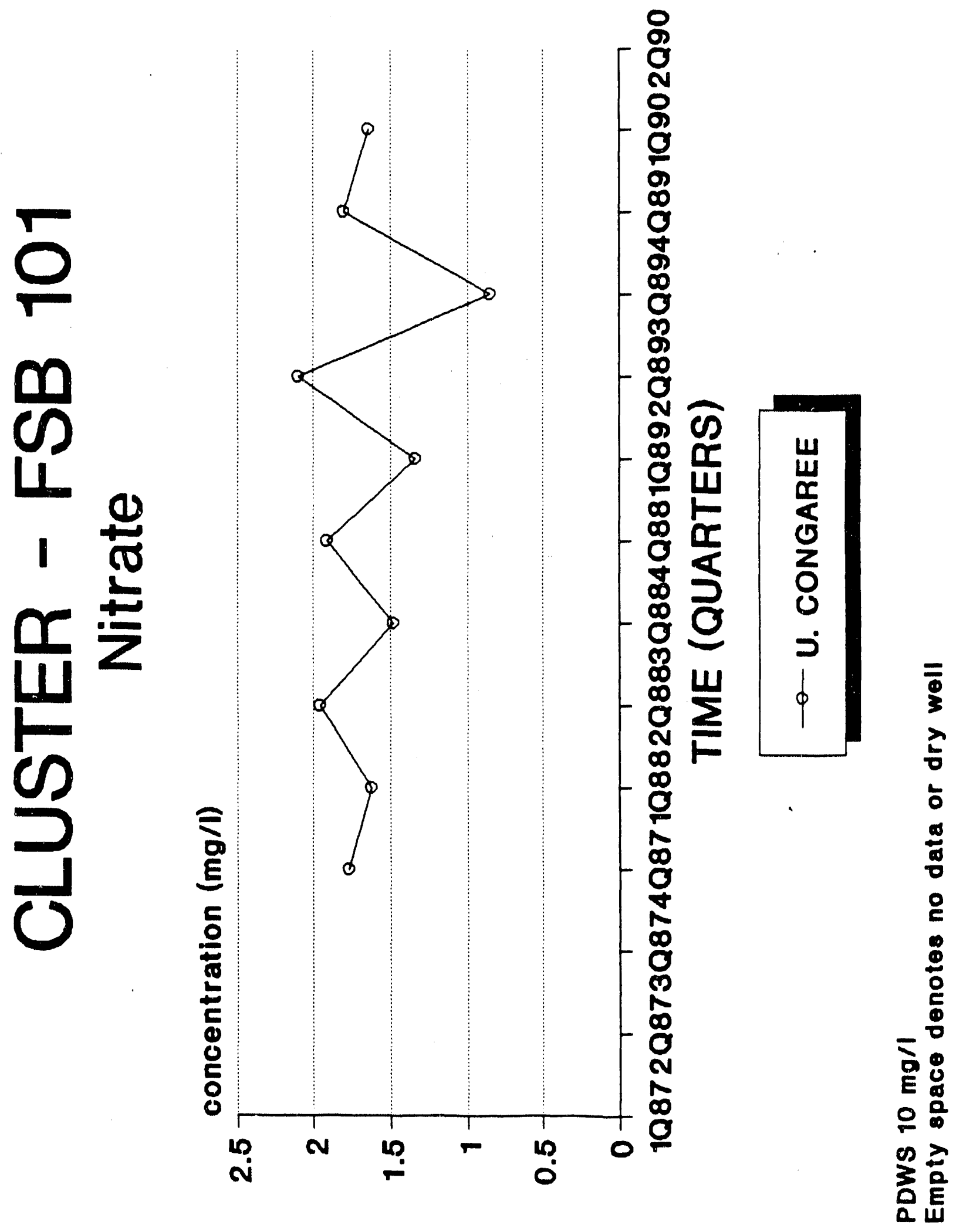




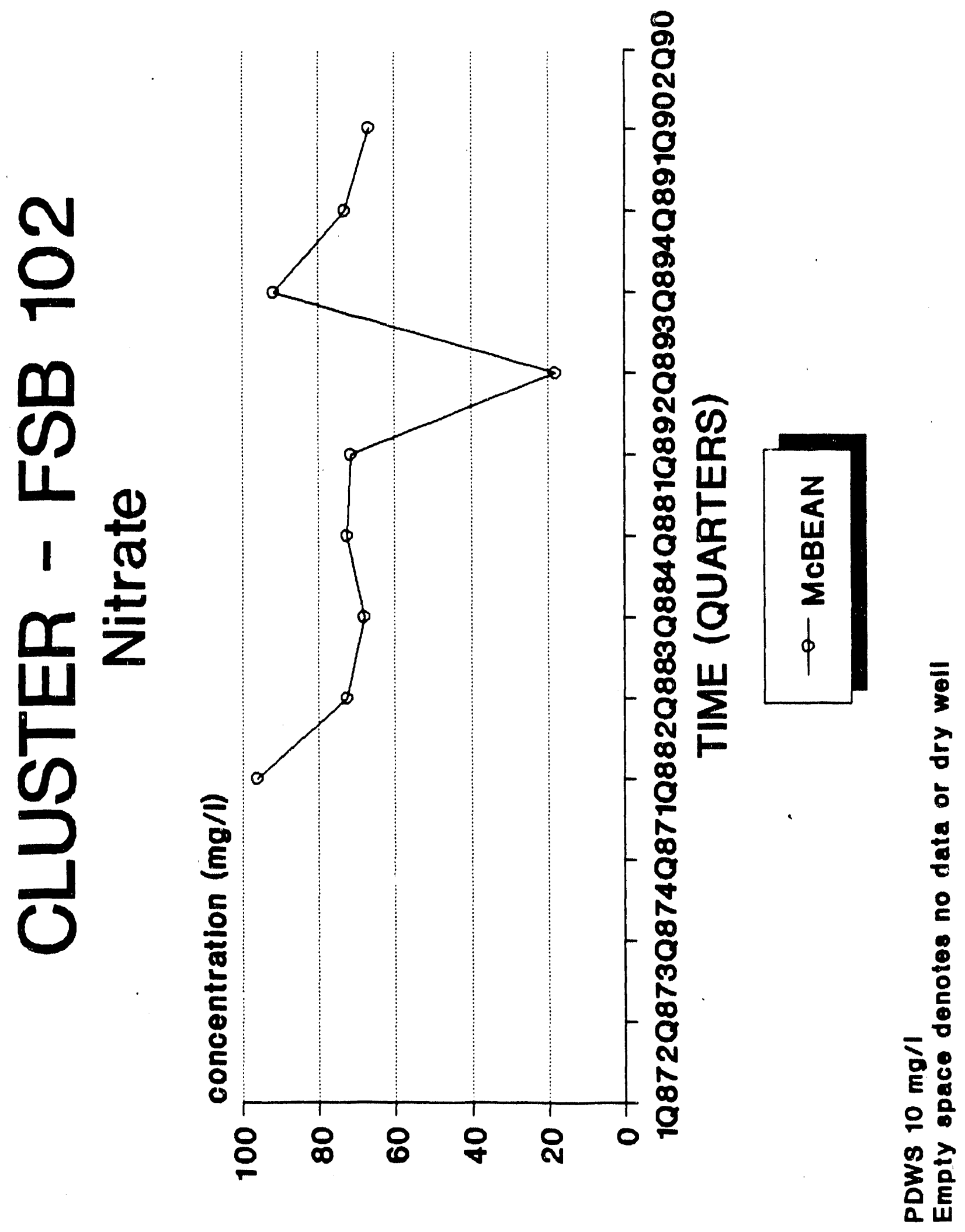




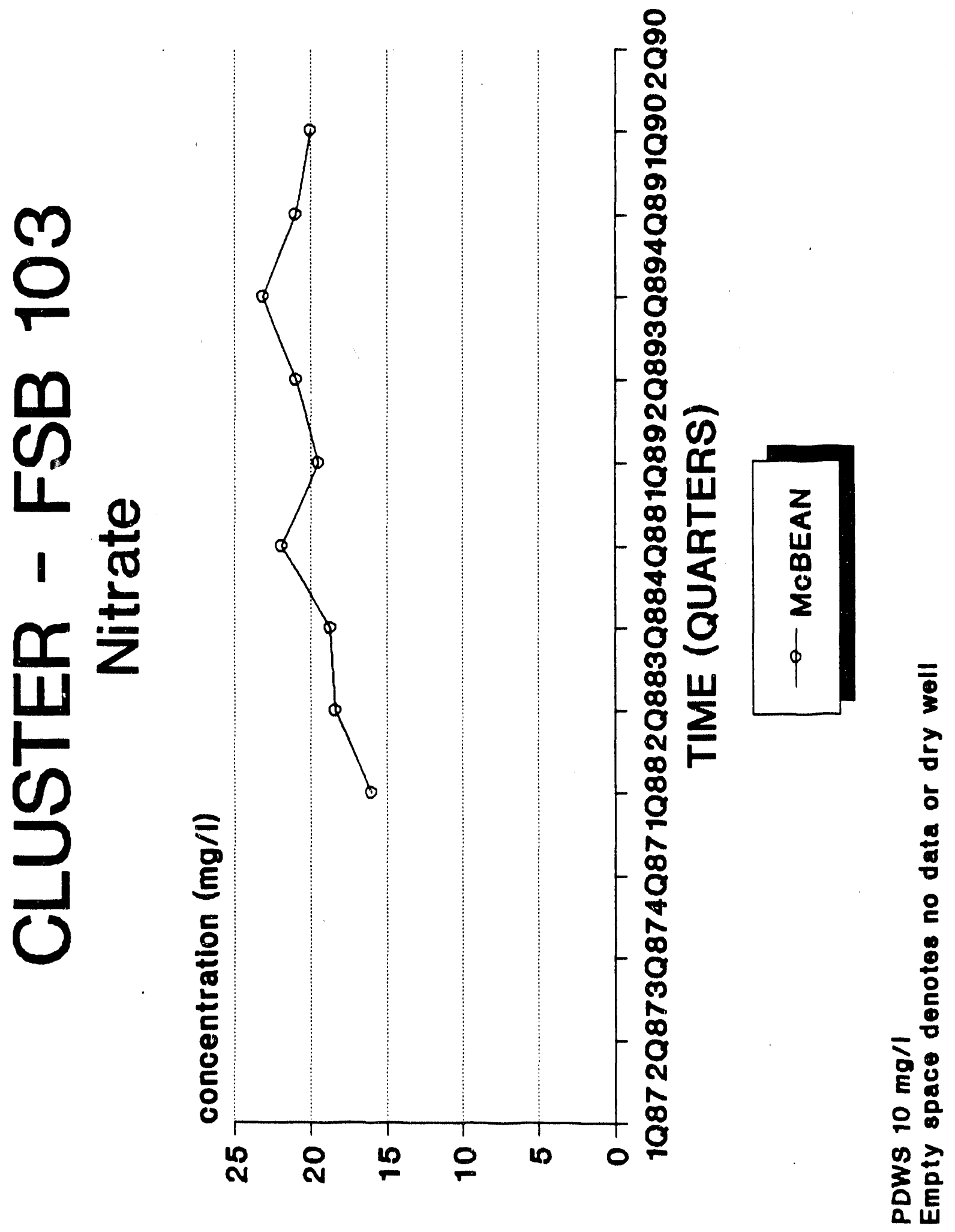




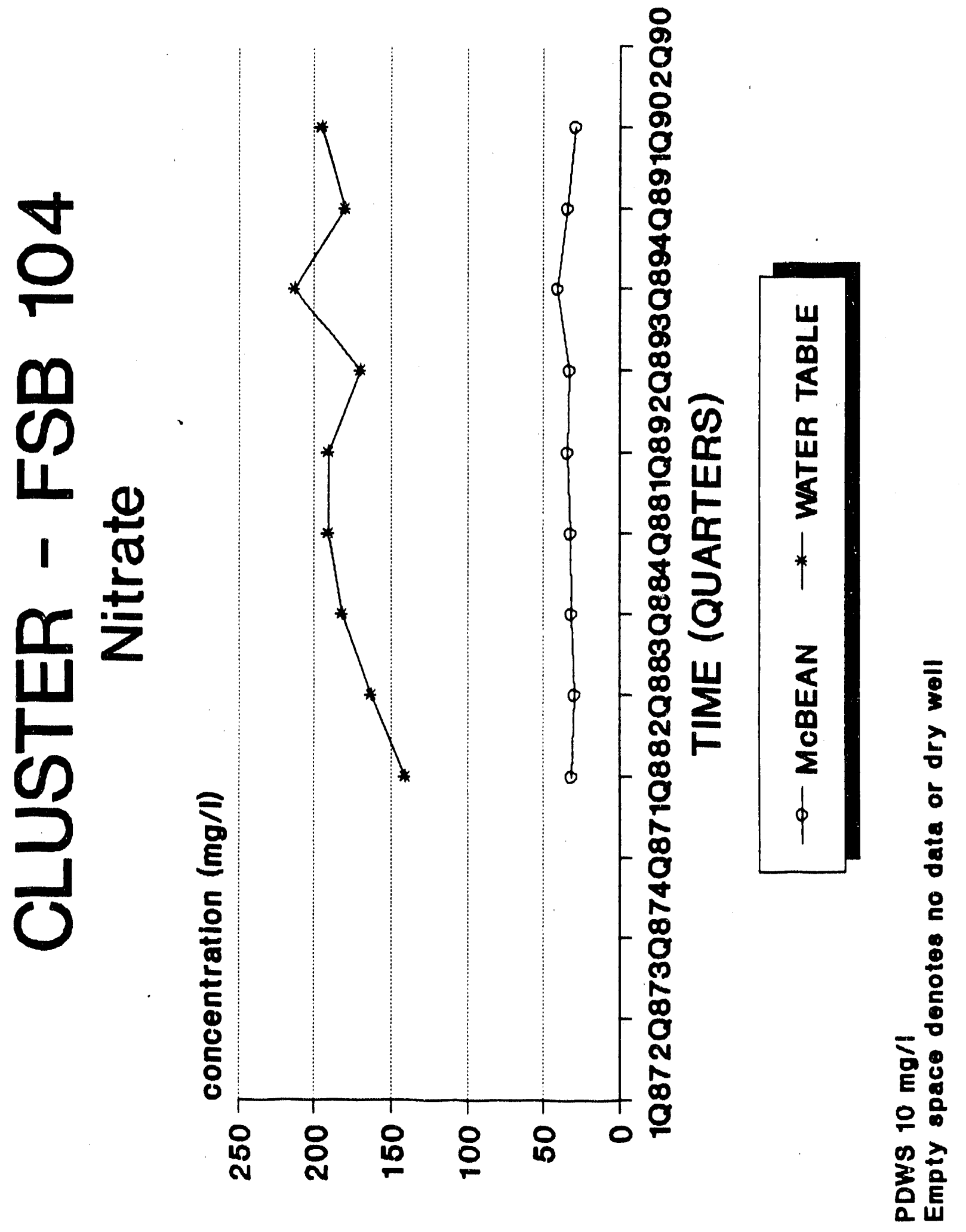




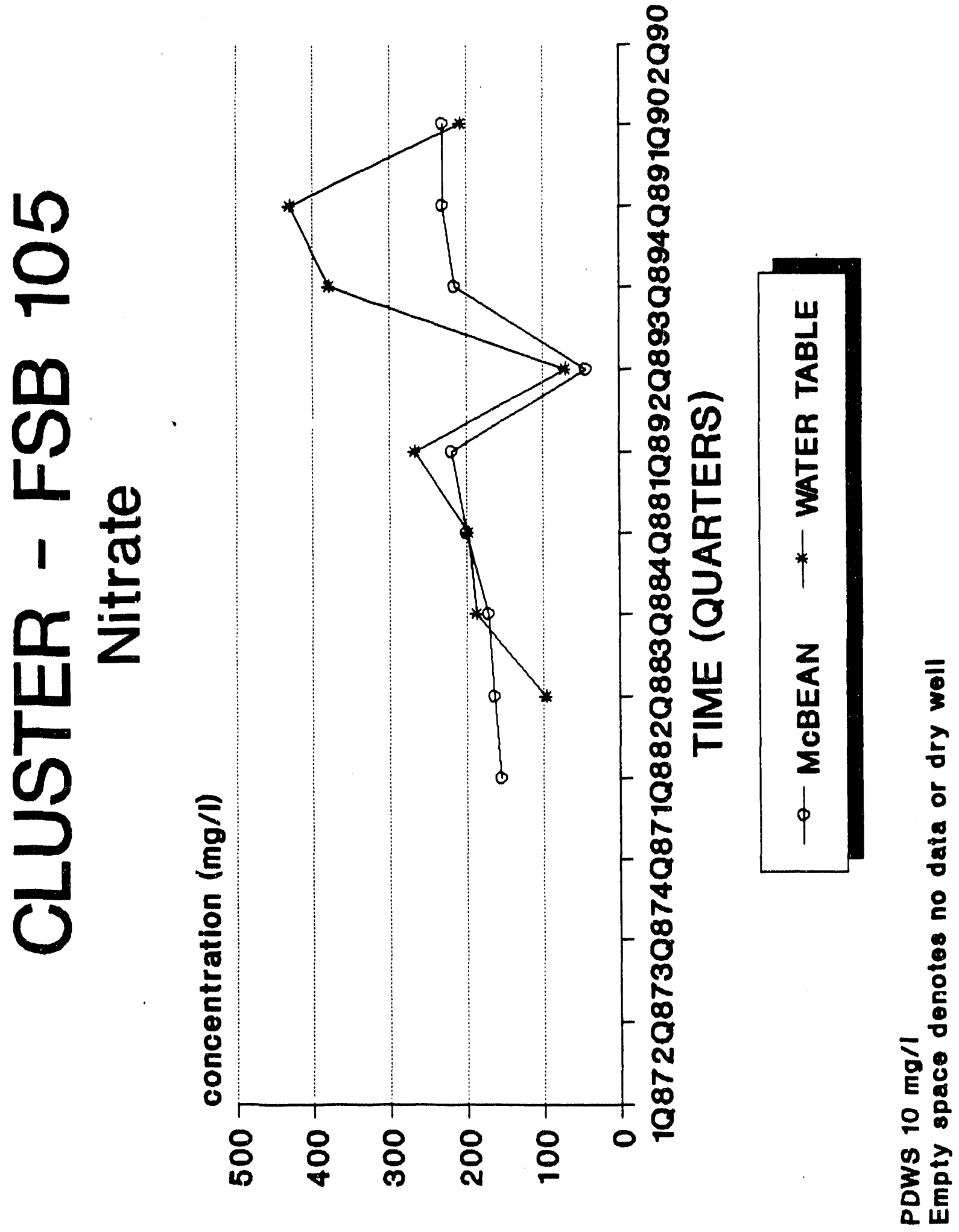




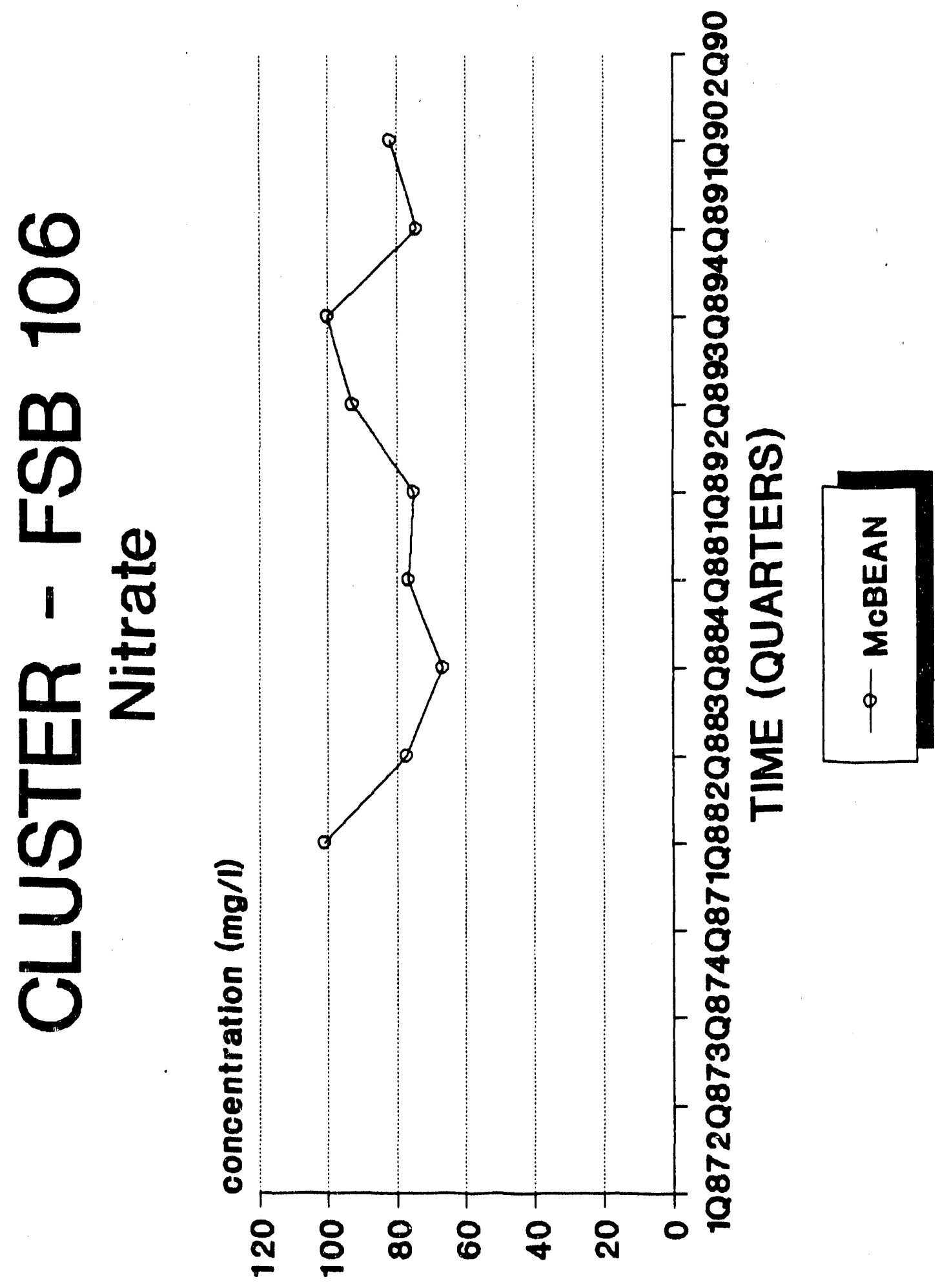

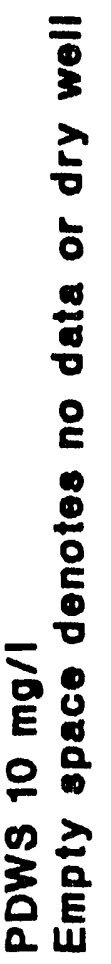




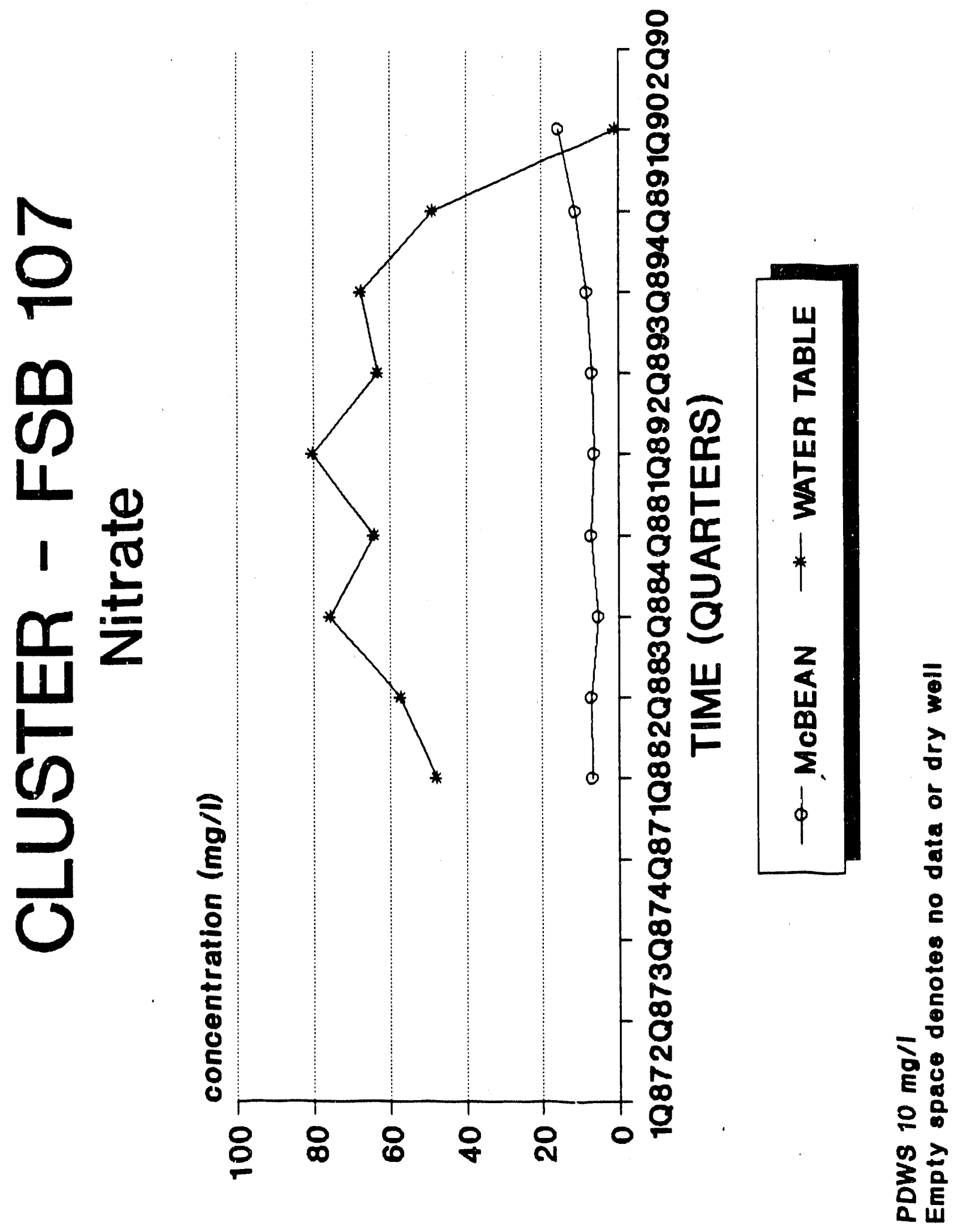




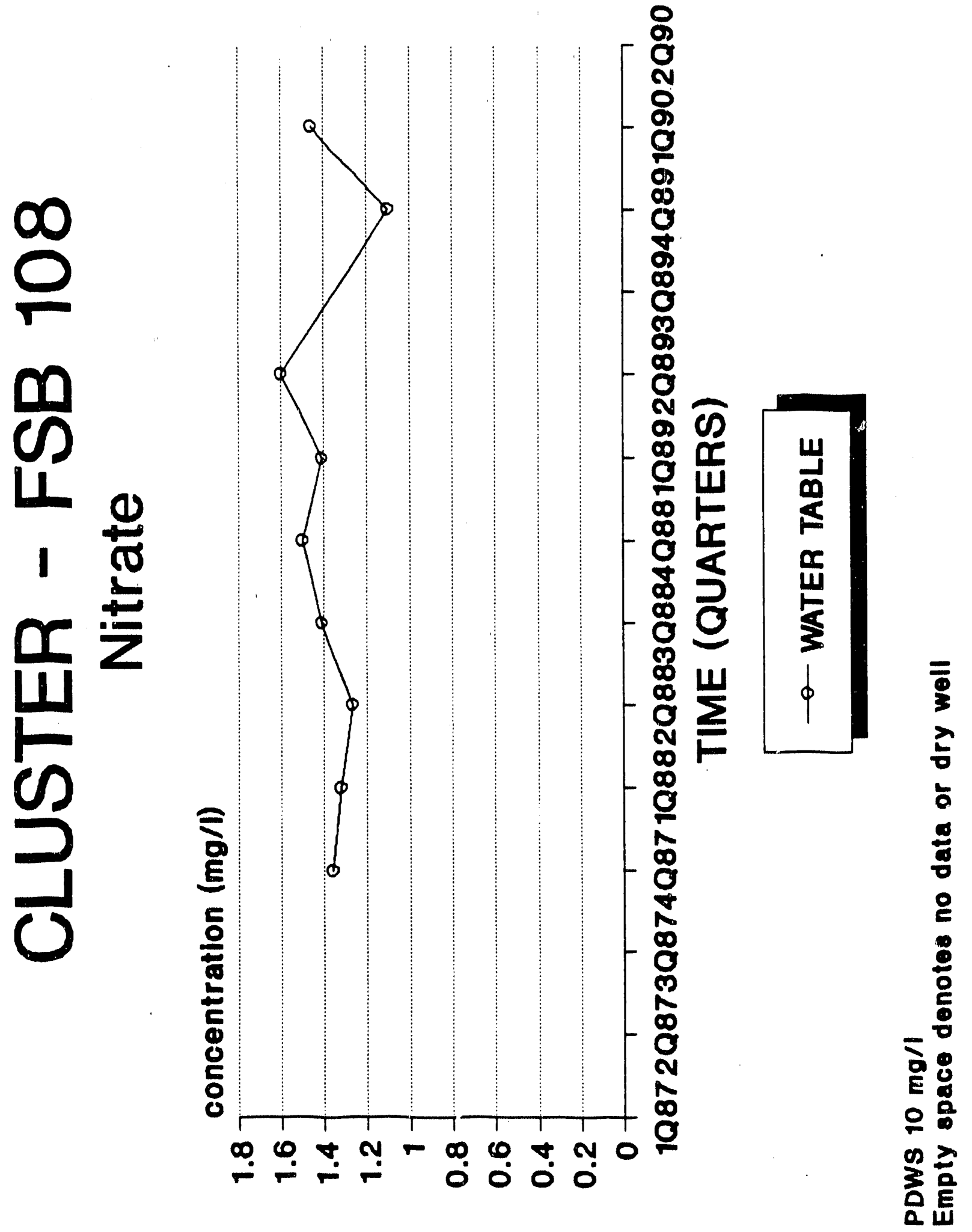




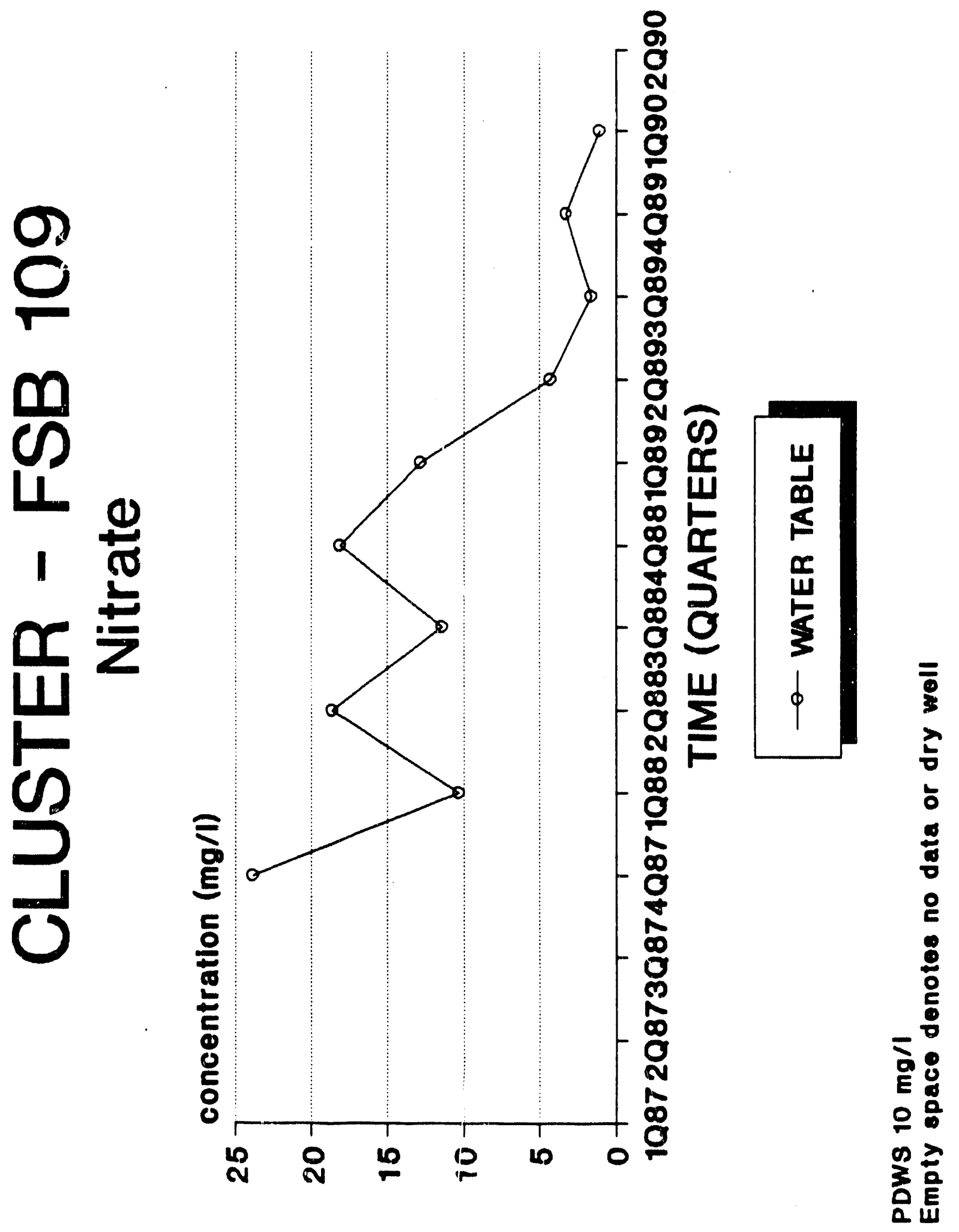




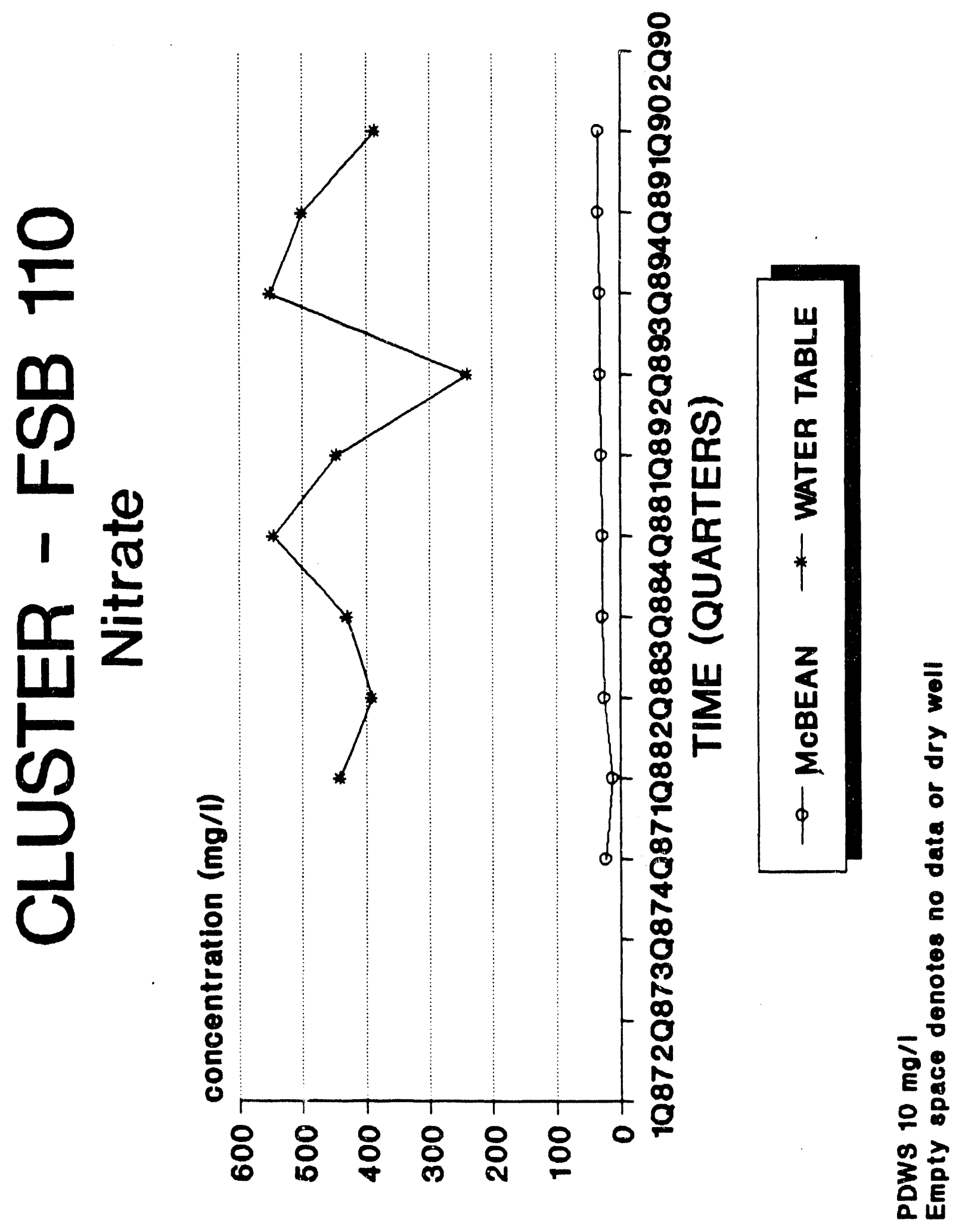




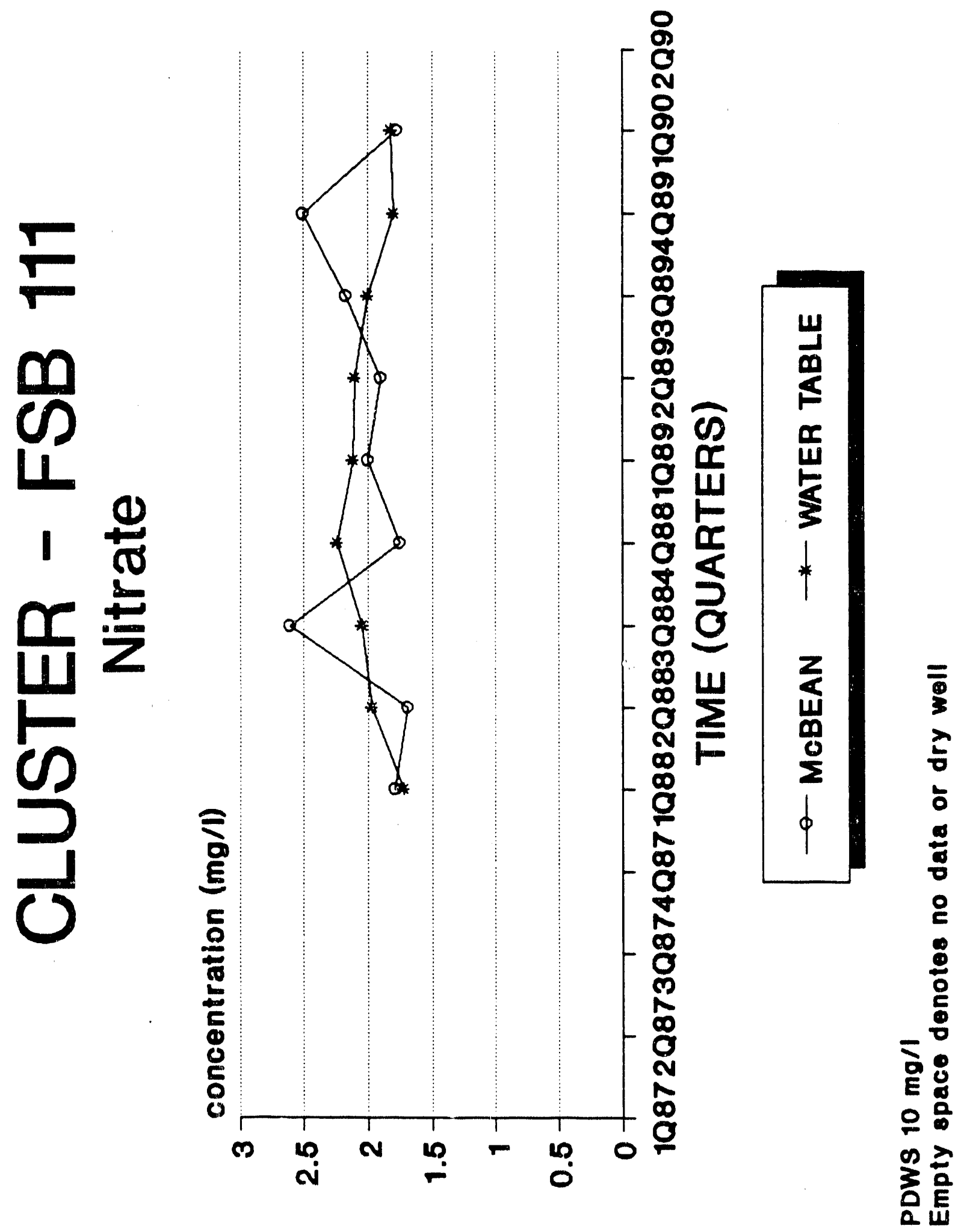




\section{APPELDIX E}

TRITIUY-TIYE TRENDS

Figures E1-E29 display the levels of tritilum measured at each unit in every well cluster as a function of time. 


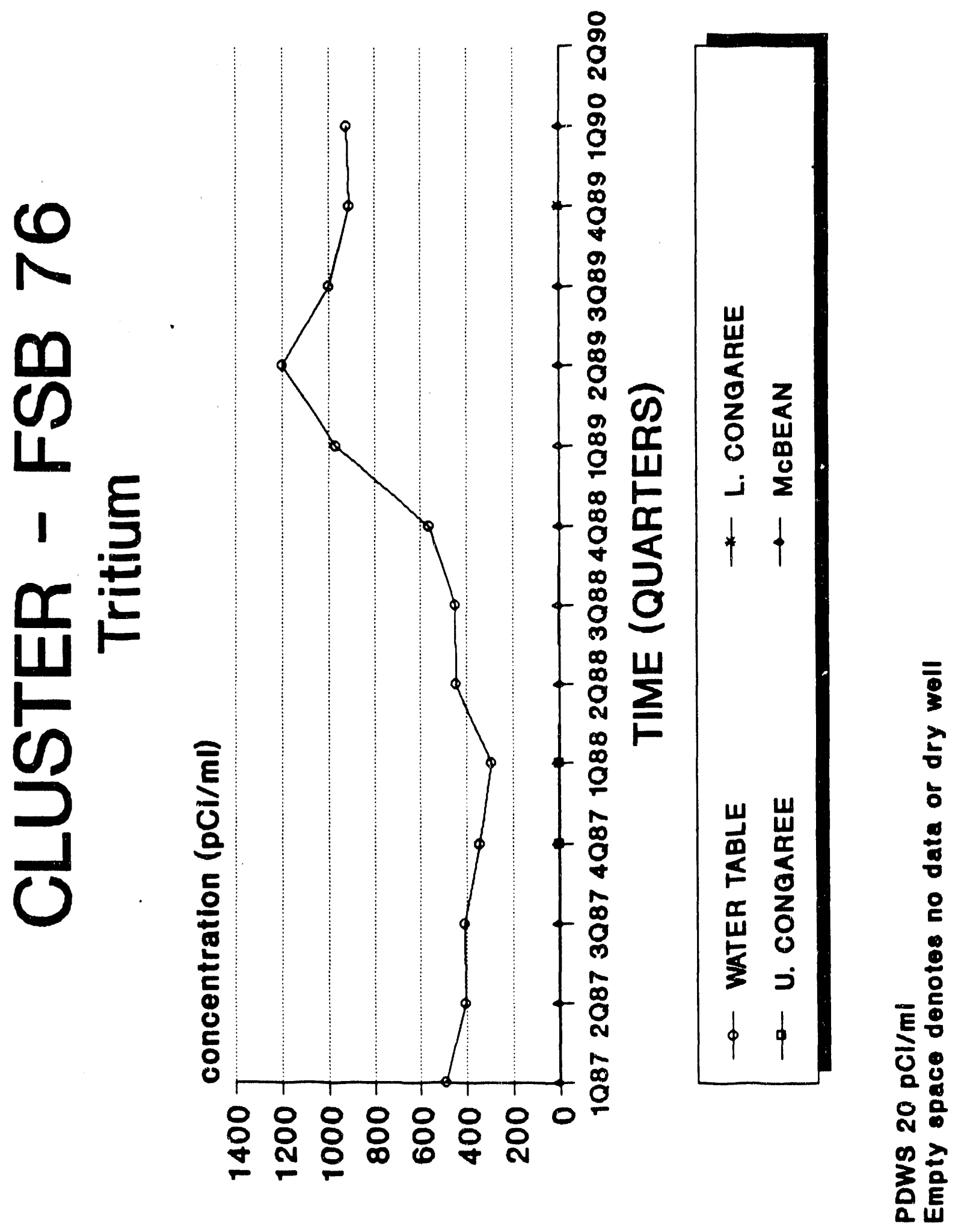




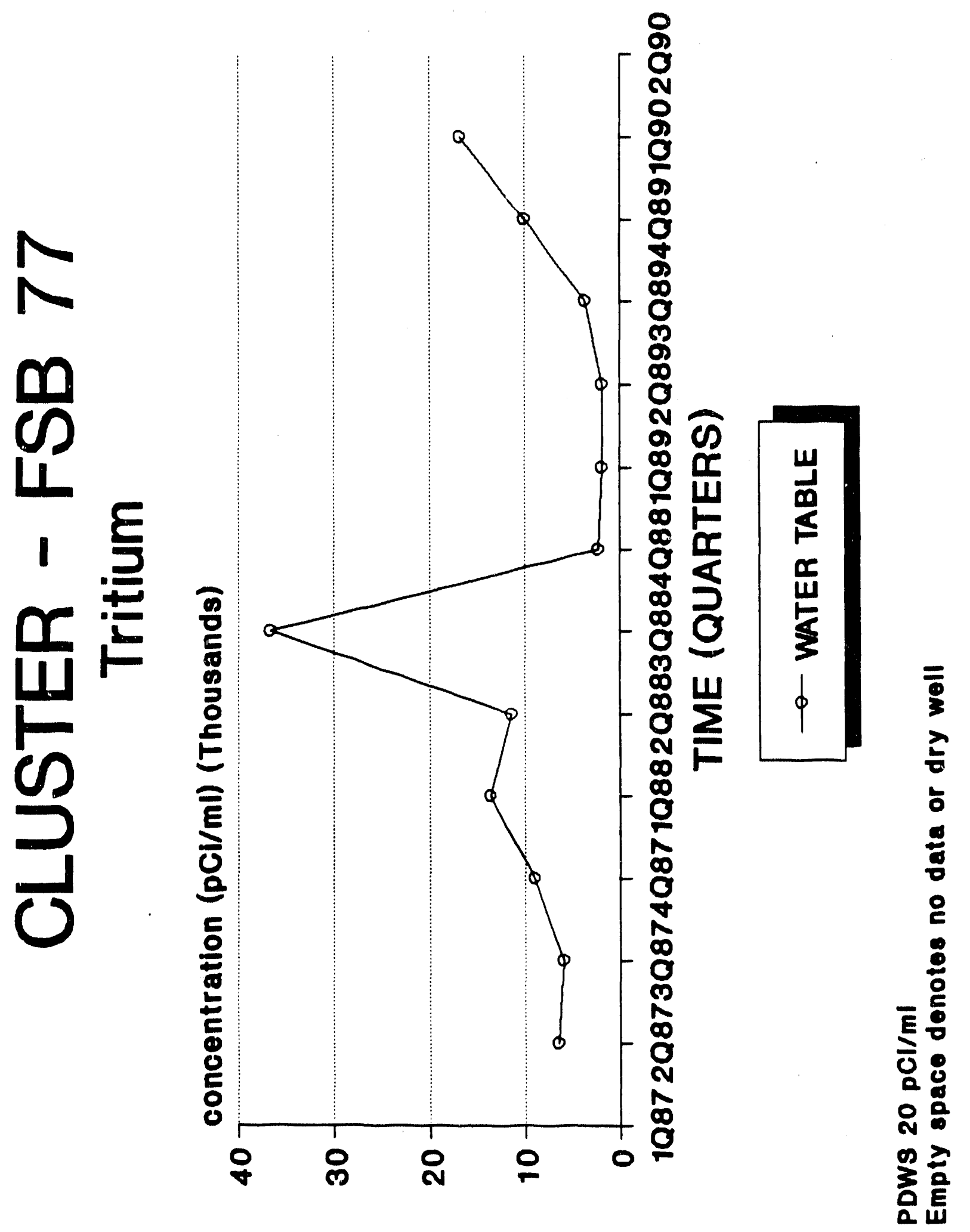




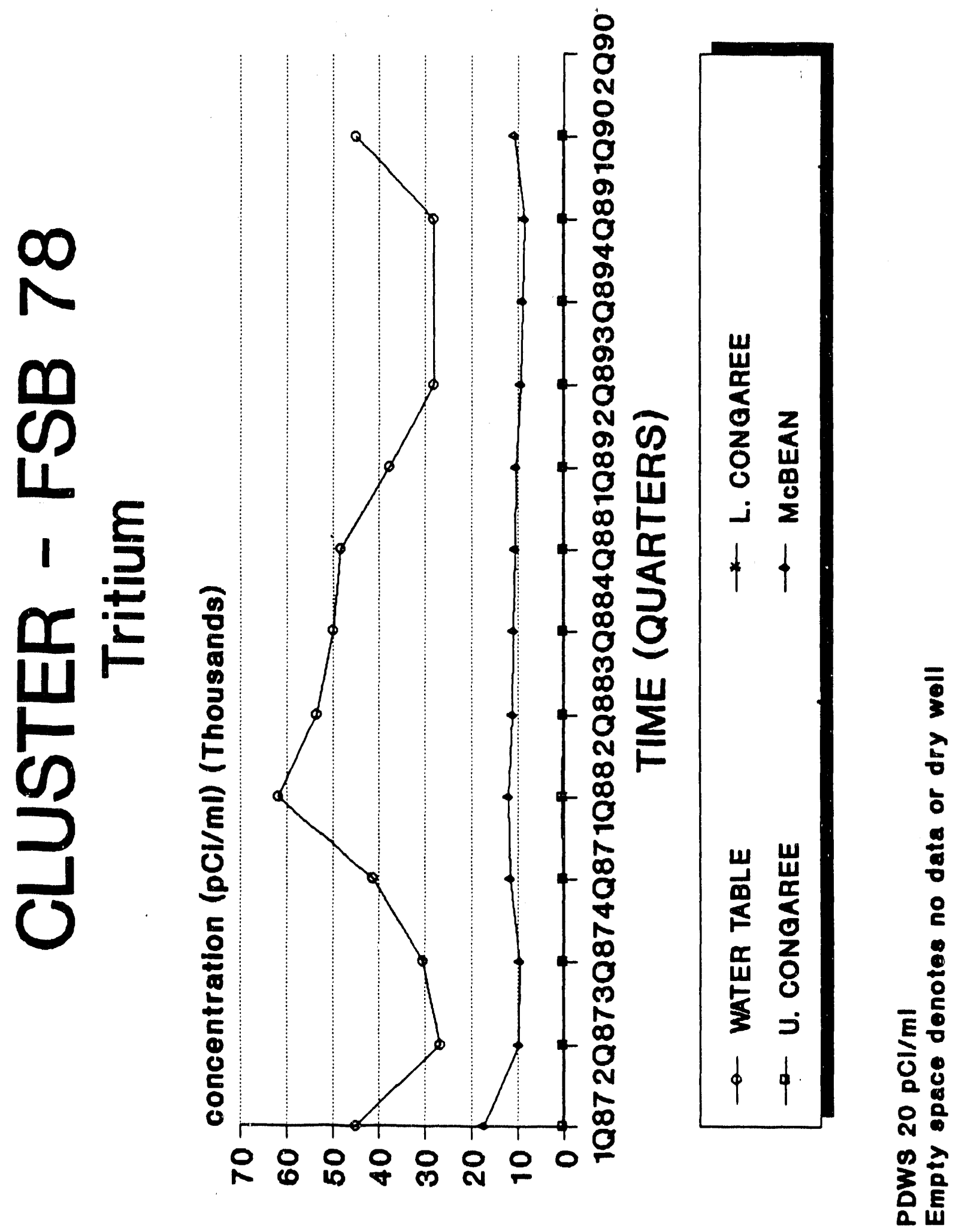




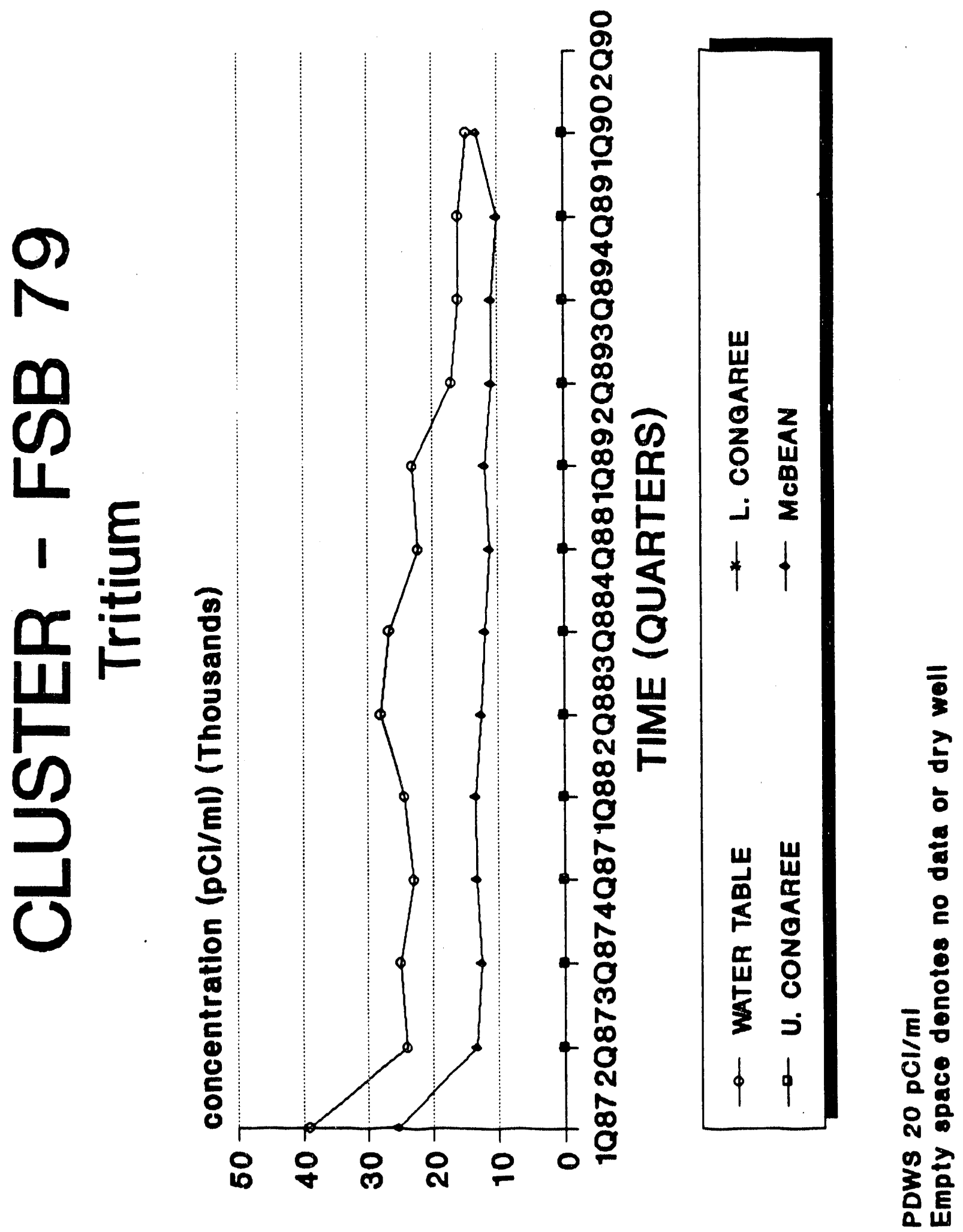




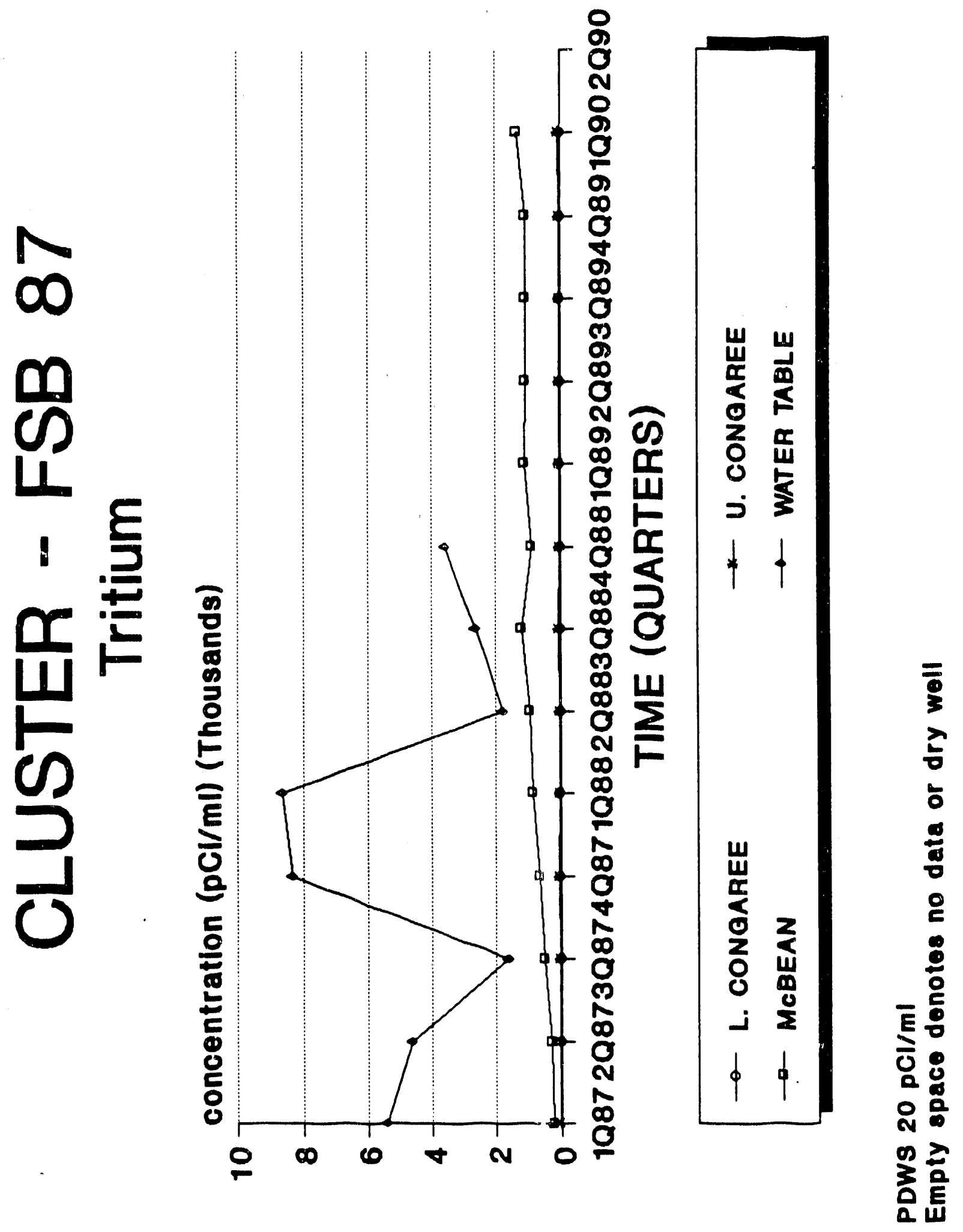




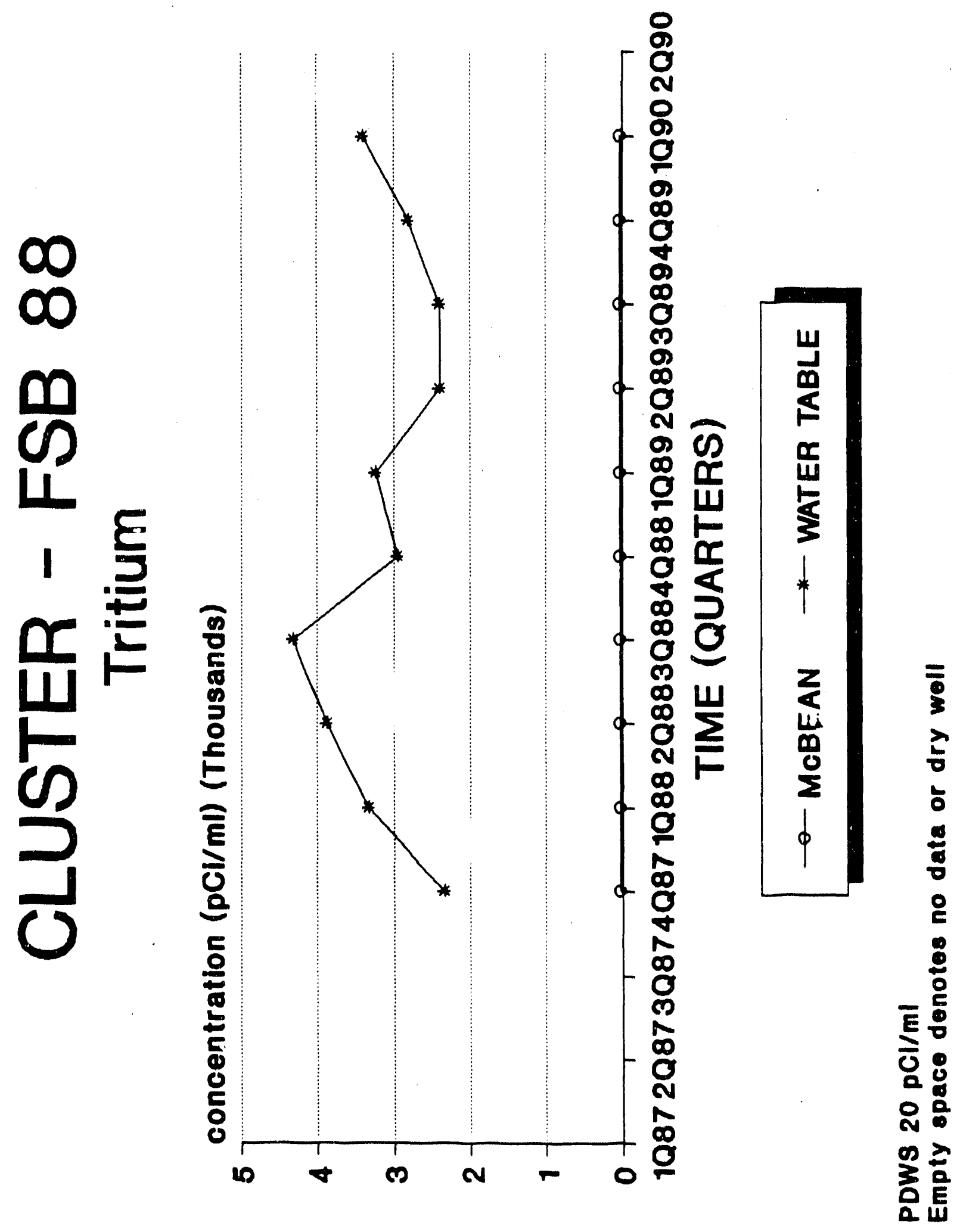




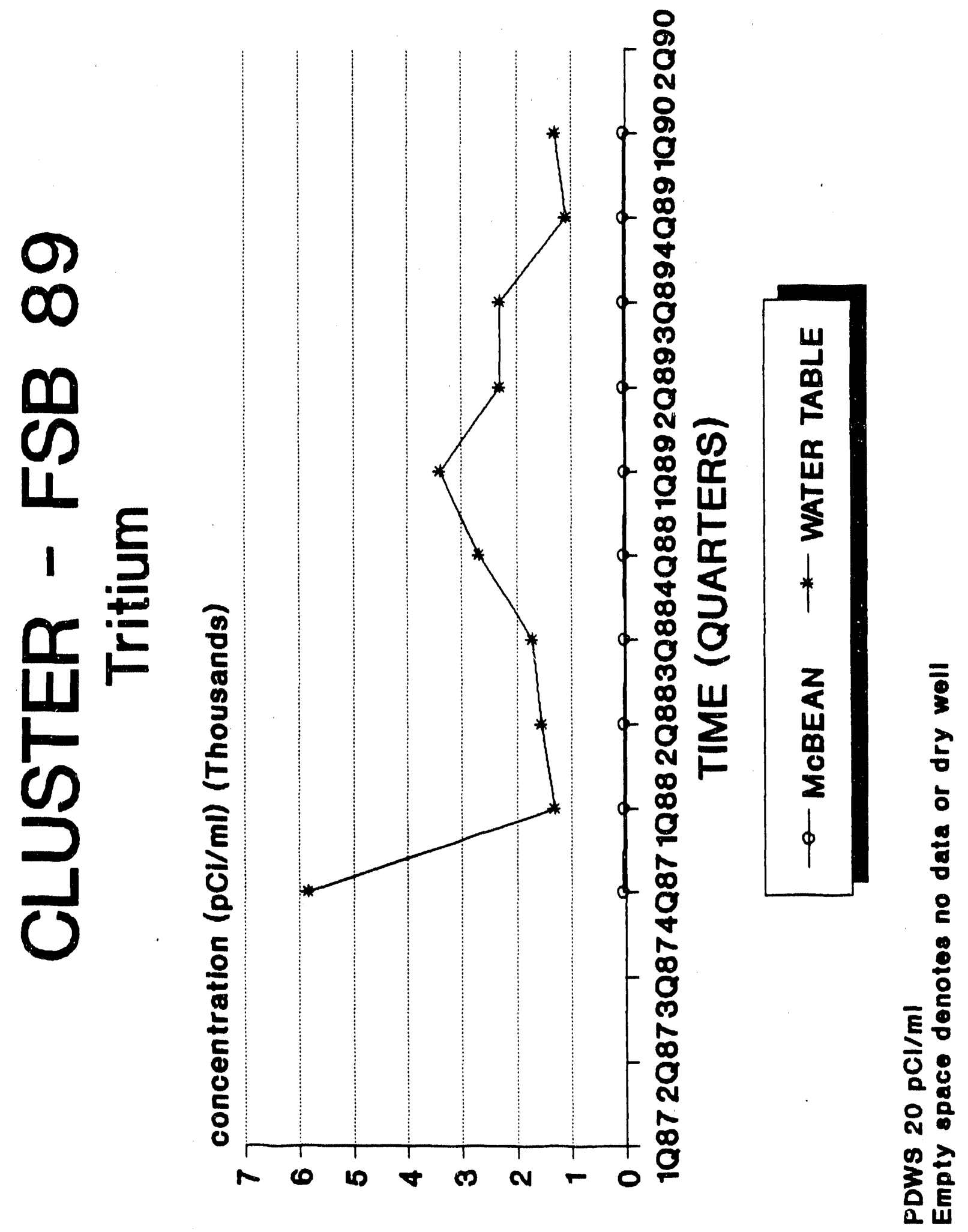




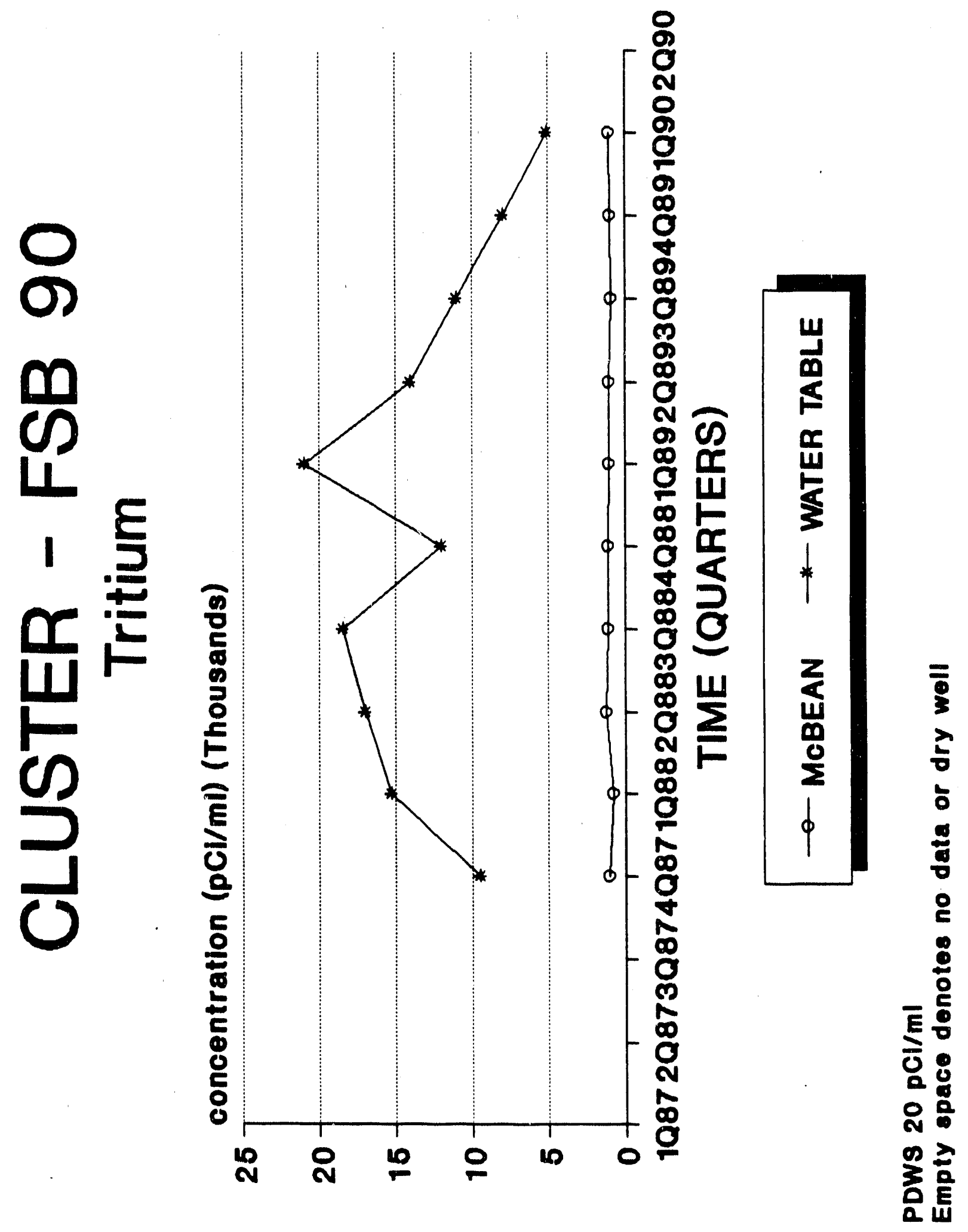




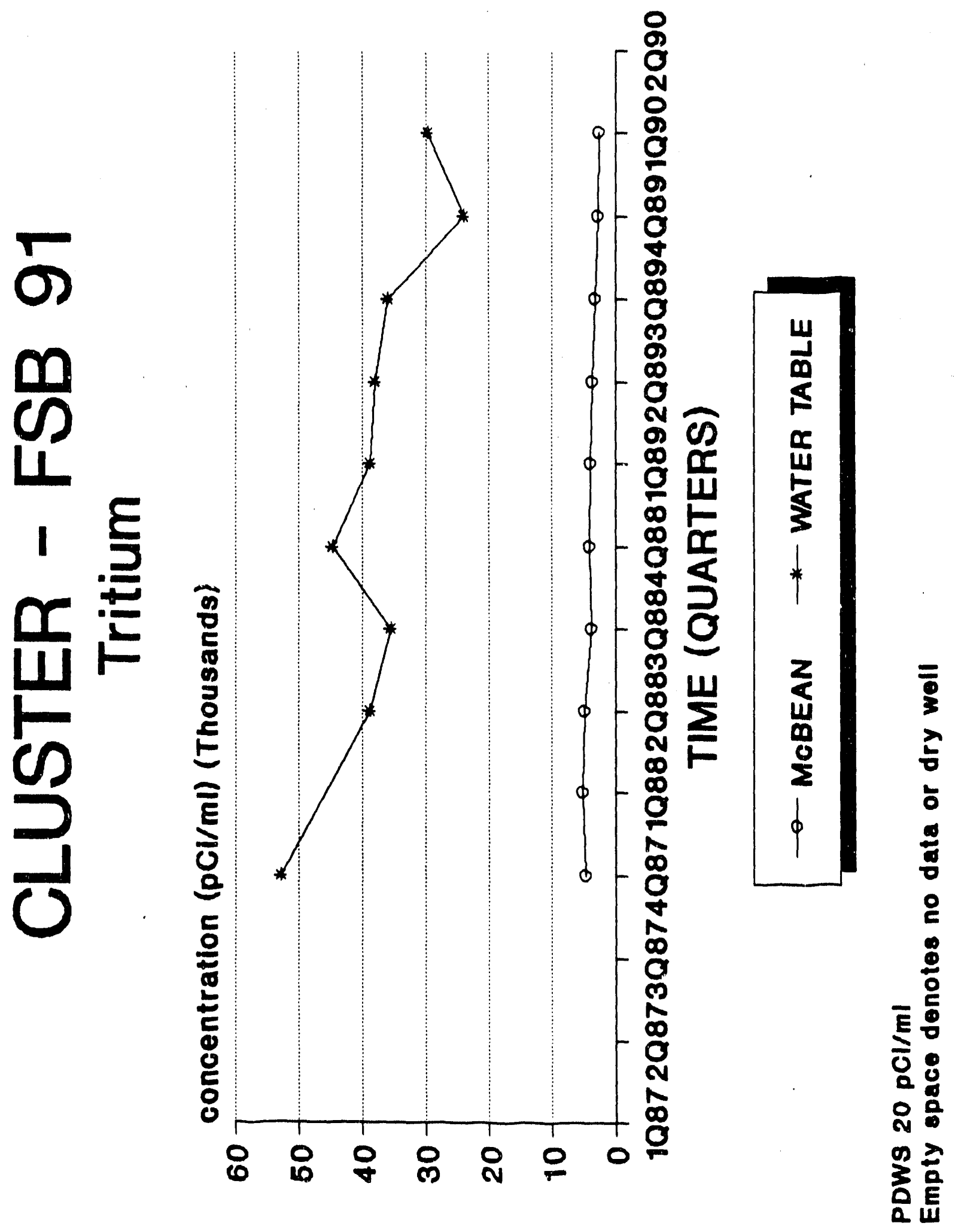




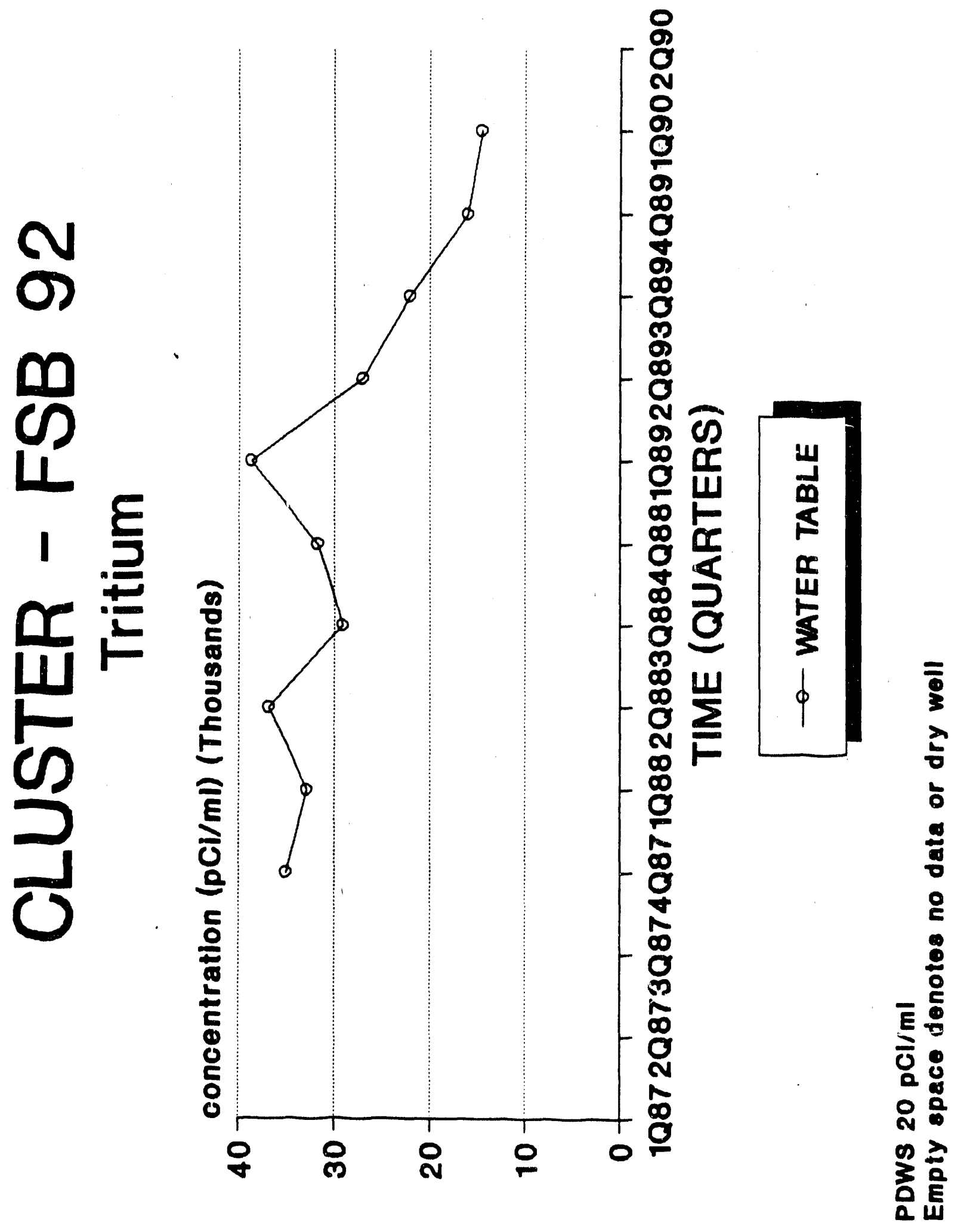




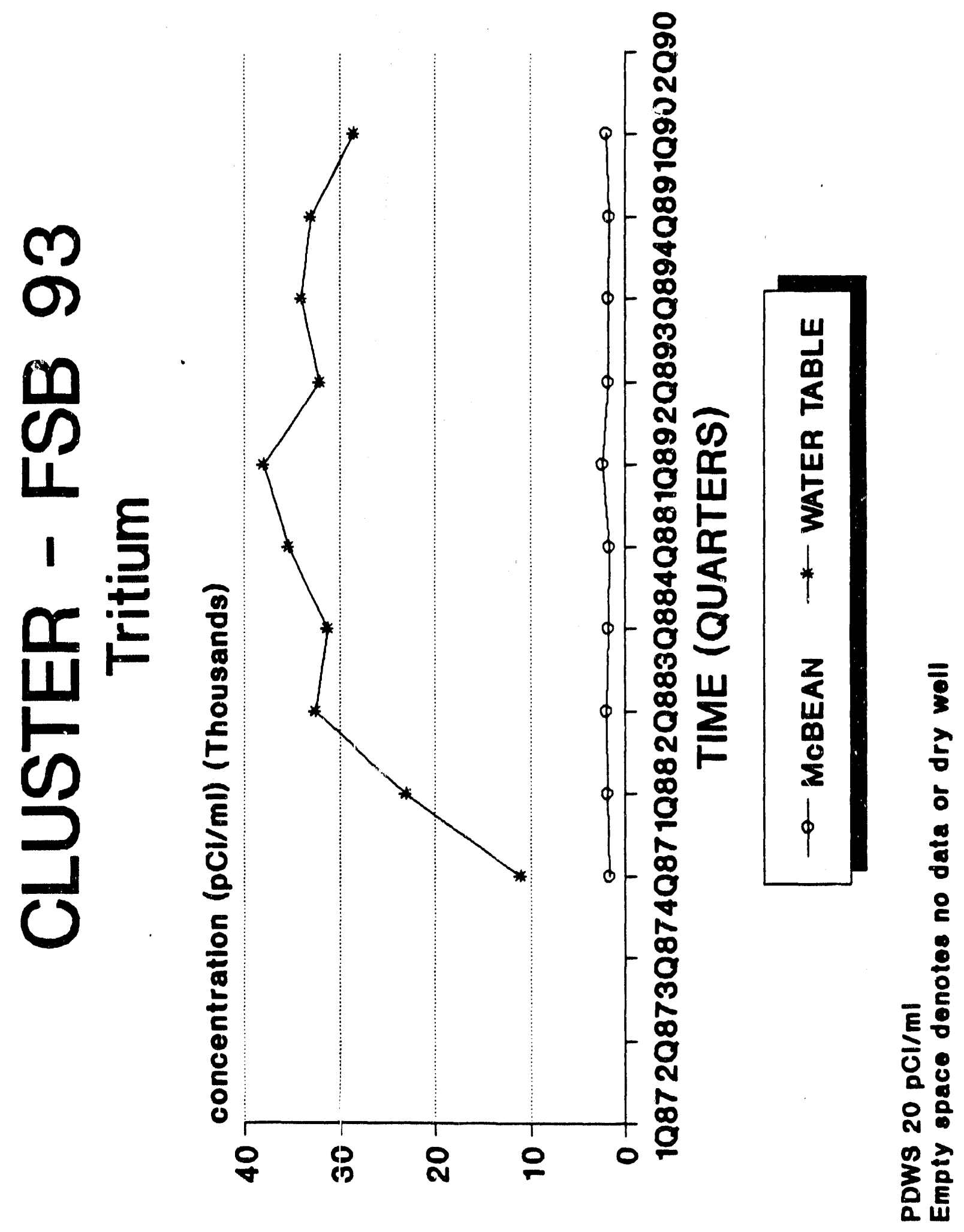




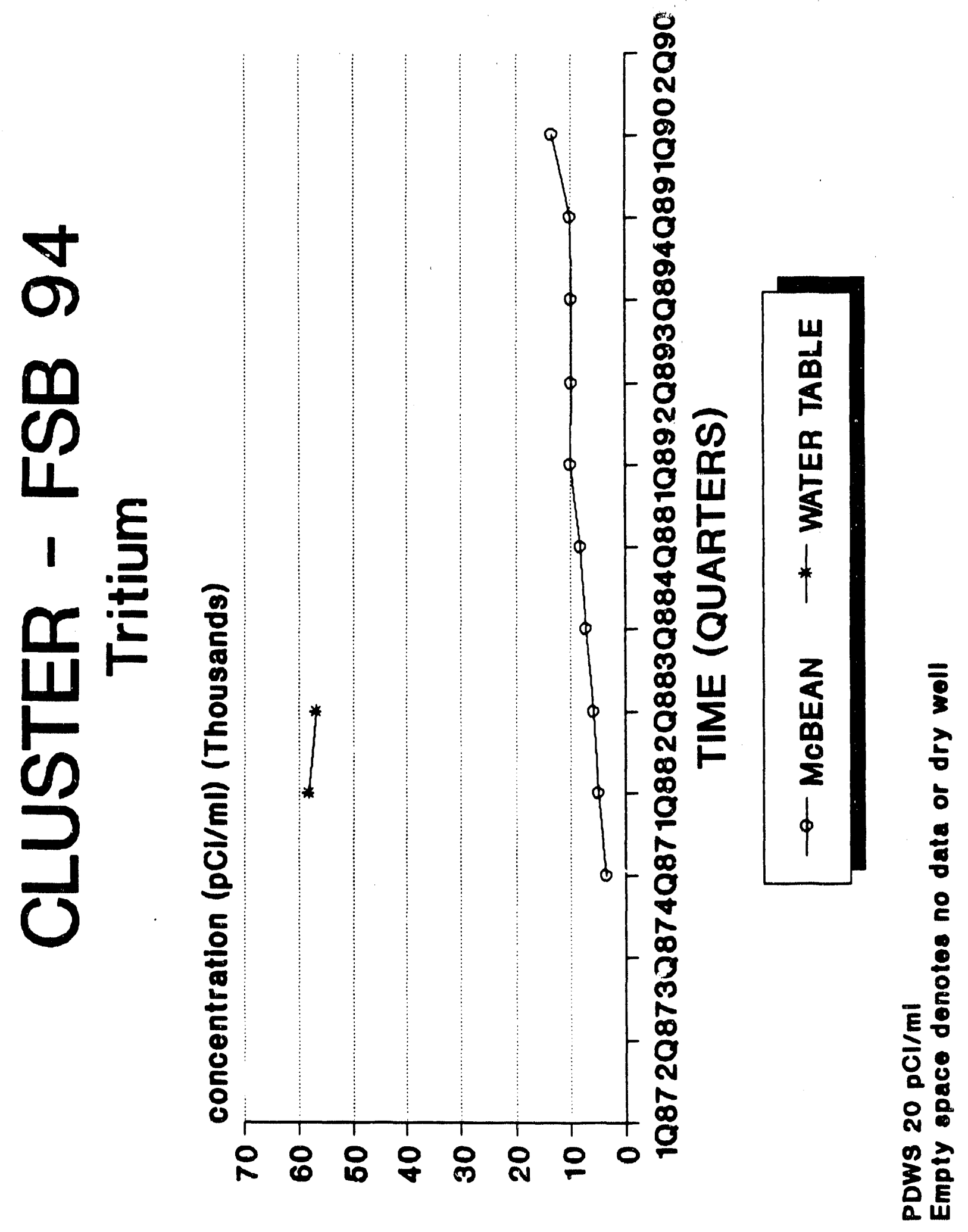




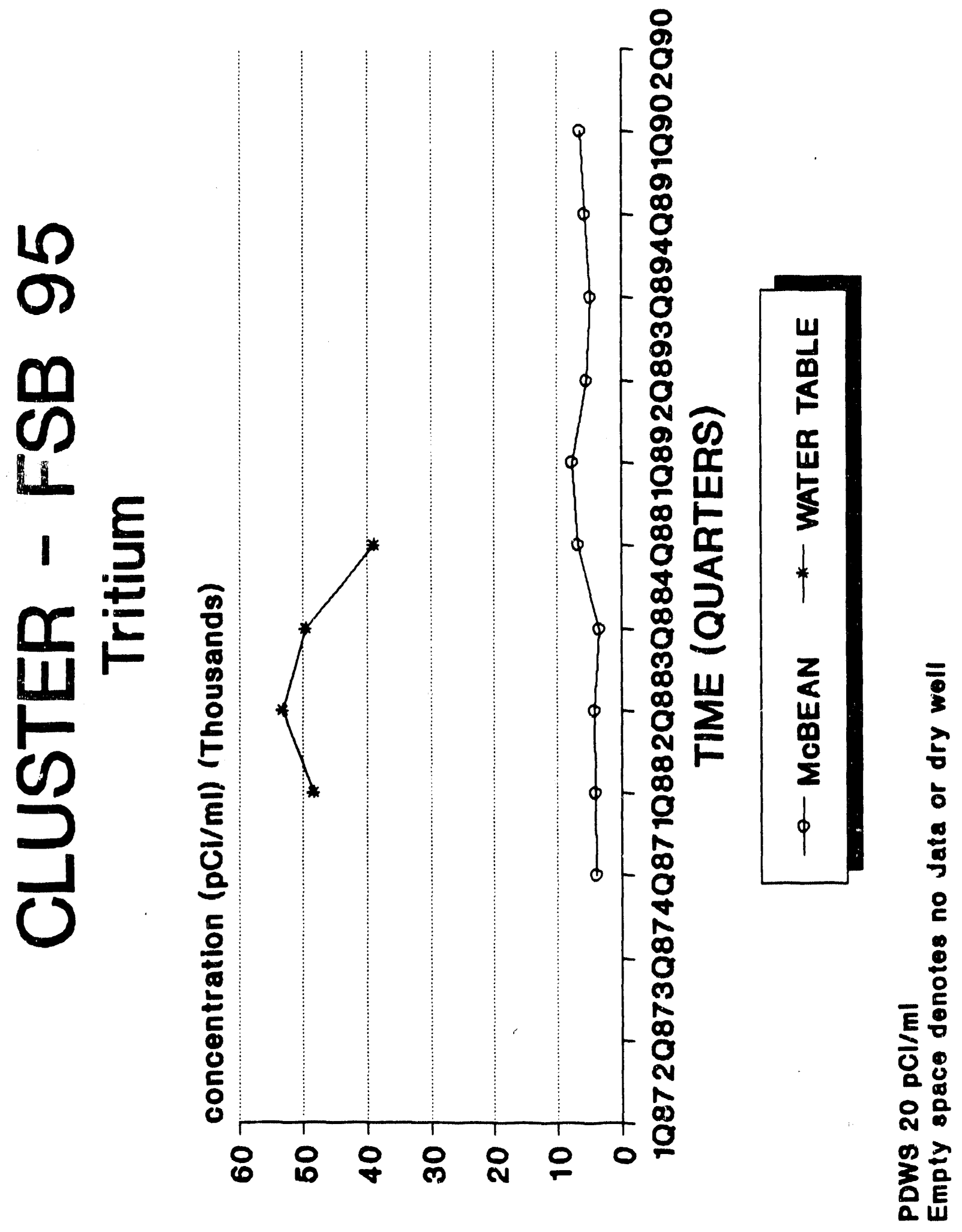




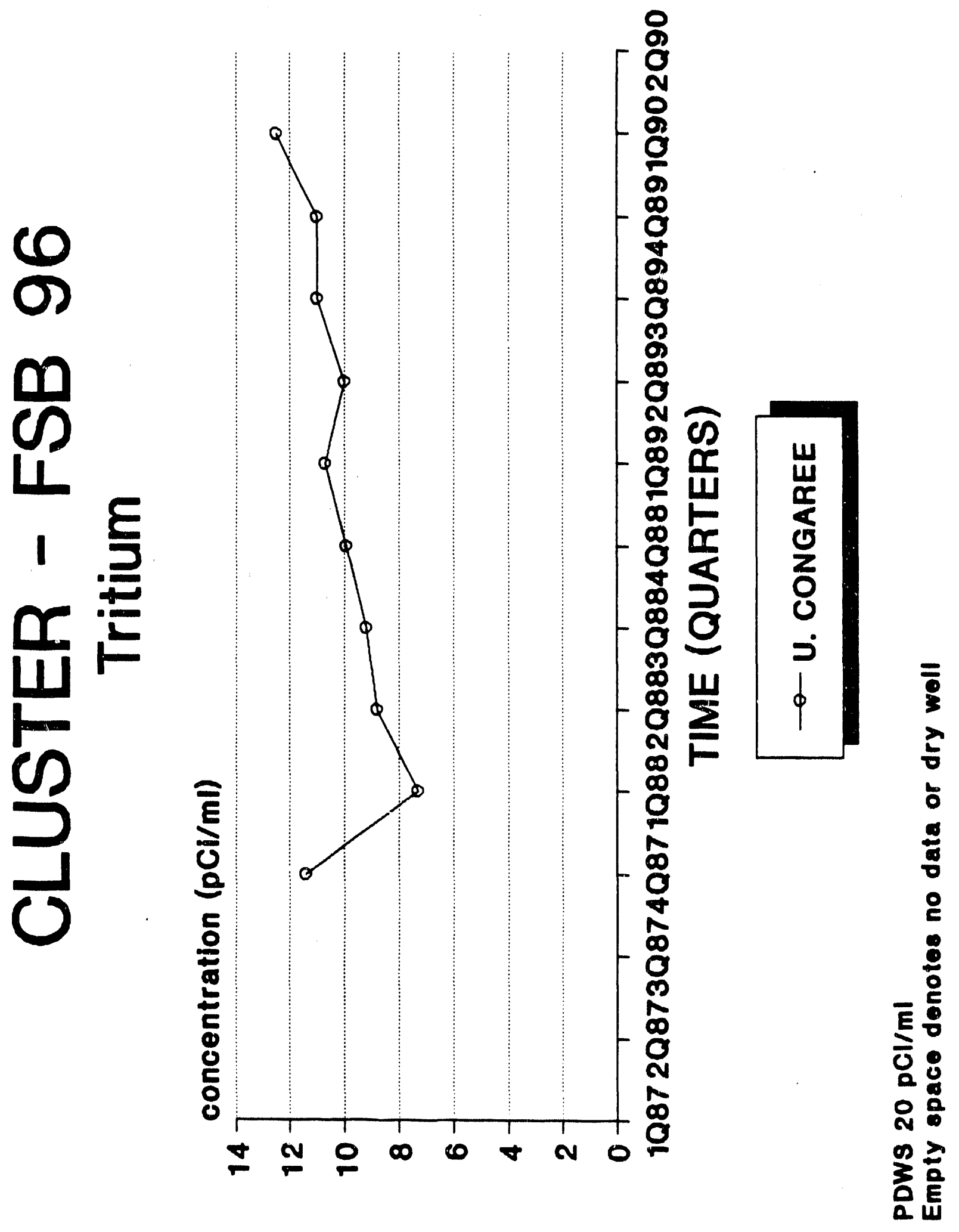




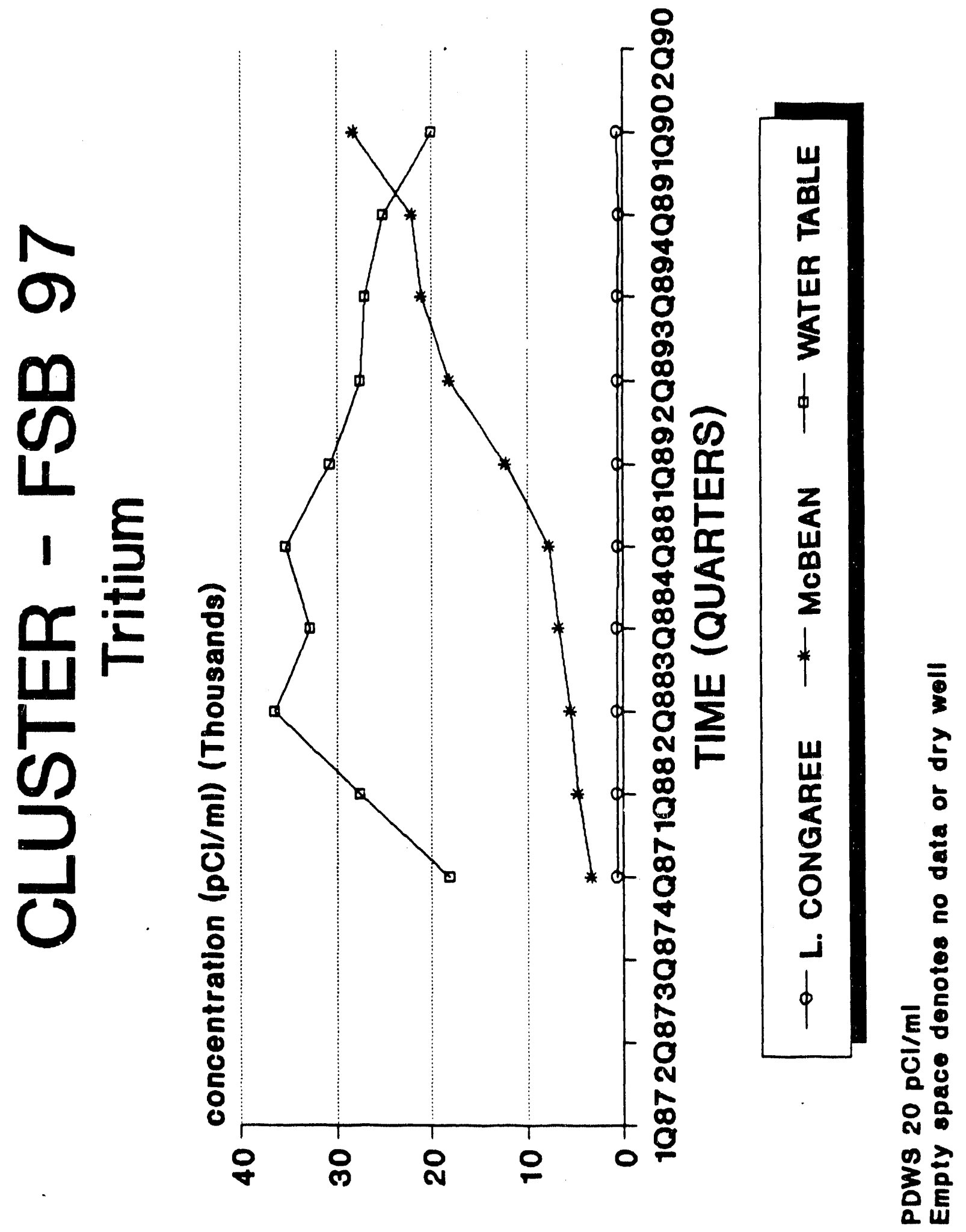




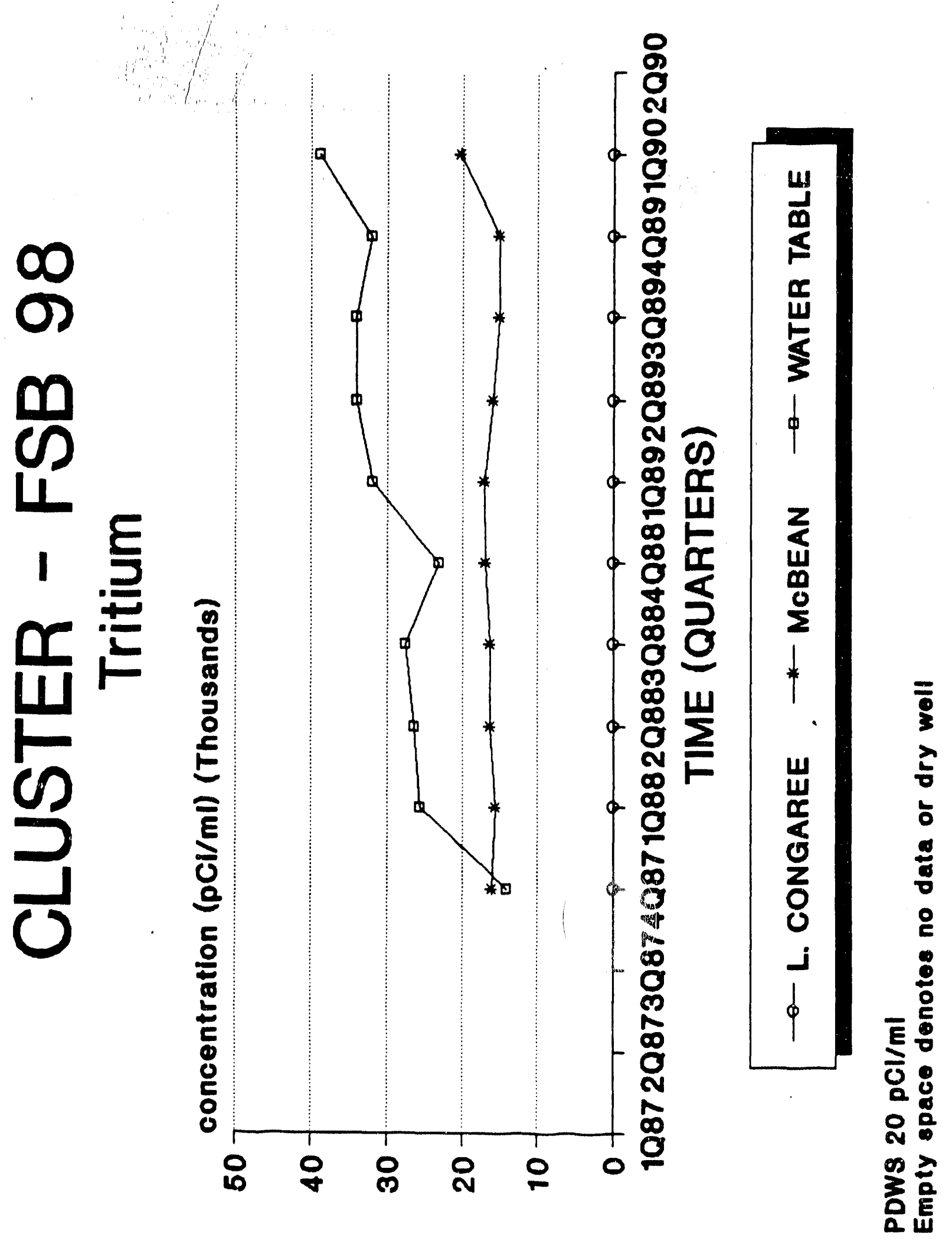




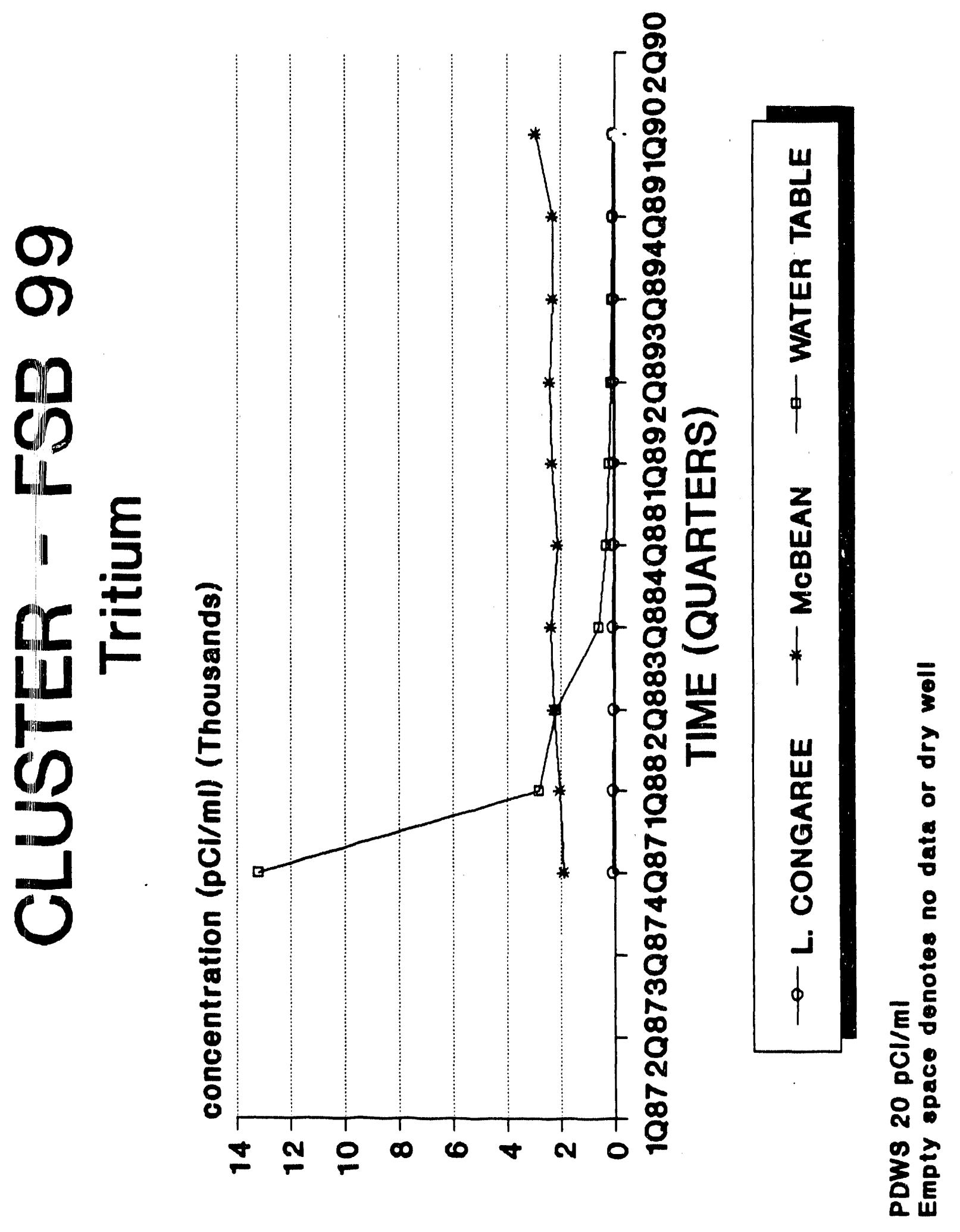




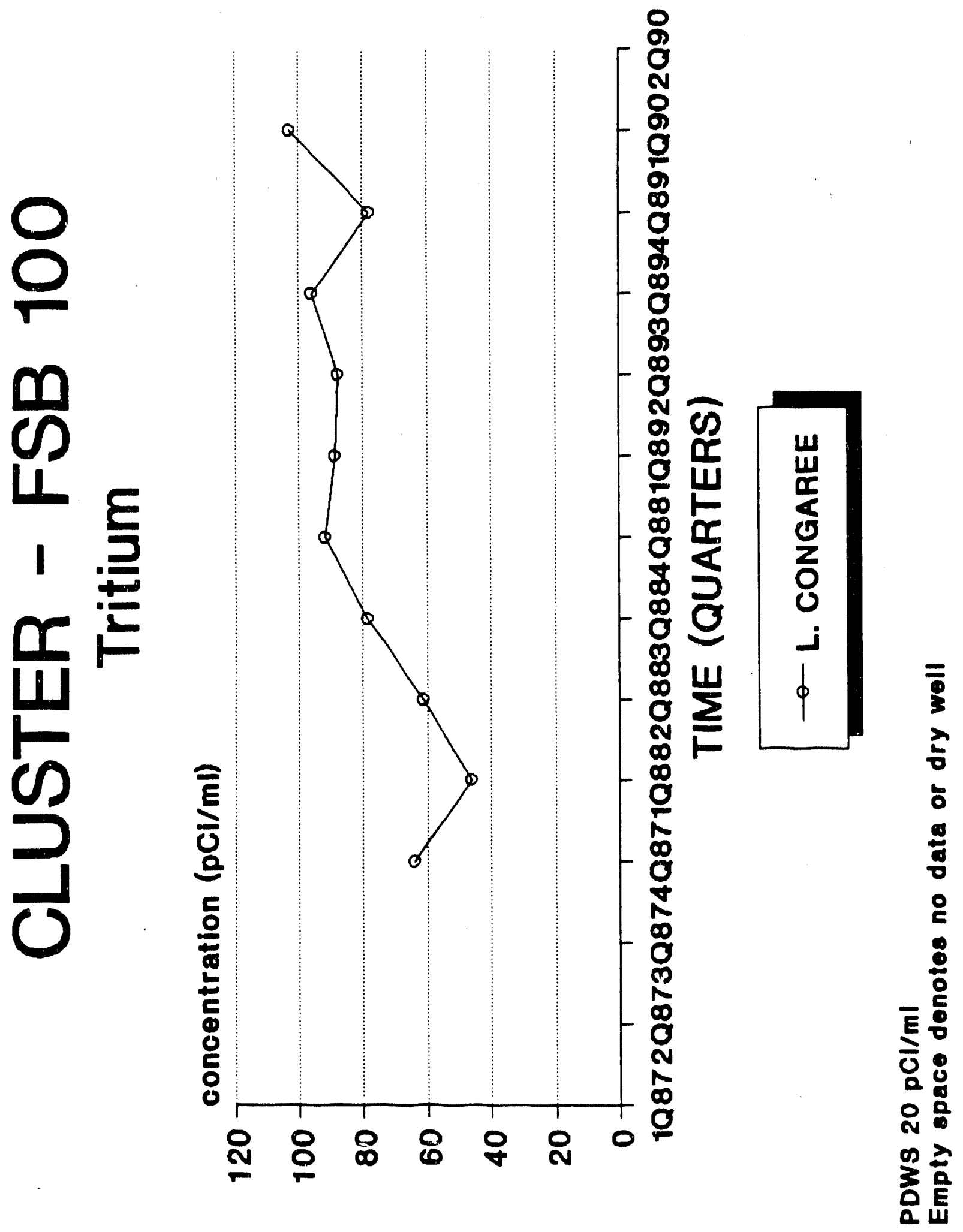




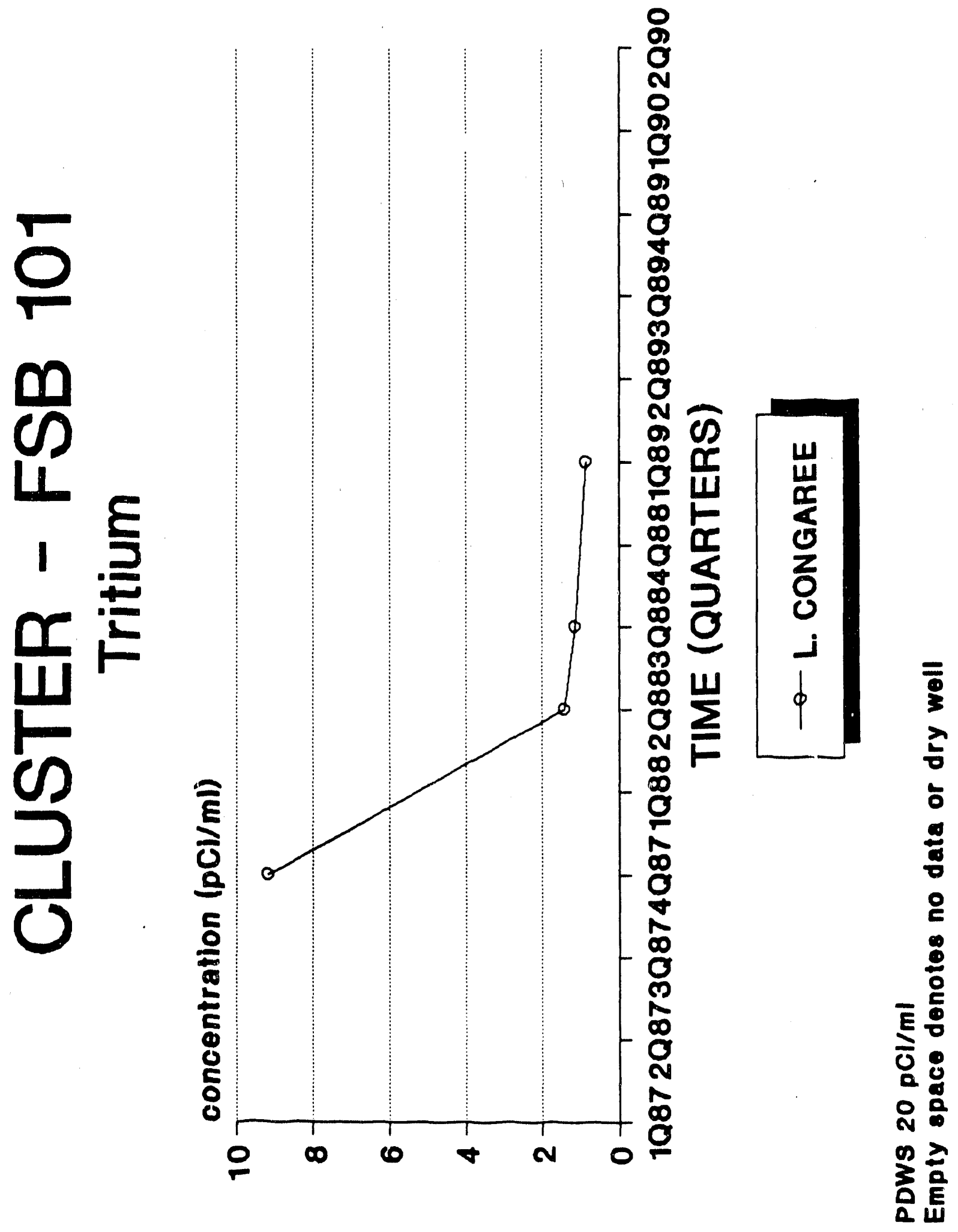




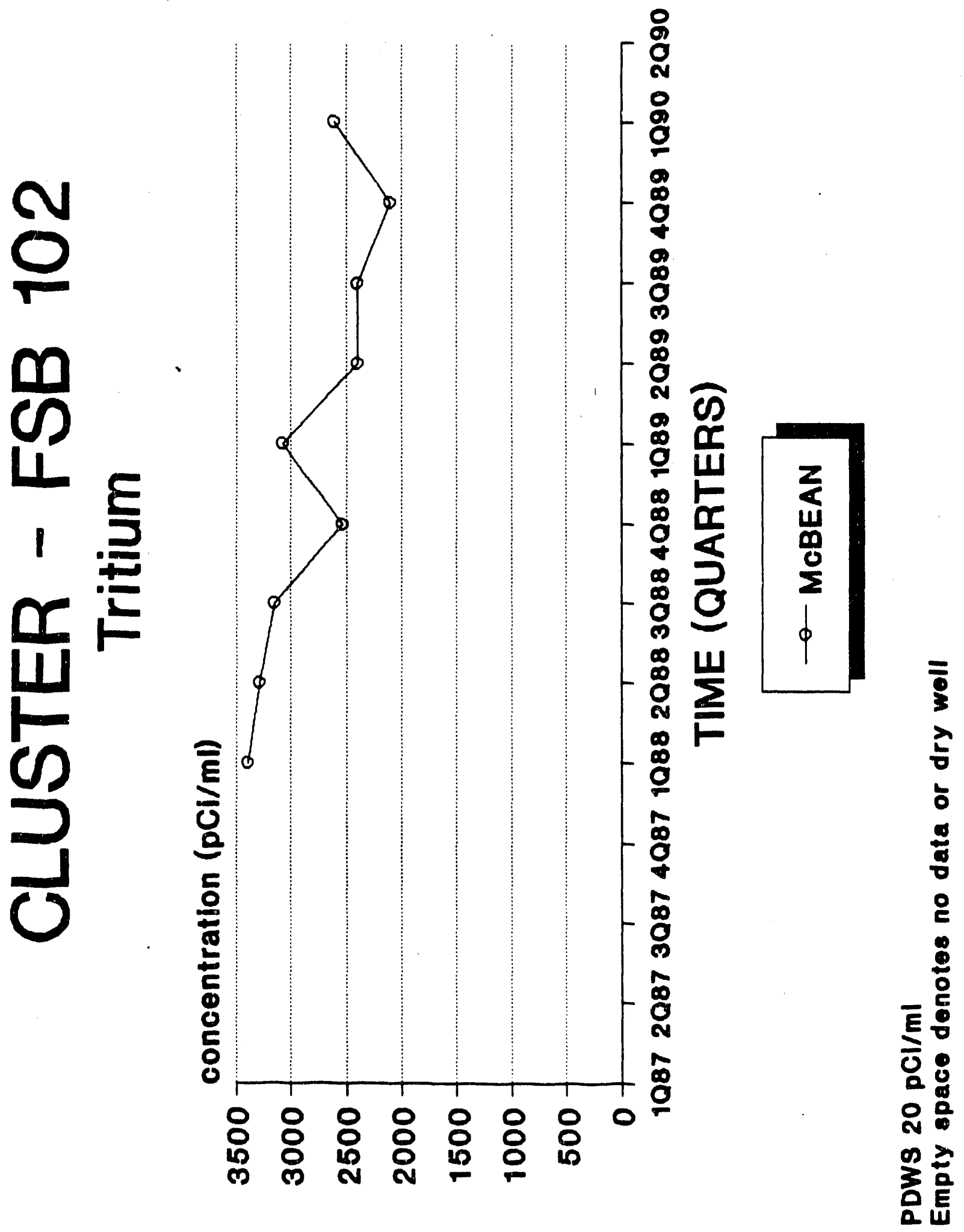




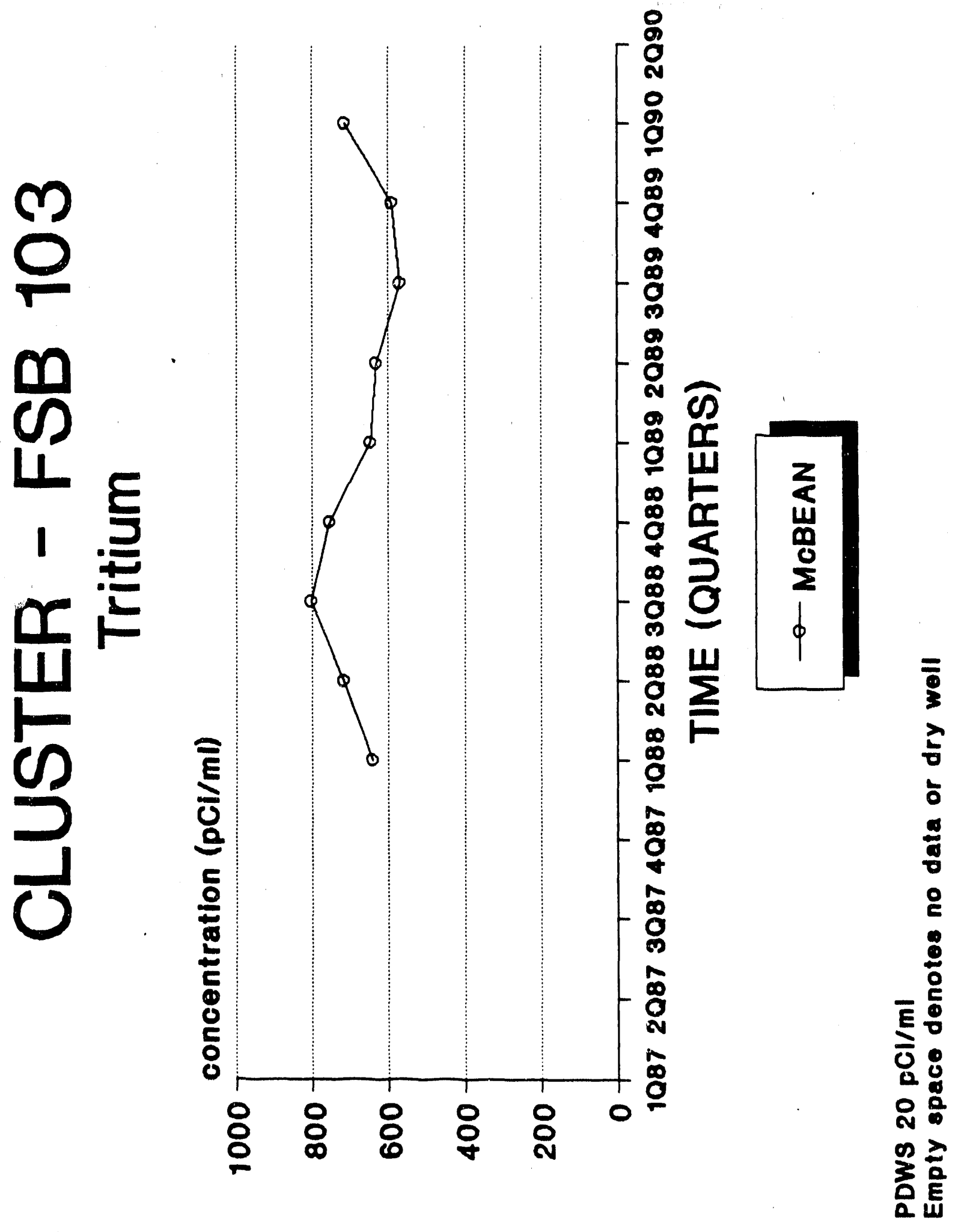




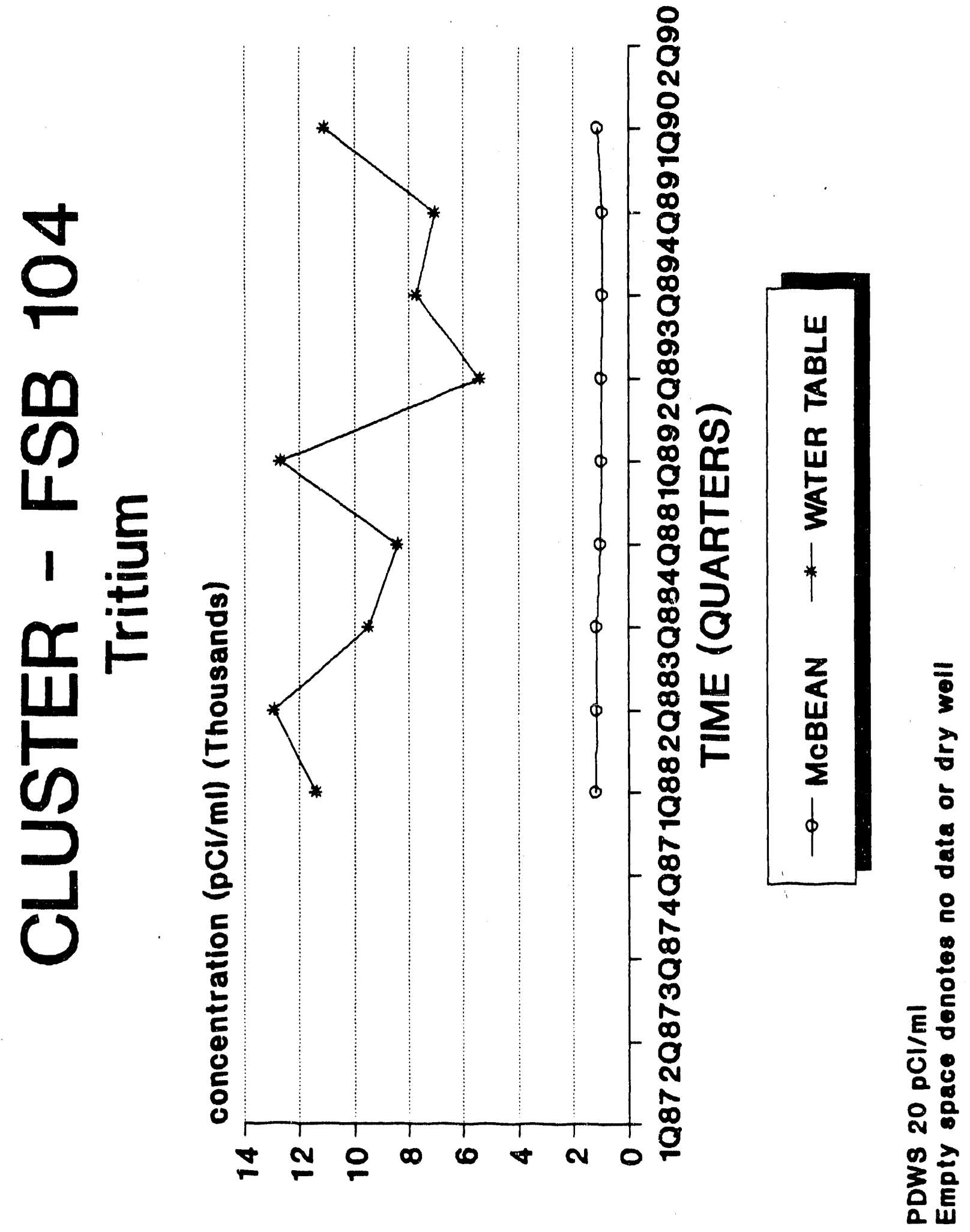




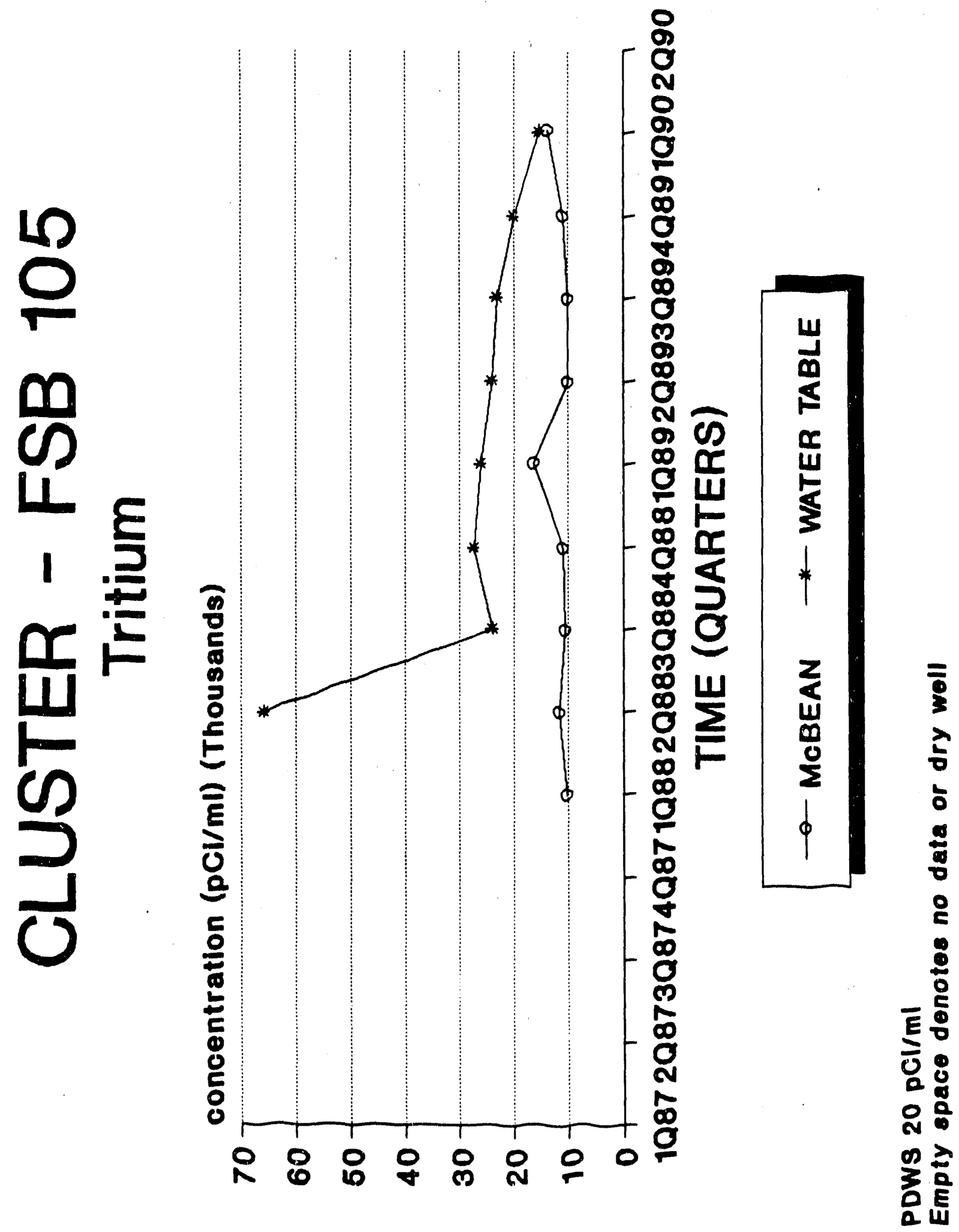




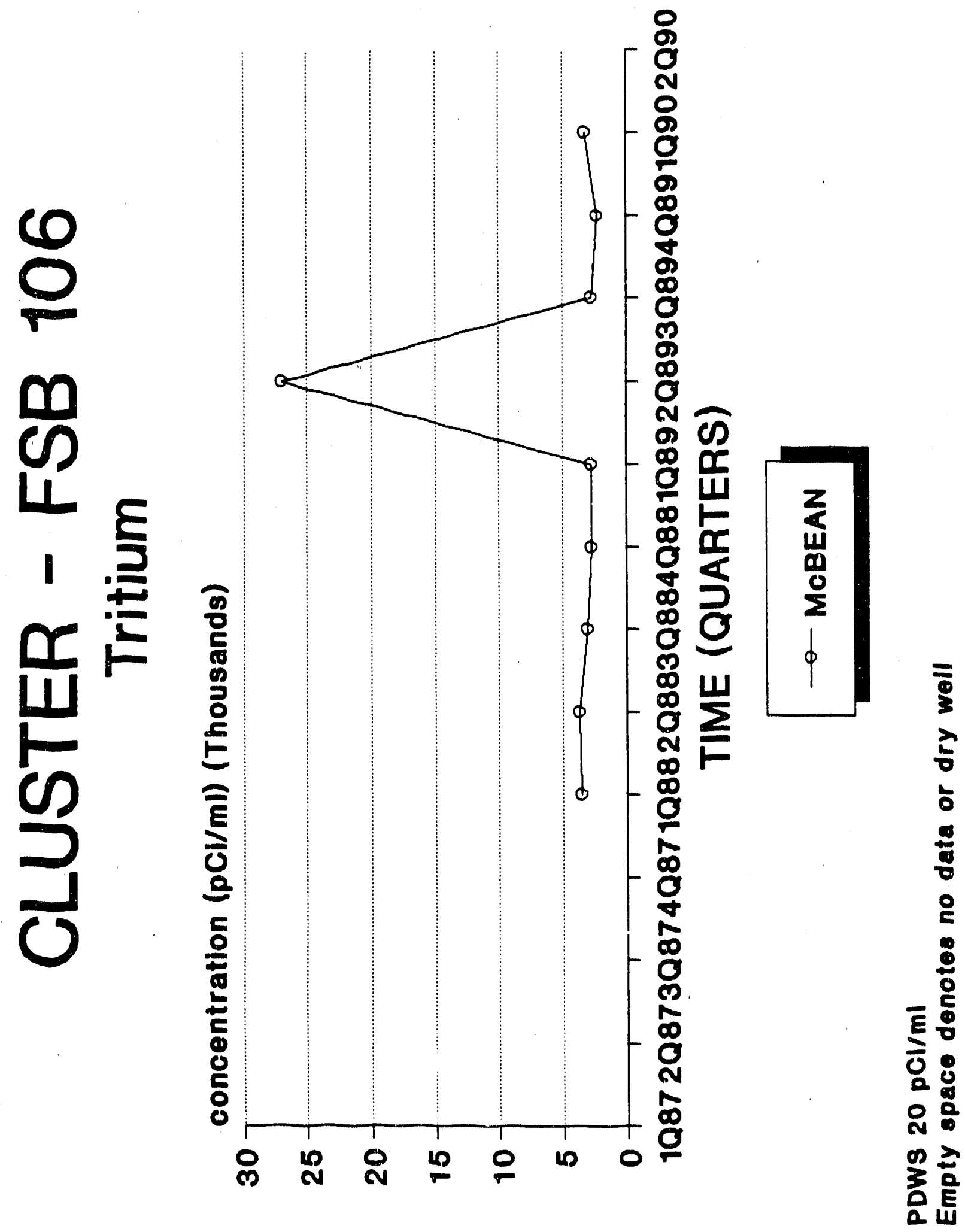




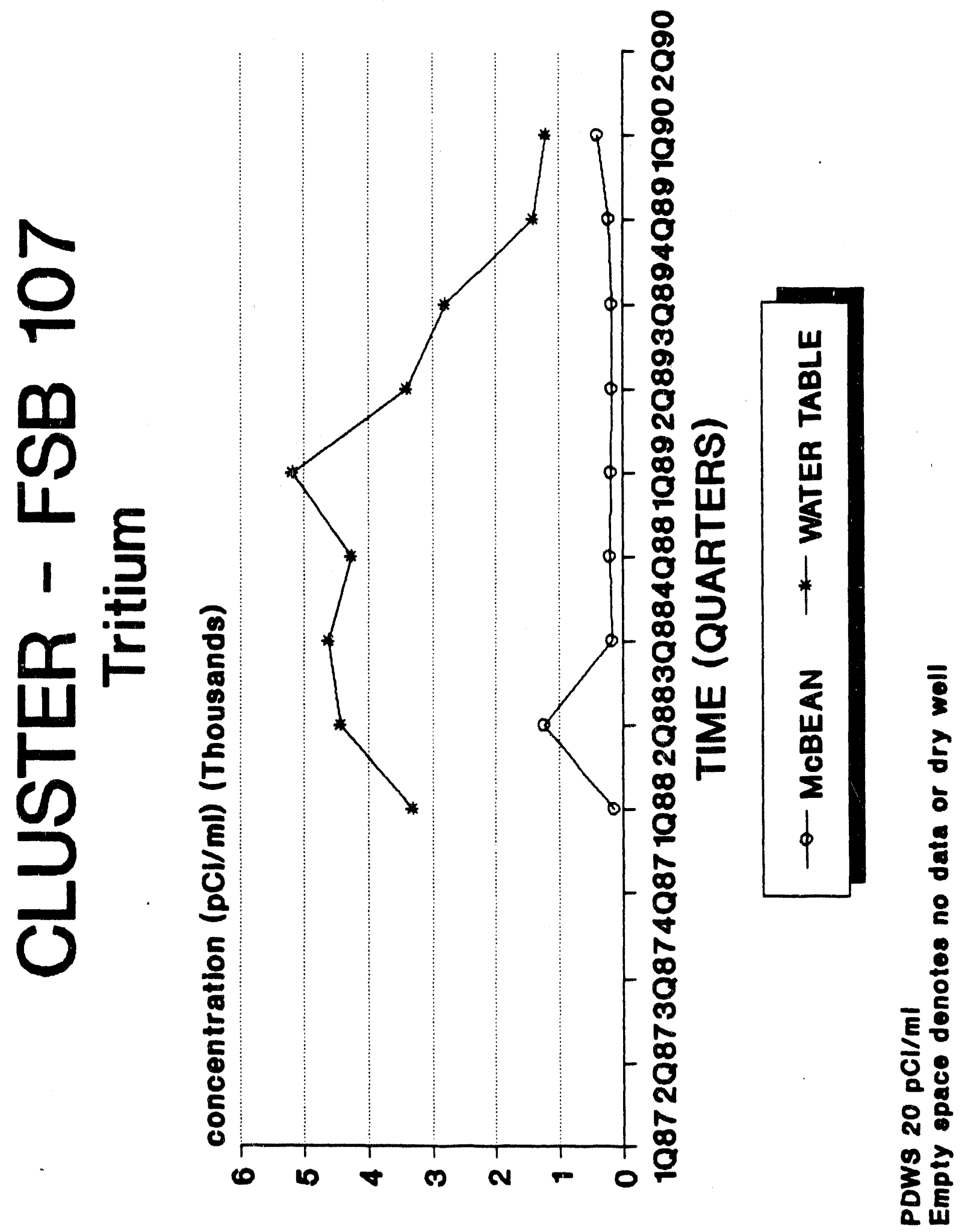




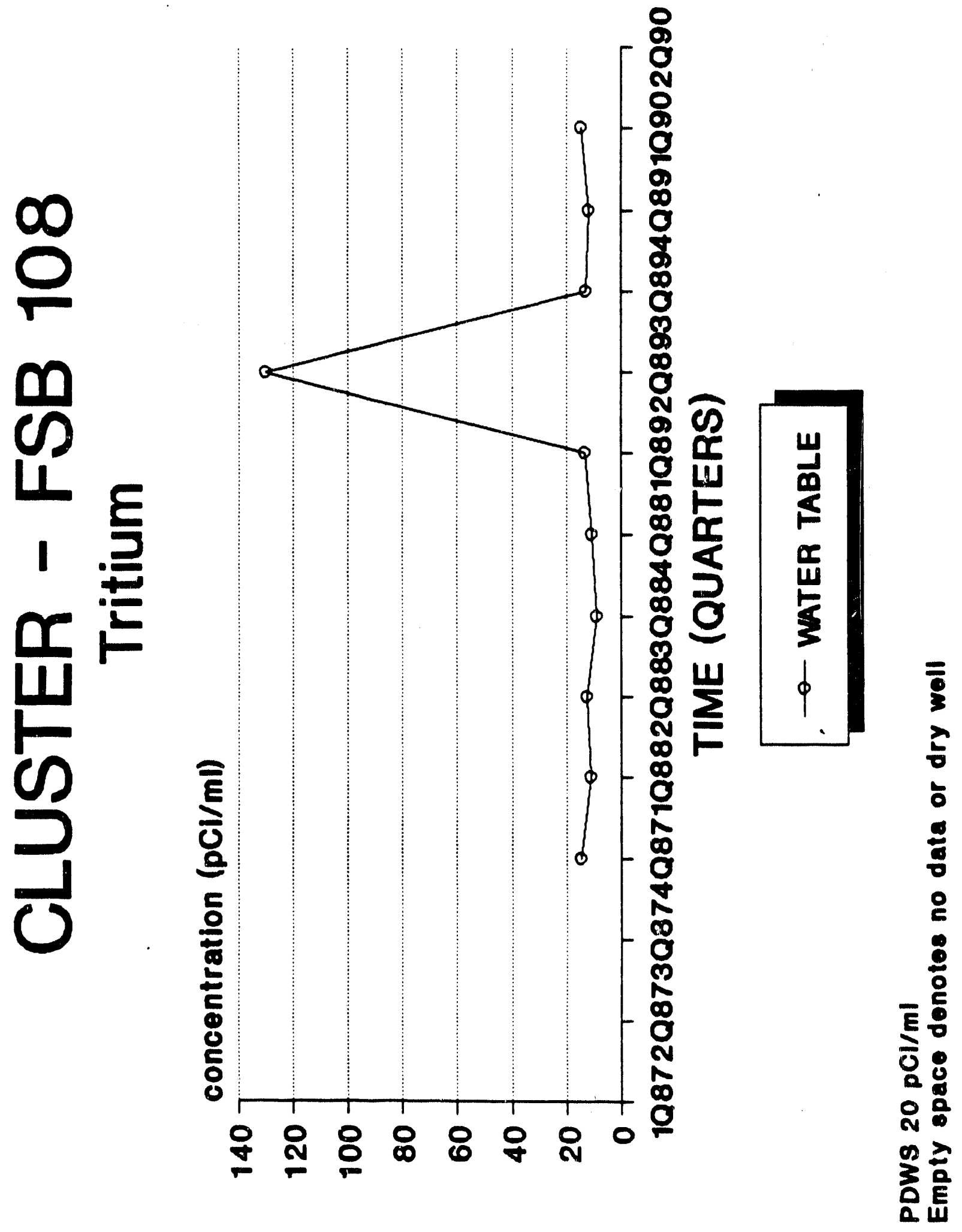




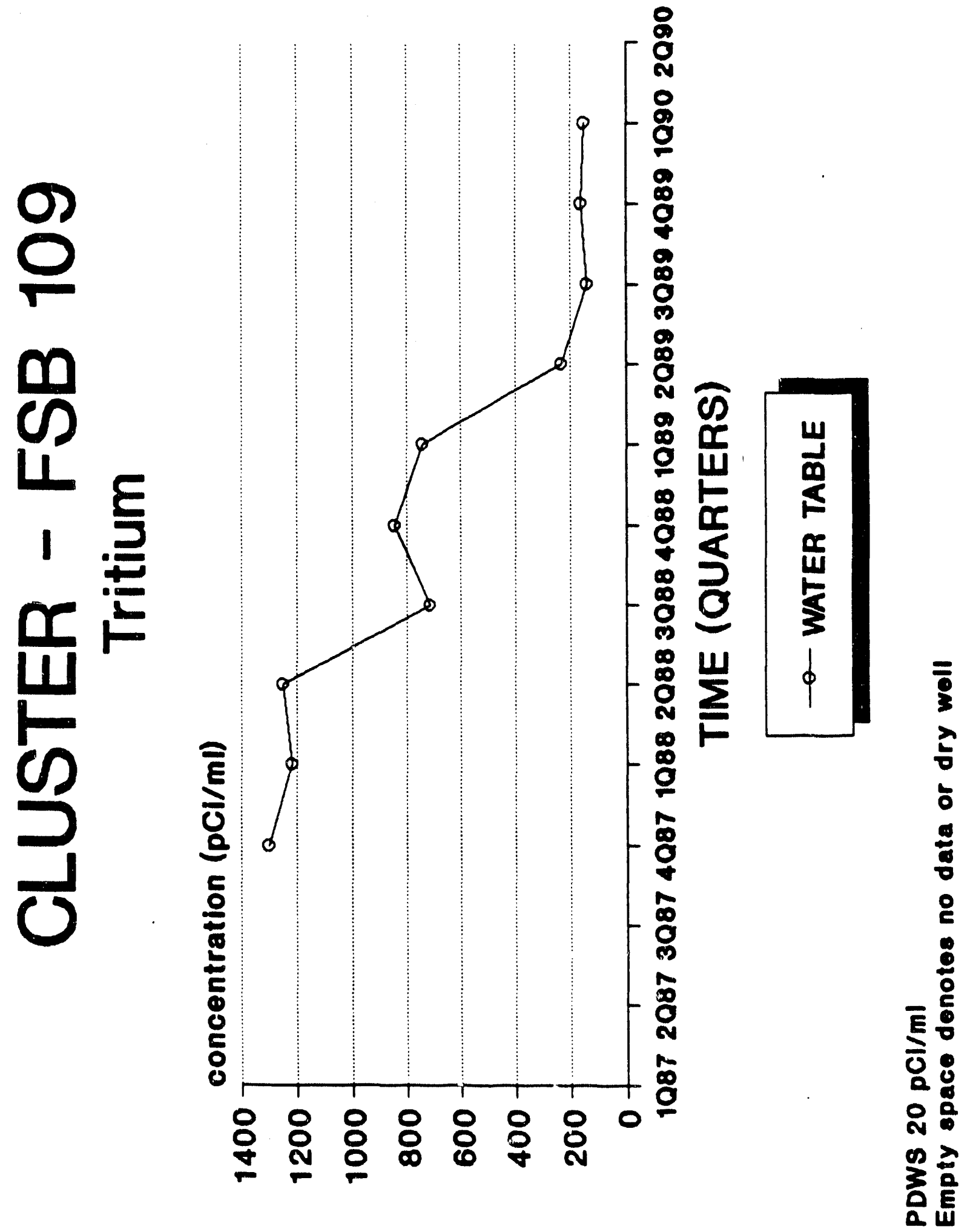




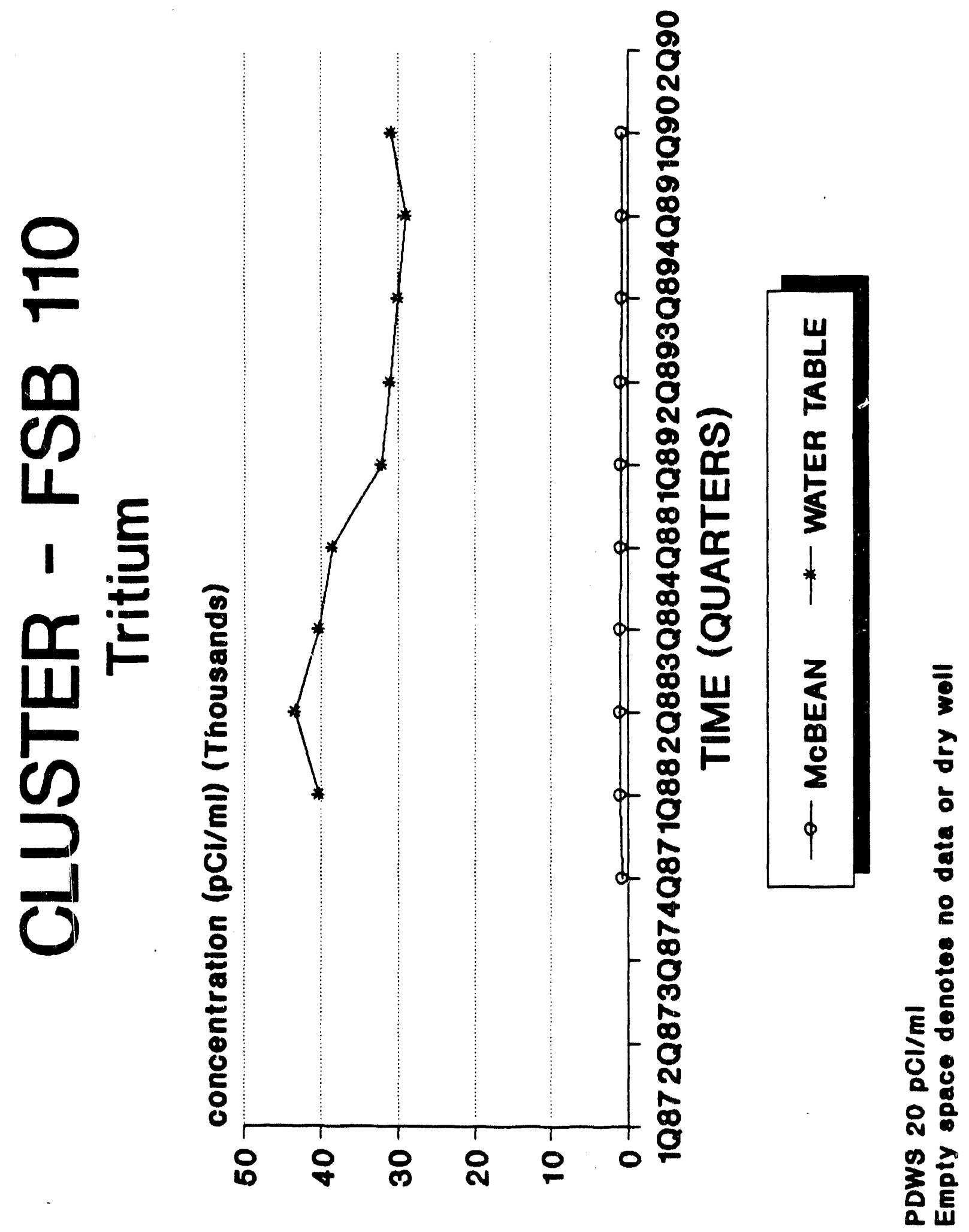




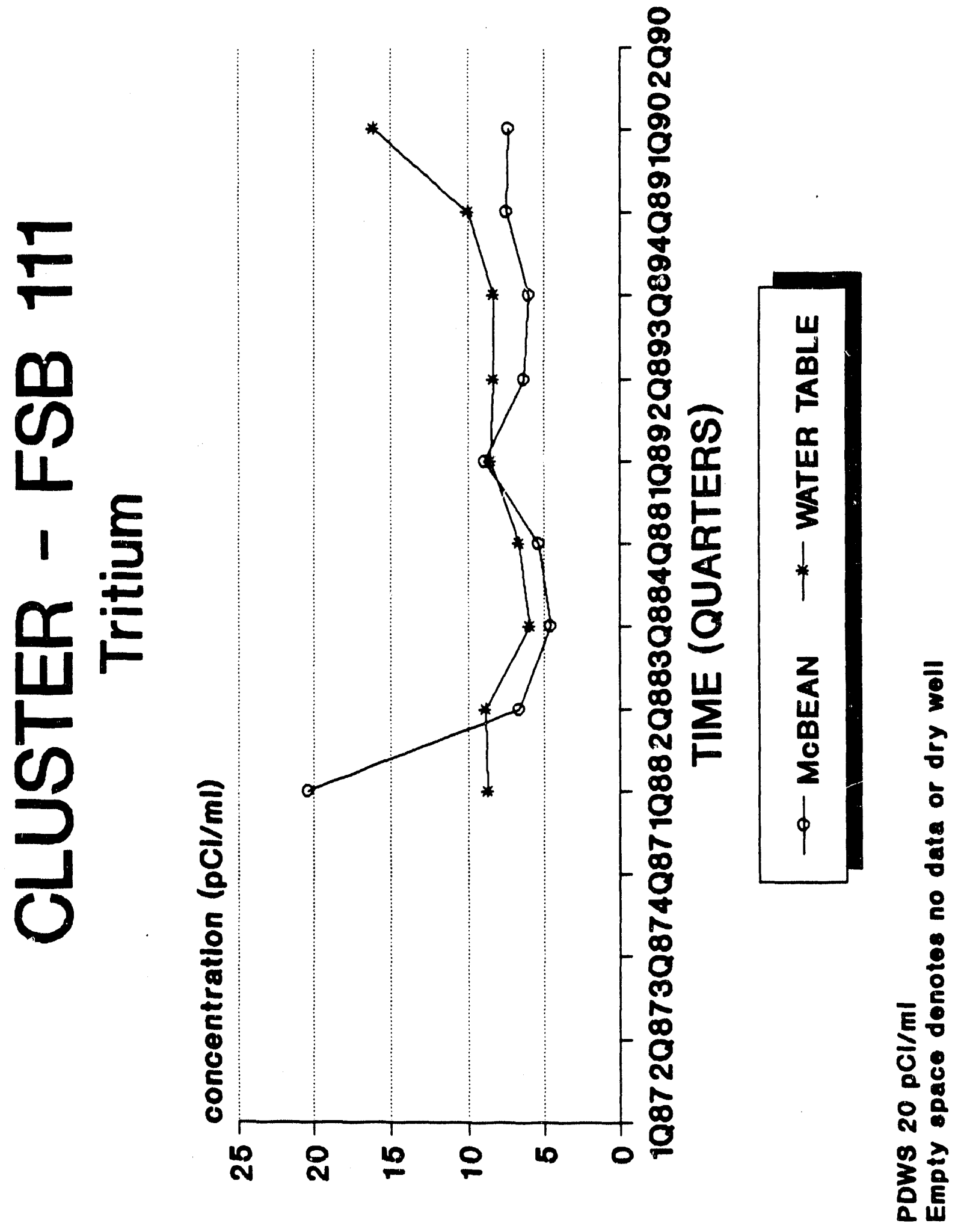


1) Seven of the wells yield sufficient water to be pumped continuously. They are FSB 99A, FSB 110C, HSB 104C, HSB 129A, HSB 120A, HSB $121 A$ and HSB 123A. These wells will be pumped continuously, using the sampling pump, for a period not to exceed 30 hours, or until the pH drops below 8.0 and stabilizes.

2) The remaining eighteen wells do not yield enough water to be continuously pumped. The pumps in these wells will have to be pulled and development methods such as swabbing and airiffting will be employed. This will proceed for a period not to exceed thirty hours or until the $\mathrm{pH}$ drops below 8.0 and stabilizes.

There are two water table welle (FSB 87D and HSB 102D) that appear to be screened correctly in relation to the water table but no water level measurements are available because the water level pipes are dry. The depths of the water level pipes in these two wells need to be checked and possibly adjusted.

FSB 94D appears to be screened correctly in relation to the water table but it is chronically reported as dry. The pump setting and the water level pipe setting in this well both need to be checked and possibly adjusted.

PHASE II - All of the wells that are not successfully rehabilitated in phase $I$, will have to be replaced. At the conclusion of Phase I, those wells will be identified and guidance will be requested from the SCDHEC on the exact locations of the replacement wells.

In addition to the Phase I failures, there are five water table wells that are screened too high and need to be abandoned and replaced. They are FSB 95D, FSB 105D, BGO 10D, BGO 13D, and BGO 14D.

There are three Congaree wells (BGO 10A, BGO 12A and HSB 124A) that exhibit anomalous water levels and high $\mathrm{pH}$ and they will require replacement. Further development has already been attempted with HSB $124 \mathrm{~A}$ and it was unsuccessful. The replacement of HSB 124A should include the abandonment of the problem well. BGO 10A and BGO 12A are thought to be screened in the base of the aquitard that separates the Congaree from the McBean. If $\mathrm{pH}$ in these two wells can be brought down and stabilized, they should remain in place and new wells that are screened in the deeper, more permeable part of the Congaree should be installed close by 
A more detailed scope concerning the well replacements can be written at the conclusion of Phase I.

\section{RESPONSIBIIITITES}

P. W. Albenesius of Waste Management Technology (7-8981) is the project coordinator. J. I. Janseen of Health Protection (5-7648) is responsible for scheduling and implementing this plan. The coneultant ueed as technical oversight is responsible for overseeing the field work and for providing a written report to Waste Managenent, detailing both phases of work. The HP driliing contractor is rasponsible for developing the wells in an efficient and effective manner and for replacing the problem wells with high quality wells in accordance with DPSOP 254. 
ATTACRIENT 1

WEIL REHABILITATION PLAN

The program outlined in the attached plan was implemented in early october of 1989. It was estimated that the rehabilitation phase would be completed by the end of the 1989 calendar year. However, the rehabilitation phase has been pushed into the 1990 calendar year and to date the plan is now completed for the $F / H$ seepage Basins but not for the Mixed Waste Management Facility (MWMF). Post closure activity has hindered the rehabilitation plan from being completed on some of the wells in the MWM. Wells that need replacing will have been identified and a program plan detailing the procedure and schedule for well replacements, will be issued (attachment 2). 
BCOPE or nORX

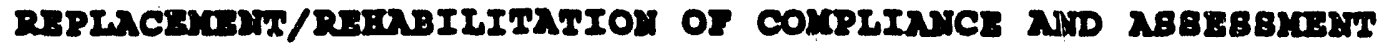

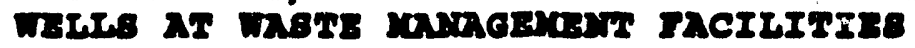

\section{INTRODUCTION}

Thirty six of the compliance and assessment wells around the $F$ and $H$ area seepage basins and the Mixed waste Management Facility, need to be replaced or rehabilitated, to be in compliance with RCRA requirements. The well names and explanations of the specific problems follow:

Five of the wells are water table wells that are screened above the water table. They are, FSB 95D, FSB 105D, BGO 10D, BGO 13D, and BGO 14D.

Twenty five of the wells have exhibited high pH through four quarters of monitoring. They are: FSB 94C, FSB 95C, FSB 96A, FSB 98A, FSB 98D, FSB 99A, FSB 100A, FSB 110C, HSB 104C HSB 115C, HSB 119A, HSB 120A, HSB 121A, HSB 123A, HSB 127C, HSB 133C, BGO 8A, BGO 10C, BCO 12C, BGO 14A, BGO 14C, BGO $16 A, B G O 16 D$, and $B G O 24 D$.

Three of the wells need to have the pump depth and/or depth of water level measuring pipe checked, to see if they are set correctly. They are FSB 87D, FSB 94D and HSB 102D.

Three of the wells exhibit both high $\mathrm{pH}$ and anomalous water levels. They are $B G O$ 10A, BGO 12A, and HSB $124 \mathrm{~A}$.

\section{OBJECTIVE}

The objective of this program plan is to rehabilitate those wells that have thus far not yielded representative groundwater samples by rigorous and conscientious well development, and to replace those wells that prove to be beyond rehabilitation, with properly installed, high quality ground water monitoring wells.

\section{PROCEDURE}

Replacement/rehabilitation of the wells will be conducted in two phases:

PHASE 1 - The twenty five welle that exhibit chronically high pH will require further well development This will be accomplished in one of two ways: 


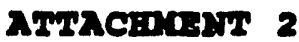

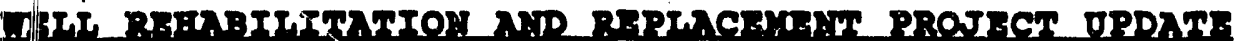

STATUS DF CONPI,IANCE WEIT, REHABIITTATION PROJECT

The profject to rehabilitate some of the compliance wells at the Mixed Waste Management Facility and the $F$ and $H$ area seepage basins, has been underway since Oct. $1,1989$.

\section{SUCCESSES}

Rehablilitation in the $F$ and $H$ Area Seepage Basins has been completed. Rehabilitation in the Mixed Waste Management Facility (WWMF) is approximately ninety percent comp' te. Access problems due to closure activity is slowing progress to have $B C$ O 12A, 14A, 14C, 16D, and 24D redeveloped. The Old Burial Ground wells are now being assessed as to which ones are to be considered problem wells.

\section{FATLURIAS}

Attempts to lower the $\mathrm{pH}$ in FSB $98 \mathrm{~A}$ were unsuccessful and it will be replaced in Phase II of the project.

\section{STATUS OF COMPILIANCE WELT REPIACFYENT PROJECT}

Those wells which were not rehabilitated are slated for replaciement. The replacement project has approval and the project to replace some of the compliance wells at the $F$ and $H$ Area Seepage Basins is nearing start date.

Wells that will be abandoned and replaced are HSB 124A, FSB 150D, 98A, 95C, 95D, 94D, BGO 8A, 10A, 10D, 12C, 13D, 14D, 16A, and 22D. This project is scheduled to start in the near future.

\section{PATH FORWARD}

of the original twenty seven wells requiring rehabilitation there are now five. The number of wells requiring replacement has grown from eight to eleven. Phase II (the replacement of bad wells) can be implemented soon, now that we have identified a large enough number of deficient wells to keep the project rolling. 

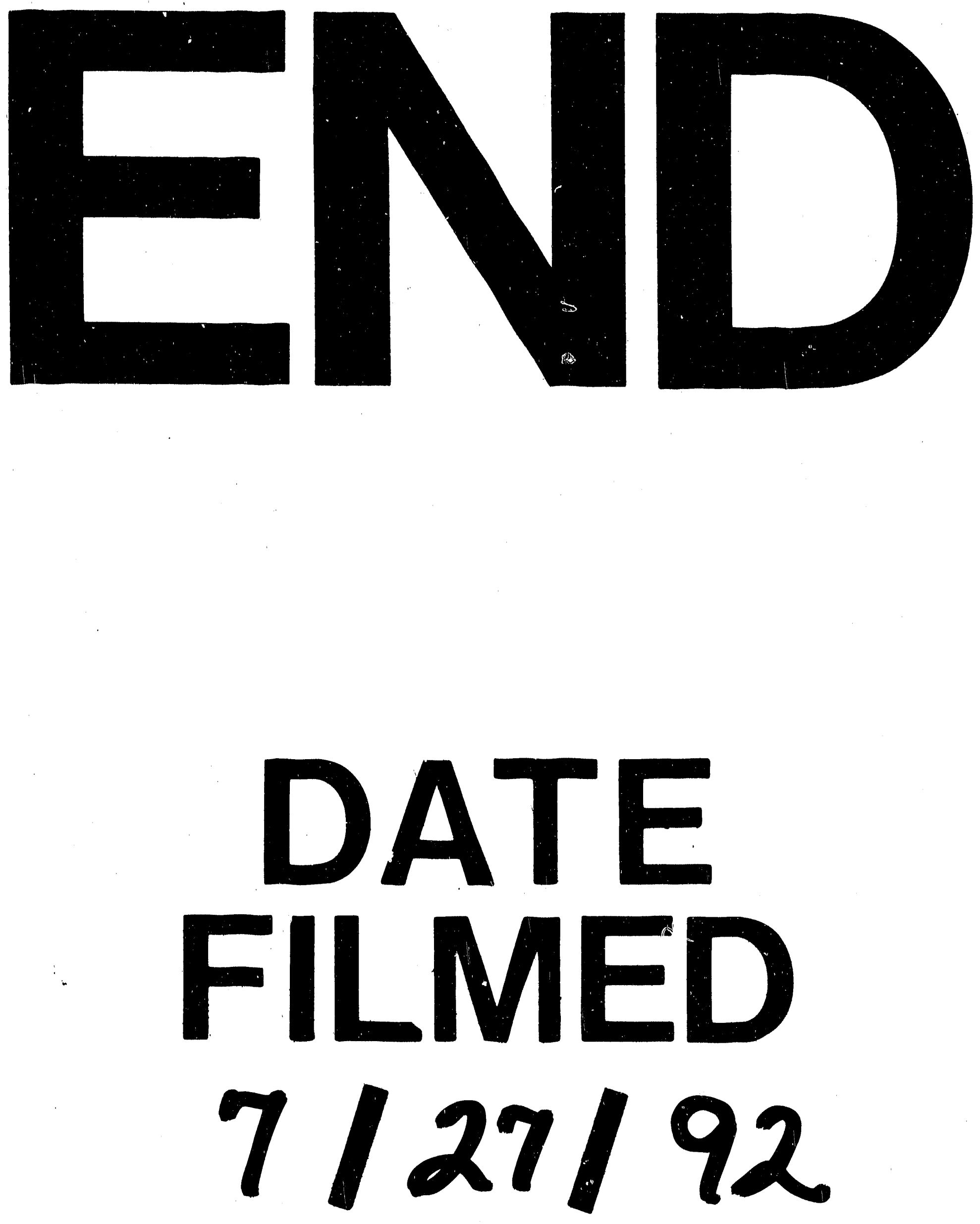
